Florida International University FIU Digital Commons

\title{
Protein-Ligand Interactions and Allosteric Regulation of Activity in DREAM Protein
}

Walter G. Gonzalez

Florida International University, wgonz002@fiu.edu

DOI: $10.25148 /$ etd.FIDC000223

Follow this and additional works at: https://digitalcommons.fiu.edu/etd

Part of the Biochemistry Commons, Biophysics Commons, Molecular Biology Commons, and the Structural Biology Commons

\section{Recommended Citation}

Gonzalez, Walter G., "Protein-Ligand Interactions and Allosteric Regulation of Activity in DREAM Protein" (2016). FIU Electronic Theses and Dissertations. 2503.

https://digitalcommons.fiu.edu/etd/2503

This work is brought to you for free and open access by the University Graduate School at FIU Digital Commons. It has been accepted for inclusion in FIU Electronic Theses and Dissertations by an authorized administrator of FIU Digital Commons. For more information, please contact dcc@fiu.edu. 


\section{FLORIDA INTERNATIONAL UNIVERSITY}

Miami, Florida

\section{PROTEIN-LIGAND INTERACTIONS AND ALLOSTERIC REGULATION OF ACTIVITY IN DREAM PROTEIN}

A dissertation submitted in partial fulfillment of the requirements for the degree of DOCTOR OF PHILOSOPHY

in CHEMISTRY

by

Walter Gabriel Gonzalez 
To: Dean Michael R. Heithaus

College of Arts, Sciences and Education

This dissertation, written by Walter Gabriel Gonzalez, and entitled Protein-Ligand Interactions and Allosteric Regulation of Activity in DREAM Protein, having been approved in respect to style and intellectual content, is referred to you for judgment.

We have read this dissertation and recommend that it be approved.

Stanislaw Wnuk

Xiaotang Wang

Yuan Liu

Prem Chapagain

Jaroslava Miksovska, Major Professor

Date of Defense: March 23, 2016

The dissertation of Walter Gabriel Gonzalez is approved.

Dean Michael R. Heithaus

College of Arts, Sciences and Education

Andrés G. Gil

Vice President for Research and Economic Development and Dean of the University Graduate School

Florida International University, 2016 
(C) Copyright 2016 by Walter Gabriel Gonzalez

All rights reserved. 


\section{ACKNOWLEDGMENTS}

I would like to express my gratitude towards all the people who have worked with me throughout the last five years, including the numerous undergraduate students, visiting students, and high school students who have made significant contributions to the work presented here. In particular, I would like to thank Andres Arango, Victoria Ramos, and Joseph Hernandez, who have contributed to published and unpublished results. I am also especially indebted to the work done by Maurizio Diaz throughout the last two years, during which he has helped me to generate and characterize all the protein mutants presented in this work. I would like to also thank members of the lab (Khoa Pham, Luisana Astudillo, David Butcher, Antonija Tangar, and Samiol Azam) for the occasional discussions and for making the laboratory an enjoyable place to work.

I am also very grateful to have had the opportunity to work with my major Professor, Dr. Jaroslava Miksovska, and to have had the privilege of working in her laboratory. I greatly appreciate the trust she often places in me and for allowing me to carry such a large array of experiment, even when in disagreement about the relevance of such experiment. I also appreciate the encouraging support of Dr. Randy Larsen from the University of South Florida and Dr. David Jameson from the University of Hawaii for their encouraging and motivational discussions at conferences and workshops.

Last but not least, I would like to thank all the professors and students of the groups with whom I have had the privilege of collaborating; and I apologize for not acknowledging them explicitly. I also would like to thank my committee members, Dr. Prem Chapagain, Dr. Stanislaw Wnuk, Dr. Xiaotang Wang, and Dr. Yuan Liu, for their time and for any critiques they may have of my work. I am very grateful and highly appreciate the financial 
support of the FIU MBRS RISE program at FIU (NIH/NIGMS R25 GM061347) for providing me with a research fellowship and research funding throughout the last three years. The content presented here is solely the responsibility of the author and does not necessarily represent the official views of the National Institutes of Health. 
ABSTRACT OF THE DISSERTATION

PROTEIN-LIGAND INTERACTIONS AND ALLOSTERIC REGULATION OF

ACTIVITY IN DREAM PROTEIN

\author{
by \\ Walter Gabriel Gonzalez
}

Florida International University, 2016

Miami, Florida

Professor Jaroslava Miksovska, Major Professor

Downstream regulatory antagonist modulator (DREAM) is a calcium sensing protein that co-assembles with $\mathrm{K}_{\mathrm{v}} 4$ potassium channels to regulate ion currents as well as with DNA in the nucleus, where it regulates gene expression. The interaction of DREAM with A-type $\mathrm{K}_{\mathrm{V}} 4$ channels and DNA has been shown to regulate neuronal signaling, pain sensing, and memory retention. The role of DREAM in modulation of pain, onset of Alzheimer's disease, and cardiac pacemaking has set this protein as a novel therapeutic target. Moreover, previous results have shown a $\mathrm{Ca}^{2+}$ dependent interaction between DREAM and $\mathrm{K}_{\mathrm{V}} 4 / \mathrm{DNA}$ involving surface contacts at the N-terminus of DREAM. However, the mechanisms by which $\mathrm{Ca}^{2+}$ binding at the C-terminus of DREAM induces structural changes at the $\mathrm{C}$ - and $\mathrm{N}$-terminus remain unknown. Here, we present the use of biophysics and biochemistry techniques in order to map the interactions of DREAM and numerous small synthetic ligands as well as $\mathrm{K}_{\mathrm{V}}$ channels. We further demonstrate that a highly conserved network of aromatic residues spanning the C- and N-terminus domains control protein dynamics and the pathways of signal transduction on DREAM. Using molecular dynamics simulations, site directed mutagenesis, and fluorescence spectroscopy 
we provide strong evidence in support of a highly dynamic mechanism of signal transduction and regulation. A set of aromatic amino acids including Trp169, Phe171, Tyr174, Phe218, Phe235, Phe219, and Phe252 are identified to form a dynamic network involved in propagation of $\mathrm{Ca}^{2+}$ induced structural changes. These amino acids form a hydrophobic network connecting the $\mathrm{N}$ - and C-terminus domains of DREAM and are well conserved in other neuronal calcium sensors. In addition, we show evidence in support of a mechanism in which $\mathrm{Ca}^{2+}$ signals are propagated towards the $\mathrm{N}$-terminus and ultimately lead to the rearrangement of the inactive EF-hand 1. The observed structural motions provide a novel mechanism involved in control of the calcium dependent $\mathrm{K}_{\mathrm{v}} 4$ and DNA binding. Altogether, we provide the first mechanism of intramolecular and intermolecular signal transduction in a $\mathrm{Ca}^{2+}$ binding protein of the neuronal calcium sensor family. 


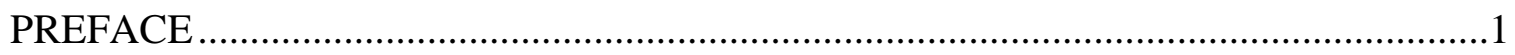

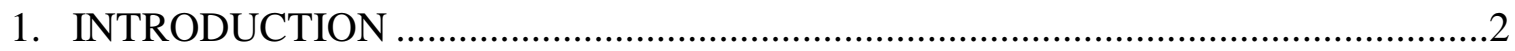

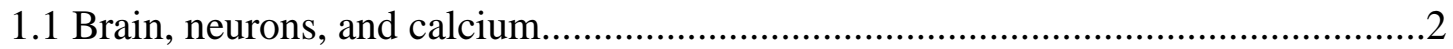

1.2 Calcium binding proteins and neuronal calcium sensors.......................................

1.3 The EF-hand $\mathrm{Ca}^{2+}$ binding motif .....................................................................

1.4 EF-hand domain organization...........................................................................18

1.5 KChIPs and DREAM................................................................................20

1.5 Objectives of this study ....................................................................................32

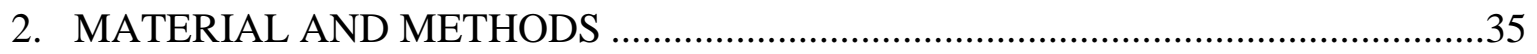

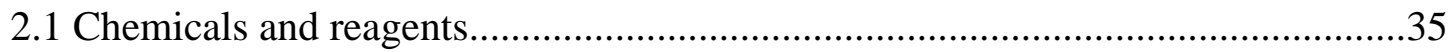

2.1 Recombinant protein expression and purification ………...................................36

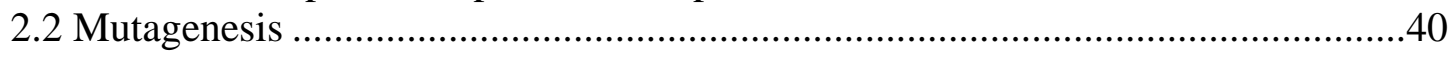

2.4 Full length DREAM expression and purification ....................................................41

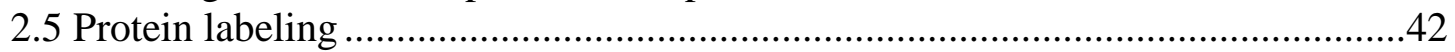

2.6 Protein sample preparation ..............................................................................4

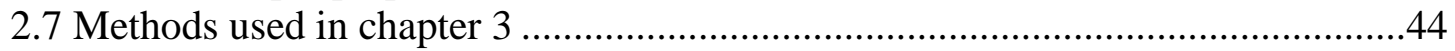

2.8 Methods used in chapter 4 ................................................................................4

2.9 Methods used in chapter 5 ............................................................................52

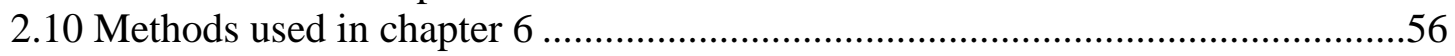

2.11 Methods used in chapter 7 .........................................................................6

2.12 Methods used in chapter 8 ............................................................................62

3. ROLE OF THE HYDROPHOBIC CAVITY AT THE C-TERMINUS DOMAIN OF DREAM IN LIGAND BINDING AND RECOGNITION ...................................71

3.1 Background and significance..............................................................................71

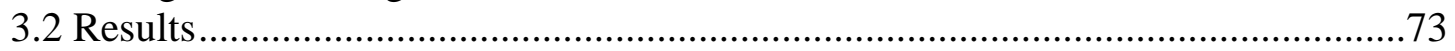

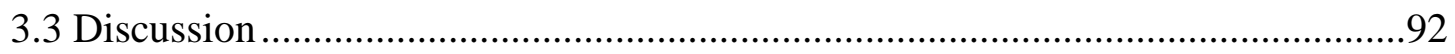

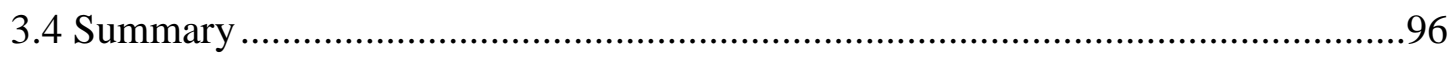

4. REGULATION OF DREAM ACTIVITY BY THE NOVEL ITO CURRENT

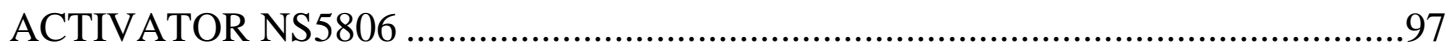

4.1 Background and significance...........................................................................

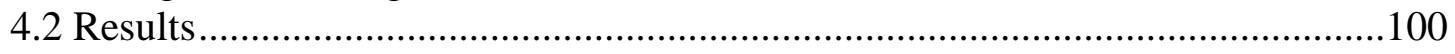

4.3 Discussion .............................................................................................116

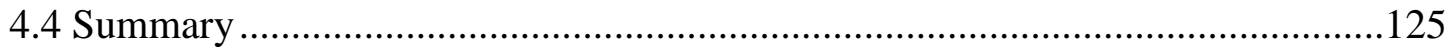

5. MODEL OF DREAM PROTEIN AND IMPLICATION IN PROTEIN AND DNA

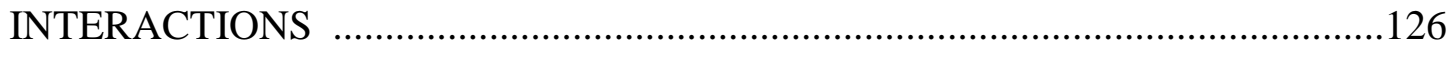

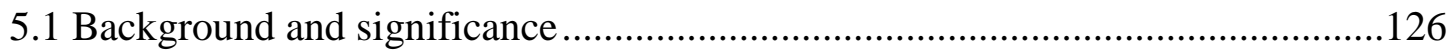




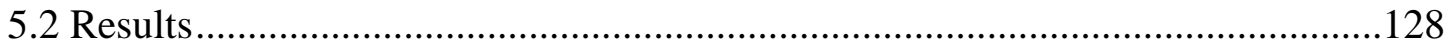

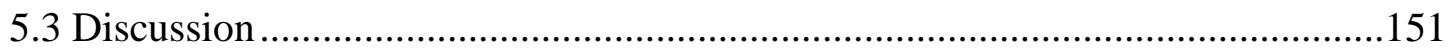

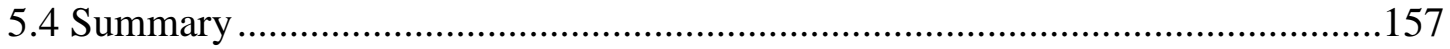

6. CHARACTERIZATION OF THE PHOTOPHYSICAL, THERMODYNAMIC AND STRUCTURAL PROPERTIES OF THE TERBIUM(III)-DREAM

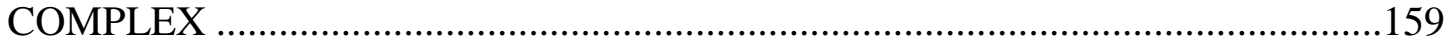

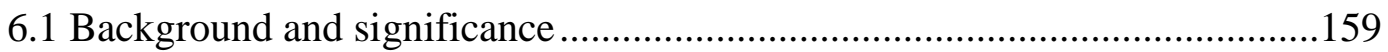

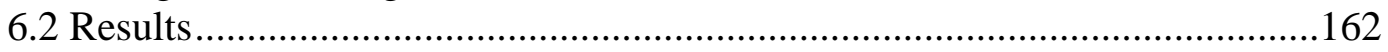

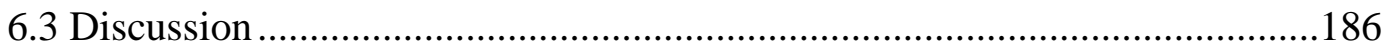

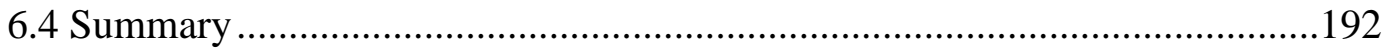

7. PHOTOTHERMAL BEAM DEFLECTION TO STUDY THE DYNAMICS OF DIVALENT ION-CAGE AND ION-PROTEIN COMPLEXES ………………......193

7.1 Background and significance .......................................................................193

7.2 Results, theory and instrumentation ............................................................196

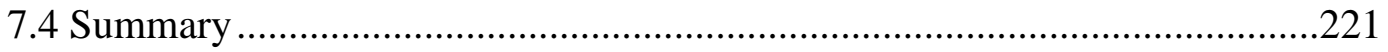

8. ROLE OF AROMATIC AMINO ACIDS IN ALLOSTERIC REGULATION OF

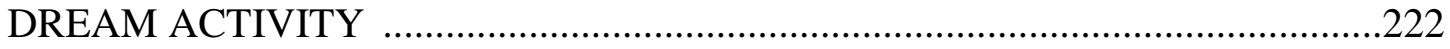

8.1 Background and significance ...............................................................222

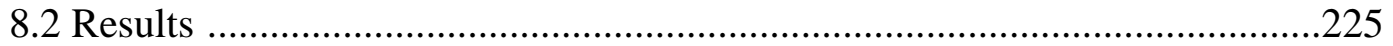

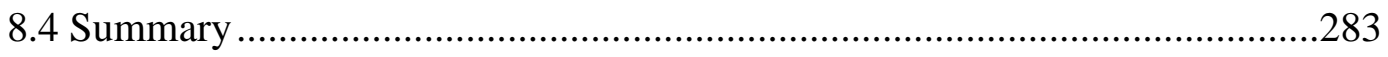

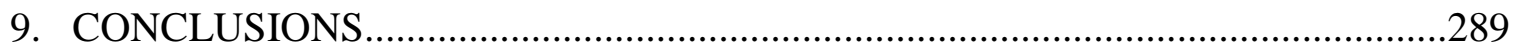

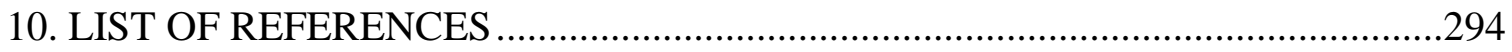

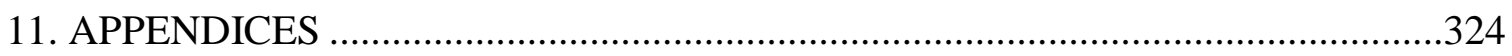

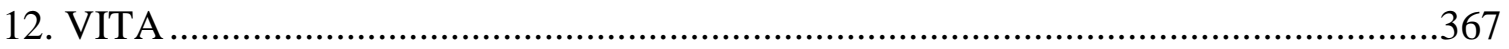




\section{LIST OF TABLES}

TABLE

PAGE

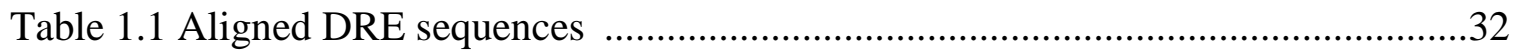

Table 2.1a List of chromophore, extinction coefficient, and manufacturer......................37

Table 2.1b List of chromophore, extinction coefficient, and manufacturer ....................38

Table 2.2 Primers used for site directed mutagenesis ..................................................40

Table 3.1 Equilibrium dissociation constants for 1,8-ANS binding to DREAM ............76

Table 3.2 Decay parameters for ANS-DREAM complexes ......................................78

Table 3.3. Anisotropy decay parameters of 1,8-ANS-DREAM $(\Delta 65)$ and 2,6-ANS$\operatorname{DREAM}(\Delta 65)$

Table 4.1 Overlap integrals and Förster distances between DREAM and NS5806 ........103

Table 4.2. Thermodynamic parameters for NS5806 binding to $\mathrm{Ca}^{2+} \mathrm{KChIP3}$ constructs determined using ITC

Table 4.3 Dissociation constant for Kv4.3(2-22) “site 1”and Kv4.3(70-90) “site 2” binding to DREAM constructs

Table 4.4. Kinetic parameter recovered for Kv4.3(2-21) “site 1” binding to DREAM constructs in the presence of NS5806

Table 5.1. Activation parameters for CaM:DRE29 and CaMRR2:DREAM dissociation

Table 5.2 Dissociation kinetics of calcium bound CaM:DRE29 and CaMRR2

DREAM complexes upon addition of EDTA as a function of temperature .141

Table 5.3. Photophysical properties of DRE29 and CaM-IAEDANS upon complex formation

Table 5.4. Anisotropy decay parameters of DRE29 and CaM-IAEDANS upon complex formation Table 1.1 Aligned DRE sequences

Table 6.1. Fluorescence and anisotropy decay parameters of $\operatorname{DREAM}(\Delta 64)$ bound to $\mathrm{Ca}^{2+}$ or $\mathrm{Tb}^{3+}$. 
Table 6.2. ITC parameters recovered for $\mathrm{Tb}^{3+}$ displacement of $\mathrm{Ca}^{2+}$ from EF hands of CaM and DREAM using a sequential model

Table 6.3. Terbium(III) luminescence decay parameters recovered upon binding to DREAM and DREAM constructs

Table 6.4. Experimental and theoretical ion-neutral collision cross section for the $\operatorname{DREAM}(\Delta 64): \mathrm{Ca}^{2+} / \mathrm{Tb}^{3+}$ molecular ions.

Table 7.1 Volume and enthalpy following photo-cleavage of caged compounds

Table 8.1 Fluorescence and anisotropy decay parameters of 1,8-ANS bound DREAMNCS1.

Table 8.2. Equilibrium dissociation constants for $\mathrm{Ca}^{2+}$ binding to DREAM 254

Table 8.3 Parameters recovered from fitting of the unfolding curves of DREAM mutants .260

Table 8.4 Parameters recovered from fitting of the unfolding curves of DREAM mutants .261

Table 8.5 Summary of observed unfolding effects on DREAM mutants .265 


\section{LIST OF FIGURES}

FIGURE

PAGE

Figure 1.1: Three dimensional structures the most common families of $\mathrm{Ca}^{2+}$ binding proteins shown as a carton representation, with calcium metal shown as tallow spheres. (a) Synaptotagmin 1 with $\mathrm{Ca}^{2+}$ bound to the C2-domain, (b) Annexin III with $\mathrm{Ca}^{2+}$ bound at the Annexin domain, and (c) calmodulin with $\mathrm{Ca}^{2+}$ bound at the the EF-hand

Figure 1.2: Three dimensional structure of a representative EF-hand motif (PDB: 2pvb). (a) Cartoon representation of the helix-loop-helix motif of the EF-hand with $\mathrm{Ca}^{2+}$ bound and shown as a yellow sphere. (b) The same structure as in (a) but shown as a licorice model, without hydrogens.

Figure 1.3. Dendrogram showing the relationship between members of the intracellular calcium binding proteins.

Figure 1.4. (left) NMR structure of $\mathrm{Ca}^{2+}$ free recoverin (PDB: 1iku, conformation 1) with EF-hand 1 shown in orange, EF-hand 2 in green, EF-hand 3 in blue, and EF-hand 4 in red. The myristoyl group is shown as VDW spheres. (Right) NMR structure of Ca2+ bound to EF-hand 2 and 3 of recoverin (PDB: 1jsa, conformation 1). Calcium is shown as yellow spheres.

Figure 1.5. (a) Crystal structure of Parvalbumin (PBD: 2pvb). Helices are shown in different colors and turn-loop involved in Ca2+ chelation is shown in licorice model. The $\mathrm{E}$ and $\mathrm{F}$ hand helices forming the EF-hand are shown in blue and red, respectively. (b) EF-hand formed by the helix $\mathrm{E}$ and $\mathrm{F}$ of the $\mathrm{Ca}^{2+}$ bound parvalbumin (PBD: 1b8r). The entering helix is colored in blue and exiting helix in red, with calcium shown as a yellow sphere.

Figure 1.6. Coordination of $\mathrm{Ca}^{2+}$ at the $\mathrm{x}, \mathrm{y}, \mathrm{z},-\mathrm{y}$, and -z by: (a) Asp73, Gln75, Asp75, Thr79, and Glu84 of EF-hand 2 in recoverin (PDB: 1jsa); (b) Asp109, Asp111, Asp113, Thr115, and Glu120 of EF-hand 3 in recoverin (PDB: 1jsa); (c) Asp131, Asp133, Asp135, His140, and Glu129 of EF-hand 3 in calmodulin (PDB: 1exr); (d) Asp93, Asp95, Gln97, Leu99, and Glu104 in EF-hand 4 of calmodulin (PDB: 1exr). 
Figure 1.7. Crystal structure of calmodulin with $\mathrm{Mg}^{2+}$ bound at EF-1 (PDB: 3ucw). Coordinating amino acid involved in metal binding are shown in licorice model. Water molecules shown as red spheres.

Figure 1.8. EF-hand pair showing the formation of a $\beta$-sheet connecting the adjacent $\mathrm{Ca}^{2+}$ binding loops (PDB: 2jul).

Figure 1.9. Reorientation of the EF-hands of calmodulin in the $\mathrm{Ca}^{2+}$ bound (PDB: 1cll) shown in solid color and $\mathrm{Ca}^{2+}$ free forms shown in diffuse color with backbone in thick cyan lines (PDB: 1cfc, conformation 1). (a) EF-hand 1, (b) EF-hand 2, (c) EF-hand 3, and

(d) EF-hand 4.

Figure 1.10. Reorientation of the EF-hands of myristoylated recoverin in the $\mathrm{Ca}^{2+}$ bound (PDB: 1iku) shown in solid color and $\mathrm{Ca}^{2+}$ free forms shown in diffuse color with backbone in in thick cyan lines (PDB: 1jsa). (a) EF-hand 2 and (b) EF-hand 3.

Figure 1.11. Reorientation of the EF-hands of myristoylated NCS-1 in the $\mathrm{Ca}^{2+}$ bound (PDB: 1fpw) shown in solid color and $\mathrm{Ca}^{2+}$ free nonmyristoylated NCS-1 shown in diffuse color with backbone in in thick cyan lines (PDB: 2l2e). (a) EF-hand 2, (b) EF-hand 3, and (c) EF-hand 4.

Figure 1.12. Domain organization of CaBPs, EF-hand pairs near the Nterminus shown in blue and near the C-terminus shown in red. Calcium is shown a yellow sphere. (a) Calbindin D9k (PDB: 3icb), (b) parvalbumin (PDB: 1b8r), (c) calmodulin (PDB: 1cll), (d) recoverin (PDB: $1 \mathrm{jsa})$.

Figure 1.13. Interdomain structural dynamics of (a) $\mathrm{Ca}^{2+}$ free CaM (PDB: 1cfc), where the C-terminus EF-hand 3 (blue) and EF-hand 4 (red) are aligned for clarity and the flexible N-terminus is shown as diffuse orange and green ribbons. (b) The NMR structure of $\mathrm{Ca}^{2+}$ bound myristoylated recoverin (PDB: $1 \mathrm{jsa}$ ). EF-hand 1,2,3, and 4 are colored in orange, green, blue, and red, respectively. The EF-hand 3 and 4 were aligned to show the flexibility of the N-terminus.

Figure 1.14. Sequence alignment of KChIP1 (panel A) and KChIP2 (panel B) for different isoforms. Identical amino acid positions shown in bold blue color. The turn-loop amino acids of each EF-hand are underlined. The position of the EF-hands are shown in colored cartoons for reference. Isoform 4 and 8 of KChIP2 not included for clarity. 
Figure 1.15. Sequence alignment of KChIP3 (panel C) and KChIP4 (panel D) for different isoforms. Identical amino acid positions shown in bold blue color. The turn-loop amino acids of each EF-hand are underlined. For EF-hand that are active, the amino acids providing the coordination to the metal ion are shown in red. The position of the EF-hands are shown in colored cartoons for reference. Isoform 4 and 8 of KChIP2 not included for clarity.

Figure 1.16. Cartoon representation of a hypothetical potassium channel macromolecular complex, dotted lines represent the transmembrane thickness. The model was constructed by structural alignment of the full length shaker potassium channel Kv1.2 (PDB: 3lut) and the crystal structure of the KChIP1-Kv4.3 T1 domain complex (PDB: 2i2r). (a) Side view: the transmembrane domain containing the $\mathrm{K}^{+}$selectivity pore is shown in green with potassium as solid purple spheres. The T1domain is shown in light blue and KChIP1 bound to $\mathrm{Ca}^{2+}$ (yellow sphere) is show in dark blue. The $\beta$ subunit is shown in orange. (b) Top vie of the same complex.

Figure 1.17. Cartoon representation of the KChIP1:Kv4.3 T1 domain protein complex (PDB: 2nz0). (a) Octameric cross shaped complex of four KChIP1 (dark blue) and four T1 domain monomers (orange). (b) Close up of the interaction between the N-terminus hydrophobic region of the T1 domain ("Site 1") and the hydrophobic surface of KChIP1. Residues 1 to 10 shown in yellow and 10 to 20 in red. (c) Contact between the Nterminus of KChIP1 and "Site 2" (residues 70 to 90,shown in green licorice) of Kv4.3.

Figure 1.18. (a) Cartoon representation of KhIP1 (PDB: 2nz0) using the same coloring scheme as in Figure 1.4 with residues forming the interface with the N-terminus of Kv4.3 shown as licorice model. (b) Alpha-helix at the $\mathrm{N}$-terminus of the $\mathrm{K}_{\mathrm{V}} 4.3$ potassium channel with hydrophobic amino acids involved in binding of KChIP1 shown as licorice model.

Figure 1.19. Cartoon representation of the second interface "Site 2" between KhIP1 and the N-terminus of Kv4.3 (PDB: 2nz0) using the same coloring scheme as in figure 1.x. The T1 domain is shown in brown. The inset show the hydrophobic (top) and electrostatic (bottom) interactions.

Figure 1.20. Sequence alignment of human KChIP1, KChIP2, KChIP3, and KChIP4. Turn loops of EF-hand 1, 2, 3, and 4 are shown in bold and underlined. the negatively charged amino acids involved in metal coordination shown in red. Asterisks indicate identical residues, semicolon indicates very similar residues, and the period is for somewhat similar residues. Amino acids involved in Kv4 binding are shown in green. 
Figure 1.21. Cartoon representation of the NMR structures of (a) $\mathrm{Ca}^{2+}$ bound mouse DREAM (first 75 amino acids not resolved, PDB: 2jul) and (b) $\mathrm{Ca}^{2+}$ bound human DREAM (first 160 amino acids not resolved, PDB: 2e6w). The helices numbers are shown for clarity, and the EF-hand 1, 2, 3, and 4 are shown in orange, green, blue, and red, respectively. Calcium is shown as a yellow sphere. (c) Amino acid sequence of mouse DREAM (residues 76 to 256) following the same coloring scheme as in figure 1.X. The cartoon representation of the secondary structure is shown.

Figure 1.22. Cartoon representation of the proposed DREAM dimer in which Leu155, Leu158, Leu159, and Leu251 are shown in licorice model with a diffuse surface. Color scheme as in figure 1.12 .

Figure 1.23. Cartoon representation of the NMR structures of (a) $\mathrm{Ca}^{2+}$ bound mouse DREAM (PDB: 2jul). Positively charged amino acids proposed to mediate DNA binding are shown in licorice and transparent blue surface.

Figure 2.1. Amino acid sequence of plasmid purchased. The mouse DREAM, $\operatorname{DREAM}(\Delta 65)$, and $\operatorname{DREAM}(\Delta 160)$ plasmids contain a start methionine residue (verified by mass spectrometry) and a Cterminus His-tag (blue) connected by tripeptide linker (green). The DREAM $(\Delta 65, C 4)$ plasmid contains an N-terminus His-tag (blue) and a C-terminus tetracysteine tag. The DREAM-NCS chimera plasmid is identical to the $\operatorname{DREAM}(\Delta 65)$ plasmid but residues Leu158, Leu159 and the linker between EF-hand 3 and 4 were replaced by the amino acid sequence of human NCS-1 (shown in red). Lastly, a GST-Kv4.3 T1 domain fusion construct was generated (GST in red). The enterokinase cleavage site shown in red and underlined. The T1-domain of human Kv4.3 residues 2 to 152 shown in black. The biarsenical tag is shown in underlined red color.

Figure 2.2. Increase in fluorescence intensity of FlAsH-EDT 2 and ReAsH$\mathrm{EDT}_{2}$ upon specific covalent attachment to a small CCPGCC peptide. Green trace functions as a control to show that there is no direct interaction of the biarsenical probe and DREAM, even though 5 cysteine are present in this protein.

Figure 3.1. Normalized fluorescence emission spectra of 1,8-ANS (top panel) and 2,6-ANS (bottom panel) bound to DREAM. Conditions: $40 \mu \mathrm{M}$ DREAM in $20 \mathrm{mM}$ Tris $\mathrm{pH} 7.4$ and $1 \mathrm{mM}$ DTT. The excitation wavelength was $350 \mathrm{~nm}$ and $319 \mathrm{~nm}$ for 1,8-ANS and 2,6-ANS, respectively. 
Figure 3.2 Calcium titration to $7 \mu \mathrm{M}$ DREAM in the presence of $10 \mu \mathrm{M} 1,8$ ANS. Calcium titration into $\mathrm{Mg}^{2+}$ bound DREAM (squares) and apostate DREAM(circles). Samples were prepared in 20mM Tris $\mathrm{pH}$ 7.4 100mM NaCl 1mM DTT which was decalsified by passing through an equilibrated CHELEX resin column (BioRad) and delivered into a decalsified $1 \mathrm{~cm} x 1 \mathrm{~cm}$ quartz cuvette. The average of three trials is shown, and nonlinear fit with a Hill equation (solid lines) yields $\mathrm{K}_{\mathrm{d}}=8.0 \pm 0.9 \mu \mathrm{M}$ with a hill coefficient of $\mathrm{n}=1.22$.

Figure 3.3. Titration of $10 \mu \mathrm{M}$ 1,8-ANS by apoDREAM (squares), $\mathrm{Mg}^{2+} \mathrm{DREAM}$ (circles), $\mathrm{Ca}^{2+} \mathrm{DREAM}$ (up-triangles), and $\mathrm{Mg}^{2+} \mathrm{Ca}^{2+} \mathrm{DREAM}$ (down triangles). The solid lines correspond to the fit of experimental data.

Figure 3.4. Titration of 2,6-ANS by DREAM. ANS concentration was kept constant $(10 \mu \mathrm{M})$ and emission was collected from $405 \mathrm{~nm}$ to $600 \mathrm{~nm}$ using excitation wavelength of $319 \mathrm{~nm}$. The data were corrected for scattering as described in Material and Methods section. The solid lines correspond to the fit of experimental data using a hill equation. 76

Figure 3.5: Frequency-domain intensity decay of ANS bound to apoDREAM (squares), and $\mathrm{Mg}^{2+}$ (circles), $\mathrm{Ca}^{2+}$ (up-triangle), and $\mathrm{Mg}^{2+} \mathrm{Ca}^{2+}$ (down triangle).Phase delay is shown as empty symbols, modulation ratio as solid symbols and the solid lines represent the calculated fitting curves. Protein concentration was $47.5 \mu \mathrm{M}$ with $30 \mu \mathrm{M} 1,8-\mathrm{ANS}$ or $30 \mu \mathrm{M}$ 2,6-ANS.

Figure 3.6. Phasor plot representation of frequency domain data for 1,8-ANS (solid symbols) and 2,6-ANS (open symbols) bound to by apoDREAM (squares), $\mathrm{Mg}^{2+} \mathrm{DREAM}$ (circles), $\mathrm{Ca}^{2+} \mathrm{DREAM}$ (uptriangles), and $\mathrm{Mg}^{2+} \mathrm{Ca}^{2+} \mathrm{DREAM}$ (down triangles). Conditions: 64 $\mu \mathrm{M}$ DREAM and $30 \mu \mathrm{M}$ ANS, modulation frequency $(\omega)$ was 70 $\mathrm{MHz}$.

Figure 3.7. Titration curves for 1,8-ANS binding to site 1 and site 2 of $\mathrm{Ca}^{2+}$ DREAM. The concentration of the free 1,8-ANS bound to site 1 ( $\tau_{1} \sim 6 \mathrm{~ns}$ ) and binding site 2 ( $\tau_{2} \sim 17 \mathrm{~ns}$ ) was determined based on the pre-exponential parameters $\alpha_{0}, \alpha_{1}$, and $\alpha_{2}$. The solid line corresponds to the fit using Hill equation. 
Figure 3.8. Titration of $28.2 \mu \mathrm{M}$ 1,8-ANS by DREAM using pre-exponential factors. The concentration of ANS bound to site 1 (a) and site 2 (b) was determined based on the pre-exponential parameters $\alpha_{0}, \alpha_{1}$, and $\alpha_{2}$.

Figure 3.9. Differential angle and modulation ratio data for 1,8-ANS (left) and 2,6-ANS (right) in complex with DREAM. The solid lines correspond to the NLLS fit of the data as described in the text. Conditions were the same as in Figure 3.5.

Figure 3.10. Fractional depolarization recovered from NLLS analysis of the anisotropy decay of 1,8-ANS-DREAM complexes at different protein concentration, with $28.2 \mu \mathrm{M}$ 1,8-ANS. The rotational correlation times $(\Phi)$ were fixed to those shown on Table 3 for the dimer, tetramer, and free dye; while the fractional depolarization and limiting anisotropy were the variable parameters. In all cases the global chi squared was less than 2, the phase and modulation error set to $\leq 0.2$ and $\leq 0.002$, respectively.

Figure 3.11. Position of three docking site for 1,8-ANS on the surface of $\mathrm{Ca}^{2+}$ bound DREAM as predicted using AutoDock 4.2 (Panel A). The binding sites were found in the vicinity of EF-4 (panel B, left), at the interface between EF-3 and EF-4 (panel B, middle) and at the hydrophobic crevice between the EF-1 and EF-2 (panel B, right). Cartoon representation of monomeric calcium bound DREAM (pdb: 2JUL) with EF-1 shown in green, EF-2 in orange, EF-3 in blue and EF-4 in red while calcium is shown as solid spheres and 1,8-ANS as licorice model.

Figure 3.12. Position of three docking site for 2,6-ANS on the surface of $\mathrm{Ca}^{2+}$ bound DREAM as predicted using AutoDock 4.2 (Panel A). The binding sites were found in the vicinity of EF-4 (panel B, top), at the interface between EF-3 and EF-4 (panel B, middle) and at the hydrophobic crevice between the EF-1 and EF-2 (panel B, bottom)

Figure 3.13. Normalized absorption spectrum of 1,8-ANS bound to $\mathrm{Ca}^{2+}$ DREAM, normalized emission spectrum of $\mathrm{Ca}^{2+}$ DREAM and the overlap integral for the Trp169 - 1,8-ANS FRET pair.

Figure 3.14. (Top left) Quenching of the DREAM fluorescence emission by addition of 1,8-ANS (Top right) resulting efficiency plot calculated using the decrease in fluorescence emission at $328 \mathrm{~nm}$ as a function of 1,8-ANS concentration. Protein concentration was kept at $53 \mu \mathrm{M}$ and aliquots containing $53 \mu \mathrm{M}$ protein and $260 \mu \mathrm{M}$ 1,8-ANS were added to the solution. (Bottom left) Equations used for the analysis 
of the ANS-W169 distances, where $r_{i}$ represents the steady state anisotropy of the fluorophore in the absence of rotational diffusion and $r_{0}$ represents the limiting anisotropy determined from the modulated anisotropy extrapolated a high frequencies. (Bottom right) Steady state anisotropy determined and limiting anisotropy determined for the ANS-W169 FRET pair used to calculate the lower and upper limits for the kappa squared parameter.

Figure 3.15: Decrease in the emission intensity of 1,8-ANS bound to $\mathrm{Ca}^{2+}$ DREAM upon addition of arachidonic acid (a). The change in 1,8-ANS fluorescence intensity as a function of arachidonic acid concentration. The solid line corresponds to the fit of the experimental data using a single binding site model (solid line) (b). The position of the docking site for arachidonic acid in the Cterminal domain of DREAM (c).

Figure 3.16. A cartoon model depicting DREAM interactions with $\mathrm{Mg}^{2+}$ and $\mathrm{Ca}^{2+}$ ions and arachidonic acid. Association of the $\mathrm{Mg}^{2+}$ ions to apoDREAM tetramer does not lead to substantial changes in DREAM structure (step 1). Subsequent binding of $\mathrm{Ca}^{2+}$ to EF-3 and EF-4 promotes tetramer dissociation and concomitant exposure of the hydrophobic cavity located in the C-terminal domain by repositioning of the C-terminal $\alpha$-helix (step 2) allowing binding of the arachidonic acid to the C-terminus hydrophobic cavity (step 3).

Figure 4.1. (a) Fluorescence emission of $15 \mu \mathrm{M}$ apoDREAM( $\Delta 65)$ and $15 \mu \mathrm{M} \mathrm{Ca}^{2+}$ $\operatorname{DREAM}(\Delta 65)$ in the absence and presence of $30 \mu \mathrm{M}$ NS5806, $\lambda_{\text {exc }}=$ $295 \mathrm{~nm}$. (b) Titration of DREAM $(\Delta 65)$ with NS5806 by monitoring the change in tryptophan fluorescence in the apo and calcium bound form. The recovered dissociation constants were determined using Eqn. 2.8.1 (solid lines) and are listed in Table 4.1. (c) Average tryptophan fluorescence lifetime of $15 \mu \mathrm{M} \operatorname{DREAM}(\Delta 65)$ and $15 \mu \mathrm{M}$ $\operatorname{DREAM}(\Delta 160)$ in the $\mathrm{Ca}^{2+}$ bound state as a function of NS5806. Solid lines represent the best non-linear fit using a single site Hill equation. .

Figure 4.2. (a) Quenching of the tryptophan fluorescence of $\operatorname{DREAM}(\Delta 65)$ and $\operatorname{DREAM}(\Delta 160))$ in the apo-state as a function of NS5806, analyzed using a modified Stern-Volmer equation (inset). The linear fit recovered a slope of $45 \pm 2 \mu \mathrm{M}$ and intercept of $1.0 \pm 0.2$ for $\operatorname{DREAM}(\Delta 65)$ and slope of 80 $\pm 4 \mu \mathrm{M}$ and intercept of $1.8 \pm 0.2$ for $\operatorname{DREAM}(\Delta 160)$. The inverse of the intercept for DREAM $(\Delta 160)$ indicates that only $56 \%$ of the tryptophan residues are quenched at saturating amounts of NS5806. After correction for the fraction quenched, both constructs result in identical 
dissociation constant values of $\sim 40 \mu \mathrm{M}$ in the apoform. (b) The

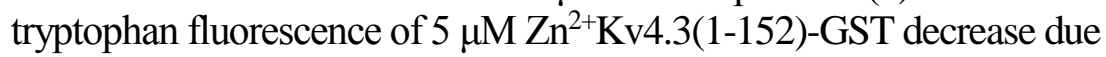
to binding of NS5806, inset shows the structural formula of NS5806.

(c) Fluorescence intensity change as a function of NS5806 concentration, solid line represents best fit using eq. 1 with recovered $\mathrm{K}_{\mathrm{d}}=11 \pm 2 \mu \mathrm{M}$. .

Figure 4.3. (a) Fluorescence emission of 1,8-ANS:DREAM $(\Delta 65)$ complexes as a function of increasing concentrations of NS5806, inset shows the shift in emission spectra maximum as a function of NS5806. (b) Replacement curves for the 1,8-ANS- DREAM $(\Delta 65)$ complex in the apo and calcium bound states as a function of NS5806 concentration. The dissociation constants recovered using Eq. 2.8.2 are $\mathrm{K}_{\mathrm{d}}=23 \pm 7 \mu \mathrm{M}$ and $\mathrm{K}_{\mathrm{d}}=2.5 \pm 0.3 \mu \mathrm{M}$ for apo and calcium bound forms, respectively. Conditions: $95 \mu \mathrm{M}$ 1,8-ANS, $10 \mu \mathrm{M} \mathrm{Ca}^{2+} \operatorname{DREAM}(\Delta 65)$ in $20 \mathrm{mM}$ MOPS, $\mathrm{pH} 7.4$, and $100 \mathrm{mM} \mathrm{NaCl}$. (c) Change in fluorescence lifetime preexponential parameters $\alpha_{1}$ and $\alpha_{2}$ as a function of NS5806 concentration. Pre-exponential parameters were recovered using a triple discrete exponential decay model from analysis of emission decay data of $10 \mu \mathrm{M} 1,8$-ANS bound to $10 \mu \mathrm{M}$ $\operatorname{DREAM}(\Delta 65)$ in the apoform and $\mathrm{Ca}^{2+}$ bound state. The lifetime of unbound ANS was set as a fixed value at $0.28 \mathrm{~ns}$ (measured separately), while the second and third decay lifetimes were set as linked variables with lifetimes of $5.7 \pm 0.4$ ns and $15.4 \pm 0.9$ ns in the apoform $\left(\chi^{2}=2.7\right)$ and $6.3 \pm 0.5 \mathrm{~ns}$ and $16.7 \pm 0.5 \mathrm{~ns}$ in the calcium bound state $\left(\chi^{2}=1.7\right)$.

Figure 4.4. ITC binding isotherms for NS5806 association to $\mathrm{Mg}^{2+} \mathrm{Ca}^{2+} \mathrm{DREAM}(\Delta 65)$ (left) and to $\mathrm{Mg}^{2+} \mathrm{Ca}^{2+} \operatorname{DREAM}(\Delta 160)$ (right). The upper panels of each profile reflects the thermal power expressed in units of $\mu \mathrm{cal} \cdot \mathrm{s}^{-1}$. The lower panel shows integrated reaction heats $(\Delta \mathrm{H})$ normalized for bound NS5806 and expressed in units of $\mathrm{kcal} \cdot \mathrm{mol}^{-1}$. The solid lines represent fitting curves using the two-set of sites model. Conditions: $10 \mu \mathrm{M}$ $\mathrm{Mg}^{2+} \mathrm{Ca}^{2+}$ DREAM in $20 \mathrm{mM}$ MOPS, $\mathrm{pH}$ 7.4, and $100 \mathrm{mM}$ $\mathrm{NaCl}, 1 \mathrm{mM} \mathrm{CaCl} 2$ and $5 \mathrm{mM} \mathrm{MgCl}_{2}$. 107

Figure 4.5. Titration curve for (a) Site $1(\mathrm{Kv} 4.3(2-21))$ binding to $\operatorname{apoDREAM}(\Delta 65)$ (open symbols) and apoDREAM $(\Delta 160)$ (solid symbols) in the absence (circles) and presence of $200 \mu \mathrm{M}$ NS5806 (up triangle). Conditions: $0.5 \mu \mathrm{M} \mathrm{Kv} 4.3(2-22)$ and $200 \mu \mathrm{M}$ 
NS5806 in $20 \mathrm{mM}$ MOPS, pH 7.4, and $100 \mathrm{mM} \mathrm{NaCl}$. (b)

Titration curves of $\mathrm{Ca}^{2+}$ (open symbols) or NS5806 (solid symbols) binding to the DREAM-site 1 complex in the presence of NS5806 or $\mathrm{Ca}^{2+}$. Solid lines represent fits to a Hill equation, with dissociation constants for NS5806 binding to $\mathrm{Ca}^{2+}$ DREAMsite 1 of $\mathrm{K}_{\mathrm{d}}=51 \pm 2 \mu \mathrm{M}$ and to apoDREAM-site $1 \mathrm{~K}_{\mathrm{d}}=21 \pm 1$ $\mu \mathrm{M}$. The recovered $\mathrm{Ca}^{2+}$ dissociation constants do not reflect the actual apparent calcium affinities since EDTA was present in the samples. Conditions: $1 \mu \mathrm{M} \mathrm{Kv} 4.3(2-22)$ and $20 \mu \mathrm{M}$ $\operatorname{DREAM}(\Delta 65)$ in $20 \mathrm{mM}$ MOPS, $\mathrm{pH} 7.4,100 \mathrm{mM} \mathrm{NaCl}$ with $1 \mathrm{mM} \mathrm{Ca}^{2+}$ or $100 \mu \mathrm{M}$ EDTA. (c) Time profile of the change in fluorescence intensity of $100 \mathrm{nM} \mathrm{Kv} 4.3(2-22)$ peptide upon binding to $5 \mu \mathrm{M} \mathrm{Ca}^{2+} \operatorname{DREAM}(\Delta 65)$ in the absence and presence of $200 \mu \mathrm{M}$ NS5806. Solid line represents the NLLS fit using a double exponential decay model, recovered parameters listed on Table 4.

Figure 4.6. (a) Docking site for NS5806 on DREAM $(\Delta 65)$ and (b) $\operatorname{DREAM}(\Delta 160)$. Solid line indicates the distance between tryptophan 169 and the dibromophenyl ring of NS5806. The initial NMR structures of DREAM $(\Delta 65)$ (pdb: 2JUL) and $\operatorname{DREAM}(\Delta 160)$ (pdb:2E6W) were used as docking macromolecules, EF-1 shown in green, EF-2 in orange, EF-3 in blue, EF-4 in red and NS5806 shown as licorice model. (c) Mutation of Tyr174 or Phe218 to alanine completely abolishes the affinity enhancement observed in the absence of calcium upon binding of NS5806. Conditions as described in figure 4.5a.

Figure 4.7. Titration curve for calcium binding to $1 \mu \mathrm{M} \operatorname{DREAM}(\Delta 65)$ labeled with the biarsenical fluorophore FlAsH-EDT 2 in the absence (squares) and in the presence (circles) of NS5806. The solid line represents the NLLS fitting using equation 4. Conditions: $1 \mu \mathrm{M}$ DREAM( $\Delta 65), 200 \mu \mathrm{M}$ NS5806 in $20 \mathrm{mM}$ MOPS, pH 7.4, and $100 \mathrm{mM} \mathrm{NaCl}$. 116

Figure 4.8. Reorganization of the hydrophobic pocket in the DREAM Cterminus upon binding of NS5806 and/or site 1. Top left: Position of helix-10 (shown in yellow) in the $\mathrm{C}$ terminus hydrophobic pocket of $\operatorname{DREAM}(\Delta 65)$ is stabilized by stacking interactions among F218, F252 and Y174. Top right: Binding of NS5806 to the $\operatorname{DREAM}(\Delta 65)$ leads to the displacement of F252 and NS5806 is stabilized by hydrophobic interactions with F218 and Y174. Bottom left: Orientation of site 1 in the C-terminus hydrophobic pocket of DREAM $(\Delta 65)$ with focus on the interaction of W19 from site 
1 with hydrophobic residues from $\operatorname{DREAM}(\Delta 65)$. Bottom right: The reorientation of the hydrophobic domain at the DREAM Cterminus upon concomitant binding of site 1 and NS5806.

Figure 5.1. (a) Bioinformatics analysis of the first 100 residues at the Nterminus of mouse DREAM indicating regions of helical structure and charge distribution. Proposed sites for posttranslational modification and ligand interactions are shown. (b) Helical plot analysis of residues 29 to 44 of DREAM. (c) DREAM amino acid sequence disorder prediction using the PONDR-FIT and VL3 prediction servers. (c) Potential energy surface of apoDREAM (lacking the first 77 residues) calculated using the published NMR structure and the APBS plugin of the VMD software with CHARMM parameters at $150 \mathrm{mM}$ mobile ion concentration.

Figure 5.2. Titration curves for calmodulin binding to (a) $100 \mathrm{nM}$ of a FITC labeled peptide analogous to residues 29-44 of mouse DREAM "DRE29" in the absence (circles) or present (squares) of $1 \mathrm{mM}$ calcium ( $\mathrm{Kd}=136 \pm 10 \mathrm{nM})$ and in the presence of calcium and $30 \mu \mathrm{M}$ Trifluoperazine (up triangles). (b) Fluorescence emission spectra of $5 \mu \mathrm{M}$ DRE29 peptide in the presence or absence of 10 $\mu \mathrm{M} \mathrm{Ca}^{2+} \mathrm{CaM}$, sample was excited at $300 \pm 4 \mathrm{~nm}$ which resulted in negligible contribution from tyrosine on CaM. Buffer background emission was subtracted to decrease Rayleigh, Raman and $2^{\text {nd }}$ harmonic peaks contributing to the signal. (c) Observed shift and increase in tryptophan fluorescence upon calmodulin binding. (d) Normalized tryptophan fluorescence change at $370 \mathrm{~nm}$ of DREAM(1-256) upon addition of calmodulin in the absence (circles) and presence (squares) or $1 \mathrm{mM}$ calcium $(\mathrm{Kd}=3.3 \pm 0.9 \mu \mathrm{M})$.

Figure 5.3. (a) Fluorescence intensity quenching of $1 \mu \mathrm{M}$ DRE29 upon association to $\mathrm{Ca}^{2+} \mathrm{CaMRR} 2$. (b) Plot of the FRET efficiency between DRE29 and $\mathrm{Ca}^{2+}$ CaMRR2 complex, a dissociation constant of $134 \pm 16 \mathrm{nM}$ was recovered using a Hill equation. (c) Fluorescence anisotropy of $100 \mathrm{nM}$ DRE29 versus calculated TFP free calmodulin. The drug free calmodulin concentration was determined assuming that a single TFP molecule is bound to calmodulin with an affinity of $1 \mu \mathrm{M}, 30 \mu \mathrm{M}$ TFP was held constant while the concentration of CaM was increased.

Figure 5.4. Anisotropy change upon addition of DREAM and DREAM $(\Delta 65)$ to $1 \mu \mathrm{M}$ DRE29 peptide in the presence of $\mathrm{CaCl}_{2}$ (solid symbols) or EDTA (open symbols). Line represent the best fit using Eqn. 
1, with dissociation constant for DREAM of $57 \mu \mathrm{M}$ while $\operatorname{DREAM}(\Delta 65)$ resulted in a $\mathrm{K}_{\mathrm{d}} \sim 30 \mu \mathrm{M}$ in the absence of salt and $\mathrm{K}_{\mathrm{d}} \sim 0.3 \mathrm{mM}$ in the presence of $100 \mathrm{mM} \mathrm{NaCl}$.

Figure 5.5. (a) Modified Stern-Volmer analysis of tryptophan fluorescence quenching upon binding of NS5806 to apo and $\mathrm{Ca}^{2+}$ DREAM, solid lines illustrate the expected traces for complete quenching and dashed lines show the intercept a $2.83 \pm 0.12$. (b) Association of Kv4.3(1-22)-FITC with apo and $\mathrm{Ca}^{2+}$ DREAM monitored using fluorescence anisotropy; solid lines represent the best fit to the data using a Hill binding model with the recovered parameters shown in the inset. (c) Fluorescence spectra of 1,8-ANS bound to apoDREAM with $\lambda_{\max }=478 \mathrm{~nm}$ and $\mathrm{Ca}^{2+} \mathrm{DREAM}$ with $\lambda_{\max }$ $=475 \mathrm{~nm}$. These results support the idea that the accessibility of the hydrophobic cavity at the C-terminus of DREAM is not compromised by the presence of the $\mathrm{N}$-terminus residues 1-65.

Figure 5.6. Eight $10 \mathrm{~ns}$ MD simulation at $313 \mathrm{~K}$ of DREAM N-terminus (residues 1-66) showing the overall stability of the predicted $\alpha_{0}$ helix. Bulky hydrophobic residues which form part of the CaM binding region (shown in red) are drawn as licorice model.

Figure 5.7. (a) Far-UV CD spectra of DREAM $(\Delta 65)$ and DREAM in the presence of $100 \mu \mathrm{M}$ calcium in $5 \mathrm{mM}$ phosphate buffer $\mathrm{pH} 7.4$. (b) Near-UV CD spectra of DREAM $(\Delta 65)$ and (c) DREAM in the absence and presence of calcium.

Figure 5.8. Dissociation kinetics of (a) $10 \mu \mathrm{M}$ CaM and $1 \mu \mathrm{M}$ DRE29 and (b) $1 \mu \mathrm{M}$ CaMRR2 and $10 \mu \mathrm{M}$ DREAM upon addition of $1 \mathrm{mM}$ EDTA monitored using T-format fluorescence anisotropy as a function of temperature. (c) Eyring plot analysis of the dissociation constant as a function of temperature, solid lines represent best linear fit to the data.

Figure 5.9. Frequency-domain fluorescence decay of apo and $\mathrm{Ca}^{2+} \mathrm{CaM}-$ IAEDANS (b) Frequency-domain anisotropy decay of $\mathrm{Ca}^{2+}$ bound CaM-IAEDANS in the absence and presence of DREAM. Phase delay and modulation ratio are shown as open and solid symbols, respectively. Solid lines represent the best fit using multi-exponential decay models, recovered parameters are listed in Table 5.3 and 5.4.

Figure 5.10. (a) Fluorescence emission spectra transition of $5 \mu \mathrm{M}$ CaMIAEDANS upon association with $20 \mu \mathrm{M}$ DREAM. (b) Global fluorescence decay lifetime $\chi^{2}$ surface for the two component 
exponential decay of CaM-IAEDANS in the presence (red) or absence (black) of $\mathrm{Ca}^{2+}$ and $\mathrm{Ca}^{2+} \mathrm{CaM}$-IAEDANS:DREAM (blue) and $\mathrm{Ca}^{2+}$ free complex (green). Rotational correlation time $\chi^{2}$ surface from the global analysis of the anisotropy decay data using a two component exponential decay for CaM-IAEDANS and triple exponential decay model for the CaMIAEDANS:DREAM complex, color scheme as in panel b. 146

Figure 5.11. Initial structure of DREAM built using the protocol described in the Material and Methods section, where the structure derived from the published NMR is shown in green and the predicted structure of residues 1 to 75 is shown in orange with the CaM binding site highlighted in red licorice model. Calcium atoms are shown as yellow spheres.

Figure 5.12. Molecular dynamics simulation of DREAM using an initial model constructed by combining a peptide model for residues 1 to 76 with the published NMR structure of DREAM (residues 76 to 256) shown in green. The alpha-helical section of the Nterminus domain was predicted using secondary prediction tools and shown in orange, the CaM binding region corresponding to residues 29-44 is shown in red licorice. All structures are aligned using residues 76 to 256; calcium is shown as yellow spheres. (a) Two final structures of DREAM after $80 \mathrm{~ns}$ classical MD simulations. (b) Three final structures of DREAM after 30 ns accelerated MD simulations. (c) Three independent structures of DREAM after 1000ns of coarse grained molecular dynamics simulation in the absence of $\mathrm{Ca}^{2+}$, showing a collapsed $\mathrm{N}$-terminus. The hydrophobic surface known to mediate ligand binding on DREAM is highlighted by a blue ellipse, the electronegative surface identified on Figure 5.1 is highlighted in black.

Figure 5.13. Fluorescence anisotropy increase due to $0.5 \mu \mathrm{M}$ FITC labeled dsDNA homologous to the DRE sequence of human prodynorphin gene binding to $5 \mu \mathrm{M}$ DREAM in the presence or absence of $\mathrm{Ca}^{2+}$. Addition of CaM in increasing concentrations $(0 \mu \mathrm{M}, 20 \mu \mathrm{M}, 40 \mu \mathrm{M}$ and $60 \mu \mathrm{M})$ results in a concomitant decrease in fluorescence anisotropy. Chelation of $\mathrm{Ca}^{2+}$ by $2 \mathrm{mM}$ EDTA results in an increase in anisotropy due to dsDNA association with DREAM, even in the presence of $60 \mu \mathrm{M} \mathrm{CaM}$. .150

Figure 5.14. Carton representation of the proposed model for a heterotetrameric DREAM:CaM complex. The DREAM dimer is 
shown in cyan and calmodulin is shown in orange, calcium is shown as yellow spheres.

Figure 6.1. (a) NMR structure of DREAM monomer with highlighted hydrophobic residues (gray mesh) and charged residues (red mesh) (pdb entry 2JUL) ${ }^{7}$. The four EF-hand of DREAM are shown in green (EF-hand 1), orange (EF-hand 2), blue (EF-hand 3 ), and red (EF-hand 4). Calcium are shown as an orange sphere. (b) Coordination geometry of calcium bound to EF-hand 3 (left) and EF-hand 4 (right). Residues involved in coordination of $\mathrm{Ca}^{2+}$ are shown in licorice model. EF-hand 3 shows a clear pentagonal bipyramidal coordination whereas EF-hand 4 is distorted. (c) Geometry and consensus sequence of EF-hand binding loops as well as sequence homology between neuronal calcium sensors and calmodulin at the metal binding loops.

Figure 6.2: Intrinsic fluorescence changes of $40 \mu \mathrm{M}$ (a) CaM and (b) $\operatorname{DREAM}(\Delta 65)$ upon binding of $1 \mathrm{mM}$ calcium or $160 \mu \mathrm{M} \mathrm{Tb}{ }^{3+}$ on CaM or $80 \mu \mathrm{M} \mathrm{Tb}{ }^{3+}$ for $\operatorname{DREAM}(\Delta 65)$, excited at $280 \pm 4$ $\mathrm{nm}$. The observed tyrosine fluorescence change upon $\mathrm{Tb}^{3+}$ binding to CaM shows a small deviation from that observed for $\mathrm{Ca}^{2+}$, likely due to quenching of the fluorescence by energy transfer to $\mathrm{Tb}^{3+}$. The $\mathrm{Tb}^{3+}$ induced transition for $\operatorname{DREAM}(\Delta 65)$ is identical to that observed in the presence of $\mathrm{Ca}^{2+}$. Sensitized emission of (c) $160 \mu \mathrm{M} \mathrm{Tb}^{3+}$ bound to CaM and (d) $80 \mu \mathrm{M}$ $\mathrm{Tb}^{3+}$ bound to DREAM $(\Delta 65)$, excited at $280 \mathrm{~nm}$ with and without $2 m M$ EDTA. The background emission of the $\mathrm{Tb}^{3+}$ :EDTA complex is shown as reference. The observed sensitized emission of $\mathrm{Tb}^{3+}$ shows the characteristic sharp bands at 489, 544 (major), 586 and $622 \mathrm{~nm}$ due to the ${ }^{5} \mathrm{D}_{4}-{ }^{7} \mathrm{~F}_{6},{ }^{5} \mathrm{D}_{4}-{ }^{7} \mathrm{~F}_{5},{ }^{5} \mathrm{D}_{4}-{ }^{7} \mathrm{~F}_{4},{ }^{5} \mathrm{D}_{4}-{ }^{7} \mathrm{~F}_{3}$ transitions of $\mathrm{Tb}^{3+}$, respectively. The major peak at $545 \mathrm{~nm}$ for $\operatorname{DREAM}(\Delta 65)$ is 40 $\%$ smaller than that observed for CaM. Addition of $2 \mathrm{mM}$ EDTA to either $\operatorname{CaM}$ or $\operatorname{DREAM}(\Delta 65)$ resulted in an emission identical to that of $\mathrm{Tb}^{3+}$ in solution.

Figure 6.3: (a) Excitation spectra of DREAM constructs bound to $\mathrm{Tb}^{3+}, 20$

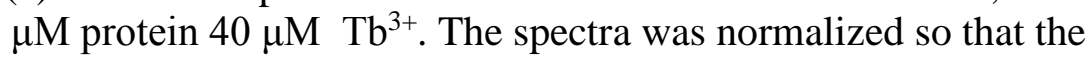
intensity corresponding to backbone to $\mathrm{Tb}^{3+}$ energy transfer at $240 \mathrm{~nm}$ is the same for $\operatorname{DREAM}(\Delta 65), \operatorname{DREAM}(\Delta 160)$, and DREAM(Y174A) while the magnitude is half for DREAM(E186Q) and DREAM(E234Q). (b) Calcium/terbium binding sites of EFhand 3 and 4, only EF-hand 3 is shown (in blue) for clarity. (c) Titrations of $\mathrm{Tb}^{3+}$ into DREAM $(\Delta 65)$ and DREAM $(\Delta 160)$ in 20 $\mathrm{mM}$ TRIS $\mathrm{pH} 7.4$ and $100 \mu \mathrm{M} \mathrm{Ca}^{2+}$ with and without $5 \mathrm{mM} \mathrm{Mg}^{2+}$. 
(d) Titration of $\mathrm{Tb}^{3+}$ into DREAM(E186Q) and DREAM(E234Q) under identical conditions as panel (c). Solid lines represent the best fit using the quadratic equation for N-binding sites.

Figure 6.4. (a) Tryptophan fluorescence lifetime distribution of $\operatorname{DREAM}(\Delta 64)$ in the presence of $\mathrm{Ca}^{2+}$ or $\mathrm{Tb}^{3+}$, decay data was modeled as a Gaussian distribution and a discrete decay, parameters listed in Table 1. (b) Circular dichroism spectra of 40 $\mu \mathrm{M}$ DREAM $(\Delta 64)$ in the presence of $1 \mathrm{mM} \mathrm{Ca}^{2+}, 2 \mathrm{mM}$ EDTA or $160 \mu \mathrm{M} \mathrm{Tb}^{3+}$ in $5 \mathrm{mM}$ TRIS pH 7.4. (b) Binding of $\mathrm{Tb}^{3+}$ or $\mathrm{Ca}^{2+}$ to $10 \mu \mathrm{M}$ DREAM( $(\Delta 64)$ in the presence of $40 \mu \mathrm{M}$ 1,8-ANS.

Figure 6.5: ITC isotherms for $\mathrm{Tb}^{3+}$ displacement of $\mathrm{Ca}^{2+}$ from CaM, $\operatorname{DREAM}(\Delta 64)$ and $\operatorname{DREAM}(\Delta 160)$. The upper panels of each profile reflects the thermal power expressed in units of $\mu \mathrm{cal} \cdot \mathrm{s}^{-1}$. The lower panel shows integrated reaction heats $(\Delta \mathrm{H})$ expressed in units of $\mathrm{kcal} \cdot \mathrm{mol}^{-1}$. The solid lines present the best fitting curve with parameters shown in Table 6.2.

Figure 6.6: (a) Luminescence decay of $\mathrm{Tb}^{3+}$ bound to CaM and constructs of DREAM, with stoichiometric ratios of $4: 1 \mathrm{~Tb}^{3+}: \mathrm{CaM}$ and 2:1 $\mathrm{Tb}^{3+}$ :DREAM. Solid lines represent the best fit using Globals software analysis, recovered parameters listed in Table 3. (b) Luminescence intensity decay profiles as a function of $\mathrm{Tb}^{3+}$ binding to $10 \mu \mathrm{M} \operatorname{DREAM}(\Delta 64)$. The luminescence decays in (b) were analyzed using a triple discrete model and the fractional intensity contribution of the two slowest lifetimes is shown in (c). The fastest decay component had a lifetime of $\sim 80 \mu$ s with less than $10 \%$ contribution and is not shown. (d) Luminescence decay $\mathrm{Tb}^{3+}$ bound to DREAM and DREAM $(\Delta 64)$ as a function of the hydrophobic molecules TFP (top) bound to DREAM and NS5806 (bottom) bound to DREAM. 180

Figure 6.7: (a) Typical nanoESI mass spectra of DREAM $(\Delta 64)$ in the presence of $\mathrm{Ca}^{2+}$ and of DREAM $(\Delta 64)$ in the presence of $\mathrm{Ca}^{2+}$ and $\mathrm{Tb}^{3+}$. The isotopic distribution of the $\operatorname{DREAM}(\Delta 64): 2 \mathrm{Ca}^{2+}$ and $\operatorname{DREAM}(\Delta 64): 2 \mathrm{~Tb}^{3+}$ are shown for clarity. (b) NanoESI TIMS mobility spectra of DREAM $(\Delta 64)$ bound to $\mathrm{Ca}^{2+}$ and/or $\mathrm{Tb}^{3+}$.

Figure 6.8: Plot of $\mathrm{T} \Delta \mathrm{S}$ versus $\Delta \mathrm{H}$ for $\mathrm{Tb}^{3+}$ displacement of $\mathrm{Ca}^{2+}$ from $\operatorname{DREAM}(\Delta 64), \operatorname{DREAM}(\Delta 161), \operatorname{DREAM}(\mathrm{E} 186 \mathrm{Q})$, DREAM(E234Q) and CaM. Solid line represents the best linear fit to the data. 
Figure 7.1. Jablonski-Perrin diagram highlighting the two major pathways by which the excited state of a chromophore can be depopulated.

Figure 7.2. Boundary conditions used in solving the Green's function. Yellow represents the heat posited including any additional process.

Figure 7.3. Experimental set-up for the photo-thermal beam deflection measurements in the collinear configuration.

Figure 7.4. (Top) PBD noise on long timescales with and without air insulation. (Bottom) The PBD amplitude distribution of the noise.

Figure 7.5. (a) PBD signal amplitude of $\mathrm{Na}_{2}\left(\mathrm{CrO}_{4}\right)_{2}$ in $20 \mathrm{mM}$ MOPS pH 7.4 and $100 \mathrm{mM} \mathrm{NaCl}$ as a function of absorbance at $355 \mathrm{~nm}$.

(b) Linear plots of the PBD data as a function of $\left[\left(\frac{\mathrm{dn}}{\mathrm{dT}}\right)\left(\frac{1}{\rho \mathrm{Cp}}\right)\right]^{-1}$ at different optical absorbance at $355 \mathrm{~nm}$. The inset shows the deviation from 0-interecept as a function of optical density at 355 $\mathrm{nm}$. (c) PBD signal amplitude of the same sample as a function of pump laser power at an absorbance of 0.701 at $355 \mathrm{~nm}$.

Figure 7.6. (a) PBD signal of Na2(CrO4)2 in 20 mM MOPS pH 7.4 upon excitation with $1100 \mu \mathrm{J}$ power at $355 \mathrm{~nm}$. (b) PBD signal as a function of pump laser repetition rate at $20^{\circ} \mathrm{C}$. Inset shows the magnitude of the heat diffusion effect as a function of frequency.

Figure 7.7. A representative trace highlighting the kinetics of a photo triggered processes and the amplitude of the slow and fast phases used to calculate the enthalpy and volume changes.

Figure 7.8. (a) Differences in calculated $\epsilon\left[\left(\frac{\mathrm{dn}}{\mathrm{dT}}\right)\left(\frac{1}{\rho \mathrm{Cp}}\right)\right]^{-1}$ values. In method 1 , the values are obtained by fitting the $\left(\frac{\mathrm{dn}}{\mathrm{dT}}\right), \frac{1}{\rho}$, and $\frac{1}{\mathrm{Cp}}$ terms individually to a polynomial of rank 3 . In method 2 , the values of $\left(\frac{\mathrm{dn}}{\mathrm{dT}}\right), \frac{1}{\rho}$, and $\frac{1}{\mathrm{Cp}}$ are first multiplied and the trace fitted with polynomial of rank 5 . These polynomials are used to calculate the $\epsilon\left[\left(\frac{\mathrm{dn}}{\mathrm{dT}}\right)\left(\frac{1}{\rho \mathrm{Cp}}\right)\right]^{-1}$ at the temperature shown. (b) The deviation from linearity becomes more obvious at lower temperatures (near 0 on the $\mathrm{x}$-axis). This plot was obtained using $\mathrm{Na}_{2}\left(\mathrm{CrO}_{4}\right)_{2}$ in $20 \mathrm{mM}$ MOPS pH $7.4100 \mathrm{mM} \mathrm{NaCl}$ with a laser power of $1100 \mu \mathrm{J}$. (c) Effect on intercept due to increase in ionic strength, conditions are the same as in (b). .208 
Figure 7.9. (a) PBD signal of $\mathrm{Na}_{2}\left(\mathrm{CrO}_{4}\right)_{2}$ (reference) in $20 \mathrm{mM}$ MOPS pH 7.4 $100 \mathrm{mM} \mathrm{NaCl}$. (b) Plot of the reference PBD signal as a function of $\epsilon\left[\left(\frac{\mathrm{dn}}{\mathrm{dT}}\right)\left(\frac{1}{\rho \mathrm{Cp}}\right)\right]^{-1}$ used to determine the instrument constant $A E_{a}$. (c) Linear fit of the PBD signal as a function temperature used to identify the temperature at which the PBD signal for the reference is equal to 0 .

Figure 7.10. Titration curves for $\mathrm{Ca}^{2+}$ and $\mathrm{Tb}^{3+}$ binding to DM-nitrophen. The titrations were conducted in 20mM MOPS pH 6.5 and $100 \mu \mathrm{M}$ DM-nitrophen. The absorbance at $350 \mathrm{~nm}$ is plotted.

Figure 7.11. (a) Representative ITC isotherms for displacement of $\mathrm{Ca}^{2+}$ by $\mathrm{Tb}^{3+}$ upon binding to DM-nitrophen. (b) Heat due to $\mathrm{Tb}^{3+}$ binding to DM-nitrophen (black symbols), $\mathrm{Tb}^{3+}$ displacement of $\mathrm{Ca}^{2+}$ from DM-nitrophen (red symbols), and (c) from EDTA. The solid lines represent the best fit to the data using a one-site model.

Figure 7.12. (a) PBD signal of $\mathrm{H}_{2}$ Cage (dark lines) in $20 \mathrm{mM}$ MOPS pH 7.1 $100 \mathrm{mM} \mathrm{NaCl}$ as a function of temperature. (b) PBD signal of $\mathrm{Cu}\left(\mathrm{OH}_{2}\right)$ Cage at different temperatures. The reference PBD signal of $\mathrm{Na}_{2}\left(\mathrm{CrO}_{4}\right)_{2}$ (light lines) at $30{ }^{\circ} \mathrm{C}$ (red) and $10{ }^{\circ} \mathrm{C}$ (black) are shown for comparison in plots a and b. (c) Plot of the sample to reference PBD signal ratio as a function of $\epsilon\left[\left(\frac{\mathrm{dn}}{\mathrm{dT}}\right)\left(\frac{1}{\rho \mathrm{Cp}}\right)\right]^{-1}$ for the fast and slow phases of $\mathrm{H}_{2}$ Cage and $\mathrm{Cu}(\mathrm{OH})_{2}$ Cage used to determine $\Delta \mathrm{H}$ and $\Delta \mathrm{V}$, following eqn. 8.37. The solid red lines represent the linear fit of the data. Recovered parameters listed in Table 8.1.

Figure 7.13. Eyring plot of $\mathrm{H}_{2} \mathrm{Cage}$ and $\mathrm{Cu}\left(\mathrm{OH}_{2}\right)$ Cage aci-nitro intermediate decay as determined by PBD. Solid lines represent the best linear fit to the data.

Figure 7.14. (a) PBD traces for DREAM constructs at $200 \mu \mathrm{M}$ concentration, solid green line represent best NLLS fit to an exponential decay. Trace for DREAM(1-256) not shown. (b) PBD traces of $\mathrm{Ca}^{2+} \mathrm{DM}-$ nitrophen from different manufacturers.

Figure 8.1. Calcium induced dissociation of DREAM from DNA. The $\mathrm{Ca}^{2+}$ free DREAM shown as a tetrameric blue pentagon, upon $\mathrm{Ca}^{2+}$ binding a dimer is formed (green shape). The dimeric form of DREAM has lower affinity for DNA. Dissociation of DREAM from DNA induces the expression of DRE regulated genes. .223 
Figure 8.2. Association of ligand with DREAM (shown in the center as a cartoon model). The electrostatic and hydrophobic regions of DREAM are highlighted in a red surface or gray surface, respectively. Ligand known to interact with DREAM are shown, (counterclockwise) calcium, terbium(III), 1,8-ANS, NS5806, arachidonic acid, site 1 of $K_{v} 4.3$, site 2 of $K_{v} 4.3$, DRE DNA, and magnesium.

Figure 8.3. Cartoon representation of the three dimensional structures of (a) NCS1 (PDB: 1fpw), (b) recoverin (PDB: 1jsa), and (c) DREAM (PDB: 2jul) in the $\mathrm{Ca}^{2+}$ bound form.

Figure 8.4. (a) Root mean square deviation (RMSD) of the alpha carbon $\left(\mathrm{C}_{\alpha}\right)$ DREAM (residues 76 to 256) as a function of simulation time. Notice that the classical simulation must undergo over $400 \mathrm{~ns}$ to reach a deviation similar to that observed using aMD. Heat map plot showing the RMSD of the $\mathrm{C}_{\alpha}$ of each residue as a function of simulation time for the cMD simulation (b) and for the aMD simulation (c). Note the similar patter or reorganization between residue 76 to 96 on both simulations (marked as 1 ). The region 2 corresponds to residues connecting the exiting helix of EF-hand 3 and entering helix of EF-hand 4.

Figure 8.5. Global structural changes following 100 ns of aMD simulation.

(a) Initial structure of DREAM following minimization and equilibration (b) after 100 ns of aMD. (c) Overlaid of the initial structure shown in (a) with the N-terminus of structure shown in (b) represented as geometric cartoons. The C-terminus EF-hand 3 and 4 were used in the alignment.

Figure 8.6. (a) Reorientation of the EF-hands of DREAM in the absence of $\mathrm{Ca}^{2+}$ before (shown in solid colors) and after $100 \mathrm{~ns}$ of aMD simulation (shown in diffuse color with solid lines representing the backbone atoms). All alignments were conducted using the first 12 amino acids of the entering helix (shown horizontal). The EF-hand 1 residues 89 to 122 (a), EF-hand 2 residues 128 to 160 (b), EF-hand 3 residue 163 to 198 (c), EF-hand 4 residue 210 to 242 (d), and the H10 helix residues 243 to 256.

Figure 8.7. Structure of the N-terminus domain of DREAM (EF-hand 1 in orange and EF-hand 2 in green) (a) before simulation and (b) after 100 ns of aMD simulation in the absence of $\mathrm{Ca}^{2+}$. Root mean square deviation per residue of the last 10 ns compared to the initial structure of $\mathrm{Ca}^{2+}$ bound DREAM (red) and $\mathrm{Ca}^{2+}$ free DREAM (black). The approximate positions of the EF-hand 
helices shown as colored squares, EF-hand 1 in orange, EF-hand 2 in green, EF-hand 3 in blue, and EF-hand 4 in red. Heat map representation of the RMSD per residue as a function of time for $\mathrm{Ca}^{2+}$ bound DREAM during $100 \mathrm{~ns}$ of aMD simulation.

Figure 8.8. (a) Root mean square deviation (RMSD) and root mean square fluctuation (RMSF) of the EF-hand 2 (residues 163 to 198. RMSF calculated with a moving window of $1 \mathrm{~ns}$ with a step of $0.2 \mathrm{~ns}$. The RMSD (c) and RMSF(d) of EF-hand 4. White square highlights the $\mathrm{N}$-terminus of the metal binding loop.

Figure 8.9. (a) Cartoon representation of the proposed DREAM dimer in which Leu155, Leu158, Leu159, and Leu251 are shown in licorice model with a diffuse surface. The N-terminus EF-hand 1 and 2 shown in blue and the C-terminus EF-hand 3 and 4 shown in red. (b) RMSD of the $\mathrm{C}_{\alpha}$ in EF-hand 1 (residues 89 to 102) as a function of aMD simulation time for the $\mathrm{Ca}^{2+}$ free and bound DREAM as well as the dimer of DREAM. (c) Heat map of the RMSD per residue as a function of simulation time for each DREAM subunit in the dimer protein.

Figure 8.10. Residue cross correlation analysis of the cMD simulations for the (a) $\mathrm{Ca}^{2+}$ bound DREAM monomer, (b) $\mathrm{Ca}^{2+}$ bound DREAM dimer (only one subunit shown), and (c) $\mathrm{Ca}^{2+}$ free DREAM monomer. All the structure are based on the last $90 \mathrm{~ns}$ of a cMD simulation in which the RMSD changed by less than $1 \AA$. The structure of the $\mathrm{Ca}^{2+}$ free DREAM was obtained after $100 \mathrm{~ns}$ of aMD simulation. Regions of interest are marked and described in the text.

Figure 8.11. Cartoon representation $\mathrm{Ca}^{2+}$ bound DREAM monomer with the colored communities shown as ball and stick colored cartoons. The structure was obtained following 100 ns of cMD simulation. (b) Cartoon representation of DREAM with EF-hands colored and helices labeled for reference to panel (a). (c) Structure of DREAM with overlaid dynamic networks highlighting the critical amino acids connecting the $\mathrm{N}$ - and C-terminus of DREAM (amino acids shown in purple licorice, H10 and gray network not shown for clarity). (d) Networks at the C-terminus showing the connection between the H10 helix (gray network) and the entering helix of EF-hand 4 (yellow network), critical amino acids shown in green licorice model. .236

Figure 8.12. (a) Cartoon representation $\mathrm{Ca}^{2+}$ free DREAM monomer with the colored communities shown as ball and stick colored cartoons. 
The structure was obtained following 100 ns of cMD simulation.

(b) Cartoon representation of DREAM with EF-hands colored and helices labeled for reference to panel (a).

Figure 8.13. (a) Cartoon representation of the proposed DREAM dimer on which the communities have been overlaid using a ball and stock representation. Subunit A and B are highlighted for reference. The figure shows one angle the other rotated 180 degrees. (b) carton representation of DREAM with EF-hands colored and $\mathrm{Ca}^{2+}$ metal shown, LxxLL motif shown in purple licorice model.

(c) Intersubunit salt bridges formed between DREAM subunits.

Figure 8.14. Dynamic networks at the C-terminus, highlighting the strong dynamic coupling between the exiting helix of EF-hand 4 (cyan) and the entering helix of EF-hand 3 (purple), (a) in the presence of $\mathrm{Ca}^{2+}$ and (b) in the absence of $\mathrm{Ca}^{2+}$. Reorganization of the $\mathrm{C}$ terminus aromatic amino acids upon removal of $\mathrm{Ca}^{2+}$ from the EF-hands 3 and 4 of DREAM.

Figure 8.15. (a) Sequence alignment of mouse NCS-1, recoverin, VILIP-1, GCAP-1, and DREAM. Turn loops of EF-hand 1, 2, 3, and 4 are shown in bold and underlined. the negatively charged amino acids involved in metal coordination shown in red. The conserved aromatic amino acids are shown in bold underlined green letters. Asterisks indicate identical residues, semicolon indicates very similar residues, and the period is for somewhat similar residues. Amino acids involved in Kv4 binding are shown in green.

Figure 8.16. (a) Sequence alignment of mouse NCS-1, recoverin, VILIP-1, GCAP-1, and DREAM. Turn loops of EF-hand 1, 2, 3, and 4 are shown in bold and underlined. the negatively charged amino acids involved in metal coordination shown in red. The loop connecting the EF-hands 3 and 4 are shown in blue bold and underlined, the respective negatively residue with whom these amino acids interacts is shown in bold underlined orange color. The LxxLL motif is shown in blue and the the amino acid with whom they interact is shown in orange.

Figure 8.17. Far-UV circular dichroism spectra of DREAM mutants. (a) $\operatorname{DREAM}(\Delta 65)$, (b) DREAM $(\Delta 160)$, (c) $\operatorname{DREAM}(\mathrm{Y} 174 \mathrm{~A}),(\mathrm{d})$ DREAM(F218A), (e) DREAM(F235A), and (f) DREAM-NCS. The spectra are shown in the presence of $1 \mathrm{mM}$ EDTA (apo), 5 $\mathrm{mM} \mathrm{MgCl} 2$ and $1 \mathrm{mM}$ EGTA $\left(\mathrm{Mg}^{2+}\right), 1 \mathrm{mM} \mathrm{CaCL} 2\left(\mathrm{Ca}^{2+}\right)$, and $1 \mathrm{mM} \mathrm{CaCl}_{2}$ and $5 \mathrm{mM} \mathrm{MgCl} 2\left(\mathrm{Mg}^{2+} \mathrm{Ca}^{2+}\right)$. The coloring scheme 
is the same for all spectra. The protein centration was kept near $10 \mu \mathrm{M}$ for all protein constructs in $5 \mathrm{mM}$ TRIS $\mathrm{pH}$ 7.4. .248

Figure 8.18. Tryptophan fluorescence spectra of DREAM mutants. (a) $\operatorname{DREAM}(\Delta 65)$, (b) DREAM $(\Delta 160)$, (c) DREAM(Y174A), (d) DREAM(F218A), (e) DREAM(F219A), (f) DREAM(F235A), (g) DREAM(F252A), and (h) DREAM-NCS. The spectra are shown in the presence of $1 \mathrm{mM}$ EDTA (apo), $5 \mathrm{mM} \mathrm{MgCl}_{2}$ and $1 \mathrm{mM}$ EGTA $\left(\mathrm{Mg}^{2+}\right), 1 \mathrm{mM} \mathrm{CaCL} 2\left(\mathrm{Ca}^{2+}\right)$, and $1 \mathrm{mM} \mathrm{CaCl}_{2}$ and $5 \mathrm{mM} \mathrm{MgCl} 2\left(\mathrm{Mg}^{2+} \mathrm{Ca}^{2+}\right)$. The coloring scheme is the same for all spectra. The protein concentration was kept near $10 \mu \mathrm{M}$ for all protein constructs in $5 \mathrm{mM}$ TRIS $\mathrm{pH}$ 7.4. .250

Figure 8.19. Fluorescence emission spectra of 1,8-ANS bound to DREAMNCS1 in the presence of $\mathrm{Ca}^{2+}, \mathrm{Mg}^{2+}$, or both.

Figure 8.20. Calcium binding experiments monitoring the tryptophan fluorescence change upon $\mathrm{Ca}^{2+}$ binding to each DREAM mutants. (a) All the titration curves for DREAM constructs showing a decrease in tryptophan fluorescence similar to that of $\operatorname{DREAM}(\Delta 65)$ and (b) DREAM mutants whose change in fluorescence is reverse to that observed in the $\operatorname{DREAM}(\Delta 65)$ protein. Protein samples contained $5 \mu \mathrm{M}$ DREAM in $20 \mathrm{mM}$ MOPS pH $7.41 \mathrm{mM}$ EGTA and $1 \mathrm{mM}$ NTA. The concentration of calcium free was determined using the known fluorescence transition and dissociation constants of Quin-2. .253

Figure 8.21. Unfolding experiment of DREAM constructs, in which the tryptophan fluorescence maxima was monitored as a function of guanidium hydrochloride $(\mathrm{GuHCl})$ concentration. The protein was kept at $5 \mu \mathrm{M}$ for all protein construct and at all $\mathrm{GuHCl}$ concentrations. .256

Figure 8.22. Tryptophan fluorescence emission spectra as a function of $\mathrm{GuHCl}$ for (a) $\mathrm{Ca}^{2+}$ free and (b) $\mathrm{Ca}^{2+}$ bound $\operatorname{DREAM}(\Delta 65)$. The emission spectra of the native DREAM, intermediate, and unfolding protein in the absence of $\mathrm{Ca}^{2+}$ (c) and presence of $\mathrm{Ca}^{2+}$ is shown (d) .258

Figure 8.23. Representative unfolding trace of (a) DREAM $(\triangle 65)$ and (b) DREAM-NCS in the apo, $\mathrm{Mg}^{2+}, \mathrm{Ca}^{2+}$, and $\mathrm{Mg}^{2+} \mathrm{Ca}^{2+}$ bound forms. Solid lines represent the best fit using equation 8.4. .259

Figure 8.24. Unfolding plot of (a) DREAM (F218A) and (b) 
DREAM(F219A) in the apo, $\mathrm{Mg}^{2+}, \mathrm{Ca}^{2+}$, and $\mathrm{Mg}^{2+} \mathrm{Ca}^{2+}$ bound forms. Solid lines represent the best fit using equation 8.4. .264

Figure 8.25. Rotameric states of tryptophan in proteins. (a) Newman projection of the t rotamer and (b) $\mathrm{g}^{+}$rotamer with view along the $\mathrm{C} \alpha-\mathrm{C} \beta$ carbon bond. The corresponding indole conformations as observed in the aMD simulation of apoDREAM $(\Delta 161)$. The measured $C \alpha-C \beta$ and $C \beta-C \gamma$ angles are highlighted with black arrows.

Figure 8.26. Plots of the $C \beta-C \gamma$ as a function of $C \alpha-C \beta$ dihedral angle of tryptophan 169 in the $\mathrm{Ca}^{2+}$ bound (a) DREAM $(\Delta 160)$, (c) $\operatorname{DREAM}(\Delta 65)$, and (e) DREAM(Y174A), as well as the $\mathrm{Ca}^{2+}$ free of the (a) DREAM $(\Delta 160)$, (c) $\operatorname{DREAM}(\Delta 65)$, and (e) DREAM(Y174A). A total of 4000 points representing 20 ps each are plotted, obtained from the aMD simulations.

Figure 8.27. Plots of the $C \beta-C \gamma$ as a function of $C \alpha-C \beta$ dihedral angle of tryptophan 169 in the $\mathrm{Ca}^{2+}$ bound (a) DREAM(F218A), (c) DREAM(F219A), and (e) DREAM(F252A), as well as the $\mathrm{Ca}^{2+}$ free of the (b) DREAM(F218A), (d) DREAM(F219A), and (e) DREAM(F252A). A total of 4000 points representing 20 ps each are plotted, obtained from the aMD simulations. .270

Figure 8.28. Plots of the $C \beta-C \gamma$ as a function of $C \alpha-C \beta$ dihedral angle of tryptophan 169 in the $\mathrm{Ca}^{2+}$ bound (a) and $\mathrm{Ca}^{2+}$ free (b) of the DREAM(F235A). Solvent exposure of Trp169 in the (c) $t$ rotamer with $\mathrm{G}$ - indole orientation and in the (d) $\mathrm{g}^{+}$rotamer with $\mathrm{G}+$ indole orientation. Inset shows the change in SASA as a function of time.

Figure 8.29 (a) Helices of DREAM included in the analysis of fluctuations. The EF-hands 1 and 2 are shown in orange and green, respectively, and form part of the N-terminus. The EF-hands 3 and 4 are shown in blue and red, respectively, and form the C-terminus. The H1 helix, the connecting loop between EF-hand 3 and 4, and the H10 helix are not used in the calculations and are shown in diffuse cyan. (b) A representative RMSF plot of apoDREAM $(\Delta 65) \mathrm{N}$ - and C-terminus as a function of simulation time, the last $80 \mathrm{~ns}$ of simulation time was used for all constructs.

Figure 8.30. Plots of the root mean square fluctuations (RMSF) distribution for the last $80 \mathrm{~ns}$ of aMD simulation for $\mathrm{N}$ - and $\mathrm{C}$-terminus of the

(a) DREAM( $\Delta 65)$, (b) DREAM(Y174A), (c) DREAM(F218A),

(d) DREAM(F219A), (e) DREAM(F235A) and (f) 
DREAM(F252A) construct in the $\mathrm{Ca}^{2+}$ bound and free conformations.

Figure 8.31. Plots of the difference in unfolding energy $\left(\Delta \Delta \mathrm{G}=\Delta \mathrm{G}_{\mathrm{I}}-\Delta \mathrm{G}_{\mathrm{U}}\right)$ as a function of the difference in RMSF $\left(\triangle \mathrm{RMSF}=\mathrm{RMSF}_{\mathrm{N}}\right.$ terminus $\left.-\operatorname{RMSF}_{\mathrm{C}-\text { terminus }}\right)$. The results for $\operatorname{DREAM}(\Delta 65)$, DREAM(Y174A), DREAM(F218A), DREAM(F219A), DREAM(F235A), and DREAM(F252A) are shown in the apo (symbols with green outline) and $\mathrm{Ca}^{2+}$ bound form.

Figure 8.32. Binding site of the $\mathrm{K}_{\mathrm{v}} 4.3$ site 2 peptide at the $\mathrm{N}$-terminus of DREAM. .278

Figure 8.33. Emission spectra of dansylated $\mathrm{K}_{\mathrm{v}} 4.3$ site 2 peptide in the absence and in the presence of $\mathrm{Ca}^{2+}$ bound (a) $\operatorname{DREAM}(\Delta 65)$, (b) DREAM(Y174A), (c) DREAM(F218A), (d) DREAM(F235A), and € DREAM-NCS1. .279

Figure 8.34. (a) Structural alignment of the EF-hand 1 exiting helix of the $\mathrm{Ca}^{2+}$ bound DREAM structure (PDB: 2jul) and the $\mathrm{Ca}^{2+}$ bound structure of KChIP1 bound to the T1-domain of Kv4.3, shown in yellow (PDB: 2nz0). The exiting helix of EF-hand 1 was used for aliment. (b) Alignment of the exiting helix of EF-hand 1 of $\mathrm{Ca}^{2+}$ free DREAM (structure obtained after $432 \mathrm{~ns}$ of cMD simulation) and the exiting helix of KChIP1 in complex with the Kv4.3 channel.

Figure 8.35. Structural reorganization of EF-hand 1 in the $\mathrm{Ca}^{2+}$ free form of DREAM obtained from: (a) $435 \mathrm{~ns}$ cMD simulation of apoDREAM and (b) $1 \mu$ s coarse-grained simulation. The inset shows the rotation Tyr97 into the hydrophobic cavity lining the $\mathrm{N}$-terminus domain. The EF-hand 1 was aligned using the backbone atoms of residues 89 to 160. (c) Structure of DREAM after $1 \mu$ s coarse-grained simulation showing the rotation of Arg98 and Lys101 in the absence of $\mathrm{Ca}^{2+}$. .281

Figure 8.36. Summary of the mechanism by which binding of Kv4.3 site 1 at the hydrophobic surface of DREAM is regulated by $\mathrm{Ca}^{2+}$ binding and changes in structural dynamics. (a) Structure of KChIP1:Kv4.3 complex (PDB: 2nz0) highlighting the role of the $\mathrm{N}$ - and $\mathrm{C}$-terminus hydrophobic cavity in ligand binding. (b) Dynamic network analysis which shows the collapse of the hydrophobic cavities on DREAM and the enhanced dynamic coupling of the H10 helix (black network). 


\section{LIST OF ABREVIATIONS AND ACRONYMS}

\begin{tabular}{|c|c|}
\hline ABBREVIATION & FULL NAME \\
\hline aMD & Accelerated molecular dynamics \\
\hline$\Delta \mathrm{H}^{*}$ & Activation enthalpy change \\
\hline $\mathrm{Ka}$ & Association constant \\
\hline $\mathrm{BZF}$ & Bezafibrate \\
\hline $\mathrm{Ca}^{2+}$ & Calcium \\
\hline cMD & Classical molecular dynamics \\
\hline $\mathrm{CD}$ & Circular dichroism \\
\hline $\mathrm{E}_{\text {dihed }}$ & Dihedral energy \\
\hline DMSO & Dimethyl sulfoxide \\
\hline $\mathrm{Kd}$ & Dissociation constant \\
\hline DTT & Dithiothreitol \\
\hline DREAM & Downstream regulatory element antagonist modulator \\
\hline EFX & EF-hand X \\
\hline$\Delta \mathrm{H}$ & Enthalpy change \\
\hline$\lambda \max$ & Emission maxima \\
\hline EGTA & $\begin{array}{l}\text { Ethylene glycol-bis(2-aminoethylether)-N,N,N', } \mathrm{N}^{\prime}- \\
\text { tetraacetic }\end{array}$ \\
\hline $\mathrm{GuHCl}$ & Guanidine hydrochloride \\
\hline $\mathrm{Ki}$ & Inhibition constant \\
\hline IPTG & Isopropyl $\beta$-D-1-thiogalactopyranoside \\
\hline
\end{tabular}




\begin{tabular}{|c|c|}
\hline ITC & Isothermal Calorimetry \\
\hline $\mathrm{Mg}^{2+}$ & Magnesium \\
\hline MS & Mass spectrometry \\
\hline MEM & Maximum entropy method \\
\hline NLLS & Non-linear least squares \\
\hline NS5806 & $\begin{array}{l}\text { N-[3,5-Bis(trifluoromethyl)phenyl]-N'-[2,4-dibromo-6- } \\
\text { (2H-tetrazol-5-yl)phenyl]urea }\end{array}$ \\
\hline PAC & Photoacoustic calorimetry \\
\hline PBD & Photothermal beam deflection \\
\hline KChIP & Potassium channel interaction protein \\
\hline$\Phi$ & Quantum yield \\
\hline CaM & Rat calmodulin \\
\hline RMSD & Root mean square deviation \\
\hline SDS & Sodium dodecyl sulfate \\
\hline SASA & Solvent accessible surface area \\
\hline $\mathrm{Tb}^{3+}$ & Terbium \\
\hline TIMS & Trapped-ion mobility spectrometer \\
\hline $\mathrm{E}_{\text {total }}$ & Total energy \\
\hline$\Delta \mathrm{V}$ & Volume change \\
\hline 1,8-ANS & 1-anilinonaphthalene-8-sulfonic acid \\
\hline PIPES & 1,4-Piperazinediethanesulfonic acid \\
\hline DM-nitrophen & $\begin{array}{l}\text { ((1-(2-nitro-4, 5-dimethoxyphenyl)-N, N, N', N'-tetrakis } \\
\text { [ (oxy-carbonyl) methyl]-1, 2-ethanediamine) }\end{array}$ \\
\hline 2,6-ANS & 2-anilinonaphthalene-6-sulfonic acid \\
\hline
\end{tabular}


EDTA

L35

TRIS

TnC

MOPS

HEPES

1,5-IAEDANS
2-(\{2-[Bis(carboxymethyl)amino]ethyl $\}$

(carboxymethyl)amino)acetic acid

2-[4-(3,5-dichlorophenyllureido)phenoxyl]-2methylpropionic acid

2-Amino-2-hydroxymethyl-propane-1,3-diol

Troponin C

3-Morpholinopropane-1-sulfonic acid

4-(2-hydroxyethyl)-1-piperazineethanesulfonic

(5-((((2-Iodoacetyl)amino)ethyl)amino)Naphthalene-1Sulfonic Acid) 


\section{PREFACE}

This dissertation is a compilation of research studies carried out from January 2011 to April 2016 by Walter G. Gonzalez. During this time, some of these results have been published in peer-reviewed journals. In particular, Chapter 3 (Gonzalez, Miksovska 2014), Chapter 4 (Gonzalez, Pham \& Miksovska 2014), Chapter 5 (Gonzalez, Arango \& Miksovska 2015), and Chapter 7 (Gonzalez, Miksovska 2014). The data presented in Chapter 6 has been accepted for publication in the Biochemistry journal of the America Chemical Society (Gonzalez, et al. 2016). Chapter 8 is presented in a format common in the field of biophysics and biochemistry, and will likely be submitted as two separate research articles. 


\section{INTRODUCTION}

\subsection{Brain, neurons, and calcium}

The vertebrate central nervous system (CNS) is the most complex biological system known and its morphological complexity is only overshadowed by the exceptional array of behaviors that it creates and controls. Among the numerous cells that actively regulate the CNS function, neurons are the essential building block controlling activity (y Cajal 1995, Hodgkin, Huxley 1952). It is estimated that there are on average about 130 billion neurons in the brain, each interconnected with thousands of other neurons in a maze of about 150 trillion synapses (Saver 2006). Despite the enormous complexity of the CNS at the system level; at the molecular level, a certain level of organization and hierarchy begins to emerge. Indeed, decades of research have allowed a detailed (albeit, not yet complete) understanding of the signaling mechanisms controlling communication among distinct classes of neurons and other excitable cells, such as cardiac cells in the heart. The signaling mechanism is known as the action potential, and it involves the coordinated firing of numerous membrane bound ion channels and transporters (Barnett, Larkman 2007). Action potentials control cell excitability by effectively changing the normal negative polarity of the transmembrane voltage of the cell by allowing the influx of positive ions such as sodium and calcium, thus causing a membrane depolarization. The influx of positive ions is counteracted by the efflux of potassium or chloride ions, causing the hyperpolarization of the cell. Even though these are gross simplifications of the ion transport underlying the creation of the action potential, they provide a brief insight into the relevance of ion homeostasis in the cell. Indeed, it is the ion concentration inside the cell which controls the transmembrane voltage which in turns controls the gating of numerous voltage-gated ion 
channels. If well, the prevalence of $\mathrm{Na}+, \mathrm{K}^{+}, \mathrm{Cl}^{-}, \mathrm{Ca}^{2+}$, or other voltage-gated channels depends on the cell type, there is a widespread prevalence of $\mathrm{K}^{+}$ions in the cytosol of excitable cells (Olaf 2009). On the other hand, the concentration of ions such as $\mathrm{Ca}^{2+}$ and $\mathrm{Na}^{+}$is highly regulated (Carafoli 2002, Clapham 2007). More interestingly, excitable cells such neurons and myocardiocytes are partially devoid of $\mathrm{Ca}^{2+}$ in the resting state. The lack of $\mathrm{Ca}^{2+}$ ion in the cytosol of cells may have initially arisen in cellular organisms to prevent the precipitation of phosphate, a widely used molecular building block for all life forms (Clapham 2007). Alternatively, it seems that whatever necessary role a divalent cationic ion could have served in the cell, this role has been achieved by use of $\mathrm{Mg}^{2+}$ (Laires, Monteiro \& Bicho 2004, Agus, Morad 1991). Unlike $\mathrm{Ca}^{2+}$, magnesium concentration in the cell is largely unregulated, with concentrations well in the 2-5 $\mathrm{mM}$ range in different compartments of the cell. This is in stark contrast to the $100 \mathrm{nM}$ concentration of $\mathrm{Ca}^{2+}$ in resting neurons and cardiomyocytes (Clapham 2007).

The energetic costs of maintaining low cytosolic concentration of $\mathrm{Ca}^{2+}$ is not without its benefits. In fact, vertebrates have evolved mechanisms that not only exploit the high extracellular concentration of $\mathrm{Ca}^{2+}$ for production of bone and teeth, but have also taken advantage of the chemical gradient of this ion that is present across the membrane. Calcium is maintained at about $2 \mathrm{mM}$ in the extracellular environment, providing a strong chemiosmosis force that facilitates the fast influx of this ion. Additionally, the resting transmembrane voltage of $-70 \mathrm{mM}$ to $-90 \mathrm{mV}$ present in neurons and cardiomyocytes further enhances the force driving influx of $\mathrm{Ca}^{2+}$ into the cell (YELLEN 1998). Whenever a route of passage across the plasma membrane is activated, calcium ions can diffuse into the cell at rates exceeding $10^{6}$ ion/sec (Naraghi, Neher 1997). These calcium influx routes 
can be formed by voltage-gate calcium channels (Cav), which are part of over a dozen other voltage-gated ion channels controlling action potentials in neurons (Bean 2007). In neurons, about 4 to 5 voltage-gated calcium channels are expressed, which include, the low-voltage activated T-type channels $\left(\mathrm{Ca}_{\mathrm{v}} 3\right)$ and the high-voltage activated L-type (Cav1), among other channels (Bean 2007). However, the slower dynamics of these channels preclude $\mathrm{Ca}^{2+}$ from inducing depolarization of of the action potential, a role which is fulfilled by the much faster sodium channels. Thus, calcium entry is maximal during the falling phase of the action potential. More importantly, the rich repertoire of $\mathrm{Ca}^{2+}$ channels result in a wide range of temporally and spatially controlled influx of this ion. Furthermore, cytosolic increases of calcium concentrations can also be triggered by release of $\mathrm{Ca}^{2+}$ from intracellular stores, such as the endoplasmic reticulum (Berridge, Lipp \& Bootman 2000, Gifford, Walsh \& Vogel 2007b). The precise pattern and frequency of calcium spikes (also referred as "blips", "puffs", or "waves”) is used by neurons to regulate numerous cellular processes. Indeed, the spatiotemporal regulation of $\mathrm{Ca}^{2+}$ spikes has made this metal an essential player in the regulation of neuronal signaling in the brain.

\subsection{Calcium binding proteins and neuronal calcium sensors}

Despite calcium playing a pivotal role during the evolution of the action potential in both neurons and cardiomyocytes, these cells have evolved mechanisms to make further use of this ion. The influx of $\mathrm{Ca}^{2+}$ in the cell is further utilized to monitor and control numerous physiological processes. For instance, under hypoxic conditions, depletion of ATP induces reduction of ATP-dependent $\mathrm{Ca}^{2+}$ pumps activity and reduction in $\mathrm{Ca}^{2+}$ removal. Accumulation of $\mathrm{Ca}^{2+}$ in the cell has salso been shown to trigger apoptosis among 
other processes (Jordán et al. 2000). Additionally, $\mathrm{Ca}^{2+}$ plays an essential role in regulating neurotransmitter release via interaction of this metal ion with synaptotagmins and SNARE proteins (Jahn, Scheller 2006, Hu et al. 2002). Gene regulation, morphological remodeling of cells (Babich, Burkhardt 2013), tumor progression (Chen et al. 2013), and cancer (Braunewell 2005), have also been known to be regulated by $\mathrm{Ca}^{2+}$.(Bito, Deisseroth \& Tsien 1997).

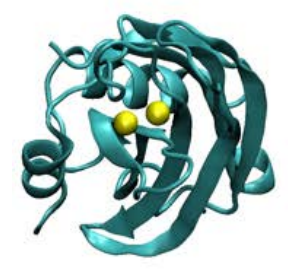

a)

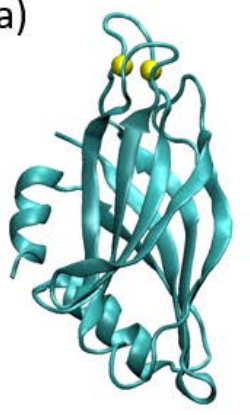

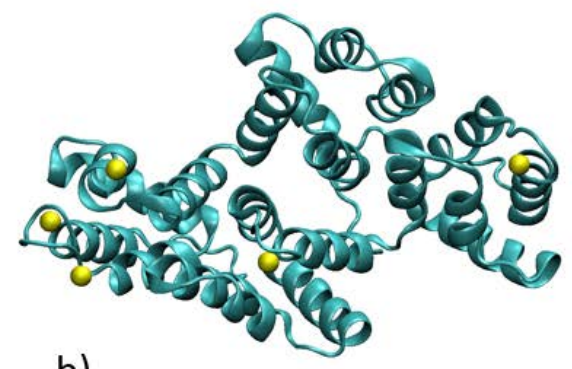

b)

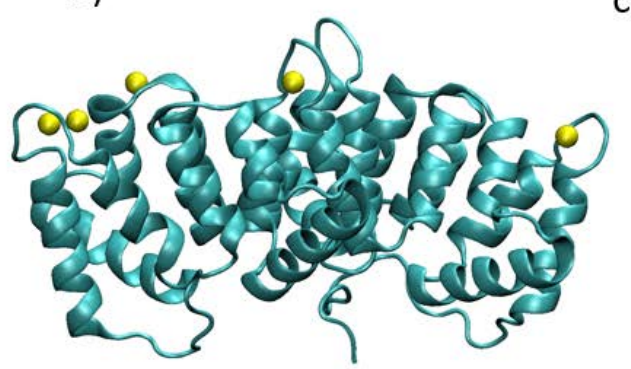

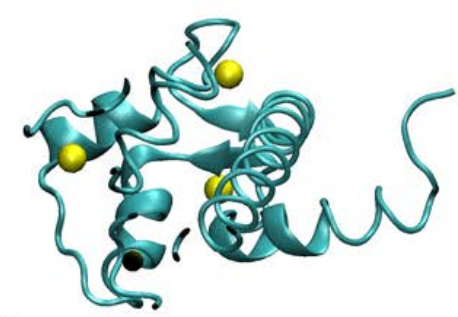

c)

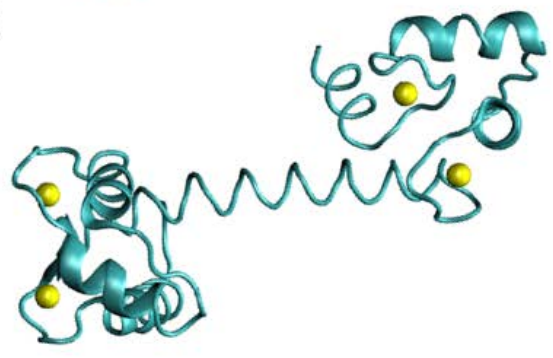

Figure 1.1: Three dimensional structures the most common families of $\mathrm{Ca}^{2+}$ binding proteins shown as a carton representation, with calcium metal shown as tallow spheres. (a) Synaptotagmin 1 with $\mathrm{Ca}^{2+}$ bound to the C2-domain, (b) Annexin III with $\mathrm{Ca}^{2+}$ bound at the Annexin domain, and (c) calmodulin with $\mathrm{Ca}^{2+}$ bound at the the EF-hand.

However, calcium itself would be devoid of any biological activity were it not for the numerous proteins that associate with this metal. Indeed, despite the drastic thousand-fold increase in $\mathrm{Ca}^{2+}$ concentration during repolarization of the action potential, most of the cytosolic $\mathrm{Ca}^{2+}$ is readily bound to proteins. The extent to which cells exploit $\mathrm{Ca}^{2+}$ signals is highlighted by the large number of calcium-binding proteins (CaBPs) which use this metal to trigger and/or regulate about every aspect of biological function (Clapham 2007, Berridge, Bootman \& Roderick 2003, Berridge, Bootman \& Lipp 1998). 
The ever-growing list of CaBPs known to directly interact with $\mathrm{Ca}^{2+}$ is only overshadowed by the numerous biological processes they regulate. During evolution, these CaBPs evolved at least three common $\mathrm{Ca}^{2+}$ binding motifs: the $\mathrm{C} 2$ domain (Nalefski, Falke 1996), the annexin domain (Raynal, Pollard 1994), and the EF-hand domain (Kuster et al. 1991). The structure, amino acid composition, and affinity for $\mathrm{Ca}^{2+}$ of these three domains are markedly different (Figure 1.1).
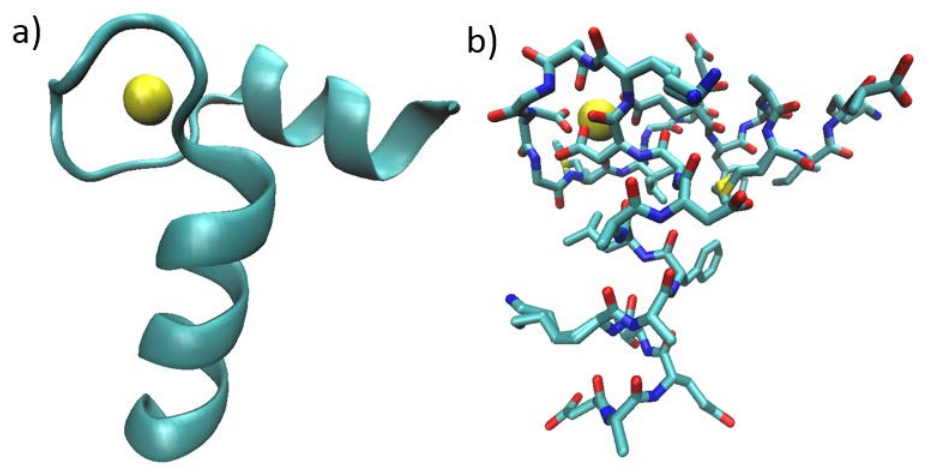

Figure 1.2: Three dimensional structure of a representative EF-hand motif (PDB: 2pvb). (a) Cartoon representation of the helix-loop-helix motif of the EF-hand with $\mathrm{Ca}^{2+}$ bound and shown as a yellow sphere. (b) The same structure as in (a) but shown as a licorice model, without hydrogens.

The EF-hand is the most widespread $\mathrm{Ca}^{2+}$ binding motif (Figure 1.2), with over 250 proteins belonging to this superfamily (Braunewell, Gundelfinger 1999). Proteins in this superfamily have been organized by those that function as calcium buffers and those which function as $\mathrm{Ca}^{2+}$ sensors (Yap et al. 1999, Ikura 1996). The $\mathrm{Ca}^{2+}$ buffer subfamily of EFhand proteins is exemplified by their ability to bind $\mathrm{Ca}^{2+}$ with the sole purpose of regulation of the $\mathrm{Ca}^{2+}$ levels inside the cell. Member of this subfamily include, parvalbumin, which was the first EF-hand protein whose $\mathrm{Ca}^{2+}$ binding loop was investigated in detail (Moews, Kretsinger 1975, Kretsinger, Nockolds 1973). In fact, the name of the EF-hand arose from the observation that the $\mathrm{E}$ and $\mathrm{F}$ helices of parvalbumin are orientated around the $\mathrm{Ca}^{2+}$ 
coordinating loop in a manner which resembles the thumb and index finger of a hand. Other members of this subfamily include Calbindin D9k, Calbindin D28k, and Calretin (Ikura 1996). The $\mathrm{Ca}^{2+}$ sensor subfamily is further divided into $\mathrm{Ca}^{2+}$ sensing proteins and neuronal calcium sensors (NCS) (Figure 1.3).

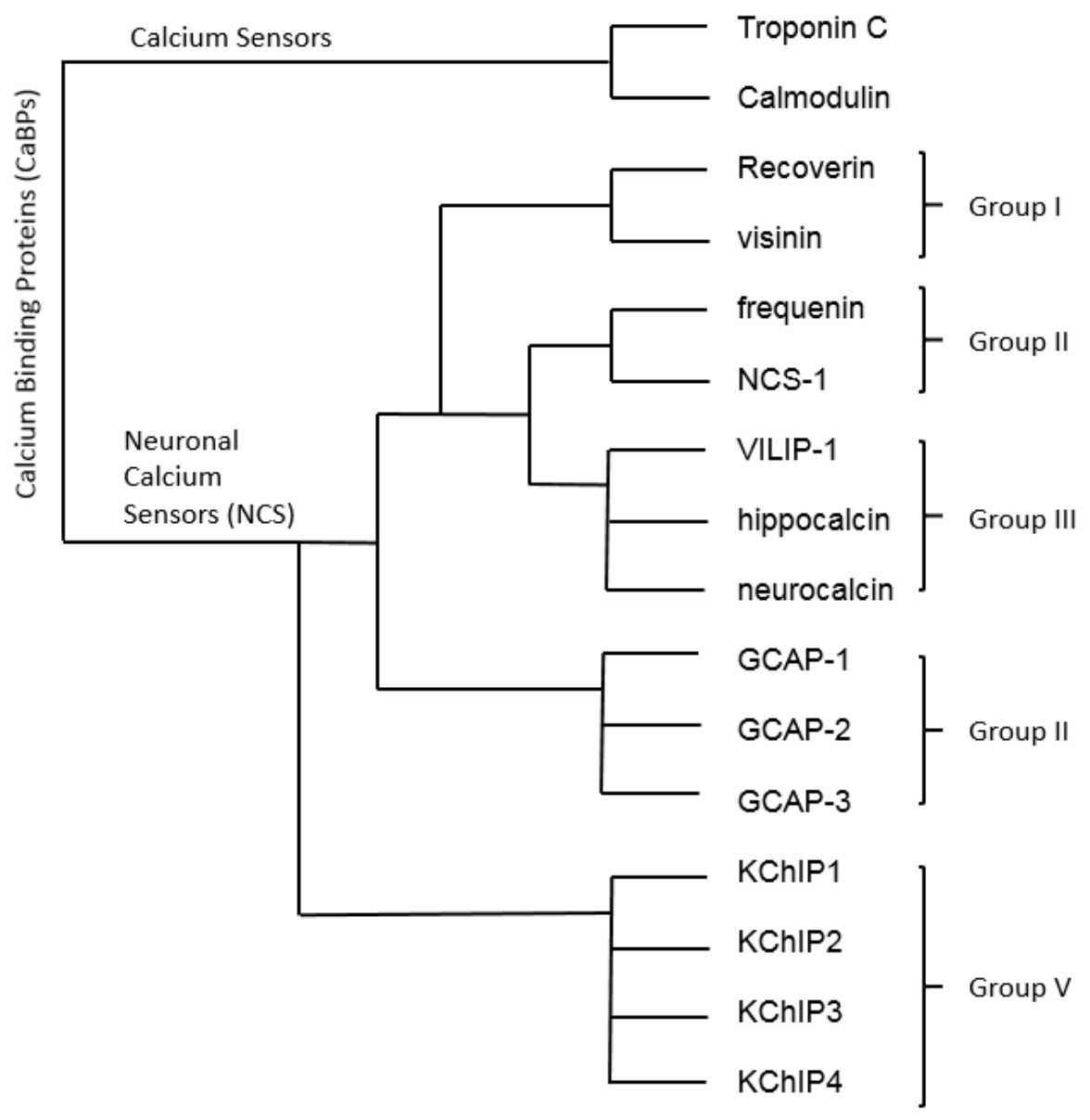

Figure 1.3. Dendrogram showing the relationship between members of the intracellular calcium binding proteins.

Member of the $\mathrm{Ca}^{2+}$ sensor group include Calmodulin, Troponin C, Calcineurin B, and the myosin light chains (McCue, Haynes \& Burgoyne 2010). The second group, the neuronal calcium sensors, are characterized by five subgroups, including recoverin (group I), NCS-1 (group II), visinin-like proteins (group III), guanylyl cyclase-activating proteins (group IV), and the potassium channel interacting proteins (group V) (Braunewell, 
Gundelfinger 1999, Yap et al. 1999). Of the NCS proteins, recoverin has been the most extensively studied (Ames et al. 1996, Ames, Hamasaki \& Molchanova 2002, Ames et al. 2006).
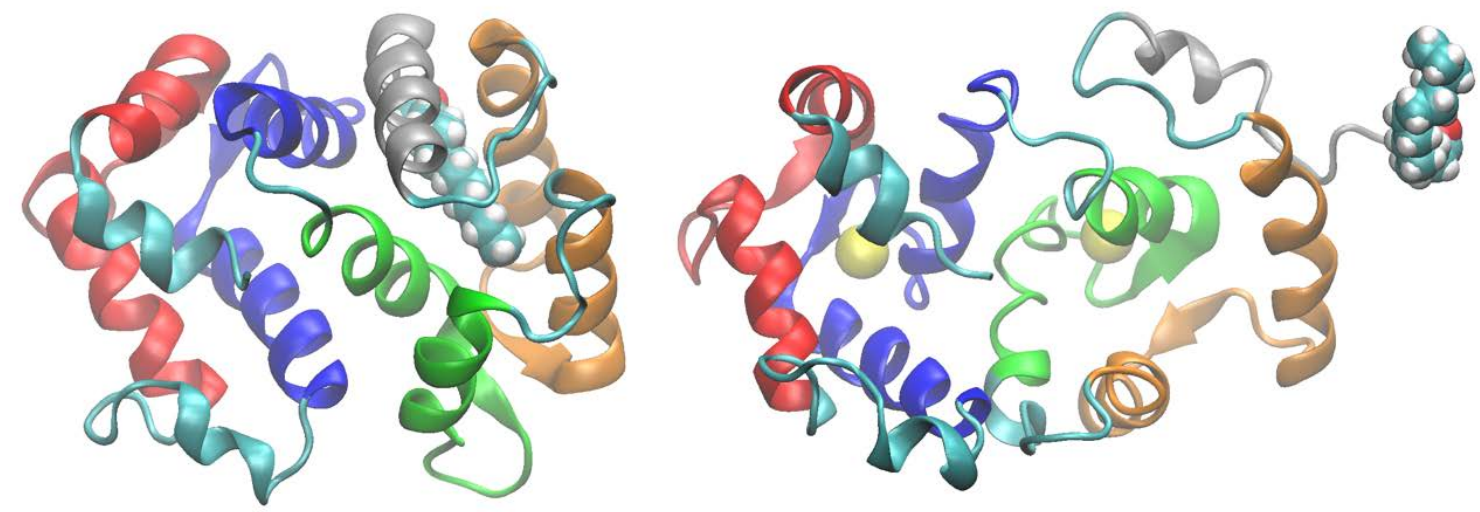

Figure 1.4. (left) NMR structure of $\mathrm{Ca}^{2+}$ free recoverin (PDB: 1iku, conformation 1) with EF-hand 1 shown in orange, EF-hand 2 in green, EF-hand 3 in blue, and EF-hand 4 in red. The myristoyl group is shown as spheres. (Right) NMR structure of $\mathrm{Ca}^{2+}$ bound to EF-hand 2 and 3 of recoverin (PDB: 1jsa, conformation 1). Calcium is shown as yellow spheres.

Most member of the NCS group are exclusively expressed in the brain and retina, with the exception of the newly discovered KChIP subgroup (Braunewell, Gundelfinger 1999, Paterlini et al. 2000, Polans, Baehr \& Palczewski 1996). The NCS proteins are characterized by the presence of four EF-hand domains of which the most N-terminal one (EF-hand 1) is always inactive and does not bind $\mathrm{Ca}^{2+}$, EF-hand 2 and 3 always bind calcium, and EF-hand 4 is functional in some members (Ames et al. 2006). Despite lack of $\mathrm{Ca}^{2+}$ binding to EF-hand 1 at the N-terminus, some NCS members are known to incorporate a myristoyl or palmitoyl group near this hand (Burgoyne et al. 2004). The hydrophobic prosthetic group has been shown to be effectively exposed upon $\mathrm{Ca}^{2+}$ binding to the Cterminus (Figure 1.4) (Ames et al. 1996, Takimoto, Yang \& Conforti 2002, Zozulya, Stryer 1992, Hughes et al. 1995). Numerous studies have also highlighted the submicromolar 
affinity for $\mathrm{Ca}^{2+}$ of EF-hands in NCS proteins, some of which can show a sequential, noncooperative, or cooperative behaviors (Cox et al. 1994, Ladant 1995, Osawa et al. 2005).

\subsection{The EF-hand $\mathrm{Ca}^{2+}$ binding motif}

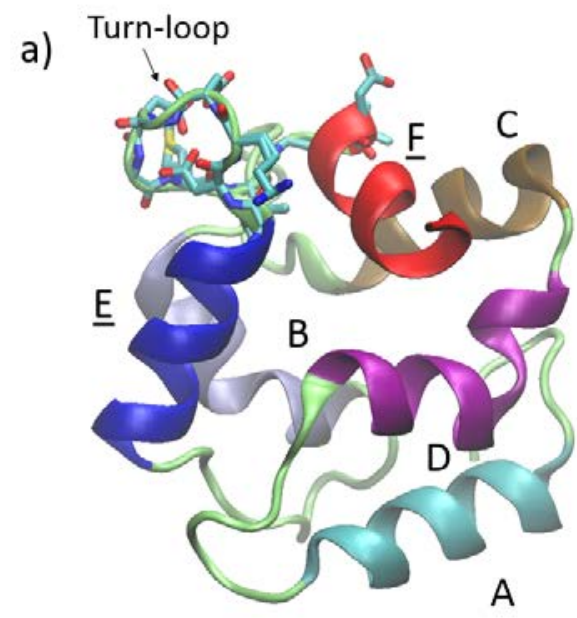

b)

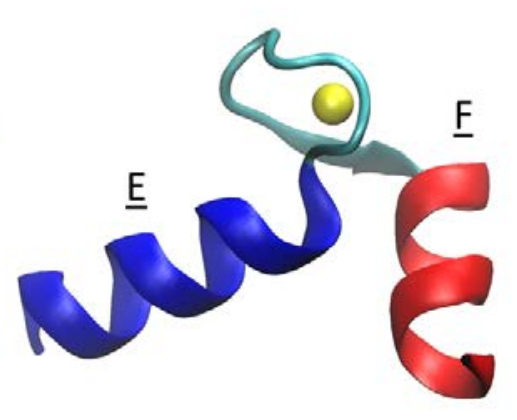

Figure 1.5. (a) Crystal structure of Parvalbumin (PBD: 2pvb). Helices are shown in different colors and turn-loop involved in $\mathrm{Ca}^{2+}$ chelation is shown in licorice model. The $\mathrm{E}$ and $\mathrm{F}$ hand helices forming the EF-hand are shown in blue and red, respectively. (b) EFhand formed by the helix E and $\mathrm{F}$ of the $\mathrm{Ca}^{2+}$ bound parvalbumin (PBD: 1b8r). The entering helix is colored in blue and exiting helix in red, with calcium shown as a yellow sphere.

Following the initial identification of the EF-hand motif in parvalbumin (Kretsinger, Nockolds 1973), this motif was fund to be present in troponin C (Collins et al. 1973), myosin light chains (Collins 1974), calmodulin (Stevens et al. 1976), and recoverin (Flaherty et al. 1993), to name a few. In fact, bioinformatics analysis indicate that this motif may be present in over 3000 other proteins (Grabarek 2006). The canonical EF-hand motif consist of a helix-loop-helix structure of roughly 30 amino acids, of which 12 comprise the $\mathrm{Ca}^{2+}$ binding loop (Figure 1.5) (Kretsinger, Nockolds 1973, Grabarek 2006). Analysis of numerous EF-hand containing proteins structures has revealed that: i) EF-hands are often present in pairs (Yap et al. 1999), ii) $\mathrm{Ca}^{2+}$ is always coordinated by the oxygen atoms of 
aspartic, glutamic, and backbone carbonyls, (Cates et al. 1999), and that iii) association of $\mathrm{Ca}^{2+}$ induces stabilization and/or structural changes on these proteins (Gifford, Walsh \& Vogel 2007b, Gifford, Walsh \& Vogel 2007a).

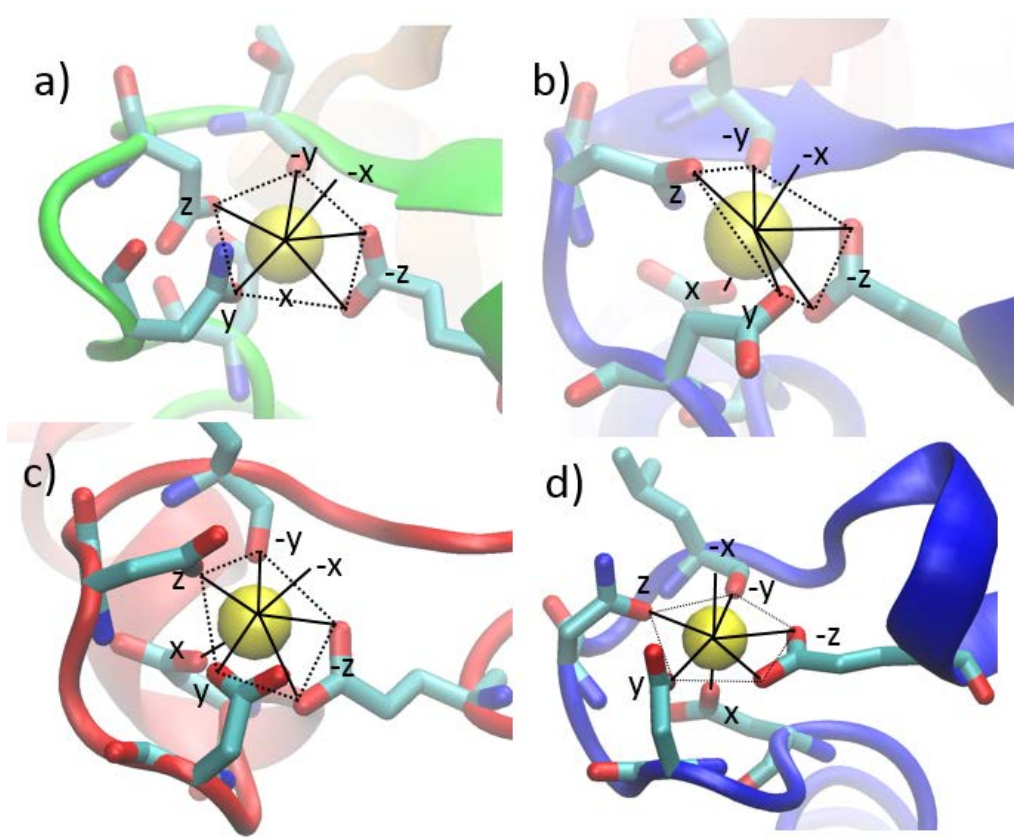

Figure 1.6. Coordination of $\mathrm{Ca}^{2+}$ at the $\mathrm{x}, \mathrm{y}, \mathrm{z},-\mathrm{y}$, and $-\mathrm{z}$ by: (a) Asp73, Gln75, Asp75, Thr79, and Glu84 of EF-hand 2 in recoverin (PDB: 1jsa); (b) Asp109, Asp111, Asp113, Thr115, and Glu120 of EF-hand 3 in recoverin (PDB: 1jsa); (c) Asp131, Asp133, Asp135, His140, and Glu129 of EF-hand 3 in calmodulin (PDB: 1exr); (d) Asp93, Asp95, Gln97, Leu99, and Glu104 in EF-hand 4 of calmodulin (PDB: 1exr).

1.3.1 EF-hand metal coordination

The coordination of $\mathrm{Ca}^{2+}$ and small chelating compounds, such as EDTA, is octahedral (Barnett 1979). However, the coordination of $\mathrm{Ca}^{2+}$ with oxygen atoms in EFhands varies among CaBPs, with a hepta-coordination being the most common (Grebarek 2006) (Figure 1.6). The coordination bonds in most EF-hands also follow a pentagonal bipyramidal organization. The heptahedral coordination of $\mathrm{Ca}^{2+}$ allows the assignment of a coordinated system, with $\mathrm{Ca}^{2+}$ as the origin, in which the first three ligands are assigned the $\mathrm{X}, \mathrm{Y}$, and $\mathrm{Z}$ axes, respectively. 


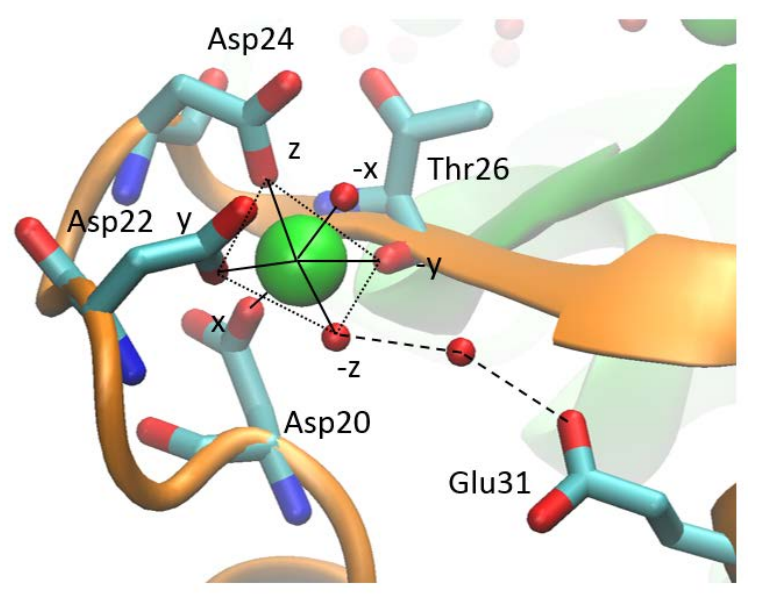

Figure 1.7. Crystal structure of calmodulin with $\mathrm{Mg}^{2+}$ bound at EF-1 (PDB: 3ucw). Coordinating amino acid involved in metal binding are shown in licorice model. Water molecules shown as red spheres.

Sequence analysis of numerous EF-hand loops has revealed that glutamic acid at the $-\mathrm{Z}$ coordination is common among $\mathrm{Ca}^{2+}$ selective EF-hands. At the $12^{\text {th }}$ position of the metal binging loop (-Z coordination), glutamic acid provides two coordination bonds to $\mathrm{Ca}^{2+}$. Nonetheless, in the absence of $\mathrm{Ca}^{2+}$ and millimolar concentrations of $\mathrm{Mg}^{2+}$, EF-hands can bind $\mathrm{Mg}^{2+}$ as shown in Figure 1.7 (Senguen, Grabarek 2012). The EF-hand loops containing aspartic acid at the $-\mathrm{Z}$ position often show higher selectivity for $\mathrm{Mg}^{2+}$ ions thank $\mathrm{Ca}^{2+}$ (da Silva, Kendrick-Jones \& Reinach 1995). The preference of $\mathrm{Mg}^{2+}$ over $\mathrm{Ca}^{2+}$ arises from the shorter length of the sidechain of asparagine, which is ideally suited for the hexacoordination necessary to chelate $\mathrm{Mg}^{2+}$. On the other hand, the hepta-coordination of $\mathrm{Ca}^{2+}$ by EF-hand loops with aspartic acid at position 12 is satisfied by addition of a water molecule bridging the second oxygen (Senguen, Grabarek 2012). At the center of the EFhand loop, the $-\mathrm{Y}$ coordination is provided by a backbone carbonyl, for example, threonine in Figure 1.7. Next to the $-Y$ coordination, a bulky hydrophobic amino acid stabilizes intermotifs interactions with the neighboring EF-hand (Grabarek 2006, Gifford, Walsh \& Vogel 
2007a). The interaction between adjacent EF-hands has been shown to play a role in regulation of $\mathrm{Ca}^{2+}$ binding cooperativity (Strynadka, James 1989). The EF-hand loop has been observed to form a short $\beta$-sheet with the adjacent EF-hand named the EF-hand- $\beta$ scaffold (Figure 1.8) (Grabarek 2006).

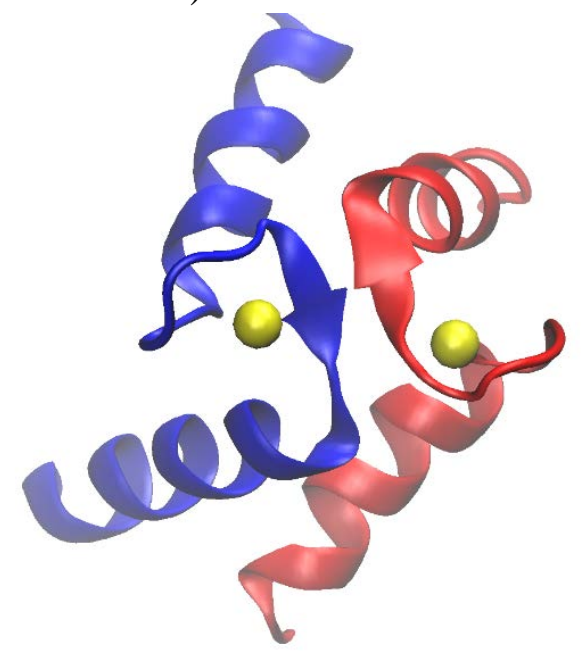

Figure 1.8. EF-hand pair showing the formation of a $\beta$-sheet connecting the adjacent $\mathrm{Ca}^{2+}$ binding loops (PDB: 2jul).

\subsubsection{EF-hand structural changes}

Structural analysis of CaM (Grabarek 2005) and TnC (Gagne 1997) mutants have revealed that $\mathrm{Ca}^{2+}$ binding follows a multi-step mechanism in which the $\mathrm{N}$-terminus of the EF-hand binding loop initially interacts with $\mathrm{Ca}^{2+}$. The initial interaction allows the subsequent coordination between $\mathrm{Ca}^{2+}$ and the bidentate Glu-12 at the $-\mathrm{Z}$ coordination. The coordination of the $-\mathrm{Z}$ amino acid positioned at $\sim 2 \AA$ triggers the structural rearrangement of the exiting helix and exposure of a hydrophobic cavity. The magnitude of the structural rearrangements induced by the $-\mathrm{Z}$ coordination is largely dependent on the EF-hand loop sequence as well as the inter-helical contacts between the entering and exiting helices of adjacent EF-hands (Yap et al. 1999, Nelson, Chazin 1998). In the model 
presented above, the EF-hand- $\beta$-scaffold functions as a hinge connecting the N- and Cterminus of the EF-hand loop.
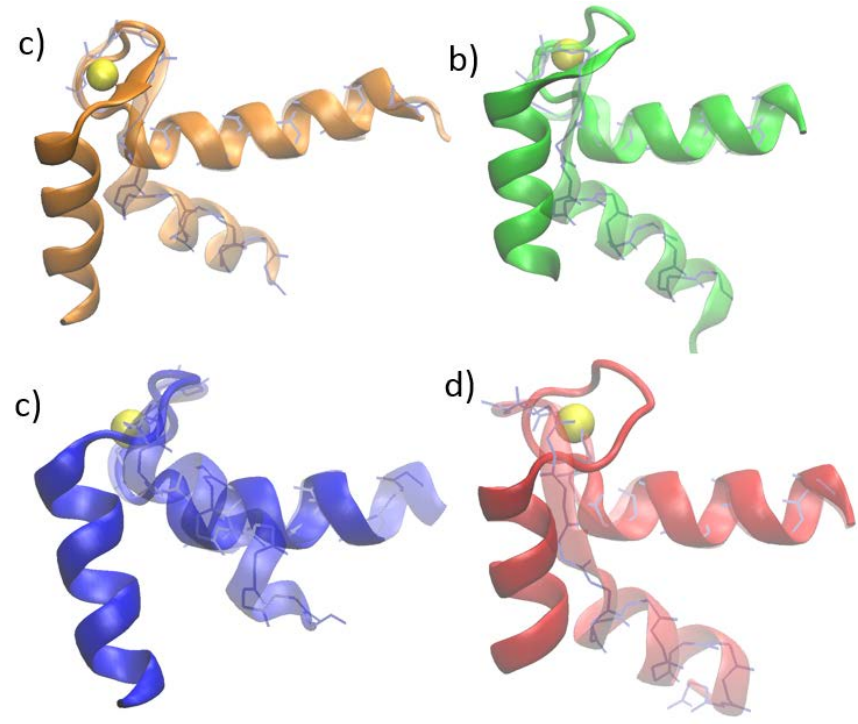

Figure 1.9. Reorientation of the EF-hands of calmodulin in the $\mathrm{Ca}^{2+}$ bound (PDB: 1cll) shown in solid color and $\mathrm{Ca}^{2+}$ free forms shown in diffuse color with backbone in thick cyan lines (PDB: 1cfc, conformation 1). (a) EF-hand 1, (b) EF-hand 2, (c) EF-hand 3, and (d) EF-hand 4.

The structural changes associated with $\mathrm{Ca}^{2+}$ binding to the turn-loop of EF-hands was first simplified by observations of the $\mathrm{Ca}^{2+}$ free and $\mathrm{Ca}^{2+}$ bound calmodulin and troponin C (Sun, Brandmeier \& Irving 2006, Yamniuk, Vogel 2004). These proteins revealed that $\mathrm{Ca}^{2+}$ induces conformational changes in the packing of the helices forming each EF-hand. The structural reorganization observed in $\mathrm{TnC}$ and $\mathrm{CaM}$ is often not observed in CaBPs that function as buffers, such as parvalbumin. The reorientation of the entering and exiting helices of the CaBPs that function as calcium sensors has been shown to follow a transition from an open to closed conformation (Figure 1.9). In the absence of $\mathrm{Ca}^{2+}$, the entering and exiting helices are oriented in antiparallel conformation with extensive hydrophobic contacts between helices. Coordination of $\mathrm{Ca}^{2+}$ by the EF-hand loop results in the repositioning of the helices to the open position in which the entering and 
exiting helices are in an almost perpendicular orientation. Even though this closed to open transition is observed in many calcium sensor proteins, the magnitude of the reorientation varies among proteins and between EF-hands in the same protein (Nelson, Chazin 1998).

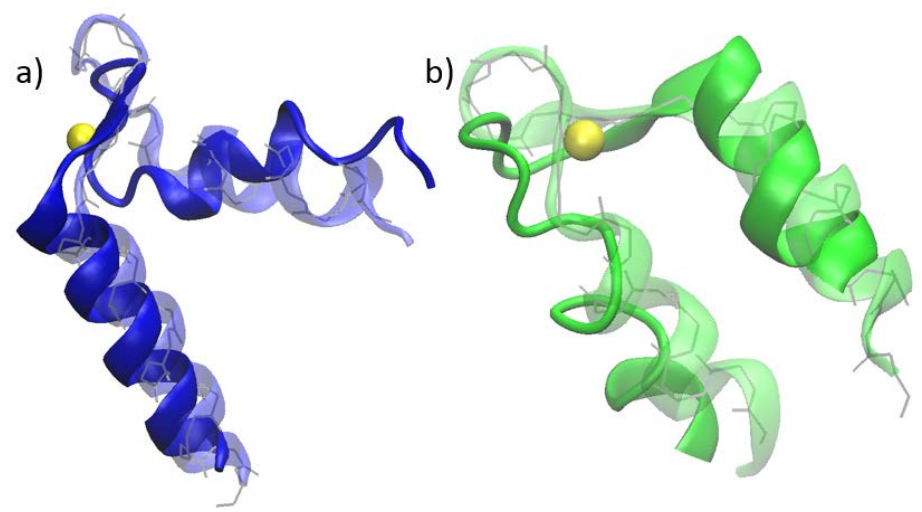

Figure 1.10. Reorientation of the EF-hands of myristoylated recoverin in the $\mathrm{Ca}^{2+}$ bound (PDB: 1iku) shown in solid color and $\mathrm{Ca}^{2+}$ free forms shown in diffuse color with backbone in thick cyan lines (PDB: 1jsa). (a) EF-hand 2 and (b) EF-hand 3.

Moreover, analysis of the $\mathrm{Ca}^{2+}$ bound and $\mathrm{Ca}^{2+}$ free structures of myristoylated recoverin reveals the lack of significant structural rearrangement of the helices in this protein (Figure 1.10). The lack of structural information on the $\mathrm{Ca}^{2+}$ free form of additional members of the NCS subfamily of EF-hand proteins precludes the interpretation of whether the static nature of EF-hand is specific to recoverin, or common among all NCS members. However, unpublished NMR data by Ames, et al. on the $\mathrm{Ca}^{2+}$ free myristoylated structure for neuronal calcium sensor-1 from fission yeast (PDB: 2l2e) shows a minimal reorganization of the EF-hand helices upon binding of calcium (Figure 1.11) (Ames et al. 2000)). Structural and molecular dynamics simulations have also highlighted the flexibility of the EF-hand conformation. Solution studies of a CaM mutant in which Glu-12 was mutated to glutamine in the loop of EF-hand 4 showed that this mutant can undergo a global conformational exchange between an open and closed conformation (Evenäs, Malmendal 
\& Akke 2001). Similar transitions were observed in the wild-type bovine CaM (Vigil et al. 2001, Chattopadhyaya et al. 1992).

a)

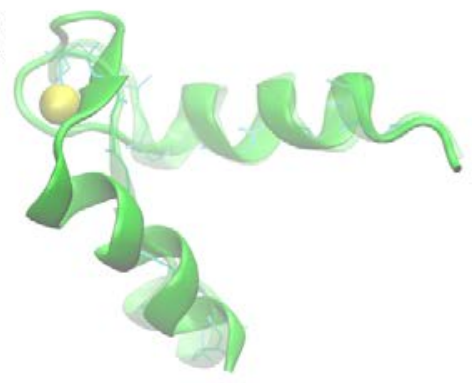

b)

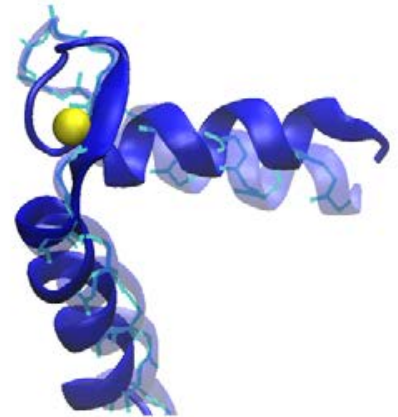

c)

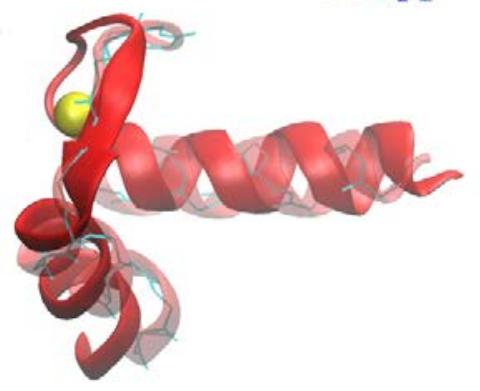

Figure 1.11. Reorientation of the EF-hands of myristoylated NCS-1 in the $\mathrm{Ca}^{2+}$ bound (PDB: 1fpw) shown in solid color and $\mathrm{Ca}^{2+}$ free non-myristoylated NCS-1 shown in diffuse color with backbone in in thick cyan lines (PDB: 2l2e). (a) EF-hand 2, (b) EF-hand 3, and (c) EF-hand 4.

\subsection{3 $\mathrm{Ca}^{2+}$ affinity of EF-hands}

The regulation of $\mathrm{Ca}^{2+}$ affinity in EF-hands can be understood by considering the energetic costs of structural changes and the biochemical energy provided by $\mathrm{Ca}^{2+}$ binding (Gifford, Walsh \& Vogel 2007a). In this aspect, as described below, $\mathrm{Ca}^{2+}$ binding and the induced structural changes can be analyzed in terms of increase of disorder of the system (increase in entropy) or due to the formation of new favorable interactions (decrease in enthalpy). Thus, thermodynamic analysis of the processes occurring during $\mathrm{Ca}^{2+}$ binding can provide some insight into the molecular mechanism controlling the metal affinity of EF-hands. 
Initially, both the protein and $\mathrm{Ca}^{2+}$ are solvated by a shell of water molecules. In particular, $\mathrm{Ca}^{2+}$ will have about 6 to 8 water molecules coordinating it while the negatively charged loop of the EF-hand will be solvated as well. In order for $\mathrm{Ca}^{2+}$ to associate with the chelating loop, both of them must release the water molecules. In the event that the water lost by $\mathrm{Ca}^{2+}$ is larger than that loss by dehydration of the loop, then the entropic gain will be substantial (Linse, Forsen 1995).

The correlation between water loss and affinity can be used to explain the lower affinity of EF-hand loops in which position 12 is replaced by aspartic acid instead of glutamic acid. In these loops, aspartic acid is only able to provide a single direct coordination bond to $\mathrm{Ca}^{2+}$, while the additional bond is bridged by a water molecule. The decrease in entropic gain has been used to explain the lower affinity of the EF-hands containing aspartic acid at position 12 in oncomodulin and parvalbumin (Henzl, Hapak \& Likos 1998, Henzl, Hapak \& Likos 1998, Lee et al. 2004). However, the flexibility of the EF-hand loop in the absence of $\mathrm{Ca}^{2+}$ can also negatively impact the entropic gain upon calcium binding. In addition, the exposure of hydrophobic amino acids upon $\mathrm{Ca}^{2+}$ binding will also result in rearrangement of water molecules around the protein, and decrease in entropy. However, as mentioned above, not all CaBPs undergo significant structural changes. Therefore, it is possible that the interplay of these entropic factors plays a direct role in $\mathrm{Ca}^{2+}$ affinity (Gifford, Walsh \& Vogel 2007a).

The contribution of enthalpy to $\mathrm{Ca}^{2+}$ affinity is less clear; however, a model termed the 'charge-ligand-balanced model' has been proposed to explain the energetic contribution of metal coordination in metal affinity (Ye et al. 2005). In the charge-ligandbalanced thermodynamic model, both the number of residues coordinating $\mathrm{Ca}^{2+}$ as well as 
the repulsion of adjacent residues and stabilization of the chelation geometry can modulate metal affinity. In addition, the extensive presence of hydrogen bonds stabilizing the EFhand loops can have significant contribution to the enthalpy. These electrostatic interactions can be extensively modified by the presence of hydrophobic amino acids in the flanking helices of EF-hands. Hydrophobic amino acids can induce changes in the dielectric environment, which in turn will modify the electrostatic forces between residues. The electrostatic shielding has been implicated in increasing the $\mathrm{Ca}^{2+}$ affinity of the Cterminus EF-hands of TnC, compared to that of calmodulin (Wang et al. 1998).

a)

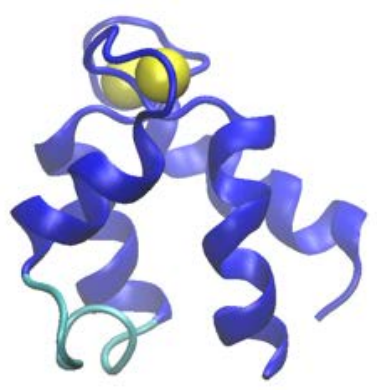

c)

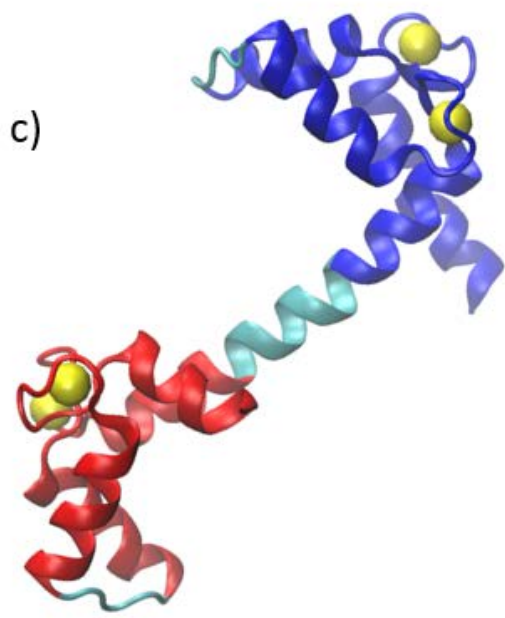

b)
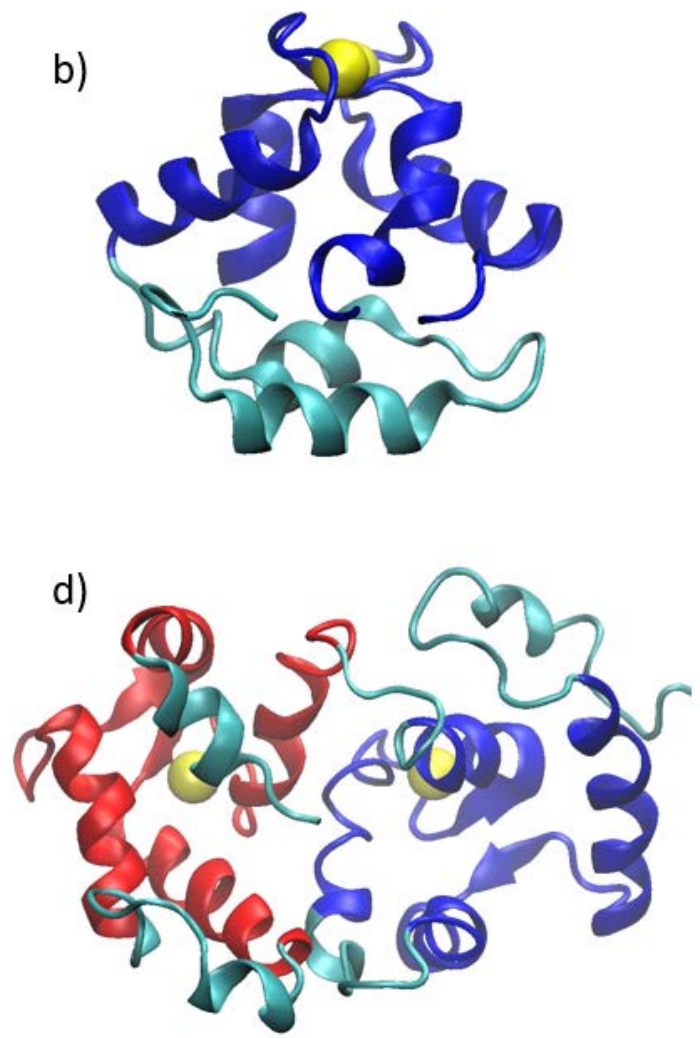

Figure 1.12. Domain organization of CaBPs, EF-hand pairs near the N-terminus shown in blue and near the C-terminus shown in red. Calcium is shown as a yellow sphere. (a) Calbindin D9k (PDB: 3icb), (b) parvalbumin (PDB: 1b8r), (c) calmodulin (PDB: 1cll), (d) recoverin (PDB: 1jsa). 


\subsection{EF-hand domain organization}

The local organization of the EF-hands (open vs closed) are not the only factors that control the activity and classification of CaBPs. Another aspect which separates CaBPs, is the interdomain organization of the EF-hand pairs. At least five types of domain organization of the EF-hand pairs have been observed (Figure 1.12). The simples domain consist of a single active EF-hand pair as observed in calbindin D9k (Szebenyi, Moffat 1986) and parvalbumin (Declercq et al. 1999). Second, in proteins such as TnC and CaM, two EF-hand pairs are connected via a flexible linker. The flexibility of the linker region plays a significant role in ligand recognition of these proteins (Sun, Brandmeier \& Irving 2006, Yamniuk, Vogel 2004). Third, proteins of the NCS subfamily contain short linkers, which position the EF-hand pairs at the $\mathrm{N}$ - and C-terminus is close contact. The orientation of the EF-hand domains in these proteins leads the formation of a large hydrophobic surface which mediates ligand recognition (McCue, Haynes \& Burgoyne 2010, Ames, Lim 2012). Fourth, the EF-hand domain organization in the sarcoplasmic calcium-binging proteins positions the $\mathrm{Ca}^{2+}$ binding loops at opposite sides of a tightly held hydrophobic cavity (Cook et al. 1993). In the globular domain organization, a hydrophobic cluster of hydrophobic amino acids lining the entering and exiting helices of each EF-hand form a large core. The overall globular fold of these proteins is quite distinct than that observed in the extended conformation of calmodulin. Lastly, proteins such as calbindin $\mathrm{D}_{28 \mathrm{~K}}$ and celretinin contain odd number of EF-hands (Kojetin et al. 2006). The presence of these unpaired EF-hand drives the homodimerization of these proteins in the presence of $\mathrm{Ca}^{2+}$, resulting in three pairs of EF-hands. 
Furthermore, small angle x-ray scattering and NMR have shown that the linker connecting the EF-hand domains of calmodulin is flexible (Barbato et al. 1992, Heidorn, Trewhella 1988). These observation have been supported by molecular dynamics simulations of $\mathrm{Ca}^{2+}$ bound calmodulin which revealed that the extended structure of the protein can collapse to form a compact structure similar to that observed in the presence of binding peptides (Shepherd, Vogel 2004). Similarly, the NMR structure of $\mathrm{Ca}^{2+}$ bound recoverin shows multiple conformations in which the N-terminus myristoyl group is largely free to move (Figure 1.13) (Ames et al. 1997).

a)

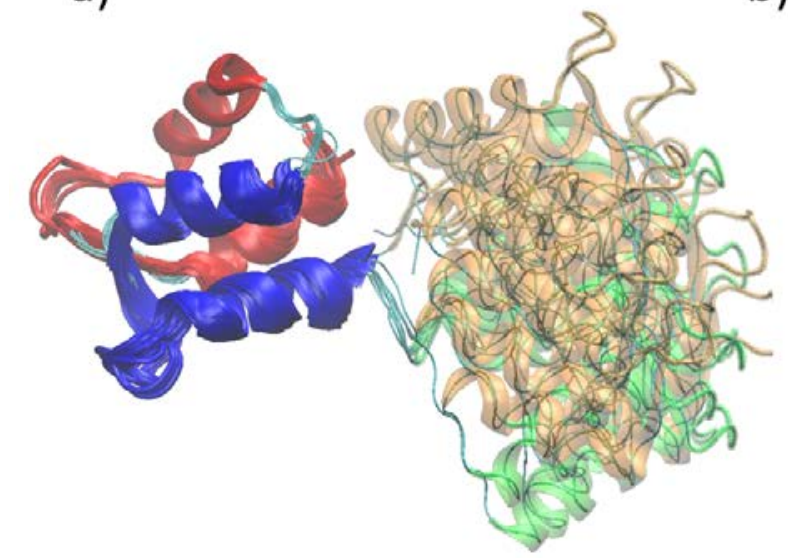

b)

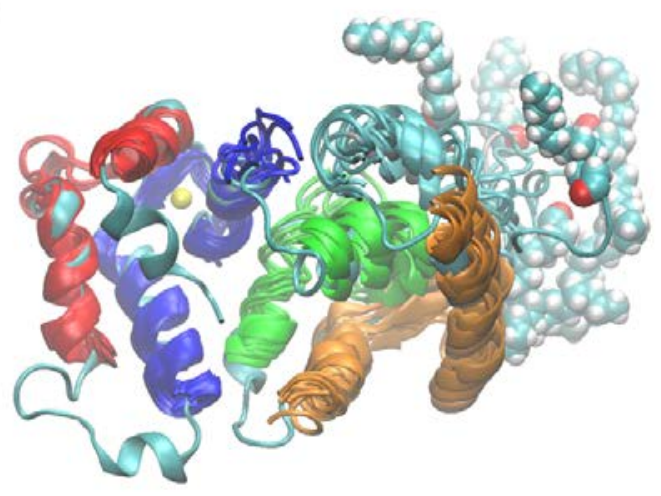

Figure 1.13. Interdomain structural dynamics of (a) $\mathrm{Ca}^{2+}$ free CaM (PDB: $1 \mathrm{cfc}$ ), where the C-terminus EF-hand 3 (blue) and EF-hand 4 (red) are aligned for clarity and the flexible $\mathrm{N}$-terminus is shown as diffuse orange and green ribbons. (b) The NMR structure of $\mathrm{Ca}^{2+}$ bound myristoylated recoverin (PDB: 1jsa). EF-hand 1,2,3, and 4 are colored in orange, green, blue, and red, respectively. The EF-hands 3 and 4 were aligned to show the flexibility of the N-terminus.

Molecular dynamics simulations of NCS-1 also support the idea that in solution, NCS-1 is more flexible than observed in crystal structures (Bellucci et al. 2013). In addition, unfolding experiments have shown that interdomain communications in the neuronal calcium sensor subfamily can regulate the stability of these proteins (Heidarsson et al. 2013). However, the role of interdomain dynamics in calcium binding proteins, especially 
the neuronal calcium sensors, have not been investigated in detail. Future work investigating the molecular mechanism by which domain organization affects protein activity will provide invaluable insight into their function.

\subsection{KChIPs and DREAM}

\subsubsection{Potassium channel interacting proteins}

The $\mathrm{K}^{+}$channel interacting proteins (KChIPs) subgroup are the newest member of the NCS family. This group was discovered by pull down search of proteins which could interact with the N-terminus of Kv4 channels (An et al. 2000). Association of these proteins with the $\alpha$-subunit of $K_{V} 4$ channels was found to reconstitute several biophysical features of A-type currents measured in vivo. Northern blot analysis also revealed that KChIP1 is highly expressed in the brain, KChIP2 is expressed in the brain, heart, and lung; while KChIP3 is expressed mainly in the brain (An et al. 2000). The last member of this group, KChIP4, was discovered by database search of proteins with similar sequence homology to KChIP1/2/3 (Holmqvist et al. 2002). All four members of this group, KChIP1, KChIP2, KChIP3, and KChIP4, are known to interact with the N-terminus domain of $\mathrm{K}^{+}$voltagegated channels and regulate the fast ISA and $\mathrm{I}_{\mathrm{TO}}$ currents in the brain and heart, respectively (An et al. 2000). Interaction of KChIP1, KChIP2, and KChIP3 with $\mathrm{K}_{v} 4$ channels expressed in xenopus oocytes results in an increase in current density, acceleration of recovery from inactivation, and slower inactivation kinetics (An et al. 2000). Similar effects on the biophysical properties of neural fast inactivating $\mathrm{K}^{+}$currents have been observed for KChIP1 (Bourdeau et al. 2011) and KChIP3 (Witzel, Fischer \& Bähring 2012). 


\section{Panel A: KChIP1 isoforms}

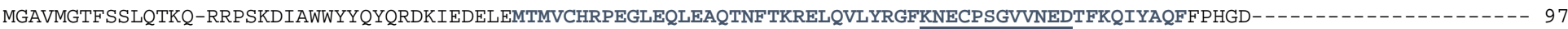
MGAVMGTFSSLQTKQR - - _. MGAVMGTFSSLQTKQR -

- - MSGCSKRCKLGFVKFAOTIFKLITGTLSKDKIEDELEMTMVCHRPEGLELLEAOTNFTKRELOVLYRGFKNECPSGVVNEDTFKOIYAOFFPHGD $\ldots$ MGAVMGTFSSLQTKQR - - ....-RPSKDKIEDELEMTMVCHRPEGLQLEAQTNFTKRELQVLYRGFKNECPSGVVNEDTFKQIYAQFFPHGALPCLEGSPCVEFLPPSPALLFC 108 EF-hand 1

- - -ASTYAHYLFNAFDTTQTGSVKFEDFVTALSILLRGTVHEKLRWTFNLYDINKDGYINKEEMMDIVKAIYDMMGKYTYPVLKEDTPRQHVDVFFQKMDKNKDGIVTLDEFLESCQEDD 214 (165 - - ASTYAHYLFNAFDTTQTGSVKFEDFVTALSILLRGTVHEKLRWTFNLYDINKDGYINKEEMMDIVKAIYDMMGKYTYPVLKEDTPRQHVDVFFQKMDKNKDGIVTLDEFLESCQEDD 175 - - ASTYAHYLFNAFDTTQTGSVKFEDFVTALSILLRGTVHEKLRWTFNLYDINKDGYINKEEMMDIVKAIYDMMGKYTYPVLKEDTPRQHVDVFFQKMDKNKDGIVTLDEFLESCQEDD 212 LVDASTYAHYLFNAFDTTQTGSVKFEDFVTALSILLRGTVHEKLRWTFNLYDINKDGYINKEEMMDIVKAIYDMMGKYTYPVLKEDTPRQHVDVFFQKMDKNKDGIVTLDEFLESCQEDD 228 EF-hand 2 EF-hand 3

NIMRSLOLFONVM 227 KChIP1.1 NIMRSLOLFONVM $178 \mathrm{KChIP1.2}$ NIMRSLOLFONVM $188 \mathrm{KChIP1.3}$ NIMRSLQLFQNVM 225 KChIP1.4 (⿸丆口

Figure 1.14. Sequence alignment of KChIP 1 (panel A) and KChIP2 (panel B) for different isoforms. Identical amino acid positions shown in bold blue color. The turn-loop amino acids of each EF-hand are underlined. The position of the EF-hands are shown in colored cartoons for reference. Isoform 4 and 8 of KChIP2 not included for clarity.

\section{Panel B: KChIP2 isoforms}

MRGQGRKESLSDSRDLDGSYDQLTGHPPGPTKKALKQRFLKLLPCCGPQALPSVSE- - _ _ - _ - - TLAAPASLRPHRPRLLDPDSVDDEFELSTVCHRPEGLEQLEQTKFTRK 105 MRGQGRKESLSDSRDLDGSYDQLTGHPPGPTKKALKQRFLKLLPCCGPQALPSVSE - $\ldots$ MRGQGRKESLSDSRDLDGSYDQLTMRGQGKESLSDSRDLDGSYDLT MRGQGRKESLSDSRDLDGSYDQLTGHPPGPTKKALKQRFLKLLPCCGPQALPSVSEIGRVFRFLGDSSLPSALAAPASLRPHRPRLLDPDSVDDEFELSTVCHRPEGLEQLQEQTKFTRK 120 MRGQGRKESLSDSRDLDGSYDQLTMRGQGRKESLSDSRLDGSYDQLT $\ldots$

ELOVLYRGFKN- - - - -ECPSGIVNEENFKOIYSOFFPOGDSSTYATFLFNAFDTNHDGSVSFEDFVAGLSVILRGTVDDRLNWAFNLYDLNKDGCITKEEMLDIMKSIYDMMGKYTYP 218 ELOVLYRGFKN - - - - -ECPSGIVNEENFKOIYSOFFPOGDSSTYATFLFNAFDTNHDGSVSFEDFVAGLSVILRGTVDDRLNWAFNLYDLNKDGCITKEEMLDIMKSIYDMMGKYTYP 200 ELOVLYRGFKN- - - -ECPSGIVNEENFKQIYSOFFPOGDSSTYATFLFNAFDTNHDGSVSFEFVAGLSVILRGTVDDRLNWAFNLYDLNKDGCITKEEMLDIMKSIYDMMGKYTYP 168 (168) ELVW ELQVYR ELQVLYRGKN ELQVLYRGFKN- - - -ECPSGIVNEENFKQIYSQFFPQGDSSTYATFLFNAFDTNHDGSVSFE EF-hand 1

EF-hand 2

EF-hand 3

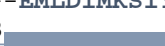

ALREEAPREHVESFFQKMDRNKDGVVTIEEFIESCQKDENIMRSMQLFDNVI 270 KChIP2.1

ALREEAPREHVESFFQKMDRNKDGVVTIEEFIESCQKDENIMRSMQLFDNVI 252 KChIP2.2

ALREEAPREHVESFFQKMDRNKDGVVTIEEFIESCQKDENIMRSMQLFDNVI 220 KChIP2.3

ALREEAPREHVESFFQKMDRNKDGVVTIEEFIESCQKDENIMRSMQLFDNVI 216 KChIP2.5

ALREEAPREHVESFFQKMDRNKDGVVTIEEFIESCQKDENIMRSMQLFDNVI 285 KChIP2.6

ALREEAPREHVESFFQKMDRNKDGVVTIEEFIESCQKDENIMRSMQLFDNVI 227 KChIP2.7

ALREEAPREHVESFFQKMDRNKDGVVTIEEFIESCQKVQLPALYITLTWTQA 184 KChIP2.9

$$
\text { EF-hand } 4
$$




\section{Panel C: KChIP3 isoforms}

MOPAKEVTKASDGSLLGDLGHTPLSKKEGIKWQRPRLSRQALMRCCLVKW - - _ - _ - _ ILSSTAPOGSDSSDSELELSTVRHOPEGLDQLQAQTKFTKKELOSLYRGFKNECPTGLVD 110 MQPAKEVTKASDGSLLGDLGHTPLSKKEGIKWQRPRLSRQALMRCCLVKW- - - - - - ILSSTAPQGSDSSDSELELSTVRHQPEGLDQLQAQTKFTKKELQSLYRGFKN - - - - 102 8. EF-hand 1

EDTFKLIYAQFFPQGDATTYAHFLFNAFDADGNGAIHFEDFVVGLSILLRGTVHEKLKWAFNLYDINKDGYITKEEMLAIMKSIYDMMGRHTYPILREDAPAEHVERFFEKMDRNQDGVV 230 - - - GDATTYAHFLFNAFDADGNGAIHFEDFVVGSILLRGTVHEKLKWAFNLYDINKDGYITKEEMLAIMKSIYDMMGRHTYPILREDAPAEHVERFFEKMDRNQDGVV 208 EDTFKLIYAQFFPOGDATTYAHFLFNAFDADGNGAIHFEDFVVGLSILLRGTVHEKLKWAFNLYDINKDGYITKEEMLAIMKSIYDMMGRHTYPILREDAPAEHVERFFEKMDRNODGVV 204
EF-hand 2
hand 2
EF-hand 3
EF-hand 4

TIEEFLEACQKDENIMSSMQLFENVI 256 KChIP3.1
TIEEFLEACQKDENIMSSMQLFENVI $234 \mathrm{KChIP3.2}$

\begin{tabular}{l} 
TIEEFLEACKDENIMSSMQLFENVI $230 \mathrm{KChIP3.3}$ \\
\hline TIEEFLEACQKDENIMS
\end{tabular}

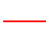

\section{Panel D: KChIP4 isoforms}

MNVRRVESISAQLEEASSTGGFLYAQNSTKRSIKERLMKLLPCSAAKTSSPAIQNSVEDELEMATVRHRPEALELLEAQSKFTKKELQILYRGFKNECPSGVVNEETFKEIYSQFFPQGD 120 MNVRRVESISAQLEEASSTG-1-10 DSVEDELEMATVRHRPEALELLEAQSKFTKKELQILYRGFKNECPSGVVNEETFKEIYSQFFPQGD 86 (1) MNLEGLEMIAVLIVMSGCRKRCREILKFAQYLLRLLTGSLHTDSVEDELEMATVRHRPEALELLEAQSKFTKKELQILYRGFKNECPSGVVNEETFKEIYSQFFPQGD 95

$$
\text { EF-hand } 1
$$

STTYAHFLFNAFDTDHNGAVSFEDFIKGLSILLRGTVQEKLNWAFNLYDINKDGYITKEEMLDIMKAIYDMMGKCTYPVLKEDAPRQHVETFFQKMDKNKDGVVTIDEFIESCQKDENIM 240 STTYAHFLFNAFDTDHNGAVSFEDFIKGLSILLRGTVOEKLNWAFNLYDINKDGYITKEEMLDIMKAIYDMMGKCTYPVLKEDAPROHVETFFQKMDKNKDGVVTIDEFIESCQKDENIM 206 STTYAHFLFNAFDTDHNGAVSFEDFIKGLSILLRGTVQEKLNWAFNLYDINKDGYITKEEMLDIMKAIYDMMGKCTYPVLKEDAPRQHVETFFQKMDKNKDGVVTIDEFIESCQKDENIM 178 STTYAHFLFNAFDTDHNGAVSFEDFIKGLSILLRGTVQEKLNWAFNLYDINKDGYITKEEMLDIMKAIYDMMGKCTYPVLKEDAPROHVETFFOKMDKNKDGVVTIDEFIESCQKDENIM 219 STTYAHFLFNAFDTDHNGAVSFEDFIKGLSILLRGTVQEKLNWAFNLYDINKDGYITKEEMLDIMKAIYDMMGKCTYPVLKEDAPROHVETFFOKMDKNKDGVVTIDEFIESCOKDENIM 215

EF-hand 2
EF-hand 3

RSMQLFENVI 250 KChIP4.1

RSMQLFENVI 216 KChIP4.2

RSMQLFENVI 188 KChIP4.3

RSMQLFENVI 229 KChIP4.4

RSMQLFENVI 225 KChIP4.5

Figure 1.15. Sequence alignment of KChIP3 (panel C) and KChIP4 (panel D) for different isoforms. Identical amino acid positions shown in bold blue color. The turn-loop amino acids of each EF-hand are underlined. For EF-hand that are active, the amino acids providing the coordination to the metal ion are shown in red. The position of the EF-hands are shown in colored cartoons for reference. 
The repertoire of KChIPs is further expanded by the presence numerous KChIP splice variants (Figure 1.14 and 1.15) (Deschênes et al. 2002, Van Hoorick et al. 2003). In total, twenty two isomers have been identified ( 5 for KChIP1, 9 for KChIP2, 3 for KChIP3, and 5 for KChIP4), the most common difference among these isomers is the deletion or insertion of amino acid sequences near the N-terminus. In particular, KChIP1.2 and KChIP3.2 isoforms which have a deletion of the metal binding loop of EF-hand 2. Interestingly, a minimal KChIP2 isoform has been discovered, in which only EF-hand 3 and 4 are present; this isoform was shown to still effectively regulate potassium currents (Patel, Campbell \& Strauss 2002).
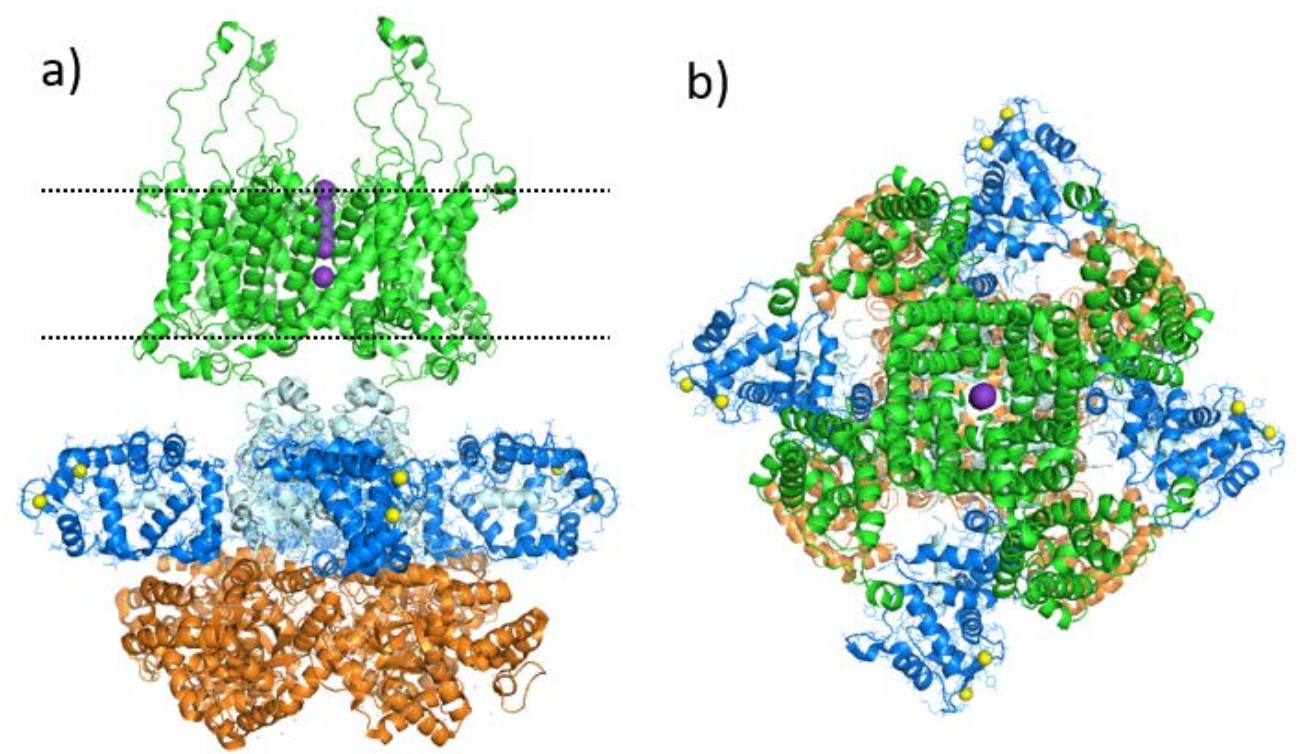

Figure 1.16. Cartoon representation of a hypothetical potassium channel macromolecular complex, dotted lines represent the transmembrane thickness. The model was constructed by structural alignment of the full length shaker potassium channel $\mathrm{K}_{\mathrm{v}} 1.2$ (PDB: 3lut) and the crystal structure of the KChIP1-Kv4.3 T1 domain complex (PDB: 2i2r). (a) Side view: the transmembrane domain containing the $\mathrm{K}^{+}$selectivity pore is shown in green with potassium as solid purple spheres. The T1-domain is shown in light blue and $\mathrm{KChIP1}$ bound to $\mathrm{Ca}^{2+}$ (yellow sphere) is show in dark blue. The $\beta$ subunit is shown in orange. (b) Top vie of the same complex. 
This minimal construct highlights the importance of the C-terminus of KChIPs in $\mathrm{K}^{+}$channel regulation. KChIPs have also been shown to associate with Kv4 channels in many different combination of different KChIPs/isomers, which results in a wider range of $\mathrm{K}^{+}$channel tuning (Bourdeau et al. 2011, Kunjilwar, Qian \& Pfaffinger 2013b, Liss et al. 2001).
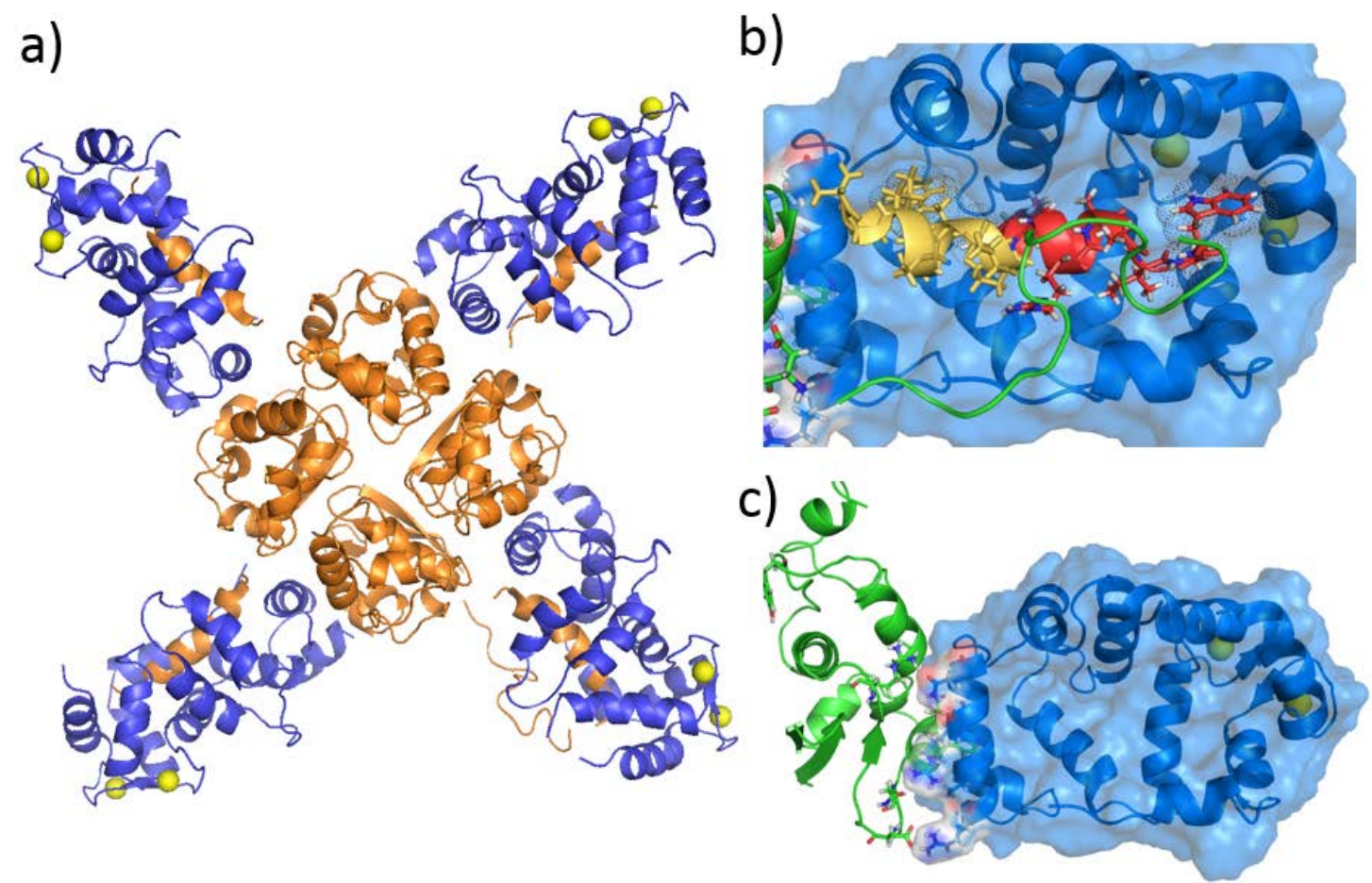

Figure 1.17. Cartoon representation of the KChIP1:Kv4.3 T1 domain protein complex (PDB: 2nz0). (a) Octameric cross shaped complex of four KChIP1 (dark blue) and four T1 domain monomers (orange). (b) Close up of the interaction between the $\mathrm{N}$-terminus hydrophobic region of the T1 domain ("Site 1") and the hydrophobic surface of KChIP1. Residues 1 to 10 shown in yellow and 10 to 20 in red. (c) Contact between the N-terminus of KChIP1 and "Site 2" (residues 70 to 90 , shown in green licorice) of Kv4.3.

Structural studies have shown that KChIP1.1 associates with the cytosolic portion of the $\alpha$ subunit of the Kv4 potassium channels, to form octameric complexes (Figure 1.16) (Pioletti et al. 2006, Scannevin et al. 2004, Wang et al. 2007). The KChIP1:Kv4 interaction is mediated by two surface contacts on KChIP1 and the cytosolic T1 domain of the Kv4 channels (Scannevin et al. 2004). The KChIP1:Kv4 protein complex forms an octameric cross shaped organization (Figure 1.17) (Pioletti et al. 2006, Wang et al. 2007). The most predominant interaction is 
between a large hydrophobic surface on KChIP1 and the hydrophobic N-terminus $\alpha$-helix of the Kv4 channel. This hydrophobic interaction buries about $2300 \AA 2$ of exposed surface and involves two well conserved bulky residues on the N-terminus of Kv4 channels, Trp8 and Trp19 (Figure 1.18) (Wang et al. 2007).

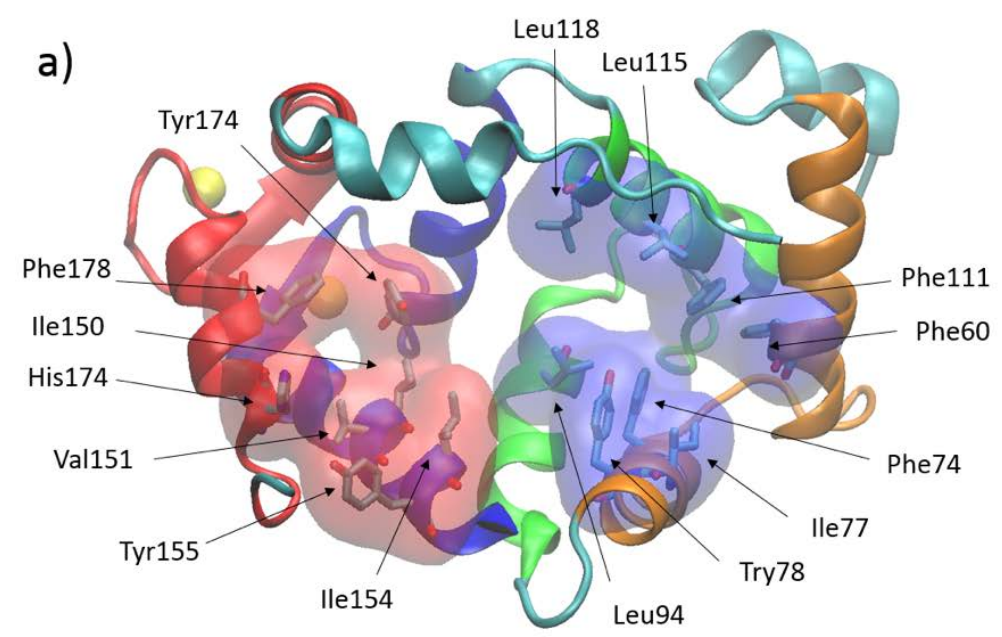

b)

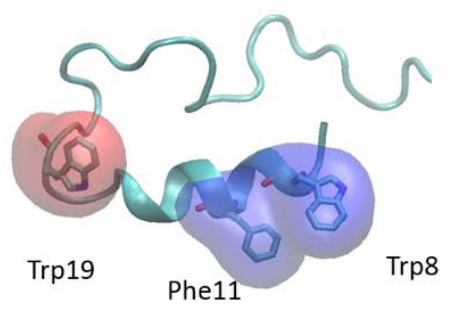

Figure 1.18. (a) Cartoon representation of KhIP1 (PDB: 2nz0) using the same coloring scheme as in Figure 1.4 with residues forming the interface with the N-terminus of Kv4.3 shown as licorice model. (b) Alpha-helix at the $\mathrm{N}$-terminus of the $\mathrm{K}_{\mathrm{v}} 4.3$ potassium channel with hydrophobic amino acids involved in binding of KChIP1 shown as licorice model.

The hydrophobic surface on KChIP1 is lined by two groups of amino acids at the N- and C-terminus. The C-terminus cluster includes Tyr134, Ile150, Val151, Tyr155, Ile154, Phe178, and His174, which interact with Trp19 of the Kv4 channel (Figure 1.18). At the N-terminus, residues Ile77, Tyr78, Phe74, Leu94, Phe111, Leu115, Leu118, Phe60 and Gly59 are in direct contact with Trp8 and Phe11 of the N-terminus of the Kv4 channel. The second contact interface between KChIP1 and the T1 domain involves hydrophobic and charged residues at the entering helix of EFhand 1 on KChIP1 and residues 70 to 78 of the T1 domain (Figure 1.19) (Scannevin et al. 2004, Wang et al. 2007). In this interface Phe73 of the T1 domain interacts through stacking interactions with Leu39, Leu42, Leu43, Tyr57 and Phe108 of KChIP1. In addition, two salt bridges between Glu77 and Asp78 of the T1 domain and Lys50 and Arg51 on KChIP1 stabilize this interaction. 
Even though all members of the KChIP group of NCS proteins associate with $\mathrm{K}_{\mathrm{v}} 4$ channels, only the structure of KChIP1 has been obtained in complex with these channels (Pioletti et al. 2006, Liang et al. 2009). However, all KChIPs share a high sequence homology (58 \% among residues 60 to 256) at the core domain spanning EF-hands 1, 2, 3, and 4 (Figure 1.20). Thus it has been proposed that similar structural organization of the KChIP:Kv4.3 complex may be present in other KChIPs (Liang et al. 2009). Indeed, all amino acids involved in stabilizing the KChIP1:Kv4 complex are conserved among all KChIP proteins (Figure 1.20, highlighted in green).

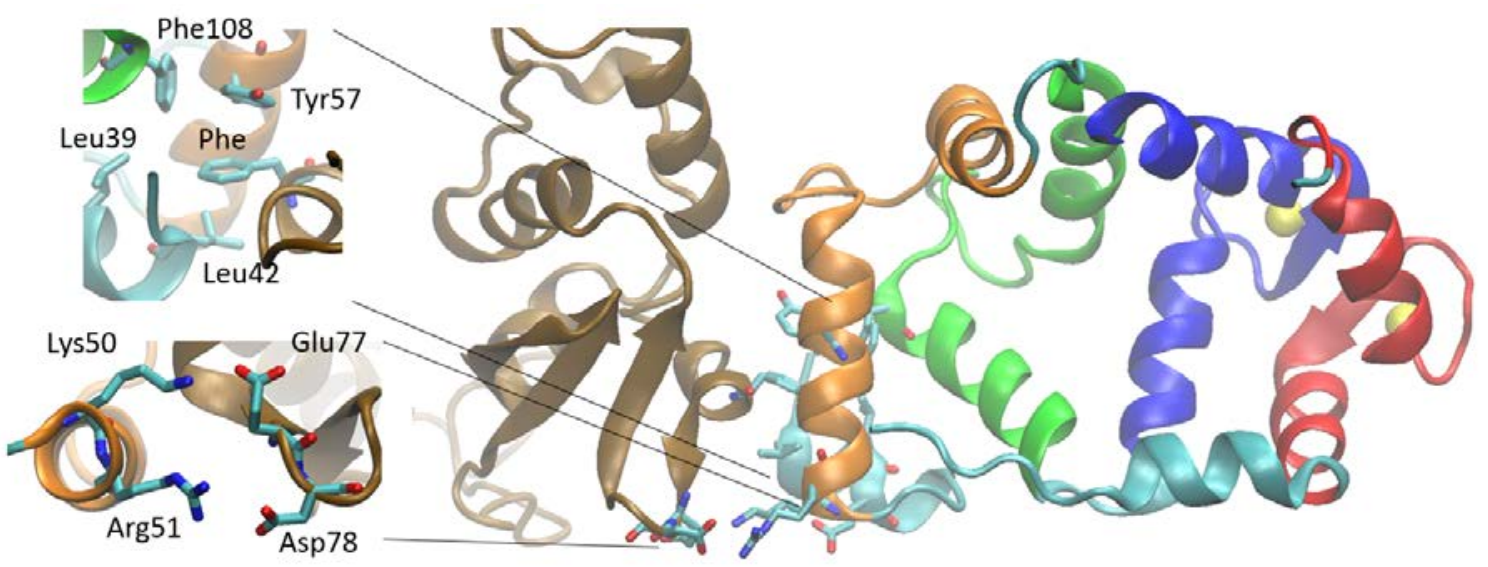

Figure 1.19. Cartoon representation of the second interface "Site 2" between KhIP1 and the N-terminus of Kv4.3 (PDB: 2nz0) using the same coloring scheme as in Figure 1.4. The T1 domain is shown in brown. The inset show the hydrophobic (top) and electrostatic (bottom) interactions.

All members of the KChIP subgroup contain four EF-hand domains, with EF-hand 3 and 4 showing the strongest affinity for $\mathrm{Ca}^{2+}$, while EF-hand 2 is proposed to bind $\mathrm{Mg}^{2+}$ preferentially (Osawa et al. 2005, Lusin et al. 2008). The selectivity for $\mathrm{Mg}^{2+}$ over $\mathrm{Ca}^{2+}$, is believe to arise due to the mutation of glutamic acid at position 12 of the EF-hand loop to aspartic acid. However, it is not known whether the $\mathrm{Ca}^{2+}$ affinity varies among the member of the KChIP group. Nonetheless, calcium binding studies on KChIP3 have revealed that this construct can bind two calcium ions 
with higher affinity than that observed for other NCS proteins, $(\mathrm{Kd} \sim 1$ to $10 \mu \mathrm{M})$ (Osawa et al. 2005, Craig et al. 2002).

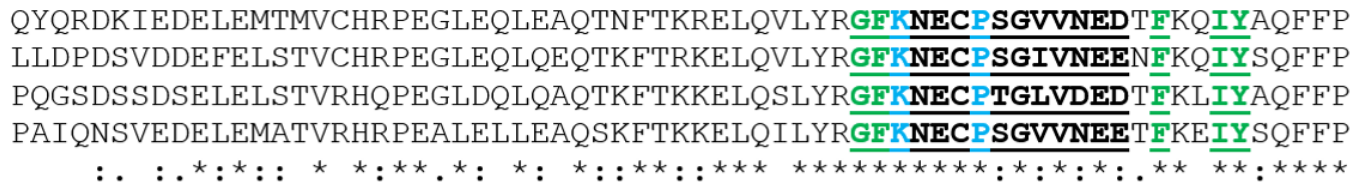
HGDASTYAHYLFNAFDTTQTGSVKFEDFVTALS ILLRGTVHEKLRWTFNLYDINKDGYINKEEMMDI

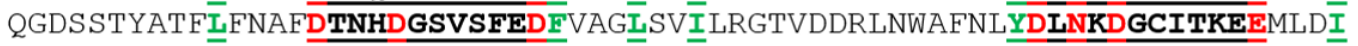
QGDATTYAHFIFNAFDADGNGAIHFEDFVVGISILLRGTVHEKLKWAFNLYDINKDGYITKEEMLAI QGDSTTYAHF'

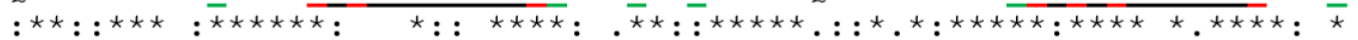

VKAIYDMMGKYTYPVLKEDTPRQHVDVFFQKMDKNKDGIVTLDEFLESCQEDDNIMRSLQLFQNVM MKSIYDMMGKYTYPALREEAPREFHVESFFEKMDRNKDGVVTIEEFIESCQKDENIMRSMQLFDNVI MKSIY DMMGRHTYPILREDAPAE MKAIYDMMGKCTYPVLKEDAPRQHVETFEKMDKNKDGVVTIDEFIESCQKDENIMRSMQLFENVI

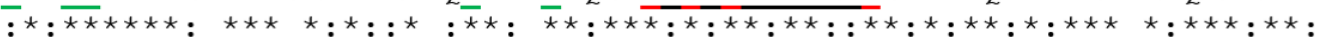

KChIP1 KChIP2 KChIP3 KChIP4

Figure 1.20. Sequence alignment of human KChIP1, KChIP2, KChIP3, and KChIP4. Turn loops of EF-hand 1, 2, 3, and 4 are shown in bold and underlined. the negatively charged amino acids involved in metal coordination shown in red. Asterisks indicate identical residues, semicolon indicates very similar residues, and the period is for somewhat similar residues. Amino acids involved in Kv4 binding are shown in green.

\subsubsection{DREAM/KChIP3}

In contrast with other members of the neuronal calcium sensor family, KChIPs are not only prominently expressed in the brain, but also in cardiac tissue. In the heart, they play a major role in regulation of the fast ITo currents generated by the fast opening of voltage gated $\mathrm{K}^{+}$channels (An et al. 2000). For instance, the expression gradient of KChIP2 in the endocardium and epicardium have been implicated in generation of the transmural current necessary for proper pacemaking (Rosati et al. 2001). More importantly, KChIP2 has been shown to mediate the dose dependent effect of drugs known to regulate heart pacemaking (Bett et al. 2006). Thus, KChIPs have been proposed to be an innovative target for the treatment of cardiovascular diseases (Calloe et al. 2009, Lundby et al. 2010). In the brain, KChIP2 and KChIP3 have been shown to play a major role in regulation of the fast IsA 
current arising from $\mathrm{K}^{+}$escape through the $\mathrm{Kv} 4$ voltage sensitive channels (Amarillo et al. 2008, Jerng, Pfaffinger 2008).

In addition, KChIP3 has been shown to be involved in regulation of gene expression. Association of KCHIP3 in the absence of calcium with the downstream regulatory element (DRE) of some genes has been directly shown to inhibit expression of downstream genes (Carrion et al. 1999). On the basis of the antagonist modulation of gene expression by KChIP3, Carrion et al. named this protein as DREAM (downstream regulatory element antagonist modulator). Association of $\mathrm{Ca}^{2+}$ with DREAM induces dissociation from the DRE DNA sequence and subsequent expression of the downstream gene (Carrion, Mellstrom \& Naranjo 1998). These findings have set KChIP3/DREAM as the first calcium binding protein able to directly associate with DNA and regulate gene expression in a calcium dependent manner (Carrion et al. 1999, Carrion, Mellstrom \& Naranjo 1998). Calmodulin is also known to regulate gene expression, but in this case, regulation is indirect since calmodulin must associate with basic helix-loop-helix nucleoproteins (Corneliussen et al. 1994). Indirect regulation of gene expression has also been observed in DREAM via interactions with $\alpha$-CREAM and CREB proteins (Ledo et al. 2000, Ledo et al. 2002). The DREAM-CREB interactions allows the regulations of genes with promoters different than the DRE, thus expanding the role of DREAM in gene regulation. Furthermore, the role of KChIP3/DREAM in regulation of additional biological processes has been highlighted by the initial findings that this protein was able to associate with presenilin and regulate the activity of the $\gamma$-secretase protein complex (Buxbaum et al. 1998). The calcium dependent manner in which KChIP3/DREAM associates with presenilin led the authors to name this protein calsenilin. Thus, KChIP3 has been 
independently found by three groups to associate with three different ligands and has been named with three different names, KChIP3/DREAM/calsenilin. It is fortunate that despite DREAM being found to interact with and regulate the activity of over 40 other proteins in the membrane, cytosol, and nucleus, no additional names have been assigned to the protein (Ramachandran et al. 2012).

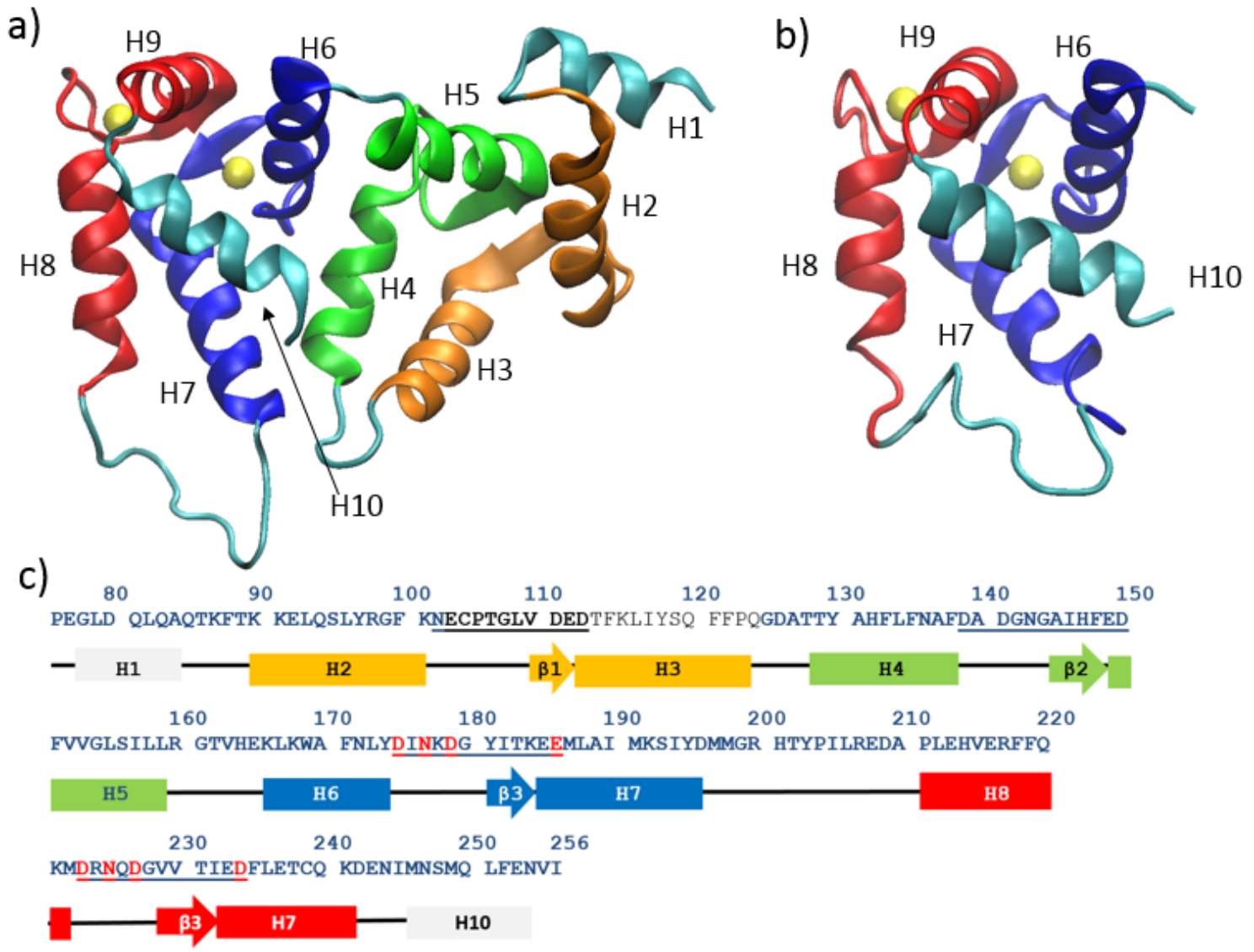

Figure 1.21. Cartoon representation of the NMR structures of (a) $\mathrm{Ca}^{2+}$ bound mouse DREAM (first 75 amino acids not resolved, PDB: 2jul) and (b) $\mathrm{Ca}^{2+}$ bound human DREAM (first 160 amino acids not resolved, PDB: 2e6w). The helices numbers are shown for clarity, and the EF-hand 1, 2, 3, and 4 are shown in orange, green, blue, and red, respectively. Calcium is shown as a yellow sphere. (c) Amino acid sequence of mouse DREAM (residues 76 to 256) following the same coloring scheme as in Figure 1.4. The cartoon representation of the secondary structure is shown.

Structural studies of DREAM have provided some insight into the potential mechanisms of DNA and potassium channel regulation. The NMR structure of $\mathrm{Ca}^{2+}$ bound 
mouse DREAM $(\Delta 65)$ reveals an EF-hand domain organization similar to that of recoverin (Figure 1.21) (Lusin et al. 2008). In DREAM, EF-hands 1 through 4 are organized as two closely packed domains, where the $\mathrm{N}$-terminus contains the inactive EF-hand 1 and the $\mathrm{Mg}^{2+}$ sensitive EF-hand 2, while the C-terminus contains EF-hand 3 and 4. The first helix (H1, residues 77 to 89) and last helix (H10, residue 242 to 256) are not part of the EFhands, but may be involved in ligand recognition (Lusin et al. 2008). Calcium binds with high affinity (1 to $10 \mu \mathrm{M}$ ) at EF-hand 3 and 4 (Osawa et al. 2005). The coordination of $\mathrm{Ca}^{2+}$ by the turn-loop of EF-hand 3 and 4 follows that observed in other NCS protein, as shown in Figure 1.6.

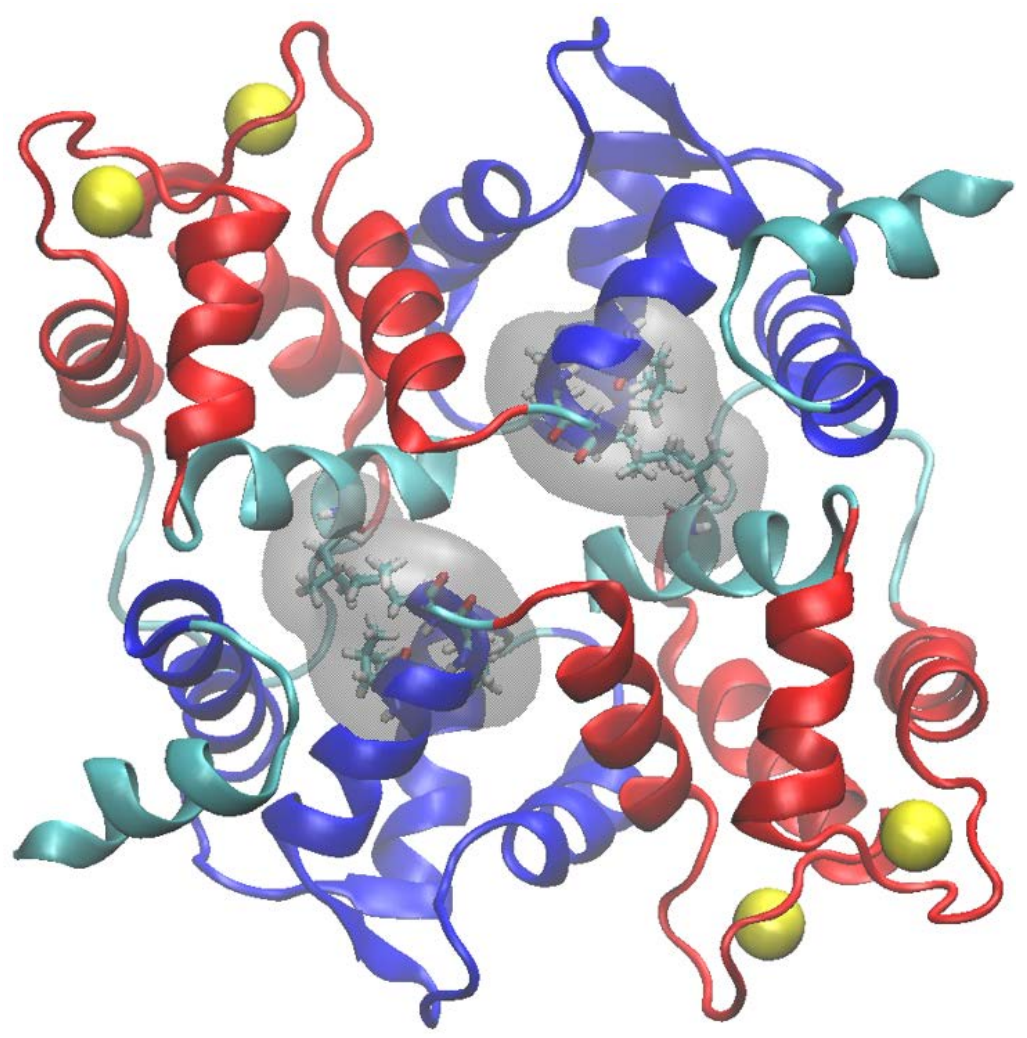

Figure 1.22. Cartoon representation of the proposed DREAM dimer in which Leu155, Leu158, Leu159, and Leu251 are shown in licorice model with a diffuse surface. Color scheme as in Figure 1.12. 
In the absence of $\mathrm{Ca}^{2+}$, DREAM has been proposed to form tetramers, whereas upon binding of $\mathrm{Ca}^{2+}$ a transition to dimers has been observed (Lusin et al. 2008, Osawa et al. 2001). The role of dimerization in ligand recognition is not known, but dimerization upon binding of calcium is widely present in NCS proteins. Indeed, the crystal structures of neurocalcin (Vijay-Kumar, Kumar 1999), recoverin (Weiergräber et al. 2003), VILIP-1 (Li et al. 2011), and KChIP1 (Zhou et al. 2004b) reveal the formation of dimers upon $\mathrm{Ca}^{2+}$ binding.

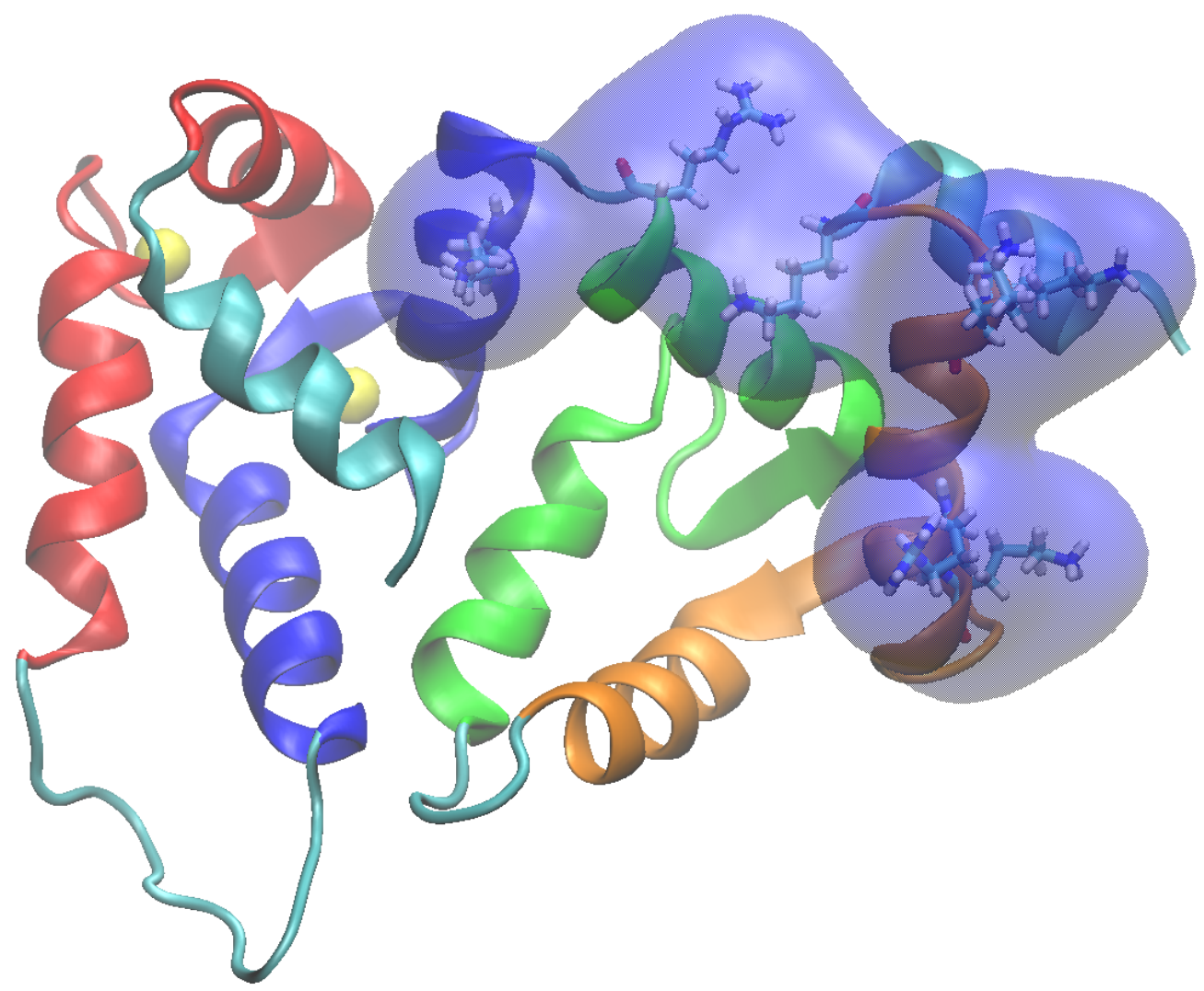

Figure 1.23. Cartoon representation of the NMR structures of (a) $\mathrm{Ca}^{2+}$ bound mouse DREAM (PDB: 2jul). Positively charged amino acids proposed to mediate DNA binding are shown in licorice and transparent blue surface.

The crystal structure of KChIP1 also shows that a hydrophobic crevice lining one side of the protein stabilizes the dimer in the calcium bound form (Zhou et al. 2004b). Similarly, a proposed model for the dimer of DREAM also highlights the role of hydrophobic amino 
acids in stabilizing the dimer (Lusin et al. 2008). In particular, two leucine rich regions at the N-terminus (Leu155, Leu158, and leu159) and C-terminus (Leu251) have been implicated in dimerization and protein-protein interactions (Figure 1.22) (Lusin et al. 2008, Ledo et al. 2000).

\begin{tabular}{lcl}
\hline Table 1.1 Aligned DRE sequences \\
\hline Consensus sequence & \multicolumn{1}{c}{ ANGTCAAAG } \\
& G $\quad$ GG & \\
NPFF DRE 1 & GCTTTGTCAGAGCCAGGC & antisense \\
NPFF DRE 2 & CTCCTGTCAGGATTGAGC & antisense \\
Human DynDRE & CCGGAGTCAAGGAGGCC & sense \\
Mouse DynDRE & CTAGAGTCAGGGTACCT & sense \\
Human c-fos DRE & GAAGAGTCAACGAGCGA & inverse sense \\
Mouse c-fos DRE & GAAGAGTCAACGAGCGA & inverse sense \\
\hline \multicolumn{2}{l}{ Adapted from (Nystedt et al. 2006) } \\
\hline
\end{tabular}

Structural analysis and deletion studies have also revealed the potential role of basic amino acids at the entering helix of EF-hand 1 (helix H2) in DNA binding (Osawa et al. 2005, Lusin et al. 2008, Ledo et al. 2000). These basic amino acids (Lys87, Lys90, Lys91, Arg98, Lys101, Arg160, and Lys166) are clustered at the N-terminus protein surface and may mediate electrostatic interactions with DNA (Figure 1.23). Furthermore, analysis of the DRE DNA sequence to which DREAM binds (Table 1.1) as well as pull down experiments have highlighted the role of the core section of the DRE sequence (GTCA) in binding of DREAM (Nystedt et al. 2006, Ledo et al. 2000).

\subsection{Objectives of this study}

Overall, the studies presented above have highlighted DREAM as a novel type of NCS protein with the ability to modulate numerous biological processes. However, the precise mechanism by which this protein regulates these processes and the molecular 
reorganization triggered by $\mathrm{Ca}^{2+}$ binding which controls these mechanisms remains to be discovered.

The role of KChIPs in regulation of ITO currents in the heart and ISA currents in the brain has widespread implications in terms of treatment and understanding of cardiovascular and neurological diseases. Moreover, the tissue specific expression of KChIPs is in sharp contrast to the widespread expression of Kv4 channels, thus providing a novel avenue for the development of drug therapies aimed at treatment of abnormal activity in the heart or brain. However, the development of novel therapies is hindered by the lack of detailed understanding of the molecular mechanisms controlling the interactions of KChIPs and their biological partners. Also, the interaction of KChIPs and NCS protein with small synthetic and natural ligands remains unexplored. Similarly, information on the molecular mechanisms by which $\mathrm{Ca}^{2+}$ regulates the activity of these proteins remains scarce.

Here, we propose to use KChIP3/DREAM as a model neuronal calcium sensor in order to understand the role of $\mathrm{Ca}^{2+}$ in regulating the structural mechanism responsible in controlling ligand recognition. First, we seek to characterize the role a hydrophobic surface on DREAM in regulating association of small fluorescent ligands. This information is further used to identify the mechanism by which small non-fluorescent molecules can regulate the activity of DREAM and its ability to interact with biological $\mathrm{K}_{\mathrm{V}}$ channels. We further seek to identify role of the N-terminus of DREAM in regulation of protein-protein and protein-DNA interactions, and the mechanism by which the C-terminus mediates protein-metal coordination. Lastly, through the use of theoretical and experimental biophysics, we seek to identify the role of specific amino acids in regulation of protein 
activity, stability, and ligand recognition. We envision that the combination of experimental and theoretical biophysics would allow us to decipher the precise molecular reorganization triggered by $\mathrm{Ca}^{2+}$ binding to DREAM. 


\section{MATERIALS AND METHODS}

\subsection{Chemicals and reagents}

The hydrophobic fluorescent probes 1,8-AN, 2,6-ANS and nile red were purchased from Invitrogen and used without further purification. The 1,5-IAEDANS, fluorescein 5maleimide, and rhodamine red C2 maleimide were purchased from Invitrogen. DMnitrophen was purchased from Invitrogen Life Sciences or Calbiochem as described in the text. The caged proton, 2-nitrobenzaldehyde was purchased from sigma and dissolved in ultrapure water and protected from light by using light resistive microcentrifuge tubes and/or aluminum foil. The compound NS5806 (1-[2,4-dibromo-6-(1H-tetrazol-5-yl)phenyl]-3-(3,5-bis-trifluoromethyl-phenyl)-urea) (purity>99\%) was purchased from Tocris Bioscience. Trifluoperazine (TFP) was purchased from Sigma-Aldrich. All other reagents and solvents were purchased from Sigma-Aldrich or Fischer scientific and were of analytical grade. Maleimide labeling fluorophores were solubilized in DMSO and stored at $-20^{\circ} \mathrm{C}$. Concentrated TFP, $1,8-\mathrm{ANS}$, and 2,6-ANS stocks were prepared in ultrapure (18.2 $\mathrm{M} \Omega$ ) water and stored at $-20^{\circ} \mathrm{C}$. The concentration of NS5806, TFP and 1,8-ANS stocks were determined spectrophometrically using the extinctions coefficients listed in table 2.1. All reagents required for the synthesis of the ReAsH-EDT 2 and FlAsH-EDT 2 biarsenical probes were purchased from the vendors recommended in the reference (Adams, Tsien 2008). Arachidonic acid was purchased from Sigma, dissolved in DMSO and stored under anaerobic conditions at $-30{ }^{\circ} \mathrm{C}$. Cholesterol, benzafibrate, and LS35 were obtained from sigma and stocks were prepared in 20mM MOPS pH 7.4. The terbium(III) lanthanide was obtained as a hydrated chloride salt $\left(\mathrm{TbCl}_{3} \cdot 6 \mathrm{H}_{2} \mathrm{O}\right)$ from Sigma-Aldrich and was used without further purification. Terbium stocks of $\sim 0.5 \mathrm{M}$ were 
prepared gravimetrically in decalsified ultrapure $18 \mathrm{M} \Omega$ water, the concentration of $\mathrm{Tb}^{3+}$ stocks were confirmed by titrations against EDTA standards.

\subsection{Recombinant protein expression and purification}

The DREAM and human Kv4.3 protein constructs were expressed in E. coli BL21(DE3) cells and purified according to previously published procedures (Scannevin et al. 2004, Lusin et al. 2008, Osawa et al. 2001). The plasmid for mouse DREAM, $\operatorname{DREAM}(\Delta 65), \quad \operatorname{DREAM}(\Delta 160), \quad \operatorname{DREAM}(\Delta 65, \quad \operatorname{E} 186 \mathrm{Q}), \quad \operatorname{DREAM}(\Delta 65, \quad \mathrm{E} 234 \mathrm{Q})$, $\operatorname{DREAM}(\Delta 65)$ with a CCPGCC peptide tag at the C-terminus, DREAM-NCS, and Kv4.3(1-152) were purchased from Genecopoeia. The rat calmodulin plasmid (herein referred as CaM) was a kind gift of Dr. J. P. Davis (Ohio State University). The CaM plasmid was subcloned in BL21(DE3) cells and purified as previously described (George et al. 1993). The amino acid sequence of all purchased plasmids is shown in Figure 2.1. Cells were disrupted by sonication (Fisher Scientific, Model 100). The DREAM( $\Delta 65)$ with a CCPGCC peptide tag and Kv4.3(1-152) protein constructs were recovered as insoluble inclusion bodies which were collected by centrifugation. Inclusion bodies were washed extensively with $50 \mathrm{mM}$ Tris buffer $\mathrm{pH}$ 8.0, $1 \mathrm{M} \mathrm{NaCl}, 0.5 \%$ Triton-X100, $0.1 \%$ $\mathrm{NaN}_{3}, 1 \mathrm{mM}$ DTT, and $5 \mathrm{mM}$ EDTA followed by a final wash with $50 \mathrm{mM}$ Tris buffer $\mathrm{pH}$ 8.0 and $2.0 \mathrm{M} \mathrm{NaCl}$. Washed inclusion bodies were solubilized in $100 \mathrm{mM}$ Tris buffer $\mathrm{pH}$ 12.5, 2.0 M urea, and 5 mM DTT. Refolding was achieved by fast dilution of solubilized inclusion bodies into a 10x volume of $100 \mathrm{mM}$ Tris buffer $\mathrm{pH}$ 7.4, $250 \mathrm{mM}$ sucrose, $30 \%$ glycerol, $20 \mathrm{mM}$ LDAO, $1 \mathrm{mM}$ 2-mercaptoethanol on an ice bath with constant stirring. All proteins were stored as concentrated stocks ranging in concentrations from $250 \mu \mathrm{M}$ to $850 \mu \mathrm{M}$ at $-32{ }^{\circ} \mathrm{C}$ in $20 \mathrm{mM}$ TRIS pH 7.4. 


\begin{tabular}{|c|c|c|c|c|c|}
\hline Chemical & $\begin{array}{c}\lambda \\
(\mathrm{nm})\end{array}$ & $\begin{array}{c}\text { Extinction } \\
\text { coefficient } \\
\text { (M-1cm- } \\
1)\end{array}$ & Solvent & Reference & Manufacturer \\
\hline NS5806 & 295 & 2200 & MOPS pH 7.4 & & Tocris Bioscience \\
\hline TFP & 306 & 3300 & Water & (Hart et al. 1983) & Sigma \\
\hline 1,8-ANS & 350 & 5000 & water & (Stryer 1965) & Calbiochem/Sigma \\
\hline 2,6-ANS & 350 & 5000 & Water & (Diwu et al. 1997) & Molecular Probes \\
\hline Nile red & 552 & 19600 & DMSO & (Castro et al. 2005) & Molecular Probes \\
\hline IAEDANS & 332 & 6100 & $\begin{array}{l}\text { Phosphate } \\
\text { pH } 7.4\end{array}$ & (Hudson, Weber 1973b) & Molecular Probes \\
\hline Dansyl chloride & 340 & 3300 & $\begin{array}{l}\text { Phosphate } \\
\text { pH } 7.4\end{array}$ & (Weber 1952) & Sigma \\
\hline $\begin{array}{l}\text { Rhodamine } \\
\text { maleimide }\end{array}$ & 560 & 52000 & $\mathrm{DMF}$ & (Beija, Afonso \& Martinho 2009) & Molecular Probes \\
\hline $\begin{array}{l}\text { Fluorescein } \\
\text { maleimide }\end{array}$ & 495 & 78000 & $0.1 \mathrm{M} \mathrm{NaOH}$ & (Bigelow, Inesi 1991) & Molecular Probes \\
\hline
\end{tabular}




\begin{tabular}{|c|c|c|c|c|c|}
\hline Chemical & $\begin{array}{c}\lambda \\
(\mathrm{nm})\end{array}$ & $\begin{array}{l}\text { Extinction } \\
\text { coefficient } \\
\left(\mathrm{M}^{-1} \mathrm{~cm}^{-1}\right)\end{array}$ & Solvent & Reference & Manufacturer \\
\hline FITC & 495 & 80000 & $0.1 \mathrm{M} \mathrm{NaOH}$ & (Bigelow, Inesi 1991) & Sigma \\
\hline $\mathrm{FlAsH}_{-\mathrm{EDT}_{2}}$ & 508 & 63500 & $0.1 \mathrm{M} \mathrm{NaOH}$ & (Adams, Tsien 2008) & \\
\hline $\mathrm{ReAsH}_{-} \mathrm{EDT}_{2}$ & 5579 & 63000 & $0.1 \mathrm{M} \mathrm{NaOH}$ & (Adams, Tsien 2008) & \\
\hline DM-nitrophen & 350 & 4330 & pH 7.1 & (Kaplan, Ellis-Davies 1988) & $\begin{array}{l}\text { Molecular } \\
\text { probes }\end{array}$ \\
\hline 2-nitrobenzaldehyde & 313 & 1590 & water & (Galbavy et al. 2010) & Aldrich \\
\hline Arachidonic acid & 369 & 4.72 & DMSO & & Sigma \\
\hline Benzafibrate & 280 & 660 & MOPS pH 7.4 & & Sigma \\
\hline Quin-2 & 240 & 42000 & MOPS pH 7.4 & (Linse et al. 1987) & Sigma \\
\hline $\mathrm{TbCl}_{3} \cdot 6 \mathrm{H}_{2} \mathrm{O}$ & 265 & 0.052 & MOPS pH 7.4 & & Sigma \\
\hline
\end{tabular}




\begin{abstract}
DREAM
MQRTKEAVKASDGNLLGDPGRI PLSKRESIKWQRPRFTRQALMRCCLIKWILSSAAPQGSDSSDSELELST VRHQPEGLDQLQAQTKFTKKELQSLYRGFKNECPTGLVDEDTFKLIYSQFFPQGDATTYAHFLFNAFDADG NGAIHFEDFVVGLS ILLRGTVHEKLKWAFNLYDINKDGCITKEEMLAIMKS IYDMMGRHTYP ILREDAPLE HVERFFQKMDRNQDGVVTIDEFLETCQKDENIMNSMQLFENVIYLEHHHHHH

$\operatorname{DREAM}(\Delta 65)$

MELELSTVRHQPEGLDQLQAQTKFTKKELQSLYRGFKNECPTGLVDEDTFKLIYSQFFPQGDATTYAHFLF NAFDADGNGAIHFEDFVVGLS ILLRGTVHEKLKWAFNLYDINKDGCITKEEMLAIMKS IYDMMGRHTYPIL REDAPLEHVERFFQKMDRNQDGVVT IDEFLETCQKDENIMNSMQLFENVIYLEHHHHHH

$\operatorname{DREAM}(\Delta 160)$

MGTVHEKLKWAFNLYDINKDGCITKEEMLAIMKS IYDMMGRHTYP ILREDAPLEHVERFFQKMDRNQDGVV TIDEFLETCQKDENIMNSMQLFENVIYLEHHHHHH

$\operatorname{DREAM}(\Delta 65, \mathrm{C} 4)$

HHHHHHELELSTVRHQPEGLDQLQAQTKFTKKELQSLYRGFKNECPTGLVDEDTFKLIYSQFFPQGDATTY AHFLFNAFDADGNGAIHFEDFVVGLS ILLRGTVHEKLKWAFNLYDINKDGCITKEEMLAIMKS IYDMMGRH TYPILREDAPLEHVERFFQKMDRNQDGVVT IDEFLETCQKDENIMNSMQLFENVICCPGCC

DREAM-NCS

MELELSTVRHQPEGLDQLQAQTKFTKKELQSLYRGFKNECPTGLVDEDTFKLIYSQFFPQGDATTYAHFLF NAFDADGNGAIHFEDFVVGLSITSRGTVHEKLKWAFNLYDINKDGCITKEEMLAIMKS IYDMMGNTVELPE EEDAPLEHVERFFQKMDRNQDGVVTIDEFLETCQKDENIMNSMQLFENVIYLEHHHHHH

Kv4.3 T1 domain with FlAsH tag

HHHHHHSSGMSPILGYWKIKGLVQPTRLLLEYLEEKYEEHLYERDEGDKWRNKKFELGLEFPNLPYYIDGD VKLTQSMAI IRYIADKHNMLGGCPKERAEISMLEGAVLDIRYGVSRIAYSKDFETLKVDFLSKLPEMLKMF EDRLCHKTYLNGDHVTHPDFMLYDALDVVLYMDPMCLDAFPKLVCFKKRIEAIPQIDKYLKSSKYIAWPLQ GWQATFGGGDHPPKSDLGHTGHRSGTDDDDKAMAAGVAAWLPFARAAAIGWMPVANCPMPLAPADKNKRQD ELIVLNVSGRRFQTWRTTLERYCCPGCCDTLLGSTEKEFFFNEDTKEYFFDRDPEVFRCVLNFYRTGKLHY PRYECISAYDDELAFYGILPEIIGDCCYEEYKDRKRENAERLMDDNDLGHHHHHH
\end{abstract}

Figure 2.1. Amino acid sequence of plasmid purchased. The mouse DREAM, $\operatorname{DREAM}(\Delta 65)$, and $\operatorname{DREAM}(\Delta 160)$ plasmids contain a start methionine residue (verified by mass spectrometry) and a C-terminus His-tag (blue) connected by tripeptide linker (green). The DREAM $(\Delta 65, \mathrm{C} 4)$ plasmid contains an N-terminus His-tag (blue) and a Cterminus tetracysteine tag. The DREAM-NCS chimera plasmid is identical to the $\operatorname{DREAM}(\Delta 65)$ plasmid but residues Leu158, Leu159 and the linker between EF-hand 3 and 4 were replaced by the amino acid sequence of human NCS-1 (shown in red). Lastly, a GST-Kv4.3 T1 domain fusion construct was generated (GST in red). The enterokinase cleavage site shown in red and underlined. The T1-domain of human Kv4.3 residues 2 to 152 shown in black. The biarsenical tag is shown in underlined red color.

Labeling of DREAM $(\Delta 65)$ containing a CysCysProGlyCyCys amino acid sequence at the C-terminus with the biarsenical probe FlAsH-EDT 2 was conducted by adding $2 \mathrm{x}$ stoichiometric excess probe under identical condition as reported before (Adams, Tsien 2008). Unbound probe was removed by size exclusion chromatography and labeled protein was further purified through a Ni-NTA followed by extensive dialysis in 20 mM MOPS pH 7.4 $100 \mathrm{mM} \mathrm{NaCl}$. 
The labeling efficiency and stock concentration was determined by comparing the protein concentration using the bicinchroninic colorimetric method (Thermo Scientific) to the protein concentration determined using the FlAsH and ReAsH absorbance in $0.1 \mathrm{M} \mathrm{NaOH}$. Protein purity and proper folding prior to FlAsH labeling was assayed using SDS-page electrophoresis, CD spectroscopy, and the $\mathrm{Zn}^{2+}$ and $\mathrm{Ca}^{2+}$ induced oligomerization changes as previously reported.

\subsection{Mutagenesis}

\begin{tabular}{ll}
\hline \multicolumn{2}{l}{ Table 2.2 Primers used for site directed mutagenesis } \\
\hline Mutant name & 5' to 3' \\
\hline CaM(A148C) & GCTGACTGAAGAACAGATTTGCGAATTCAAGGAAGCTTTCTC \\
DREAM(K87A) & CTACAAGCTCAGACCGCGTTCACCAAGAAGG \\
DREAM(E92A) & CAAGTTCACCAAGAAGGCGCTGCAGTCCCTTTAC \\
DREAM(F171A) & GAGAAGCTCAAGTGGGCCGCGAATCTCTATGACATTAAC \\
DREAM(Y174A) & GTGGGCCTTCAATCTCGAGGACATTAACAAGGATGG \\
DREAM(F218A) & GGAGCATGTGGAGAGGGCGTTTCAGAAAATGGACAG \\
DREAM(F219A) & GCATGTGGAGAGGTTCGCGCAGAAAATGGACAGG \\
DREAM(F235A) & GTGGTGACCATTGATGAAGCGCTGGAGACTTGTCAGAAG \\
DREAM(F252A) & GAACTCCATGCAGCTGGCGGAGAACGTCATCTAC \\
\hline
\end{tabular}

A CaM(A148C) mutant was obtained by site directed mutagenesis of the CaM plasmid using the QuickChange Lightning kit (Agilent Technologies). The specific procedure described in detail in the QuickChange Lightning procedure provided by the manufacturer was strictly followed. Similarly, a small library of $\operatorname{DREAM}(\Delta 65)$ proteins in which specific amino acids were mutated to alanine was generated following the same protocols. The primer sequence used for the mutagenesis were selected using results from the PrimerX webserver and are shown in Table 2.2. Mutation of the selected amino acids was confirmed by DNA sequencing (Eurofins Genomics) of plasmid extracted with the 
StrataPrep Plasmid Miniprep kit (Agilent). Following sequencing, these plasmids were subcloned in BL21(DE3) E. coli cells; protein expression and purifications was carried out as described above. We have observed that not all plasmid extractions yield plasmid of enough purity for sequencing. For instance, we have observed that large concentrations usually larger than $100 \mathrm{ng} / \mathrm{mL}$ with absorbance ratios of $1.8>260 / 280<2.1$ and $1.2<260 / 240<1.7$ usually result in successful sequencing; however, in some instances we have obtained good enough sequencing for samples which did not satisfy these conditions. In all instances, sequencing allowed unequivocal identification of the mutations being introduced (see appendix 2). Overall, almost all plasmid mutations were a success using the protocols described, with only one failed transformation.

\subsection{Full length DREAM expression and purification}

We have also developed a CaM and DREAM low temperature co-expression protocol which has allowed us to recover milligram quantities of soluble full-length DREAM. Briefly, E. coli BL21(DE3) competent cells were transformed with CaM and DREAM plasmids and grown in $\mathrm{LB}$ medium at $37^{\circ} \mathrm{C}$ in the presence of $100 \mu \mathrm{g} / \mathrm{mL}$ ampicillin until reaching an optical density of 2 at $600 \mathrm{~nm}$. The medium was then quickly chilled on an ice bath to $4^{\circ} \mathrm{C}$ and protein expression was induced by adding $0.2 \mathrm{mM}$ IPTG. The medium was placed back in the shaker at $250 \mathrm{rpm}$ for an additional 6 hours at $18^{\circ} \mathrm{C}$ followed by harvest of the cells by centrifugation for 15 minutes at 5000 RPM. The cell pellet was resuspended in $100 \mathrm{mM}$ Tris buffer $\mathrm{pH}$ 8.0, $0.5 \mathrm{M} \mathrm{NaCl}, 1 \mathrm{mM} \mathrm{CaCl}_{2}$, $5 \mathrm{mM}$ $\mathrm{MgCl}_{2}, 5 \mathrm{mM}$ imidazole, $1.0 \%$ Triton-X100, $0.5 \%$ Tween 20 supplemented with 20 $\mu \mathrm{g} / \mathrm{mL}$ DNAse, $200 \mu \mathrm{g} / \mathrm{mL}$ lysozyme, and $0.5 \mathrm{mM}$ phenylmethylsulfonyl fluoride and sonicated on an ice bath for 30 cycles at $30 \%$ duty cycle. The cell lysate was centrifuged 
at $11,000 \mathrm{~g}$ for 50 minutes and the supernatant was loaded on a Ni-NTA column equilibrated with buffer A (20 mM Tris pH 7.4, $300 \mathrm{mM} \mathrm{NaCl}, 0.5 \mathrm{mM} \mathrm{CaCl} 2$ and $10 \mathrm{mM}$ LDAO). The Ni-NTA column loaded with DREAM was washed extensively with buffer A and eluted in buffer B (20 mM Tris pH 7.4 and 10 mM LDAO) supplemented with 250 mM imidazole. Selected fractions were loaded onto a DEAE-sepharose anion exchange column previously equilibrated with buffer B, washed with buffer B and $80 \mathrm{mM} \mathrm{NaCl}$ then eluted with $180 \mathrm{mM} \mathrm{NaCl}$, the final protein was stored in $5 \mathrm{mM}$ Tris $\mathrm{pH}$ 8.0. The purity of all protein constructs was assessed using SDS-PAGE. The proper secondary structure and tertiary structure of all protein constructs was confirmed by using the known far-UV CD transition of DREAM protein as well as changes in tryptophan fluorescence upon calcium binding (Osawa et al. 2005, Carrion et al. 1999, Osawa et al. 2001).

\subsection{Protein labeling}

Labeling of CaM(A148C) with 1,5-IAEDANS or rhodamine red C2 (RRC2) maleimide was conducted after reduction of cysteine residues with $5 \mathrm{mM}$ TCEP in 200 mM Tris $\mathrm{pH} 7.4$ followed by extensive purging with argon and addition of (10-50x) excess fluorophore freshly prepared in DMF. The reaction was allowed proceed for 24 hours at $4^{\circ} \mathrm{C}$ with constant stirring in the dark. Unbound fluorophore was removed by size exclusion chromatography through a Sephadex G10 column. Labeled protein fractions were selected based on their absorption and fluorescence anisotropy and were extensively dialyzed in 5 $\mathrm{mM}$ Tris $\mathrm{pH}$ 7.4. The fraction of labeled protein was determined by dividing the fluorophore concentration (1,5-IAEDANS $\varepsilon_{336 \mathrm{~nm}}=6100 \mathrm{M}^{-1} \mathrm{~cm}^{-1}$ in buffer (Hudson, Weber 1973a) or RRC2 $\varepsilon_{560 \mathrm{~nm}}=119000 \mathrm{M}^{-1} \mathrm{~cm}^{-1}$ in DMSO) by the protein concentration 
$\left(\mathrm{CaM}(\mathrm{A} 148 \mathrm{C}) \varepsilon_{280 \mathrm{~nm}}=3140 \mathrm{M}^{-1} \mathrm{~cm}^{-1}\right)$ after correction by the absorption of the fluorophore at $280 \mathrm{~nm}$. Protein samples were prepared by dilution and the final protein concentration was measured using $\varepsilon_{280 \mathrm{~nm}}=31,200 \mathrm{M}^{-1} \mathrm{~cm}^{-1}$ for DREAM and $\varepsilon_{280 \mathrm{~nm}}=19,000 \mathrm{M}^{-1} \mathrm{~cm}^{-1}$ for $\operatorname{DREAM}(\Delta 65)$. The extrinsic fluorophore 1,8-ANS was added to protein samples directly from a concentrated stock. Calcium was added at 10x excess for DREAM and $1 \mathrm{mM}$ for DRE29 samples and $2 \mathrm{mM}$ EDTA was used to remove calcium; $50 \mathrm{mM} \mathrm{CaCl} 2$ and 200 mM EDTA standard stocks at pH 7.4 were used. CaM labeled with IAEDANS or RRC2 are referred as CaM-IAEDANS and CaMRR2, respectively. The amount of DMSO in the protein samples, whenever needed, did not exceed $1.2 \% \mathrm{v} / \mathrm{v}$.

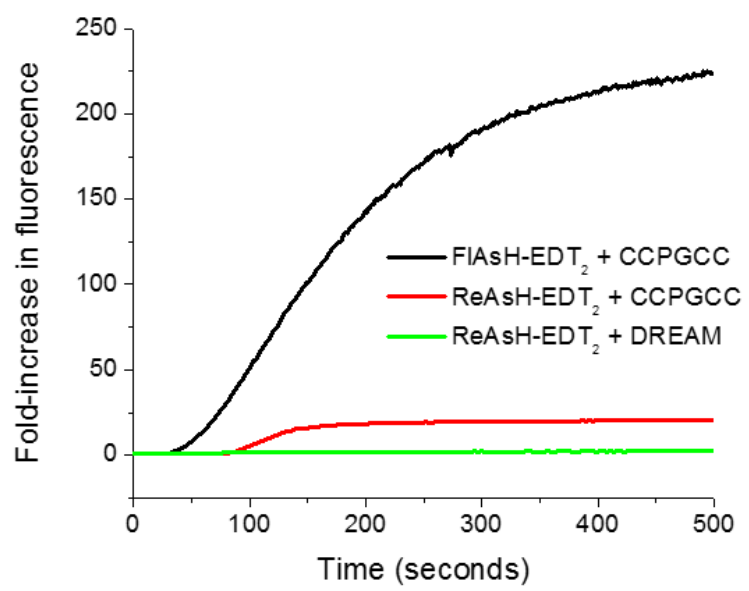

Figure 2.2. Increase in fluorescence intensity of FlAsH-EDT 2 and ReAsH-EDT 2 upon specific covalent attachment to a small CCPGCC peptide. Green trace functions as a control to show that there is no direct interaction of the biarsenical probe and DREAM, even though 5 cysteine are present in this protein.

The ReAsH-EDT 2 and FlAsH-EDT 2 synthesis and purification was carried out as described in detail by (Adams et al. 2002). The selectivity of the purified products was tested by monitoring the fluorescence enhancement upon binding to the CCPGCC test peptide. A representative plot of total fluorescence vs. time is shown in Figure 2.2. Proteins were labeled with FlAsH and ReAsH following previous protocols (Adams, Tsien 2008). 
Unbound fluorophores were removed by size exclusion chromatography using a G10 Sephandex column (Sigma) followed by Ni-NTA purification and extensive dialysis.

\subsection{Protein sample preparation}

Protein samples were prepared by diluting a concentrated protein stock in buffered aqueous solutions at $\mathrm{pH}$ and ionic strength as described throughout the text. Final protein concentration was measured spectrophotometrically using $\varepsilon_{280 \mathrm{~nm}}=19,000 \mathrm{M}^{-1} \mathrm{~cm}^{-1}$ for $\operatorname{DREAM}(\Delta 65), \varepsilon_{280 \mathrm{~nm}}=10,500 \mathrm{M}^{-1} \mathrm{~cm}^{-1}$ for $\operatorname{DREAM}(\Delta 160), \varepsilon_{280 \mathrm{~nm}}=71,500 \mathrm{M}^{-1} \mathrm{~cm}^{-1}$ for GST-Kv4.3(1-152), and $\varepsilon_{508 \mathrm{~nm}}=63,500 \mathrm{M}^{-1} \mathrm{~cm}^{-1}$ for DREAM $(\Delta 65)$ labeled with FlAsH or ReAsH (Adams, Tsien 2008). All samples were prepared and measured at room temperature, unless specified otherwise. Protein stocks were kept in small concentrated aliquots at $-30{ }^{\circ} \mathrm{C}$ immediately after purification, these stocks were thawed on ice only once prior to use. The $\mathrm{Ca}^{2+}$ free or $\mathrm{Mg}^{2+}$ DREAM samples were prepared by adding $1 \mathrm{mM}$ EDTA or $1 \mathrm{mM}$ EGTA and $5 \mathrm{mM} \mathrm{MgCl}$, respectively. The $\mathrm{Ca}^{2+}$ and $\mathrm{Ca}^{2+} \mathrm{Mg}^{2+}$ samples were prepared by adding $1 \mathrm{mM} \mathrm{Ca}^{2+}$ or $1 \mathrm{mM} \mathrm{Ca}^{2+}$ and $5 \mathrm{mM} \mathrm{Mg}^{2+}$. All EDTA, EGTA, $\mathrm{CaCl}_{2}$, and $\mathrm{MgC}_{12}$ stocks were prepared in ultrapure $18 \mathrm{M} \Omega$ water and adjusted to $\mathrm{pH} 7.4$ using $\mathrm{NaOH}$ or $\mathrm{HCl}$. Throughout the text, the metal free protein is referred as $\mathrm{Ca}^{2+}$ free, apo-, or apoform.

\subsection{Methods used in chapter 3}

\subsubsection{Steady-state fluorescence}

Steady-state emission measurements were carried out on a custom PC1 spectrofluorometer (ISS, Champaign, IL) with bandwidth and slit width for excitation/emission set at $8 \mathrm{~nm}$ and $0.5 \mathrm{~mm}$, respectively. The emission spectra for 1,8- 
ANS:DREAM $(\Delta 65)$ and 2,6-ANS:DREAM $(\Delta 65)$ complexes were measured using $\lambda_{\text {exc }}=$ $350 \mathrm{~nm}$ and $319 \mathrm{~nm}$, respectively. Titration were carried out at $20{ }^{\circ} \mathrm{C}$ and in a $2 \mathrm{~mm}$ x 10 $\mathrm{mm}$ path length quartz cuvette with excitation along the $2 \mathrm{~mm}$ path. Dissociation constants were determined either by titrating a small amount of concentrated ANS or arachidonic acid stock solution into $7 \mu \mathrm{M} \operatorname{DREAM}(\Delta 65)$ sample or keeping ANS concentration constant and adding aliquots of $\operatorname{DREAM}(\Delta 65)$ stock solution. In order to avoid dilution effects, ANS or protein was kept in both titrant and titrand at identical concentration. The intensity was corrected for the inner filter effect (Lakowicz 2010). The signal from unbound dye was subtracted and the emission intensity was determined by integrating the emission signal. Titration curves were analyzed using the Hill equation (Hawe, Sutter \& Jiskoot 2008):

$$
F_{\text {corrected }}=\frac{F_{\text {maximum }}[D R E A M]^{n}}{K d_{s s}^{n}+[D R E A M]^{n}}+y_{0}
$$

where, $\mathrm{K}_{\mathrm{dss}}$ is the dissociation constant, $\mathrm{n}$ represents the Hill coefficient, and $\mathrm{y}_{\mathrm{o}}$ is an offset associated with small uncorrected light scattering.

2.7.2 Fluorescence decay lifetime

Frequency-domain fluorescence lifetime and anisotropy decay measurements were performed using a ChronosFD spectrofluorimeter. The samples containing 1,8-ANS or 2,6ANS were excited with a $305 \mathrm{~nm}$ diode or $370 \mathrm{~nm}$ laser diode and the emission was collected using a 400-600 $\mathrm{nm}$ band pass filters (Andover). The lifetime reference used was 1,4-bis(5-phenyloxazol-2-yl)benzene (POPOP) in ethanol ( $\tau=1.35 \mathrm{~ns}$ ) with polarizers set at a magic angle configuration (Spencer, Weber 1970). Modulation ratio and phase change data were analyzed using GlobalsWE software and the $\chi^{2}$ parameter was used as 
criterion for goodness of the fit (Lakowicz 2010). Fluorescence intensity decays were analyzed using a multiple-exponential decay model and multiple frequency-domain data from at least three different protein batches were simultaneously fitted using the global analysis approach with the lifetime parameters set as linked variables. The theory behind frequency-domain depolarization has been described elsewhere (Badea 1979, Lakowicz et al. 1993). We implemented a triple associative decay model since multiple correlations lifetimes due to oligomerization equilibrium of $\operatorname{DREAM}(\Delta 65)$ and due to heterogeneous binding of the ANS probes. The validity of the model was judged on the basis of the closeness of the $\chi^{2}$ value to 1 . Frequency-domain depolarization data were analyzed using global analysis between data sets obtained with $305 \mathrm{~nm}$ and $370 \mathrm{~nm}$ excitation while keeping the rotational time as a linked-variable.

2.7.3 Calculation of Förster distances and energy transfer

The energy transfer efficiency between Trp donor and 1,8-ANS acceptor was determined according to Eqn. 2.7.2 (Blackman, Piston \& Beth 1998, Runnels, Scarlata 1995)

$$
E=\frac{R_{0}^{6}}{R_{0}^{6}+D^{6}}
$$

where $\mathrm{E}$ represents efficiency of energy transfer and $\mathrm{D}$ is the distance between the donor and acceptor (D). The variable $\mathrm{R}_{0}$ describes the critical distance at which rate of energy transfer equals the rate of fluorescence decay can be readily calculated using (Steinberg 1971):

$$
R_{0}^{6}=\frac{9000(\ln 10) \kappa^{2} \Phi_{D}}{128 \pi^{5} N n^{4}} \int_{0}^{\infty} F_{D}(\lambda) \varepsilon(\lambda) \lambda^{4} d \lambda
$$


where $\kappa$ is the dipole orientation factor (set to $2 / 3$ ), $\Phi_{\mathrm{D}}$ is the quantum yield of the donor, $\mathrm{N}$ is Avogadro's number, $\mathrm{n}$ is the index of refraction of water, $\mathrm{F}_{\mathrm{D}}(\lambda)$ is the normalized fluorescence emission of the donor and $\varepsilon(\lambda)$ is the extinction coefficient spectra of the acceptor.

\subsubsection{ANS displacement studies}

For experiments investigating the displacement of 1,8-ANS by arachidonic acid, aliquots of the arachidonic acid stock solution (1.6 mM arachidonic acid in $20 \mathrm{mM}$ Tris, $\mathrm{pH} 7.4$, and $100 \mathrm{mM} \mathrm{NaCl}$ ) were titrated into $7 \mu \mathrm{M}$ apo- or $\mathrm{Ca}^{2+}$ DREAM solubilized in $20 \mathrm{mM}$ Tris, pH 7.4, and $100 \mathrm{mM} \mathrm{NaCl}, 1 \mathrm{mM}$ DTT and $20 \mu \mathrm{M}$ 1,8-ANS. The change in 1,8-ANS fluorescence intensity as a function of arachidonic acid was analyzed by a single binding site model as described by (Epps, Raub \& Kezdy 1995).

2.7.5 Molecular docking modeling

The ANS binding sites on DREAM surface were identified using AutoDock 4.2 software (http://autodock.scripps.edu/) (Morris et al. 1998). The structure of $\mathrm{Ca}^{2+}$ bound DREAM (PDB: 2jul) was used as a rigid macromolecule (Lusin et al. 2008). The most plausible docking model was selected on the basis of the energetic ranking provided by AutoDock 4.2.

\subsection{Methods used in chapter 4}

\subsubsection{Steady-state fluorescence}

Steady-state emission spectra were recorded using a PC1-ChronosFD spectrofluorometer (ISS, Illinois). Tryptophan emission spectra were measured using a 295 $\mathrm{nm}$ excitation, and titrations were carried out in a $0.1 \times 1 \mathrm{~cm}$ path length quartz cuvette 
with excitation along the $0.1 \mathrm{~cm}$ path. The DREAM( $\Delta 65)$ :NS5806 dissociation constants were determined by non-linear fit of the change in integrated fluorescence (310 nm to 400 $\mathrm{nm}$ ) using a single binding site model:

$$
\Delta F=\frac{\left(K_{d}+\left[n P_{t}\right]+\left[L_{t}\right]\right)-\sqrt{\left(K_{d}+\left[n P_{t}\right]+\left[L_{t}\right]\right)^{2}-4\left[n P_{t}\right]\left[L_{t}\right]}}{2 c}
$$

where $\Delta \mathrm{F}$ is the intensity change, $\mathrm{K}_{\mathrm{d}}$ is the dissociation constant, $\mathrm{n}$ is the number of binding sites, $\mathrm{P}_{\mathrm{t}}$ is the total protein concentration, $\mathrm{L}_{\mathrm{t}}$ is the total ligand concentration, and $\mathrm{c}$ is a proportionality constant. For 1,8-ANS displacement studies, the excitation wavelength was set at $350 \mathrm{~nm}$ along the $2 \mathrm{~mm}$ path of a $0.2 \times 1 \mathrm{~cm}$ cuvette, and the integrated emission intensity (410 nm to $500 \mathrm{~nm}$ ) of 1,8-ANS bound to $\operatorname{DREAM}(\Delta 65)$ was used to calculate the extent of displacement. The dissociation constant of NS5806 for DREAM $(\Delta 65)$ was recovered using a single binding site displacement equation (Chuang et al. 2008):

$$
F_{\text {measured }}=F_{\max }\left[\alpha+\frac{\beta\left[L_{t}\right]}{\left(K_{i}+\left[L_{t}\right]\right)\left(1+\frac{\left[I_{t}\right]}{K_{d}}\right)}\right]
$$

where, $\alpha$ and $\beta$ are proportionality constants, $K_{i}$ is the dissociation constant of 1,8-ANS (measured separately), $\mathrm{I}_{\mathrm{t}}$ is the total concentration of NS5806 and $\mathrm{K}_{\mathrm{d}}$ is its dissociation constant.

To investigate the association between DREAM $(\Delta 65)$ constructs and $\mathrm{K}_{\mathrm{V}} 4.3(2-22)$ “Site 1” or $\mathrm{K}_{\mathrm{V}} 4.3(70-90)$ “Site 2”, fluorescently labeled peptides were purchased from ThinkPeptides (Sarasota, FL, USA). The peptides with the sequence of the Site 1 of human $\mathrm{K}_{\mathrm{V}} 4.3$ (Dansyl-Ahx-EKEFFFNEDTKEYFFDRDPEV) and Site 2 of human $\mathrm{K}_{\mathrm{V}} 4.3$ (FITC-Ahx-AAWLPFARAAAIGWMPV) were received at higher than $98 \%$ purity. The 
fraction of FITC-Ahx-KV4.3(2-22) bound to DREAM $(\Delta 65)$ constructs was determined based on the increase in steady state anisotropy and corrected for the increase in total intensity upon complex formation using:

$$
f_{B}=\frac{r-r_{F}}{\left(r-r_{F}\right)+\frac{I_{B}}{I_{F}}\left(r_{B}-r\right)}
$$

where $f_{\mathrm{B}}$ is the fractional concentration of peptide bound, $\mathrm{r}$ is the measured anisotropy, $\mathrm{r}_{\mathrm{F}}$ and $\mathrm{r}_{\mathrm{B}}$ are the anisotropy of the free and fully bound peptide, respectively (Lakowicz 2010). As a consequence of the large change in fluorescence intensity of Dansyl-Ahx-Kv4.3(7090) peptide upon binding to $\operatorname{DREAM}(\Delta 65)$ constructs the fraction bound was determined by the change in integrated fluorescence intensity (450 nm to $650 \mathrm{~nm}$ ) normalized by the maximum intensity change. The resulting titration curves were analyzed using a single site binding model, Eqn. 2.8.1.

For the determination of the calcium dissociation constants, a sample containing 1 $\mu \mathrm{M}$ of DREAM $(\Delta 65)$ containing the biarsenical binding sequence (CCPGCC) at the $\mathrm{C}$ terminus and labeled with FlAsH was prepared in 20 mM MOPS pH 7.4, 100 mM NaCl, 1 mM DTT, 1 mM EGTA, and 1 mM NTA. All solutions were previously decalsified by treatment with CHELEX resin (BioRad). The amount of residual calcium was determined using Quin-2 and calculated to be $200 \mathrm{nM}$. Calcium binding was monitored by the change in integrated fluorescence emission (510 nm to $580 \mathrm{~nm}$ ) upon calcium binding to $\operatorname{DREAM}(\Delta 65)$-FlAsH. Calcium was added from a buffered $50 \mathrm{mM} \mathrm{CaCl} 2$ stock prepared from a $100 \mathrm{mM} \mathrm{CaCl}_{2}$ standard (Fisher Scientific) and the free calcium concentration was determined by obtaining the saturation curves for the calcium indicators Quin-2 $\left(\mathrm{K}_{\mathrm{d}}=120\right.$ $n M)$, Calcium Green-5N $\left(K_{d}=14 \mu \mathrm{M}\right)$ and CaGreen-2 $\left(K_{d}=550 \mathrm{nM}\right)$. The determined 
free calcium concentrations were in agreement with the values calculated using the $\mathrm{pCa}$ calculator software (Dweck, Reyes-Alfonso Jr. \& Potter 2005). The macroscopic calcium binding constants in the presence of NS5806 were obtained under identical conditions. The FlAsH labeled DREAM $(\Delta 65)$ was excited with a $470 \mathrm{~nm}$ laser diode and the change in fluorescence intensity was used to probe the extent of calcium binding. The resulting titration curves were analyzed using a double-Hill equation:

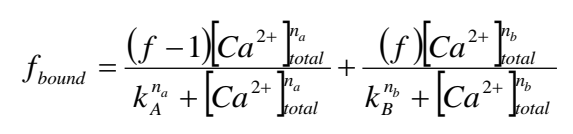

where $\mathrm{f}_{\text {bound }}$ is the fraction of proteins bound to calcium calculated from the intensity change, $\mathrm{f}$ is the fraction of sites $\mathrm{B}$ with dissociation constant $\mathrm{k}_{\mathrm{B}}$ and showing a Hill coefficient $\mathrm{n}_{\mathrm{b}}$, with remaining sites showing a dissociation constant of $\mathrm{k}_{\mathrm{A}}$ and Hill coefficient $\mathrm{n}_{\mathrm{a}}$.

\subsubsection{Fluorescence and anisotropy decay}

Tryptophan fluorescence decay lifetimes were measured using a ChronosFD spectrofluorometer (ISS, Champaign Illinois) in the frequency domain mode. Tryptophan was excited with a frequency modulated light ( $280 \mathrm{~nm}$ diode) and emission was collected using a $305 \mathrm{~nm}$ long pass filter and $400 \mathrm{~nm}$ short pass filter (Andover). The modulated anisotropy of tryptophan was calculated from the amplitude ratio of the parallel and perpendicular components of the modulated emission. The fluorescence decay lifetime of 1,8-ANS was determined by excitation with a $370 \mathrm{~nm}$ output of a frequency modulated laser diode and the emission was collected through a 400-600 nm wide pass filters (Andover). Modulation-phase data were best fitted by a multiple-exponential decay model 
using GlobalsWE software and the $\chi^{2}$ parameter was used as criterion for goodness of fit (Beechem 1989).

\subsubsection{Isothermal Titration Calorimetry (ITC)}

Thermodynamic parameters for NS5806 binding to $\operatorname{DREAM}(\Delta 65)$ and $\operatorname{DREAM}(\Delta 160)$ were determined using a VP-ITC titration calorimeter (Microcal Inc. Northampton, MA). The ITC buffer (20 mM MOPS pH 7.4, 0.25 mM DTT, and $100 \mathrm{mM}$ $\mathrm{NaCl}$ ) was prepared using decalcified water filtered through Chelex-100 resin (Bio-Rad). The protein stock solution was dialyzed against ITC buffer overnight. To minimize artifacts from mismatched buffers, stock NS5806 solutions were prepared in the final ITC dialysate buffer. The cell sample and injection syringe were extensively cleaned with decalcified water and then with ITC buffer. The reaction cell was loaded with $10 \mu \mathrm{M} \operatorname{DREAM}(\Delta 65)$ or $\operatorname{DREAM}(\Delta 160)$ solution and the concentration of NS5806 in syringe was $473 \mu \mathrm{M}$. Thirty injections ( $9 \mu \mathrm{L}$ each) of NS5806 were titrated into protein solution with 2 min intervals between injections. Parallel experiments were carried out for titration of NS5806 into the dialysate buffer as a control for heats of dilution of ligand. The temperature was kept at $25{ }^{\circ} \mathrm{C}$ and stirring speed was at $307 \mathrm{rpm}$. ITC data were analyzed using Origin 7 ITC data analysis software (OriginLab Corp. Northampton, MA). The data obtained for NS5806 binding to both KChIP3 constructs were analyzed using a two binding site model (Jelesarov, Bosshard 1999).

\subsubsection{Molecular modeling}

The NS5806 binding sites on DREAM $(\Delta 65)$ surface were identified using AutoDock 4.2 software (http://autodock.scripps.edu/) (Morris et al. 1998). The structure of $\mathrm{Ca}^{2+}$ bound DREAM( $\Delta 75)$ (PDB: 2jul, conformation \# 1) and DREAM( $\Delta 160)$ (PDB: 2E6W) 
were used as a rigid macromolecule and docking grids were set to cover the entire protein surface (Lusin et al. 2008, Yu et al. 2007b). The most favorable docking site among 200 docking simulation was selected based on their energy rankings. The structure of the NS5806- DREAM( $\Delta 65)$ complex was further refined by running 10 ns of molecular dynamic simulation using AMBER03 force fields on the YASARA interface at $298 \mathrm{~K}$, with a dynamic time step of 1.25 fs and trajectories recorded every $25 \mathrm{ps}$ for further analysis (Krieger, Koraimann \& Vriend 2002). The molecular model for the DREAM:K ${ }_{v} 4.3$ T1 domain was constructed by combining the crystal structure of KChIP1:Kv $4.3 \mathrm{~T} 1$ domain (PDB: 2NZ0) (Wang et al. 2007) and the DREAM( 475$)$ or the NS5806:DREAM(765) complex prior to molecular dynamic simulation. The energy minimized $\operatorname{DREAM}(\Delta 75): \mathrm{K}_{\mathrm{V}} 4.3 \mathrm{~T} 1$ domain or NS5806:DREAM $(\Delta 75): \mathrm{K}_{\mathrm{V}} 4.3 \mathrm{~T} 1$ domain complexes were then subjected to a $10 \mathrm{~ns}$ molecular dynamics simulation. The resulting protein and drug-protein complexes were aligned using the MUSTANG algorithm on the YASARA interface.

\subsection{Methods used in chapter 5}

\subsubsection{Steady-state fluorescence measurements}

Fluorescence emission measurements were recorded using a custom PC1 spectrofluorimeter (ISS, Illinois). Tryptophan emission spectra were measured using 295 nm excitation, and titrations were carried out in a $0.2 \times 1 \mathrm{~cm}$ path length quartz cuvette with excitation along the $0.2 \mathrm{~cm}$ path. The dissociation constants for CaM binding to DREAM were determined by a non-linear fit of the change in normalized fluorescence emission spectra at $370 \mathrm{~nm}$ using a single binding site model (Equation 2.8.1). 
To study the interaction between CaM and the N-terminus of DREAM we employed a fluorescently labeled peptide homologous to DREAM(29-44) and the fluorescence anisotropy increase upon complex formation was used to determine the affinity. A peptide homologous to DREAM(29-44) with a FITC label at the N-terminus (SIKWQRPRFTRQALMRK-FITC) was purchased from ThinkPeptides, USA. Additional titrations of apo and $\mathrm{Ca}^{2+} \mathrm{CaMRR} 2$ and DREAM(29-44)-FITC peptides were conducted by monitoring the quenching of FITC fluorescence by rhodamine through energy transfer. Equation 2.8.1 was used for fitting of the anisotropy and quenching data.

The effect of CaM on DREAM interaction with the DRE sequence of human prodynorphin was probed by steady-state fluorescence anisotropy. A synthetic oligonucleotide analogous to the DRE sequence (5-GAAGCCGGAGTCAAGGAGGCCC CTG-3) labeled at the 5' end with FITC was purchased (Eurofins, USA) and used without further purification, the complementary strand was not labeled. Samples were prepared as previously reported (Osawa et al. 2005), in the absence of LDAO.

\subsubsection{Circular dichroism measurements}

All CD spectra were measured using a Jasco J-815 CD spectropolarimeter. The far UV-CD spectra were measured at $10 \mu \mathrm{M}$ protein concentration through a $1 \mathrm{~mm}$ path in 5 mM Tris buffer $\mathrm{pH}$ 7.4. Near-UV CD spectra were measured along a $1 \mathrm{~mm}$ path using 160 $\mu \mathrm{M}$ DREAM and $560 \mu \mathrm{M}$ DREAM $(\Delta 65)$ in $20 \mathrm{mM}$ Tris $\mathrm{pH} 7.4$ and $10 \mathrm{mM}$ LDAO. All measurements were conducted at $16{ }^{\circ} \mathrm{C}$ and traces represent an average of 20 scans.

\subsubsection{Stopped-flow kinetics}

A PC1 spectrofluorometer (ISS, Illinois) with an SFA-20 stopped-flow kinetics accessory (Hi-Tech, Salisbury, UK) was used to determine the dissociation rate constants 
by monitoring the change in anisotropy upon complex dissociation. Experiments between CaM and DREAM(29-44)-FITC, as well as CaMRR2 and DREAM, were conducted by mixing 1:1 volumetric ratio of the protein complexes in the presence of $100 \mu \mathrm{M}$ calcium with $2 \mathrm{mM}$ EDTA in $20 \mathrm{mM}$ Tris $\mathrm{pH}$ 7.4. Samples were excited using $470 \mathrm{~nm}$ for DREAM(29-44)-FITC and $520 \mathrm{~nm}$ excitation for CaMRR2. The change in fluorescence anisotropy upon complex dissociation was determined by collecting the fluorescence at $520 \pm 10 \mathrm{~nm}$ for FITC and $600 \pm 10 \mathrm{~nm}$ for RRC2 using a T-format configuration. The dissociation of $\mathrm{Ca}^{2+}$ from CaM was monitored using $285 \mathrm{~nm}$ excitation and the intrinsic tyrosine fluorescence in the L-format through a $305 \mathrm{~nm}$ long pass filter. The temperature was controlled using a circulating water bath (Fisher, Isotemp M9100) and measured at the sample holder to $\pm 0.5^{\circ} \mathrm{C}$. Dissociation kinetic data were best described by single or double-exponential decay equations using Origin 8.0 software (OriginLab Corp.).

2.9.4 Fluorescence lifetime and anisotropy decay measurements

Fluorescence decay data were obtained using a ChronosFD spectrofluorometer (ISS) in the frequency domain mode. Fluorescence decay lifetime of IAEDANS or FITC complexes was determined by excitation with a $370 \mathrm{~nm}$ and $470 \mathrm{~nm}$ modulated laser diode, respectively. Fluorescence was collected through a $400 \mathrm{~nm}$ long pass filter for IAEDANS samples or $500 \mathrm{~nm}$ long pass filter for FITC samples. POPOP (1,4-bis(5-phenyloxazol-2yl)benzene) in ethanol $(\tau=1.35 \mathrm{~ns})$ or rhodamine B in water $(\tau=1.35 \mathrm{~ns})$ were used as lifetime references; polarizers were set at magic angle configuration. Anisotropy decay measurements were conducted under identical conditions. Modulation-phase data were fitted using GlobalsWE software and the $\chi^{2}$ parameter was used as a criterion for goodness of fit (Beechem 1989). 


\subsubsection{Molecular modeling and dynamic simulations}

The initial structure of $\mathrm{Ca}^{2+}$ bound DREAM( $\left.\Delta 75\right)$ (PDB: 2jul, conformation \# 1) was employed as a starting model of the core domain of the full-length protein(Lusin et al. 2008). The secondary structure of the N-terminal residues of DREAM not resolved in the NMR structure and those comprising helix 1 (residues 76-86) were predicted by a combination of six secondary structure prediction software (Jpred, Porter, PSIPRED, PredictProtein, PROF - secondary structure prediction, and CFSSP). To decrease the variability of the helix prediction, a helical conformation was only accepted if it was predicted by at least 4 of the algorithms. A model for the full-length protein was constructed by modeling the first 90 residues as an extended helix or loop according to the secondary structure prediction followed by 10 ns molecular dynamics simulation to equilibrate the structure. The helical structure of residues 76-86 (helix 1) was used to align the peptide to the published NMR structure to obtain the structure of DREAM from residue 1 to 256 .

The DREAM model was solvated and ionized as described below (resulting in a total of 122639 atoms) followed by classical and accelerated molecular dynamics simulations (cMD and aMD, respectively) to identify the dynamics and folding of the Nterminus. The parameters for the aMD were determined from the total energy and dihedral energy from a cMD simulation $\left(<\mathrm{E}_{\text {total }}>=-330141.1 \mathrm{kcal} / \mathrm{mol}\right.$ and $\left\langle\mathrm{E}_{\text {dihed }}>=973.1\right.$ $\mathrm{kcal} / \mathrm{mol})$. The dihedral boost was applied above a threshold energy set to $1993.1 \mathrm{kcal} / \mathrm{mol}$ with a dihedral alpha value of $204 \mathrm{kcal} / \mathrm{mol}$. All-atom explicit solvent molecular dynamics simulations were obtained using the molecular dynamics simulation package NAMD (Phillips et al. 2005) with the CHARMM27 force fields (Brooks et al. 1983). All simulated systems were solvated in a periodic TIP3P water box with $10 \AA$ margins and ionized with 
150 mM NaCl (Humphrey, Dalke \& Schulten 1996). Long range interactions were treated with the PME method (Essmann et al. 1995) with a $12 \AA$ cutoff. Energy was minimized with a conjugate gradient algorithm, followed by heating at $1 \mathrm{~K} / \mathrm{ps}$ from 0 to $310 \mathrm{~K}$. After reaching $310 \mathrm{~K}$, a 100 ps equilibration step in the NVT ensemble using Langevin dynamics with a $1 \mathrm{fs}$ integration time was conducted. Production runs were in the NPT ensemble (1 atm and $310 \mathrm{~K}$ ) both with Langevin coupling and with a 2 fs integration time. All simulations were performed on a i7-4770/ GTX-780Ti system using NAMD 2.9. Trajectory files were saved every 20 ps for further analysis. The initial structure used for cMD and aMD in the absence of $\mathrm{Ca} 2+$ was modeled as coarse grained spheres using the VMD plugin and MARTINI parameters (Marrink et al. 2007). The structure was minimized and simulation were run in the NPT ensemble. Solvation, ionization and forcefield parameters were identical to those utilized in CMD and aMD simulation, but with an increase time step of $20 \mathrm{fs}$.

\subsection{Methods used in chapter 6}

2.10.1 Photophysics of the DREAM:Tb ${ }^{3+}$ complex

Fluorescence experiments were conducted on a custom PC1-ChronosFD instrument (ISS, Champaign, IL) in steady-state mode for excitation and emission spectra and in frequency domain mode for fluorescence decay measurements. The intrinsic protein fluorescence arising from tyrosine residues in CaM and tryptophan on DREAM $(\Delta 65)$ was obtained by exciting the sample with $280 \pm 2 \mathrm{~nm}$ and $295 \pm 2 \mathrm{~nm}$ light, respectively. The fluorescence of 1,8-ANS was monitored by exciting the sample with $350 \pm 4 \mathrm{~nm}$ light, through a vertically oriented polarizer. The sensitized emission spectra of terbium(III) 
bound proteins were obtained by exciting the sample at $280 \pm 4 \mathrm{~nm}$ while collecting the emission through a $400 \mathrm{~nm}$ long pass filter to minimize contribution of the protein intrinsic fluorescence and the $2^{\text {nd }}$ harmonic peaks. Displacement of $\mathrm{Ca}^{2+}$ from $\operatorname{DREAM}(\Delta 65)$ was monitored by adding small aliquots of a $2.0 \mathrm{mM} \mathrm{Tb}^{3+}$ in $20 \mathrm{mM}$ Tris $\mathrm{pH} 7.4$ stock solution into 10-20 $\mu \mathrm{M}$ DREAM $(\Delta 65)$ construct in the same buffer with $5 \mathrm{mM} \mathrm{Mg}^{2+}$ and/or 100 $\mu \mathrm{M} \mathrm{Ca}{ }^{2+}$. The resulting spectra were normalized by dividing the intensity at each wavelength by the background value at $530 \mathrm{~nm}$ and the resulting titration plots were fitted using a non-cooperative n-site quadratic equation which assumes similar affinity for all the sites (see Chapter 4). All spectra were corrected for the PMT wavelength dependent response as well as the lamp wavelength dependent changes in intensity. The intrinsic fluorescence lifetime of $\operatorname{DREAM}(\Delta 65)$ was measured by exciting the sample with the modulated light of a $280 \mathrm{~nm}$ diode and the fluorescence collected through a $320 \mathrm{~nm}$ long pass filter with 2,5-diphenyloxazole (PPO) in ethanol $(\tau=1.40 \mathrm{~ns})$ used a lifetime reference.

Circular dichroism measurements were conducted in a Jasco J-815 CD spectrometer along the $1 \mathrm{~mm}$ path of a quartz cuvette (J-815 Jasco, Easton, MD). Luminescence measurements were conducted on a custom-built instrument where the sample was placed in a 2x10 mm quartz cuvette in a temperature controlled sample holder (Quantum Northwest, Liberty Lake, WA) and excited along the $10 \mathrm{~mm}$ path. The $355 \mathrm{~nm}$ line of a Nd:YAG laser (Minilite II Continuum, San Jose, CA) was used to directly excite $\mathrm{Tb}^{3+}$ ions while the luminescence was measured perpendicularly through a $550 \pm 10 \mathrm{~nm}$ band pass filter and detected by a H7360-01 PMT (Hamamatsu, Japan). The signal was digitized by a $400 \mathrm{MHz}$ oscilloscope (WaveSurfer 42Xs, Teledyne Lecroy, NY) and the 
initial $100 \mu$ seconds of each trace were deleted to eliminate contribution from scattered light and PMT recovery time. The fluorescence modulation-phase plots and luminescence decay traces were fit using Globals for spectroscopy software (LFD, Irvine CA).

2.10.2 Thermodynamics of the DREAM:Tb ${ }^{3+}$ complex

Isothermal calorimetry titrations were employed to determine the thermodynamics of $\mathrm{Tb}^{3+}$ displacement of $\mathrm{Ca}^{2+}$ from $\operatorname{DREAM}(\Delta 65)$ or $\mathrm{CaM}$ and they were carried out using a VP-ITC isothermal calorimeter (Microcal Inc. Northampton, MA). Protein constructs were dialyzed overnight in $5 \mathrm{mM}$ Tris $\mathrm{pH}$ 7.4, $100 \mathrm{mM} \mathrm{NaCl}, 100 \mu \mathrm{M} \mathrm{CaCl}_{2}$ with/without $5 \mathrm{mM} \mathrm{MgCl}_{2}$. The use of $10 \mathrm{mM}$ EDTA during purification of calmodulin required multiple overnight dialysis steps to ensure complete removal of contaminating EDTA. Terbium stock solutions were prepared in ITC dialysate buffer. The reaction cell was loaded with $\sim 10 \mu \mathrm{M}$ protein solution, determined spectrophotometrically prior to the ITC experiment, the concentration of $\mathrm{Tb}^{3+}$ in the syringe $(297 \mu \mathrm{L})$ was $1.00 \mathrm{mM}$. Thirty injections of increasing volume were titrated into the protein solution with increasing time intervals between injections. Isotherms were corrected for the heat of dilution of ligand and all ITC experiments were conducted in triplicates. The recovered thermodynamic parameters were

obtained by fitting the isotherms modeled either with an N-set-of-sites model or a sequential model using the Microcal ITC analysis plug in Origin 7.0.

2.10.3 Trapped ion mobility spectrometry—mass spectrometry studies (TIMS-MS) 2.10.4 Experimental:

Details regarding the TIMS operation and specifics compared to traditional IMS can be found elsewhere (Fernandez-Lima, Kaplan \& Park 2011, Fernandez-Lima et al. 2011, Molano-Arevalo et al. 2014, Hernandez et al. 2014). Briefly, in TIMS, the mobility 
separation is achieved by holding the ions stationary with an electric field against a moving gas. The separation in a TIMS device can be described by the center of the mass frame using the same principles as in a conventional IMS drift tube (McDaniel, Mason 1973). The TIMS analyzer was coupled to a maXis Impact Q-UHR-ToF (Bruker Daltonics Inc., Billerica, MA). Data acquisition was controlled using in-house software, written in National Instruments Lab VIEW (2012, v. 12.0f3), and synchronized with the maXis Impact acquisition program. The TIMS separation was performed using nitrogen as a bath gas at ca. $300 \mathrm{~K}$ and typical P1 (pressure at the ramp electrode) and P2 (pressure at the outlet electrode) values are 1.8 and 0.6 mbar, respectively. The pressure difference drives the input of the sample into the analyzer. The same RF (880 kHz and 200-350 Vpp) was applied to all electrodes including the entrance funnel, the mobility separating section, and the exit funnel. The purpose of the voltage potentials is to generate an electromagnetic field able to trap the ions in the TIMS analyzer and minimize loos of the charged ions through diffusion. Protein samples were prepared at $15 \mu \mathrm{M}$ concentration protein and $15 \mu \mathrm{M}$ $\mathrm{TbCl}_{3} \cdot 6 \mathrm{H}_{2} \mathrm{O}$ using HPLC grade solvents from Thermo Fisher Scientific Inc. (Waltham, MA) in $10 \mathrm{mM}$ ammonium acetate at $\mathrm{pH}=6.7$. A custom built, nano electrospray ionization source was coupled to the TIMS-MS analyzer and was used for all analyses. A typical source voltage of 600-1200 V was used and analyses were performed in positive ion mode.

\subsubsection{Theoretical}

Theoretical CCS were calculated for the previously reported 2JUL NMR structure of DREAM (Lusin et al. 2008) using the IMoS (v1.04b) (Larriba, Hogan Jr 2013, Larriba, Hogan 2013, Ouyang et al. 2013) software with nitrogen as a bath gas at ca. 300K. In the 
IMoS calculations, 100 total rotations were performed using the diffuse hard sphere scattering method with a Maxwell distribution. In addition, I have also developed a MATLAB script designed to extract the average peak intensity, calculate calibration constants, and convert mobility to collisional cross sections for multiple peaks. This script was designed in order to expedite and facilitate the analysis of multiple ion species. For instance, using TIMS-MS we are able to identify the apo, $1 \mathrm{Ca}^{2+}$, and $2 \mathrm{Ca}^{2+}$ bound DREAM produces. In the presence of NS5806, the same peaks with additional peaks corresponding to NS5806 $+1 \mathrm{Ca}^{2+}$ and NS5806 $+2 \mathrm{Ca}^{2+}$ can be identified. Thus, if the charge states from +6 to +22 are identified (which is common), this would result in a total of 80 peaks. In addition, TIMS experiments require the use of different ramp times (often a set of five ramp times are used), resulting in a total of 400 peaks to be analyzed. It becomes obvious that a large amount of time could be spent analyzing the data. The details about this script (which is not used in the presented work) are listed in appendix 2.

\subsection{Methods used in chapter 7}

\subsubsection{Caged compounds}

The DM-nitrophen samples were prepared by dissolving DM-nitrophen in buffers as described in the text. The $\mathrm{Ca}^{2+}$-DM-nitrophen samples were prepared by adding $\mathrm{CaCl}_{2}$ to DM-nitrophen sample to achieve a desirable ratio of $[\mathrm{DM} \text {-nitrophen }]_{\text {total }}:\left[\mathrm{Ca}^{2+}\right]_{\text {total }}$. The sample preparation and measurements were carried out in a dark room to prevent unwanted uncaging and release of the chelated ligand $\left(\mathrm{H}^{+}, \mathrm{Ca}^{2+}\right.$, or $\left.\mathrm{Tb}^{3+}\right)$. Absorption spectra were recorded using a single beam UV-vis spectrophotometer (Cary 50, Varian) and the DMnitrophen concentration was determined from the absorbance value at $350 \mathrm{~nm}$. 
The synthesis of the $\mathrm{H}_{2}$ Cage and $\mathrm{Cu}\left(\mathrm{OH}_{2}\right)$ Cage compounds were performed in the laboratory of Dr. Konstantinos Kavallieratos at FIU and were done according to the procedure reported by Ciesienski et al. (2008). The purity was verified by $\mathrm{H}^{1} \mathrm{NMR}$ (400 $\mathrm{MHz}$ Bruker NMR spectrometer) and IR (Nicolet Magna-IR 560 spectrometer) spectroscopy. For all trials and experiments, both the ligand and complex were dissolved in a MOPS buffer mixture (5mM MOPS buffer, $\mathrm{pH} 7.10$ ) and then transferred to a $1 \mathrm{x} 0.5$ cm quartz cuvette. To minimize unwanted photo cleavage all samples were prepared and measured in a dark room. Absorptions spectra for both compounds were collected using a single beam UV-Vis spectrophotometer (Cary 50, Varian).

\subsubsection{Isothermal Titration Calorimetry (ITC)}

Thermodynamic parameters for Terbium binding to DM-nitrophen or EDTA were determined using a VP-ITC titration calorimeter (Microcal Inc. Northampton, MA). The ITC buffer (20 mM MOPS pH 6.5 with and without $1 \mathrm{mM} \mathrm{CaCl}_{2}$ ) was prepared using ultrapure $18 \mathrm{M} \Omega$ water which was decalcified by filtering through a Chelex-100 resin (BioRad). The cell sample and injection syringe were extensively cleaned with decalcified water and then with ITC buffer. The reaction cell was loaded with 120 $\mu$ M DM-nitrophen solution and the concentration of $\mathrm{Tb}^{3+}$ in syringe was $2 \mathrm{mM}$. Thirty injections (10 $\mu \mathrm{L}$ each) of $\mathrm{Tb}^{3+}$ were titrated into a protein solution with 2 min intervals between injections. Parallel experiments were carried out for titration of $\mathrm{Tb}^{3+}$ into buffer as a control for heats of dilution. The temperature was kept at $25{ }^{\circ} \mathrm{C}$ and stirring speed was at $307 \mathrm{rpm}$. The ITC results were analyzed using Origin 7 ITC data analysis software (OriginLab Corp. Northampton, MA). 


\subsubsection{Photothermal beam deflection instrument}

All photothermal beam deflection (PBD) measurements were performed on a custom-built instrument described in detail elsewhere (Mikšovská, Day \& Larsen 2003). The sample was held in a $1.0 \times 0.5 \mathrm{~cm}$ cuvette which was placed inside a temperature controlled holder (TC 125, Quantum Northwest). Illumination of the sample was done with a Nd: YAG laser (355 nm, 5 ns pulse, Minilite, Continuum, CA) passed through the 1.0 cm path length of the cuvette. A diode probe beam (635 nm, $5 \mathrm{~mW}$ output from He-Ne Laser, Edmund Optics) was aligned so that it is nearly collinear to the pump beam and crossed in the center of the sample cell. A mirror on the opposite side was used to align the probe beam onto the center of a split diode, so the intensity of the Gaussian laser beam is equally distributed between the two photodiodes. The detector is of our own design and the signal output was digitized by a $400 \mathrm{MHz}$ digitizer (WaveSurfer, LeCroy). All data analysis was performed off line in Origin 8.0 data analysis software (OriginLab Corp. Northampton, MA or Microsoft Excel.

\subsection{Methods used in chapter 8}

\subsubsection{Modeling of DREAM}

The initial structure of $\mathrm{Ca}^{2+}$ bound $\operatorname{DREAM}(\Delta 75)$ (PDB: 2jul, conformation \# 1) was employed as a starting model of the core domain of DREAM protein (Lusin et al. 2008). This core domain contains helix 1 through 10 and 2 calcium ions bound at EF-hand and 3 and 4. All DREAM mutants were generated by the mutate residue plug in of VMD using the conformation \#1 of DREAM as the initial structure (Humphrey, Dalke \& Schulten 1996). The DREAM models were then solvated using TIP3P waters and ionized 
with $\mathrm{Na}+$ ions to neutrality using the ionize plug in of VMD. The complete system consisted of about 29800 atoms in a $81.5 \AA$ x $68.6 \AA$ x $58.1 \AA$ rectangular box, which is big enough to prevent the protein from interacting with its own periodic image.

The dimeric form of DREAM was generated by overlaying the monomeric $\mathrm{Ca}^{2+}$ bound structure of DREAM (pdb: 2jul, conformation \#1) on each subunit of the dimeric structure observed in the structure of KChIP1 (pdb: 1s6c) obtained by x-ray crystallography (Zhou et al. 2004b). This dimeric form of DREAM is in good agreement with the dimer structure proposed previously (Lusin et al. 2008), the dimeric structure was subsequently solvated and ionized as described above.

2.12.2 All atom molecular dynamic simulations

Classical molecular dynamics (cMD) and accelerated molecular dynamics (aMD) simulations have been widely employed to study the conformational dynamics and folding of proteins at the atomic level (Liu et al. 2005, Zhou et al. 2004a, Hamelberg, Mongan \& McCammon 2004, Kern, Zuiderweg 2003). In this work, we have carried out cMD and aMD simulations using the NAMD (Phillips et al. 2005) simulation software and the CHARMM27 force field including the phi, psi, cross term map (CMAP) correction (MacKerell, Feig \& Brooks 2004). These forcefield have been shown to be able to reproduce experimental NMR and unfolding data of the neuronal calcium sensor-1 protein (Bellucci et al. 2013, Heidarsson et al. 2013, Zhu et al. 2014). Accelerate MD simulations have also been shown to enhance the conformational space sampled by proteins within shorter simulation times (Hamelberg, Mongan \& McCammon 2004). This method has been used to provide insight into the allosteric mechansims of calcium binding in calcium binding proteins (Kekenes-Huskey et al. 2012). 
In both simulation approaches, the long range interactions were treated with the PME method (Essmann et al. 1995) with a 12 A cutoff. The system described above was energy minimized with a conjugate gradient algorithm, followed by: 1) heating at $1 \mathrm{~K} / \mathrm{ps}$ from 0 to $155 \mathrm{~K}$, 2) 200 ps of simulation using constant pressure, temperature, and number of particles (NPT), and 3) a second heating step from $155 \mathrm{~K}$ to $310 \mathrm{~K}$ at $1 \mathrm{~K} / \mathrm{ps}$. We have noticed that performing the short NPT equilibration step between the heating steps eliminates the formation of vacuum bubbles during the simulation. After reaching $310 \mathrm{~K}$, a 100 ps equilibration step in the NVT ensemble using Langevin dynamics with a $1 \mathrm{fs}$ integration time was conducted. Production runs were in the NPT ensemble (1 atm and 310 K) both with Langevin coupling and a 2 fs integration time. All simulations were performed on a i7-4770/ GTX-780Ti system using NAMD 2.10. Trajectory files were saved every 20 ps for further analysis.

Accelerated molecular dynamics (aMD) is an enhanced sampling technique in which the energy of the system is modified throughout the simulation in order to allow the protein to transition into structural conformations not readily sampled by classical molecular simulations (cMD). The acceleration in aMD arises from introduction of a nonnegative energy to the dihedral angles of all amino acids in the protein whenever the total dihedral energy of the system goes below a predefined threshold (Miao et al. 2015). This concept of acceleration can be best explained by the following equations:

$$
\begin{gathered}
V_{\text {boost }}(t)=V(t) \quad \text { if } \quad V(t) \geq E_{\text {reference }} \\
V_{\text {boost }}(t)=V(t)+\Delta V(t) \quad \text { if } \quad V(t)<E_{\text {reference }}
\end{gathered}
$$

In which $\mathrm{V}(\mathrm{t})$ is the potential of the system at simulation time $\mathrm{t}$, $\mathrm{E}_{\text {reference }}$ is the reference threshold potential, and $\mathrm{V}_{\text {boost }}(\mathrm{t})$ is the modified potential. In our case, the $\mathrm{E}_{\text {reference }}$ value 
has been obtained from a 10 ns simulation of the system using classical molecular dynamics using:

$$
E_{\text {reference }}=\left[V_{\text {dihedral }}\right]+a * N_{\text {residue }}
$$

Where $\left[\mathrm{V}_{\text {dihedral }}\right]$ is the average dihedral energy of the cMD simulation, $\mathrm{N}_{\text {residue }}$ is the number of amino acids in the protein, and a is an empirically determined constant usually near 4. The parameter $\Delta \mathrm{V}(\mathrm{t})$ represents the boost potential, which takes the form of:

$$
\Delta V(t)=\frac{\left(E_{\text {reference }}-V(t)\right)^{2}}{\alpha+E_{\text {reference }}-V(t)}
$$

In which the $\alpha$ value has been introduced as an acceleration factor that must be either adjusted based on simulation results. In our work, we have set the $\alpha$ value as previously reported using (Miao et al. 2015):

$$
\alpha=\frac{a}{5} * N_{\text {residue }}
$$

We have observed that setting value for a near 4 would result in the $\mathrm{N}$ - and C-terminus helices of DREAM to become unstable, thus in our simulations we have used a value of a $=3.26$. The scripts used are described in detail in appendix 3 .

\subsubsection{Coarse grained simulations}

In order to explore conformational changes in DREAM which may occur on longer timescales, we have performed coarse-grained molecular dynamics simulations (cgMD). In this approach, the atomic composition of amino acids in DREAM is reduced by clustering of atom into larger bead models. These models have been optimized to maintain the biophysical characteristics of the protein, while reducing the computational cost of performing long simulations. We have performed simulation on DREAM using the same model described above, in which the all atom representation was reduced from about 29800 
atoms to about 6900 beads. The model was protonated using the pdb2pqr webserver (Dolinsky et al. 2004), solvated with WT4 waters and neutralized with NaW sodium-water coarse-grained ion representations. These simulations were performed on the GROMACS 5.0.7 software package (Hess et al. 2008) and the SIRAH coarse-grained force fields (Darré et al. 2015). The simulations protocol included 100000 minimization steps using the steeped descent algorithm, followed by 250000 steps of equilibration with $10 \mathrm{fs}$ time step and positional restrain applied to all the protein beads. Production runs were performed in the NPT ensemble at 20 fs time step for $1 \mu$ s (Darré et al. 2015).

\subsection{Analysis methods}

Analysis of the cMD, aMD, nd cgMD simulations were carried using the VMD 1.9.2 software. Root mean square deviations (RMSD) were calculated for the alpha carbon of the whole protein (residue 76 to 256) using the structure of DREAM after minimization and equilibration as the frame of reference. ge of 500 frames (10 ns) of the alpha carbons of selected amino acids. The amino acids included in calculation of the N-terminus RMSF included residues 86 to 160 and residues 163 to 242 for the C-terminus included. The solvent accessible surface of Trp169 was calculated using a rolling sphere of $1.4 \AA$ radius.

Analysis of the conformational dynamics of DREAM were analyzed by community network analysis of trajectories in which the equilibration time was deleted. We compared the RMSD of all simulations (apo and $\mathrm{Ca}^{2+}$ bound separately) and observed that all simulations were equilibrated within 20 ns. Therefore, we deleted the first 1000 frames of every simulation, in order to analyze only the dynamic networks present in the equilibrated 
apo or $\mathrm{Ca}^{2+}$ bound DREAM. The network analysis was performed using the VMD plug in NetworkSetup and visualized using Network View (Sethi et al. 2009). In this representation, each node represents the alpha carbon of an amino acids, and the edges connecting non-neighboring alpha carbons represents atoms which interacted over $75 \%$ of the time within $4.5 \AA$. The thickness of the edges represents the degree of correlation between the nodes. These networks were further classified by the Girvan-Newman algorithm in order to split the networks into communities of dynamically coupled residues (Girvan, Newman 2002). In these communities, amino acids belonging to one communities are more correlated to each other than to amino acids of other communities. Furthermore, the connection between these communities, represent dynamic hubs through which most dynamic fluctuations propagate from one community to the rest of the protein.

\subsubsection{Circular dichroism and Emission spectra}

All CD spectra were measured using a Jasco J-815 CD spectropolarimeter. The far UV-CD spectra were measured at $10 \mu \mathrm{M}$ protein concentration through a $1 \mathrm{~mm}$ path in 5 $\mathrm{mM}$ phosphate buffer $\mathrm{pH}$ 7.4. Near-UV CD spectra were measured along a $1 \mathrm{~mm}$ path using $160 \mu \mathrm{M}$ DREAM and $560 \mu \mathrm{M} \operatorname{DREAM}(\Delta 65)$ in $20 \mathrm{mM}$ Tris $\mathrm{pH} 7.4$ and $10 \mathrm{mM}$ LDAO. All measurements were conducted at $16{ }^{\circ} \mathrm{C}$ and traces represent an average of 20 scans.

\subsubsection{Tryptophan fluorescence}

The tryptophan fluorescence of DREAM was monitored by placing $20 \mu \mathrm{M}$ to 40 $\mu \mathrm{M}$ DREAM protein in 20mM MOPS pH 7.4 and $1 \mathrm{mM}$ DTT buffer in a 2x10 mm quartz cuvette. The sample was placed in the sample holder of a ChronosFD-PC1 custom spectrofluorometer, with $295 \mathrm{~nm}$ excitation along the short $2 \mathrm{~mm}$ path and emission 
collected along the $10 \mathrm{~mm}$ path. The bandwidth was set at $\pm 4 \mathrm{~nm}$. All measurements were conducted at room temperature.

\subsection{4 $\mathrm{Ca}^{2+}$ binding experiments}

The binding of $\mathrm{Ca}^{2+}$ to all the DREAM constructs was carried out in a ChronosFDPC1 custom spectrofluorometer (ISS). A protein concentrations between $4 \mu \mathrm{M}$ to $6 \mu \mathrm{M}$ was used throughout, and the sample was prepared by dilution of a concentrated DREAM stock (concentrations ranging from $450 \mu \mathrm{M}$ to $800 \mu \mathrm{M}$ ) stored in 20mM Tris $\mathrm{pH}$ 7.4. No more than $50 \mu \mathrm{L}$ of DREAM was added to $3 \mathrm{~mL}$ of $20 \mathrm{mM}$ MOPS pH 7.4 supplemented with $1 \mathrm{mM}$ EGTA and 1mM NTA. This buffer was prepared by dilution of $100 \mathrm{mM}$ EGTA and $100 \mathrm{mM}$ NTA standard solution prepared separately to an accuracy of $\pm 2 \%$, as determined by electrochemical titration using a $\mathrm{Ca}^{2+}$ electrode. In the event that the DREAM stock was highly concentrated, an additional volume of 20mM Tris pH 7.4 was added so that the total volume added to the 3mL EGTA-NTA buffer solution was always $50 \mu \mathrm{L}$. The addition of $50 \mu \mathrm{L}$ of solution to all samples ensured that any dilution of EGTA and NTA was constant throughout all the experiments. The protein in EGTA-NTA buffer was placed in a $1 \mathrm{x} 1 \mathrm{~cm}$ quartz cuvette and the sample was kept at room temperature throughout $\left(\sim 17^{\circ} \mathrm{C}\right)$. The excitation wavelength was set to $295 \mathrm{~nm}$ with a bandwidth of \pm $4 \mathrm{~nm}$, while the emission was collected through a monochromatic with bandwidth of \pm $4 \mathrm{~nm}$. The emission spectra was collected from $305 \mathrm{~nm}$ to $450 \mathrm{~nm}$ at a step of $2 \mathrm{~nm}$. A total of twenty two samples were prepared, ranging from $0 \mathrm{mM}$ to $1 \mathrm{mM} \mathrm{CaCl}_{2}$, with injection volumes ranging from $0.9 \mu \mathrm{L}$ to $33 \mu \mathrm{L}$. The contribution associated with dilutions were not corrected, as we observe that this correction induced an erroneous increase in fluorescence; however, this effect should only be present in the last 3 to 4 point as most, since the dilution 
of the first 18 points was not more $3 \%$ in total. Each injection was added with a Hamilton syringe (Sigma-Aldrich). The change in tryptophan fluorescence was determined by integrating the emission spectra between $309 \mathrm{~nm}$ to $423 \mathrm{~nm}$. The data were analyzed by a NLLS fit to the data using origin 8.0 software and a simple one site HILL equation.

\subsubsection{DREAM stability studies}

The guanidium hydrochloride $(\mathrm{GuHCl})$ induced protein unfolding was monitored by measuring the tryptophan fluorescence spectra as a function of increasing $\mathrm{GuHCl}$ concentration. The samples for fluorescence measurements were prepared by solubilizing a $80 \mu \mathrm{M}$ DREAM protein stock in $20 \mathrm{mM}$ MOPS $\mathrm{pH} 7.4,1 \mathrm{mM}$ DTT to a final concentration of $5 \mu \mathrm{M}$. The samples were then incubated in the presence of $\mathrm{GuHCl}$ for 18 to 24 hours prior to measurements. A total of twenty four samples were measured, each sample $(\sim 100 \mu \mathrm{L})$ was placed in a $3 \times 3 \times 5$ (width-length-height) quartz cuvette. The emission spectra were recorded using a FluoroMax-3 spectrofluorometer (Horiba, Japan) at room temperature $\left(\sim 17^{\circ} \mathrm{C}\right)$. The excitation was set a $295 \mathrm{~nm}$ with an excitation and emission bandwidth of $4 \mathrm{~nm}$. A total of three scans were average for each sample. DREAM unfolding traces were analyzed as a one-step or two-step process involving an intermediate state:

$$
\begin{aligned}
& \mathrm{N} \leftrightarrow \mathrm{U} \quad \text { Eqn. } 8.2 \\
& \mathrm{~N} \leftrightarrow \mathrm{I} \leftrightarrow \mathrm{U} \quad \text { Eqn. } 8.3
\end{aligned}
$$

where $\mathrm{N}$ corresponds to the native state, I to the intermediate state, and $\mathrm{U}$ to the unfolded state. The unfolding curves were fit using the following equation (Hung et al. 2003, Santoro, Bolen 1988): 


$$
\lambda(G u H C l)=\frac{\lambda_{N}+\lambda_{I} \exp \frac{\left(-\Delta G_{I}^{\circ}-m_{I}[D]\right)}{R T}+\lambda_{U} \exp \frac{-\left(\Delta G_{I}^{\circ}-m_{I}[D]+\Delta G_{U}^{\circ}-m_{U}[D]\right)}{R T}}{1+\exp \frac{-\left(\Delta G_{I}^{\circ}-m_{I}[D]\right)}{R T}+\exp \frac{-\left(\Delta G_{I}^{\circ}-m_{I}[D]+\Delta G_{U}^{\circ}-m_{U}[D]\right)}{R T}}
$$

Eqn. 8.4

where $\lambda(\mathrm{GuHCl})$ is the wavelength of the emission maxima of the tryptophan fluorescence, $\Delta \mathrm{G}$ is the standard free energy for the transition, $\mathrm{m}$ is the dependence of the free energy on the denaturant concentration, $T$ is the temperature ( $T=290 \mathrm{~K}$ ), [D] is the concentration of GuHCl and $\mathrm{R}$ is the ideal gas constant, $\mathrm{R}=8.314 \mathrm{JK}^{-1} \mathrm{~mol}^{-1}$. The parameters $\lambda_{\mathrm{N}}, \lambda_{\mathrm{I}}$, and $\lambda_{U}$ represent the wavelength of the tryptophan emission maxima of each molecular species at zero GuHCl concentration.

\subsubsection{Site 2 peptide binding to DREAM constructs}

Binding of DREAM to the site 2 peptide of the voltage gated potassium channel $\mathrm{K}_{\mathrm{v}} 4.3$ were carried out using the same spectrofluorometer as in section 2.12.3. In these experiments a $10 \mu \mathrm{M}$ solution of Kv4.3 peptide (described in section 2.8.2) was solubilized in $20 \mathrm{mM}$ Tris pH 7.4 supplemented with 1mM DTT in the presence of 1mM EDTA or 500 $\mu \mathrm{M} \mathrm{CaCl}_{2}$. The sample was placed in a $2 \times 10 \mathrm{~mm}$ quartz cuvette and the dansyl chloride covalently bound to the peptide was excited with $330 \mathrm{~nm}$ light. The dansyl fluorescence was collected through a $400 \mathrm{~nm}$ longpass filter and a vertically oriented polarizer placed in front of a monochromator. The presence of the polarizer in the configuration described above eliminates strong contribution from scattering as well as the Wood's anomaly of our instrument, which happens at $500 \mathrm{~nm}$. The DREAM construct was added to the sample from a concentrated stock at concentration ranges between 0 and $100 \mu \mathrm{M}$, without exceeding $10 \%$ of the total volume of the sample $(450 \mu \mathrm{L})$. Dilution effects were corrected. 


\section{ROLE OF THE HYDROPHOBIC CAVITY AT THE C-TERMINUS DOMAIN OF DREAM IN LIGAND BINDING AND RECOGNITION}

\subsection{Background and significance}

The function of DREAM and its interactions with various target proteins are strongly dependent on its cellular localization. In the nucleus, DREAM functions as an agonist transcriptional modulator of the specific DRE (downstream regulatory element) sequence found in prodynorphin and c-fos genes (Carrion et al. 1999, Carrion, Mellstrom \& Naranjo 1998, Ledo et al. 2000). DREAM regulates gene expression in a $\mathrm{Ca}^{2+}$ dependent manner with apoDREAM binding to DRE sequence as a tetramer whereas $\mathrm{Ca}^{2+}$ bound DREAM forms a stable dimer with a decreased affinity for DRE sequence (Osawa et al. 2005, Carrion et al. 1999). In the cytoplasm, DREAM binds to the $\mathrm{K}_{\mathrm{v}} 4$ voltage channels through the N-terminus in a calcium independent fashion (An et al. 2000, Bowlby et al. 2005). In addition, in vitro and in vivo studies have proposed DREAM involvement in regulating apoptotic processes, accumulation of amyloid $\beta$ peptides, and $\mathrm{N}$-cadherin processing through interactions with presenilin 1 (Buxbaum et al. 1998). It was recently also shown that DREAM forms a heterodimer with calmodulin in a $\mathrm{Ca}^{2+}$ dependent manner and regulates calmodulin function (Ramachandran et al. 2012).

However, the molecular basis of DREAM - target proteins/DNA association and the mechanism of how $\mathrm{Ca}^{2+}$ binding modulates DREAM - target protein/DNA interactions remains unclear. Based on the NMR structure of $\mathrm{Ca}^{2+}$ loaded DREAM, it has been proposed that solvent exposed basic residues located at the DREAM N-terminal domain may form the DNA binding pocket and that solvent exposed leucine residues stabilize the $\mathrm{Ca}^{2+}$ DREAM dimer (Lusin et al. 2008). The leucine residue rich domain found close to 
EF-hand 2 may also mediate DREAM interactions with leucine zipper proteins such as $\alpha$ CREM (Ledo et al. 2000). The structure of the $\mathrm{Ca}^{2+}$ bound DREAM reveals the presence of a large hydrophobic cavity located between EF-hand 1 and EF-hand 2 which extends into the C-terminal domain. In addition, solvent exposed hydrophobic residues were shown to be involved in DREAM interactions with $\mathrm{K}_{\mathrm{v}} 4$ channels and possibly with other intracellular partners. For example, the structure of the KChIP1-Kv4.3 complex reveals two binding sites for the T1 domain; the first binding site on KChIP1 is formed by a hydrophobic cavity extending from the N-terminal domain to the C-terminal domain. The second binding site has a more polar character and contains residues located at the $\mathrm{N}$ terminal domain (Scannevin et al. 2004). In addition, the functional properties of the KChIP-K 4 complexes have been observed to be modulated by association of small hydrophobic molecules, such as arachidonic acid (Holmqvist et al. 2001) and NS5806 (Witzel, Fischer \& Bähring 2012).

Considering the multifunctional role of DREAM in neuronal cells and its interactions with diverse intracellular targets, we have investigated the structural transitions induced by $\mathrm{Ca}^{2+}$ and/or $\mathrm{Mg}^{2+}$ binding to DREAM. In particular, we are interested in identifying the hydrophobic binding sites that may be involved in stabilization of DREAM oligomers, specific recognition of intracellular partners, and providing binding sites for small hydrophobic molecules. Using the extrinsic fluorescent probes 8-anilino 1naphathalene sulfonate (1,8-ANS) and 6-anilino 2-naphthalene sulfonate (2,6-ANS), both of which are highly sensitive to their immediate environment, two distinct hydrophobic sites with high affinity for ANS probes were identified. These sites were located at the Cterminal domain and were characterized using time-resolved fluorescence and docking 
studies. In addition, 1,8-ANS displacement studies show that site 2 located between helix 7, 8, and 10 serves as a binding site for arachidonic acid and possibly for other hydrophobic modulators of $\mathrm{K}_{\mathrm{v}} 4$ :KChIP complexes. The identification of high affinity hydrophobic sites on DREAM surface may provide the structural insight necessary for development of small molecules for modulation of DREAM interactions with other proteins.

\subsection{Results}

3.2.1 $\mathrm{Ca}^{2+}$ triggers changes in DREAM surface hydrophobicity
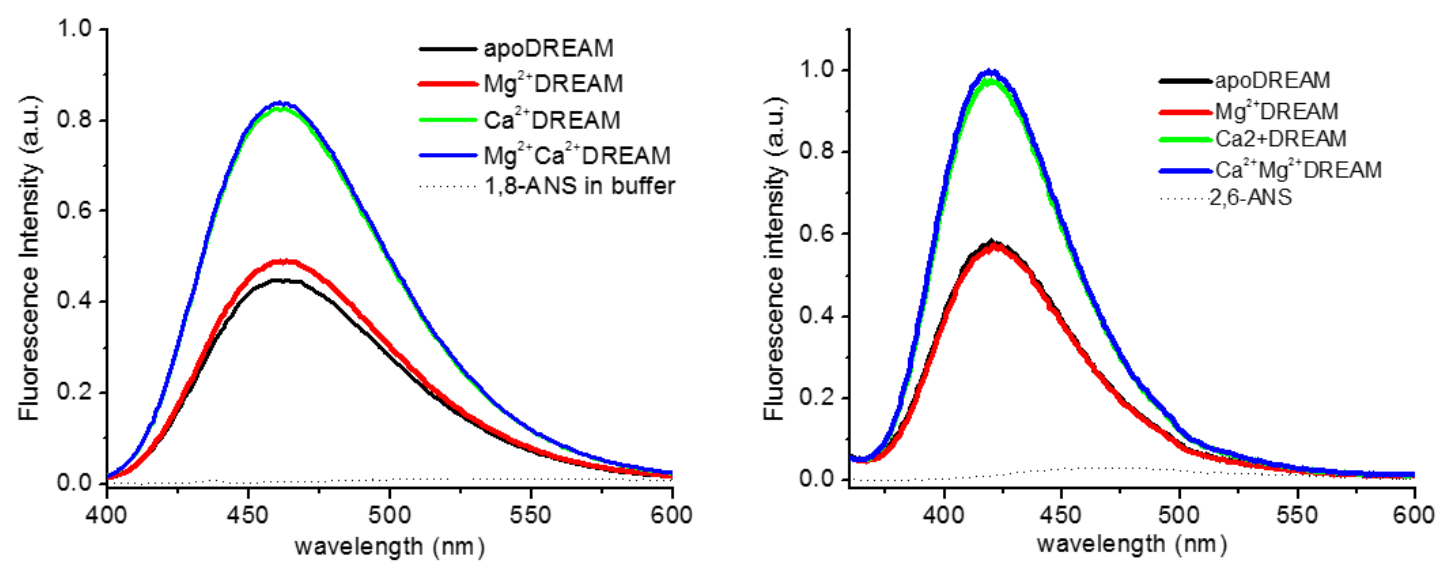

Figure 3.1. Normalized fluorescence emission spectra of 1,8-ANS (left panel) and 2,6ANS (right panel) bound to DREAM. Conditions: $40 \mu \mathrm{M}$ DREAM in $20 \mathrm{mM}$ Tris pH 7.4 and $1 \mathrm{mM}$ DTT. The excitation wavelength was $350 \mathrm{~nm}$ and $319 \mathrm{~nm}$ for 1,8-ANS and 2,6ANS, respectively. 


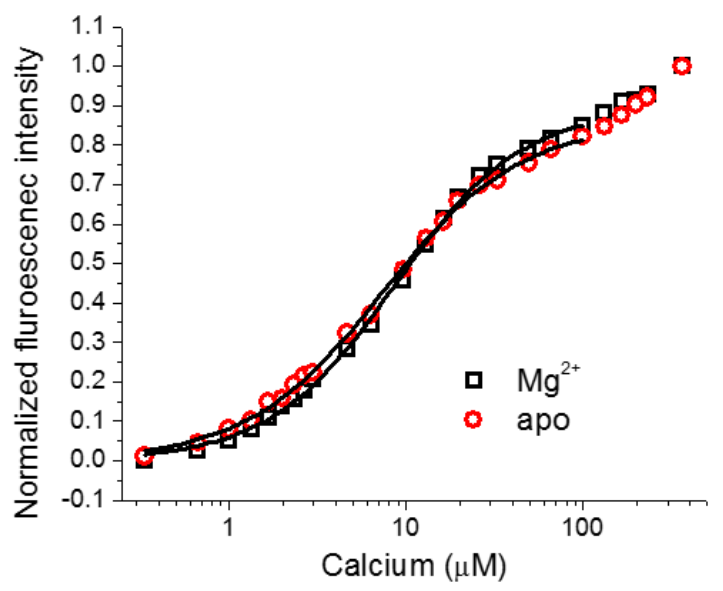

Figure 3.2 Calcium titration to $7 \mu \mathrm{M}$ DREAM in the presence of $10 \mu \mathrm{M} 1,8$-ANS. Calcium titration into $\mathrm{Mg}^{2+}$ bound DREAM (squares) and apostate DREAM(circles). Samples were prepared in 20mM Tris $\mathrm{pH} 7.4,100 \mathrm{mM} \mathrm{NaCl}$, and $1 \mathrm{mM}$ DTT which was decalsified by passing through an equilibrated CHELEX resin column (BioRad) and delivered into a decalsified $1 \mathrm{cmx} 1 \mathrm{~cm}$ quartz cuvette. The average of three trials is shown, and nonlinear fit with a Hill equation (solid lines) yields $K_{d}=8.0 \pm 0.9 \mu \mathrm{M}$ with a Hill coefficient of $n=1.22$.

The ANS emission intensity increases in the presence of DREAM due to ANS association to the protein, a further increase in intensity is observed upon $\mathrm{Ca}^{2+}$ addition to both apo- and $\mathrm{Mg}^{2+} \mathrm{DREAM}$ indicating that ANS binds to hydrophobic cavities on the DREAM surface (Figure 3.1). The identical emission spectra for 1,8-ANS bound to $\mathrm{Ca}^{2+} \mathrm{DREAM}$ and $\mathrm{Mg}^{2+} \mathrm{Ca}^{2+} \mathrm{DREAM}$ suggest that the change in surface polarity due to $\mathrm{Ca}^{2+}$ binding to apo or $\mathrm{Mg}^{2+} \mathrm{DREAM}$ are identical. On the other hand, association of $\mathrm{Mg}^{2+}$ to apoDREAM leads to a small but reproducible increase in the 1,8-ANS emission compared to the apoform. This change is consistent with a more stable tertiary structure of $\mathrm{Mg}^{2+}$ bound DREAM, which may induce slight changes of the ANS binding sites (Osawa et al. 2005). Nonetheless the hydrophobic core is not fully exposed in the $\mathrm{Mg}^{2+}$ bound form of the protein. The results for 2,6-ANS are consistent with those obtained using 1,8-ANS probe. We further observes that ANS association to DREAM does not impair the binding of $\mathrm{Ca}^{2+}$, since the $\mathrm{K}_{\mathrm{d}}$ value determined for $\mathrm{Ca}^{2+}$ association to DREAM-ANS complexes, 
$\mathrm{K}_{\mathrm{d}}=8.0 \pm 0.9 \mu \mathrm{M}$ (Figure 3.2) is comparable to that determined by ITC, $\mathrm{K}_{\mathrm{d}}=1-10 \mu \mathrm{M}$ (Osawa et al. 2005).

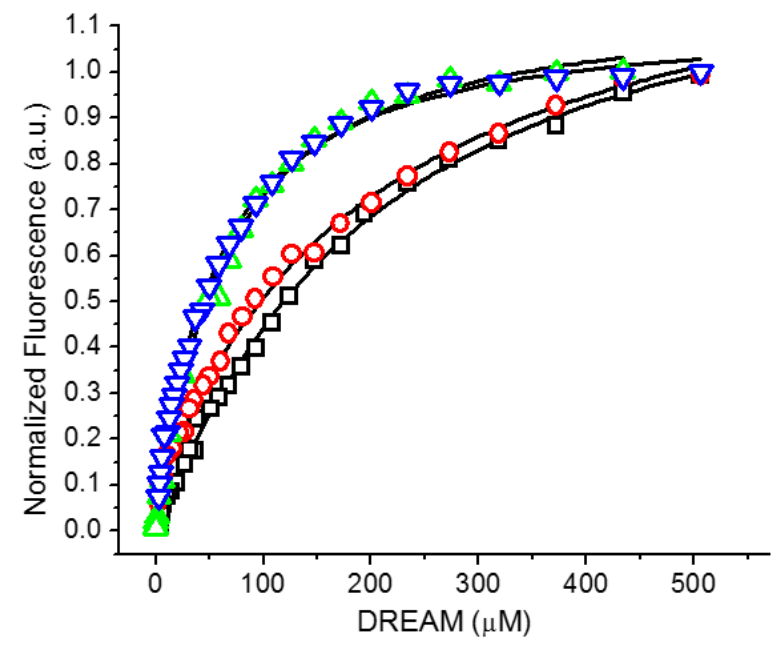

Figure 3.3. Titration of $10 \mu \mathrm{M} 1,8-\mathrm{ANS}$ by apoDREAM (squares), $\mathrm{Mg}^{2+} \mathrm{DREAM}$ (circles), $\mathrm{Ca}^{2+}$ DREAM (up-triangles), and $\mathrm{Mg}^{2+} \mathrm{Ca}^{2+} \mathrm{DREAM}$ (down triangles). The solid lines correspond to the fit of experimental data.

Affinity of the ANS probes for DREAM was determined by conducting a series of titrations, with ANS titrated into a constant amount of protein (data not shown) or with protein titrated into a constant amount of ANS (Figure 3.3). Both approaches provided identical $K_{d}$ values, within the experimental error. The titration curve shown in Figure 3.3 were analyzed using a single binding site model according to eqn. 2.7.1. Approximately three times higher affinity for 1,8-ANS was observed for $\mathrm{Ca}^{2+}$ bound DREAM (Table 3.1) than in the apoform or in the presence of $\mathrm{Mg}^{2+}$. The affinity of 2,6-ANS for apoDREAM $\left(\mathrm{K}_{\mathrm{dss}} \sim 330 \mu \mathrm{M}\right)$ is significantly lower than that for 1,8-ANS but it is also promoted by $\mathrm{Ca}^{2+}$ association to DREAM ( $\left.\mathrm{K}_{\mathrm{dss}} \sim 154 \mu \mathrm{M}\right)$ (Figure 3.4). Dynamic light scattering and gel filtration studies have shown that in the absence of calcium, DREAM adopts a tetrameric 
conformation whereas $\mathrm{Ca}^{2+}$ bound DREAM forms dimers (Carrion et al. 1999, Osawa et al. 2001).

\begin{tabular}{lcccccc}
\hline \multicolumn{6}{c}{ Table 3.1 Equilibrium dissociation constants for 1,8-ANS binding to DREAM } \\
\hline DREAM & $\begin{array}{l}\mathrm{Kd}_{\mathrm{ss}} \\
(\mu \mathrm{M})^{\mathrm{a}}\end{array}$ & $\mathrm{nss}^{\mathrm{b}}$ & $\begin{array}{l}\mathrm{Kd}_{\alpha 1} \\
(\mu \mathrm{M})^{\mathrm{c}}\end{array}$ & $\begin{array}{l}\mathrm{Kd}_{\alpha 2} \\
(\mu \mathrm{M})^{\mathrm{c}}\end{array}$ & $\begin{array}{l}\mathrm{Kd}_{\text {tetramer }} \\
(\mu \mathrm{M})\end{array}$ & $\begin{array}{l}\mathrm{Kd}_{\text {dimer }} \\
(\mu \mathrm{M})\end{array}$ \\
\cline { 2 - 7 } apo & $195 \pm 20$ & 1.10 & $235 \pm 36$ & $158 \pm 27$ & $46 \pm 5$ & \\
$\mathrm{Mg}^{2+}$ & $198 \pm 28$ & 1.00 & $217 \pm 20$ & $131 \pm 30$ & $59 \pm 9$ & $31 \pm 3$ \\
$\mathrm{Ca}^{2+}$ & $62 \pm 4$ & 1.09 & $68 \pm 13$ & $63 \pm 12$ & & $30 \pm 2$ \\
$\mathrm{Mg}^{2+} \mathrm{Ca}^{2+}$ & $60 \pm 3$ & 1.11 & $107 \pm 16$ & $49 \pm 30$ & & 30 \\
\hline
\end{tabular}

(a) Dissociation constants calculated from steady-state fluorescence. (b) Errors are less than 0.1 units. (c) Calculated from fluorescence decay parameters $\alpha_{1}$ and $\alpha_{2}$.

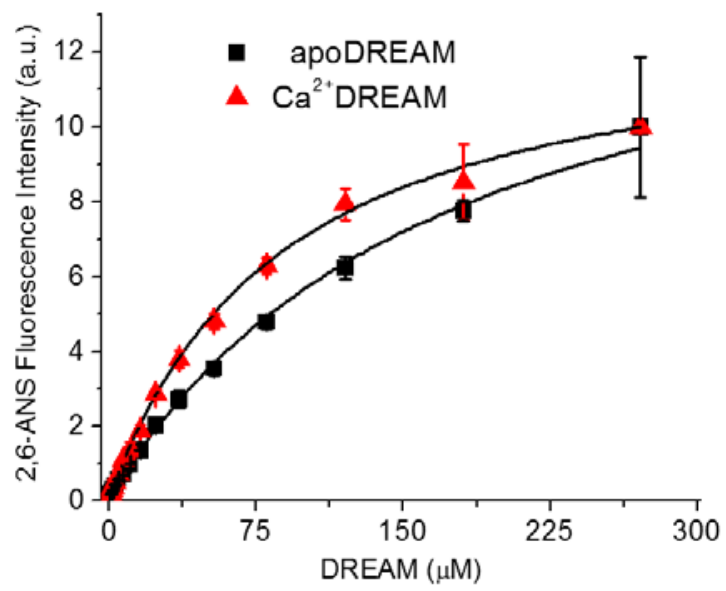

Figure 3.4. Titration of 2,6-ANS by DREAM. The concentration of 2,6-ANS was kept constant $(10 \mu \mathrm{M})$ and emission was collected from $405 \mathrm{~nm}$ to $600 \mathrm{~nm}$ using excitation wavelength of $319 \mathrm{~nm}$. The data were corrected for scattering as described in Material and Methods section. The solid lines correspond to the fit of experimental data using a hill equation.

The dissociation constants show a stronger ANS binding to DREAM monomer in the dimeric $\mathrm{Ca}^{2+}$ bound form than in the tetrameric $\mathrm{Ca}^{2+}$ free form of the protein. The increased accessibility of ANS binding sites could be due to a conformational change in each 
monomer and/or due to the tetramer dissociation into individual dimers. The equilibrium constants were also determined by plotting the ANS emission intensity as a function of the tetramer concentration (for apo- and $\mathrm{Mg}^{2+} \mathrm{DREAM}$ ) and dimer concentration (for $\mathrm{Ca}^{2+}$ - and $\mathrm{Ca}^{2+} \mathrm{Mg}^{2+} \mathrm{DREAM}$ ) (Table 3.1). The affinity of the DREAM dimer for 1,8-ANS is approximately two times larger than that of the DREAM tetramer which is consistent with an increased accessibility of the ANS binding sites upon tetramer dissociation into the individual dimers.

\subsection{2 $\mathrm{Ca}^{2+}$ association to the EF-hand 3 and EF-hand 4 increases accessibility of ANS} binding sites
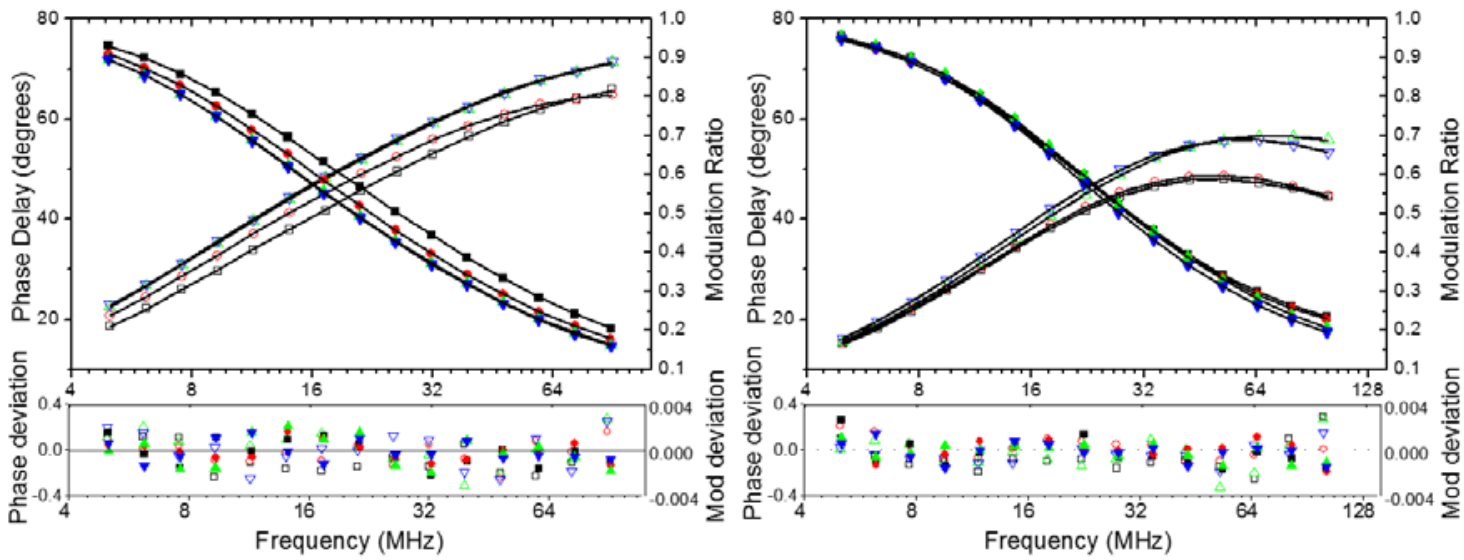

Figure 3.5: Frequency-domain intensity decay of ANS bound to apoDREAM (squares), and $\mathrm{Mg}^{2+}$ (circles), $\mathrm{Ca}^{2+}$ (up-triangle), and $\mathrm{Mg}^{2+} \mathrm{Ca}^{2+}$ (down triangle). Phase delay is shown as empty symbols, modulation ratio as solid symbols and the solid lines represent the calculated fitting curves. Protein concentration was $47.5 \mu \mathrm{M}$ with $30 \mu \mathrm{M} 1$,8-ANS or $30 \mu \mathrm{M} 2,6-\mathrm{ANS}$.

The lifetimes for 1,8-ANS and 2,6-ANS DREAM complexes were determined using frequency domain fluorometry (Figure 3.5), and the decay parameters are summarized in Table 3.2. The lifetime of 1,8-ANS in $20 \mathrm{mM}$ Tris $\mathrm{pH} 7.4$ was measured to be $0.27 \mathrm{~ns}$ with a minor fraction ( $\sim 2 \%$ ) of a 4 ns lifetime due to the non-planar excited state decay 
(Upadhyay et al. 1995, Someya, Yui 2010). The lifetime of 2.6-ANS was determined to be 0.35 ns in agreement with a previously determined value (León et al. 2009).

In the presence of $\operatorname{DREAM}(\Delta 65)$, two additional lifetimes were resolved and attributed to ANS bound to at least two binding sites with distinct polarity. Heterogeneous binding is common and was reported for other ANS-protein systems (Parul et al. 2000, Togashi, Ryder 2008, Gasymov, Glasgow 2007). We associate the longer lifetime ( 16 ns) to 1,8-ANS bound to a nonpolar solvent restricted binding site, labeled site 2, that promotes the decay of the excited state by populating the long lived non-planar conformation of 1,8-ANS (Upadhyay et al. 1995). The faster lifetime can be attributed to the 1,8-ANS probe bound to a partially solvent exposed binding surface, site 1 , which facilitates rotation of the ANS aniline group into a lower energy planar configuration with respect to the naphthalene group (Gao, Mammen \& Whitesides 1996).

\begin{tabular}{lllllc}
\hline \multicolumn{7}{l}{ Table 3.2 Decay parameters for ANS-DREAM complexes } \\
\hline 1,8-ANS & $\tau_{1}(\mathrm{~ns})$ & $\alpha_{1}(\%)$ & $\tau_{2}(\mathrm{~ns})$ & $\alpha_{2}(\%)$ & $\chi^{2}$ \\
\cline { 2 - 6 } apo & $5.9 \pm 0.1$ & $13.1 \pm 0.5$ & $16.2 \pm 0.7$ & $22.5 \pm 0.3$ & 1.16 \\
$\mathrm{Mg}^{2+}$ & $5.7 \pm 0.2$ & $13.9 \pm 0.6$ & $16.2 \pm 0.9$ & $26.1 \pm 0.4$ & 1.13 \\
$\mathrm{Ca}^{2+}$ & $5.9 \pm 0.2$ & $17.9 \pm 0.9$ & $17.6 \pm 0.5$ & $41.9 \pm 0.6$ & 1.13 \\
$\mathrm{Mg}^{2+} \mathrm{Ca}^{2+}$ & $5.9 \pm 0.2$ & $17.8 \pm 0.9$ & $17.5 \pm 0.5$ & $44.5 \pm 0.5$ & 0.95 \\
\hline $2,6-\mathrm{ANS}$ & $\tau_{1}(\mathrm{~ns})$ & $\alpha_{1}(\%)$ & $\tau_{2}(\mathrm{~ns})$ & $\alpha_{2}(\%)$ & $\chi^{2}$ \\
\cline { 2 - 6 } $\mathrm{apo}$ & $4.6 \pm 0.2$ & $9.8 \pm 0.3$ & $10.3 \pm 0.3$ & $39.9 \pm 0.3$ & 0.87 \\
$\mathrm{Mg}^{2+}$ & $4.5 \pm 0.9$ & $9.6 \pm 0.6$ & $10.5 \pm 0.6$ & $42.5 \pm 0.4$ & 0.83 \\
$\mathrm{Ca}^{2+}$ & $4.6 \pm 0.9$ & $9.9 \pm 0.1$ & $10.6 \pm 0.3$ & $60.0 \pm 0.7$ & 1.44 \\
$\mathrm{Mg}^{2+} \mathrm{Ca}^{2+}$ & $4.9 \pm 1.1$ & $11.1 \pm 0.5$ & $10.7 \pm 0.9$ & $56.0 \pm 0.6$ & 0.98 \\
\hline
\end{tabular}

Data recovered from Globals software using a sum of three discrete exponential decays with fixed 1,8-ANS lifetime ( $\left.\tau_{0}=0.27 \mathrm{~ns}\right)$ and 2,6-ANS lifetime ( $\tau_{0}=0.35$ ns). Phase and modulation errors were set at $\leq 0.2^{\circ}$ and $\leq 0.002$ respectively.

The fluorescence decay parameters are also sensitive to structural changes associated with $\mathrm{Ca}^{2+}$ binding to $\operatorname{DREAM}(\Delta 65)$. 1,8-ANS bound to $\mathrm{Ca}^{2+} \mathrm{Mg}^{2+}$ or 
$\mathrm{Ca}^{2+} \operatorname{DREAM}(\Delta 65)$ shows a small increase in its long lifetime and almost twofold increase in the pre-exponential parameter $\alpha_{2}$ compared to the apoprotein or $\operatorname{Mg}^{2+} \operatorname{DREAM}(\Delta 65)$. The fact that the individual 1,8-ANS lifetimes are not significantly altered by $\mathrm{Ca}^{2+}$ association to $\operatorname{DREAM}(\Delta 65)$ is consistent with 1,8-ANS binding to identical binding sites in the $\mathrm{Ca}^{2+}$ free and $\mathrm{Ca}^{2+}$ bound DREAM $(\Delta 65)$. The small increase of the longer lifetime, $\tau_{2}$, indicates that $\mathrm{Ca}^{2+}$ induced structural changes in the vicinity of site 2 further restrict the rotation of the aniline ring. We associate the increase in the pre-exponential factors (Table 3.2) with an increased accessibility of the ANS binding sites due to $\mathrm{Ca}^{2+}$ triggered change in the $\operatorname{DREAM}(\Delta 65)$ monomer structure and concomitant tetramer dissociation into dimers. Minor changes in the lifetime or pre-exponential factor values were measured for $\mathrm{Mg}^{2+}$ addition to either apoDREAM $(\Delta 65)$ or $\mathrm{Ca}^{2+}$ bound $\operatorname{DREAM}(\Delta 65)$, supporting the idea that $\mathrm{Mg}^{2+}$ association to $\operatorname{DREAM}(\Delta 65)$ does not significantly alter surface hydrophobicity and the structural changes due to $\mathrm{Mg}^{2+}$ association are localized in the vicinity of the EF-2. Two lifetimes were also resolved for 2,6-ANS bound to apoDREAM $(\Delta 65)$ (Table 3.2) in agreement with two different binding environments for hydrophobic ANS probes. The increase in the pre-exponential parameters for 2,6-ANS$\mathrm{Ca}^{2+} \operatorname{DREAM}(\Delta 65)$ complexes is smaller compared to those for 1,8-ANS$\mathrm{Ca}^{2+} \operatorname{DREAM}(\Delta 65)$ complexes. This is expected since the elongated structure of 2,6-ANS could represents a larger steric hindrance for binding to hydrophobic pockets on the protein surface.

The phase and modulation data presented in Figure 3.5 were also analyzed using a phasor approach (Figure 3.6) (James et al. 2011). The resulting phasor plots clearly show a significant change in the signal between ANS in Tris buffer solution and in the presence 
of apoDREAM and $\mathrm{Ca}^{2+}$ bound DREAM. Interestingly, a small difference was observed between experimental data for apoDREAM $(\Delta 65)$ and $\mathrm{Mg}^{2+} \operatorname{DREAM}(\Delta 65)$; this shift is consistent with the small increase in the emission intensity due to the $\mathrm{Mg}^{2+}$ binding to $\operatorname{DREAM}(\Delta 65)$. The phasor plot for 2,6-ANS bound to $\operatorname{DREAM}(\Delta 65)$ shows similar results as those observed for 1,8-ANS DREAM( $\Delta 65)$ complex (data not shown).

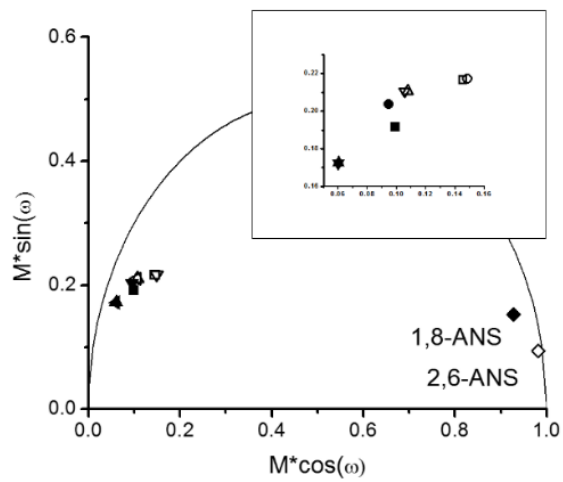

Figure 3.6. Phasor plot representation of frequency domain data for 1,8-ANS (solid symbols) and 2,6-ANS (open symbols) bound to by apoDREAM (squares), $\mathrm{Mg}^{2+}$ DREAM (circles), $\mathrm{Ca}^{2+} \mathrm{DREAM}$ (up-triangles), and $\mathrm{Mg}^{2+} \mathrm{Ca}^{2+} \mathrm{DREAM}$ (down triangles). Conditions: $64 \mu \mathrm{M}$ DREAM and $30 \mu \mathrm{M}$ ANS, modulation frequency $(\omega)$ was $70 \mathrm{MHz}$.

The pre-exponential parameters $\left(\alpha_{0}, \alpha_{1}, \alpha_{2}\right)$ corrected by their quantum yields correlate with the concentration of unbound ANS $\left(\alpha_{0}\right)$ and ANS associated to individual binding sites $\left(\alpha_{1}, \alpha_{2}\right)$ with lifetimes $\tau_{1}$ and $\tau_{2}$, respectively. These pre-exponential factors can be used to calculate the fraction of ANS bound to site 1 and site 2 (Robinson et al. 1978, Kosower, Kanety 1983) and determine ANS binding constants for individual binding sites. Since titrations of DREAM into $28 \mu \mathrm{M}$ ANS solution resulted in very small deviations in individual lifetimes $(<0.5 \mathrm{~ns})$, the lifetime data obtained at different $\operatorname{DREAM}(\Delta 65)$ concentrations were analyzed using the global analysis approach, i.e. the lifetimes corresponding to ANS bound to protein were treated as linked variables and the lifetime for unbound ANS was fixed. The plots of $\alpha_{1}, \alpha_{2}$ as a function of 
$\mathrm{Ca}^{2+} \operatorname{DREAM}(\Delta 65)$ concentration are shown in Figure 3.7 and the recovered dissociation constants are presented in Table 3.1.

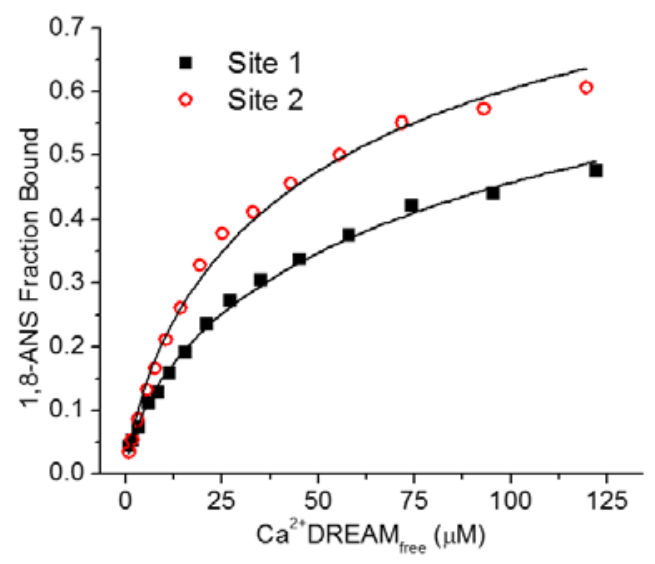

Figure 3.7. Titration curves for 1,8-ANS binding to site 1 and site 2 of $\mathrm{Ca}^{2+} \mathrm{DREAM}$. The concentration of the free 1,8-ANS bound to site $1\left(\tau_{1} \sim 6 \mathrm{~ns}\right)$ and binding site $2\left(\tau_{2} \sim 17\right.$ ns) was determined based on the pre-exponential parameters $\alpha_{0}, \alpha_{1}$, and $\alpha_{2}$. The solid line corresponds to the fit using a Hill equation.
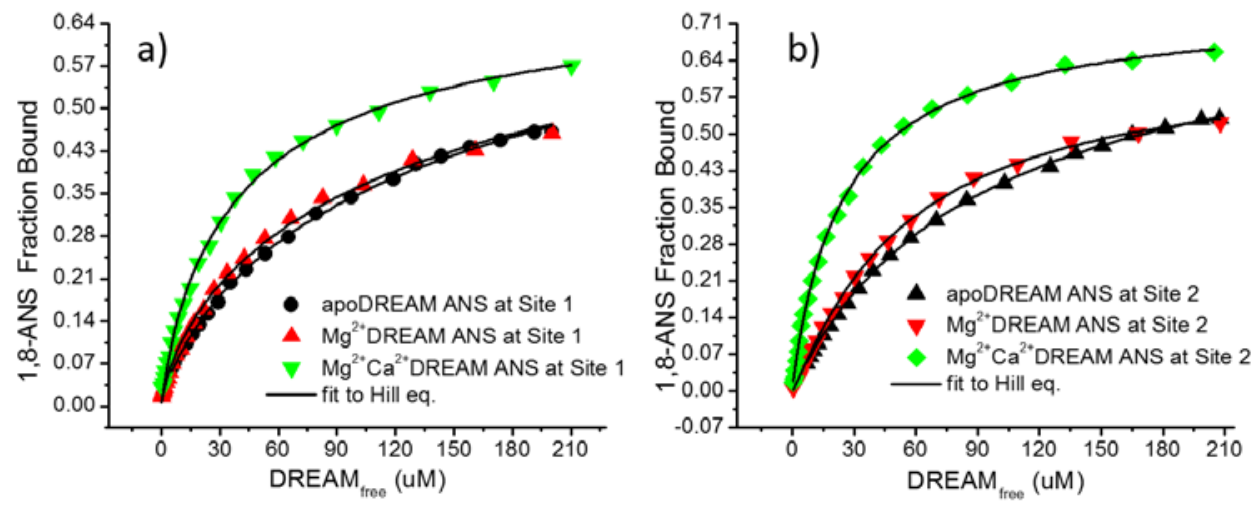

Figure 3.8. Titration of $28.2 \mu \mathrm{M}$ 1,8-ANS by DREAM using pre-exponential factors. The concentration of ANS bound to site $1(a)$ and site $2(b)$ was determined based on the preexponential parameters $\alpha_{0}, \alpha_{1}$, and $\alpha_{2}$.

The plots for the titrations of apoDREAM, $\mathrm{Mg}^{2+}$ DREAM and $\mathrm{Ca}^{2+} \mathrm{Mg}^{2+}$ DREAM are provided in Figure 3.8. Interestingly, the binding constants for 1,8-ANS binding to apoDREAM are similar with $\mathrm{K}_{\mathrm{d}}=235 \mu \mathrm{M}$ for site 1 and $\mathrm{K}_{\mathrm{d}}=158 \mu \mathrm{M}$ for site 2 . The $\mathrm{Ca}^{2+}$ bound DREAM exhibits an approximately 2-3 times increase in affinity for 1,8-ANS 
binding to both sites which is in agreement with the overall binding constants determined in steady-state emission measurements.
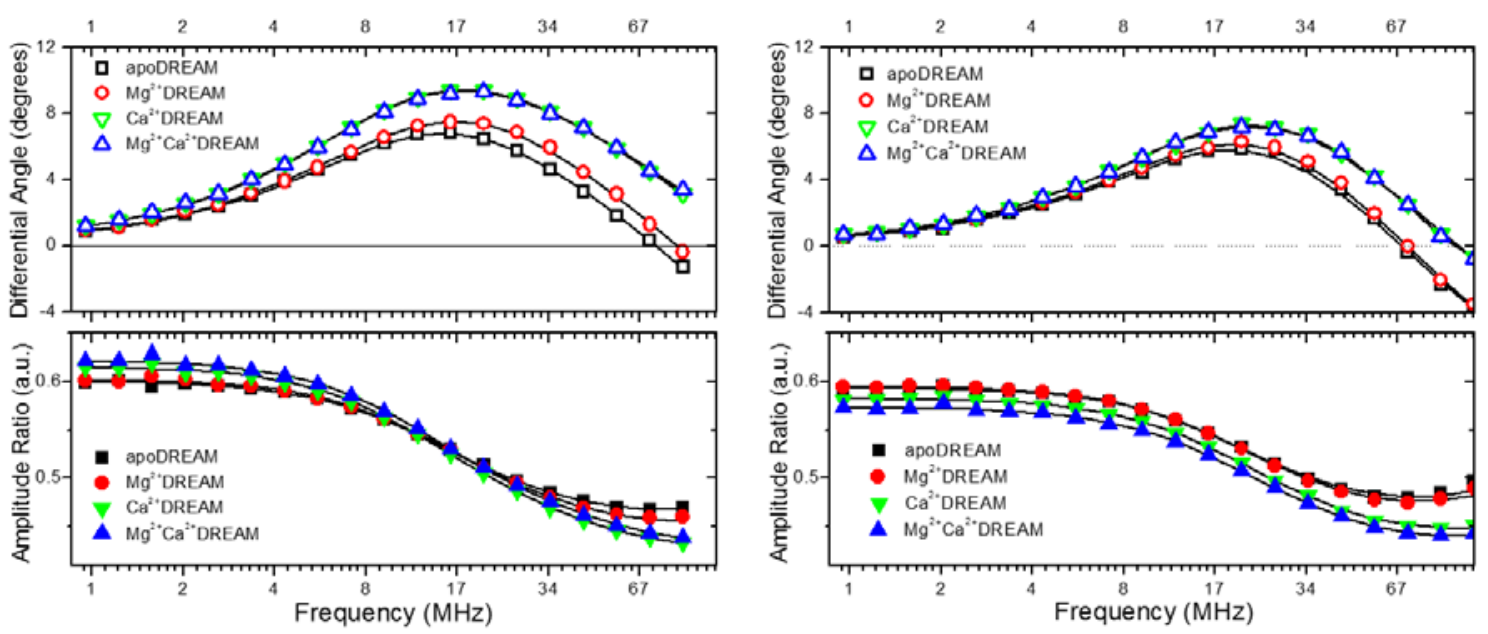

Figure 3.9. Differential angle and modulation ratio data for 1,8-ANS (left) and 2,6-ANS (right) in complex with DREAM. The solid lines correspond to the NLLS fit of the data as described in the text. Conditions were the same as in Figure 3.5.

\subsubsection{Changes in DREAM oligomerization}

We have taken further advantage of the long fluorescence lifetime of 1,8-ANS and 2,6-ANS DREAM complexes by monitoring the changes in DREAM oligomerization by probing the rotational diffusion of the ANS-DREAM complexes in time-resolved anisotropy measurements. The frequency-domain anisotropic decay of ANS-DREAM complexes in the presence of $\mathrm{Mg}^{2+}$ and/or $\mathrm{Ca}^{2+}$ are shown in Figure 3.9. Results from the non-linear least square analysis of the differential angle and amplitude ratio are listed in Table 3.3. The differential angle plots show a transition towards negative values at high modulation frequencies. This is characteristic for complexes with a fluorophore located in dissimilar environments with distinct depolarization contributions (Szmacinski et al. 1987). Under our experimental conditions, ANS is bound to at least two binding sites on 
the surface of DREAM, while residual unbound ANS also contributes to the observed signal.

Table 3.3. Anisotropy decay parameters of 1,8-ANS$\operatorname{DREAM}(\Delta 65)$ and $2,6-\mathrm{ANS}-\mathrm{DREAM}(\Delta 65)$

\begin{tabular}{cccccc}
\hline 1,8-ANS & $\Phi_{1}{ }^{\mathrm{a}}(\mathrm{ns})$ & $f_{1}{ }^{\mathrm{b}}$ & $\Phi_{2}{ }^{\mathrm{a}}(\mathrm{ns})$ & $f_{2}{ }^{\mathrm{b}}$ & $\chi^{2}$ \\
\cline { 2 - 6 } apo & $55.0 \pm 6.1$ & 0.29 & $13.9 \pm 0.6$ & 0.38 & 1.15 \\
$\mathrm{Mg}^{2+}$ & $57.6 \pm 5.8$ & 0.25 & $12.3 \pm 0.4$ & 0.38 & 0.96 \\
$\mathrm{Ca}^{2+}$ & $26.4 \pm 0.7$ & 0.48 & $6.7 \pm 0.4$ & 0.17 & 0.99 \\
$\mathrm{Mg}^{2+} \mathrm{Ca}^{2+}$ & $23.8 \pm 0.5$ & 0.51 & $5.2 \pm 0.4$ & 0.11 & 1.16 \\
\hline $2,6-\mathrm{ANS}$ & $\Phi_{1}^{\mathrm{a}}(\mathrm{ns})$ & $f_{1}{ }^{\mathrm{b}}$ & $\Phi_{2}{ }^{\mathrm{a}}(\mathrm{ns})$ & $f_{2}{ }^{\mathrm{b}}$ & $\chi^{2}$ \\
\cline { 2 - 6 } apo & $55.8 \pm 7.7$ & 0.20 & $9.4 \pm 1.7$ & 0.39 & 0.83 \\
$\mathrm{Mg}^{2+}$ & $54.9 \pm 6.9$ & 0.13 & $12.1 \pm 1.9$ & 0.39 & 0.97 \\
$\mathrm{Ca}^{2+}$ & $22.3 \pm 1.5$ & 0.69 & $5.7 \pm 2.4$ & 0.23 & 1.17 \\
$\mathrm{Mg}^{2+} \mathrm{Ca}^{2+}$ & $21.8 \pm 1.2$ & 0.43 & $6.9 \pm 2.1$ & 0.15 & 1.71 \\
\hline Conditions: $40 \mu \mathrm{M}$ ANS and 53 $\mu \mathrm{M}$ DREAM $(\Delta 65)$, rotational $^{\text {correlation for unbound ANS was fixed, } \Phi_{0}=0.105 \text { ns. (a) }}$ \\
Recovered rotational correlation lifetime. (b) Recovered \\
fractional depolarization, errors are less than 0.05 units.
\end{tabular}

Nearly identical experimental data were observed for 1,8-ANS bound to $\mathrm{Ca}^{2+} \mathrm{DREAM}$ and

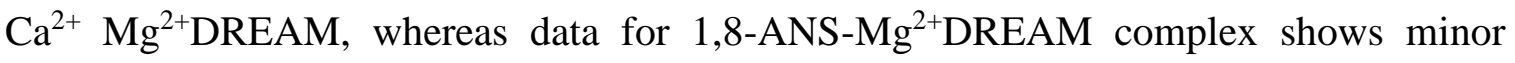
deviations from 1,8-ANS bound to apoDREAM. Non-linear analyses of experimental data yielded satisfactory $\chi^{2}$ values only when a triple associative decay model was used with the rotational lifetime for freely rotating 1,8-ANS or 2,6-ANS being fixed at $\Phi_{0}=0.105$ ns. This value was determined independently and is consistent with the value of $0.092 \mathrm{~ns}$ reported previously (Szmacinski et al. 1987). In addition to the rotational correlation time for unbound 1,8-ANS or 2,6-ANS, two rotational correlation times, $\Phi_{1}$ and $\Phi_{2}$, were resolved and are associated with a global and a local motion of the ANS-DREAM complexes, respectively, and are reported in Table 3.3. The rotational lifetimes of 1,8-ANS 
bound to DREAM closely matches those for 2,6-ANS, confirming that the measured correlation times reflect properties of the macromolecule. The rotational correlation times $\Phi_{1} \sim 56 \mathrm{~ns}$ and $\Phi_{2} \sim 13 \mathrm{~ns}$ are similar for apoDREAM and $\mathrm{Mg}^{2+}$ DREAM complexes with the fractional depolarizations, $f_{1}$ and $f_{2}$ accounting for $29 \%$ and $38 \%$ of the total depolarization. Upon $\mathrm{Ca}^{2+}$ binding, the longer rotational correlation time decreases to 26 ns for $\mathrm{Ca}^{2+} \mathrm{DREAM}$ and to $\sim 24$ ns for $\mathrm{Ca}^{2+} \mathrm{Mg}^{2+} \mathrm{DREAM}$ complexes and accounts for $~$ $50 \%$ of the anisotropy decay. The faster rotational time, $\Phi_{2}$, reaches 6.7 ns and 5.2 ns for 1,8-ANS bound to $\mathrm{Ca}^{2+} \mathrm{DREAM}$ and $\mathrm{Ca}^{2+} \mathrm{Mg}^{2+} \mathrm{DREAM}$, respectively. Analogous values were determined for the 2,6-ANS-DREAM complexes (Table 3.3).

The experimentally determined rotational correlation times can be compared to expected values for a spherical protein calculated by using the Stokes-Einstein equation $\left(\Phi_{1}{ }^{\text {calc }}=\eta \mathrm{V} / \mathrm{RT}\right)($ Lakowicz 2010$)$ where $\eta$ is the viscosity of water $(\eta=1.02 \mathrm{cP})$ (Korson, Drost-Hansen \& Millero 1969), V is the hydrodynamic molecular volume, $\mathrm{R}$ is the ideal gas constant, and $\mathrm{T}$ is the temperature $\left(20^{\circ} \mathrm{C}\right)$. Based on the molecular mass of $23 \mathrm{kDa}$ for truncated mouse DREAM, and accounting for protein hydration we estimate the rotational correlation time for DREAM tetramer and dimer to be 48 ns and 24 ns, respectively. The rotational correlation time observed for $\mathrm{Ca}^{2+}$ DREAM and $\mathrm{Ca}^{2+} \mathrm{Mg}^{2+} \mathrm{DREAM}$ matches well the estimated rotational time for the DREAM dimer confirming that $\mathrm{Ca}^{2+}$ binding to either apoDREAM or $\mathrm{Mg}^{2+} \mathrm{DREAM}$ stabilizes the protein in its dimeric form. The measured correlation rotational time for apo- and $\mathrm{Mg}^{2+} \mathrm{DREAM}, \Phi_{1} \sim 56 \mathrm{~ns}$, indicates that in the absence of $\mathrm{Ca}^{2+}$, the protein adopts a tetrameric form. The observed rotational correlation times are somehow larger than the rotational correlation time calculated for the DREAM tetramer and the discrepancy between the observed and calculated values indicates that the 
spherical model approximation applied here may not accurately describe the properties of the DREAM tetramer.

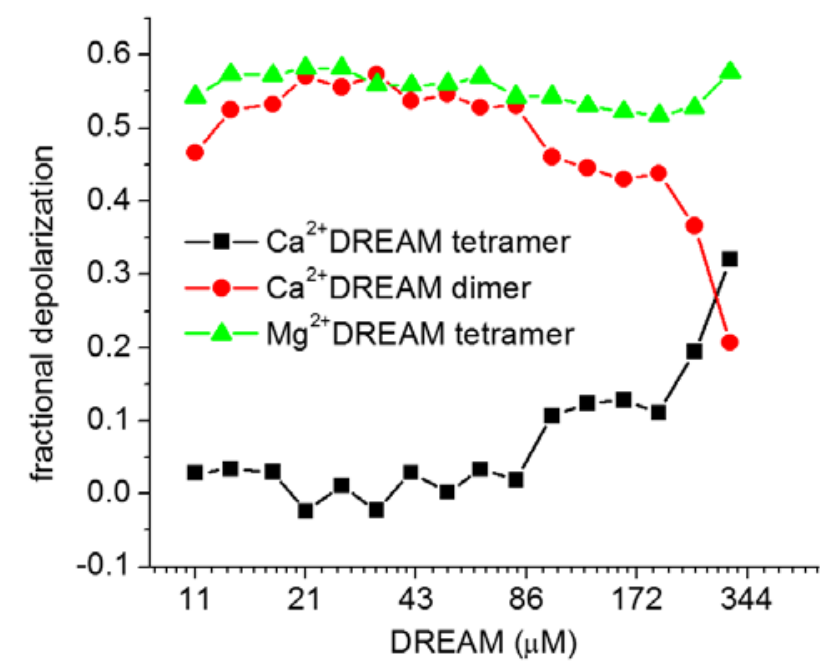

Figure 3.10. Fractional depolarization recovered from NLLS analysis of the anisotropy decay of 1,8-ANS-DREAM complexes at different protein concentration, with $28.2 \mu \mathrm{M}$ 1,8-ANS. The rotational correlation times $(\Phi)$ were fixed to those shown on Table 3 for the dimer, tetramer, and free dye; while the fractional depolarization and limiting anisotropy were the variable parameters. In all cases the global chi squared was less than 2 , the phase and modulation error set to $\leq 0.2$ and $\leq 0.002$, respectively.

To probe the impact of protein concentration on the DREAM oligomerization state, we have determined $f_{1}$ and $\Phi_{1}$ values for the 1,8 -ANS-Mg ${ }^{2+} \operatorname{DREAM}(\Delta 65)$ and 1,8-ANS$\mathrm{Ca}^{2+} \operatorname{DREAM}(\Delta 65)$ complex in the protein concentration range from $5 \mu \mathrm{M}$ to $300 \mu \mathrm{M}$ (Figure 3.10). Both values remain constant with $\Phi_{1}=58 \mathrm{~ns}$ confirming that $\mathrm{Mg}^{2+}$ $\operatorname{DREAM}(\Delta 65)$ retains tetrameric conformation in a broad concentration range and that the tetramer dissociation into individual dimers and/or monomers occurs at sub-micromolar concentration. On the other hand, the time-resolved anisotropy study of $\mathrm{Ca}^{2+} \operatorname{DREAM}(\Delta 65)$ as a function of protein concentration revealed an additional rotational correlation time that corresponds to the rotational correlation time of the tetramer ( $\sim 55 \mathrm{~ns})$ appearing at $\operatorname{DREAM}(\Delta 65)$ concentrations above $170 \mu \mathrm{M}$. These data indicate that the $\mathrm{K}_{d}$ value for the 
$\mathrm{Ca}^{2+} \operatorname{DREAM}(\Delta 65)$ dimer to $\mathrm{Ca}^{2+} \operatorname{DREAM}(\Delta 65)$ tetramer transition occurs around $170 \mu \mathrm{M}$ $\operatorname{DREAM}(\Delta 65)$. This is consistent with pre-exponential parameters recovered from ANS titration data showing a decrease in ANS affinity at $\mathrm{Ca}^{2+} \operatorname{DREAM}(\Delta 65)$ concentration above $170 \mu \mathrm{M}$ (data not shown).

The shorter rotational correlation time, $\Phi_{2} \sim 6$ ns determined for $\mathrm{Ca}^{2+} \operatorname{DREAM}(\Delta 65)$ and $\mathrm{Ca}^{2+} \mathrm{Mg}^{2+} \operatorname{DREAM}(\Delta 65)$, is too short to be attributed to the $\operatorname{DREAM}(\Delta 65)$ monomer $\left(\Phi^{\text {calc }}=12 \mathrm{~ns}\right)$. Considering the molecular mass of the C-terminal domain to be $12 \mathrm{kDa}$ and the location of ANS binding sites on the C-terminal domain of the protein (see below) we associate this rotational correlation time to the local motion of an isolated C-terminal domain of $\mathrm{Ca}^{2+} \operatorname{DREAM}(\Delta 65)$. In the case of apoDREAM $(\Delta 65)$ and $\operatorname{Mg}^{2+} \operatorname{DREAM}(\Delta 65)$, the value of $\Phi_{2}$ was determined to be similar, 14 ns and $12 \mathrm{~ns}$, respectively. This value may reflect either the presence of the monomeric form of apoDREAM or $\mathrm{Mg}^{2+} \operatorname{DREAM}(\Delta 65)$ in addition to the tetramer or it may indicate the slower rotation of the C-terminal domain in the $\operatorname{DREAM}(\Delta 65)$ tetramer. $\operatorname{DREAM}(\Delta 65)$ was detected as a monomer, dimer and tetramer in the nuclear extract, and as a dimer and tetramer in cytosolic extract using Western blot (Buxbaum et al. 1998). On the other hand, dynamic light scattering studies revealed that apoDREAM $(\Delta 65)$ adopts a tetrameric form in the absence and presence of DRE sequence (Osawa et al. 2001). Thus we associate the slower rotational correlation time observed for both, $\mathrm{Ca}^{2+}$ bound and $\mathrm{Ca}^{2+}$ free $\operatorname{DREAM}(\Delta 65)$ to the local motion of the C-terminal domain. This motion becomes slower in the tetrameric form of $\operatorname{DREAM}(\Delta 65)$ due to the interaction of the individual monomers. 


\subsubsection{Identification of ANS binding sites}

In order to identify the ANS binding site(s) we have performed ligand docking simulations using AutoDock software version 4.2 (Morris et al. 1998). The NMR structure of $\mathrm{Ca}^{2+}$ bound DREAM $(\Delta 75)$ was used in the simulations and treated as a static macromolecule whereas ANS ligands were treated as flexible molecules. Dockings were conducted with binding grids covering the whole protein surface to include all possible binding sites. The ANS binding sites were selected based on the lowest binding energy as well as on favorable ANS interactions with protein such as the presence of hydrogen bonds between the sulfonate group and amino acids or hydrophobic interactions. Additional low affinity sites identified in docking simulations represent nonspecific ANS binding and are unlikely to be populated under experimental concentrations.

Two sites for 1,8-ANS and for 2,6-ANS were identified in the C-terminal domain and one site was found at the interface of the EF-hand 1 and EF-hand 2 (Figure 3.11 and Figure 3.12). In the case of 1,8-ANS one site is located between the entering and exiting helix of EF-hand 4, and shows a high affinity (Figure 3.11). This site is buried within the protein matrix and 1,8-ANS is surrounded by several hydrophobic residues (Try174, Ile182, Ile190, Ile194, Val215, Phe218, and Phe252). In addition to hydrophobic residues, three polar residues (M187, M191, and H214) form the binding site. The ANS sulfonate group points towards the solvent and forms a hydrogen bond with H214.

The second binding site was identified at the interface of EF-3 and EF-4. This site is more polar and the ANS molecule is surrounded by V163, L167, C239, N247 and Q250 and the ANS sulfonate group forms a salt bridge with K166. The third binding site identified in the docking study partially overlays with the solvent exposed hydrophobic 
cavity between EF-2 and EF-1. However, in the model structure of DREAM dimer this hydrophobic cavity is part of the dimerization interface and may not be accessible for 1,8ANS binding. Identical binding sites were found for 2,6-ANS (Figure 3.12).

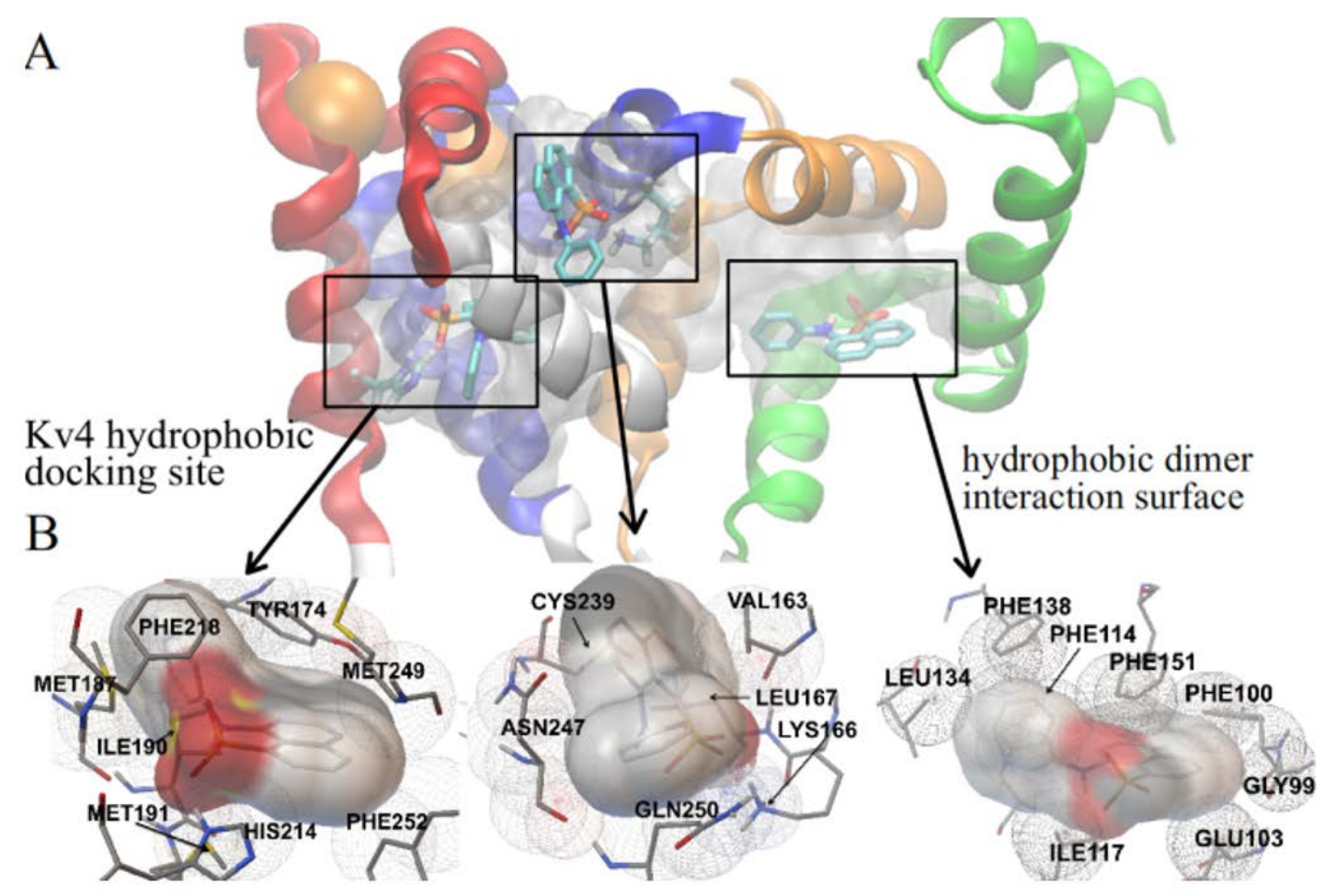

Figure 3.11. Position of three docking site for 1,8-ANS on the surface of $\mathrm{Ca}^{2+}$ bound DREAM as predicted using AutoDock 4.2 (Panel A). The binding sites were found in the vicinity of EF-4 (panel B, left), at the interface between EF-3 and EF-4 (panel B, middle) and at the hydrophobic crevice between the EF-1 and EF-2 (panel B, right). Cartoon representation of monomeric calcium bound DREAM (pdb: 2JUL) with EF-1 shown in green, EF-2 in orange, EF-3 in blue and EF-4 in red while calcium is shown as solid spheres and 1,8-ANS as licorice model. 

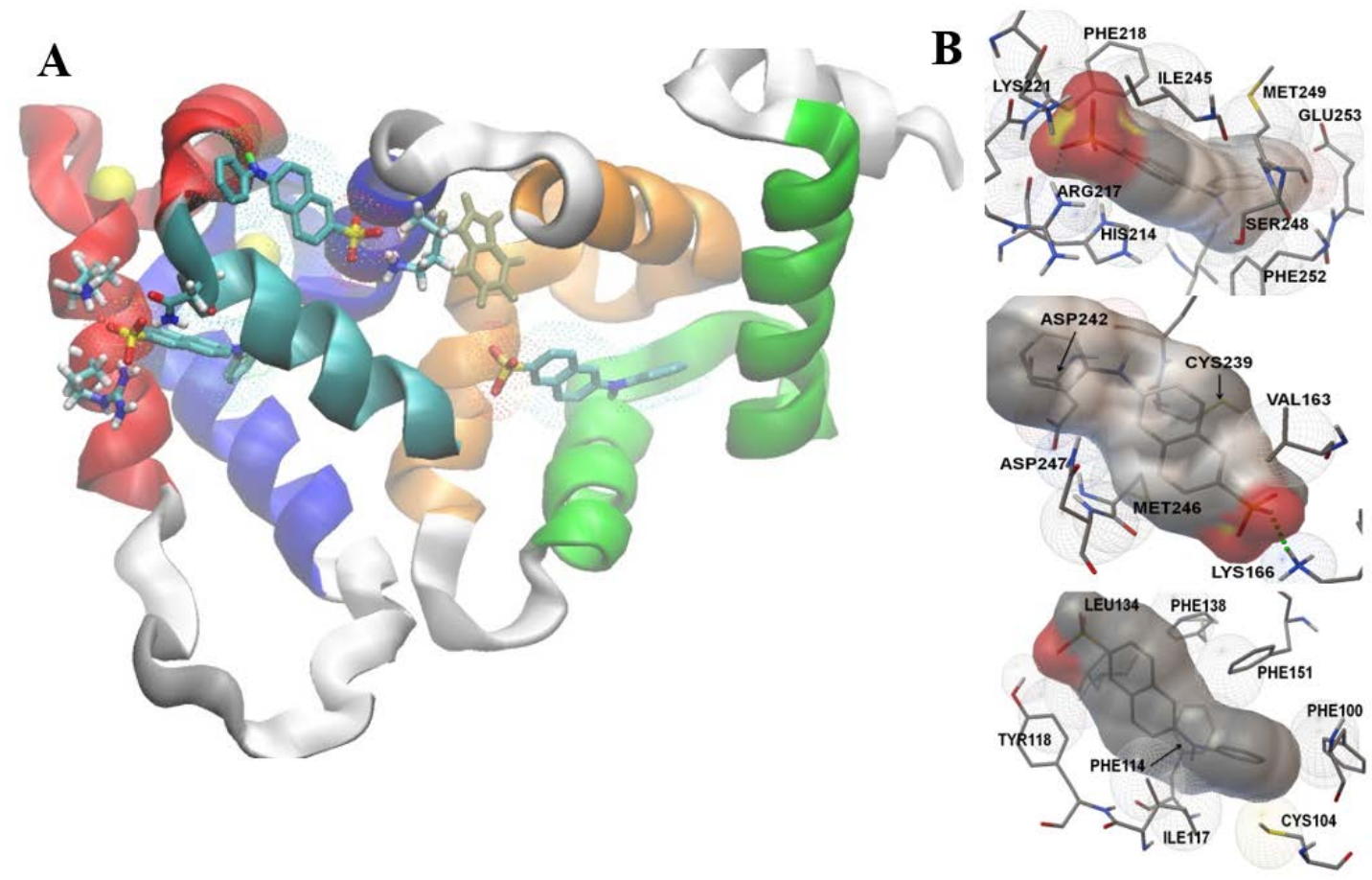

Figure 3.12. Position of three docking site for 2,6-ANS on the surface of $\mathrm{Ca}^{2+}$ bound DREAM as predicted using AutoDock 4.2 (Panel A). The binding sites were found in the vicinity of EF-4 (panel B, top), at the interface between EF-3 and EF-4 (panel B, middle) and at the hydrophobic crevice between the EF-1 and EF-2 (panel B, bottom).

\subsubsection{RET from Trp to 1,8-ANS}

Taking advantage of the spectral overlap between the emission spectrum of Trp169 and the absorption spectrum of 1,8-ANS (Figure 3.13), the distance between the 1,8-ANS binding sites and Trp residue can be determined based on the efficiency of the Förster energy transfer between the excited Trp169 donor and the 1,8-ANS acceptor. The emission intensity of Trp 169 is efficiently quenched upon addition of 1,8-ANS (Figure 3.14). The FRET efficiencies at saturating 1,8-ANS concentrations were determined to be $\sim 0.97$ for both, apoDREAM:1,8-ANS complex and the $\mathrm{Ca}^{2+}$ DREAM: 1,8-ANS complex. Using Eq. 2.7.3 the Förster distance, $\mathrm{R}_{0}$, between Trp169 in $\mathrm{Ca}^{2+}$ free and $\mathrm{Ca}^{2+}$ bound DREAM and 1,8-ANS was determined to be $25 \AA \AA$. 


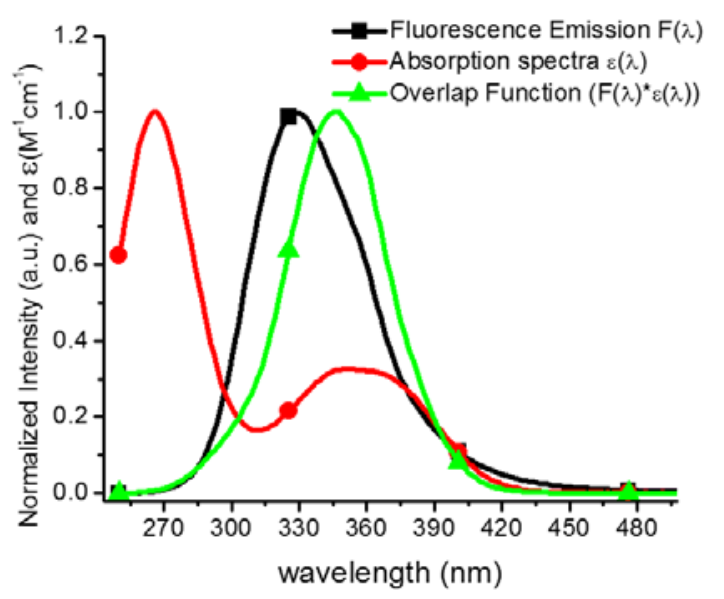

Figure 3.13. Normalized absorption spectrum of 1,8 -ANS bound to $\mathrm{Ca}^{2+} \mathrm{DREAM}$, normalized emission spectrum of $\mathrm{Ca}^{2+}$ DREAM and the overlap integral for the Trp169 1,8-ANS FRET pair.

Based on the FRET efficiency and accounting for the degree of flexibility of both 1,8-ANS and Trp 169 (Eq. 2.7.2 and Figure 3.14), we estimate the distance between 1,8-ANS and Trp169 to be between $8 \AA$ and $16 \AA$, which matches well the position of the 1,8-ANS sites determined in docking studies. The flexibility of ANS and Trp 169 was determined from the steady state anisotropy and the limiting anisotropy as described in Figure 3.14. Notably, the Eff $_{50}$ value matches the value of $K_{d}$ for 1 ,8-ANS biding to apo- and $\mathrm{Ca}^{2+}$ DREAM suggesting that the FRET efficiency is determined by the 1,8-ANS site occupancy. Also the similar distance between bound 1,8-ANS and the Trp residue in apo and $\mathrm{Ca}^{2+}$ DREAM indicates that the size and overall shape of DREAM monomer is similar in the apo and $\mathrm{Ca}^{2+}$ bound form of the protein. 

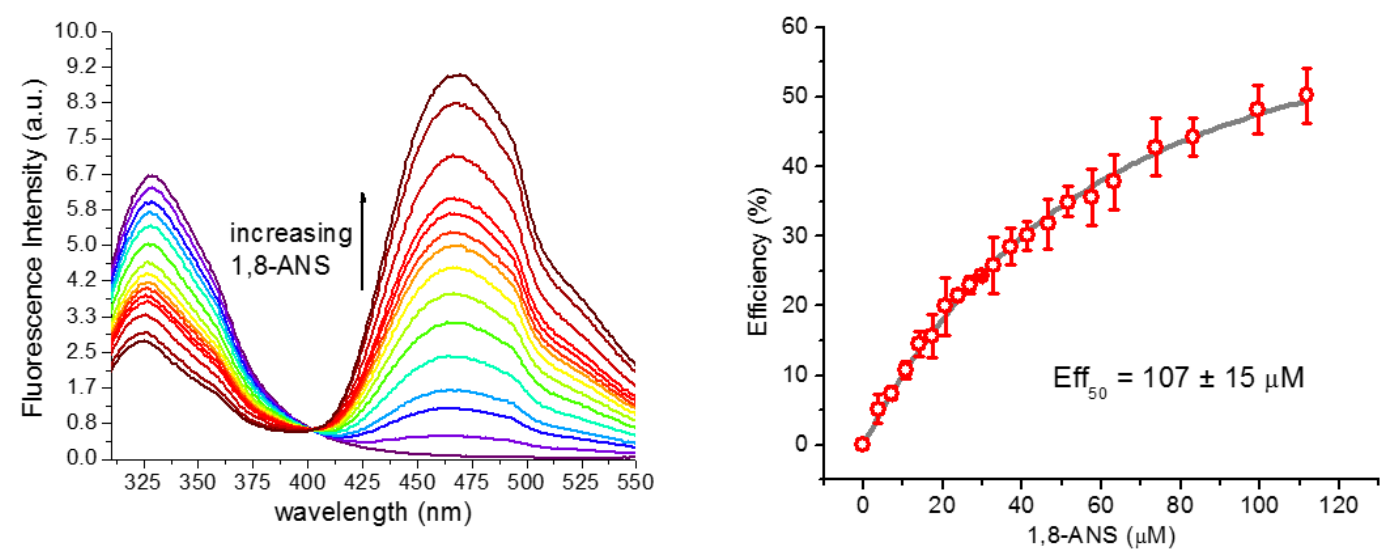

$$
\begin{aligned}
d_{i}=\left(\frac{r_{i}}{r_{0}}\right)^{1 / 2} & \text { Eqn. 3.1 } \\
\kappa_{\max }^{2}=\frac{2}{3}\left(1+d_{D}+d_{A}+3 d_{D} d_{A}\right) & \text { Eqn. 3.2 } \\
\kappa^{2}=\frac{2}{3}\left(1-\frac{\left(d_{D}+d_{A}\right)}{2}\right) & \text { Eqn. 3.3 }
\end{aligned}
$$$$
\text { Limiting and steady state }
$$$$
\text { anisotropy of DREAM-ANS and W169 }
$$$$
\begin{array}{lcc} 
& r_{i} & r_{0} \\
\text { 1,8-ANS } & 0.263 & 0.300 \\
\text { W169 } & 0.144 & 0.215
\end{array}
$$

Figure 3.14. (Top left) Quenching of the DREAM fluorescence emission by addition of 1,8-ANS (Top right) resulting efficiency plot calculated using the decrease in fluorescence emission at $328 \mathrm{~nm}$ as a function of 1,8-ANS concentration. Protein concentration was kept at $53 \mu \mathrm{M}$ and aliquots containing $53 \mu \mathrm{M}$ protein and $260 \mu \mathrm{M}$ 1,8-ANS were added to the solution. (Bottom left) Equations used for the analysis of the ANS-W169 distances, where $r_{i}$ represents the steady state anisotropy of the fluorophore in the absence of rotational diffusion and $\mathrm{r}_{0}$ represents the limiting anisotropy determined from the modulated anisotropy extrapolated a high frequencies. (Bottom right) Steady state anisotropy determined and limiting anisotropy determined for the ANS-W169 FRET pair used to calculate the lower and upper limits for the kappa squared parameter.

\subsubsection{Hydrophobic probe displacement studies}

To determine if the hydrophobic sites occupied by ANS molecules can serve as binding sites for small hydrophobic modulators of $\mathrm{K}_{\mathrm{v}}$ channels:KChIP complexes, the emission intensity of 1,8-ANS:DREAM $(\Delta 65)$ complex was monitored upon addition of arachidonic acid. Addition of the fatty acid results in a decrease in the emission intensity in $\mathrm{Ca}^{2+} \operatorname{DREAM}(\Delta 65)$ and in $\operatorname{apoDREAM}(\Delta 65)$, although to a smaller extent in the apoform (Figure 3.15). Also, the emission maximum of 1,8-ANS:DREAM $(\Delta 65)$ complex 
undergoes a bathochromic shift from $467 \mathrm{~nm}$ to $475 \mathrm{~nm}$ suggesting that the arachidonic acid preferentially displaces 1,8-ANS bound to a hydrophobic site 2 . This was confirmed by the docking studies which show that the fatty acid molecule is positioned between $\alpha$ helices 7, 8, and 9 with the carboxy group oriented towards the guanidino group of Arg217 (Figure 3.15c). The titration curves were analyzed using a competitive binding model (Epps, Raub \& Kezdy 1995). Arachidonic acid binds to $\mathrm{Ca}^{2+}$ DREAM with a dissociation constant of $19 \pm 1 \mu \mathrm{M}$, whereas an approximately 10 time smaller affinity was determined for arachidonic acid association to the apo protein $\left(\mathrm{K}_{\mathrm{i}}=160 \mu \mathrm{M}\right)$.
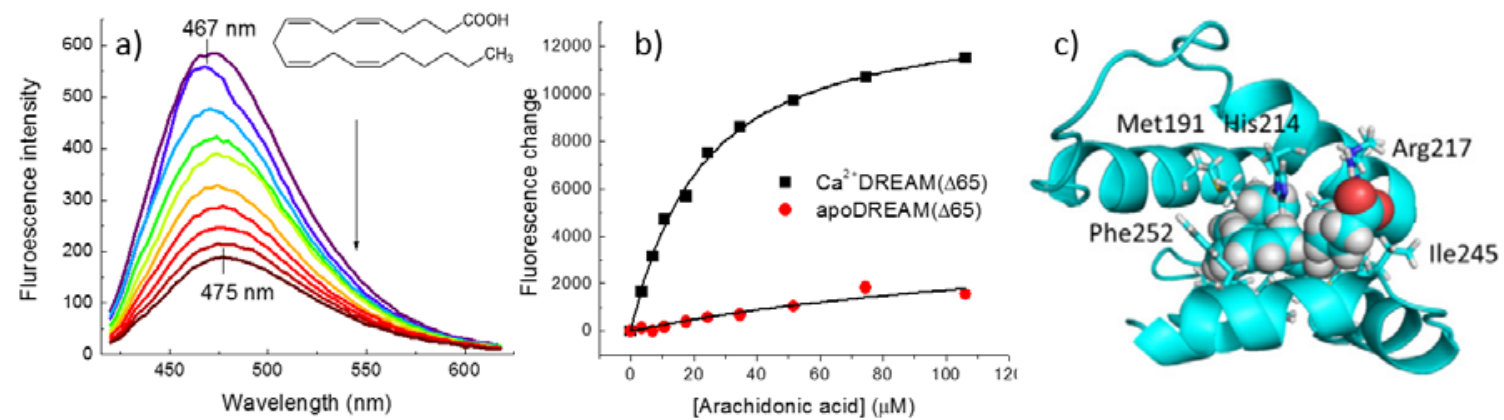

Figure 3.15: Decrease in the emission intensity of 1,8-ANS bound to $\mathrm{Ca}^{2+}$ DREAM upon addition of arachidonic acid (a). The change in 1,8-ANS fluorescence intensity as a function of arachidonic acid concentration. The solid line corresponds to the fit of the experimental data using a single binding site model (solid line) (b). The position of the docking site for arachidonic acid in the C-terminal domain of DREAM (c).

\subsection{Discussion}

In consideration of its multifunctional role in neuronal tissue, DREAM represents a unique model to study the mechanism of calcium signal transduction by neuronal calcium sensors. Unlike other members of the NCS family, DREAM adopts distinct oligomerization states depending on the $\mathrm{Ca}^{2+} / \mathrm{Mg}^{2+}$ occupation of the EF-hands and possibly on DREAM interactions with other intracellular proteins. Fluorescence properties of hydrophobic probes such as 1,8-ANS and 2,6-ANS offer several advantages in studying conformational changes in proteins that undergo oligomerization. Besides the traditional 
application of ANS probes to identify putative hydrophobic binding sites on the protein surface, the long fluorescence lifetime of ANS-protein complexes allows a direct characterization of the DREAM oligomerization by probing the rotational correlation time in time-resolved anisotropy studies.

Previous studies reported that DREAM forms a monomer, dimer, and tetramer in nuclear extracts, and as a dimer and tetramer in cytosolic extract (Carrion et al. 1999). In vitro, $\mathrm{Ca}^{2+}$ association to EF-hands stabilizes the dimeric form of DREAM (Osawa et al. 2001); however, a discrepancy about the oligomeric state of $\mathrm{Mg}^{2+}$ bound DREAM remains. Initially, Carrion et al. proposed that in the absence of $\mathrm{Ca}^{2+}$, DREAM forms a stable tetramer with a high affinity for DNA (Carrion et al. 1999). On the other hand, using size exclusion chromatography, Ames et al. have reported that $\mathrm{Mg}^{2+}$ stabilizes DREAM in the monomeric form and that the presence of $\mathrm{Mg}^{2+}$ facilitates the specific recognition of the DRE sequence (Osawa et al. 2005). The long fluorescence life-time of ANS:DREAM complexes enable us to probe the DREAM oligomerization state in solution as a function of protein concentration. The time-resolved anisotropy data presented here demonstrate that $\mathrm{Mg}^{2+} \mathrm{DREAM}$ forms a stable tetramer at the concentrations range from $8 \mu \mathrm{M}$ to 230 $\mu \mathrm{M}$ and the $\mathrm{Mg}^{2+} \mathrm{DREAM}$ tetramer dissociates into dimers or monomers at concentrations below $8 \mu \mathrm{M}$ indicating a strong affinity between the individual dimers or monomers of $\mathrm{Mg}^{2+}$ DREAM. Indeed, attempts to separate $\mathrm{Mg}^{2+}$ bound DREAM tetramer into individual monomers or dimers using low concentration of denaturant (up to $1 \mathrm{M} \mathrm{GuHCl}$ ), high salt concentrations (up to $200 \mathrm{mM}$ ), or a temperature increase (up to $33^{\circ} \mathrm{C}$ ) were unsuccessful. The concentration of free $\mathrm{Mg}^{2+}$ in the nuclei and cytoplasm of neuronal cells was determined to be $\sim 110 \mu \mathrm{M}$ and close to $1 \mathrm{mM}$, respectively (Gotoh et al. 1999). 
Considering the $\mathrm{K}_{\mathrm{d}}$ value for $\mathrm{Mg}^{2+}$ binding to DREAM of $13 \mu \mathrm{M}$ (Osawa et al. 2005), in vivo $\mathrm{Mg}^{2+}$ would constitutively bind to DREAM in the cytoplasm as well as in the nuclei and stabilizes its tetrameric structure.

Using fluorescence and computational studies, two ANS binding sites of comparable affinity were identified in the DREAM C-terminal domain; namely, in the vicinity of EF-hand 4 (site 2) and between EF-hand 3 and EF-hand 4 (site 1). The Cterminal domain carries both $\mathrm{Ca}^{2+}$ binding EF-hands in DREAM and other KChIPs and this domain was reported to be essential for the KChIP1 binding to phosphatidylserine as well as for protein association to the membrane (Liao, Chen \& Chang 2009). In addition, the crystal structure of $\mathrm{K}_{\mathrm{v}} 4.3$ channel in complex with KChIP-1 reveals that the site 1 of the T1 domain of the $\mathrm{K}_{\mathrm{v}} 4.3$ channel interacts with several hydrophobic residues in the Cterminal domain of KChIP-1 including Tyr144, Ile150, Ile154 and Tyr155 (Pioletti et al. 2006) and the replacement of Tyr144 by Glu prevents KChIP1 association to $\mathrm{K}_{\mathrm{v}} 4.3$ channels (Wang et al. 2007). Tyrosine 144 in KChIP1 is analogous to Tyr174 in DREAM, an amino acid which we found to be in direct contact with 1,8-ANS bound at the hydrophobic site. This observation confirms the role of the hydrophobic site 2 (Figure 3.11) in interactions with the $\mathrm{N}$-terminus of $\mathrm{K}_{\mathrm{v}} 4$ channels. Interestingly, the 1,8-ANS displacement study shows that arachidonic acid binds to the hydrophobic site 2 between helix 7, 8 and 10 in the C-terminal domain in presence of $\mathrm{Ca}^{2+}$. Previously it was shown that arachidonic acid modulates inactivation kinetics of $\mathrm{K}_{\mathrm{v}} 4$ channels in complex with KChIPs (Boland, Drzewiecki 2008). Both the N-terminus of the $\mathrm{T} 1$ domain and arachidonic acid bind at the hydrophobic crevice at the C-terminus of DREAM. We hypothesize that the association of arachidonic acid to the hydrophobic C-terminus of 
KChIPs triggers reorientation of the contacts between the T1 domain and the KChIP leading to modulation of the inactivation kinetics.
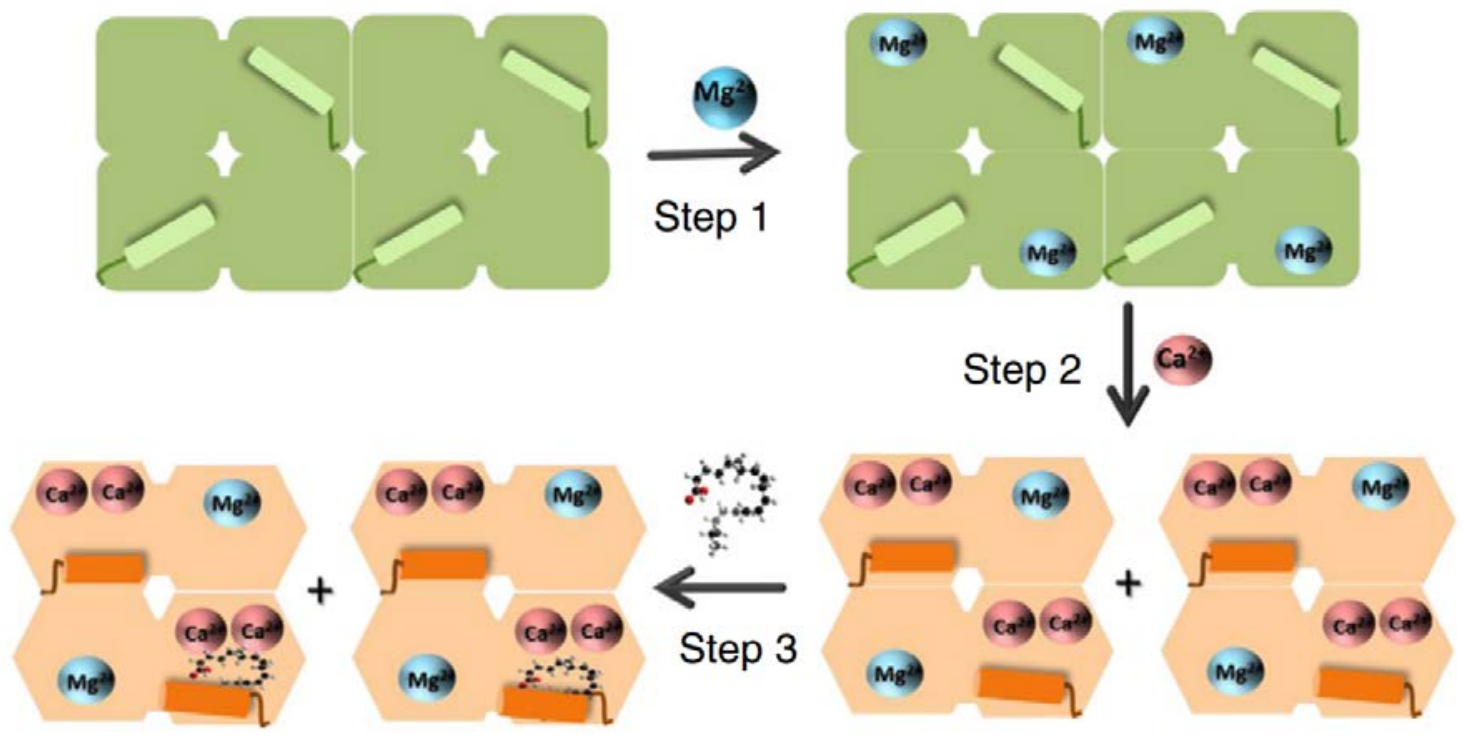

Figure 3.16. A cartoon model depicting DREAM interactions with $\mathrm{Mg}^{2+}$ and $\mathrm{Ca}^{2+}$ ions and arachidonic acid. Association of the $\mathrm{Mg}^{2+}$ ions to apoDREAM tetramer does not lead to substantial changes in DREAM structure (step 1). Subsequent binding of $\mathrm{Ca}^{2+}$ to EF-hand 3 and EF-hand 4 promotes tetramer dissociation and concomitant exposure of the hydrophobic cavity located in the C-terminal domain by repositioning of the C-terminal $\alpha$ helix (step 2) allowing binding of the arachidonic acid to the C-terminus hydrophobic cavity (step 3).

To understand the mechanism of the $\mathrm{Ca}^{2+}$ triggered increased occupancy of the site 2 by hydrophobic molecules, we have overlaid the structure of $\mathrm{Ca}^{2+}$ DREAM with the structure of recoverin in its apoform (Tanaka et al. 1995). The C-terminal helix of recoverin partially restricts the solvent exposure of site 2 whereas this site is more solvent accessible in the $\mathrm{Ca}^{2+}$ bound form of DREAM. We propose that a similar displacement of the Cterminal helix occurs in DREAM and thus concomitant reorganization of the C-terminal hydrophobic cavity contributes to the increase in 1,8-ANS and arachidonic acid binding to $\mathrm{Ca}^{2+}$ DREAM. Since the hydrophobic cavity in the DREAM C-terminal domain serves as a binding sites for the $\mathrm{N}$-terminus of the $\mathrm{T} 1$ domain, we propose that $\mathrm{Ca}^{2+}$ induced exposure 
of the hydrophobic cavity modulates interactions within the T1 domain:DREAM complex and such structural rearrangement represent an initial step in $\mathrm{Ca}^{2+}$ dependent regulation of current amplitudes and kinetics properties of $\mathrm{K}_{\mathrm{V}} 4$ - DREAM complexes (Zhou et al. 2004b). Also, the $\mathrm{Ca}^{2+}$ triggered increase in the exposure of the DREAM C-terminal hydrophobic cavity may control the affinity of DREAM for other small hydrophobic molecules such as NS5806 or CL-888 (Bowlby et al. 2005).

\subsection{Summary}

Members of the NCS family including DREAM share a cluster of highly conserved hydrophobic residues that form solvent exposed hydrophobic patches at the $\mathrm{N}$-terminal domain and at the interface between the $\mathrm{N}$ - and $\mathrm{C}$-terminal domains. The solvent exposure of hydrophobic patches regulated by $\mathrm{Ca}^{2+}$ association to the active EF-hands may represent a key factor for NCS interactions with target proteins. Based on the fluorescence lifetime data, two 1,8-ANS binding sites were identified in the apo and $\mathrm{Ca}^{2+}$ bound form of DREAM with site 2 being more accessible in $\mathrm{Ca}^{2+}$ DREAM and $\mathrm{Ca}^{2+} \mathrm{Mg}^{2+}$ DREAM compared to apo and $\mathrm{Mg}^{2+}$ DREAM. Unlike other NCS members, $\mathrm{Ca}^{2+}$ association to DREAM and possibly other KChIP proteins regulates solvent exposure of hydrophobic patches located on the C-terminal domain. Such limited exposure of the hydrophobic patches may represent a molecular mechanism used to fine tunes specificity for intracellular target proteins as well as small modulators of $\mathrm{KChIP-}_{\mathrm{v}} 4$ function. 


\section{REGULATION OF DREAM ACTIVITY BY THE NOVEL ITO CURRENT ACTIVATOR NS5806}

\subsection{Background and significance}

Potassium is the most abundant ion in vertebrate cells, and its concentration is highly regulated by the coordinated activation and inactivation of $\mathrm{Na}^{+} / \mathrm{K}^{+}$pumps and other $\mathrm{K}^{+}$ transporters, including voltage sensitive $\mathrm{K}^{+}$channels. In excitable tissue such as brain and heart tissue, the higher intracellular concentration of $\mathrm{K}^{+}$is used to restore the resting membrane potential after depolarization; this makes the regulation of $\mathrm{K}^{+}$ions of utmost relevance to cell survival. Consequently, voltage-gated $\mathrm{K}^{+}$channels are the most diverse and widespread cation-conducting membrane proteins, and have been found to be involved in numerous biological processes such as apoptosis (Szabò, Zoratti \& Gulbins 2010), neuron signaling (Song et al. 1998), cell volume (Lang et al. 2007), and cardiac action potential (Niwa, Nerbonne 2010). In neurons and cardiomyocytes the $\mathrm{K}_{\mathrm{V}} 4$ subfamily of voltage gated $\mathrm{K}^{+}$channels regulate the fast-inactivating components of the I $\mathrm{I}_{\mathrm{SA}}$ and $\mathrm{I}_{\mathrm{TO}}$ currents, respectively (Liss et al. 2001). These channels form octameric transmembrane complexes with accessory subunits such as potassium channel interacting proteins (KChIPs) (An et al. 2000), dipeptidyl-peptidases (DPPs) (Nadal et al. 2003), and $K_{V} \beta$ proteins (Martens, Kwak \& Tamkun 1999) . Interaction of KChIP2 with $K_{V} 4$ channels in the heart results in an increase in current density, acceleration of recovery from inactivation, and slower inactivation kinetics (An et al. 2000). Similar effects on the biophysical properties of neural fast inactivating $\mathrm{K}^{+}$currents have been observed for KChIP1 (Bourdeau et al. 2011) and KChIP3 (Witzel, Fischer \& Bähring 2012). Even though the molecular structures of the $\mathrm{K}_{\mathrm{v}} 4 \mathrm{~T} 1$ domain in presence and absence of the regulatory KChIP1 is known, the mechanism by which these auxiliary proteins mediate channel gating remains unknown. Patch clamp studies using 
heterologous expressed $\mathrm{K}_{\mathrm{V}} 4$ channels highlight the relevance of the T1 domain in gating and mediating ancillary subunit interaction. Two regions on the $\mathrm{K}_{\mathrm{v}} 4 \mathrm{~T} 1$ domain (amino acids 7-11 “site 1” and 71-90 “site 2”) have been identified as being necessary for KChIP1 current regulation (Scannevin et al. 2004).

KChIP3 also known as DREAM or calsenilin is expressed in the brain/testis and heart and has been shown to interact with presenilin (Buxbaum et al. 1998), DNA (Carrion et al. 1999) and $\mathrm{K}_{\mathrm{v}} 4.3$ (An et al. 2000). KChIP3DREAM contains four calcium binding domains "EFhands", of which EF-3/4 binds $\mathrm{Ca}^{2+}$ with high affinity, EF-2 binds $\mathrm{Mg}^{2+}$ preferentially, and EF-1 is inactive (Craig et al. 2002). Similarly to other KChIPs this protein contains a relatively polar surface while the opposite side is predominantly hydrophobic with a large nonpolar cavity (Lusin et al. 2008). The formation of the $K_{V} 4-K C h I P$ complex has been shown to be necessary for proper regulation of the fast component of the ITO currents in the heart (Niwa, Nerbonne 2010) and ISA in the brain (Bourdeau et al. 2011). Demodulation of $\mathrm{I}_{\text {TO }}$ currents is a characteristic trait in hypertrophied and failing hearts (Kääb et al. 1998) as well as in Brugada syndrome (Calloe et al. 2009). Normal functioning heart tissue displays an increasing $I_{T O}$ current density gradient from endocardium to epicardium, which has been shown to correlate with a transmural KChIPs density (Zicha et al. 2004). Studies using canine models of failing hearts have shown that heart failure results in a decrease of phase 1 repolarization due to a decrease in $\mathrm{I}_{\mathrm{TO}}$ current as well as a remodeling of the gating kinetics of this current (Cordeiro et al. 2012). Although numerous drugs have been shown to down regulate the amplitude or modify the gating kinetics of $\mathrm{I}_{\mathrm{TO}}$ current, drugs that directly upregulate the ITO current amplitude have not been reported.

Recently, Calloe et al. have introduced a new compound named NS5806 (Calloe et al. 2009) which was shown to modulate the $\mathrm{I}_{\mathrm{TO}}$ current kinetics and reverse the $\mathrm{I}_{\mathrm{TO}}$ current decrease induced 
by heart failure in canine models (Calloe et al. 2010). Interestingly, this ITO activator has also been shown to decrease ISA currents in cultured hippocampal neurons where its effects on gating kinetics are comparable to those observed in heart tissue (Witzel, Fischer \& Bähring 2012). Moreover, the induced potassium current modulation by NS5806 has been shown to depend on the presence of $\mathrm{K}_{\mathrm{V}} 4$ auxiliary KChIP protein (Witzel, Fischer \& Bähring 2012, Lundby et al. 2010).

In this study we investigate the mechanism of NS5806 $\mathrm{I}_{\mathrm{TO}} / \mathrm{I}_{\mathrm{SA}}$ current regulation by determining the interaction between NS5806 and KChIP3 (herein referred as DREAM), and how this interaction modulates the DREAM-Kv4.3 association. We show that the Iто activator NS5806 binds to the $\mathrm{K}_{\mathrm{v}} 4$ auxiliary protein DREAM at the C-terminus near the calcium binding sites, EF-hand 3 and EF-hand 4, with an affinity comparable to the previously determined EC50 for current potentiation. We also characterize the association constants between DREAM and peptide fragments of the $\mathrm{T} 1$ domain of $\mathrm{K}_{\mathrm{v}} 4.3$ to show that calcium binding induces an increase in the affinity between DREAM the T1 domain of $\mathrm{K}_{\mathrm{v}} 4.3$. In the presence of NS5806, the interaction between DREAM and the hydrophobic N-terminus of $\mathrm{K}_{\mathrm{v}} 4.3$ is enhanced and the calcium dependency of this interaction is abolished. Furthermore, anisotropy data suggest that the complex between DREAM and the T1 domain adopts an altered quaternary structure in the presence of NS5806. Kinetic data indicates that NS5806 decreases the rate of dissociation between DREAM and site 1 of $\mathrm{K}_{\mathrm{v}} 4.3$, which results in an overall increasing in affinity. These results support the idea that NS5806 binds at the interface between the hydrophobic N-terminus of $\mathrm{K}_{\mathrm{v}} 4.3$ and the hydrophobic cavity at the C-terminus of DREAM and stabilizes the protein complex. These results are further supported by docking, molecular dynamic simulations and site directed mutagenesis. 
Overall, the studies shown here provide a roadmap for the elucidation of the precise mechanism by which $\mathrm{K}_{\mathrm{V}} 4$ current kinetics are regulated by KChIPs and NS5806.
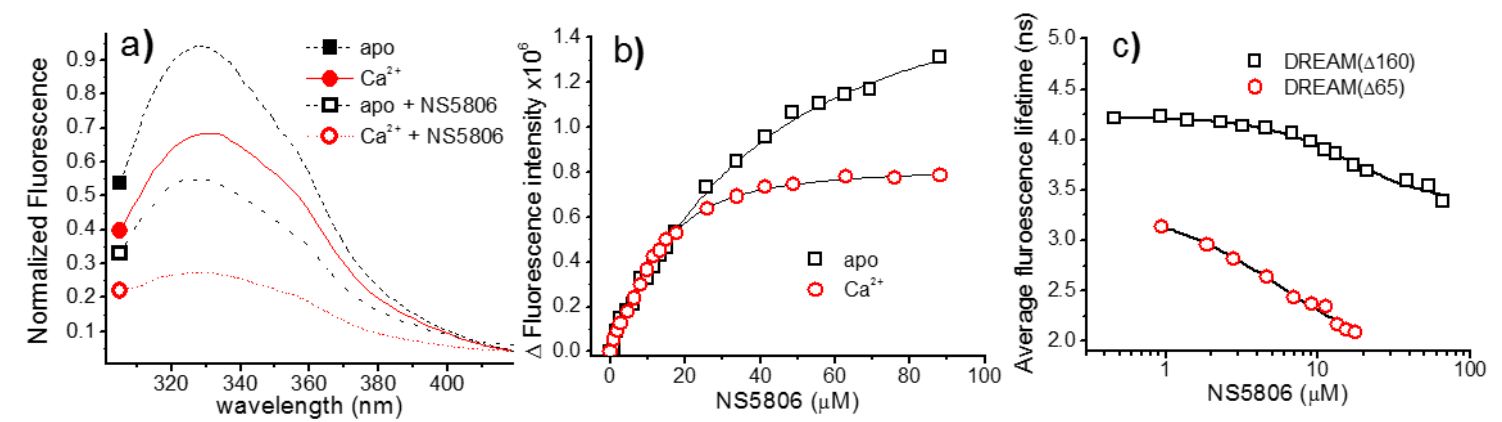

Figure 4.1. (a) Fluorescence emission of $15 \mu \mathrm{M}$ apoDREAM( $(465)$ and $15 \mu \mathrm{M} \mathrm{Ca}{ }^{2+}$ $\operatorname{DREAM}(\Delta 65)$ in the absence and presence of $30 \mu \mathrm{M}$ NS5806, $\lambda_{\text {exc }}=295 \mathrm{~nm}$. (b) Titration of $\operatorname{DREAM}(\Delta 65)$ with NS5806 by monitoring the change in tryptophan fluorescence in the apo and calcium bound form. The recovered dissociation constants were determined using Eqn. 2.8.1 (solid lines) and are listed in Table 4.1. (c) Average tryptophan fluorescence lifetime of $15 \mu \mathrm{M}$ $\operatorname{DREAM}(\Delta 65)$ and $15 \mu \mathrm{M}$ DREAM $(\Delta 160)$ in the $\mathrm{Ca}^{2+}$ bound state as a function of NS5806. Solid lines represent the best non-linear fit using a single site Hill equation.

\subsection{Results}

\subsubsection{NS5806 binds at the C-terminus of DREAM}

The $\mathrm{K}_{\mathrm{v}} 4$ mediated current amplitude increase and slowing of inactivation induced by NS5806 have been reported to be enhanced or dependent on the presence of the auxiliary KChIP proteins (Witzel, Fischer \& Bähring 2012, Lundby et al. 2010). In this study we probe whether this dependency is due to direct interaction between NS5806 and the neuronal calcium sensor $\operatorname{DREAM}(\Delta 65)$. The interaction of NS5806 with $\operatorname{DREAM}(\Delta 65)$ was initially characterized by monitoring the steady-state tryptophan fluorescence emission of $\operatorname{DREAM}(\Delta 65)$ upon addition of NS5806. The single tryptophan residue (Trp169) found in the DREAM $(\Delta 65)$ protein is located at the interface between EF-hand 3 and EF-hand 2 and thus its emission may probe NS5806 association to either N- or C-terminal domains. Upon addition of two -fold excess of NS5806, a significant decrease in tryptophan emission intensity was observed (Figure 4.1a). The quenching 
effect was present in the apo-state as well as in the calcium bound state of $\operatorname{DREAM}(\Delta 65)$. The absorption spectrum of NS5806 in 20 mM MOPS buffer shows an absorption peak at $340 \mathrm{~nm}$ with an extinction coefficient of $105 \mathrm{M}^{-1} \mathrm{~cm}^{-1}$. This absorption band overlaps with the Trp169 emission
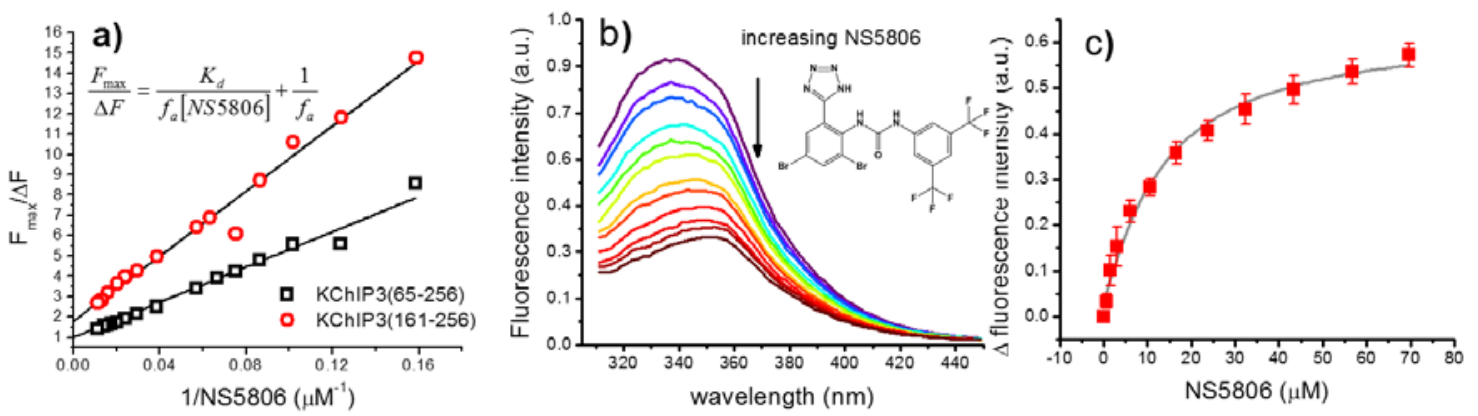

spectrum suggesting that resonance energy transfer (RET) between Trp169 and NS5806 is responsible for the observed Trp emission quenching.

Figure 4.2. (a) Quenching of the tryptophan fluorescence of $\operatorname{DREAM}(\Delta 65)$ and $\operatorname{DREAM}(\Delta 160))$ in the apo-state as a function of NS5806, analyzed using a modified Stern-Volmer equation (inset). The linear fit recovered a slope of $45 \pm 2 \mu \mathrm{M}$ and intercept of $1.0 \pm 0.2$ for $\operatorname{DREAM}(\Delta 65)$ and slope of $80 \pm 4 \mu \mathrm{M}$ and intercept of $1.8 \pm 0.2$ for DREAM $(\Delta 160)$. The inverse of the intercept for $\operatorname{DREAM}(\Delta 160)$ indicates that only $56 \%$ of the tryptophan residues are quenched at saturating amounts of NS5806. After correction for the fraction quenched, both constructs result in identical dissociation constant values of $\sim 40 \mu \mathrm{M}$ in the apoform. (b) The tryptophan fluorescence of $5 \mu \mathrm{M}$ $\mathrm{Zn}^{2+} \mathrm{Kv} 4.3(1-152)-G S T$ decrease due to binding of NS5806, inset shows the structural formula of NS5806. (c) Fluorescence intensity change as a function of NS5806 concentration, solid line represents best fit using eq. 1 with recovered $\mathrm{K}_{\mathrm{d}}=11 \pm 2 \mu \mathrm{M}$.

To determine the DREAM $(\Delta 65)$ affinity for NS5806, the Trp emission intensity was plotted as a function of NS5806 concentration of DREAM $(\Delta 65)$ (Figure 4.1b), the dissociation constants were recovered using Eq. 2.8.1 and are listed on Table 4.1. The stoichiometry of the model was set to one since analysis of the tryptophan fluorescence by the continuous variation method yielded a stoichiometry of one to one (data not shown). The recovered dissociation constants implies that in the $\mathrm{Ca}^{2+}$ bound form, $\operatorname{DREAM}(\Delta 65)$ binds NS5806 with a dissociation constant of $4.4 \pm 0.3 \mu \mathrm{M}$. Separate experiment using the truncated C-terminus domain, $\operatorname{DREAM}(\Delta 160)$, shows roughly a 
two-fold affinity increase to $\mathrm{K}_{\mathrm{d}}=2.7 \pm 0.3 \mu \mathrm{M}$ (data not shown). Upon $\mathrm{Ca}^{2+}$ removal, the affinity of DREAM $(\Delta 65)$ for NS5806 decreases, $\mathrm{K}_{d}=30 \pm 2 \mu \mathrm{M}$, similarly the affinity of DREAM $(\Delta 160)$ is reduced to $\mathrm{K}_{\mathrm{d}}=26 \pm 4 \mu \mathrm{M}$. Since both constructs show similar affinities for NS5806 in the calcium bound form and apo-state we propose that the C-terminus of DREAM is the binding site for NS5806.

It is interesting to note that the decrease in fluorescence of $\operatorname{apoDREAM}(\Delta 65)$ upon addition of NS5806 is about twice as large as that observed for apo DREAM( $\Delta 160)$ (data not shown), we hypothesize that this could be due to a structurally different conformation of the isolated C-terminus in the apo-state. To investigate if this is the case, the quenching data was analyzed using a modified Stern-Volmer equation which accounts for the presence of tryptophan residues which may not be quenched by NS5806 (Figure 4.2a). The results show that both $\operatorname{DREAM}(\Delta 65)$ and $\operatorname{DREAM}(\Delta 160)$ in the apo-form bind to NS5806 with the same affinity, and that the observed differences in the extent of quenching are due to the fact that in the apo-state, $\operatorname{DREAM}(\Delta 160)$ populates a second conformation ( $40 \%$ ) which is not quenched by NS5806. This additional population may be present due to a lower stability of the truncated C-terminus domain.

To probe if RET is responsible for the observed fluorescence quenching we measured the tryptophan fluorescence lifetime of both DREAM constructs in the calcium bound form. The average lifetime of Trp169 decreases from $3.3 \mathrm{~ns}$ to approximately $1.3 \mathrm{~ns}$ in $\mathrm{Ca}^{2+} \operatorname{DREAM}(\Delta 65)$ and from 4.3 ns to $3.2 \mathrm{~ns}$ in $\mathrm{Ca}^{2+} \operatorname{DREAM}(\Delta 160)$ as the concentration of NS5806 is increased (shown in Figure. 4.1c). The fluorescence lifetime decrease indicates that the quenching mechanism happens during the excited state of tryptophan, and correlates well with a dynamic quenching process such as RET. 
Table 4.1 Overlap integrals and Förster distances between DREAM and NS5806

\begin{tabular}{|c|c|c|c|c|c|c|}
\hline $\begin{array}{l}\mathrm{J}(\lambda) \\
\left(\mathrm{M}^{-1} \mathrm{Cm}^{-}\right. \\
\left.{ }^{-} \mathrm{nm}^{4}\right)\end{array}$ & $\begin{array}{l}\mathrm{R}_{0} \\
(\AA)\end{array}$ & $\Phi^{(\mathrm{a})}$ & $\kappa_{\text {min }}^{2}$ & $\kappa^{2}$ max & $\begin{array}{l}\mathrm{r}^{(\mathrm{b})} \\
(\AA)\end{array}$ & $\begin{array}{l}\mathrm{r}^{(\mathrm{c})} \\
(\AA)\end{array}$ \\
\hline $2.44 \times 10^{12}$ & 11 & 0.039 & 0.096 & 2.76 & $5-9$ & - \\
\hline $2.36 \times 10^{12}$ & 11 & 0.037 & 0.076 & 2.87 & $7-12$ & 8-15 \\
\hline $8.38 \times 10^{11}$ & 12 & 0.190 & 0.063 & 2.96 & $7-13$ & - \\
\hline $1.57 \times 10^{12}$ & 12 & 0.125 & 0.055 & 3.00 & $7-13$ & $11-20$ \\
\hline
\end{tabular}

(a) Quantum yield of Trp169 determined relative to L-Tryptophan. Experimental distance " $r$ " determined using the energy transfer efficiency at saturating NS5806 concentration $(150 \mu \mathrm{M})$ using the tryptophan fluorescence intensity.

Furthermore, it is possible to use the fluorescence emission spectra of both DREAM constructs, the tryptophan fluorescence quantum yield, and the absorbance spectra of NS5806 while assuming a random NS5806-Trp169 orientation to obtain the overlap function and calculate the Förster distance between the NS5806-Trp169 pair for $\operatorname{DREAM}(\Delta 65)$ and $\operatorname{DREAM}(\Delta 160)$. The Förster distances were calculated to be $12 \AA$ and $14 \AA$ for the apo-form $\operatorname{DREAM}(\Delta 65)$ and $\operatorname{DREAM}(\Delta 160)$, respectively, while a Förster distance of $12 \AA$ was recovered for the $\mathrm{Ca}^{2+}$ bound form of $\operatorname{DREAM}(\Delta 65)$ and $13 \AA$ for $\mathrm{Ca}^{2+} \operatorname{DREAM}(\Delta 160)$, (Table 4.1). Due to the location of Trp169 within the protein matrix, a random orientation value for $\kappa^{2}$ is not appropriate. However, an upper $\left(\kappa^{2}\right.$ max $)$ and lower $\left(\kappa^{2} \min \right)$ limit for the orientation factor can be obtained from measurement of the frequency modulated anisotropy of Trp169 for both DREAM construct (data not shown). These values were used to calculate the upper and lower limits for NS5806-Trp169 distances as shown in Table 4.1. The recovered NS5806-Trp169 distances using fluorescence emission and fluorescence lifetime of Trp169 range from $5 \AA$ to $13 \AA$ and $8 \AA$ to $20 \AA \AA$, respectively. The larger values for the lifetime measurements support the presence of an additional static quenching process induced by binding of NS5806 which results in smaller distances being resolved in the steady state 
quenching measurements. Additional contribution due to quenching of residual tyrosine fluorescence cannot be ruled out. Overall, these results show that NS5806 binds at the C-terminus of DREAM near EF-hands 3 and 4 and quenches the single tryptophan residue through resonance energy transfer. NS5806 also showed binding and quenching of tryptophan residues on a GST-Kv4.3(1-152) fusion protein; however, the dissociation constant for binding to this construct was $11 \pm 2 \mu \mathrm{M}$ (Figure $4.2 \mathrm{~b}$ and $4.2 \mathrm{c}$ ). The low solubility of the full $\mathrm{T} 1 \mathrm{domain}$ prevented us from further characterization of this interaction and from unequivocally showing that NS5806 binds at the T1 domain. Nonetheless, based on the more favorable binding of NS5806 with DREAM, it is likely that the site on DREAM is populated before binding at the T1 domain.

4.2.2 NS5806 binding on DREAM displaces 1,8-ANS bound at the hydrophobic cavity near EF-hand 4

Previously, we have identified two binding sites for the solvent sensitive fluorophore 1,8-ANS at the hydrophobic cavities located on the C-terminus of $\operatorname{DREAM}(\Delta 65)$ as well as on $\operatorname{DREAM}(\Delta 160)$ (Chapter 3). In order to determine the nature of interactions between NS5806 and DREAM, displacement studies were carried out in the presence of the hydrophobic fluorophore 1,8-ANS. Upon binding of 1,8-ANS to calcium bound $\operatorname{DREAM}(\Delta 65)$ an intense fluorescence emission at $475 \mathrm{~nm}$ is observed. The emission intensity of bound 1,8-ANS decreased sharply as the concentration of NS5806 is increased, indicating that NS5806 displaces 1,8-ANS from the hydrophobic cavity. The decrease in 1,8-ANS emission intensity is accompanied with a redshift of the emission maxima from $475 \mathrm{~nm}$ to $484 \mathrm{~nm}$ (Figure 4.3a). Using the decrease in fluorescence intensity and the previously determined affinities of 1,8 -ANS for $\operatorname{DREAM}(\Delta 65)$ the affinities for 
NS5806 binding to $\operatorname{DREAM}(\Delta 65)$ were determined (Eq. 2.7.6) to be $\mathrm{K}_{\mathrm{d}}=23 \pm 7 \mu \mathrm{M}$ in the apo-form and $\mathrm{K}_{\mathrm{d}}=2.5 \pm 0.3 \mu \mathrm{M}$ in the $\mathrm{Ca}^{2+}$ bound form (Figure 4.3b), which are in good
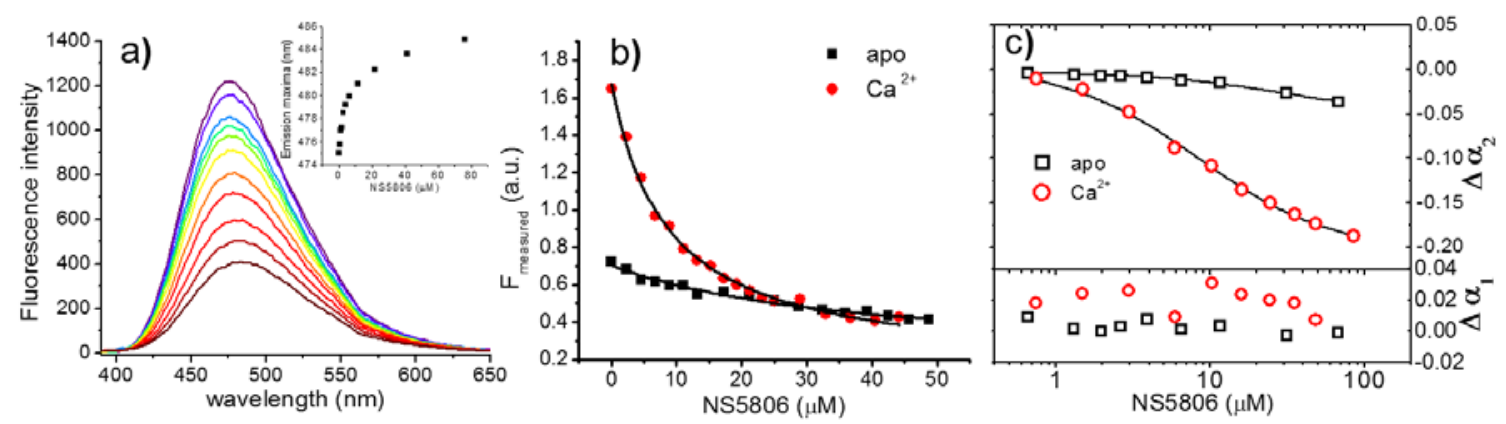

agreement with the quenching data.

Figure 4.3. (a) Fluorescence emission of 1,8-ANS:DREAM $(\Delta 65)$ complexes as a function of increasing concentrations of NS5806, inset shows the shift in emission spectra maximum as a function of NS5806. (b) Replacement curves for the 1,8-ANS- DREAM $(\Delta 65)$ complex in the apo and calcium bound states as a function of NS5806 concentration. The dissociation constants recovered using Eq. 2.8.2 are $\mathrm{K}_{d}=23 \pm 7 \mu \mathrm{M}$ and $\mathrm{K}_{\mathrm{d}}=2.5 \pm 0.3$ $\mu \mathrm{M}$ for apo and calcium bound forms, respectively. Conditions: $95 \mu \mathrm{M}$ 1,8-ANS, $10 \mu \mathrm{M}$ $\mathrm{Ca}^{2+} \operatorname{DREAM}(\Delta 65)$ in $20 \mathrm{mM}$ MOPS, $\mathrm{pH} 7.4$, and $100 \mathrm{mM} \mathrm{NaCl}$. (c) Change in fluorescence lifetime pre-exponential parameters $\alpha_{1}$ and $\alpha_{2}$ as a function of NS5806 concentration. Pre-exponential parameters were recovered using a triple discrete exponential decay model from analysis of emission decay data of $10 \mu \mathrm{M}$ 1,8-ANS bound to $10 \mu \mathrm{M} \operatorname{DREAM}(\Delta 65)$ in the apoform and $\mathrm{Ca}^{2+}$ bound state. The lifetime of unbound ANS was set as a fixed value at $0.28 \mathrm{~ns}$ (measured separately), while the second and third decay lifetimes were set as linked variables with lifetimes of $5.7 \pm 0.4 \mathrm{~ns}$ and $15.4 \pm 0.9 \mathrm{~ns}$ in the apoform $\left(\chi^{2}=2.7\right)$ and $6.3 \pm 0.5 \mathrm{~ns}$ and $16.7 \pm 0.5 \mathrm{~ns}$ in the calcium bound state $\left(\chi^{2}=1.7\right)$.

Previously, we have identified that 1,8-ANS binds to at least two different hydrophobic sites on DREAM, each bound 1,8-ANS being characterized by distinct lifetimes. Therefore it is possible to employ the fluorescence lifetime of 1,8-ANS in the presence of $\operatorname{DREAM}(\Delta 65)$ and the associated pre-exponential factors to pinpoint the NS5806 binding site. The 1,8-ANS: $\mathrm{Ca}^{2+} \operatorname{DREAM}(\Delta 65)$ complex exhibits a fluorescence decay that is best modeled by the sum of three exponential decays. We associated the fastest lifetime ( $\tau_{0}=$ 
$0.28 \mathrm{~ns})$ as corresponding to unbound 1,8-ANS. The two additional decay lifetimes correspond to 1,8-ANS binding to a partially polar cavity on the protein surface with lifetime $\tau_{1} \sim 6$ ns and 1,8-ANS bound to a predominantly hydrophobic site/cavity on the KChIP3 surface with a lifetime of $\tau_{2} \sim 16$ ns. Addition of NS5806 to the 1,8-ANS: $\mathrm{Ca}^{2+}$ $\operatorname{DREAM}(\Delta 65)$ complex did not alter the 1,8-ANS lifetimes. However, a systematic decrease in the pre-exponential factor for the long lifetime $\left(\alpha_{2}\right)$ and an increase of the preexponential parameter for $1,8-\mathrm{ANS}$ in solution $\left(\alpha_{0}\right)$ were observed. The pre-exponential factor for 1,8-ANS bound at the site with 6 ns lifetimes $\left(\alpha_{1}\right)$ remained fairly constant (Figure 4.3c). These results indicate that NS5806 binds to the hydrophobic site/cavity on DREAM, which results in a displacement of 1,8-ANS bound at that site.

\begin{tabular}{|c|c|c|c|}
\hline & $\begin{array}{l}\mathrm{K}_{\mathrm{d}} \\
(\mu \mathrm{M})\end{array}$ & $\begin{array}{l}\Delta \mathbf{H} \\
(\mathrm{kcal} / \mathrm{mol})\end{array}$ & $\begin{array}{l}-\mathbf{T} \Delta \mathbf{S} \\
(\mathrm{kcal} / \mathrm{mol})\end{array}$ \\
\hline DREAM $(\Delta 65)$ & $0.8 \pm 0.4$ & $-4.4 \pm 0.8$ & -3.9 \\
\hline $\operatorname{DREAM}(\Delta 160)$ & $2.5 \pm 1.0$ & $-6.9 \pm 0.7$ & -1.3 \\
\hline
\end{tabular}

\subsubsection{NS5806 binding to DREAM is enthalpically driven}

The thermodynamic properties associated with the formation of NS5806- DREAM complexes were probed using isothermal calorimetry. Isotherms for the titration of NS5806 into $\mathrm{Ca}^{2+} \operatorname{DREAM}(\Delta 65)$ and $\mathrm{Ca}^{2+} \operatorname{DREAM}(\Delta 160)$ in presence of $5 \mathrm{mM} \mathrm{Mg}^{2+}$ are shown in Figure 4.4. The isotherms were analyzed using a two-site binding model (solid line). The binding of NS5806 to DREAM was characterized with two dissociation constants with the specific binding site associated with a $\mathrm{K}_{\mathrm{d}}$ of $0.8 \mu \mathrm{M}$ and a non-specific binding site which showed a very weak affinity with $\mathrm{K}_{\mathrm{d}} \sim 150 \mu \mathrm{M}$ (data not shown). This weak binding 
site was not observed in the Trp169 quenching and 1,8 ANS displacement studies, probably due to an inefficient quenching or different binding site than that of 1,8-ANS. ITC thermodynamic parameters for NS5806 specifically binding to DREAM constructs are

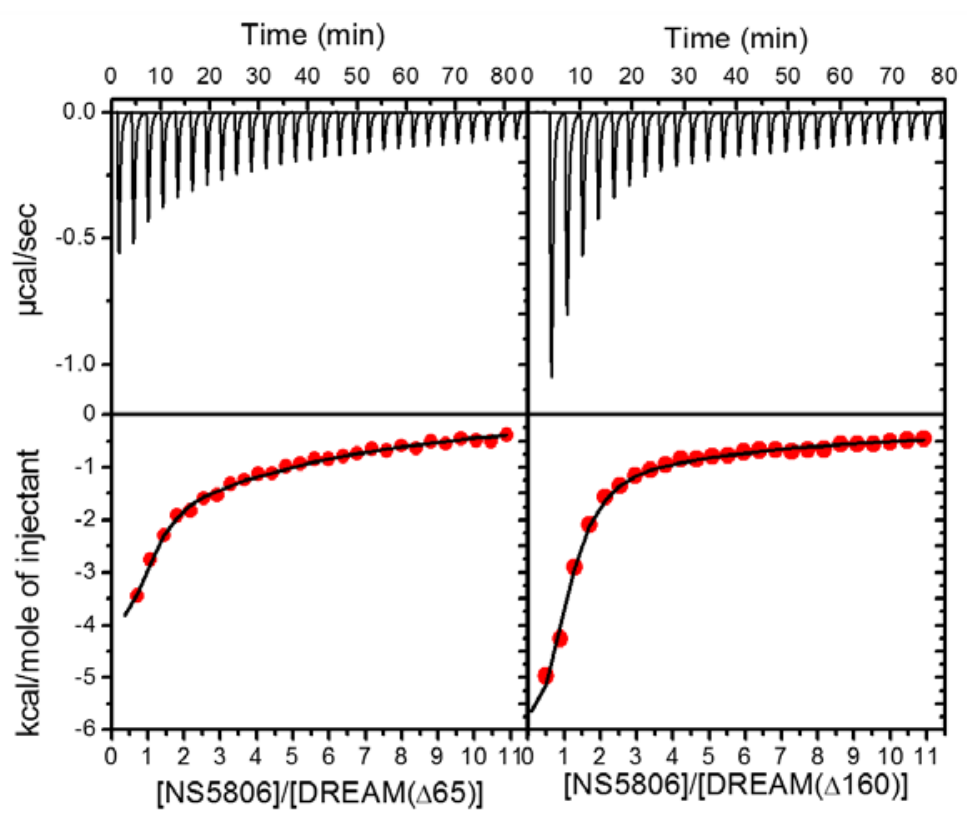

summarized in Table 4.2 .

Figure 4.4. ITC binding isotherms for NS5806 association to $\mathrm{Mg}^{2+} \mathrm{Ca}^{2+} \operatorname{DREAM}(\Delta 65)$ (left) and to $\mathrm{Mg}^{2+} \mathrm{Ca}^{2+} \operatorname{DREAM}(\Delta 160)$ (right). The upper panels of each profile reflects the thermal power expressed in units of $\mu \mathrm{cal} \cdot \mathrm{s}^{-1}$. The lower panel shows integrated reaction heats $(\Delta \mathrm{H})$ normalized for bound NS5806 and expressed in units of $\mathrm{kcal} \cdot \mathrm{mol}^{-1}$. The solid lines represent fitting curves using the two-set of sites model. Conditions: $10 \mu \mathrm{M} \mathrm{Mg}^{2+} \mathrm{Ca}^{2+}$ DREAM in $20 \mathrm{mM}$ MOPS, $\mathrm{pH}$ 7.4, and $100 \mathrm{mM} \mathrm{NaCl}$, $1 \mathrm{mM} \mathrm{CaCl}_{2}$ and $5 \mathrm{mM} \mathrm{MgCl}_{2}$.

The association of NS5806 to $\operatorname{DREAM}(\Delta 65)$ and $\operatorname{DREAM}(\Delta 160)$ exhibited an exothermic binding with $\Delta \mathrm{H}=-4.4 \mathrm{kcal} \mathrm{mol}^{-1}$ and $\Delta \mathrm{H}=-6.9 \mathrm{kcal} \mathrm{mol}^{-1}$, respectively. Association of NS5806 to $\operatorname{DREAM}(\Delta 65)$ showed different enthalpic and entropic contributions than DREAM $(\Delta 160)$, which may indicate that the structural rearrangements induced by NS5806 are not localized solely on the C-terminus domain. An exothermic 
interaction indicates that the overall interaction may involve a structural reorganization of the protein-drug complex upon association with the NS5806.

4.2.4 NS5806 increases the affinity between Kv4.3 Site 1 and DREAM and abolishes its calcium dependency
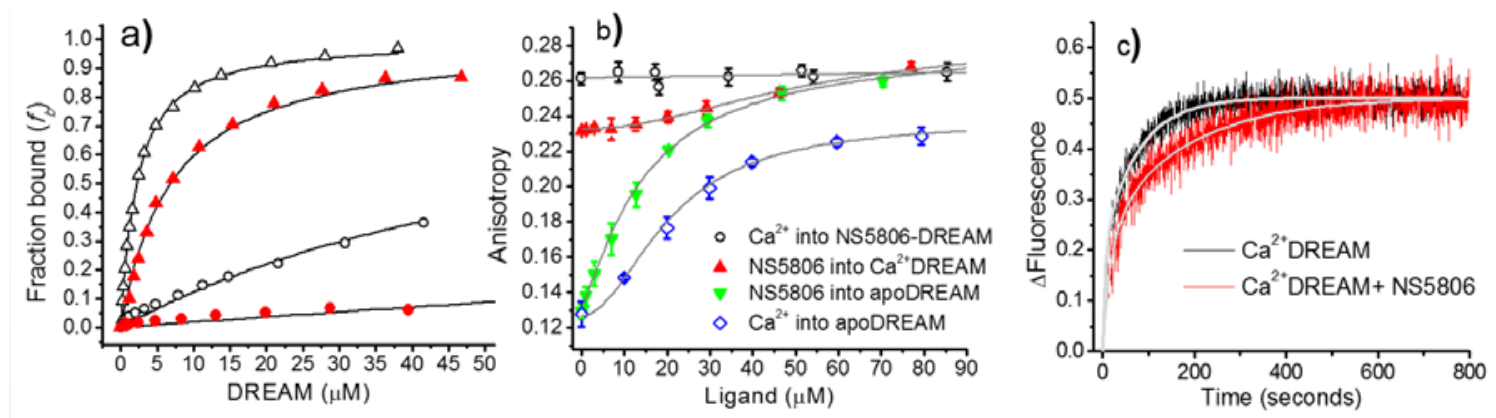

Figure 4.5. Titration curve for (a) Site 1 (Kv4.3(2-21)) binding to apoDREAM $(\Delta 65)$ (open symbols) and apoDREAM $(\Delta 160)$ (solid symbols) in the absence (circles) and presence of $200 \mu \mathrm{M}$ NS5806 (up triangle). Conditions: $0.5 \mu \mathrm{M} \mathrm{Kv} 4.3(2-22)$ and $200 \mu \mathrm{M}$ NS5806 in 20 $\mathrm{mM}$ MOPS, pH 7.4, and $100 \mathrm{mM} \mathrm{NaCl}$. (b) Titration curves of $\mathrm{Ca}^{2+}$ (open symbols) or NS5806 (solid symbols) binding to the DREAM-site 1 complex in the presence of NS5806 or $\mathrm{Ca}^{2+}$. Solid lines represent fits to a Hill equation, with dissociation constants for NS5806 binding to $\mathrm{Ca}^{2+}$ DREAM-site 1 of $\mathrm{K}_{\mathrm{d}}=51 \pm 2 \mu \mathrm{M}$ and to apoDREAM-site $1 \mathrm{~K}_{\mathrm{d}}=21 \pm 1$ $\mu \mathrm{M}$. The recovered $\mathrm{Ca}^{2+}$ dissociation constants do not reflect the actual apparent calcium affinities since EDTA was present in the samples. Conditions: $1 \mu \mathrm{M} \mathrm{Kv} 4.3(2-22)$ and 20 $\mu \mathrm{M}$ DREAM $(\Delta 65)$ in $20 \mathrm{mM}$ MOPS, $\mathrm{pH} 7.4,100 \mathrm{mM} \mathrm{NaCl}$ with $1 \mathrm{mM} \mathrm{Ca}^{2+}$ or $100 \mu \mathrm{M}$ EDTA. (c) Time profile of the change in fluorescence intensity of $100 \mathrm{nM} \mathrm{Kv} 4.3(2-22)$ peptide upon binding to $5 \mu \mathrm{M} \mathrm{Ca}^{2+} \operatorname{DREAM}(\Delta 65)$ in the absence and presence of $200 \mu \mathrm{M}$ NS5806. Solid line represents the NLLS fit using a double exponential decay model, recovered parameters listed on Table 4.

Previous structural and electrophysiological studies have identified the T1-domain at the N-terminus of Kv4 mediates binding of KChIPs (An et al. 2000, Bahring et al. 2001). The association of KChIP1 was also shown to involve two binding sites on the Kv4.2 T1 domain, site 1 comprising mainly nonpolar residues 2-22 and the more polar site 2 on the T1 domain surface consisting of residues 70-90 (Callsen et al. 2005). Due to the poor solubility of the T1 domain of Kv4.3 (residues 1-152), we decided to investigate the 
interactions by employing the fluorescently labeled peptides FITC-Ahx-Kv4.3(2-22) and Dansyl-Ahx-Kv4.3(70-90). A representative titration curve for $\operatorname{DREAM}(\Delta 65)$ binding to site 1 is shown in Figure 4.5a, with the recovered parameters listed on Table 4.3.

Binding of site 1 to $\mathrm{Ca}^{2+} \operatorname{DREAM}(\Delta 65)$ was marked by an increase in anisotropy from $r=0.072$ to $r=0.238$ and a strong calcium dependency with a dissociation constant for $\mathrm{Kv} 4.3$ site 1 in the apoform of $\mathrm{K}_{\mathrm{d}}=70 \pm 3 \mu \mathrm{M}$ and $\mathrm{K}_{\mathrm{d}}=2.7 \pm 0.1 \mu \mathrm{M}$ in the calcium bound form. A stronger calcium dependency was observed for binding of site 2 to $\operatorname{DREAM}(\Delta 65)$, with a dissociation constant of $\sim 500 \mu \mathrm{M}$ in the apo-state and $\mathrm{K}_{\mathrm{d}}=10 \pm 1$ $\mu \mathrm{M}$ upon calcium binding. Similarly, binding of Site 1 to the $\operatorname{DREAM}(\Delta 160)$ construct, which lacks the N-terminus domain, showed an increase in anisotropy to $r=0.230$ in the presence of calcium as well as a strong calcium dependency with $\mathrm{K}_{\mathrm{d}} \sim 450 \mu \mathrm{M}$ in the apoform to $\mathrm{K}_{\mathrm{d}}=24 \pm 1 \mu \mathrm{M}$ in the calcium bound form. On the other hand, binding of site 2 to calcium bound DREAM $(\Delta 160)$ was weak $\left(\mathrm{K}_{\mathrm{d}} \sim 390 \mu \mathrm{M}\right)$, indicating that binding of this peptide is specific

to the N-terminus domain as proposed previously (Pioletti et al. 2006, Wang et al. 2007).

Furthermore, upon addition of $150 \mu \mathrm{M}$ of NS5806, the binding affinity of $\operatorname{DREAM}(\Delta 65)$ and $\operatorname{DREAM}(\Delta 160)$ for site 1 becomes calcium independent and the $\mathrm{K}_{\mathrm{d}}$ values decrease to $1.9 \pm 0.2 \mu \mathrm{M}(\mathrm{r}=0.289)$ and $5.3 \pm 0.3 \mu \mathrm{M}(\mathrm{r}=0.249)$, respectively (Table 4.3). The NS5806 induced affinity increase is dose dependent and was observed in the apo and $\mathrm{Ca}^{2+}$ bound forms (Figure 4.5b). Interestingly, in the presence of $\mathrm{Ca}^{2+}$ and site 1 peptide, the affinity for NS5806 decreased significantly likely due to a restricted access to the hydrophobic cavity (Figure 4.5 and legend). On the other hand, addition of $\mathrm{Ca}^{2+}$ to the apo DREAM( $\Delta 65)$-NS5806-site 1 complex did not result in any change in anisotropy. 
In the apoform, the affinity between site 2 and $\operatorname{DREAM}(\Delta 65)$ was not observed to be modulated by NS5806. These results support the idea that the interactions between $\operatorname{DREAM}(\Delta 65)$ - and the Kv4.3 T1 domain are regulated by calcium and that in the presence of NS5806, DREAM $(\Delta 65)$ undergoes structural rearrangements that favor the association of the hydrophobic N-terminus of Kv4.3.

\begin{tabular}{|c|c|c|c|c|c|}
\hline & & \multirow[t]{2}{*}{$\begin{array}{c}\text { NS5806 } \\
K_{d}(\mu M)^{a}\end{array}$} & \multicolumn{2}{|c|}{$\begin{array}{c}\text { Site 1 } \\
K_{d}(\mu \mathrm{M})^{b}\end{array}$} & \multirow{2}{*}{$\begin{array}{l}\text { Site } 2 \\
\mathrm{~K}_{\mathrm{d}} \\
(\mu \mathrm{M})^{\mathrm{c}}\end{array}$} \\
\hline & & & $\begin{array}{c}- \\
\text { NS5806 }\end{array}$ & $\begin{array}{c}+150 \mu \mathrm{M} \\
\mathrm{NS} 5806\end{array}$ & \\
\hline \multirow[t]{2}{*}{$\operatorname{DREAM}(\Delta 65)$} & apo & $30 \pm 2$ & $70 \pm 3$ & $1.9 \pm 0.1$ & $\sim 500$ \\
\hline & $\mathrm{Ca}^{2+}$ & $4.4 \pm 0.3$ & $2.7 \pm 0.1$ & $1.9 \pm 0.2$ & $10 \pm 1$ \\
\hline \multirow[t]{2}{*}{$\operatorname{DREAM}(\Delta 160)$} & apo & $26 \pm 4$ & $\sim 450$ & $5.6 \pm 0.2$ & n.d. \\
\hline & $\mathrm{Ca}^{2+}$ & $2.7 \pm 0.3$ & $24 \pm 1$ & $5.3 \pm 0.3$ & $\sim 390$ \\
\hline \multirow[t]{2}{*}{ DREAM(Y174A) } & аро & $17 \pm 0.8$ & $90 \pm 16$ & $25 \pm 3$ & n.d. \\
\hline & $\mathrm{Ca}^{2+}$ & $8.3 \pm 0.8$ & $6.9 \pm 0.4$ & $3.1 \pm 0.2$ & n.d. \\
\hline \multirow[t]{2}{*}{ DREAM( F218A ) } & аро & $28 \pm 3$ & $90 \pm 31$ & $130 \pm 28$ & n.d. \\
\hline & $\mathrm{Ca}^{2+}$ & $13 \pm 1.3$ & $2.1 \pm 0.2$ & $7.8 \pm 0.3$ & n.d. \\
\hline
\end{tabular}

(a) Determined by the integrated intensity decrease of Trp169 and fitted using equation 1. (b) Determined using steady state anisotropy change and corrected for change in fluorescence intensity using equation 3 (c) determined by the integrated intensity increase of dansyl labeled peptide upon binding.

\subsubsection{NS5806 modulates Kv4.3 site 1 binding to KChIP3 by decreasing the rate of dissociation}

To better understand the mode of action of NS5806 we investigated its effect on the kinetics of binding between $\operatorname{DREAM}(\Delta 65)$ and site 1 of $\mathrm{Kv} 4.3$. Association of the fluorescently labeled peptide analogous to site 1 of $\operatorname{Kv} 4.3$ with $\operatorname{DREAM}(\Delta 65)$ in the apo- 
form or calcium bound form resulted in an approximately $18 \%$ increase in total fluorescence intensity. The fluorescence intensity increase can be attributed to a decrease in solvent accessibility of the fluorophore upon binding. The change in fluorescence intensity was used to probe the binding kinetics of $\operatorname{Kv} 4.3$ site 1 to $\operatorname{DREAM}(\Delta 65)$ and $\operatorname{DREAM}(\Delta 160)$, a representative trace is shown on Figure $4.5 \mathrm{c}$. The kinetic traces were best analyzed using a double exponential decay, and the recovered parameters are listed in Table 4.4. The association rate for the fast and slow phase of site 1 binding to KChIP3(65256) were similar in the apo and calcium bound state, with $\mathrm{k}_{\mathrm{on}}{ }^{\text {fast }}=17 \pm 5 \mathrm{mM}^{-1} \mathrm{~s}^{-1}$ and $\mathrm{k}_{\text {on }}{ }^{\text {slow }}=2.5 \pm 0.3 \mathrm{mM}^{-1} \mathrm{~s}^{-1}$ in the apo-state and $\mathrm{k}_{\text {on }}{ }^{\text {fast }}=28 \pm 8 \mathrm{mM}^{-1} \mathrm{~s}^{-1}$ and $\mathrm{k}_{\text {on }}{ }^{\text {slow }}=2.6 \pm$ $0.7 \mathrm{mM}^{-1} \mathrm{~s}^{-1}$ in the calcium bound form. However, the pre-exponential factors associated with the slow $\left(\alpha_{2}\right)$ phase showed an increase upon binding of calcium from 0.02 in the apostate to 0.13 in the calcium bound state. The parameters most influenced by binding of calcium were the dissociations rates of both phases, decreasing from $\mathrm{k}_{\text {off }}{ }^{\text {fast }}=1170 \pm 30$ $\mathrm{x} 10^{-3} \mathrm{~s}^{-1}$ and $\mathrm{k}_{\mathrm{off}}{ }^{\text {slow }}=2.9 \pm 0.7 \times 10^{-3} \mathrm{~s}^{-1}$ in the apoform to $\mathrm{k}_{\mathrm{off}}^{\text {fast }}=77 \pm 20 \times 10^{-3} \mathrm{~s}^{-1}$ and $\mathrm{k}_{\text {off }}^{\text {slow }}=0.2 \pm 0.06 \times 10^{-3} \mathrm{~s}^{-1}$ in the calcium bound form. Upon addition of $150 \mu \mathrm{M}$ of NS5806, the observed association rate for the fast and slow phase decreased approximately twofold; consequently, the association rate decreased but remained independent of calcium , $\mathrm{k}_{\text {on }}^{\text {fast }}=12 \pm 2 \mathrm{mM}^{-1} \mathrm{~s}^{-1}$ and $\mathrm{k}_{\text {on }}{ }^{\text {slow }}=1.1 \pm 0.8 \mathrm{mM}^{-1} \mathrm{~s}^{-1}$ in the apo-state and $\mathrm{k}_{\text {on }}{ }^{\text {fast }}=9 \pm$ $0.8 \mathrm{mM}^{-1} \mathrm{~s}^{-1}$ and $\mathrm{k}_{\text {on }}{ }^{\text {slow }}=1.1 \pm 0.3 \mathrm{mM}^{-1} \mathrm{~s}^{-1}$ in the calcium bound form. The pre-exponential factor associated with the slow phase increased were the dissociations rates of both phases, decreasing from $\mathrm{k}_{\text {off }}{ }^{\text {fast }}=1170 \pm 30 \times 10^{-3} \mathrm{~s}^{-1}$ and $\mathrm{k}_{\mathrm{off}}{ }^{\text {slow }}=2.9 \pm 0.7 \times 10^{-3} \mathrm{~s}^{-1}$ in the apoform to $\mathrm{k}_{\text {off }}{ }^{\text {fast }}=77 \pm 20 \times 10^{-3} \mathrm{~s}^{-1}$ and $\mathrm{k}_{\text {off }}{ }^{\text {slow }}=0.2 \pm 0.06 \times 10^{-3} \mathrm{~s}^{-1}$ in the calcium bound form. 


\begin{tabular}{|c|c|c|c|c|c|c|c|c|}
\hline $\operatorname{DREAM}(\Delta 65)$ & $\begin{array}{l}\tau_{1} \\
\text { (s) }\end{array}$ & $\alpha_{1}$ & $\begin{array}{l}\tau_{2} \\
\text { (s) }\end{array}$ & $\alpha_{2}$ & $\begin{array}{l}k_{\text {on }}{ }^{\text {fast }} \\
\left(\mathrm{mM}^{-1} \mathrm{~s}^{-1}\right)\end{array}$ & $\begin{array}{l}k_{\text {on }}^{\text {slow }} \\
\left(\mathrm{mM}^{-1} \mathrm{~s}^{-1}\right)\end{array}$ & $\begin{array}{c}\mathbf{k}_{\text {off }}{ }^{\text {fast }} \\
\left(\mathbf{s}^{-1}\right) \times 10^{-3}\end{array}$ & $\begin{array}{c}\mathbf{k}_{\text {off }}^{\text {slow }} \\
\left(\mathbf{s}^{-1}\right) \times 10^{-3}\end{array}$ \\
\hline apo & $12 \pm 3$ & 0.98 & $81 \pm 5$ & 0.02 & $17 \pm 5$ & $2.5 \pm 0.3$ & $1170 \pm 30$ & $2.9 \pm 0.7$ \\
\hline $\mathrm{Ca}^{2+}$ & $7 \pm 2$ & 0.87 & $76 \pm 2$ & 0.13 & $28 \pm 8$ & $2.6 \pm 0.7$ & $77 \pm 20$ & $0.20 \pm 0.06$ \\
\hline apo + NS5806 & $16 \pm 2$ & 0.92 & $181 \pm 13$ & 0.08 & $12 \pm 2$ & $1.1 \pm 0.8$ & $24 \pm 3$ & $0.03 \pm 0.01$ \\
\hline $\mathrm{Ca}^{2+}+\mathrm{NS} 5806$ & $22 \pm 2$ & 0.67 & $191 \pm 6$ & 0.33 & $9 \pm 0.8$ & $1.1 \pm 0.3$ & $17 \pm 2$ & $0.02 \pm 0.01$ \\
\hline \multicolumn{9}{|l|}{ DREAM( $\Delta 160)$} \\
\hline apo & $18 \pm 3$ & 0.85 & $156 \pm 43$ & 0.15 & $11 \pm 2$ & $1.3 \pm 0.4$ & $5050 \pm 90$ & $6.5 \pm 1.2$ \\
\hline $\mathrm{Ca}^{2+}$ & $31 \pm 2$ & 0.64 & $270 \pm 18$ & 0.36 & $6.5 \pm 0.9$ & $0.74 \pm 0.05$ & $150 \pm 10$ & $0.11 \pm 0.01$ \\
\hline apo + NS5806 & $21 \pm 2$ & 0.78 & $516 \pm 33$ & 0.22 & $9.5 \pm 0.4$ & $0.39 \pm 0.03$ & $53 \pm 5$ & $0.02 \pm 0.01$ \\
\hline $\mathrm{Ca}^{2+}+\mathrm{NS} 5806$ & $36 \pm 4$ & 0.67 & $480 \pm 43$ & 0.33 & $5.6 \pm 0.6$ & $0.42 \pm 0.04$ & $29 \pm 3$ & $0.01 \pm 0.01$ \\
\hline
\end{tabular}


Upon addition of $150 \mu \mathrm{M}$ of NS5806, the observed association rate for the fast and slow phase decreased approximately twofold; consequently, the association rate decreased but remained independent of calcium, $\mathrm{k}_{\text {on }}^{\text {fast }}=12 \pm 2 \mathrm{mM}^{-1} \mathrm{~s}^{-1}$ and $\mathrm{k}_{\text {on }}{ }^{\text {slow }}=1.1 \pm 0.8 \mathrm{mM}^{-}$ ${ }^{1} \mathrm{~s}^{-1}$ in the apo-state and $\mathrm{k}_{\text {on }}{ }^{\text {fast }}=9 \pm 0.8 \mathrm{mM}^{-1} \mathrm{~s}^{-1}$ and $\mathrm{k}_{\text {on }}{ }^{\text {slow }}=1.1 \pm 0.3 \mathrm{mM}^{-1} \mathrm{~s}^{-1}$ in the calcium bound form. The pre-exponential factor associated with the slow phase increased from 0.08 in the apo-form to 0.33 in the calcium bound form. The calculated dissociation rates decreased upon addition of NS5806 and became partially calcium independent with $\mathrm{k}_{\text {off }}^{\text {fast }}=24 \pm 3 \times 10^{-3} \mathrm{~s}^{-1}$ and $\mathrm{k}_{\text {off }}^{\text {slow }}=0.03 \pm 0.01 \times 10^{-3} \mathrm{~s}^{-1}$ in the apo-form and $\mathrm{k}_{\text {off }}{ }^{\text {fast }}=17$ $\pm 2 \times 10^{-3} \mathrm{~s}^{-1}$ and $\mathrm{k}_{\text {off }}^{\text {slow }}=0.02 \pm 0.01 \times 10^{-3} \mathrm{~s}^{-1}$ in the presence of calcium.

The change in fluorescence intensity associated with binding of Kv4.3 site 1 to $\operatorname{DREAM}(\Delta 160)$ were also best modeled with a double exponential function, Table 4.4. Similarly $\operatorname{DREAM}(\Delta 65)$, the association rate constants for the fast phase were mainly unaffected by binding of calcium. An increase in the pre-exponential factor associated with the slow phase $\left(\alpha_{2}\right)$ upon binding of calcium to $\operatorname{DREAM}(\Delta 160)$ was also observed. The calculated dissociation rates were affected by calcium binding to a larger extent than in the case of DREAM $(\Delta 65)$, with the fast phase decreasing from $\mathrm{k}_{\text {off }}{ }^{\text {fast }}=5050 \pm 90 \times 10^{-3} \mathrm{~s}^{-1}$ to $\mathrm{k}_{\text {off }}^{\text {fast }}=150 \pm 10 \times 10^{-3} \mathrm{~s}^{-1}$ and the slow phase decreasing from $\mathrm{k}_{\text {off }}{ }^{\text {slow }}=6.5 \pm 1.2 \times 10^{-3} \mathrm{~s}^{-1}$ to $\mathrm{k}_{\text {off }}{ }^{\text {slow }}=0.11 \pm 0.01 \times 10^{-3} \mathrm{~s}^{-1}$.

Addition of NS5806 resulted in a decrease in the association rate of the slow phase to $\mathrm{k}_{\text {on }}{ }^{\text {slow }}=0.39 \pm 0.03 \mathrm{mM}^{-1} \mathrm{~s}^{-1}$ in the apo-form and $\mathrm{k}_{\text {on }}{ }^{\text {slow }}=0.42 \pm 0.04 \mathrm{mM}^{-1} \mathrm{~s}^{-1}$ in the calcium bound form; as well as, a decrease in the dissociation rate of the fast and slow phases to $\mathrm{k}_{\text {off }}^{\text {fast }}=53 \pm 5 \times 10^{-3} \mathrm{~s}^{-1}$ and $\mathrm{k}_{\text {off }}{ }^{\text {slow }}=0.021 \pm 0.002 \times 10^{-3} \mathrm{~s}^{-1}$ in the apoform and $\mathrm{k}_{\text {off }}^{\text {fast }}=29 \pm 3 \times 10^{-3} \mathrm{~s}^{-1}$ and $\mathrm{k}_{\text {off }}^{\text {slow }}=0.012 \pm 0.001 \times 10^{-3} \mathrm{~s}^{-1}$ upon calcium binding. Unlike 
$\operatorname{DREAM}(\Delta 65)$, the pre-exponential parameters for the slow phase were unaffected by addition of NS5806 to apo or $\mathrm{Ca}^{2+}$ bound $\operatorname{DREAM}(\Delta 160)$. Together, these results indicate that the observed increase in affinity between $\operatorname{Kv} 4.3$ site 1 and $\operatorname{DREAM}(\Delta 65)$ or $\operatorname{DREAM}(\Delta 160)$ due to NS5806 binding can be attributed to a decrease in the dissociation rate of the site 1 peptide.
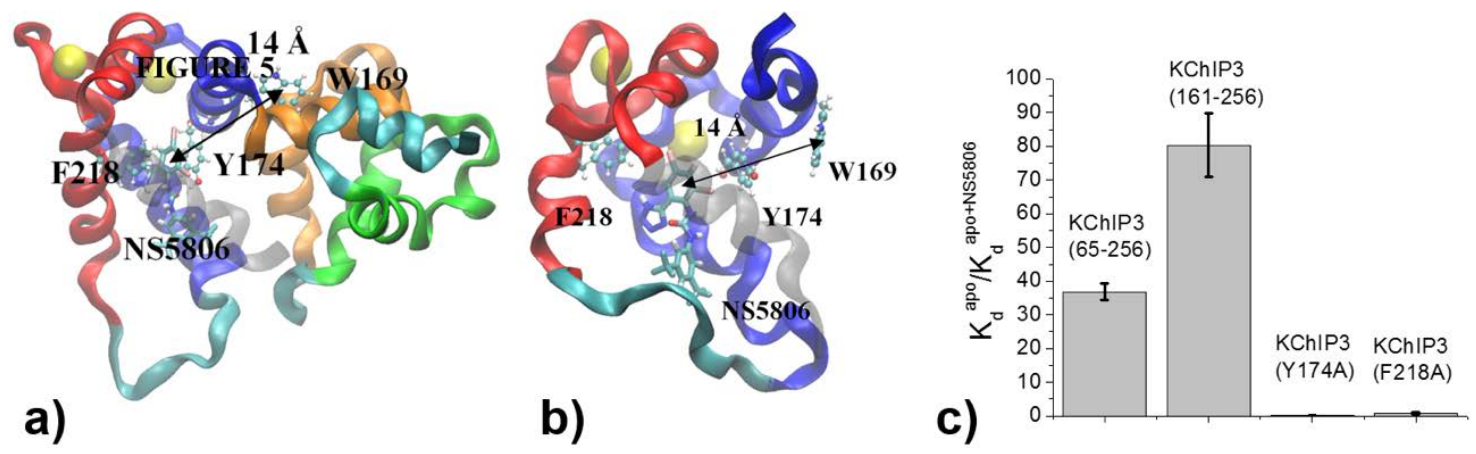

Figure 4.6. (a) Docking site for NS5806 on DREAM $(\Delta 65)$ and (b) DREAM $(\Delta 160)$. Solid line indicates the distance between tryptophan 169 and the dibromophenyl ring of NS5806. The initial NMR structures of $\operatorname{DREAM}(\Delta 65)$ (pdb: 2JUL) and $\operatorname{DREAM}(\Delta 160)$ (pdb:2E6W) were used as docking macromolecules, EF-1 shown in green, EF-2 in orange, EF-3 in blue, EF-4 in red and NS5806 shown as licorice model. (c) Mutation of Tyr174 or Phe218 to alanine completely abolishes the affinity enhancement observed in the absence of calcium upon binding of NS5806. Conditions as described in figure 4.5a.

\subsubsection{NS5806 binds at the hydrophobic cavity near EF-hand 4}

To identify the NS5806 binding site, docking simulations using AutoDock 4.2 algorithm were employed. Identical docking sites for NS5806 were identified on both constructs of DREAM (Figure 4.6). The most prominent NS5806-DREAM $(\Delta 65)$ interactions are hydrophobic, between the nonpolar brominated phenyl ring on NS5806 and Phe218, Tyr174 and Ile194 on DREAM, whereas the more electrophilic fluorinated phenyl ring faces the solvent. We have previously used a similar approach to identify the docking site of 1,8-ANS to $\operatorname{DREAM}(\Delta 65)$ and $\operatorname{DREAM}(\Delta 160)$, and found that the same 
hydrophobic cavity at the C-terminus was the most favorable binding site (Chapter 3). The fact that docking simulations identify the same docking site for 1,8-ANS and NS5806 correlates well with the 1,8-ANS displacement studies shown above. The direct role of Phe218 and Tyr174 in binding of NS5806 was confirmed by independent mutation of these residues to alanine. We observed that mutation of either residues results in a 2 to 3 -fold decrease in the affinity of NS5806 in the calcium bound state, whereas the affinity in the apostate for the Tyr174 mutant increases about 2 fold to $17 \pm 0.8 \mu \mathrm{M}$ (Table 4.3). The binding affinity in the presence of calcium of the Kv4.3 site 1 peptide decreased 3-fold for the Tyr174 mutant while remaining unchanged for the Phe218 mutant. In the presence of NS5806, DREAM(Y174A) showed an increased affinity for the site 1 peptide in the apo and calcium bound forms, but still weaker than $\operatorname{DREAM}(\Delta 65)$. On the other hand, DREAM(F218A) in the presence of NS5806 showed lower affinity for the site 1 peptide in both apo and calcium states. Overall, these results support the docking simulation and the idea that these hydrophobic residues play an important role in NS5806 binding and its observed effect on Kv4.3 site binding.

Furthermore, given that the binding site of NS5806 is located in the vicinity of the EF-hand 3 and 4 we tested whether drug association alters the DREAM interactions with $\mathrm{Ca}^{2+}$. Titration curves for $\mathrm{Ca}^{2+}$ binding to DREAM( $(465)$-FlAsH and NS5806$\operatorname{DREAM}(\Delta 65)$-FlAsH are shown in Figure 4.7, the resulting titration curves were best analyzed using a model that assumes two protein populations in the apo-state, each with at least two calcium binding sites (Eq. 2.7.8). The recovered parameters show a dual population of $\operatorname{DREAM}(\Delta 65)$-FlAsH in the apo-state with the larger population ( $\mathrm{f}=0.82$ ) having macroscopic binding constant $\mathrm{K}_{\mathrm{d}}=170 \pm 20 \mathrm{nM}$ (Hill coefficient $\mathrm{n}_{\mathrm{A}}=1.5$ ) and the 
minor population having a $\mathrm{K}_{\mathrm{d}}=10 \pm 2 \mu \mathrm{M}$ (Hill coefficient $\mathrm{n}_{\mathrm{B}}=1.1$ ). The apparent dissociation constants are comparable to the calcium binding constants reported in previous studies, ranging from $0.1 \mu \mathrm{M}$ to $10 \mu \mathrm{M}$ (Craig et al. 2002),(Osawa et al. 2005), indicating that addition of the amino acid sequence CCPGCC at the C-terminus and labeling with the biarsenical fluorophore FlAsH did not alter the calcium affinity. Titration of calcium into $\operatorname{DREAM}(\Delta 65)$-FlAsH in the presence of $150 \mu \mathrm{M}$ of NS5806 resulted in titrations curves which were also best fitted with a double Hill equation. The macroscopic binding constant recovered for the high affinity sites was $K_{d}=120 \pm 20 \mathrm{nM}$ (Hill coefficient $n_{A}=1.1$ ) with $\mathrm{f}=0.66$, and a dissociation constant for low affinity sites of $\mathrm{K}_{\mathrm{d}}=21 \pm 4 \mu \mathrm{M}$ (Hill coefficient $\left.n_{B}=1.4\right)$, indicating that the overall calcium affinity of $\operatorname{DREAM}(\Delta 65)$ is marginally affected by binding of NS5806.

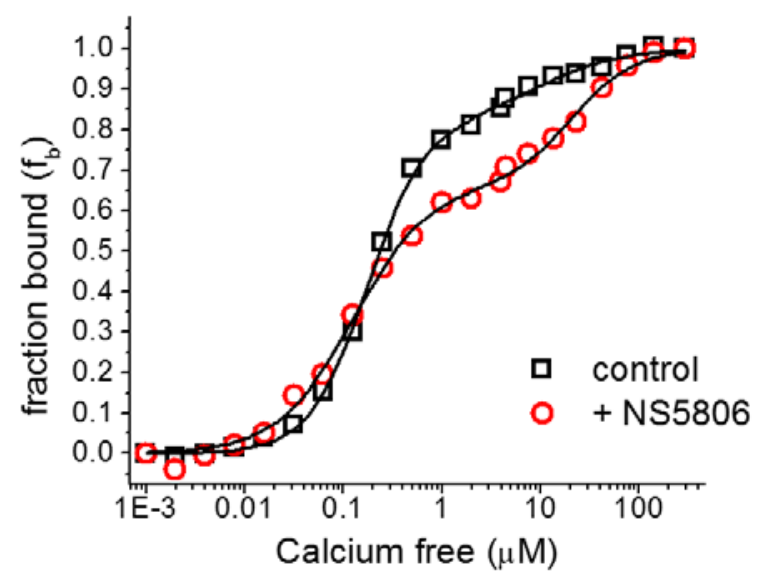

Figure 4.7. Titration curve for calcium binding to $1 \mu \mathrm{M} \operatorname{DREAM}(\Delta 65)$ labeled with the biarsenical fluorophore FlAsH-EDT 2 in the absence (squares) and in the presence (circles) of NS5806. The solid line represents the NLLS fitting using equation 4. Conditions: $1 \mu \mathrm{M}$ $\operatorname{DREAM}(\Delta 65), 200 \mu \mathrm{M}$ NS5806 in $20 \mathrm{mM}$ MOPS, pH 7.4, and $100 \mathrm{mM} \mathrm{NaCl}$.

\subsection{Discussion}

The precise mechanisms underlying the gating regulation of Kv4 mediated $\mathrm{K}^{+}$ currents are yet to be completely understood; however, it is widely accepted that these 
channels do not undergo open state inactivation involving the N-terminus (Gebauer et al. 2004, Bähring, Covarrubias 2011). It has also been shown that association of KChIPs at the N-terminus of Kv4 channels impairs open-state inactivation (Beck et al. 2002). Electrophysiological studies support the idea that both the N- and C-terminus of $\mathrm{K}_{\mathrm{v}} 4$ channels interact in order to regulate the inactivation kinetics (Jerng, Covarrubias 1997, Jerng, Shahidullah \& Covarrubias 1999), opening the possibility that such interactions are modulated by binding of KChIPs at the N-terminus (Bahring et al. 2001),(Gebauer et al. 2004). It is also proposed that the N-terminus functions as a membrane transport control, which in the absence of KChIPs, anchors Kv4 channels near the perinuclear region of the cell (Foeger, Marionneau \& Nerbonne 2010, Cui, Liang \& Wang 2008). Association of KChIPs at the $\mathrm{T} 1$ domain of $\mathrm{Kv} 4$ results in translocation to the membrane and a concomitant increase in total $\mathrm{K}^{+}$current. In addition, the presence of KChIPs is necessary for recovery of $\mathrm{K}^{+}$current with similar biophysical properties as those found in vivo.

Recent reports by Calloe and coworkers on the $\mathrm{I}_{\text {Tо }}$ activating properties of a novel diphenyl urea compound (NS5806) whose activity depends on the presence of KChIPs has highlighted the role of these auxiliary proteins in regulation of Iто currents (Calloe et al. 2009). This drug was shown to reverse the effect of induced heart failure in canine models (Calloe et al. 2009, Cordeiro et al. 2012). On the other hand, Witzel and coworkers hypothesized that the observed current inhibition in hippocampal neuronal cells was likely due to a higher concentration of NS5806 being used $(20 \mu \mathrm{M})$ compared to the concentration used in canine cardiomyocytes (Witzel, Fischer \& Bähring 2012). Indeed, at NS5806 concentrations above $100 \mu \mathrm{M}$ a reversal of activation was observed in HEK293 cells. This highlights the possibility of multiple binding sites of NS5806 on the Kv4-auxiliary subunit 
complex. Nonetheless, NS5806 still resulted in a slowing of inactivation and accelerated recovery from inactivation in both cardiac and neuronal cells at concentrations below 20 $\mu \mathrm{M}$.

Given the dependency $\mathrm{K}^{+}$current modulation by NS5806 on the presence of KChIPs, in this report we investigated whether KChIPs could be a target binding partner for NS5806. Titration experiments show that both the apo and calcium bound forms of $\operatorname{DREAM}(\Delta 65)$ were quenched by NS5806 with an affinity fivefold lower in the apoform. The hydrophobic nature of NS5806 supports the idea that the binding site on DREAM is located in a solvent restricted hydrophobic cavity, and that calcium binding to DREAM increases accessibility of the drug to this cavity. Moreover, the similar dissociation constants recovered for both DREAM constructs indicate that in the calcium bound and calcium free state of the protein, the binding site of NS5806 is located at the C-terminus. Interestingly the dissociation constants recovered for NS5806 binding to $\mathrm{Ca}^{2+}$ $\operatorname{DREAM}(\Delta 65)$ is very similar to the EC50 value observed for current potentiation in CHO$\mathrm{K} 1$ cells expressing $\mathrm{Kv} 4.3 / \mathrm{KChIP} 2(\mathrm{EC} 50=5.3 \mu \mathrm{M})(\mathrm{Lundby}$ et al. 2010) as well as in HEK293 cells expressing Kv4.2/KChIP3 (EC50 = $6.9 \mu \mathrm{M})$ (Witzel, Fischer \& Bähring 2012). Similarly, the affinity of NS5806 for the Ca2 + free form of DREAM $(\mathrm{Kd} \sim 25 \mu \mathrm{M})$ is identical to the EC50 determined for NS5806 induced accelerated recovery from inactivation $(\mathrm{EC} 50=2625 \mu \mathrm{M})$ (Lundby et al. 2010). Displacement studies of the extrinsic fluorophore 1,8-ANS bound to $\operatorname{DREAM}(\Delta 65)$ confirm that NS5806 binds at a hydrophobic cavity. The emission spectrum of 1,8-ANS bound to DREAM shows a maxima at $475 \mathrm{~nm}$. Addition of NS5806 to 1,8-ANS:DREAM complexes resulted in a fluorescence intensity decrease in the presence or absence of calcium as shown in Figure 4.3b. Interestingly, upon 
addition of $\sim 40 \mu \mathrm{M}$ NS5806 the total emission intensity of 1,8-ANS:DREAM complex is identical in the presence or absence of calcium. A fluorescence signal is still detected even at saturating amounts of NS5806 $(150 \mu \mathrm{M})$. This is likely due the presence of additional 1,8-ANS bound to a site distinct than that occupied by NS5806. Furthermore, the emission maxima of 1,8-ANS bound to calcium or apoform $\operatorname{DREAM}(\Delta 65)$ in the presence of 150 $\mu \mathrm{M}$ NS5806 shows a maxima at $485 \mathrm{~nm}$ (Figure 4.3a inset). These results are in agreement with fluorescence lifetime data, which show that addition of NS5806 results in a concomitant decrease in the pre-exponential parameter $\alpha_{2}$ associated with 1,8-ANS bound at the solvent restricted cavity (Figure 4.3c), while the pre-exponential parameter that corresponds to 1,8-ANS bound to a solvent exposed site with emission maximum at 485 nm remains constant. These results suggest that 1,8-ANS binds at a physiologically active hydrophobic cavity on DREAM. These observations also support the idea that displacement of 1,8-ANS could be used as a fluorescence based high throughput method for the discovery of new drugs that bind at this hydrophobic cavity and could induce similar effects to that observed for NS5806. Indeed, an analogous 1,8-ANS screening assay has been previously reported for other drug targets (Martin et al. 2012).

Isothermal calorimetric titrations of NS5806 into DREAM further confirm the strong association between NS5806 and DREAM. The recovered thermodynamic parameters show that both enthalpic and entropic contributions stabilize the complex formation, with the enthalpic contribution becoming dominant in the DREAM $(\Delta 160)$ construct. The large enthalpy contribution is in contrast to the idea that the binding site of NS5806 on DREAM is mainly hydrophobic (entropy driven binding); however, it is likely that binding of NS5806 triggers larger exothermic structural rearrangements on the protein. 
Characterization of the interaction of DREAM and peptides homologous to Kv4.3(2-22) and Kv4.3(70-90) indicate that the interactions between site 1/site 2 and DREAM are modulated by calcium, Table 4.3. In the presence of calcium, DREAM $(\Delta 65)$ showed a 26-fold increase in affinity for site 1, whereas, DREAM $(\Delta 160)$ showed an 18fold increase compared to the apoform. Furthermore, the dissociation constant recovered for site 1 binding to $\operatorname{DREAM}(\Delta 160)$ in the presence of calcium was about tenfold weaker than that of site 1 binding to $\operatorname{DREAM}(\Delta 160)$. The determined dissociation constants can be utilized to calculate the role of the $\mathrm{N}$ - and C-terminus domains of DREAM on binding of site 1 of Kv4.3. The energetic contribution of the $\mathrm{N}$ - and C-terminus of DREAM to the association with site 1 calculated from the dissociation constants in the calcium bound state are $-1.3 \mathrm{kcal} \mathrm{mol}^{-1}$ and $-6.1 \mathrm{kcal} \mathrm{mol}^{-1}$, respectively. The larger contribution of the Cterminus highlights the role of this domain on DREAM as mediator in protein-protein interaction. Interestingly, the Y134E mutation on KChIP1 (Y174E on DREAM) has also been shown to completely abolish current modulation by KChIP1 (Wang, Chai 2009). However, the contribution of the N-terminus hydrophobic cavity of KChIPs is necessary for Kv4 translocation to the membrane and KChIP-Kv4 complex formation (Zhou et al. 2004b, Kunjilwar, Qian \& Pfaffinger 2013a). This is in agreement with structural studies, where the N-terminus of Kv4.3 was determined to bind across the hydrophobic face of KChIP1 contacting residues spanning both the N- and C-terminus of KChIP1 (Pioletti et al. 2006, Wang et al. 2007). The dissociation constant for site 2 binding to $\operatorname{DREAM}(\Delta 65)$ was also dependent on calcium, with an approximately 50 -fold increase in affinity but still weaker than the site 1 interaction. The fact that calcium binding at the C-terminus EF-3 and EF-4 of DREAM modulates binding of site 2 is remarkable, suggesting that the 
structural changes associated with the calcium binding to the C-terminal domain are propagated into the $\mathrm{N}$-terminal domain of the protein. However, it is also possible that the calcium induced dimerization of DREAM is responsible for the increase in affinity.

Addition of saturating amounts of NS5806 completely abolished the calcium dependency of site 1 association with DREAM as well as further increased its affinity. These results are surprising, given that the experiments above support the idea that NS5806 and site 1 share the same binding site at the C-terminus of DREAM. Furthermore, a clear dose dependent increase in DREAM-site 1 association is observed upon addition of NS5806 in the absence of calcium $\left(K_{d}=21 \pm 1\right)$, but no further increase is observed upon addition of excess calcium. On the other hand, at saturating amounts of calcium addition of NS5806 induces a further increase in anisotropy. Suggesting that in the presence of NS5806 the DREAM-site 1 complex adopts an altered tertiary structure, as evident from the larger anisotropy upon binding in the presence of NS5806 (Figure 4.5b). These results are comparable to those obtained for the Kv4.3(1-143)-KChIP1 complex using size exclusion chromatography in the presence of the diphenyl-urea compound CL-888 (Bowlby et al. 2005). A similar effect due to the presence of NS5806 for site 1 bound to $\operatorname{DREAM}(\Delta 65)$ on anisotropy was observed, indicating that structural rearrangement are localized on the C-terminus domain of DREAM.

A better understanding of the mechanism by which NS5806 modulates the affinity of site 1 peptide binding to DREAM construct is gained from the kinetics associated with binding, Table 4.4. The biphasic nature of the binding kinetics is potentially due to the presence of DREAM proteins which populate partially distinct conformations or oligomerization state. Overall, these results revealed that saturating amounts of NS5806 
decreased the rate of dissociation between DREAM and site 1 of Kv4.3, independent of calcium. A possible explanation for the stabilization of the protein peptide complex, is that binding of NS5806 at the C-terminus cavity destabilizes this domain and increases the accessibility of the hydrophobic residues to interact with hydrophobic residues on site 1 . Also, the fact that the affinity of $\mathrm{Ca}^{2+}$ free DREAM for NS5806 is lower than in the presence of calcium indicate that there is a larger energy barrier for access to the hydrophobic cavity at the C-terminus. However, once NS5806 binds at the hydrophobic cavity in the apoform, the resulting structural changes are comparable to those present in the calcium bound form. This results in similar affinity and dissociation constants for the $\mathrm{N}$-terminus of Kv4.3. On the other hand, the observed decrease in dissociation rate in the presence of NS5806 seem to indicate that the DREAM-NS5806 reorganizes into a conformation which is different from that of the apoform or calcium bound KChIP3 and which forms a more stable complex with site 1 of Kv4.3.

Computational simulation allowed us to pinpoint the potential docking site for NS5806 as being at a hydrophobic cavity between EF-3, EF-4 and the H10 helix. This docking site is also in good agreement with the distances from Trp169 determined using RET and with the displacement studies. However, previous docking studies of a similar diphenyl-urea compound (CL-888) identified a site on the hydrophilic surface of KChIP1 (Bowlby et al. 2005). The proposal that this is the docking site for NS5806 is not supported by the fact that similar dissociation constants were recovered for $\operatorname{DREAM}(\Delta 65)$ and $\operatorname{DREAM}(\Delta 160)$, even though the C-terminus construct lacks half of the residues involved in the proposed docking site for CL-888. We also conducted docking simulation with NS5806 where we limited the docking grid to cover only the previously proposed binding 
site at the hydrophilic surface. The predicted association energy of this site was $4 \mathrm{kcal} \mathrm{mol}^{-}$ ${ }^{1}$ weaker than predicted energy for the docking at the hydrophobic cavity near EF-3 and EF-4. Indeed, the role of Tyr174 and Phe218 identified in docking simulation as being involved in stabilization of NS5806 and Kv4.3 site 1 peptide interactions is supported by site directed mutagenesis studies, which show that mutation of either residue to alanine completely abolishes the effect of NS5806 (Figure 4.6c). We also have observed that a DREAM(F252A) mutant readily forms aggregates (data not shown), supporting the idea that this residue plays an important role in the stability of the protein. Thus, displacement of this amino acid by NS5806 could potentially play a major role in restructuring of the DREAM structure.

With these results in mind, we propose that binding of NS5806 at the C-terminus hydrophobic cavity results in an increase in flexibility of the H10 helix, which is likely facilitated by displacement of the hydrophobic residues on this helix (Fig. 4.8). This is supported by molecular dynamic simulations which show that in the absence of NS5806 the aromatic residue Phe252 on the H10 helix is stacked between Tyr174 and Phe218, inherently reducing the flexibility of this helix (Fig. 4.8). In contrast, simulations in the presence of NS5806 show a displacement of Phe252 residue and concomitant motion of the H10 helix in order to accommodate NS5806 in the hydrophobic cavity. Furthermore, simulation of the Kv4.3-DREAM complex show that Trp19 of Kv4.3 is positioned in an identical stacking position between Tyr174 and Phe218 as that found for Phe252 and NS5806; whereas, in the presence of NS5806 the cavity at the C-terminus of DREAM 
expands in order to accommodate both NS5806 and the hydrophobic N-terminus of Kv4.3 while pushing Trp19 deeper into the hydrophobic cavity (Fig. 4.8).

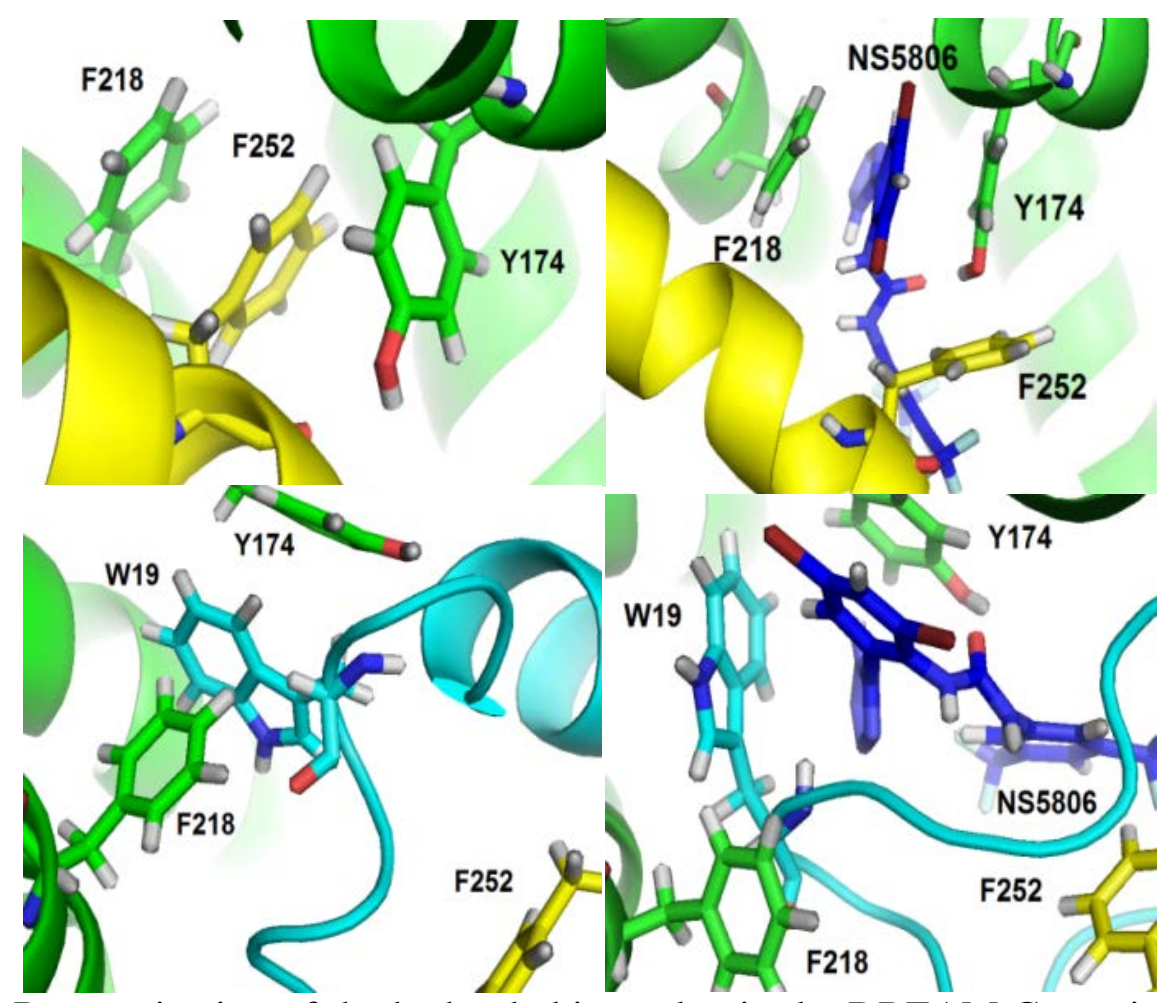

Figure 4.8. Reorganization of the hydrophobic pocket in the DREAM C-terminus upon binding of NS5806 and/or site 1. Top left: Position of helix-10 (shown in yellow) in the C terminus hydrophobic pocket of $\operatorname{DREAM}(\Delta 65)$ is stabilized by stacking interactions among F218, F252 and Y174. Top right: Binding of NS5806 to the DREAM $(\Delta 65)$ leads to the displacement of F252 and NS5806 is stabilized by hydrophobic interactions with F218 and Y174. Bottom left: Orientation of site 1 in the C-terminus hydrophobic pocket of $\operatorname{DREAM}(\Delta 65)$ with focus on the interaction of W19 from site 1 with hydrophobic residues from DREAM $(\Delta 65)$. Bottom right: The reorientation of the hydrophobic domain at the DREAM C-terminus upon concomitant binding of site 1 and NS5806.

The motion of Trp19 into the hydrophobic cavity of DREAM C-terminal may contribute to the slower rate of dissociation of the hydrophobic site 1 peptide in the presence of NS5806. The structural rearrangement observed using molecular dynamics are consistent with the idea that binding of NS5806 induces structural rearrangements in the Kv4.3DREAM protein complex. Whether these structural changes are isolated on DREAM and 
the N-terminus of Kv4.3 or they propagate across the T1 domain remains to be determined. Previous studies favor the idea that such structural changes could propagate towards the $\mathrm{K}^{+}$channel inner vestibule near the pore and modulate the $\mathrm{K}^{+}$currents (Wang, Covarrubias 2006, Cushman et al. 2000).

\subsection{Summary}

The results presented here could potentially explain the observed increase in $\mathrm{K}^{+}$ current and the slowing of inactivation in the presence of NS5806 in cardiac, neuronal, and heterologous expression studies. The elimination of the calcium dependency of association between DREAM and site 1 of Kv4.3 as well as the increase in affinity in the presence of NS5806 support the idea that the observed increase in $\mathrm{K}^{+}$current amplitude is due to an enhanced translocation of Kv4.3-DREAM protein complexes to the cell membrane induced by the presence of NS5806. We further hypothesize that the resulting decrease in inactivation kinetic is due to the decrease in accessibility of the N-terminus of Kv4.3 channel to interact with the cytoplasmic C-terminus of Kv4. Indeed, this interaction has been shown to be necessary for modulation of gating currents of Kv4.2 (Callsen et al. 2005). The fact that the dissociation constant was reduced in the presence of calcium also highlights the potential role of $\mathrm{Ca}^{2+}$ in regulation of $\mathrm{K}^{+}$currents. In support of the role of calcium in regulating $\mathrm{K}^{+}$current are studies which have shown that in rat stellate cells, a protein complex is formed involving the voltage-gated calcium channel Cav3, the potassium channel Kv4.2 and the auxiliary protein DREAM (Anderson et al. 2010). 


\section{MODEL OF DREAM PROTEIN AND IMPLICATION IN PROTEIN AND DNA INTERACTIONS}

\subsection{Background and significance}

One of the most widely studied calcium binding proteins is calmodulin (CaM), a small and ubiquitous (148 residues) acidic protein, with four active EF-hand motifs able to bind calcium with affinities between 1 to $10 \mu \mathrm{M}$ (Crivici, Ikura 1995). The structure of calcium bound CaM has been shown to follow a dumbbell arrangement of the N- and Cterminus domain, while removal of calcium induces local rearrangement of the EF-hands and an increase in flexibility of the N- and C-terminus (Chattopadhyaya et al. 1992, Zhang, Tanaka \& Ikura 1995). The interaction of CaM with proteins as well as with their peptide fragments often surpass the affinity for calcium(Rhoads, Friedberg 1997). The mechanism of calcium induced protein binding to CaM can be simplified as a calcium triggered exposure of hydrophobic surfaces which facilitates the association of the target protein. The protein fragments involved in CaM association often function as an autoinhibitory domain or are directly involved in protein activity. These CaM recognition sequences are amphipathic in nature with numerous positive and bulky hydrophobic residues forming electrostatic and hydrophobic contacts. CaM binding sequences can be often grouped by those that are enhanced by calcium or are calcium independent (Rhoads, Friedberg 1997). The fact that CaM has been shown to constitute about $0.1 \%$ of total cytosolic protein, also highlights the role of this signal transducer in calcium signaling (Chin, Means 2000).

DREAM is a relatively new member of the group of EF-hand calcium binding proteins and it has been shown to be one of the first proteins to regulate gene expression in a calcium dependent manner (Carrion et al. 1999). Additionally, this protein was identified 
to be involved in presenilin fragmentation (Buxbaum et al. 1998), as well as regulation of the $\mathrm{K}_{\mathrm{V}} 4$ subfamily of voltage gated potassium channels (An et al. 2000). DREAM is a 256 amino acid long, $29 \mathrm{kDa}$ protein highly expressed in the brain (An et al. 2000) and belongs to the superfamily of neuronal calcium sensors (NCS) with close homology to NCS-1 and recoverin (Burgoyne, Weiss 2001). Similarly to other NCS, DREAM contains four EFhand motifs of which EF-hand 1 is unable to bind calcium, EF-hand 2 binds preferentially magnesium and the two EF-hands at the C-terminus bind calcium with affinities between 1 to $10 \mu \mathrm{M}$ (Osawa et al. 2005). Three other homologous proteins with close homology to DREAM (KChIP1, KChIP2 and KChIP4) have also been identified, and together form the KChIP subgroup of NCS proteins (An et al. 2000). Despite the high homology of KChIPs at their $\mathrm{C}$-terminus, the $\mathrm{N}$-terminus region and tissue expression are highly divergent.

A recent report by Ramachandran and coworkers showed that DREAM and CaM form a complex and enhance the activation of calcineurin (Ramachandran et al. 2012). Additionally, over 40 protein partners were identified to interact with DREAM in a calcium dependent and independent manner, further highlighting the role of DREAM as a calcium sensor involved in numerous cell processes (Ramachandran et al. 2012). Consequently, given the wide spectrum of processes regulated by $\mathrm{CaM}$ and the multifunctional activity of DREAM, a better understanding of the molecular mechanism by which these two proteins interact will provide insight into the regulation of calcium signaling in neuronal cells.

Recent studies have addressed the molecular structure of DREAM and how calcium induced changes are translated into regulation of protein-protein and protein-DNA interactions (Lusin et al. 2008, Ramachandran et al. 2012, Osawa et al. 2001). NMR structures of the calcium binding C-terminus domain (EF-hand 3 and EF-hand 4, residues 
161-256, PDB: 2e6w) (Yu et al. 2007b) and of the core domain (EF-hand 1 to 4, residues 76-256, PDB: 2jul) (Lusin et al. 2008) have been resolved. Interestingly, DREAM has been shown to be cleaved at Aps61-Asp64 by caspase-3, which indicates that inside the cell there may be a subpopulation DREAM with structure analogous to that resolved by NMR(Choi et al. 2001). However, no model for the full-length DREAM protein is currently available.

In this report, we seek to identify the amino acid sequence at the $\mathrm{N}$-terminus of DREAM involved in CaM binding. We further characterize the secondary and tertiary structure of DREAM N-terminus using a combination of theoretical and experimental techniques to build a molecular model for the full-length protein. Finally, we propose a mechanism by which CaM associates to DREAM and sequesters its $\mathrm{N}$-terminus domain which could potentially modulate DREAM's interaction with binding targets. Altogether, the results presented here provide us with a molecular understanding of the CaM:DREAM interaction and underline the implication of this protein complex in calcium signaling and gene expression.

\subsection{Results}

5.2.1 Calmodulin stabilizes the N-terminus of DREAM and increases the solubility of the protein complex.

Previous reports have shown that DREAM readily forms inclusion bodies when overexpressed in BL21(DE3) E. coli cells, whereas DREAM( $\Delta 65)$ was recovered in moderate yields as a soluble protein (Lusin et al. 2008, Osawa et al. 2001). Indicating that residues 1-65 in DREAM destabilize the protein under conditions used for recombinant protein expression. It has also been shown that the association between DREAM and CaM 
is mediated by an amino acid sequence within the N-terminal 94 residues of DREAM (Ramachandran et al. 2012). We hypothesized that binding of CaM could stabilize DREAM and lead to an increase in solubility during recombinant expression. To test this hypothesis we co-expressed CaM and DREAM in BL21-DE3 E. coli at low temperature (see materials and methods). Under such conditions, DREAM was recovered as a soluble protein with yields of $\sim 40 \mathrm{mg}$. Interestingly, we have observed that $10 \mathrm{mM}$ LDAO abolishes the CaM:DREAM interaction and prevents DREAM aggregation in the presence of saturating calcium, which facilitates the purification of soluble DREAM. We envision this protocol being more robust than the previously employed method of inclusion body refolding (Osawa et al. 2001) or GST fusion tags (Ramachandran et al. 2012). These results support the role of CaM as a protein cofactor that stabilizes the N-terminus of DREAM and potentially facilitates its translocation in the cell.

5.2.2 Residues 29-44 at the N-terminus of DREAM mediate CaM binding in a calcium dependent manner.

Bioinformatics analysis of the N-terminus of DREAM revealed the presence of numerous basic, acidic and hydrophobic residues. Of particular interest is a short amphipathic 16-residue peptide segment (residues 29-44, sequence: SIKWQRPRFTRQALMR) with a high density of positively charged residues and hydrophobic residues (Figure 5.1). 
a)

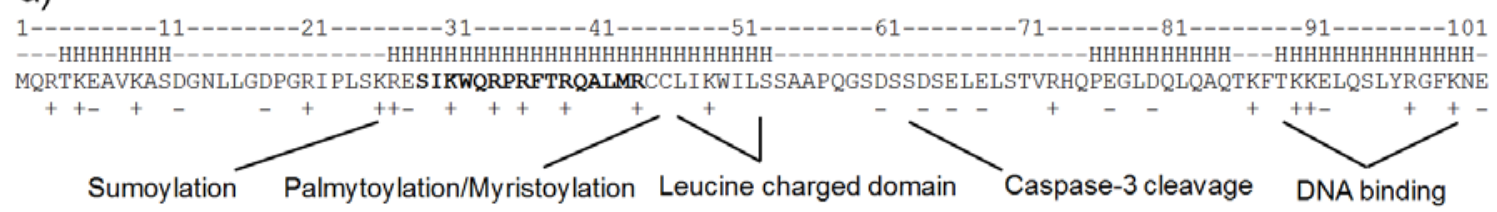

b)

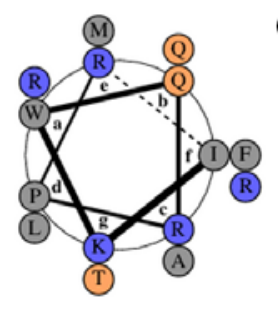

c)

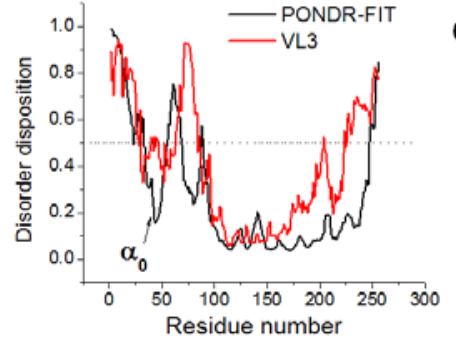

d)

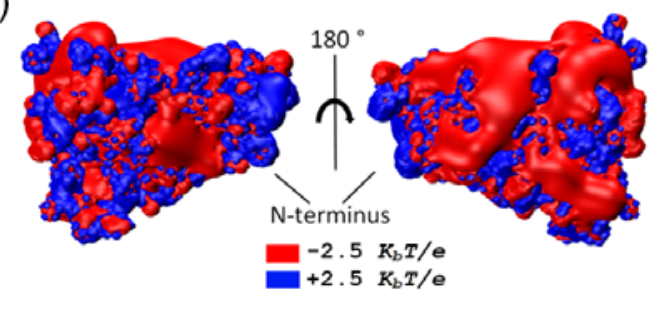

Figure 5.1. (a) Bioinformatics analysis of the first 100 residues at the N-terminus of mouse DREAM indicating regions of helical structure and charge distribution. Proposed sites for posttranslational modification and ligand interactions are shown. (b) Helical plot analysis of residues 29 to 44 of DREAM. (c) DREAM amino acid sequence disorder prediction using the PONDR-FIT and VL3 prediction servers. (c) Potential energy surface of apoDREAM (lacking the first 77 residues) calculated using the published NMR structure and the APBS plugin of the VMD software with CHARMM parameters at $150 \mathrm{mM}$ mobile ion concentration.

This short amino acid sequence shows close similarity with the 1-8-14 Type A binding motif associated with other calmodulin binding peptides(Rhoads, Friedberg 1997); with a total charge of +5 and bulky hydrophobic residues Ile30, Trp32, Phe37, Leu42 and Met43. Secondary structure prediction also shows this sequence as having a moderate helical propensity. Helical wheel diagram of DREAM(29-44) shows an arrangement of dispersed positively and hydrophobic residues (Figure 5.1b). This small helical region is surrounded by extended disordered domains (Figure 5.1c), which may enhance the flexibility of the Nterminus. The core domain of DREAM (residues 76 to 256) exhibits a strong 
electronegative surface opposite to a hydrophobic groove which has been shown to be involved in ligand binding (Chapter 3 ) (Figure 5.1d).
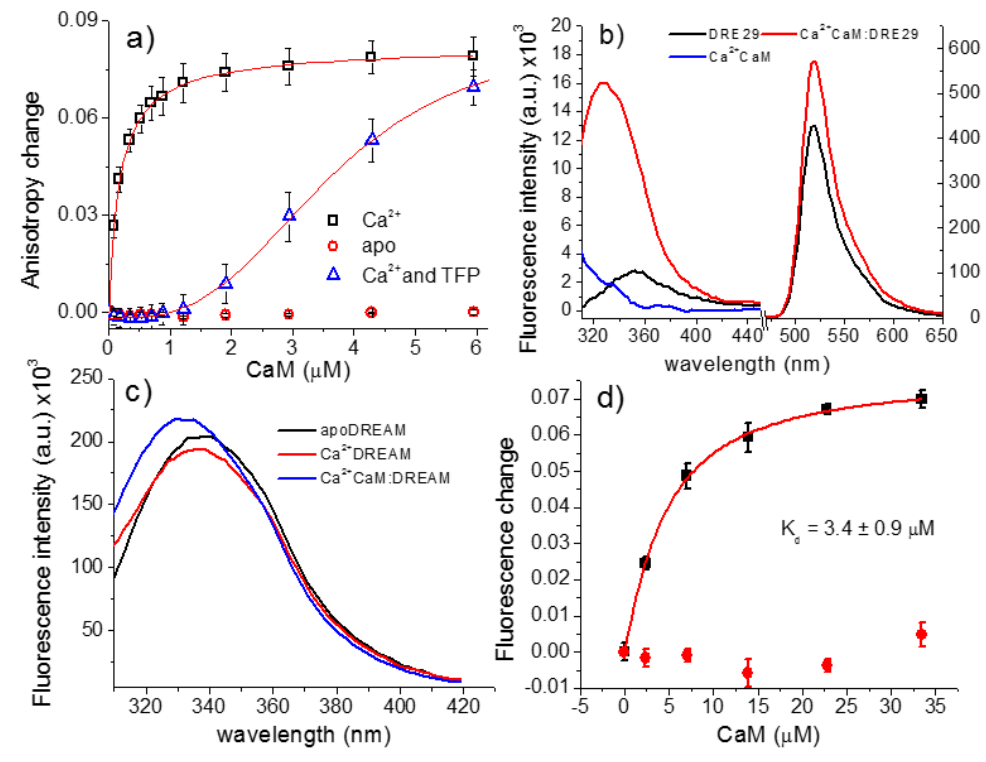

Figure 5.2. Titration curves for calmodulin binding to (a) $100 \mathrm{nM}$ of a FITC labeled peptide analogous to residues 29-44 of mouse DREAM "DRE29" in the absence (circles) or present (squares) of $1 \mathrm{mM}$ calcium $(\mathrm{Kd}=136 \pm 10 \mathrm{nM})$ and in the presence of calcium and $30 \mu \mathrm{M}$ Trifluoperazine (up triangles). (b) Fluorescence emission spectra of $5 \mu \mathrm{M}$ DRE29 peptide in the presence or absence of $10 \mu \mathrm{M} \mathrm{Ca}^{2+} \mathrm{CaM}$, sample was excited at 300 $\pm 4 \mathrm{~nm}$ which resulted in negligible contribution from tyrosine on CaM. Buffer background emission was subtracted to decrease Rayleigh, Raman and $2^{\text {nd }}$ harmonic peaks contributing to the signal. (c) Observed shift and increase in tryptophan fluorescence upon calmodulin binding. (d) Normalized tryptophan fluorescence change at $370 \mathrm{~nm}$ of DREAM(1-256) upon addition of calmodulin in the absence (circles) and presence (squares) or $1 \mathrm{mM}$ calcium $(\mathrm{Kd}=3.3 \pm 0.9 \mu \mathrm{M})$.

Calmodulin is well known to bind positively charged amphipathic protein segments in a calcium dependent manner; therefore, we hypothesize that a similar binding mechanism is present on DREAM. To test this hypothesis we studied binding of CaM to a fluorescently labeled peptide homologous to residues 29 to 44 of DREAM (herein named DRE29). Upon addition of calcium bound CaM to DRE29 a large increase in fluorescence anisotropy was measured, indicative of binding. The binding curves show a clear calcium dependent binding of the DRE29 peptide to calmodulin, with a dissociation constant in the 
calcium bound form of $\mathrm{K}_{\mathrm{d}}=136 \pm 10 \mathrm{nM}$ and negligible binding in the absence of calcium (Figure 5.2a). Similar results were obtained using FRET experiments between DRE29 and CaMRR2, with a dissociation constant of $132 \pm 16 \mathrm{nM}$ (Figure 5.3a-b). The binding of CaM to DRE29 was inhibited in the presence of the antipsychotic drug and known calmodulin inhibitor trifluoperazine (TFP). Analysis of the binding using a competitive model in the presence of $30 \mu \mathrm{M}$ TFP, using the reported affinity of TFP for CaM of 1 $\mu \mathrm{M}$,(Levin, Weiss 1977) shows that a single bound TFP is sufficient to inhibit binding of the peptide (Figure 5.3c). Since the stronger affinity binding sites of TFP on CaM are at the C-terminus domain, the data shows that this domain is mainly responsible for binding the DRE29 peptide(Cook, Walter \& Walter 1994).
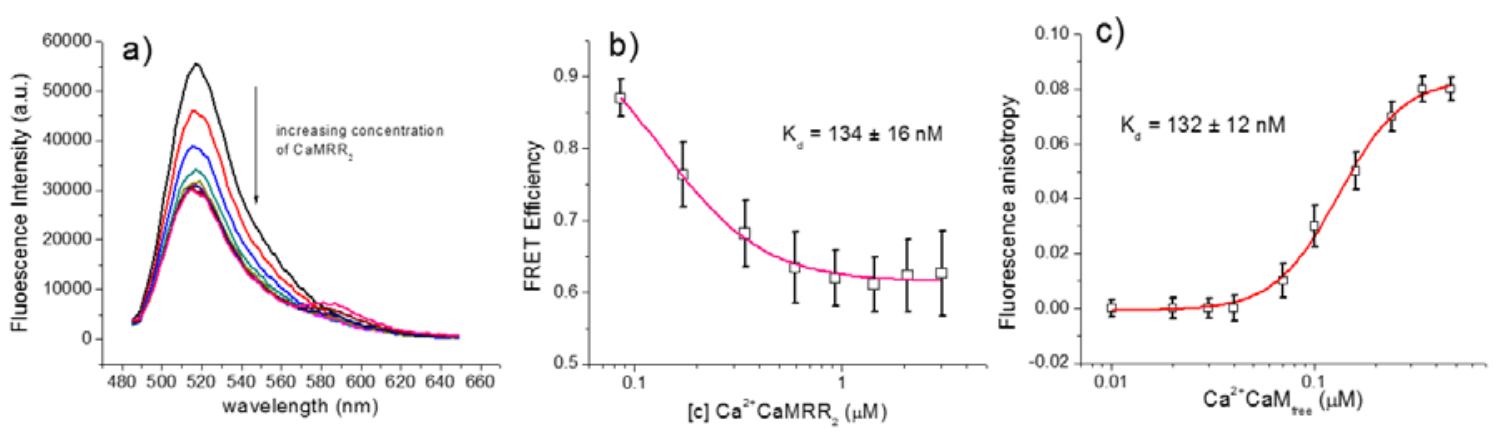

Figure 5.3. (a) Fluorescence intensity quenching of $1 \mu \mathrm{M}$ DRE29 upon association to $\mathrm{Ca}^{2+}$ CaMRR2. (b) Plot of the FRET efficiency between DRE29 and $\mathrm{Ca}^{2+} \mathrm{CaMRR} 2$ complex, a dissociation constant of $134 \pm 16 \mathrm{nM}$ was recovered using a Hill equation. (c) Fluorescence anisotropy of $100 \mathrm{nM}$ DRE29 versus calculated TFP free calmodulin. The drug free calmodulin concentration was determined assuming that a single TFP molecule is bound to calmodulin with an affinity of $1 \mu \mathrm{M}, 30 \mu \mathrm{M}$ TFP was held constant while the concentration of $\mathrm{CaM}$ was increased.

The emission spectra of DRE29 in the presence or absence of $\mathrm{Ca}^{2+} \mathrm{CaM}$ show two peaks corresponding to Trp32 and FITC (Figure 5.2b). The weak fluorescence of Trp32 is due to an efficient energy transfer to the FITC label; however, upon association with CaM an increase in fluorescence and blue shift from $351 \mathrm{~nm}$ to $329 \mathrm{~nm}$ is observed. The increase 
in FITC emission is expected based on the increase in quantum yield of Trp32. It is also possible that restructuring of the DRE29 peptide in the CaM:DRE29 complex increases the energy transfer efficiency. Overall, these results identify the amphipathic region in the Nterminus of DREAM spanning residues 29 to 44 as mediating the calcium dependent binding of CaM. It also shows that upon association with $\mathrm{Ca}^{2+} \mathrm{CaM}$ the solvent accessibility of Trp32 decreases.

\subsubsection{The N-terminus domain of DREAM does not block its hydrophobic cavity.}

The intrinsic tryptophan fluorescence of DREAM decreases upon calcium binding, a transition similar to that observed for $\operatorname{DREAM}(\Delta 65)$ (Carrion et al. 1999, Pham et al. 2015). Upon association of $\mathrm{Ca}^{2+} \mathrm{CaM}$ to DREAM a $5 \mathrm{~nm}$ blue shift from $337 \mathrm{~nm}$ to 332 $\mathrm{nm}$ is observed along with an increase in fluorescence intensity (Figure 5.2c). Similar to our peptide studies, CaM interacts with DREAM only in the presence of calcium (Kd = 3.4 $\pm 0.9 \mu \mathrm{M}$ ) with no detectable interaction in the absence of $\mathrm{Ca}^{2+}$ (Figure 5.2d), in good agreement with the previously reported value of $3 \mu \mathrm{M}$ (Ramachandran et al. 2012). The decreased affinity of CaM for the N-terminus of DREAM compared to the affinity of the isolated residues 29 to 44 may indicate that the $\mathrm{N}$-terminus is not completely solvent accessible. This could be easily explained by a model in which the N-terminus collapses on the DREAM core domain (residues 76 to 256) and reduces the affinity of CaM. Indeed, a similar folding was observed in the structure of calcium bound KChIP4 and $\mathrm{Mg}^{2+}$ bound caldendrin (Liang et al. 2009)'(Reddy et al. 2014). Therefore, to test whether the Nterminus of DREAM (residues 29-44) physically interacts with the core domain we conducted titrations of DRE29 with $\operatorname{DREAM}(\Delta 65)$. We observe an interaction between 
DRE29 and DREAM $(\Delta 65)$ with $\mathrm{Kd} \sim 30 \mu \mathrm{M}$; however, a similar affinity with DREAM was observed (Figure 5.4). In the presence of $100 \mathrm{mM} \mathrm{NaCl}$, the affinity of the DRE29 peptide with $\operatorname{DREAM}(\Delta 65)$ decreased to $\mathrm{K}_{\mathrm{d}} \sim 0.3 \mathrm{mM}$. These results indicate that the observed association between residues 29-44 and the core domain of DREAM are nonspecific and mediated by electrostatic interactions.

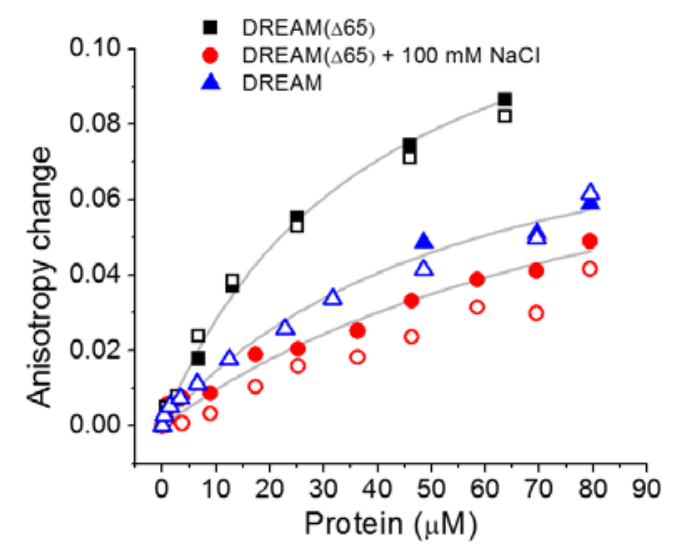

Figure 5.4. Anisotropy change upon addition of DREAM and DREAM $(\Delta 65)$ to $1 \mu \mathrm{M}$ DRE29 peptide in the presence of $\mathrm{CaCl}_{2}$ (solid symbols) or EDTA (open symbols). Line represent the best fit using Eqn. 1, with dissociation constant for DREAM of $57 \mu \mathrm{M}$ while $\operatorname{DREAM}(\Delta 65)$ resulted in a $\mathrm{K}_{\mathrm{d}} \sim 30 \mu \mathrm{M}$ in the absence of salt and $\mathrm{K}_{\mathrm{d}} \sim 0.3 \mathrm{mM}$ in the presence of $100 \mathrm{mM} \mathrm{NaCl}$.

$\operatorname{DREAM}(\Delta 65)$ protein has been shown to interact with small hydrophobic ligands such as arachidonic acid, the $\mathrm{K}^{+}$current activator NS5806 and the hydrophobic probes 1,8ANS and 2,6-ANS (Chapter 3). These ligands associate with a hydrophobic cavity at the C-terminus of DREAM whose accessibility increases upon $\mathrm{Ca}^{2+}$ association to EF-3 and EF-4. Association of the hydrophobic N-terminus of $\mathrm{K}_{\mathrm{V}} 4$ channels with DREAM and other KChIPs is also mediated by a hydrophobic groove which extends from C- to the Nterminus (Gonzalez, Pham \& Miksovska 2014, Pioletti et al. 2006, Li et al. 2014). Therefore, we investigated whether the presence of residues $1-65$ in DREAM can affect the binding of these hydrophobic ligands. Titrations of a peptide homologous to the 
hydrophobic N-terminus of Kv4.3 (residues 2-22) show that the affinity is not only independent of calcium but also identical to the previously reported value for binding to the $\mathrm{Ca}^{2+}$ bound DREAM $(\Delta 65)$ construct (Figure 5.5) (Gonzalez, Pham \& Miksovska 2014). Similarly, the hydrophobic probe 1,8-ANS and NS5806 compound showed interactions with DREAM which are similar to those observed for $\operatorname{DREAM}(\Delta 65)$ (Gonzalez, Miksovska 2014, Gonzalez, Pham \& Miksovska 2014) .
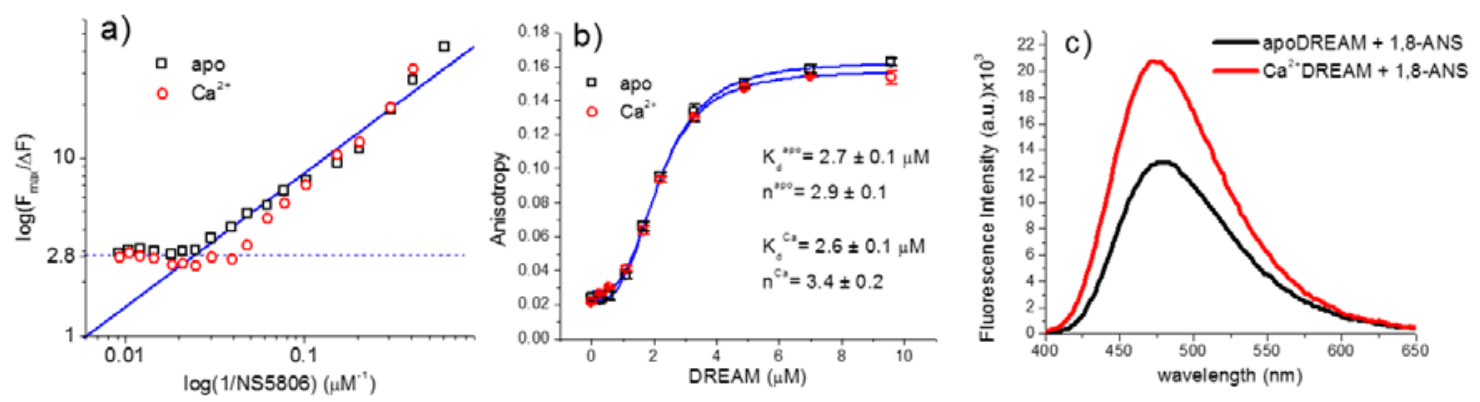

Figure 5.5. (a) Modified Stern-Volmer analysis of tryptophan fluorescence quenching upon binding of NS5806 to apo and $\mathrm{Ca}^{2+}$ DREAM, solid lines illustrate the expected traces for complete quenching and dashed lines show the intercept a $2.83 \pm 0.12$. (b) Association of Kv4.3(1-22)-FITC with apo and $\mathrm{Ca}^{2+}$ DREAM monitored using fluorescence anisotropy; solid lines represent the best fit to the data using a Hill binding model with the recovered parameters shown in the inset. (c) Fluorescence spectra of 1,8-ANS bound to apoDREAM with $\lambda_{\max }=478 \mathrm{~nm}$ and $\mathrm{Ca}^{2+}$ DREAM with $\lambda_{\max }=475 \mathrm{~nm}$. These results support the idea that the accessibility of the hydrophobic cavity at the C-terminus of DREAM is not compromised by the presence of the $\mathrm{N}$-terminus residues 1-65.

These results support the idea that the N-terminus of DREAM does not function as an autoinhibitory domain of the hydrophobic cavity as observed for the isoform 4 of KChIP4(Liang et al. 2009). Analysis of the NS5806 tryptophan quenching data using a modified Stern-Volmer plot shows that unlike $\operatorname{DREAM}(\Delta 65)$, where Trp169 is completely quenched (see Chapter 4), only one out of three tryptophans is quenched in DREAM (Figure 5.5a). This observation can easily be explained if the N-terminus of DREAM is not near the hydrophobic C-terminus where Kv4.3 N-terminus, 1,8-ANS or NS5806 bind or if the association of the hydrophobic ligand induces the displacement of the N-terminus. 
5.2.4 Calcium binding to DREAM induces a structural rearrangement at the N-terminus

The structure of the N-terminus of DREAM (residues 1-75) has not been resolved and based on NMR results, it has been proposed to form a random coil (Lusin et al. 2008). In order to better understand the mechanism of CaM binding, we set forth to identify the potential secondary structure of the N-terminal residues of DREAM using structure prediction tools and CD. The N-terminus of DREAM show the potential formation of two alpha helices between residues 4 and 11 and between residues 27 and 53, herein named $\alpha$ 1 and $\alpha_{0}$ (Fig 1). Two additional alpha helices comprising residues 76-85 and 88-102 were also identified, these helices correspond to $\alpha_{1}$ and $\alpha_{2}$ previously identified by NMR (Lusin et al. 2008). Further analysis of the N-terminus of DREAM using protein disorder prediction tools also shows residues surrounding $\alpha 0$ as having high propensity to form disordered regions (Xue et al. 2010, Obradovic et al. 2003). Molecular dynamics simulations support that both $\mathrm{N}$-terminus helices retain their secondary structure and are flexible relative to each other (Figure 5.6), with $\alpha_{0}$ showing an expected bend at Pro36. Overall, these bioinformatics analyses and simulations of the N-terminus domain of DREAM indicate that this domain forms a disordered region with a small helical structure connected to the core of DREAM by a 21 residues long disordered region rich in small hydrophilic residues (residues 54-75). 


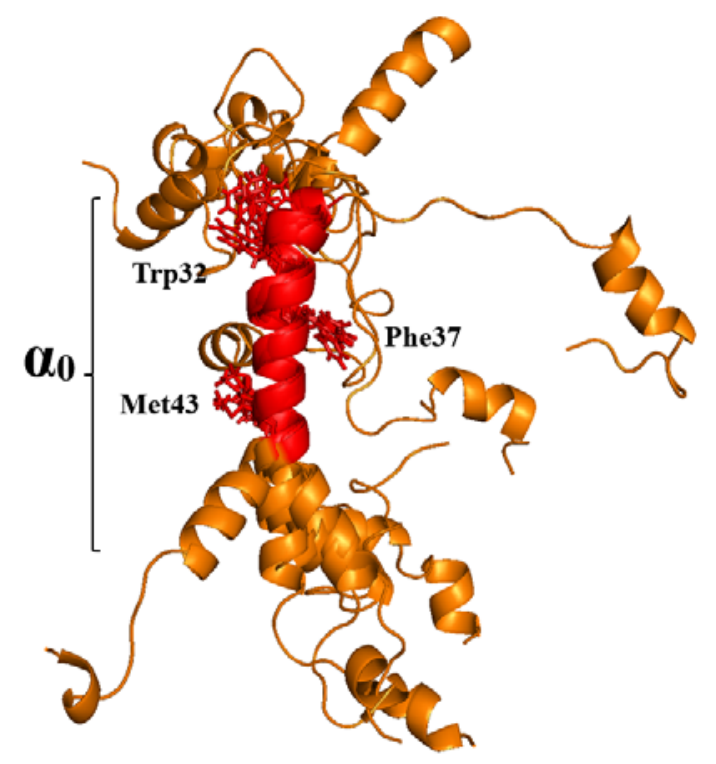

Figure 5.6. Eight $10 \mathrm{~ns}$ MD simulation at $313 \mathrm{~K}$ of DREAM N-terminus (residues 1-66) showing the overall stability of the predicted $\alpha_{0}$ helix. Bulky hydrophobic residues which form part of the CaM binding region (shown in red) are drawn as licorice model.

To gain insight into the secondary structure of DREAM and its N-terminus, we also performed near and far UV circular dichroism measurements on DREAM and $\operatorname{DREAM}(\Delta 65)$. The far-UV CD signal for both constructs show the characteristic spectra of alpha-helical proteins, with negative peaks at $220 \mathrm{~nm}$ and $207 \mathrm{~nm}$ and a larger positive peak near $190 \mathrm{~nm}$ (Figure 5.7a). The magnitude of the CD signal was dependent on $\mathrm{Ca}^{2+}$ for both constructs with an increase in $190 \mathrm{~nm}$ and decrease in 220/207 nm peaks upon calcium binding (data not shown). However, the magnitude of the positive and negative peaks of DREAM in the absence or presence of calcium was smaller than that of $\operatorname{DREAM}(\Delta 65)$, indicative of less alpha-helical content in the full-length construct. Analysis of the far-UV CD spectra with the K2D secondary structure prediction tools shows $68 \%$ and $50 \%$ alpha-helical content for calcium bound DREAM $(\Delta 65)$ and DREAM, respectively. A fully unfolded N-terminus domain in DREAM would result in a 47\% alpha- 
helical content, which is slightly lower than the value determined here. Therefore, the Nterminus domain of DREAM likely forms a disordered domain with a small region of alpha-helical structure near the CaM binding sequence.
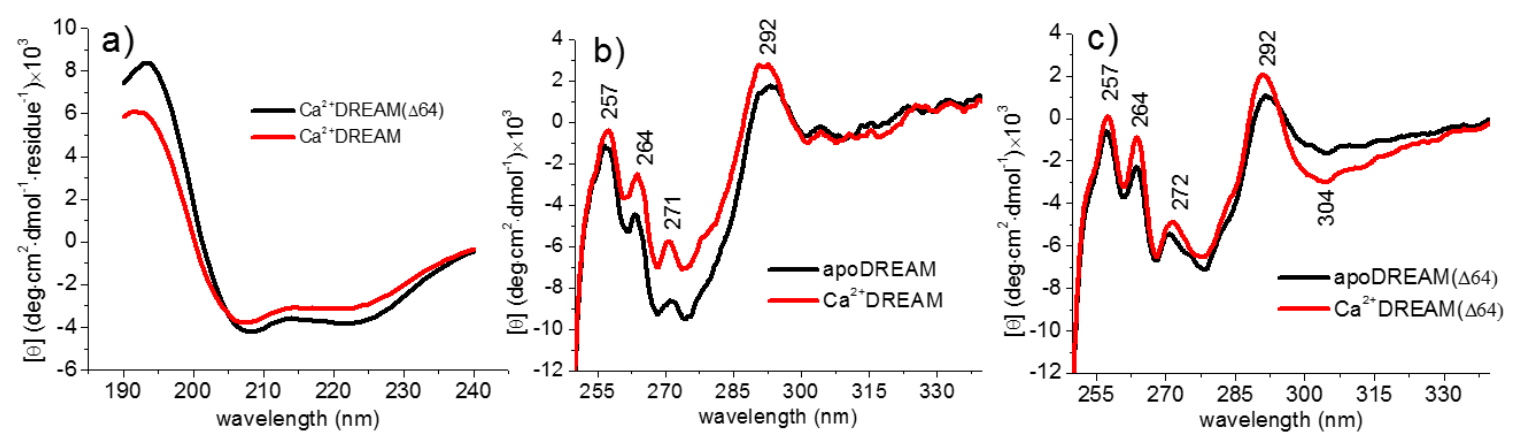

Figure 5.7. (a) Far-UV CD spectra of DREAM $(\Delta 65)$ and DREAM in the presence of 10 $0 \mu \mathrm{M}$ calcium in $5 \mathrm{mM}$ phosphate buffer $\mathrm{pH}$ 7.4. (b) Near-UV CD spectra of DREAM $(\Delta 6$ 5) and (c) DREAM in the absence and presence of calcium.

On the other hand, the near-UV CD spectra for both DREAM constructs showed complex and distinct positive and negative peaks. In the absence of calcium, DREAM showed a broad negative band with fine structured positive and negative peaks. The peaks between $256 \mathrm{~nm}$ and $272 \mathrm{~nm}$ are likely due to phenylalanine residues whereas the peak between $275 \mathrm{~nm}$ and $305 \mathrm{~nm}$ are associated with tyrosine and tryptophan residues (Strickland 1974, Woody 2012). Calcium binding resulted in an increase in ellipticity signal between $256 \mathrm{~nm}$ and $295 \mathrm{~nm}$ (Figure 5.7b), without a significant shift of the observed vibronic structures. The near UV CD spectra of DREAM $(\Delta 65)$ in the absence of calcium showed many similarities in the phenylalanine and tyrosine peaks to DREAM, whereas a larger peak at $290 \mathrm{~nm}$ was observed in the tryptophan region. Binding of calcium to $\operatorname{DREAM}(\Delta 65)$ did not induce major changes in the spectra, with the exception of a decrease in ellipticity near $305 \mathrm{~nm}$ (Figure 5.7c). The presence of only three additional aromatic residues in DREAM compared to the construct lacking the first 65 residues 
indicates that the increase in the ellipticity between $270 \mathrm{~nm}$ and $290 \mathrm{~nm}$ likely arises from Phe37, Trp32 and/or Trp50. Additionally, the broad changes in ellipticity upon binding of calcium in DREAM but not in $\operatorname{DREAM}(\Delta 65)$ indicates that the N-terminus residues undergo a structural rearrangement which restricts solvent accessibility of Trp32 and/or Trp50 (Nagai et al. 2012). These results support the idea that the conformation of the Nterminal residues of DREAM may be affected by calcium binding.

\subsubsection{The core domain of DREAM facilitates dissociation of the CaM:DREAM complex}

To better understand the mechanism of CaM:DREAM interaction, we determined the kinetics of CaM:DREAM complex formation by monitoring the fluorescence change of CaM and CaMRR2 upon dissociation from DRE29 and DREAM. Initial attempts to resolve the association rate by mixing $1: 1$ volumetric ratio of $10 \mu \mathrm{M} \mathrm{Ca}{ }^{2+} \mathrm{CaM}$ and $0.5 \mu \mathrm{M}$ DRE29 or $10 \mu \mathrm{M}$ apoDREAM and $0.5 \mu \mathrm{M}$ CaMRR2 with $1 \mathrm{mM} \mathrm{CaCl}_{2}$ showed that the protein complex was formed within the dead time of our stopped-flow instrument $(\sim 20$ ms), indicating that similarly to other CaM binding peptides and proteins (Brown, Martin \& Bayley 1997, Kasturi, Vasulka \& Johnson 1993), the bimolecular association rate constant is near the diffusion limit, with $\mathrm{k}_{\text {on }}$ in excess of $10^{8} \mathrm{M}^{-1} \mathrm{~s}^{-1}$. Dissociation of $\mathrm{Ca}^{2+}$ from $2.5 \mu \mathrm{M}$ CaM by $1 \mathrm{mM}$ EDTA monitored using tyrosine fluorescence showed a monophasic decay with $\mathrm{k}_{\text {off }}=7.9 \pm 0.6 \mathrm{~s}^{-1}$ at $25^{\circ} \mathrm{C}$, as previously reported (Brown, Martin \& Bayley 1997). The calcium dissociation from $0.5 \mu \mathrm{M}$ CaMRR2 determined by monitoring the anisotropy change of the rhodamine label at Cys148 was four times slower than that observed for CaM, with $\mathrm{k}_{\text {off }}=1.9 \pm 0.1 \mathrm{~s}^{-1}$ at $25^{\circ} \mathrm{C}$. The slow $\mathrm{Ca}^{2+}$ dissociation from CaMRR2 could be likely due to stabilization of the calcium exposed hydrophobic 
cavity at the C-terminus by the rhodamine label or due to measurement of a structural change sequential to calcium dissociation.
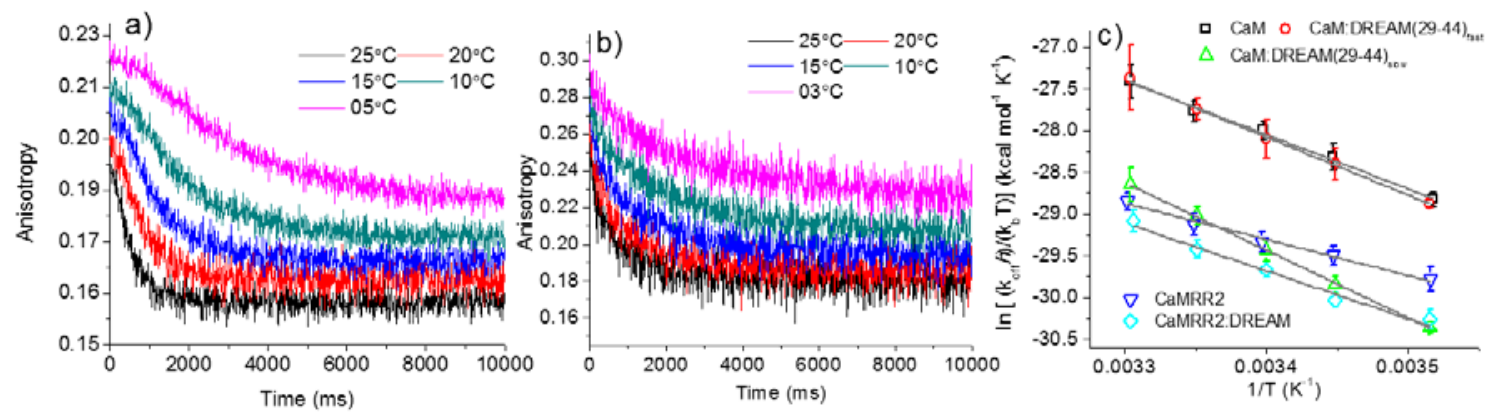

Figure 5.8. Dissociation kinetics of (a) $10 \mu \mathrm{M}$ CaM and $1 \mu \mathrm{M}$ DRE29 and (b) $1 \mu \mathrm{M}$ CaMRR2 and $10 \mu \mathrm{M}$ DREAM upon addition of $1 \mathrm{mM}$ EDTA monitored using T-format fluorescence anisotropy as a function of temperature. (c) Eyring plot analysis of the dissociation constant as a function of temperature, solid lines represent best linear fit to the data.

On the other hand, mixing of $\mathrm{Ca}^{2+} \mathrm{CaM}$ :DRE29 and $\mathrm{Ca}^{2+} \mathrm{CaMRR} 2$ :DREAM with 2 mM EDTA resulted in a well-resolved decrease in fluorescence anisotropy indicative of complex dissociation upon $\mathrm{Ca}^{2+}$ removal (Figure 5.8a-b). The dissociation of CaM:DRE29 and CaMRR2:DREAM were best fitted by a double and single exponential decay functions, respectively, Table 5.1. The dissociation rate of CaM with the DRE29 peptide and DREAM protein were koff $=2.3 \pm 0.2 \mathrm{~s}^{-1}$ and $\mathrm{koff}=1.5 \pm 0.1 \mathrm{~s}^{-1}$, respectively. The amplitude of the lag phase observed in the CaM:DRE29 accounted for about $22 \%$ of the signal change with koff $=8.3 \pm 3.5 \mathrm{~s}^{-1}$. The dissociation of $\mathrm{Ca}^{2+}$ from $\mathrm{CaM}$ and CaMRR2 as well as CaM:DRE29 and CaMRR2:DREAM complexes was measured in the temperature range of $6.5^{\circ} \mathrm{C}$ to $24.7^{\circ} \mathrm{C}$ and the decay times are listed in Table 5.2. Analysis of the dissociation rates as a function of temperature using Eyring plots(Eyring 1935) shows a larger contribution of the enthalpic component to the activation parameters of $\mathrm{Ca}^{2+}$ dissociation from CaM and CaM:DRE29, Table 5.1. 
Table 5.1. Activation parameters for CaM:DRE29 and CaMRR2:DREAM dissociation

\begin{tabular}{|c|c|c|c|}
\hline & $\begin{array}{l}k_{\text {off }} \\
\left(\mathrm{s}^{-1}\right)^{a}\end{array}$ & $\begin{array}{c}\Delta \mathbf{H}^{\ddagger} \\
(\mathrm{kccl} / \mathrm{mol})\end{array}$ & $\begin{array}{c}\mathrm{T} \Delta \mathrm{S}^{\ddagger} \\
\text { (kcal/mol)* }\end{array}$ \\
\hline $\mathrm{CaM}$ & $7.9 \pm 0.6$ & $12.9 \pm 0.5$ & $-3.5 \pm 0.5$ \\
\hline CaM-DRE29 $9_{\text {fast }}$ & $8.3 \pm 3.5$ & $13.8 \pm 0.4$ & $-2.7 \pm 0.2$ \\
\hline CaM-DRE29 ${ }_{\text {slow }}$ & $2.3 \pm 0.16$ & $16.1 \pm 0.3$ & $-1.1 \pm 0.2$ \\
\hline CaMRR2 & $1.9 \pm 0.1$ & $8.6 \pm 0.6$ & $-8.6 \pm 0.6$ \\
\hline CaMRR2:DREAM $^{b}$ & $1.5 \pm 0.1$ & $13.5 \pm 1.2$ & $-4.0 \pm 0.7$ \\
\hline \multicolumn{4}{|c|}{$\begin{array}{l}{ }^{(a)} \text { At } 298 \mathrm{~K}^{(b)} \text { Activation enthalpy and entropy corrected for } \\
\text { the fraction of DREAM bound, } f_{\mathrm{b}}=0.57 \text { based on the } \\
\text { concentration of DREAM of } 5 \mu \mathrm{M} \text { and a } \mathrm{K}_{\mathrm{d}}=3.3 \mu \mathrm{M} \text {. }\end{array}$} \\
\hline
\end{tabular}

Table 5.2 Dissociation kinetics of calcium bound CaM:DRE29 and CaMRR2:DREAM complexes upon addition of EDTA as a function of temperature

\begin{tabular}{cccccc}
\hline & CaM & $\begin{array}{c}\text { CaM:DRE2 } \\
9\end{array}$ & $\begin{array}{c}\text { CaM:DRE2 } \\
9\end{array}$ & CaMRR2 & $\begin{array}{c}\text { CaMRR2 } \\
\text { DREAM }\end{array}$ \\
$\begin{array}{c}\text { Temp. } \\
\left({ }^{\mathbf{C}} \mathbf{)}\right.\end{array}$ & $\begin{array}{c}\boldsymbol{\tau}_{\text {off }} \\
(\mathbf{m s})\end{array}$ & $\begin{array}{c}\boldsymbol{\tau}_{\text {off }} \\
\mathbf{( m s}^{\boldsymbol{a}}\end{array}$ & $\begin{array}{c}\boldsymbol{\tau}_{\text {off }} \\
\mathbf{( m s}^{\boldsymbol{a}}\end{array}$ & $\begin{array}{c}\boldsymbol{\tau}_{\text {off }} \\
(\mathbf{m s})\end{array}$ & $\begin{array}{c}\boldsymbol{\tau}_{\text {off }} \\
(\mathbf{m s})\end{array}$ \\
\hline 24.7 & $127 \pm 9$ & $121 \pm 51$ & $438 \pm 30$ & $532 \pm 19$ & $675 \pm 31$ \\
20.4 & $184 \pm 8$ & $179 \pm 26$ & $645 \pm 25$ & $713 \pm 32$ & $960 \pm 36$ \\
16.1 & $235 \pm 9$ & $260 \pm 64$ & $992 \pm 43$ & $898 \pm 38$ & $1247 \pm 41$ \\
12.0 & $328 \pm 18$ & $355 \pm 71$ & $1514 \pm 53$ & $1066 \pm 44$ & $1823 \pm 55$ \\
6.5 & $555 \pm 16$ & $586 \pm 26$ & $2590 \pm 59$ & $1442 \pm 74$ & $2336 \pm 92$ \\
\hline
\end{tabular}

${ }^{a}$ Decay parameters recovered by using a double exponential decay function, where the fast and slow decay lifetimes correspond to $\mathrm{Ca}^{2+}$ and peptide dissociation, respectively. 
The activation parameters for $\mathrm{Ca}^{2+} \mathrm{CaM}\left(\Delta \mathrm{H}^{\ddagger}=12.9 \pm 0.5 \mathrm{kcal} / \mathrm{mol}\right.$ and $\mathrm{T} \Delta \mathrm{S}^{\ddagger}=-3.5 \pm 0.5$ $\mathrm{kcal} / \mathrm{mol})$ and the fast phase of $\mathrm{Ca}^{2+} \mathrm{CaM}: \operatorname{DRE} 29\left(\Delta \mathrm{H}^{\ddagger}=13.8 \pm 0.4 \mathrm{kcal} / \mathrm{mol}\right.$ and $\mathrm{T} \Delta \mathrm{S}^{\ddagger}=$ $-2.7 \pm 0.2 \mathrm{kcal} / \mathrm{mol}$ ) are identical, and likely correspond to the same process. On the other hand, $\mathrm{Ca}^{2+}$ dissociation activation parameters for CaMRR2 show enthalpy and entropy contributions of $\Delta \mathrm{H}^{\ddagger}=8.6 \pm 0.6 \mathrm{kcal} / \mathrm{mol}$ and $\mathrm{T} \Delta \mathrm{S}^{\ddagger}=-8.6 \pm 0.6 \mathrm{kcal} / \mathrm{mol}$. The slow phase of $\mathrm{Ca}^{2+}$ dissociation observed in the presence of the DRE29 peptide also shows a large enthalpy barrier with $\Delta \mathrm{H}^{\ddagger}=16.1 \pm 0.3 \mathrm{kcal} / \mathrm{mol}$ and $\mathrm{T} \Delta \mathrm{S}^{\ddagger}=-1.1 \pm 0.2 \mathrm{kcal} / \mathrm{mol}$. In the presence of the full-length DREAM protein, the dissociation kinetics from CaMRR2 were not significantly different (Table 5.1) than those in the absence of DREAM.
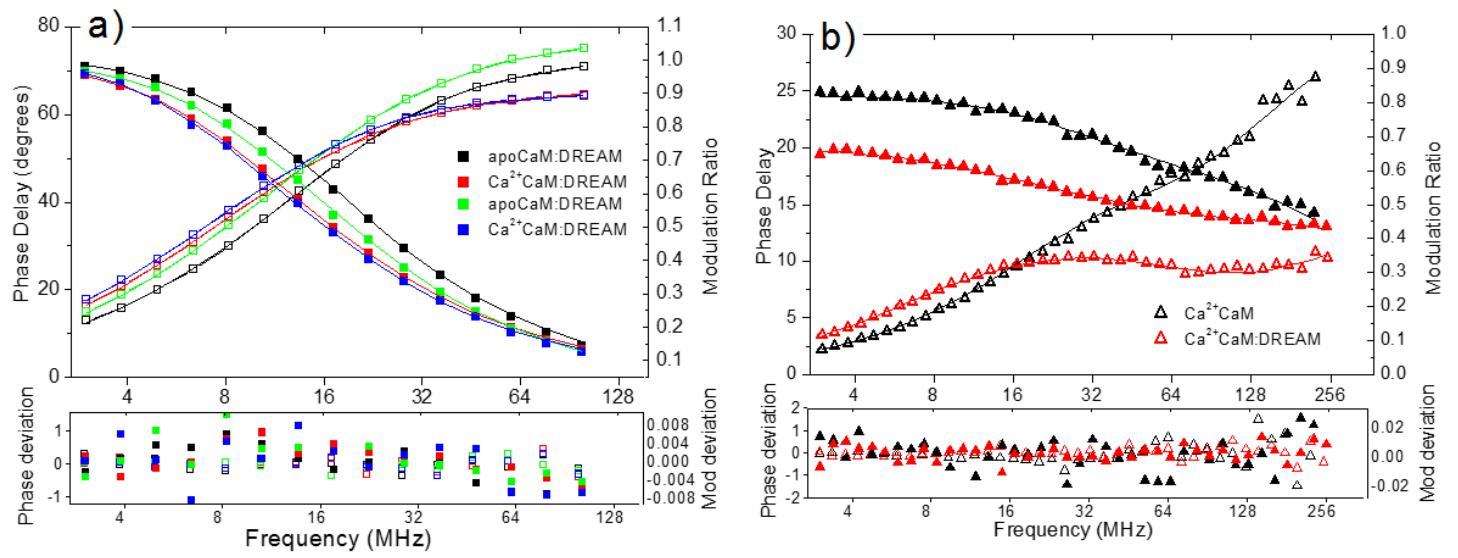

Figure 5.9. Frequency-domain fluorescence decay of apo and $\mathrm{Ca}^{2+} \mathrm{CaM}-\mathrm{IAEDANS}$ (b) $\mathrm{Fr}$ equency-domain anisotropy decay of $\mathrm{Ca}^{2+}$ bound CaM-IAEDANS in the absence and pre sence of DREAM. Phase delay and modulation ratio are shown as open and solid symbols , respectively. Solid lines represent the best fit using multi-exponential decay models, rec overed parameters are listed in Table 5.3 and 5.4.

Therefore, the recovered CaMRR2 activation parameters likely arise from a combination of $\mathrm{Ca}^{2+}$ and protein dissociation activation barriers. The protein dissociation activation parameters can be approximated by subtracting the activation barrier for CaMRR2 from those of CaMRR2:DREAM, which result in $\Delta \mathrm{H}^{\ddagger}=4.9 \pm 0.5 \mathrm{kcal} / \mathrm{mol}$ and $\mathrm{T} \Delta \mathrm{S}^{\ddagger}=4.6 \pm$ 
$0.8 \mathrm{kcal} / \mathrm{mol}$. These results show that the core domain of DREAM facilitates the dissociation of residues 29-44 from CaMRR2 by lowering the enthalpic dissociation activation barrier.

Table 5.3. Photophysical properties of DRE29 and CaM-IAEDANS upon complex formation

\begin{tabular}{|c|c|c|c|c|c|c|c|}
\hline & $\mathbf{r}_{\mathrm{ss}}$ & $\begin{array}{l}\lambda_{\max } \\
(\mathbf{n m})\end{array}$ & $\begin{array}{c}\tau_{1} \\
\text { (ns) }\end{array}$ & $\begin{array}{c}\alpha_{1} \\
(\%)\end{array}$ & $\begin{array}{c}\tau_{2} \\
(\mathrm{~ns})\end{array}$ & $\begin{array}{c}\alpha_{2} \\
(\%)\end{array}$ & $\chi^{2}$ \\
\hline $\operatorname{DRE29}^{a}$ & 0.010 & 517 & 3.6 & & & & 1.0 \\
\hline DRE29:CaM ${ }^{a}$ & 0.090 & 516 & 3.9 & & & & 0.7 \\
\hline IAEDANS & 0.006 & 505 & 4.1 & 14 & 10.5 & 86 & 1.7 \\
\hline apoCaM & 0.049 & 495 & 10.8 & 80 & 16.9 & 20 & 1.1 \\
\hline $\mathrm{Ca}^{2+} \mathrm{CaM}$ & 0.081 & 484 & 10.6 & 55 & 18.9 & 45 & 1.3 \\
\hline apoCaM:DREAM & 0.113 & 486 & 10.6 & 89 & 21.1 & 11 & 0.9 \\
\hline $\mathrm{Ca}^{2+} \mathrm{CaM}: D R E A M$ & 0.132 & 475 & 11.3 & 51 & 21.9 & 49 & 0.9 \\
\hline \multicolumn{8}{|c|}{$\begin{array}{l}\text { Recovered parameters from global analysis of at least three independent } \\
\text { measurements of the phase delay and modulation ratio using a Gaussian or } \\
\text { double exponential decay model. The lifetime parameters ( } \boldsymbol{\tau}_{1} \text { and } \tau_{2} \text { ) were set as linked } \\
\left.\text { variables, and the pre-exponential parameters ( } \boldsymbol{\alpha}_{1} \text { and } \boldsymbol{\alpha}_{2}\right) \text { as free variables; } \boldsymbol{\chi}^{2} \text { represents the goodness of the } \\
\text { fit. (a) A Gaussian lifetime distribution was used, with recovered width of } \mathbf{0 . 5} \text { ns for DRE29 and } \mathbf{1 . 0} \text { ns for } \\
\text { DRE29:CaM complex. Sample concentrations were: } 0.5 \mu \mathrm{M} \text { DRE29 and } 10 \mu \mathrm{M} \text { CaM } \\
\text { or } 10 \mu \mathrm{M} \text { CaM-IAEDANS and } 20 \mu \mathrm{M} \text { DREAM. }\end{array}$} \\
\hline
\end{tabular}

5.2.6 The DREAM-CaM protein complex forms a near spherical heterotetramer.

We employed time-resolved fluorescence anisotropy decay to recover the rotational correlation time of calmodulin and DRE29 as well as the CaM:DREAM complex. Fluorescence emission spectra and fluorescence lifetime decay of FITC labeled DREAM(29-44) show no changes upon binding of calmodulin. Analysis of the frequency domain fluorescence decay using a Gaussian model yields satisfactory fits for the DRE29 peptide and CaM:DRE29 complex, frequency domain data and decay parameters shown in Figure 5.9 and Table 5.3, respectively. A bimodal model was necessary to accurately fit 
the anisotropy decay data and the recovered rotational correlation times for the CaM:DRE29 complex are listed in Table 5.4. The fast rotational correlation time of 0.57

ns for DRE29 accounts for $57 \%$ of the depolarization and can be attributed to a local motion of the fluorophore. The second rotational correlation time of 2.0 ns for the DRE29 peptide is about twofold larger than that expected for a spherical peptide of $1.0 \mathrm{~ns}$, calculated using the Einstein-Stokes equation (2.6 kDa and $0.3 \mathrm{~mL} / \mathrm{g}$ hydration) (Einstein 1905).

Table 5.4. Anisotropy decay parameters of DRE29 and CaM-

IAEDANS upon complex formation

\begin{tabular}{lccccccc}
\hline & $\begin{array}{c}\boldsymbol{\theta}_{1} \\
(\mathbf{n s})\end{array}$ & $\boldsymbol{f}_{\mathbf{1}}$ & $\begin{array}{c}\boldsymbol{\theta}_{2} \\
(\mathbf{n s})\end{array}$ & $\boldsymbol{f}_{\mathbf{2}}$ & $\begin{array}{c}\boldsymbol{\theta}_{3} \\
(\mathbf{n s})\end{array}$ & $\boldsymbol{f}_{3}$ & $\chi^{2}$ \\
\hline $\mathrm{DRE} 29$ & 0.57 & 0.72 & 2.0 & 0.28 & & & \\
\hline $\mathrm{Ca}^{2+} \mathrm{CaM}$ : DRE29 & 0.61 & 0.42 & 10.8 & 0.58 & & & 0.6 \\
\hline $\mathrm{apoCaM}$ & 0.51 & 0.80 & 6.7 & 0.20 & & & 1.0 \\
\hline $\mathrm{Ca}^{2+} \mathrm{CaM}$ & 0.64 & 0.52 & 8.1 & 0.48 & & & 0.7 \\
\hline apoCaM:DREAM & 0.59 & 0.79 & 8.1 & 0.20 & 56 & 0.01 & 0.7 \\
\hline $\mathrm{Ca}^{2+} \mathrm{CaM}: D R E A M$ & 0.62 & 0.31 & 8.9 & 0.31 & 47 & 0.38 & 0.8 \\
\hline
\end{tabular}

Recovered from global analysis of at least three independent measurements of the phase and modulation data using a double exponential decay model. Decay parameters $\left(\boldsymbol{\theta}_{1}, \boldsymbol{\theta}_{2}\right.$ and $\left.\boldsymbol{\theta}_{3}\right)$ were set as linked variables, and the fractional depolarization $\left(f_{1}, f_{2}\right.$ and $\left.f_{3}\right)$ were set as free variables, $\chi^{2}$ represents the goodness of the fit. Sample conditions identical to those on Table 2.

The CaM:DRE29 complex shows a similar local rotational time of 0.61 ns while the global motion increased to 10.8 ns. The expected rotational time of a sphere with molecular weight similar to that of the CaM:DRE29 complex $(19 \mathrm{kDa}$ and $0.30 \mathrm{ml} / \mathrm{g}$ hydration) yields a rotational diffusion time of $8.0 \mathrm{~ns}$. The discrepancies observed could be due to deviations from a spherical conformation and/or a higher protein hydration. These results show that CaM binds to DRE29 as a monomer to form a complex with nearly spherical shape, likely similar to the often observed wrap around conformation of CaM:peptide complexes (Yamniuk, Vogel 2004). 
To investigate the oligomerization state of calmodulin bound to full-length DREAM we employed a fluorescently labeled CaM-IAEDANS construct. The long fluorescence lifetime decay and solvent sensitivity of IAEDANS allow us to resolve slower correlation times as well as changes in the probe environment(Hudson, Weber 1973b). The fluorescence emission spectra of CaM-IAEDANS upon calcium binding shows an increase in fluorescence and a blue shift from $495 \mathrm{~nm}$ to $484 \mathrm{~nm}$ (Figure 5.9a). A modest increase in the steady-state anisotropy can be observed upon $\mathrm{Ca}^{2+}$ binding to CaM-IAEDANS, from $r_{s s}=0.049$ to $r_{s s}=0.081$. Additional information is obtained from lifetime measurements,

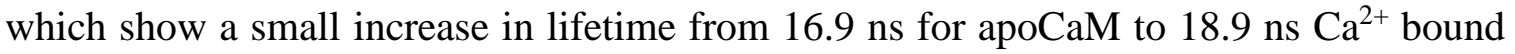
CaM and a change in pre-exponential factor $\alpha_{2}$ of $25 \%$, likely due to a decrease in solvent accessibility of the probe, Table 1 . Analysis of $\chi^{2}$ surface of the phase/modulation fits also reveal that in the absence of $\mathrm{Ca}^{2+}$, the $\mathrm{C}$-terminus domain of $\mathrm{CaM}$ is more heterogeneous and solvent exposed based on the smaller $\tau_{2}$, Figure 5.9b. The rotational correlation times recovered from the fit of the anisotropy decay yield values of $0.51 \mathrm{~ns}$ and $0.64 \mathrm{~ns}$ for the local motion of IAEDANS attached to apo and calcium bound CaM, respectively, and 6.7 ns and $8.1 \mathrm{~ns}$ for the global motion of the apo and calcium bound CaM, respectively. The increase in $\theta_{1}$ is significant (Figure 5.9c) and correlates with a decrease in local flexibility of the Cys148 region. On the other hand, increase in $\theta_{2}$ upon $\mathrm{Ca}^{2+}$ binding is likely due to a more rigid and elongated structure of $\mathrm{Ca}^{2+} \mathrm{CaM}$ in solution. Nonetheless, the rotational correlation times are in good agreement with the expected rotational time for CaMIAEDANS of $7.1 \mathrm{~ns}$ (17.2 kDa and $0.3 \mathrm{~mL} / \mathrm{g}$ hydration). Overall, these results indicate that association of calcium induces the region near Cys148 to become less flexible and solvent 
restricted, which is in good agreement with the rearrangement of CaM structure upon $\mathrm{Ca}^{2+}$ binding (Chattopadhyaya et al. 1992, Kuboniwa et al. 1995).
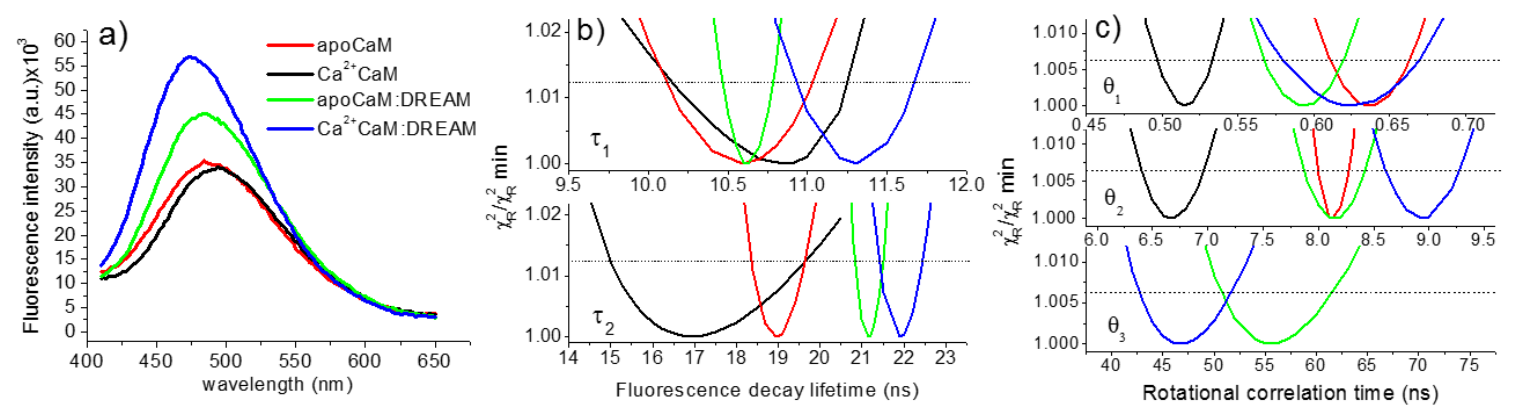

Figure 5.10. (a) Fluorescence emission spectra transition of $5 \mu \mathrm{M}$ CaM-IAEDANS upon association with $20 \mu \mathrm{M}$ DREAM. (b) Global fluorescence decay lifetime $\chi^{2}$ surface for th e two component exponential decay of CaM-IAEDANS in the presence (red) or absence ( black) of $\mathrm{Ca}^{2+}$ and $\mathrm{Ca}^{2+} \mathrm{CaM}-\mathrm{IAEDANS}$ :DREAM (blue) and $\mathrm{Ca}^{2+}$ free complex (green). $\mathrm{R}$ otational correlation time $\chi^{2}$ surface from the global analysis of the anisotropy decay data using a two component exponential decay for CaM-IAEDANS and triple exponential dec ay model for the CaM-IAEDANS:DREAM complex, color scheme as in panel b.

We also investigated the effects of CaM-IAEDANS association with DREAM and found that the emission of $\mathrm{Ca}^{2+}$ bound CaM-IAEDANS increases two-fold upon addition of DREAM with a maximum at $475 \mathrm{~nm}$ (Figure 5.10a). A small increase in intensity and blue shift to $486 \mathrm{~nm}$ was also observed for apoCaM-IAEDANS in the presence of DREAM. The fluorescence decay lifetime of $\mathrm{Ca}^{2+} \mathrm{CaM}-\mathrm{IAEDANS}$ was affected by association with DREAM, showing an increase in the long fluorescence decay lifetime from $\tau_{2}=18.9$ ns to $\tau 2=21.9$ ns. A larger increase in $\tau_{2}$ was observed for $\mathrm{Ca}^{2+}$ free CaM-IAEDANS upon addition of DREAM (from 16.9 ns to $21.1 \mathrm{~ns}$ ); however, the amplitude of this decay was about four-fold lower than that of the $\mathrm{Ca}^{2+}$ CaM-IAEDANS:DREAM complex. Further analysis of the fluorescence of CaM-IAEDANS using anisotropy decay shows that in addition to the local and global rotation of apo and $\mathrm{Ca}^{2+} \mathrm{CaM}-I A E D A N S$, a third rotation with correlation time of 56 ns and 47 ns is present (Figure 5.10c and Table 5.4). The 
rotational correlation time for the $\mathrm{Ca}^{2+} \mathrm{CaM}$-IAEDANS:DREAM complex is similar to that expected for a spherical CaM:DREAM heterotetramer (calc. $40 \mathrm{~ns}$ at $0.3 \mathrm{ml} / \mathrm{g}$ hydration). The larger rotation for the apoCaM-IAEDANS:DREAM complex could be due to the presence of a mixture of oligomers. Analysis of the $\chi^{2}$ surface reveals that the rotation of the CaM-IAEDANS:DREAM complex is heterogeneous, ranging from 43 ns to 52 ns and $51 \mathrm{~ns}$ to $61 \mathrm{~ns}$ in the presence and absence of $\mathrm{Ca}^{2+}$, respectively (Figure 5.10c). Such a heterogeneity likely arises from an extended and flexible conformation of the protein complex. These results indicate that not only calcium bound CaM can interact with DREAM but also the apo form, likely with a significantly lower affinity. The solvent accessibility of IAEDANS decreases upon protein complex formation, suggesting that Cys148 forms part of the protein interface. It also supports a model where a $\mathrm{Ca}^{2+}$ DREAM dimer associates with two $\mathrm{Ca}^{2+} \mathrm{CaM}$ to form a near spherical heterotetramer.

\subsubsection{Molecular dynamics simulations support a model in which the N-terminus of} DREAM interacts with an electrostatic patch on the surface of DREAM.

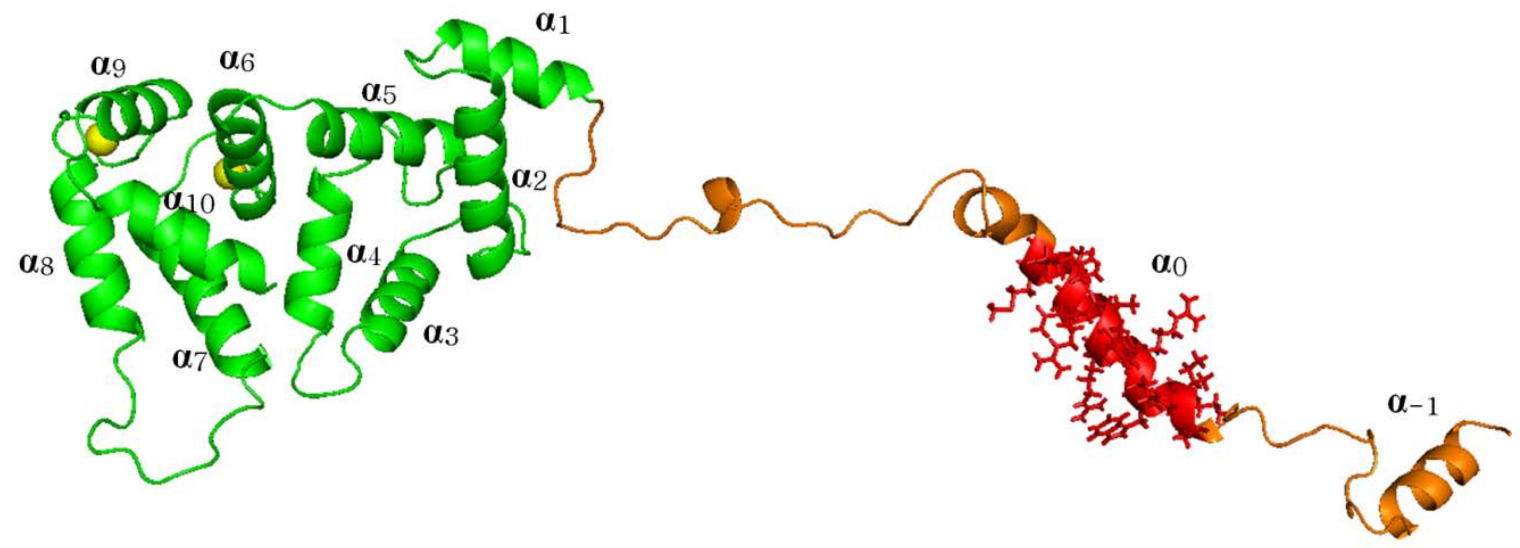

Figure 5.11. Initial structure of DREAM built using the protocol described in the Materia 1 and Methods section, where the structure derived from the published NMR is shown in $\mathrm{g}$ reen and the predicted structure of residues 1 to 75 is shown in orange with the CaM bindi ng site highlighted in red licorice model. Calcium atoms are shown as yellow spheres. 
To improve our understanding of the interaction between the $\mathrm{N}$-terminus and the core domain of DREAM, we conducted multiple molecular dynamics simulations of using three different strategies starting with the initial structure shown in Figure 5.11. Our initial approach to model the N-terminus of DREAM as an extended domain with partial alphahelical structure followed by 80 ns cMD simulation resulted in structures which are shown on Fig. 5.12a.
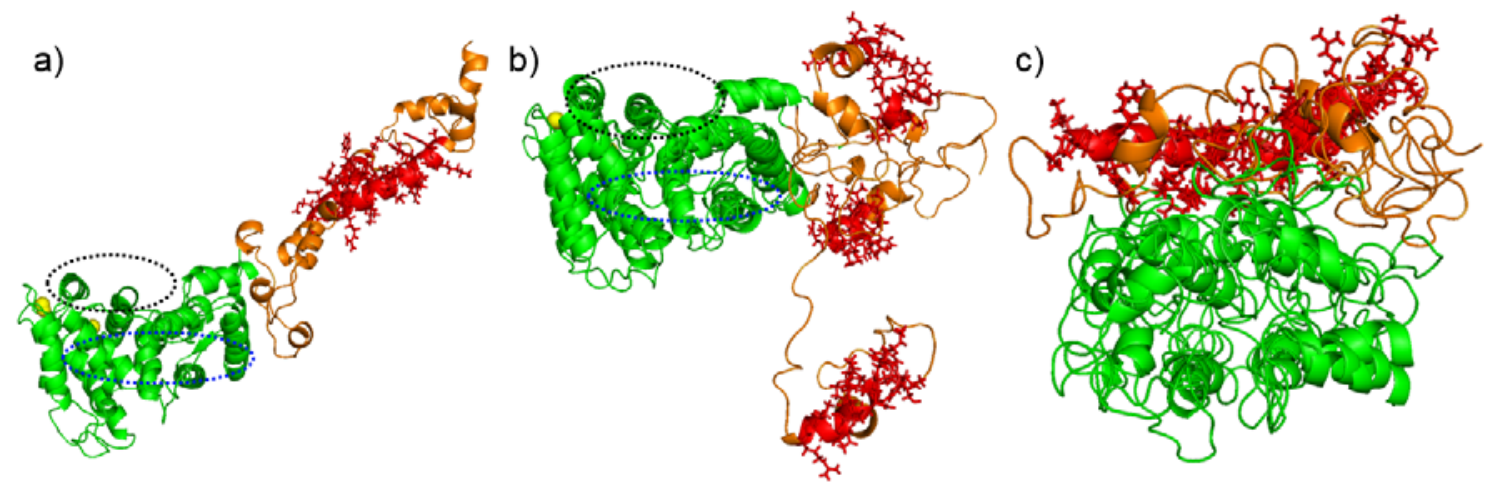

Figure 5.12. Molecular dynamics simulation of DREAM using an initial model construct ed by combining a peptide model for residues 1 to 76 with the published NMR structure 0 f DREAM (residues 76 to 256) shown in green. The alpha-helical section of the N-termin us domain was predicted using secondary prediction tools and shown in orange, the CaM binding region corresponding to residues 29-44 is shown in red licorice. All structures are aligned using residues 76 to 256; calcium is shown as yellow spheres. (a) Two final struct ures of DREAM after 80 ns classical MD simulations. (b) Three final structures of DREA $\mathrm{M}$ after 30 ns accelerated MD simulations. (c) Three independent structures of DREAM a fter 1000ns of coarse grained molecular dynamics simulation in the absence of $\mathrm{Ca}^{2+}$, show ing a collapsed $\mathrm{N}$-terminus. The hydrophobic surface known to mediate ligand binding on DREAM is highlighted by a blue ellipse, the electronegative surface identified on Figure 5.1 is highlighted in black.

We observed that during the simulation most of the alpha helices are retained and there is a partial contraction of the structure. The extended conformation of the $\mathrm{N}$-terminus likely arises from insufficient simulation time. To overcome the computational cost of longer cMD simulations, we performed three additional aMD simulations where an additional energy was applied to the dihedral angles. This type of simulation results in 
larger conformational space sampling, and as can be seen in Figure 5.12b the N-terminus of DREAM adopts a more unfolded and collapsed structure. Nonetheless, the CaM binding region is still significantly accessible to solution. Finally, we modeled DREAM using a residue based coarse grained approach, which significantly decreases computational costs and increases sampling time. In Figure 5.12c, we show three representative all-atom structures obtained from coarse grained simulations which clearly show a collapse of the $\mathrm{N}$-terminus. Furthermore, all of our simulation resulted in a collapse of the $\mathrm{N}$-terminus near the electronegative surface of DREAM (shown as black ellipse) and away from the hydrophobic cavity (blue ellipse).

\subsubsection{CaM modulates the $\mathrm{Ca}^{2+}$ dependent association of DREAM and DNA}

It has been well reported that DREAM interacts with the DRE promoter sequence of many genes, including c-fos, c-jun, and prodynorphin (Osawa et al. 2005, Carrion et al. 1999, Ledo et al. 2000). It was proposed that the interaction between DREAM and its target DNA is mediated by basic residues residing on the entering helix of EF-hand 1 (Lys101, Arg98, Lys91 and Lys90) which form an electropositive region at the N-terminus (Figure 5.1d). Additionally, $\operatorname{DREAM}(\Delta 65)$ binds DNA with about two-fold lower affinity than the full-length DREAM(Osawa et al. 2005). We hypothesize that the presence of numerous basic residues at the N-terminus of DREAM enhance its interaction with DNA and that association with CaM may result in a decrease in DNA binding affinity. 


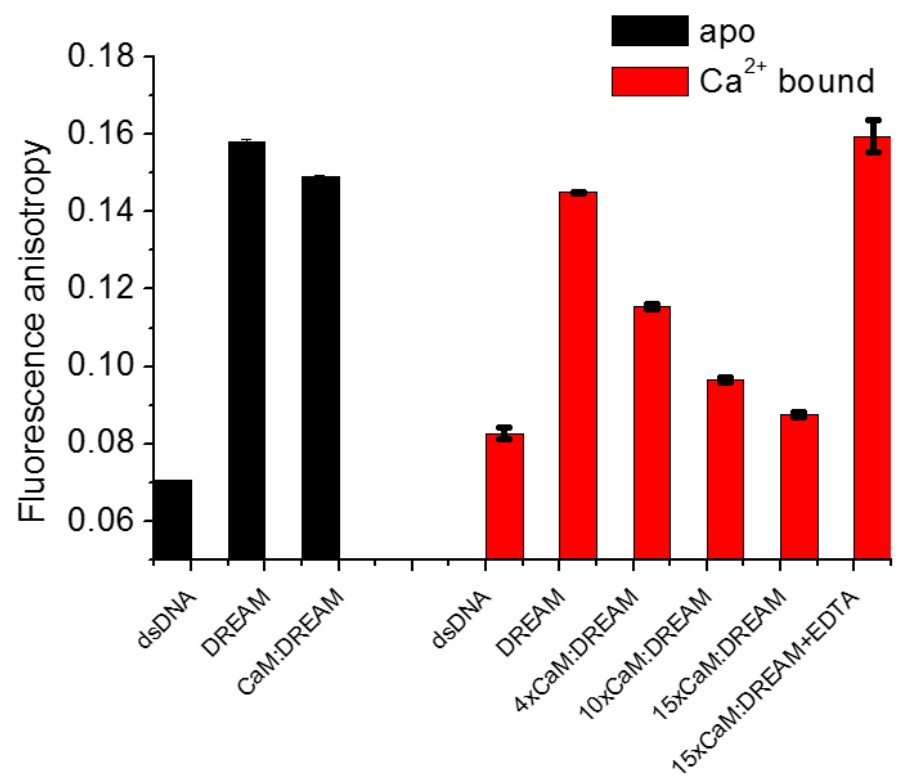

Figure 5.13. Fluorescence anisotropy increase due to $0.5 \mu \mathrm{M}$ FITC labeled dsDNA homologous to the DRE sequence of human prodynorphin gene binding to $5 \mu \mathrm{M}$ DREAM in the presence or absence of $\mathrm{Ca}^{2+}$. Addition of $\mathrm{CaM}$ in increasing concentrations $(0 \mu \mathrm{M}$, $20 \mu \mathrm{M}, 40 \mu \mathrm{M}$ and $60 \mu \mathrm{M}$ ) results in a concomitant decrease in fluorescence anisotropy. Chelation of $\mathrm{Ca}^{2+}$ by $2 \mathrm{mM}$ EDTA results in an increase in anisotropy due to dsDNA association with DREAM, even in the presence of $60 \mu \mathrm{M} \mathrm{CaM}$.

We employed a fluorescently labeled dsDNA homologous to the DRE sequence of the human prodynorphin gene and monitored the change in fluorescence anisotropy in the presence of DREAM and CaM. As shown in Figure 5.13, addition of $5 \mu \mathrm{M}$ DREAM results in a sharp increase in fluorescence anisotropy in the presence or absence of $\mathrm{Ca}^{2+}$. Furthermore, upon addition of increasing concentrations of CaM a decrease in anisotropy is observed, only in the presence of $\mathrm{Ca}^{2+}$. Addition of $2 \mathrm{mM}$ EDTA results in the reversal of the CaM induced decrease in anisotropy, to a value identical to that observed for DREAM in the absence of CaM. These results support the idea that in the presence of the $\mathrm{N}$-terminus, $\mathrm{Ca}^{2+}$ bound DREAM interacts with DNA, unless CaM is present to sequester its N-terminus domain. 


\subsection{Discussion}

The principal goal of this study is to present a model for the interface between the CaM:DREAM protein complex. Based on our results, it is clear that residues 29-44 of DREAM associate with CaM in a $\mathrm{Ca}^{2+}$ dependent manner with $\mathrm{K}_{\mathrm{d}} \sim 136 \mathrm{nM}$. Association of CaM at the N-terminus results in a blue shift and increase in tryptophan fluorescence on DREAM as well as on the DRE29 peptide, likely due to solvent restriction of Trp32 (Figure 5.2). The calcium dependency of CaM association with DRE29 peptide is similar to that of the intact DREAM protein; however, the affinity is decreased to $\mathrm{K}_{\mathrm{d}}=3.3 \pm 0.9 \mu \mathrm{M}$. This indicates that the core domain of DREAM (residues comprising helix 1 to 10) contribute to a decrease in binding energy of about $1.8 \mathrm{kcal} / \mathrm{mol}$. Such a decrease in the free energy of the complex could be due to larger steric hindrance of residues 29 to 44 in the presence of the rest of the protein, or due to a direct interaction between the N-terminus residues and the core domain of DREAM. Indeed, titration of residues $29-44$ with $\operatorname{DREAM}(\Delta 65)$ or DREAM shows the formation of a complex, with $\mathrm{K}_{\mathrm{d}} \sim 0.3 \mathrm{mM}(\sim-3$ $\mathrm{kcal} / \mathrm{mol}$ ). Such a low affinity for the $\mathrm{N}$-terminus domain with the core domain of DREAM is not irrelevant. The local concentration of the N-terminus can be calculated to be $\sim 3 \mathrm{mM}$, assuming an extended conformation of DREAM of about $100 \AA\left(0.5 \times 10^{-21} \mathrm{~L}\right)$, which would result in approximately $91 \%$ of the $\mathrm{N}$-terminus being associated with the core domain of DREAM. However, it is likely that the interaction between DRE29 and DREAM is favored by increased peptide flexibility. Therefore, using a $\mathrm{K}_{\mathrm{d}} \sim 5 \mathrm{mM}$ would be more appropriate (calculated from the decrease in binding energy of $1.8 \mathrm{kcal} / \mathrm{mol}$ ), which yields that only about $60 \%$ of the N-terminus domain is accessible to bind CaM. The collapse of the Nterminus seems to be nonspecific and mainly driven by electrostatic interactions. This 
conclusion explains why the hydrophobic cavity of DREAM at the C-terminus is not compromised and can efficiently bind small hydrophobic ligands such as 1,8-ANS and NS5806 as well as large peptides such as the N-terminus of Kv4.3 (Figure 5.5). Moreover, the puzzling calcium independent association of DREAM with Kv4.3, indicates that the Nterminus of DREAM is able to modify the accessibility of the hydrophobic groove. A similar effect was observed for NS5806 (Chapter 4), and it is possible that the presence of the N-terminus results in a destabilization of the hydrophobic groove which facilitates association with large hydrophobic peptides such as Kv4.3. A calcium independent binding of KChIPs and Kv4 channels has been observed using full-length proteins (An et al. 2000, Pioletti et al. 2006). However, we and others have reported a calcium dependent association of KChIP1 (which lacks the extended N-terminus domain) and $\operatorname{DREAM}(\Delta 65)$ with fragments of Kv4 (Gonzalez, Pham \& Miksovska 2014, Li et al. 2014, Gonzalez, Miksovska 2013). Therefore, the results presented here support a mechanism where presence of the $\mathrm{N}$-terminus in DREAM enhance association with $\mathrm{K}_{\mathrm{V}} 4$ channels, whereas its truncation by caspase-3 likely results in disruption of the DREAM: $K_{V} 4$ complex in the absence of calcium (Choi et al. 2001).

Despite the observed flexibility of the N-terminus domain, far and near UV CD spectra support a model where the first 65 residues do not form a random coil, but rather a partial helical structure. This is supported by bioinformatics analysis which highlights a region of high helical propensity, here named $\alpha_{0}$ (residues 27-53). Additionally, analysis of the disorder propensity of the DREAM amino acid sequence reveals that the N-terminus is comprised of two disordered regions connected by a structured segment, identified as $\alpha_{0}$. The N-terminus tryptophan (Trp32 and Trp50) within $\alpha_{0}$ undergo a structural 
reorganization upon calcium binding which correlates with an increase in solvent polarity(Nagai et al. 2012). However, contribution to the observed near-UV ellipticity change upon $\mathrm{Ca}^{2+}$ binding being due to the disulfide bond between Cys45 and Cys46 cannot be ruled out(Woody 2012, Kelly, Jess \& Price 2005a). We hypothesize that upon $\mathrm{Ca}^{2+}$ binding the surface charge reduction on DREAM as well as structural rearrangements may lead to a decrease in the collapse of the N-terminus, thus inducing an increase in solvent exposure of the N-terminus. Near-UV data also does not support a model where the N-terminus collapses into the hydrophobic cavity, which would result in a reversal of the observed transition.

Similarly to other CaM binding peptides, the association between CaM and the Nterminus of DREAM is fast and close to the diffusion limit(Brown, Martin \& Bayley 1997, Kasturi, Vasulka \& Johnson 1993). On the other hand, dissociation of CaM from DRE29 proceeds only after dissociation of $\mathrm{Ca}^{2+}$ from $\mathrm{CaM}$. Whether the dissociation requires dissociation of all $\mathrm{Ca}^{2+}$ ions or only those at the CaM C-terminus domain is not revealed by these experiments. However, the observed displacement of the DRE29 peptide by TFP bound at the C-terminus highlights the possibility that this domain plays a major role in complex formation. The fluorescence anisotropy change upon $\mathrm{Ca}^{2+}$ dissociation from CaMRR2 shows a much slower kinetics than that observed using tyrosine residues located at the C-terminus domain. The position of the rhodamine label at the C-terminus on the exiting helix of EF-hand 4 near the hydrophobic pocket may allow the xanthene group to stabilize the open conformation which results in much slower transition of CaM to the closed apo form. Furthermore, the temperature dependence of the dissociation kinetics allows us to extract the activation enthalpy and entropy for the dissociation of DRE29 and 
DREAM from apoCaM. These thermodynamic parameters reveal that the presence of the core domain of DREAM resulted in approximately 3-fold lower enthalpy of activation than dissociation of DRE29 $(16.1 \mathrm{kcal} / \mathrm{mol}$ versus $4.9 \mathrm{kcal} / \mathrm{mol})$. We hypothesize that this difference arises from an electrostatic attraction of the N-terminus domain involved in CaM binding to the electronegative side of DREAM, Figure 5.1. On the other hand, the positive $4.0 \mathrm{kcal} / \mathrm{mol}$ activation entropy for CaMRR2 dissociation from DREAM compared to -1.1 $\mathrm{kcal} / \mathrm{mol}$ for the DRE29 dissociation shows that the dissociation transition states are not identical. These results support our hypothesis that there is an electrostatic interaction between the N-terminus domain and the core domain of DREAM, as well as the idea that CaM decreases the disorder of the N-terminus which likely explains the increase in solubility of DREAM.

DREAM has been shown to undergo an oligomerization change upon binding of calcium; however, there seems to be some disagreement among the reported oligomerization states. We have recently reported that in the absence of calcium, the $\operatorname{DREAM}(\Delta 65)$ construct forms a stable tetramer in solution and a dimer upon calcium binding (Chapter 3). In contrast, Ramachandran and coworkers observed a dimer to monomer transition upon calcium binding to DREAM (Ramachandran et al. 2012), which is contrary to the initial finding favoring a tetramer to dimer transition (Osawa et al. 2001). The disagreement could be likely due to different expression methodologies (GST fusion vs inclusion body refolding), and/or sample conditions (high salt versus low salt and 10 mM LDAO). Therefore, to understand the impact of CaM association with DREAM we determined the structural arrangement and oligomerization of CaM:DRE29 and CaM:DREAM complexes using steady-state and time-resolved spectroscopy. 


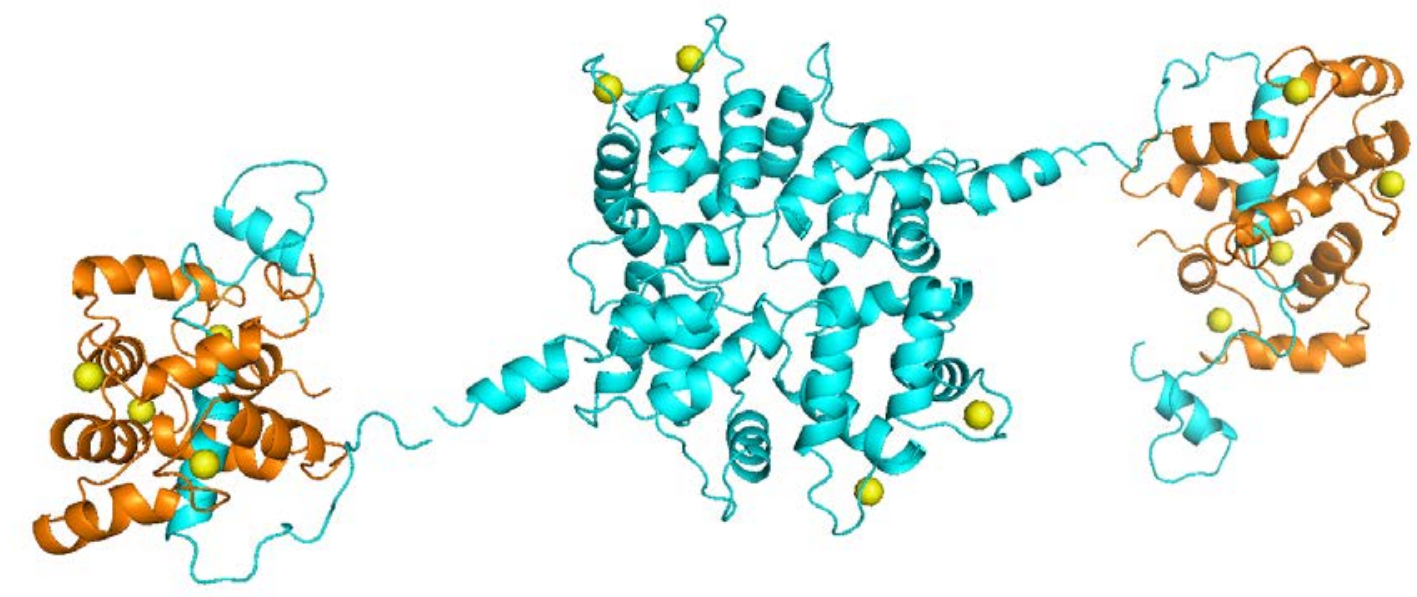

Figure 5.14. Carton representation of the proposed model for a heterotetrameric DREAM:CaM complex. The DREAM dimer is shown in cyan and calmodulin is shown in orange, calcium is shown as yellow spheres.

The fluorescence properties of the IAEDANS labeled CaM shows a clear transition upon $\mathrm{Ca}^{2+}$ binding which include an increase in fluorescence, a blue shift in emission as well as slower decay lifetime and rotational correlation time of the protein. These results are in good agreement with the known transition of CaM from a flexible conformation in the apo form to a more rigid and elongated structure in the presence of calcium with concomitant exposure of a hydrophobic surface at the C-terminus (Chattopadhyaya et al. 1992, Kuboniwa et al. 1995). Association of CaM-IAEDANS with DREAM results in an even more dramatic increased fluorescence, a larger blue shift and increased decay lifetime as well as a more complex rotational decay. Noticeably, CaM and DRE29 form a spherical heterodimer whereas a heterotetramer was observed for the CaM-IAEDANS:DREAM complex, which supports a stoichiometry of $1: 1$. Moreover, the fact that $\mathrm{Ca}^{2+} \mathrm{DREAM}$ forms a dimer in the presence of CaM supports the idea that CaM association does not disrupt the dimer interface which is partially solvent protected in calcium bound DREAM 
dimer and likely does not affect the accessibility of the hydrophobic groove on DREAM or its interaction with hydrophobic partners(Gonzalez, Miksovska 2014, Gonzalez, Pham \& Miksovska 2014, Lusin et al. 2008). With these result in mind, we propose that in the presence of $\mathrm{Ca}^{+}$, DREAM and CaM form heterotetrameric complex as shown in figure 5.14 .

Multiple simulations of a DREAM model derived from bioinformatics analysis (Figure 5.13) show that the N-terminus of DREAM likely collapses on a negative surface adjacent to the hydrophobic surface. The interaction between the core domain and the $\mathrm{N}$ terminus of DREAM is mainly mediated by Asp and Glu residues on the exiting helix of EF-2 and EE-4 and entering helix of EF-3 as well as on the loop of EF-4. The position of the N-terminus also does not conflict with the proposed structure of KChIPs bound to the T1-domain of Kv4 channels (Pioletti et al. 2006, Wang et al. 2007). It is well known that the drosophila shaker channels (homologous to Kv4 in humans) can inactivate through pore occlusion mediated by a long and flexible positively charged N-terminus (Antz et al. 1997). The positive charge and conformational flexibility of the N-terminus domain of DREAM could highlight the role of this domain in modulation of $\mathrm{Kv} 4$ channels through a mechanism similar to that of shaker channels. Moreover, the divergent amino acid sequence among the KChIP subfamily of $\mathrm{Ca}^{2+}$ binding proteins could explain the observed variation in modulation of $\mathrm{K}^{+}$currents by KChIPs.

Finally, binding of CaM to DREAM shows a clear effect on the DREAM interactions with the DRE sequence of the prodynorphin gene, an effect which likely translates to other DRE promoted genes regulated by DREAM (Ledo et al. 2000). Our observation that DREAM associates with DNA independent of calcium is in contrast to 
previous work (Osawa et al. 2005). However, we believe that the discrepancy arise from different experimental conditions since previous ITC experiments were conducted in the presence of LDAO. We observed that both LDAO and CaM increase the solubility of DREAM, and it is possible that LDAO association with DREAM modulates the interactions between DRE and DREAM and potentially inhibits the interaction between $\mathrm{Ca}^{2+}$ DREAM and DNA. Indeed, we have observed inhibition of the CaM:DREAM interaction by LDAO as well as a strong interaction between DRE29 peptide and DRE dsDNA with LDAO (data not shown). The $\mathrm{EC}_{50}$ for $\mathrm{Ca}^{2+} \mathrm{CaM}$ induced DREAM DNA dissociation observed here is relatively high, $\sim 20 \mu \mathrm{M}$. However, the high intra-cellular concentrations of $\mathrm{CaM}$, the known $\mathrm{Ca}^{2+}$ induced translocation of CaM from the cytosol to the nucleus as well as the $\mathrm{Ca}^{2+}$ induced increase in the freely diffusing CaM concentration could facilitate the formation of DREAM:CAM complex and dissociation of DREAM from DNA(Deisseroth, Heist \& Tsien 1998, Zaidi et al. 2004). It is also possible that posttranslational modification such as myristoylation/palmytoylation at Cys45/Cys46 could enhance the affinity between CaM and DREAM and decrease the observed EC50, an effect which has been observed in other CaM binding proteins(Takasaki et al. 1999).

\subsection{Summary}

We have shown that an alpha-helical region at the N-terminus of DREAM comprising residues 29 to 44 contains a $\mathrm{CaM}$ binding surface. The $\mathrm{Ca}^{2+}$ dependent association of CaM with DREAM increases its solubility in the presence of calcium. We also demonstrate that the N-terminus of DREAM forms a flexible $\mathrm{Ca}^{2+}$ sensitive domain, which can collapse on the core domain of DREAM, but does not block the hydrophobic cavity at the C-terminus. The protein complexes between CaM and DRE29 or DREAM 
follow a 1 to 1 stoichiometry, in good agreement with a heterodimer and heterotetramer oligomerization. Overall, our biochemical studies provide insight into the molecular mechanism of the CaM:DREAM complex and the impact of CaM association to DREAM and open up the possibility of CaM:DREAM complex being involved in gene regulation. The complex behavior of the N-terminus in DREAM highlights the functional diversity of members of the KChIPs group of calcium binding proteins, which share low homology in this region. These results also provide a roadmap for the design of a molecular model of CaM:DREAM complex, which may shed light on the understanding of these multifunctional proteins, and how they affect downstream binding partners such as Kv4 channels, DNA, and presenilin. 


\section{CHARACTERIZATION OF THE PHOTOPHYSICAL,}

THERMODYNAMIC AND STRUCTURAL PROPERTIES OF THE TERBIUM(III)-DREAM COMPLEX

\subsection{Background and significance}

DREAM, is a $29 \mathrm{kDa}$ multifunctional $\mathrm{Ca}^{2+}$ sensing protein found in different neuronal cell compartments (Carrion et al. 1999). Outside the nucleus, DREAM interacts with presenilin to regulate amyloid precursor protein processing and with potassium channels to regulate their membrane translocation and gating (An et al. 2000, Buxbaum et al. 1998). Moreover, DREAM represents a new class of $\mathrm{Ca}^{2+}$ sensing protein able to translocate to the nucleus and directly bind DNA (Carrion et al. 1999). In the nucleus, it regulates prodynorphin and c-fos gene expression by binding to the DRE regulatory sequence of those genes (Carrion et al. 1999, Cheng et al. ). Association of DREAM with the DRE promoter regions in the absence of calcium leads to inhibition of gene transcription. These genes have been shown to be involved in apoptosis, cell homeostasis, and pain modulation (Costigan, Woolf 2002, Fontán-Lozano et al. 2009). The role of DREAM in pain sensing, memory retention, learning and Alzheimer's disease highlight the multifunctional properties of this protein (Fontán-Lozano et al. 2009). As a calcium signal transducer, DREAM does not possess endogenous catalytic activity and its regulatory effect in biological processes arises from interaction with numerous binding partners. Therefore, understanding how calcium and other metals trigger structural changes on DREAM, and how this protein reorganization controls target recognition, would provide important insight into its mechanism of action. 
The three dimensional structure of $\mathrm{Ca}^{2+}$ bound DREAM has been obtained through NMR and is presented in Figure 6.1a (Lusin et al. 2008). DREAM has four EF-hand motifs of which EF-hand 3 and EF-hand 4 are able to bind $\mathrm{Ca}^{2+}$, while EF-hand 1 is unable to bind either $\mathrm{Mg}^{2+}$ or $\mathrm{Ca}^{2+}$. The coordination of calcium/magnesium in the EF-hand motif has been widely studied and it has been shown to form hexa- or hepta-coordination with oxygen atoms of proteins in order to form a pentagonal bipyramidal coordination (Figure 6.1b and c) (Gifford, Walsh \& Vogel 2007a, da Silva, Kendrick-Jones \& Reinach 1995). The oxygen-donating amino acids of the calcium selective metal binding loop in EF-hands follow a common organization such that the $1^{\text {st }}, 3^{\text {rd }}, 5^{\text {th }}$, positions are either an aspartic or an asparagine amino acid, while the amino acid at position $12^{\text {th }}$ is a well conserved glutamic acid (Figure 6.1b). Modulation of metal affinity and selectivity arises from distinct combinations of negatively charged amino acids at these positions. For instance, the EFhand 2 of DREAM has been proposed to selectively bind $\mathrm{Mg}^{2+}$ due to Glu $\rightarrow$ Asp mutation at the $12^{\text {th }}$ position, which eliminates the hepta-coordination necessary for strong binding of $\mathrm{Ca}^{2+}$ (Kretsinger, Nockolds 1973, Craig et al. 2002). Similarly, the presence of a lysine at the $1^{\text {st }}$, proline at the $5^{\text {th }}$, and aspartic acid at the $12^{\text {th }}$ position render the EF-hand 1 of DREAM unable to bind most metals (Craig et al. 2002, Osawa et al. 2001).

Association of calcium at the metal binding loop of the active EF-hand pair at the C-terminus of DREAM, induces a structural rearrangement which leads to exposure of a hydrophobic surface as well as changes in oligomerization state (Gonzalez, Miksovska 2014, Osawa et al. 2001). However, details about the underlying molecular mechanism by which calcium binds and induces structural changes on DREAM are not known. Nonetheless, experiments using NMR to monitor the glycine residues in the EF-hand loops 
and the associated chemical shift broadening upon metal binding would provide insight into the role of amino acids of DREAM. Of particular interest is the use of lanthanide ions, which have been shown to possess similar physical properties to calcium ions, and have been widely applied to study the metal binding properties of EF-hands (Mustafi et al. 2004)'(Martin, Richardson 1979). Additionally, lanthanide-protein complexes have been shown to undergo magnetic alignment during NMR experiments, which is of great help in elucidating the three dimensional structure of protein-metal and protein-protein complexes (Pintacuda et al. 2006)The advantage of employing lanthanides to understand the mechanism of calcium binding is their unique luminescent properties as well as their ability to effectively displace calcium from EF-hand loops. Replacement of $\mathrm{Ca}^{2+}$ by $\mathrm{Tb}^{3+}$ has also been shown to induce structural changes on the EF-hand loops which are highly homologous to those observed upon calcium binding (Rao et al. 1996).

In this chapter, we implement a combination of fluorescence, luminescence, TIMSMS , and calorimetric techniques to show that $\mathrm{Tb}^{3+}$ binds at the EF-hands of DREAM and functions as a calcium biomimetic. Moreover, we show that association of $\mathrm{Tb}^{3+}$ at EF-hand 3 and 4 of DREAM leads to a calcium-like conformation with similar hydrophobic surface exposure, oligomeric transition, and ion-neutral collisional cross section (CCS) as that observed for the $\mathrm{Ca}^{2+}$ bound protein. Nonetheless, we observe small deviations in the dynamics of the environment near Trp169 as well as secondary structure organization, indicating that not all aspects of $\mathrm{Tb}^{3+}$ binding are identical to $\mathrm{Ca}^{2+}$. Using ITC and the fluorescent properties of $\mathrm{Tb}^{3+}$ we are able to gain insight into the role of $\mathrm{Mg}^{2+}$ and ligand binding to DREAM. 


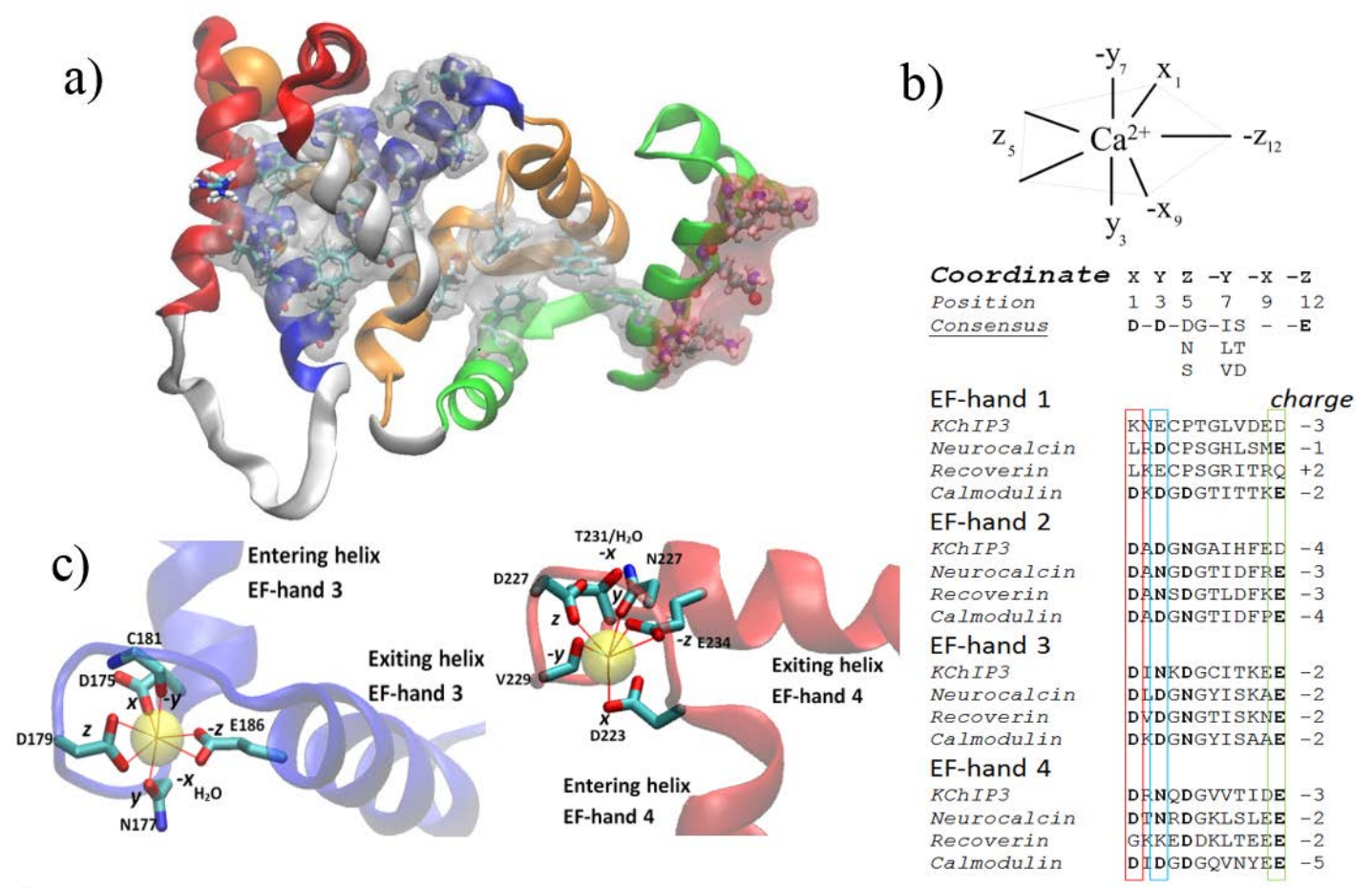

Figure 6.1. (a) NMR structure of DREAM monomer with highlighted hydrophobic residues (gray mesh) and charged residues (red mesh) (pdb entry 2JUL) ${ }^{7}$. The four EFhand of DREAM are shown in green (EF-hand 1), orange (EF-hand 2), blue (EF-hand 3), and red (EF-hand 4). Calcium are shown as an orange sphere. (b) Coordination geometry of calcium bound to EF-hand 3 (left) and EF-hand 4 (right). Residues involved in coordination of $\mathrm{Ca}^{2+}$ are shown in licorice model. EF-hand 3 shows a clear pentagonal bipyramidal coordination whereas EF-hand 4 is distorted. (c) Geometry and consensus sequence of EF-hand binding loops as well as sequence homology between neuronal calcium sensors and calmodulin at the metal binding loops.

\subsection{Results}

The well-known calcium biomimetic behavior of europium(III), terbium(III), and neodymium (III) as well as the unique spectroscopic properties of protein:lanthanide complexes have been widely employed to characterize the sequence of metal binding to calcium binding proteins (Mulqueen, Tingey \& Horrocks 1985, Chaudhuri et al. 1997, Kilhoffer, Gerard \& Demaille 1980), to observe protein conformational heterogeneity (Austin, Stein \& Wang 1987), to determine water coordination of metals bound at the EFhand motif (Horrocks, Sudnick 1979), and as binding assays (Hagan, Zuchner 2011). 
Therefore, we envisioned that the properties of lanthanides could be employed to obtain information on the biophysical properties of DREAM protein. However, the association of lanthanides with the KChIP subfamily of calcium binding proteins has not been extensively studied. Thus, we first set forth to determine whether $\mathrm{Tb}^{3+}$ is able to directly associate with the EF-hand of DREAM. This is important, since previous studies on DREAM $(\Delta 65)$ using mass spectrometry and studies on NCS-1 using sensitized emission have presented contradicting results on whether $\mathrm{Tb}^{3+}$ can bind to neuronal calcium sensors (Craig et al. 2002, Fisher et al. 2000). Additionally, we are interested in investigating whether $\mathrm{Tb}^{3+}$ binding induces structural changes on DREAM homologous to those observed for calcium.

6.2.1 Terbium(III) binds to DREAM and is sensitized by energy transfer from W169

Calmodulin and DREAM are well known to undergo distinct structural changes upon binding of calcium, these structural changes are accompanied by changes in fluorescence of tyrosine and tryptophan residues, respectively (Carrion et al. 1999, Drabikowski, Brzeska \& Venyaminov SYu 1982). These fluorescence transitions of these proteins are shown in Figure 8.2a and 8.2b. Upon calcium binding the tyrosine fluorescence of CaM increases whereas the tryptophan fluorescence of DREAM decreases, in agreement with previous reports. Of particular interest is the observation that in the presence of $\mathrm{Tb}^{3+}$, at molar ratios of 4:1 for CaM and 2:1 for DREAM, the fluorescence emission are nearly identical to that observed in the presence of saturating calcium (Figure 6.2a, b). 

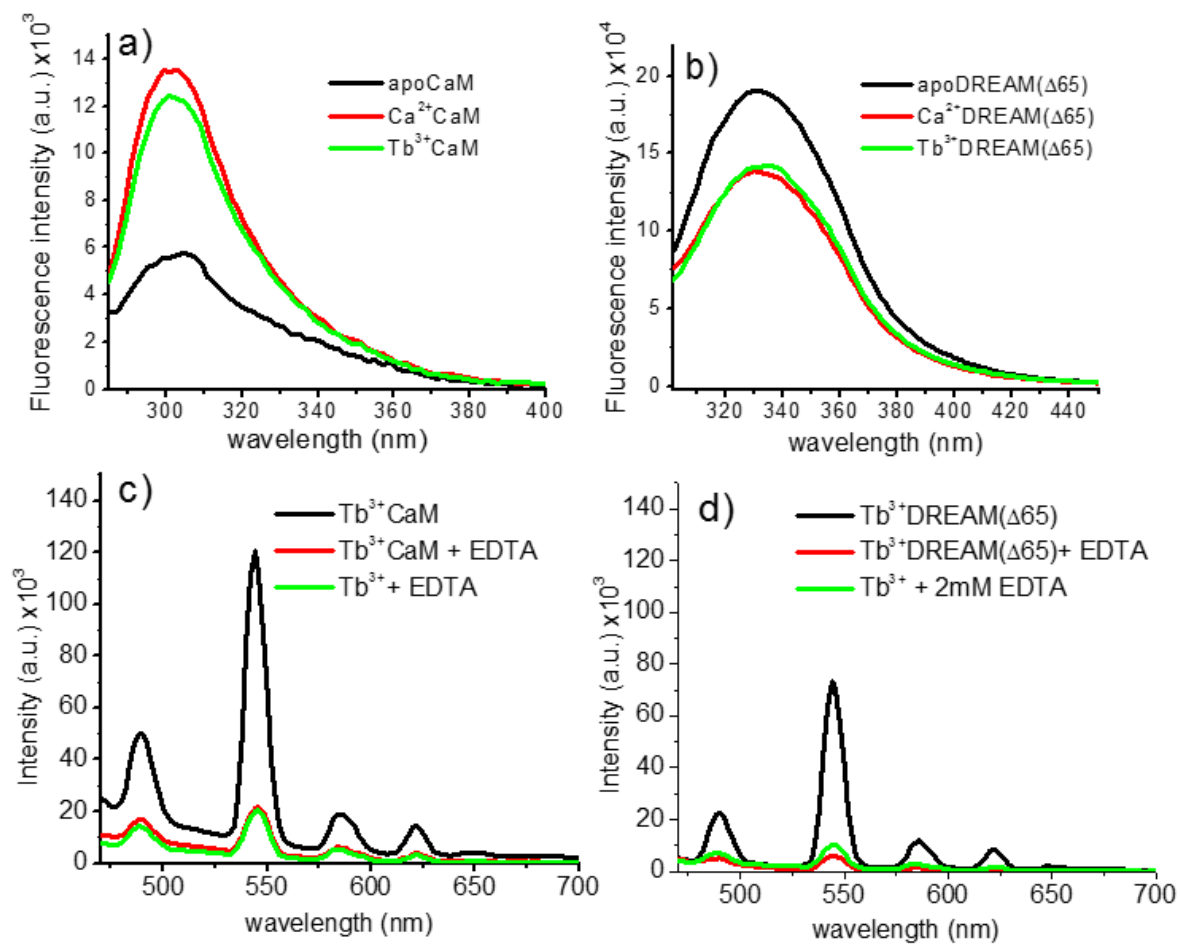

Figure 6.2: Intrinsic fluorescence changes of $40 \mu \mathrm{M}$ (a) CaM and (b) DREAM( $\triangle 65)$ upon binding of $1 \mathrm{mM}$ calcium or $160 \mu \mathrm{M} \mathrm{Tb} b^{3+}$ on CaM or $80 \mu \mathrm{M} \mathrm{Tb}^{3+}$ for DREAM( $\left.\Delta 65\right)$, excited at $280 \pm 4 \mathrm{~nm}$. The observed tyrosine fluorescence change upon $\mathrm{Tb}^{3+}$ binding to CaM shows a small deviation from that observed for $\mathrm{Ca}^{2+}$, likely due to quenching of the fluorescence by energy transfer to $\mathrm{Tb}^{3+}$. The $\mathrm{Tb}^{3+}$ induced transition for DREAM( $\left.\triangle 65\right)$ is identical to that observed in the presence of $\mathrm{Ca}^{2+}$. Sensitized emission of (c) $160 \mu \mathrm{M}$ $\mathrm{Tb}^{3+}$ bound to CaM and (d) $80 \mu \mathrm{M} \mathrm{Tb} b^{3+}$ bound to $\operatorname{DREAM}(\Delta 65)$, excited at $280 \mathrm{~nm}$ with and without $2 \mathrm{mM}$ EDTA. The background emission of the $\mathrm{Tb}^{3+}$ :EDTA complex is shown as reference. The observed sensitized emission of $\mathrm{Tb}^{3+}$ shows the characteristic sharp bands at 489, 544 (major), 586 and $622 \mathrm{~nm}$ due to the ${ }^{5} \mathrm{D}_{4}-{ }^{7} \mathrm{~F}_{6},{ }^{5} \mathrm{D}_{4}-{ }^{7} \mathrm{~F}_{5},{ }^{5} \mathrm{D}_{4}-{ }^{7} \mathrm{~F}_{4},{ }^{5} \mathrm{D}_{4}-{ }^{7} \mathrm{~F}_{3}$ transitions of $\mathrm{Tb}^{3+}$, respectively. The major peak at $545 \mathrm{~nm}$ for $\operatorname{DREAM}(\Delta 65)$ is $40 \%$ smaller than that observed for CaM. Addition of 2 mM EDTA to either CaM or DREAM $(\Delta 65)$ resulted in an emission identical to that of $\mathrm{Tb}^{3+}$ in solution.

The slightly lower tyrosine fluorescence of CaM in the presence of $\mathrm{Tb}^{3+}$ is likely due to an efficient quenching of $\mathrm{Tb}^{3+}$ by aromatic amino acid, or due to incomplete binding of this ion. We also investigated whether binding of $\mathrm{Tb}^{3+}$ on DREAM leads to energy transfer from nearby aromatic residues toward the metal ligand, as previously observed for CaM (Kilhoffer, Gerard \& Demaille 1980). The presence of the characteristic sharp emission 
bands in the sensitized emission spectra of the $\operatorname{DREAM}(\Delta 65): \mathrm{Tb}^{3+}$ complex supports the idea that aromatic residues, likely at the C-terminus of DREAM, are able to transfer energy to bound terbium (Figure 8.2c and 8.2d). The sensitized emission intensity of terbium bound to $\operatorname{DREAM}(\Delta 65)$ is about half of that observed for CaM, which is likely due to the presence of only two $\mathrm{Tb}^{3+}$ ions bound to EF-hand 3 and 4 of $\operatorname{DREAM}(\Delta 65)$, whereas four $\mathrm{Tb}^{3+}$ ions are bound to CaM (Craig et al. 2002). Nonetheless, it is also possible that the presence of Tyr-100 at position 7 of the EF-hand 3 binding loop and Tyr-139 at position 10 of EF-hand 4 on CaM provide a more efficient energy transfer to $\mathrm{Tb}^{3+}$ (Hogue et al. 1992).

Detailed analysis of DREAM $(\Delta 65)$ excitation spectra due to the ${ }^{5} \mathrm{D}_{4} \rightarrow{ }^{7} \mathrm{~F}_{5}$ transition at $545 \mathrm{~nm}$ shows a broad peak with maximum at $280 \mathrm{~nm}$ and $32 \mathrm{~nm}$ FWHM (Figure 6.3a). The ratio of terbium luminescence intensity when exciting at $295 \mathrm{~nm}$ and $280 \mathrm{~nm}$ is 0.40 , which indicates that tyrosine and tryptophan residues are able to transfer energy (Brittain, Richardson \& Martin 1976). The excitation spectra of DREAM $(\Delta 160)$, DREAM(E186Q), and DREAM(E234Q) are identical to those of DREAM( $\Delta 65)$, with a broader FWHM of $36 \mathrm{~nm}$ and larger 295/280 ratio of 0.52 for DREAM( $\Delta 160)$. Analysis of the C-terminus domain of DREAM shows the presence of three tyrosine residues (Y174, Y195 and Y203); however, only Y174 is within the range of 5 to $10 \AA$ necessary for an efficient energy transfer to $\mathrm{Tb}^{3+}$ bound at EF-hand 3 and 4 (Figure 8.3b) (Kleinerman 1969, Wallace et al. 1982). Therefore, to quantify the energy transfer contribution of Y174 we constructed a DREAM(Y174A) mutant and determined the effect of this mutation on the sensitized emission and excitation spectra. This mutant shows identical circular dichroism transition and amplitude as well as the same $\mathrm{Tb}^{3+}$ induced tryptophan fluorescence change, 
as the DREAM $(\Delta 65)$ construct (data not shown). On the other hand, the efficiency of energy transfer in the DREAM(Y174A) mutant is decreased by 60 \%, judging from the decreased sensitized emission at $545 \mathrm{~nm}$ (Figure 6.3a). The excitation spectra of this construct also shows a $5 \mathrm{~nm}$ blue shift to $275 \mathrm{~nm}$ with a FWHM of $38 \mathrm{~nm}$ and 295/280 ratio of 0.41 .
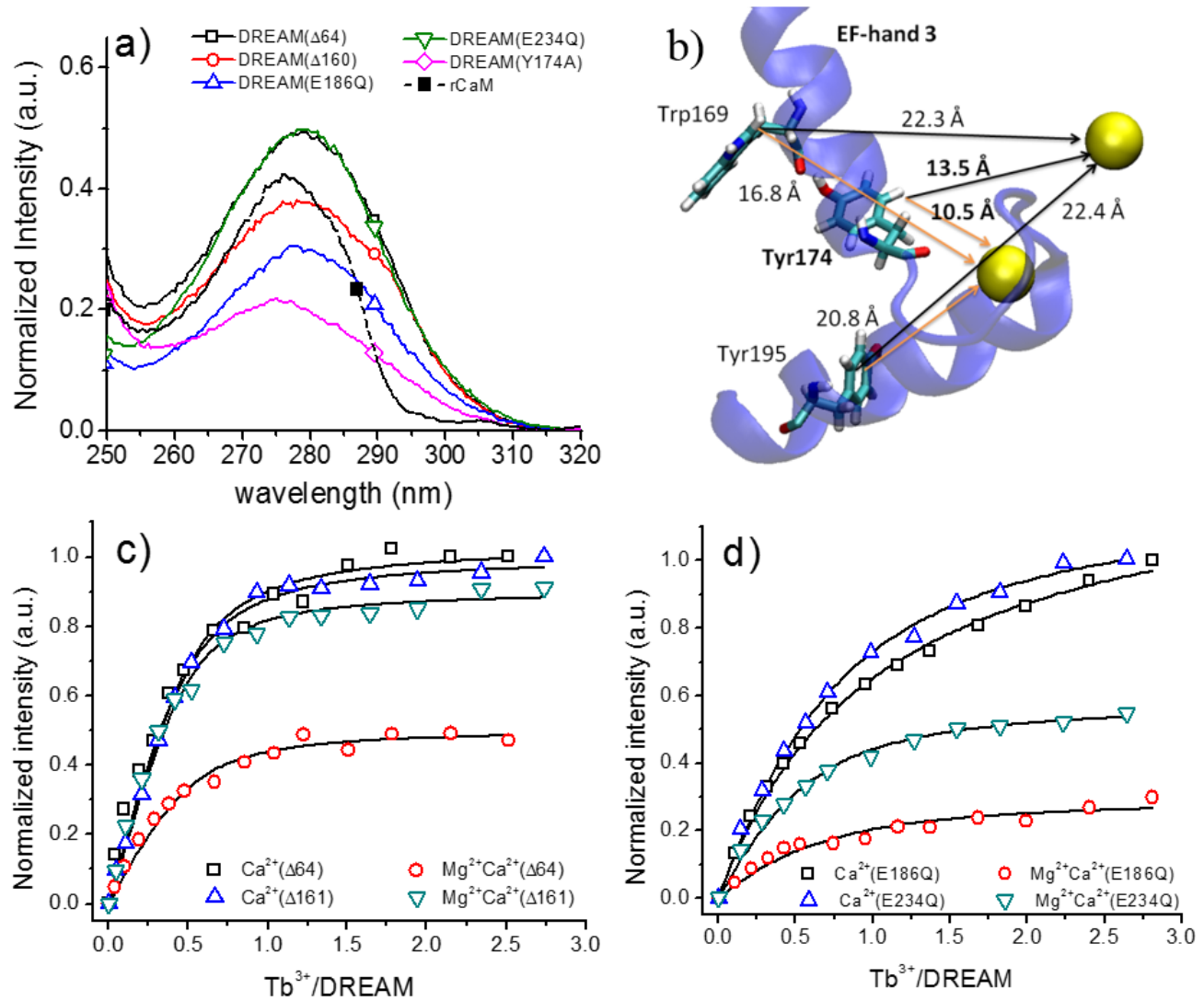

Figure 6.3: (a) Excitation spectra of DREAM constructs bound to $\mathrm{Tb}^{3+}, 20 \mu \mathrm{M}$ protein 40 $\mu \mathrm{M} \mathrm{Tb}^{3+}$. The spectra was normalized so that the intensity corresponding to backbone to $\mathrm{Tb}^{3+}$ energy transfer at $240 \mathrm{~nm}$ is the same for $\operatorname{DREAM}(\Delta 65), \operatorname{DREAM}(\Delta 160)$, and DREAM(Y174A) while the magnitude is half for DREAM(E186Q) and DREAM(E234Q). (b) Calcium/terbium binding sites of EF-hand 3 and 4, only EF-hand 3 is shown (in blue) for clarity. (c) Titrations of $\mathrm{Tb}^{3+}$ into $\operatorname{DREAM}(\Delta 65)$ and $\operatorname{DREAM}(\Delta 160)$ in $20 \mathrm{mM}$ TRIS pH 7.4 and $100 \mu \mathrm{M} \mathrm{Ca}{ }^{2+}$ with and without $5 \mathrm{mM} \mathrm{Mg}^{2+}$. (d) Titration of $\mathrm{Tb}^{3+}$ into DREAM(E186Q) and DREAM(E234Q) under identical conditions as panel (c). Solid lines represent the best fit using the quadratic equation for $\mathrm{N}$-binding sites. 
The decrease in sensitized emission and blue shift of the excitation spectra support the role of Y174 as an energy donor, while the identical 295/280 ratio for DREAM( $\Delta 65)$ and $\operatorname{DREAM}(\mathrm{Y} 174 \mathrm{~A})$ is indicative of $\operatorname{Tyr} \rightarrow \operatorname{Trp} \rightarrow \mathrm{Tb}^{3+}$ being the predominant energy transfer pathway. The similar $\mathrm{Tb}^{3+}$ sensitization observed in $\operatorname{DREAM}(\Delta 160)$ and $\operatorname{DREAM}(\Delta 65)$ constructs indicates that aromatic amino acids at the N-terminus do not transfer energy to $\mathrm{Tb}^{3+}$ and that the observed luminescence arises from terbium bound at EF-hand 3 and 4. For comparison, the excitation spectra of CaM is also shown and a characteristic maxima at $277 \mathrm{~nm}$ with FWHM of $24 \mathrm{~nm}$ and 295/280 ratio of 0.07 is observed, which is in good agreement with a $\mathrm{Tyr} \rightarrow \mathrm{Tb}^{3+}$ energy transfer. Moreover, the lack of vibronic structures on the excitation spectra on all constructs indicates that phenylalanine residues do not play a major role in energy transfer.

The closer proximity of Tyr174 to EF-hand 3 than EF-hand 4 (10.5 $\AA$ vs $13.5 \AA$ ) supports the idea that monitoring the binding of $\mathrm{Tb}^{3+}$ using the sensitized emission may allow us to identify whether aromatic amino acids on DREAM can transfer energy to $\mathrm{Tb}^{3+}$ bound at both EF-hands. To confirm this hypothesis, we employed two DREAM constructs in which glutamic acid at position 12 of the calcium binding loop has been mutated to glutamine thus inactivating either EF-hand 3 in DREAM(E186Q) or EF-hand 4 in DREAM(E234Q)(Osawa et al. 2005). As expected, the excitation spectra of $\mathrm{Tb}^{3+}$ bound at EF-hand 3 in the DREAM(E234Q) mutant shows about 39 \% larger fluorescence than that of $\mathrm{Tb}^{3+}$ bound at EF-hand 4 in the DREAM(E186Q) mutant (Figure 6.3a).

Titration of $\mathrm{Tb}^{3+}$ into $\mathrm{Ca}^{2+}$ bound $\operatorname{DREAM}(\Delta 65)$ or $\operatorname{DREAM}(\Delta 160)$ shows that both construct have similar affinity for $\mathrm{Tb}^{3+}$ in the presence of calcium. The dissociation constant obtained using the quadratic equation shows identical affinity for $\mathrm{Tb}^{3+}$ in the 
presence of $\mathrm{Ca}^{2+}$ with $\mathrm{K}_{\mathrm{d}}=1.8 \pm 0.6 \mu \mathrm{M}$ for $\operatorname{DREAM}(\Delta 65)$ and $\mathrm{K}_{\mathrm{d}}=1.6 \pm 0.2 \mu \mathrm{M}$ for $\operatorname{DREAM}(\Delta 160)$. Interestingly, in the presence of $5 \mathrm{mM} \mathrm{MgCl}_{2}$ the sensitized emission of $\mathrm{Tb}^{3+}$ is decreased by $53 \%$ for $\operatorname{DREAM}(\Delta 65)$ but no significant changes are observed for $\operatorname{DREAM}(\Delta 160)$ (Figure 4.3c). To better understand the binding mechanism of $\mathrm{Tb}^{3+}$ to EFhand 3 and 4 of DREAM, we have also conducted titrations in the presence of $\mathrm{Ca}^{2+}$ or $\mathrm{Mg}^{2+}$ and $\mathrm{Ca}^{2+}$ using the DREAM(E186Q) and DREAM(E234Q) protein mutants (Figure 6.3d). Displacement of $\mathrm{Ca}^{2+}$ from DREAM(E186Q) by $\mathrm{Tb}^{3+}$ shows a weaker dissociation constant of $\mathrm{K}_{\mathrm{d}}=11 \pm 1 \mu \mathrm{M}$ compared to DREAM(E234Q) with $\mathrm{K}_{\mathrm{d}}=5.9 \pm 0.5 \mu \mathrm{M}$. However, a similar decrease in sensitized emission upon addition of $5 \mathrm{mM} \mathrm{MgCl}_{2}$ as that for $\operatorname{DREAM}(\Delta 65)$ was observed, with an emission decrease of $55 \%$ and $80 \%$ for DREAM(E234Q) and DREAM(E186Q), respectively (Figure 6.3d). Under the conditions used for these titrations ( $500 \mu \mathrm{M} \mathrm{CaCl}_{2}$ or $500 \mu \mathrm{M} \mathrm{CaCl}_{2}$ and $5 \mathrm{mM} \mathrm{MgCl}_{2}$ ), $\mathrm{Tb}^{3+}$ binds first to the inactivated EF-hand (see ITC experiments below). Therefore, the data shown in Figure 6.3d, highlights that $\mathrm{Mg}^{2+}$ binding to EF-hand 2 induces structural rearrangements which have a larger impact on EF-hand 3. This is in good agreement with the fact that EFhand 2 is adjacent to EF-hand 3, and that the exiting helix of EF-hand 2 is in direct contact with entering helix of EF-hand 3 (Figure 6.1a). Tryptophan 169 and tyrosine 174, both of which are shown to play a major role in energy transfer, both reside on the entering helix of EF-hand 3. The presence of $\mathrm{Mg}^{2+}$ also enhanced the binding of $\mathrm{Tb}^{3+}$, with dissociations constants of $5.8 \pm 1.9 \mu \mathrm{M}$ for DREAM(E186Q) and $2.7 \pm 0.4 \mu \mathrm{M}$ for DREAM(E234Q). This increase in apparent affinity is likely due to a decrease in nonspecific binding of $\mathrm{Tb}^{3+}$ to secondary sites or to EF-hand 2. Together these results support a model in which terbium 
displaces $\mathrm{Ca}^{2+}$ from EF-hand 4 and EF-hand 3 as well as highlight the role of $\mathrm{Mg}^{2+}$ binding at EF-hand 2 as a structural cofactor in DREAM.

6.2.2 Terbium(III) binding to DREAM leads to similar structural rearrangement as those observed for $\mathrm{Ca}^{2+}$.

The terbium(III) induced tryptophan emission quenching observed in Fig. 6.2b indicates that $\mathrm{Tb}^{3+}$ is able to mimic the structural changes induced by $\mathrm{Ca}^{2+}$ binding to EFhand 3 and 4 . To test whether $\mathrm{Tb}^{3+}$ binding to DREAM induces a structural transition analog to that of $\mathrm{Ca}^{2+}$, we monitored the fluorescence and anisotropy decay of Trp169, the fluorescence of the extrinsic hydrophobic probe 1,8-ANS bound at the C-terminus of $\operatorname{DREAM}(\Delta 65)$ (see Chapter 3), and changes in secondary structure. Detailed information regarding the environment and dynamics of Trp169 and how they are affected by metal binding can be obtained by measuring the fluorescence and anisotropy decay lifetimes. As previously reported (Pham et al. 2015), the fluorescence decay of Trp169 on DREAM( $\Delta 65)$ was best fitted by a Gaussian-discrete bimodal decay model, whose parameters are listed in Table 1 and Figure 6.4a. The small discrepancies between our results and those published previously likely arise from the lack of LDAO detergent under our conditions. Nonetheless, we observe that addition of $\mathrm{Ca}^{2+}$ or $\mathrm{Tb}^{3+}$ results in a decrease in the average excited state lifetime from $4.8 \mathrm{~ns}$ to $3.8 \mathrm{~ns}$, which is due to a decrease in fractional intensity contribution of the long lifetime and a slightly faster Gaussian decay from 1.8 ns to 1.5 ns. The lack of significant lifetime quenching of Trp169 by $\mathrm{Tb}^{3+}$ is likely due to the poor efficiency of this energy transfer process. 


\begin{tabular}{|c|c|c|c|c|c|c|c|}
\hline & $\begin{array}{c}\bar{\tau}_{\mathbf{1}}\left(f_{1}\right) \\
\text { (ns) }\end{array}$ & $\begin{array}{l}\text { W1 } \\
\text { (ns) }\end{array}$ & $\begin{array}{c}\tau_{2}\left(f_{2}\right) \\
\text { (ns) }\end{array}$ & $\begin{array}{l}\langle\tau\rangle \\
\text { (ns) }\end{array}$ & $\begin{array}{c}\boldsymbol{\Theta}_{1}\left(f_{1}\right) \\
\text { (ns) }\end{array}$ & $\begin{array}{c}\boldsymbol{\Theta}_{2}\left(f_{2}\right) \\
(\mathbf{n s})\end{array}$ & $\chi^{2}$ \\
\hline $\operatorname{apoDREAM}(\Delta 64)$ & $1.8(0.39)$ & 0.69 & $5.7(0.61)$ & 4.2 & $0.40(0.35)$ & $15(0.65)$ & 0.5 \\
\hline $\mathrm{Ca}^{2+} \operatorname{DREAM}(\Delta 64)$ & $1.5(0.48)$ & 0.60 & $5.9(0.52)$ & 3.8 & $0.52(0.40)$ & $29(0.60)$ & 0.9 \\
\hline $\mathrm{Tb}^{3+} \mathrm{DREAM}(\Delta 64)$ & $1.5(0.47)$ & 0.64 & $5.8(0.53)$ & 3.8 & $0.23(0.58)$ & $27(0.42)$ & 1.5 \\
\hline
\end{tabular}

Values in parentheses represent the fraction intensity and fractional depolarization. $\tau_{1}$ is the mean decay time of the Gaussian distribution with a width of distribution $\mathrm{w}_{1}$. The lifetime of the discrete single exponential term is denoted as $\tau_{2}$. The average lifetime $<\tau>$ was calculated using Eq. 1 and Eq. 2 in Supplemental Material section. $\alpha_{1}$ and $\alpha_{2}$ are normalized pre-exponential decay and $f_{1}$ and $f_{2}$ are exponential decay fractions.

Moreover, anisotropy decay measurements were conducted to identify whether binding of $\mathrm{Tb}^{3+}$ induces dimerization of DREAM as observed for $\mathrm{Ca}^{2+}$. The frequency domain anisotropy decay data was best fitted with a double discrete model, in which the fast $\Theta_{1}$ is associated with fast local fluctuations of tryptophan and the slow $\Theta_{2}$ corresponds to the global rotation of the protein (Table 1). A clear transition from $15 \mathrm{~ns}$ to $29 \mathrm{~ns}$ upon binding of $\mathrm{Ca}^{2+}$ and $27 \mathrm{~ns}$ in the presence of $\mathrm{Tb}^{3+}$ is observed. These rotational correlation times match well with the values of $14 \mathrm{~ns}$ for a monomeric and $28 \mathrm{~ns}$ for a dimeric $\operatorname{DREAM}(\Delta 65)$ protein approximated by the Einstein-Stokes equation at $17{ }^{\circ} \mathrm{C}, \eta=0.0100 \mathrm{P}$, and $0.73 \mathrm{~g} / \mathrm{mL}$ hydration. Differences in rotational correlation times of the fast local tryptophan motion can be observed between $\mathrm{Ca}^{2+}$ and $\mathrm{Tb}^{3+}$ bound $\operatorname{DREAM}(\Delta 65)$, where a twofold faster local motion is measured in the presence of terbium (0.52 ns vs $0.23 \mathrm{~ns}$ ). 
In the metal-free $\operatorname{DREAM}(\Delta 65)$, the local flexibility accounts for $35 \%$ of the depolarization while in the $\mathrm{Ca}^{2+}$ and $\mathrm{Tb}^{3+}$ bound $\operatorname{DREAM}(\Delta 65)$ form, this rotation contributes $48 \%$ and $58 \%$ in depolarization, respectively. Altogether, excited state and anisotropy decay of Trp169 reveal that even though binding of $\mathrm{Tb}^{3+}$ can induce dimerization of $\operatorname{DREAM}(\Delta 65)$ this metal also induces a more dynamic structure near the Trp169 compared to $\mathrm{Ca}^{2+}$.
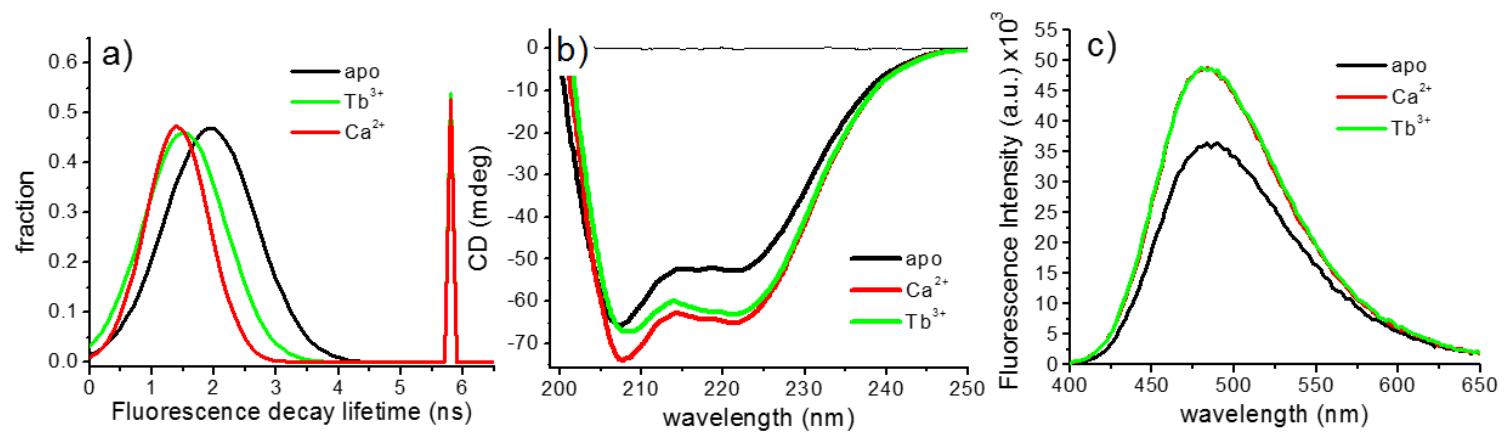

Figure 6.4. (a) Tryptophan fluorescence lifetime distribution of $\operatorname{DREAM}(\Delta 64)$ in the presence of $\mathrm{Ca}^{2+}$ or $\mathrm{Tb}^{3+}$, decay data was modeled as a Gaussian distribution and a discrete decay, parameters listed in Table 1. (b) Circular dichroism spectra of $40 \mu \mathrm{M} \operatorname{DREAM}(\Delta 64)$ in the presence of $1 \mathrm{mM} \mathrm{Ca}^{2+}, 2 \mathrm{mM}$ EDTA or $160 \mu \mathrm{M} \mathrm{Tb}^{3+}$ in $5 \mathrm{mM}$ TRIS pH 7.4. (b) Binding of $\mathrm{Tb}^{3+}$ or $\mathrm{Ca}^{2+}$ to $10 \mu \mathrm{M}$ DREAM( $\left.\Delta 64\right)$ in the presence of $40 \mu \mathrm{M}$ 1,8-ANS.

As shown in Figure 6.4b, the CD spectra of metal-free DREAM $(\Delta 65)$ shows a characteristic profile with minima at $222 \mathrm{~nm}$ and $208 \mathrm{~nm}$ (maxima at $190 \mathrm{~nm}$ is not shown) in good agreement with an alpha helical structure. Upon binding of $\mathrm{Ca}^{2+}$, an ellipticity decrease at $200-225 \mathrm{~nm}$ and increase at $190 \mathrm{~nm}$ is observed, which can be explained by an increase in alpha helical content and/or rearrangement of the alpha helices. On the other hand, in the presence of $\mathrm{Tb}^{3+}$ a CD spectra intermediate to that measured for apo and $\mathrm{Ca}^{2+}$ bound DREAM is observed. This intermediate structure was also observed when 2-fold excess of terbium was added to a sample containing $100 \mu \mathrm{M}$ calcium (not shown). The largest deviation in $\mathrm{CD}$ spectra between the $\mathrm{Ca}^{2+}$ and $\mathrm{Tb}^{3+}$ bound $\operatorname{DREAM}(\Delta 65)$ is observed near the $208 \mathrm{~nm}$ minima. Thus, the $\mathrm{CD}$ data indicates that $\mathrm{Tb}^{3+}$ is able to displace 
$\mathrm{Ca}^{2+}$ and induce a structural change distinct from those observed for metal free and $\mathrm{Ca}^{2+}$ bound $\operatorname{DREAM}(\Delta 65)$.

Despite the small differences in the protein dynamics near Trp169, as well as deviation in secondary structure upon binding of $\mathrm{Tb}^{3+}$, we observed similar binding of 1,8ANS at the C-terminal hydrophobic cavity of DREAM( $\Delta 65)$ (Gonzalez, Miksovska 2014) . The fluorescence emission of this probe has been extensively shown to be sensitive to the immediate environment, and while the total intensity upon $\mathrm{Ca}^{2+}$ binding is observed to increase, no changes in fluorescence of 1,8-ANS were observed upon displacement of $\mathrm{Ca}^{2+}$ by $\mathrm{Tb}^{3+}$ (Fig. 4c). Moreover, frequency domain analysis of the excited state of 1,8-ANS as well as the depolarization time reveal no significant differences between $\mathrm{Ca}^{2+}$ or $\mathrm{Tb}^{3+}$ bound $\operatorname{DREAM}(\Delta 65)$ (data not shown). Overall, the indication is that the hydrophobic cavity exposure induced by calcium is also triggered by terbium.

\subsubsection{DREAM binds $\mathrm{Tb}^{3+}$ through an entropy driven mechanism.}

To complement the $\mathrm{Tb}^{3+}$ :DREAM binding studies using sensitized emission we also conducted calorimetric studies where the heat associated with $\mathrm{Tb}^{3+}$ displacement of $\mathrm{Ca}^{2+}$ bound at the EF-hands of CaM and DREAM is measured. Isothermal calorimetry reveals that displacement of $\mathrm{Ca}^{2+}$ from CaM is endothermic and can be best modeled as a sequential process (Figure 6.5a). The profile of the ITC isotherm for $\mathrm{Tb}^{3+}$ displacement of $\mathrm{Ca}^{2+}$ bound to CaM is similar to that obtained for calcium binding to a plant-mammalian CaM chimera (Gilli et al. 1998), albeit with slightly different thermodynamic parameters. The association of $\mathrm{Tb}^{3+}$ ions with $\mathrm{CaM}$ at EF-hand 1 through 4 have slightly lower association constants than those determined for $\mathrm{Ca}^{2+}$ binding, which is likely due to 
competition effects. We associate the site with $\mathrm{K}_{1}$ parameters as representing the displacement of $\mathrm{Ca}^{2+}$ by $\mathrm{Tb}^{3+}$, while the two other observed binding processes $\left(\mathrm{K}_{2}\right.$ and $\left.\mathrm{K}_{3}\right)$ likely arise from a convolution of specific and nonspecific association of $\mathrm{Tb}^{3+}$ ions. The presence of nonspecific binding of $\mathrm{Tb}^{3+}$ ions is supported by the fact that addition of $5 \mathrm{mM}$ $\mathrm{Mg}^{2+}$ results in drastic changes in the thermodynamic parameters associated with $\mathrm{K}_{2}$ and $\mathrm{K}_{3}$. The observed decrease in enthalpy and entropy of $\sim 160 \mathrm{kcal} \cdot \mathrm{mol}^{-1}$ and $\sim 280 \mathrm{kcal} \cdot \mathrm{mol}^{-}$ ${ }^{1}$ for $\mathrm{K}_{2}$ and $\mathrm{K}_{3}$ also support the idea that these supplementary sites are strikingly different and may involve different ligands and/or ion displacement. We associate the thermodynamic values recovered in the presence of $\mathrm{Mg}^{2+}$ with the displacement of $\mathrm{Ca}^{2+}$ specifically bound at the loops of EF-hands 1 thru 4, convoluted with any additional protein rearrangement to accommodate $\mathrm{Tb}^{3+}$. It can be observed that in the presence of $\mathrm{Mg}^{2+}$ there is a three-fold decrease in affinity for the $\mathrm{K}_{1}$ site, with a minimal change in enthalpy and entropy; whereas sites $\mathrm{K}_{2}$ and $\mathrm{K}_{3}$ show similar affinity, with $\mathrm{K}_{2}$ having two-fold larger enthalpy and entropy than $\mathrm{K}_{3}$ sites. Altogether, these values can be interpreted as corresponding to $\mathrm{Tb}^{3+}$ displacement of $\mathrm{Ca}^{2+}$ ions from four EF-hands with $\mathrm{K}_{1}$ representing the weakest $\mathrm{Ca}^{2+}$ binding hand and $\mathrm{K}_{2}$ and $\mathrm{K}_{3}$ corresponding to the remaining three EFhands. Following previous studies in which calmodulin was shown to follow a sequential filling of EF-hands $1 \rightarrow 2 \rightarrow 3$ and 4 by $\mathrm{Tb}^{3+}$ and $\mathrm{Ca}^{2+}$, we associate the $\mathrm{K}_{1}$ binding site to that of $\mathrm{Tb}^{3+}$ binding to EF-hand 4 (Kilhoffer, Gerard \& Demaille 1980, Wang et al. 1982). Interestingly, EF-hand 4 in calmodulin shows the presence of glutamic acid at position 11 near the $-\mathrm{z}$ metal coordination (Figure 6.1), which may explain the lower enthalpic and entropic contribution upon displacement of $\mathrm{Ca}^{2+}$ by $\mathrm{Tb}^{3+}$. 

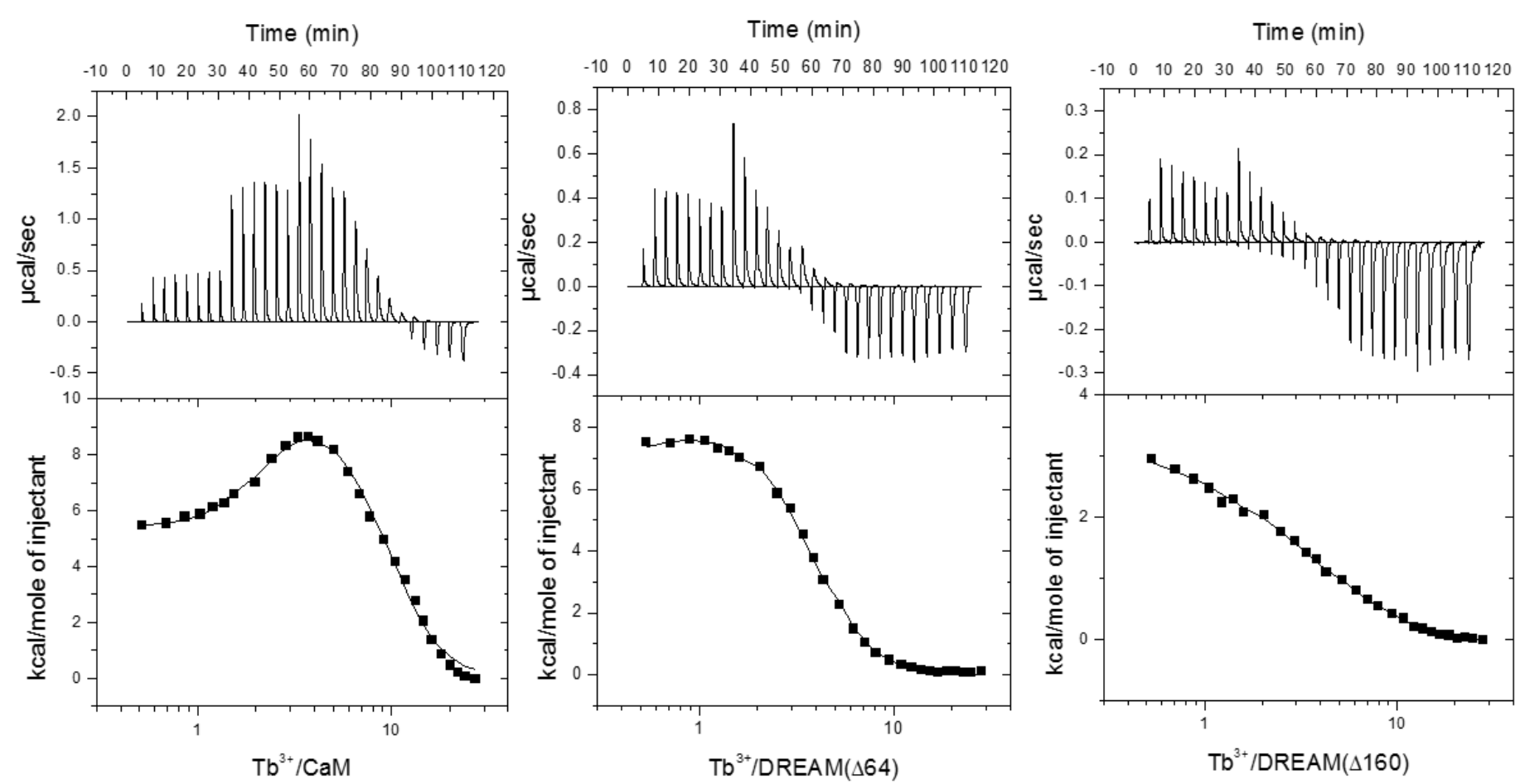

Figure 6.5: ITC isotherms for $\mathrm{Tb}^{3+}$ displacement of $\mathrm{Ca}^{2+}$ from CaM, DREAM $(\Delta 64)$ and $\operatorname{DREAM}(\Delta 160)$. The upper panels of each profile reflects the thermal power expressed in units of $\mu \mathrm{cal} \cdot \mathrm{s}^{-1}$. The lower panel shows integrated reaction heats $(\Delta \mathrm{H})$ expressed in units of $\mathrm{kcal} \cdot \mathrm{mol}^{-1}$. The solid lines present the best fitting curve with parameters shown in Table 6.2. 


\begin{tabular}{|c|c|c|c|c|c|c|c|c|c|}
\hline & $\mathbf{K}_{\mathbf{1}} \times 10^{4}$ & $\begin{array}{c}\mathbf{K}_{2} \times \\
10^{4}\end{array}$ & $\begin{array}{c}\mathbf{K}_{3} \mathbf{x} \\
10^{4}\end{array}$ & $\Delta \mathbf{H}_{1}$ & $\Delta \mathbf{H}_{2}$ & $\Delta \mathbf{H}_{3}$ & $\mathbf{T} \Delta \mathbf{S}_{1}$ & $\mathbf{T} \Delta \mathbf{S}_{2}$ & $\mathbf{T} \Delta \mathbf{S}_{3}$ \\
\hline $\mathrm{CaM}$ & $29 \pm 4.2$ & $0.7 \pm 0.4$ & $5.1 \pm 8.4$ & $12 \pm 5$ & $212 \pm 38$ & $\begin{array}{c}-307 \pm \\
127\end{array}$ & $19 \pm 3.9$ & 217 & -302 \\
\hline $\mathrm{CaM}+\mathrm{Mg}^{2+}$ & $8.9 \pm 1.8$ & $1.9 \pm 0.2$ & $1.4 \pm 0.2$ & $13 \pm 1.5$ & $47 \pm 0.6$ & $23 \pm 7$ & $20 \pm 1.4$ & $52 \pm 0.6$ & $28 \pm 7$ \\
\hline $\operatorname{DREAM}(\Delta 64)$ & $22 \pm 1.3$ & $8.1 \pm 1.6$ & $2.8 \pm 0.2$ & $13 \pm 9.0$ & $18 \pm 0.6$ & $-2.5 \pm 2.7$ & $20 \pm 9.1$ & $24 \pm 0.4$ & $3.6 \pm 2.7$ \\
\hline $\begin{array}{l}\operatorname{DREAM}(\Delta 64)+ \\
\mathrm{Mg}^{2+}\end{array}$ & $16 \pm 2.5$ & $11 \pm 1.1$ & $6.0 \pm 0.5$ & $12 \pm 1.3$ & $18 \pm 6.0$ & $3.4 \pm 3.2$ & $19 \pm 1.3$ & $25 \pm 5.9$ & $9.9 \pm 3.1$ \\
\hline DREAM $(\Delta 160)$ & $6.9 \pm 3.2$ & $8.9 \pm 9.2$ & & $13 \pm 1.7$ & $8.2 \pm 1.3$ & & $19 \pm 1.5$ & $15 \pm 1.1$ & \\
\hline $\begin{array}{l}\text { DREAM }(\Delta 160)+ \\
\mathrm{Mg}^{2+}\end{array}$ & $6.8 \pm 1.4$ & $1.8 \pm 0.6$ & & $11 \pm 2.2$ & $7.7 \pm 2.4$ & & $17 \pm 2.1$ & $14 \pm 2.4$ & \\
\hline $\begin{array}{l}\text { DREAM (E186Q) } \\
+\mathrm{Mg}^{2+}\end{array}$ & $14.0 \pm 0.9$ & $8.6 \pm 1.3$ & $8.8 \pm 2.2$ & $7.5 \pm 1.2$ & $15 \pm 1.7$ & $2.2 \pm 2.6$ & $14 \pm 0.8$ & $22 \pm 1.8$ & $9 \pm 2.7$ \\
\hline $\begin{array}{l}\text { DREAM (E234Q) } \\
+\mathrm{Mg}^{2+}\end{array}$ & $5.7 \pm 0.9$ & $27 \pm 1.5$ & $16 \pm 6.2$ & $11 \pm 1.9$ & $3.6 \pm 2.7$ & $4.0 \pm 1.3$ & $18 \pm 1.9$ & $9.6 \pm 2.5$ & $11 \pm 0.9$ \\
\hline
\end{tabular}


The $\mathrm{Tb}^{3+}$ binding sites corresponding to $\mathrm{K}_{2}$ show thermodynamic parameters for $\Delta \mathrm{H}_{2}$ and $\Delta \mathrm{S}_{2}$ which are about two-fold larger than those of $\Delta \mathrm{H}_{3}$ and $\Delta \mathrm{S}_{3}$, which is likely due to two $\mathrm{Tb}^{3+}$ binding sites being reported by the $\mathrm{K}_{2}$ parameters. This allows us to approximate a value of $\Delta \mathrm{H} \sim 23 \mathrm{kcal} \cdot \mathrm{mol}^{-1}$ and $\mathrm{T} \Delta \mathrm{S} \sim 27 \mathrm{kcal} \cdot \mathrm{mol}^{-1}$ for $\mathrm{Tb}^{3+}$ displacement of $\mathrm{Ca}^{2+}$ bound at EF-hands 1, 2, and 3 and $\Delta \mathrm{H}=13 \mathrm{kcal} \cdot \mathrm{mol}^{-1}$ and $\mathrm{T} \Delta \mathrm{S}=28 \mathrm{kcal} \cdot \mathrm{mol}^{-1}$ for EF-hand 4 .

Furthermore, displacement of $\mathrm{Ca}^{2+}$ bound to $\operatorname{DREAM}(\Delta 65)$ by $\mathrm{Tb}^{3+}$ shows a simpler isotherm (Figure 6.5b), which is similar to that obtained for $\mathrm{Ca}^{2+}$ displacement by $\mathrm{Tm}^{3+}$ in the Entamoeba histolytica calcium binding protein(Mustafi et al. 2004). Despite the ability to model the isotherm using the simpler one-set of sites model, we decided to present the results obtained from a three sites sequential model based on three accounts. First, it is expected that binding of $\mathrm{Tb}^{3+}$ is not associated with a cooperative behavior since the protein transitions from a calcium bound structure to a calcium-like structure. Second, replacement of glutamic acid at position 12 of the loop in EF-hand 3 resulted in an isotherm which could not be fitted by a one-set of sites model. Lastly, displacement of $\mathrm{Ca}^{2+}$ by $\mathrm{Tb}^{3+}$ in other calcium binding proteins with known calcium cooperativity have been observed to follow a sequential mechanism (Mustafi et al. 2004, Gilli et al. 1998). The recovered parameters using the sequential model are shown in Table 6.2. Interpretation of the data reveals that three $\mathrm{Tb}^{3+}$ ions can bind to $\operatorname{DREAM}(\Delta 65)$, with slightly different affinities, such that $\mathrm{K}_{1}>\mathrm{K}_{2}>\mathrm{K}_{3}$. Interestingly, the associated enthalpy and entropy of sites $\mathrm{K}_{1}$ and $\mathrm{K}_{2}$ are similar to those found for $\mathrm{Ca}^{2+}$ displacement of $\mathrm{K}_{1}$ sites on $\mathrm{CaM}$, while the enthalpy and entropy of the site corresponding to $\mathrm{K}_{3}$ shows significantly lower enthalpic and entropic contributions. Titration of $\mathrm{Tb}^{3+}$ in the presence of $\mathrm{Ca}^{2+}$ and $5 \mathrm{mM} \mathrm{Mg}^{2+}$ in $\operatorname{DREAM}(\Delta 65)$ increase $\Delta \Delta \mathrm{H}_{3}$ and $\mathrm{T} \Delta \Delta \mathrm{S}_{3}$ by $\sim 6 \mathrm{kcal} \cdot \mathrm{mol}^{-1}$ while the enthalpy and entropy 
of sites $\mathrm{K}_{1}$ and $\mathrm{K}_{2}$ remain unchanged. Unlike CaM, in which all four EF-hands are believed to have binding preference for $\mathrm{Ca}^{2+}$ over $\mathrm{Mg}^{2+}$ (Wang et al. 1982); EF-hand 2 on DREAM is proposed to bind $\mathrm{Mg}^{2+}$ preferentially(da Silva, Kendrick-Jones \& Reinach 1995, Craig et al. 2002). Therefore, we assign the recovered thermodynamic parameters for sites with $\mathrm{K}_{1}$ and $\mathrm{K}_{2}$ association constants as representing $\mathrm{Ca}^{2+}$ displacement from EF-hand 3 or 4 , while those recovered for site with $\mathrm{K}_{3}$ being representative of $\mathrm{Tb}^{3+}$ binding to EF-hand 2. The assignment of $\mathrm{K}_{3}$ to EF-hand 2 is also supported by the displacement of $\mathrm{Ca}^{2+}$ by $\mathrm{Tb}^{3+}$ in a $\operatorname{DREAM}(\Delta 160)$ construct lacking the $\mathrm{Mg}^{2+}$ binding EF-hand 2, which shows only two sites with affinities and thermodynamic parameters similar to those of $K_{1}$ and $K_{2}$ on $\operatorname{DREAM}(\Delta 65)$. The recovered association constant and thermodynamic parameter for $\mathrm{Tb}^{3+}$ binding to $\operatorname{DREAM}(\Delta 160)$ are also independent of $\mathrm{Mg}^{2+}$, in agreement with the sensitized emission titrations. However, despite the similarities, a decrease of $\sim 10 \mathrm{kcal} \cdot \mathrm{mol}^{-1}$ in the enthalpy and entropy for the $K_{2}$ site is observed for $\operatorname{DREAM}(\Delta 160)$ compared to $\operatorname{DREAM}(\Delta 65)$. This decrease in energetics could be due to a distinct metal coordination or loss structural rearrangement at the missing $\mathrm{N}$-terminus. It is tempting to assign the $\mathrm{K}_{2}$ parameters of DREAM $(\Delta 160)$ as representing binding of $\mathrm{Tb}^{3+}$ to EF-hand 3, due to closer proximity to the now deleted N-terminus domain, but these results do not permit unequivocal assignment. Together, the recovered thermodynamic parameters for $\operatorname{DREAM}(\Delta 65)$ and $\operatorname{DREAM}(\Delta 160)$ show that $\mathrm{Tb}^{3+}$ can efficiently displace $\mathrm{Ca}^{2+}$ from the C-terminus EF-hand 3 and 4, and that deletion of the N-terminus amino acids (1 to 160) results in modification of the metal binding properties of EF-hand 3 and 4. Thus, we can approximate a value of $\Delta \mathrm{H} \sim 15 \mathrm{kcal} \cdot \mathrm{mol}^{-1}$ and $\mathrm{T} \Delta \mathrm{S} \sim 22 \mathrm{kcal} \cdot \mathrm{mol}^{-1}$ for $\mathrm{Tb}^{3+}$ displacement of $\mathrm{Ca}^{2+}$ bound at EF-hand 3 and 4 of $\operatorname{DREAM}(\Delta 65)$. 
Additional experiments in which we monitored the thermodynamics of $\mathrm{Tb}^{3+}$ association with $\mathrm{Ca}^{2+}$ bound DREAM(E186Q) with inactivated EF-hand 3 and DREAM(E234Q) with inactivated EF-hand 4 were also conducted and the recovered parameters are listed in Table 2. Displacement of $\mathrm{Ca}^{2+}$ by $\mathrm{Tb}^{3+}$ on the DREAM(E186Q) construct is best modeled as a three site sequential binding process, in which the $\mathrm{K}_{1}$ site has the highest affinity, while $\Delta \mathrm{H}_{1}$ and $\Delta \mathrm{S}_{1}$ are $\sim 5 \mathrm{kcal} \cdot \mathrm{mol}^{-1}$ lower than those obtained for DREAM $(\Delta 65)$. The $\mathrm{Tb}^{3+}$ binding at site with $\mathrm{K}_{2}$ shows identical affinity as the observed for $\mathrm{K}_{2}$ on $\operatorname{DREAM}(\Delta 65)$ in the presence of $\mathrm{Mg}^{2+}$, and similar enthalpic and entropic contributions. The third site, $\mathrm{K}_{3}$, shows similar parameters as those recovered for $\mathrm{Tb}^{3+}$ binding at the EF-hand 2 of $\operatorname{DREAM}(\Delta 65)$ and likely corresponds to the same binding process. The decrease in enthalpy and entropy upon binding of $\mathrm{Tb}^{3+}$ at site with $\mathrm{K}_{1}$ as well as its stronger binding affinity compared to $\mathrm{K}_{2}$ and $\mathrm{K}_{3}$, seem to indicate that this site likely corresponds to the inactivated EF-hand 3. The fact that only small changes are observed upon mutation of glutamic acid at position 12 (-z coordination) to glutamine supports the

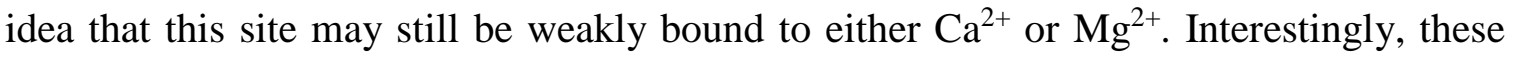
results also show that the E186Q substitution does not affect the binding of $\mathrm{Tb}^{3+}$ at EFhand 3 which is interesting, but not unexpected. This effect is likely due to weaker binding of $\mathrm{Ca}^{2+}$ which in turn facilitates $\mathrm{Tb}^{3+}$ association.

The DREAM(E234Q) construct, in which EF-hand 4 is inactivated, is also able to bind three $\mathrm{Tb}^{3+}$ ions with ranges of affinities similar to those obtained for the $\operatorname{DREAM}(\Delta 65)$ in the presence of $\mathrm{Mg}^{2+}$. In this construct, the site with association constant $\mathrm{K}_{2}$ binds stronger, followed by $\mathrm{K}_{3}$ and then $\mathrm{K}_{1}$ sites. However, the enthalpy for site $\mathrm{K}_{2}$ on $\operatorname{DREAM}(\mathrm{E} 234 \mathrm{Q})$ is five-fold lower than those obtained for $\mathrm{K}_{2}$ on $\operatorname{DREAM}(\Delta 65)$. The sites 
with $\mathrm{K}_{3}$ association constant show identical enthalpy and entropy as the site corresponding to $K_{3}$ on $\operatorname{DREAM}(\Delta 65)$. In fact, the enthalpy and entropy for sites $K_{2}$ and $K_{3}$ are much closer to those obtained for $\mathrm{Tb}^{3+}$ binding to the EF-hand 2 of $\operatorname{DREAM}(\Delta 65)$ in the presence of $\mathrm{Mg}^{2+}$, with $\Delta \mathrm{H} \sim 4 \mathrm{kcal} \cdot \mathrm{mol}^{-1}$ and $\Delta \mathrm{S} \sim 10 \mathrm{kcal} \cdot \mathrm{mol}^{-1}$. These results suggest that inactivation of EF-hand 4 likely results in a weak binding of $\mathrm{Ca}^{2+}$ or $\mathrm{Mg}^{2+}$, both of which can be easily displaced by $\mathrm{Tb}^{3+}$ (based on higher $\mathrm{K}_{2}$ and $\mathrm{K}_{3}$ ) with a concomitant lower enthalpic and entropic contribution. Overall, ITC demonstrates that in the presence of saturating amounts of calcium, the Glu $\rightarrow$ Gln mutation at position 12 of the EF-hand metal binding loop actually facilitates binding of $\mathrm{Tb}^{3+}$.

6.2.4 Luminescence decay of $\mathrm{Tb}^{3+}$ bound DREAM is sensitive to ligand binding.

Sensitized emission studies show that $\mathrm{Mg}^{2+}$ is able to induce changes in the emission of bound $\mathrm{Tb}^{3+}$; to further study this effect, we decided to determine if $\mathrm{Mg}^{2+}$ can induce structural changes within the coordination sphere of $\mathrm{Tb}^{3+}$. We employed direct excitation of $\mathrm{Tb}^{3+}$ using the $355 \mathrm{~nm}$ line of a Nd:YAG pulsed laser and measured the luminescence decay of terbium bound to the EF-hands of CaM and DREAM constructs. This approach permits analysis of the local environment at the binding site of $\mathrm{Tb}^{3+}$ ions, namely the EF-hand loops. Additionally, this method has been widely used to monitor changes in the coordination sphere of lanthanides as well as the effects of water coordination (Hungerford et al. 2010). Following the studies above, we first characterized the luminescence decay of $\mathrm{Tb}^{3+}$ bound to $\mathrm{CaM}$; and as expected, the luminescence decay shown in Figure 6.6a follows a mono-exponential decay with $\tau_{1}=1.38 \mathrm{~ms}$. A single exponential decay of approximately $1 \mathrm{~ms}$ has been reported for the $\mathrm{Tb}^{3+}$ : CaM complex 
and double exponential decays has been observed for complexes of $\mathrm{Eu}^{3+}$ and calmodulin (Mulqueen, Tingey \& Horrocks 1985, Austin, Stein \& Wang 1987).
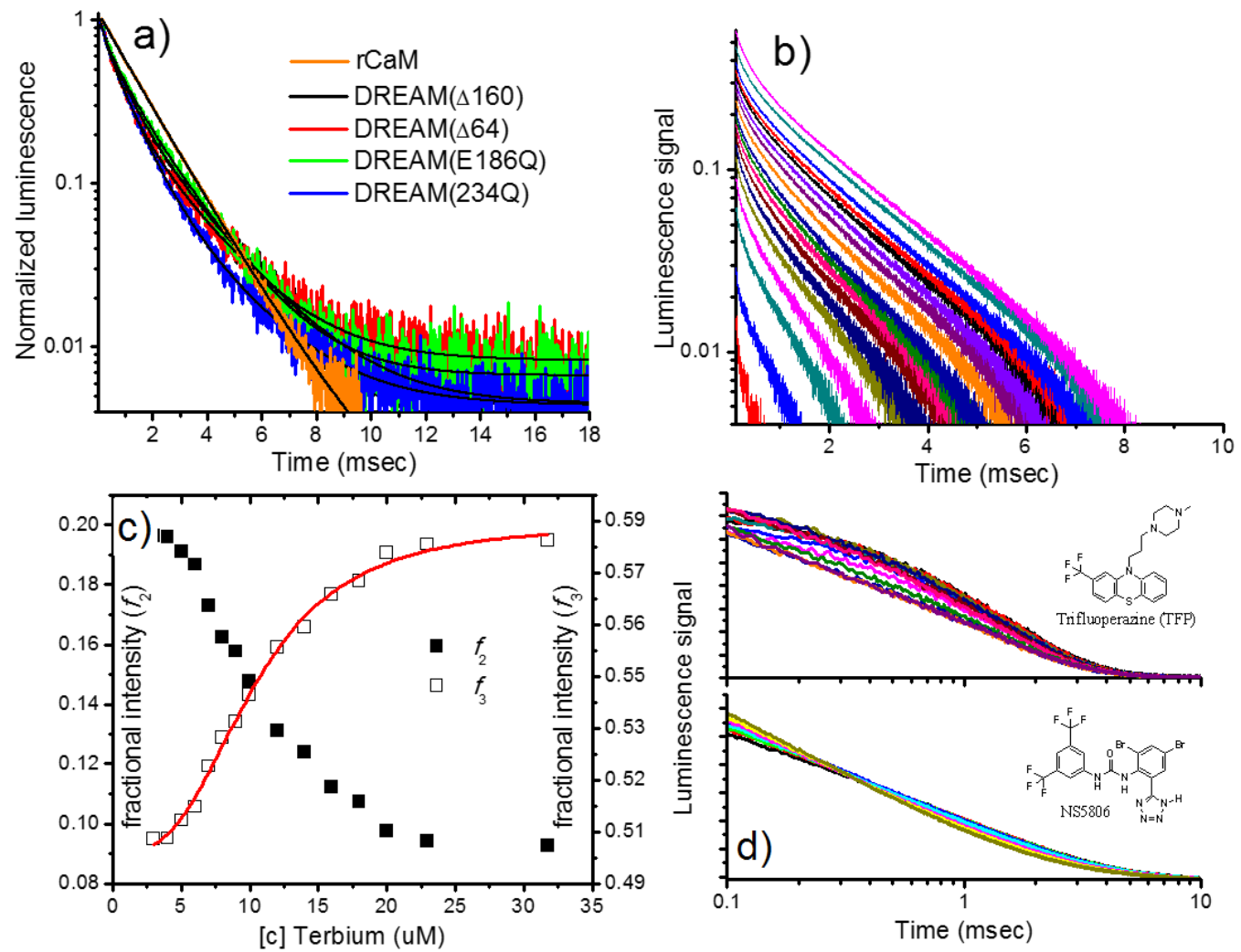

Figure 6.6: (a) Luminescence decay of $\mathrm{Tb}^{3+}$ bound to CaM and constructs of DREAM, with stoichiometric ratios of $4: 1 \mathrm{~Tb}^{3+}: \mathrm{CaM}$ and $2: 1 \mathrm{~Tb}^{3+}$ :DREAM. Solid lines represent the best fit using Globals software analysis, recovered parameters listed in Table 3. (b) Luminescence intensity decay profiles as a function of $\mathrm{Tb}^{3+}$ binding to $10 \mu \mathrm{M}$ $\operatorname{DREAM}(\Delta 64)$. The luminescence decays in (b) were analyzed using a triple discrete model and the fractional intensity contribution of the two slowest lifetimes is shown in (c). The fastest decay component had a lifetime of $\sim 80 \mu$ s with less than $10 \%$ contribution and is not shown. (d) Luminescence decay $\mathrm{Tb}^{3+}$ bound to DREAM and DREAM $(\Delta 64)$ as a function of the hydrophobic molecules TFP (top) bound to DREAM and NS5806 (bottom) bound to DREAM.

Luminescence decay of $\mathrm{Tb}^{3+}$ bound to $\operatorname{DREAM}(\Delta 65)$ shows lifetimes of $\tau_{1}=0.86$ ms $(59 \%)$ and $\tau_{2}=2.20 \mathrm{~ms}(14 \%)$, whereas $\mathrm{Tb}^{3+}$ bound to $\operatorname{DREAM}(\Delta 160)$ decays with lifetimes of $\tau_{1}=0.74 \mathrm{~ms}(54 \%)$ and $\tau_{2}=2.16 \mathrm{~ms}$ ( $\left.29 \%\right)$. The faster lifetime likely corresponds to contribution from partially coordinated $\mathrm{Tb}^{3+}$ ions, and is similar to the 780 
$\mu$ decay obtained for $\mathrm{Tb}^{3+}$ bound to NTA in water (Horrocks, Sudnick 1979). We and others have determined the lifetime of $\mathrm{Tb}^{3+}$ in buffer at $\mathrm{pH} 7.4$ to be $450 \pm 30 \mu \mathrm{s}$; therefore, we associate $\tau_{1}$ to represent $\mathrm{Tb}^{3+}$ bound to a low coordination site on DREAM (Bunzli, Eliseeva 2011). The second lifetime is much longer than that determined for $\mathrm{Tb}^{3+}$ bound to parvalbumin (1.3 ms) or CaM (1.38 ms) but is similar to that of $\mathrm{Eu}^{3+}$ bound to these proteins or complexes of $\mathrm{Tb}^{3+}$ and bimetallic ligand in which no water coordination is observed (Mulqueen, Tingey \& Horrocks 1985, Horrocks, Sudnick 1979, Deiters et al. 2009). Longlived excited state decays are observed for lanthanides with little phonon quenching which often is due to limited water access (Bunzli, Eliseeva 2011). Interestingly, addition of 100 $\mathrm{mM} \mathrm{NaCl}$ reduces the fast lifetime to values similar to those of $\mathrm{Tb}^{3+}$ in water and increases the fractional intensity of the long lifetime component $\sim$ three-fold (Table 6.3). The luminescence decay of $\mathrm{Tb}^{3+}$ was not significantly affected by LDAO, a detergent which has been proposed to stabilize DREAM in a single conformation (Osawa et al. 2001). Titration of $\mathrm{Tb}^{3+}$ into DREAM $(\Delta 65)$ in the presence of $100 \mathrm{mM} \mathrm{NaCl}$ and $10 \mathrm{mM}$ LDAO shows that the fractional intensity of the $\tau_{2}$ lifetime increases in a dose dependent manner and saturates at a stoichiometric ratio of two to one, whereas the fractional intensity of $\tau_{1}$ decreases (Figure 6.6b and 6.6c).

Additional measurements of the luminescence decay of DREAM construct with inactivated EF-hands show similar bimodal exponential decays. One of the most salient observations is that the decay lifetime of DREAM(E234Q) is significantly shorter than that observed for DREAM(E186Q), $\tau_{2}=1.75 \mathrm{~ms}(27 \%)$ vs $\tau_{2}=2.02 \mathrm{~ms}$ (46 \%), respectively. This indicates that binding of $\mathrm{Tb}^{3+}$ at EF-hand 4 induces a restructuring of the coordination sphere similar to that induced when both EF-hand 3 and 4 are bound to $\mathrm{Tb}^{3+}$. On the other 
hand, the structural arrangement of the binding loop at EF-hand 3 when $\mathrm{Tb}^{3+}$ is bound at this site is significantly different, with a much more solvent exposed $\mathrm{Tb}^{3+}$ ion. However, it could also be possible that these two lifetimes cannot be separately resolved in the $\operatorname{DREAM}(\Delta 65)$ decays.

Table 6.3. Terbium(III) luminescence decay parameters recovered upon binding to DREAM and DREAM constructs

\begin{tabular}{|c|c|c|c|c|}
\hline & $\tau_{1}(\mathrm{~ms})$ & $f_{1}$ & $\tau_{2}(\mathrm{n}$ & $f_{2}$ \\
\hline CaM & 1.38 & 1.00 & & \\
\hline CaM + TFP* & 46 & 0.31 & 1.36 & 0.69 \\
\hline DREAM $(\Delta 64)$ & 0.86 & 0.59 & 2.22 & 0.14 \\
\hline $\operatorname{DREAM}(\Delta 64)+\mathrm{Mg}^{2+}$ & 74 & 0.54 & 2.05 & 0.24 \\
\hline $\operatorname{DREAM}(\Delta 64)(a)^{\ddagger}$ & .58 & 0.27 & 2.00 & 0.69 \\
\hline $\operatorname{DREAM}(\Delta 64)(b)^{\ddagger}$ & 0.46 & 0.17 & 2.04 & 0.77 \\
\hline $\begin{array}{l}\operatorname{DREAM}(\Delta 64)(c)^{\ddagger}+ \\
\text { NS5806 }\end{array}$ & 0.43 & 0.58 & 1.83 & 0.39 \\
\hline DREAM $(\Delta 160)$ & 0.75 & 0.47 & 2.16 & 0.29 \\
\hline DREAM $(\Delta 160)+\mathrm{N}$ & 0.63 & 0.41 & 2.03 & 0.37 \\
\hline DREAM (E186Q) & 0.62 & 0.50 & 2.02 & 0.27 \\
\hline DREAM (E186Q) + & 0.48 & 0.47 & 1.58 & 0.42 \\
\hline DREAM (E234Q) & 0.63 & 0.27 & 1.75 & 0.46 \\
\hline DREAM (E234Q) $+\mathrm{Mg}^{2+}$ & 0.45 & 0.31 & 1.70 & 0.48 \\
\hline \multicolumn{5}{|c|}{ 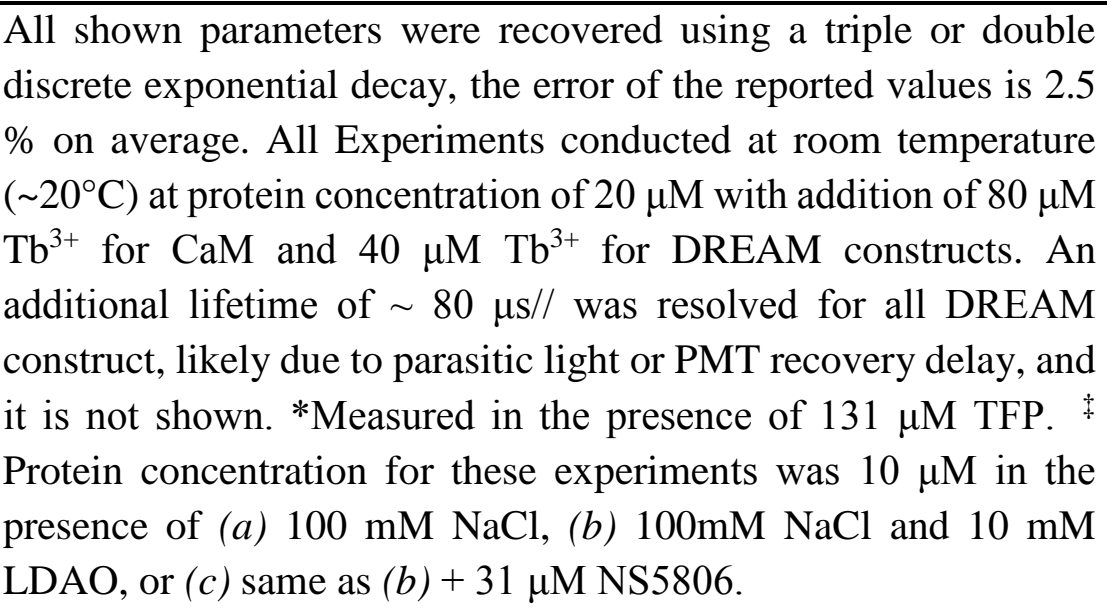 } \\
\hline
\end{tabular}


The lifetime of $\mathrm{Tb}^{3+}$ bound at the solvent exposed site is identical on both constructs, with $\tau_{1}=0.62 \mathrm{~ms}(50 \%)$ for DREAM(E186Q) and $\tau_{1}=0.63 \mathrm{~ms}(27 \%)$ for DREAM(E234Q), but faster than that observed for the fully active proteins. Interestingly, in the presence of $5 \mathrm{mM} \mathrm{Mg}^{2+}$ a decrease in the decay lifetimes and increase of intensity contribution of the long-lived decay is observed for all constructs, except for DREAM(E234Q). The observed changes are largest for DREAM(E186Q) where a decrease of $0.44 \mathrm{~ms}$ in $\tau_{2}$ is also accompanied by a $15 \%$ increase in contribution of this decay component. The decay lifetimes of $\tau_{1}=0.74 \mathrm{~ms}$ for $\operatorname{DREAM}(\Delta 65)$ and $\tau_{1}=0.63 \mathrm{~ms}$ for $\operatorname{DREAM}(\Delta 160)$ are still longer than for $\mathrm{Tb}^{3+}$ in water, while those of DREAM(E186Q) and DREAM(E234Q) are identical to that of $\mathrm{Tb}^{3+}$ in water. The effect of $\mathrm{Mg}^{2+}$ on the luminescent data is in good agreement with the changes observed in the sensitized emission titrations (Figure 6.3d).

Finally, we examined whether $\mathrm{Tb}^{3+}$ luminescence decay would be sensitive to structural changes induced on CaM and DREAM upon binding of small hydrophobic ligands. To test this hypothesis we chose trifluoperazine (TFP) and a novel biphenyl-urea compound named NS5806. These compounds have been shown to bind at hydrophobic cavities on CaM and DREAM, respectively, in a calcium dependent manner (Gonzalez, Pham \& Miksovska 2014, Cook, Walter \& Walter 1994). Upon titration of each ligand into $\mathrm{Tb}^{3+}$ bound CaM and DREAM $(\Delta 65)$ we observe a strong dose dependent modulation of the luminescence decay (Figure 6.6d). Global analysis of the luminescence decay of the $\mathrm{Tb}^{3+} \mathrm{CaM}$ and $\mathrm{Tb}^{3+} \operatorname{DREAM}(\Delta 65)$ complexes reveals that binding of these small hydrophobic ligands induces a decrease in intensity contribution from $\tau_{2}$ on both construct while also decreasing the long lifetime on $\operatorname{DREAM}(\Delta 65)$. Plotting the change in $f_{2}$ as a function of TFP or NS5806 yields dissociation constants of $\sim 37 \mu \mathrm{M}$ for both ligands (data 
not shown), which is larger than those observed in the $\mathrm{Ca}^{2+}$ bound form of these proteins $\left(\mathrm{K}_{\mathrm{d}} \sim 1 \mu \mathrm{M}\right.$ for TFP and $\mathrm{K}_{\mathrm{d}}=5 \mu \mathrm{M}$ for NS5806). The discrepancies could be due to secondary sites being populated (TFP has been shown to bind at four sites on CaM with dissociation constants between $1 \mu \mathrm{M}$ and $5 \mathrm{mM}$ ), as well as due to distinct conformation of the hydrophobic cavity of the proteins in the $\mathrm{Tb}^{3+}$ bound form.

6.2.5 Displacement of $\mathrm{Ca}^{2+}$ by $\mathrm{Tb}^{3+}$ induces minimal changes in collisional cross section of DREAM

To further study the displacement of $\mathrm{Ca}^{2+}$ ions from the EF-hand domains of DREAM, as well as to determine the magnitude of the structural differences between the $\mathrm{Ca}^{2+}$ and $\mathrm{Tb}^{3+}$ bound DREAM structures, ion mobility measurements were conducted to determine the ion-neutral collisional cross section using a TIMS-MS analyzer (see Table 6.4).

\begin{tabular}{|c|c|c|c|}
\hline \multicolumn{4}{|c|}{$\begin{array}{l}\text { Table 6.4. Experimental and theoretical ion-neutral } \\
\text { collision cross section for the } \operatorname{DREAM}(\Delta 64): \mathrm{Ca}^{2+} / \mathrm{Tb}^{3+} \\
\text { molecular ions. }\end{array}$} \\
\hline Theoretical $^{a}$ & $\begin{array}{c}+7 \\
\left(\AA^{2}\right)\end{array}$ & $\begin{array}{c}+\mathbf{+ 8} \\
\left(\AA^{2}\right)\end{array}$ & $\begin{array}{c}+\mathbf{+ 9} \\
\left(\AA^{2}\right)\end{array}$ \\
\hline DREAM $(\Delta 64): \mathrm{Ca}^{2+} \mathrm{Ca}^{2+}$ & 2773 & 2798 & 2825 \\
\hline \multicolumn{4}{|l|}{ Experimental } \\
\hline $\operatorname{DREAM}(\Delta 64): \mathrm{Ca}^{2+} \mathrm{Ca}^{2+}$ & 2225 & 2243 & 2297 \\
\hline $\operatorname{DREAM}(\Delta 64): \mathrm{Ca}^{2+} \mathrm{Tb}^{3+}$ & 2205 & 2231 & 2260 \\
\hline $\operatorname{DREAM}(\Delta 64): \mathrm{Tb}^{3+} \mathrm{Tb}^{3+}$ & 2200 & 2228 & 2238 \\
\hline
\end{tabular}

The mass spectrum of DREAM $(\Delta 65)$ under native nanoESI conditions shows a narrow charge state distribution $(+7-+9)$, with multiple $\mathrm{Ca}^{2+}$ and $\mathrm{Tb}^{3+}$ adducts. A closer look at 
the charge distribution shows the presence of the apo form $[\mathrm{M}+\mathrm{nH}]^{+\mathrm{n}}$ as well as three adduct series: $\left[\mathrm{M}+\mathrm{Ca}^{2+}{ }_{\mathrm{x}=1-3}+(\mathrm{n}-\mathrm{x}) \mathrm{H}\right]^{+\mathrm{n}},\left[\mathrm{M}+\mathrm{Ca}^{2+}{ }_{\mathrm{x}}+\mathrm{Tb}^{3+}{ }_{\mathrm{y}}+(\mathrm{n}-\mathrm{x}-\mathrm{y}) \mathrm{H}\right]^{+\mathrm{n}}$ and $[\mathrm{M}+$ $\left.\mathrm{Tb}^{3+}{ }_{\mathrm{x}=1-2}+(\mathrm{n}-\mathrm{x}) \mathrm{H}\right]^{+\mathrm{n}}$. (see example of the +8 charge state distribution of DREAM $(\Delta 65)$ in the presence of $\mathrm{Ca}^{2+}, \mathrm{Ca}^{2+}$ and $\mathrm{Tb}^{3+}$, and $\mathrm{Tb}^{3+}$ in Figure 6.7a). Inspection of the mobility profiles for each adduct series show that as the charge state increases from +7 to +9 , a decrease in mobility (and increase in CCS) is observed as a consequence of the interaction of the molecular ion with the external electric field, and not necessarily an indication of conformational changes.
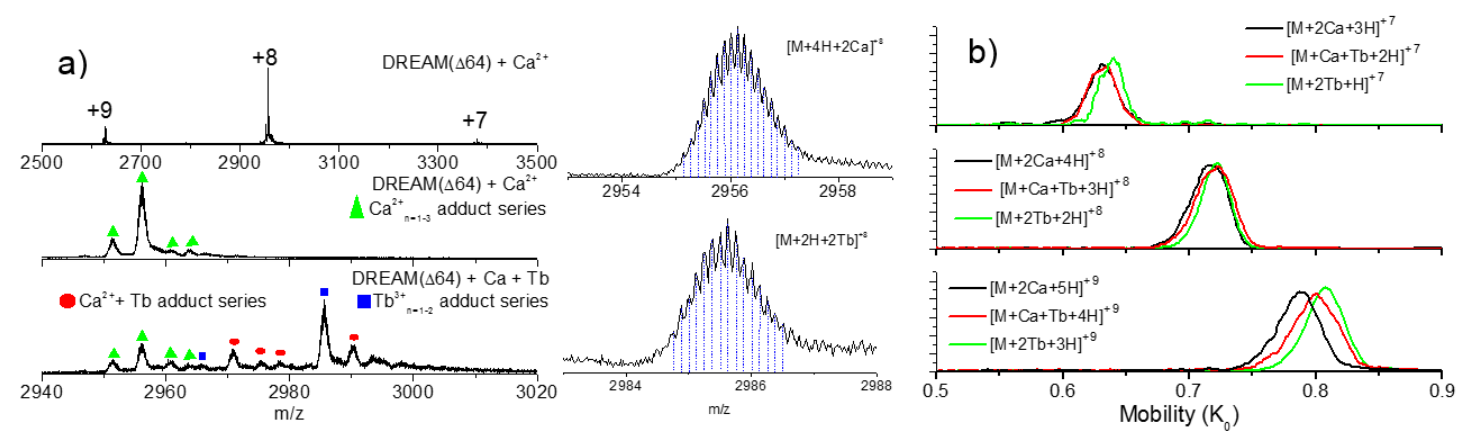

Figure 6.7: (a) Typical nanoESI mass spectra of DREAM $(\Delta 64)$ in the presence of $\mathrm{Ca}^{2+}$ and of DREAM $(\triangle 64)$ in the presence of $\mathrm{Ca}^{2+}$ and $\mathrm{Tb}^{3+}$. The isotopic distribution of the $\operatorname{DREAM}(\Delta 64): 2 \mathrm{Ca}^{2+}$ and DREAM $(\Delta 64): 2 \mathrm{~Tb}^{3+}$ are shown for clarity. (b) NanoESI TIMS mobility spectra of DREAM $(\Delta 64)$ bound to $\mathrm{Ca}^{2+}$ and/or $\mathrm{Tb}^{3+}$.

However, small changes in the CCS are observed as a function of the adduct series within each charge state. For example, a decrease in the CCS is observed for DREAM $(\Delta 65)$ bound to $\mathrm{Tb}$ when compare to $\operatorname{DREAM}(\Delta 65)$ bound to $\mathrm{Ca}^{2+}$ and $\operatorname{DREAM}(\Delta 65)$ bound to $\mathrm{Ca}^{2+}+\mathrm{Tb}^{3+}$ (see Figure 6.7b). Comparison of the observed CCS profiles $\left(\mathrm{CCS}_{+7-+9}=2200-\right.$ $2300 \AA^{2}$ ) to that of a previously reported 2JUL NMR structure of DREAM (Lusin et al. 2008) $\left(\mathrm{CCS}_{\text {theo }}=2773 \AA^{2}\right)$ suggest that the gas-phase conformations are more compact to that observed in solution, probably as a consequence of the adduct interaction with the EFhands domains in the absence of the solvent. In addition, the higher affinity of $\mathrm{Tb}^{3+}$ when 
compared to $\mathrm{Ca}^{2+}$ may induce a more compact structure (smaller CCS) for the $\operatorname{DREAM}(\Delta 65)$ bound to $\mathrm{Tb}^{3+}$ when compare to $\operatorname{DREAM}(\Delta 65)$ bound to $\mathrm{Ca}^{2+}$ and $\operatorname{DREAM}(\Delta 65)$ bound to $\mathrm{Ca}^{2+}+\mathrm{Tb}^{3+}$ in the absence of the solvent.

\subsection{Discussion}

In this report we present conclusive evidence that $\mathrm{Tb}^{3+}$ is able to bind at the $\mathrm{C}$ terminus of DREAM and displace $\mathrm{Ca}^{2+}$ from the binding loop at the EF-hands 3 and 4 . Using circular dichroism as well as fluorescence intensity and anisotropy decay of intrinsic fluorescent probes, we demonstrate that $\mathrm{Tb}^{3+}$ is able to induce identical structural changes on DREAM as those observed upon $\mathrm{Ca}^{2+}$ binding. Circular dichroism shows a small deviation of the spectra near $208 \mathrm{~nm}$, a region which is sensitive to the presence of antiparallel $\beta$-sheets (Kelly, Jess \& Price 2005b). DREAM is mainly alpha helical, and the only region where a small antiparallel $\beta$-sheets are formed is between the metal binding loops of each EF-hand pairs (Lusin et al. 2008). Therefore, it is possible that association of $\mathrm{Tb}^{3+}$ induces structural changes at the metal binding loop results in loss of these short $\beta$-sheet regions. Small differences between $\mathrm{Tb}^{3+}$ and $\mathrm{Ca}^{2+}$ bound DREAM, were also observed by anisotropy decay measurements. Anisotropy decay revealed that dimers are formed either in the $\mathrm{Ca}^{2+}$ or $\mathrm{Tb}^{3+}$ bound form of DREAM, but binding of $\mathrm{Tb}^{3+}$ induces a more dynamic environment near Trp169. Using molecular dynamics, we have previously shown that Trp169 in DREAM $(\Delta 65)$ can populate two rotamers, and it is possible that $\mathrm{Tb}^{3+}$ binding could enhance this rotamer transition, which would result in an increase of local dynamics (Pham et al. 2015).

Similarly to other calcium binding proteins, we observe that aromatic amino acids at the C-terminus are able to transfer energy to the bound $\mathrm{Tb}^{3+}$. Specifically, we show that 
mutation of Y174 to alanine results in a significant reduction ( $\sim 6 \%$ \%) in the sensitized emission of $\mathrm{Tb}^{3+}$ at $545 \mathrm{~nm}$, while maintaining identical 280/295 ratios compared to $\operatorname{DREAM}(\Delta 65)$. Together, these results highlight the presence of an antenna effect, in which $\mathrm{W} 169$ is the main donor to $\mathrm{Tb}^{3+}$. Attempts to use sensitized emission and $\mathrm{Tb}^{3+}$ titrations in order to elucidate the lanthanide binding sequence to EF-hands 3 and 4 on DREAM indicate that both EF-hands bind $\mathrm{Tb}^{3+}$ with similar affinity (DREAM(E234Q) with $\mathrm{K}_{\mathrm{d}}=$ 5.9 $\mu \mathrm{M}$ and DREAM(E186Q) with $\left.\mathrm{K}_{\mathrm{d}}=11 \mu \mathrm{M}\right)$. Since these experiments were conducted in the presence of excess calcium and magnesium, inactivation of each EF-hand actually favors binding to the mutated loop (see ITC results). Therefore, we are unable to identify whether the slightly lower affinity of $\mathrm{Tb}^{3+}$ with the DREAM(E186Q) mutant is due to inherent lower affinity to this hand or because a metal ion $\left(\mathrm{Ca}^{2+}\right.$ or $\left.\mathrm{Mg}^{2+}\right)$ is loosely bound at this mutated site. Nonetheless, one of the most salient observations of these $\mathrm{Tb}^{3+}$ titration experiments is that in the presence of $\mathrm{Mg}^{2+}$, the sensitized emission of $\mathrm{Tb}^{3+}$ decreases by more than $50 \%$. This decrease in intensity at $545 \mathrm{~nm}$, could be due to either $\mathrm{Mg}^{2+}$ induced structural rearrangements of the environment near Trp169 or to rearrangement of the $\mathrm{Tb}^{3+}$ binding loop at EF-hands 3 and 4. Indeed, we observe that EF-hand 3, which is next to EFhand 2, is more sensitive to magnesium binding. These results support the idea that $\mathrm{Mg}^{2+}$ plays a structural role on DREAM and that at physiological concentrations it may act as a functional cofactor through interaction with EF-hand 2.

Isothermal calorimetry experiments show that $\mathrm{Tb}^{3+}$ displacement of $\mathrm{Ca}^{2+}$ from EFhands 3 and 4 on $\operatorname{DREAM}(\Delta 65)$ in the presence of $5 \mathrm{mM} \mathrm{Mg}^{2+}$ is associated with a $\Delta \mathrm{G}=$ $-14 \mathrm{kcal} \cdot \mathrm{mol}^{-1}$, while the displacement energy for the DREAM $(\Delta 160)$ construct is $\Delta \mathrm{G}=$ $12 \mathrm{kcal} \cdot \mathrm{mol}^{-1}$. The same displacement process for EF-hands 1 through 4 in $\mathrm{CaM}$ is 
associated a free energy of $\Delta \mathrm{G}=-18 \mathrm{kcal} \cdot \mathrm{mol}^{-1}$. The more favorable displacement of $\mathrm{Ca}^{2+}$ from CaM EF-hands compared to $\operatorname{DREAM}(\Delta 65)$ highlights the stronger association of $\mathrm{Ca}^{2+}$ in the latter (Osawa et al. 2005). Throughout this report we correlate the $\mathrm{K}_{1}$ thermodynamic parameters with displacement of $\mathrm{Ca}^{2+}$ from EF-hand 3 and $\mathrm{K}_{2}$ with displacement from EF-hand 4 in the $\operatorname{DREAM}(\Delta 65)$, DREAM(E186Q), and DREAM(E234Q) constructs. Even thought, these correlations are not unequivocally proven by our results, they are supported by the associated changes in enthalpy and entropy under different conditions. Following this assignment, we can approximate the enthalpy and entropy associated with coordination of $\mathrm{Tb}^{3+}$ by Glu at position 12 of EF-hand 3 and 4 by calculating $\Delta \mathrm{H}_{\mathrm{Glu}}{ }^{\text {(Mutant) }}=\Delta \mathrm{H}_{\mathrm{i}}^{\operatorname{DREAM}(\Delta 65)}-\Delta \mathrm{H}_{\mathrm{i}}{ }^{\mathrm{DREAM}(M u t a n t)}$ and $\mathrm{T} \Delta \mathrm{S}_{\mathrm{Glu}}$ (Mutant) $=$ $\mathrm{T} \Delta \mathrm{S}_{\mathrm{i}}{ }^{\text {DREAM( } \triangle 65)}-\mathrm{T} \Delta \mathrm{S}_{\mathrm{i}}{ }^{\text {DREAM(Mutant) }}$. Where the subscript $\mathrm{i}$ is 1 for DREAM(E186Q) and $\mathrm{i}$ = 2 for DREAM(E234Q). These calculations reveal that coordination of $\mathrm{Tb}^{3+}$ by Glu186 of EF-hand 3 is associated with $\Delta \mathrm{H}_{\mathrm{Glu}}{ }^{(\mathrm{E} 186 \mathrm{Q})}=4.5 \mathrm{kcal} \cdot \mathrm{mol}^{-1}$ and $\mathrm{T} \Delta \mathrm{S}_{\mathrm{Glu}}{ }^{(\mathrm{E} 186 \mathrm{Q})}=5.0$ $\mathrm{kcal} \cdot \mathrm{mol}^{-1}$. In contrast, Glu234 of EF-hand 4 has a three-fold larger enthalpy and entropy contribution, with $\Delta \mathrm{H}_{\mathrm{Glu}}{ }^{(\mathrm{E} 234 \mathrm{Q})}=14 \mathrm{kcal} \cdot \mathrm{mol}^{-1}$ and $\mathrm{T} \Delta \mathrm{S}_{\mathrm{Glu}}{ }^{(\mathrm{E} 234 \mathrm{Q})}=15 \mathrm{kcal} \cdot \mathrm{mol}^{-1}$. In both cases, coordination of the metal ion is driven by favorable entropy contributions, likely due to release of a water molecule upon coordination of Glu at position 12. These results are similar to those observed for $\mathrm{Ca}^{2+}$ binding to $\mathrm{CaM}$ and $\mathrm{DREAM}(\Delta 65)$ in which entropy was observed to be the main driving force (Osawa et al. 2005, Osawa et al. 2005, Gilli et al. 1998). In contrast, the enthalpic contribution due to metal binding to the EF-hands of $\mathrm{CaM}$ and $\operatorname{DREAM}(\Delta 65)$ has been shown to be very small. It is possible that the unfavorable endothermic process observed here, is due to additional structural changes associated with Glu-12 coordination. The larger enthalpy and entropy associated with coordination of Glu- 
12 on EF-hand 4 highlights the role of this EF-hand in controlling the activation of DREAM. Indeed, Glu-12 is positioned on the exiting helix of EF-hand 4 which is immediately adjacent to a hydrophobic cavity that mediates the calcium regulated coassembly with potassium channels and small ligands (Gonzalez, Pham \& Miksovska 2014, Pioletti et al. 2006, Wang et al. 2007).

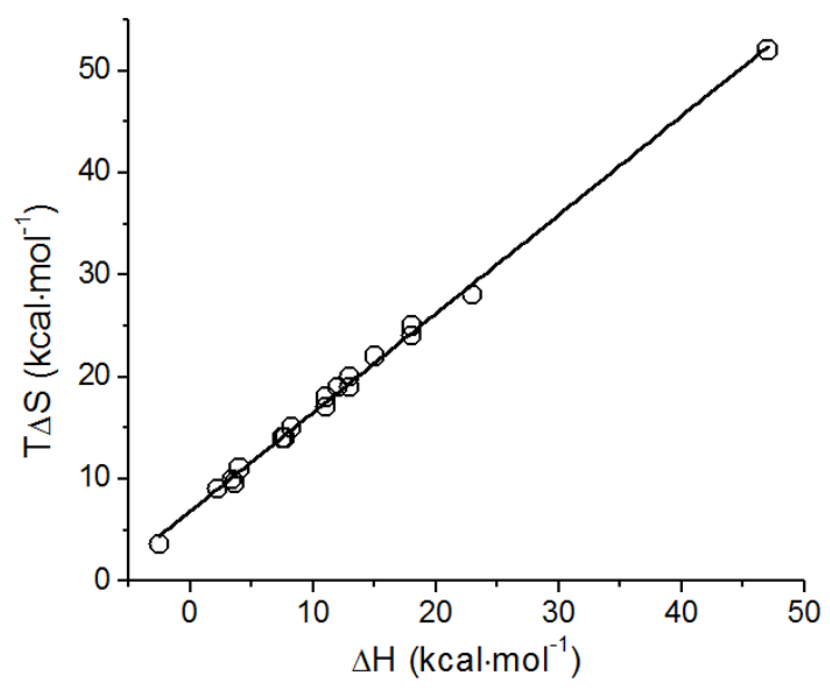

Figure 6.8: Plot of $\mathrm{T} \Delta \mathrm{S}$ versus $\Delta \mathrm{H}$ for $\mathrm{Tb}^{3+}$ displacement of $\mathrm{Ca}^{2+}$ from $\operatorname{DREAM}(\Delta 64)$, $\operatorname{DREAM}(\Delta 160)$, DREAM(E186Q), DREAM(E234Q) and CaM. Solid line represents the best linear fit to the data.

Another interesting aspect of the recovered thermodynamic parameters shown in table 2 is that the enthalpy and entropy recovered for all the titrations are highly correlated. This correlation has been observed before for $\mathrm{Ca}^{2+}$ binding to $\mathrm{CaM}$ (Gilli et al. 1998) as well as in other systems (Kuroki, Yutani 1998). More importantly, the linear relationship of $\mathrm{T} \Delta \mathrm{S}$ as a function of $\Delta \mathrm{H}$ allow us to extrapolate the entropy associated with displacement of $\mathrm{Ca}^{2+}$, prior to any structural change induced by $\mathrm{Tb}^{3+}$ (Figure 6.8). This is based on the idea that displacement of $\mathrm{Ca}^{2+}$ by $\mathrm{Tb}^{3+}$ is not associated with the formation or breakage of any new bond since the coordination of $\mathrm{Ca}^{2+}$ and $\mathrm{Tb}^{3+}$ in bulk water are identical. Extrapolating the linear relationship yields a value of $\mathrm{T} \Delta \mathrm{S}_{\text {displacement }}=6.9 \pm 0.2$ 
$\mathrm{kcal} \cdot \mathrm{mol}^{-1}$, which is similar to the value obtained for $\mathrm{Ca}^{2+}$ binding to metal free CaM in which $\mathrm{T} \Delta_{\text {sbind }}=7.2 \pm 0.1 \mathrm{kcal} \cdot \mathrm{mol}^{-1}$ (Gilli et al. 1998). A possible explanation for the entropic gain, could be due to an increase in the dynamics of the protein, something which is supported by the anisotropy decay data. The correlation of enthalpy and entropy can also be explained by a process which involves the release of water following the association of two ions of opposite charges in solution. The resulting charge neutralization would facilitate the mobilization of solvent molecules from the surface of the protein into the bulk water (Bowman-James, Bianchi \& García-Espana 2012). This release of water molecules is associated with an unfavorable endothermic process (breakage of water-protein hydrogen bonds) and favorable positive change in entropy. Indeed, $\mathrm{Ca}^{2+}$ bound $\operatorname{DREAM}(\Delta 65)$ (net charge -3 at $\mathrm{pH}$ 7.4) and $\mathrm{Ca}^{2+}$ bound $\mathrm{CaM}$ (net charge -16 at $\mathrm{pH}$ 7.4) would be neutralized by 2 and 8 units upon $\mathrm{Tb}^{3+}$ displacement, respectively. This charge neutralization effect, could also partially account for the decreased enthalpy and entropy contribution of the DREAM(E186Q) and DREAM(E234Q) mutants, both of which have one less negative charge compared to $\operatorname{DREAM}(\Delta 65)$.

Sensitized emission, circular dichroism, TIM-MS, and ITC experiment provide information on global structural changes of the protein upon displacement of $\mathrm{Ca}^{2+}$ by $\mathrm{Tb}^{3+}$. On the other hand, luminescent studies allow us to gain a better insight into the immediate coordination sphere of $\mathrm{Tb}^{3+}$ ion, and how this environment is affected by inactivation of EF-hands, $\mathrm{Mg}^{2+}$ binding, ionic strength, and/or ligand binding. Measurements of the luminescent decay of $\mathrm{Tb}^{3+}$ bound to different constructs highlight the idea that $\mathrm{Tb}^{3+}$ bound at EF-hand 3 is more solvent exposed than $\mathrm{Tb}^{3+}$ bound at EF-hand 4. Nonetheless, the decay observed for DREAM $(\Delta 65)$ is much slower than that observed for CaM, indicating 
that the coordination around $\mathrm{Tb}^{3+}$ in DREAM highly restricts the accessibility of water. We also observe that the luminescence decays are widely affected by addition of excess $\mathrm{Mg}^{2+}$, supporting the idea that binding of $\mathrm{Tb}^{3+}$ at some secondary sites, including EF-hand 2 on DREAM, are identical to those for $\mathrm{Mg}^{2+}$ binding. The high sensitivity of EF-hand 3 to $\mathrm{Mg}^{2+}$ binding could also be due to propagated rearrangement of EF-hand 2 upon association of $\mathrm{Mg}^{2+}$. It is also possible that binding of $\mathrm{Mg}^{2+}$ at these secondary sites is responsible for the observed rearrangement of the EF-hand loops. The overall picture that emerges from these observations is that $\mathrm{Mg}^{2+}$ is able to modulate the protein structure and that secondary metal sites may play a role in modulating protein conformation. Indeed, previous work and our unpublished results support the idea that two $\mathrm{Ca}^{2+}$ specific sites and additional secondary sites are found on $\operatorname{DREAM}(\Delta 160)$ (Craig et al. 2002). Lastly, titration of small hydrophobic ligands also reveal that the environment near the metal binding loops of EF-hand 3 and 4 are sensitive to ligand association with DREAM. Altogether, these observations highlight the idea that association of these small molecules at the hydrophobic surfaces of CaM and DREAM induce conformational changes which can not only distort the coordination geometry of $\mathrm{Tb}^{3+}$ at the binding loops, but could also potentially facilitate $\mathrm{Tb}^{3+}$ dissociation from the protein.

Finally, ion mobility and mass spectrometry measurement support the hypothesis that $\mathrm{Tb}^{3+}$ can displace $\mathrm{Ca}^{2+}$ from EF-hand 3 and 4 and that the resulting folded conformation of the $\operatorname{Tb}^{3+}$ bound $\operatorname{DREAM}(\Delta 65)$ protein is almost identical to that of the calcium bound protein during native ESI conditions. Comparison of the observed CCS profiles to that of a previously reported NMR structure of DREAM (Lusin et al. 2008) suggested that the gas-phase conformations are more compact to that observed in solution, 
probably as a consequence of the adduct interaction with the EF-hands domains in the absence of the solvent. In addition, the higher affinity of $\mathrm{Tb}^{3+}$ when compared to $\mathrm{Ca}$ resulted in more compact structures (smaller CCS) for the $\operatorname{DREAM}(\Delta 65)$ bound to $\mathrm{Tb}^{3+}$ when compare to $\operatorname{DREAM}(\Delta 65)$ bound to $\mathrm{Ca}^{2+}$ and $\operatorname{DREAM}(\Delta 65)$ bound to $\mathrm{Ca}^{2+}+\mathrm{Tb}^{3+}$ in the absence of the solvent.

\subsection{Summary}

In this chapter we show compelling evidence supporting the specific association of $\mathrm{Tb}^{3+}$ with the EF-hands 3 and 4 of DREAM. We also demonstrate that replacement of $\mathrm{Ca}^{2+}$ by $\mathrm{Tb}^{3+}$ leads to an increase in the dynamics of the protein; however, the structural and functional properties between DREAM bound to either metal are highly similar. We show that similarly to $\mathrm{CaM}, \mathrm{Tb}^{3+}$ bound to DREAM can be sensitized by aromatic amino acids at the C-terminus, with tryptophan 169 being the main energy donor. The high affinity of the EF-hands for $\mathrm{Tb}^{3+}$ and the fluorescent properties of this lanthanide has allowed us to highlight the role of $\mathrm{Mg}^{2+}$ as a structural cofactor, which can bind to EF-hand 2 and modify the immediate environment near the calcium binding loops of EF-hands 3 and 4. Isothermal calorimetry also highlights the role of EF-hand 4 in mediating calcium regulated ligand recognition in DREAM. These findings provide structural information on DREAM and will facilitate future structural NMR studies and lanthanide resonance energy transfer experiments aimed at studying the association of DREAM with other proteins. 


\section{PHOTOTHERMAL BEAM DEFLECTION TO STUDY THE DYNAMICS OF DIVALENT ION-CAGE AND ION-PROTEIN COMPLEXES}

\subsection{Background and significance}

Fluorescence spectroscopy has proven to be exceptionally useful to obtain detailed kinetic data for ligand induced structural changes in proteins. Spectroscopic techniques allow the study of processes from a distance, often with minimal perturbation of the system. In fact, the use of light in science is so pervasive, that there are few techniques today which do not relay on it. Current development in fluorescence microscopy have allowed the study of brain processes in freely behaving animal (Ghosh et al. 2011, Markowitz et al. 2015), cellular processes beyond the diffraction limit (Betzig et al. 2006), and biomolecular dynamic at the single-molecule level (Roy, Hohng \& Ha 2008, Lee et al. 2005). Nonetheless, the need of efficient fluorophores and their lack of stability are often a major limitation in these studies (Cordes, Blum 2013, Schermelleh, Heintzmann \& Leonhardt 2010). A simplified Jablonski-Perrin diagram highlights two competing pathways following excitation of a chromophore (Figure 7.1).

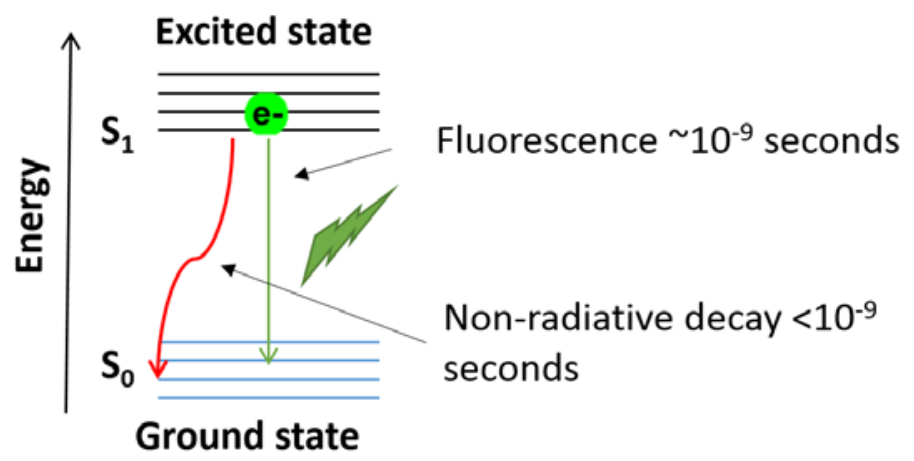

Figure 7.1. Jablonski-Perrin diagram highlighting the two major pathways by which the excited state of a chromophore can be depopulated. 
An ideal fluorophore would not only need to have a large probability of absorbing a photon (a large extinction coefficient, $\varepsilon>80,000 \mathrm{M}^{-1} \mathrm{~cm}^{-1}$ ), but it would also need to have a large probability of emitting this absorbed energy as a photon (a quantum yield near unity). On the other hand, photothermal methods, such as thermal grating (TG) (Fayer 1982), photothermal beam deflection (PBD) (Jackson et al. 1981b), and photoacoustic calorimetry (PAC) (Braslavsky, Heibel 1992) are capable of detecting the non-radiative processes following absorption of energy by a chromophore. An obvious advantage of these techniques, is that they do not require the use of fluorophores. In fact, the best signal would be obtained from a photochemical process in which all the energy absorbed is used only to drive a specific reaction, without the production of a photon. Thus, these techniques require that the photo-triggered process have a large probability of proceeding forward, as in eqn. 7.1:

$$
\begin{aligned}
& A+B \rightarrow A^{*}+B \rightarrow C \\
& \phi=\frac{C}{A^{*}} \leq 1
\end{aligned}
$$

Where A represents the ground state of the reactant and $\mathrm{A} *$ its excited state following laser excitation. These methods rely on the premise that the triggered processed would result in a detectable amount of heat being removed or added from their immediate environment. However, unlike the widely applied isothermal calorimetry titration (ITC) technique, photothermal methods have the advantage of being able to measure fast processes occurring in nanoseconds to milliseconds (Laman, Falvey 1996). This temporal resolution makes these techniques ideally suited to study fast biological processes in proteins with endogenous chromophores involved in photosynthesis (Schulenberg, Gaertner \& Braslavsky 1995) or oxygen transport (Mikšovská, Day \& Larsen 2003). Additional 
approaches to study the activity of proteins have been developed through the use of cagedcompounds (Gurney 1994). In these studies, a fast pulse of light is used to trigger the photochemical reaction of a compound whose byproducts are ligands for the protein of interest.

Despite the fact that all of these techniques measure the heat changes accompanying a photochemical process, their main differences arise from the way in which heat is measured, which in turn controls the temporal resolution of each technique. For instance, in photoacoustic calorimetry, the change in temperature of the environment results in a thermal contraction of the medium which then propagates as an acoustic wave and is measured with a tightly coupled microphone (Braslavsky, Heibel 1992, Astudillo et al. 2010). On the other hand, TG and PBD rely on the use of a probe laser in order to measure changes in diffraction patterns arising from the change in index of refraction of the media triggered by subtle increases in temperature (Fayer 1982, Falvey 1997). The advantage of PBD over TG are twofold: 1) the laser used to generate the transient change in index of refraction (pump laser) does not need to be Gaussian in shape, and 2) the probe beam does not need to be collinear with the pump laser. Furthermore, in PBD, the use of a probe beam allows the measurement of samples at a distance, which allows easy exchange of samples. However, in PAC, care must be taken to minimize changes in the interface between the piezoelectric microphone and the cuvette containing the sample to be measured. In PBD, the time resolution is limited by the detection sensors to be over $10 \mu \mathrm{s}$, compared to the 50 ns resolution of PAC. On long timescales, PBD resolution is limited to less than $\sim 100 \mathrm{~ms}$, dues to significant contribution of heat diffusion, air drift, and vibrations as described below. However, the time resolution of $10 \mu \mathrm{s}-100 \mathrm{~ms}$ is ideally suited to study the 
dynamics of structural changes in proteins. Finally, PBD allows the determination of thermodynamic parameters $(\Delta \mathrm{H}, \Delta \mathrm{V}$, and activation energies) which are not easily determined by other techniques.

Of particular interest is the use of PBD and the photosensitive compound DMnitrophen to study the thermodynamics and dynamics of metal binding to calcium binding proteins. In this chapter, we explore the thermodynamics of ligand release from cagecompounds following ultrafast (5 ns) illumination. In addition, following our previous study on $\mathrm{Tb}^{3+}$ binding to DREAM, we investigate whether this lanthanide can be chelated by DM-nitrophen and photo released upon photolysis. These studies are complemented by PBD experiment where we monitor the enthalpy and volume changes associated with $\mathrm{Ca}^{2+}$ binding to DREAM following photo cleavage of $\mathrm{Ca}^{2+} \mathrm{DM}$-nitrophen complexes.

7.2 Results, theory and instrumentation

The instrumental design and theoretical principles of PBD have been reviewed in (Laman, Falvey 1996, Falvey 1997, Larsen, Mikšovská 2007, Whinnery 1974). Following these reports, here we present a complete derivation of the equations used in PBD as well as the design of a photothermal beam deflection instrument. To derive the theoretical aspect of PBD, we briefly follow the work by Jackson et al. (1981a) and Falvey (1997). The problem involves calculating the deviation of a beam of light passing through a medium in which a temperature gradient is present. The distribution of temperature in a medium at time t after deposition of heat at distance r can be derived from the conservation of energy and the heat conduction equation.

$$
\begin{aligned}
& \frac{\partial T(r, t)}{\partial t}=-\nabla \cdot T(r, t) \overrightarrow{\boldsymbol{u}} \\
& T(r, t) \overrightarrow{\boldsymbol{u}}=-D \overline{\boldsymbol{\nabla}} T(r, t)
\end{aligned}
$$


Where the diffusion constant is $\mathrm{D}=\kappa / \rho \mathrm{Cp}, \mathrm{K}$ is the thermal conductivity, $\rho$ is the density, and $\mathrm{Cp}$ is the heat capacity. Thus, the temperature response at point $\mathrm{r}$ and time $\mathrm{t}$ resulting from a $\delta$ heat source at the origin at time $\mathrm{t}=0$ yields:

$$
T(x, t)=\frac{T_{0}}{2(\pi \kappa t)^{\frac{1}{2}}} e^{-x^{2} / 4 D t} \text { at } T(r, 0)=T_{0} \delta(r)
$$

And in radial coordinates:

$$
T(r, t)=\frac{T_{0}}{8(\pi \kappa t)^{\frac{3}{2}}} e^{-r^{2} / 4 D t}-\infty<r, x<\infty
$$

However, if the heat source does not follow a $\delta$-function and/or there is an additional heat sink or source, then the above equation is not a solution. To include this scenario, the heat diffusion must be modified so that:

$$
\frac{\partial T(r, t)}{\partial t}=D \nabla^{2} T(r, t)+f(r, t)
$$

Where the $f(r, t)$ is due to the additional heat source/sink. This equation can be rearranged so that:

$$
\frac{\partial T(r, t)}{\partial t}-D \nabla^{2} T(r, t)=f(r, t)
$$

Which allows the use Green's theorem, so that if $\boldsymbol{L} u=f$ then $\boldsymbol{L} G=\delta$ where $\boldsymbol{L}=\frac{\partial}{\partial t}-$ $D \nabla^{2}$ and $u=T(r, t)$. This leads to:

$$
\boldsymbol{L} G\left(r^{\prime}, r, t\right)=\delta\left(r^{\prime}\right) \delta\left(r^{\prime}\right) \delta(t)
$$




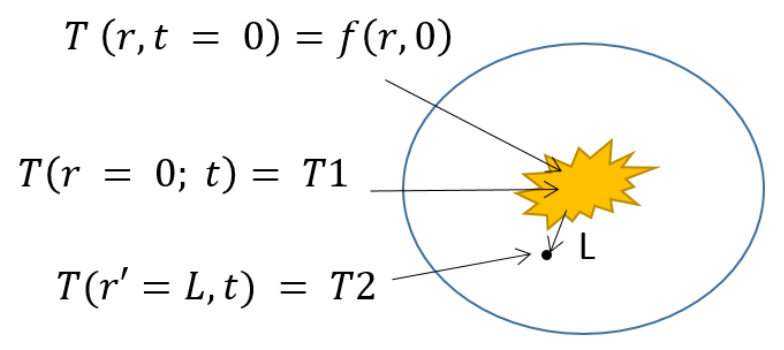

Figure 7.2. Boundary conditions used in solving the Green's function. Yellow represents the heat posited including any additional process.

Application of the Fourier transform together with the boundary conditions shown in Figure 7.2 leads to:

$$
G\left(r^{\prime}, r, t\right)=\frac{1}{(4 \pi \kappa t)} e^{-\frac{r^{2}+r^{\prime 2}}{4 D t}} I_{0}\left(r r^{\prime} / D t\right)
$$

Since eqn. 7.11 is a solution of eqn. 7.10 , then the sum of solutions is also a solution.

$$
T(r, t)=2 \pi \int_{0}^{t} d t^{\prime} \int_{0}^{\infty} d r^{\prime} r^{\prime} G\left(r^{\prime}, r, t^{\prime}\right) f\left(r^{\prime}, t\right)
$$

In order to find a complete solution to this equation, the function $f\left(r^{\prime}, t\right)$ must be defined. This can be done if we first assume this function represents the convolution of the energy absorbed, the energy of the heat source (i.e. a Gaussian laser pulse), and the heat contribution of any additional processes. So that, $f(r, t)=$ amount of energy abs x pump pulse distribution $\mathrm{x}$ heat of others which leads to:

$$
f(r, t)=\left(\frac{\alpha E a}{\hbar \omega}\right) \frac{2}{\pi \omega_{0}^{2}} e^{\frac{-2 r^{2}}{\omega_{0}^{2}}} \sum^{i} \frac{d Q i}{d t}
$$

where $\alpha$ is the absorption coefficient, $E_{a}$ the energy of the photons per mole, $\hbar \omega$ is the energy per photon, $\omega_{0}$ is the full width half maxima, and $Q$ is the heat released.

Combining eqn. 7.13 and 2.11 into eqn. 7.12 yield: 
$T(r, t)=2 \pi \int_{0}^{t} d t \int_{0}^{\infty} d r^{\prime} r^{\prime}\left(\frac{1}{(4 \pi \kappa t)} e^{-\frac{r^{2}+r^{\prime 2}}{4 D t}} I_{0}\left(r r^{\prime} / D t\right)\right)\left(\left(\frac{\alpha E a}{\hbar \omega}\right) \frac{2}{\pi \omega_{0}^{2}} e^{\frac{-2 r^{2}}{\omega_{0}^{2}}} \sum^{i} \frac{d Q i}{d t}\right)$

Eqn. 7.14

Which can be integrated

$$
\begin{aligned}
& T(r, t)=(2 \pi)\left(\frac{1}{(4 \pi \kappa t)}\right)\left(\frac{\alpha E a}{\hbar \omega}\right)\left(\frac{2}{\pi \omega_{0}^{2}}\right) \int_{0}^{t} d t \int_{0}^{\infty} d r^{\prime} r^{\prime} e^{-\frac{r^{2}+r^{\prime 2}}{4 D t} e^{\frac{-2 r^{2}}{\omega_{0}^{2}}}} I_{0}\left(\frac{r r^{\prime}}{D t}\right)^{\sum^{i} \frac{d Q i}{d t}} \text { Eqn. } 7.15
\end{aligned}
$$

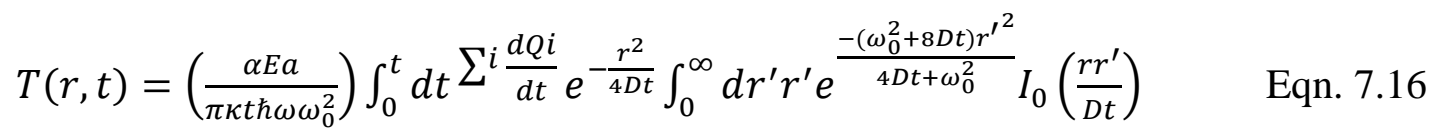

$$
\begin{aligned}
& D=\frac{\kappa}{\rho C p} \\
& T(r, t)=\left(\frac{\alpha E a}{\pi \kappa t \hbar \omega \omega_{0}^{2}}\right) \int_{0}^{t} d t \sum^{i} \frac{d Q i}{d t} e^{-\frac{r^{2}}{4 D t} \int_{0}^{\infty} d r^{\prime} r^{\prime}} e^{\frac{-\left(\omega_{0}^{2}+8 D t\right) r^{\prime 2}}{4 D t+\omega_{0}^{2}}}
\end{aligned}
$$

The last term on right hand side is of the form,

$$
\int x e^{-c x^{2}} d x=-\frac{1}{2 c} e^{-c x^{2}}
$$

whose solution is:

$$
\int_{0}^{\infty} d r^{\prime} r^{\prime} e^{-c r^{\prime} 2}=\frac{\left(8 D t+2 \omega_{0}^{2}\right) e^{\frac{-\left(\omega_{0}^{2}+8 D t\right) r^{2}}{4 D t+\omega_{0}^{2}}}}{\left(8 D t+\omega_{0}^{2}\right)}
$$

which once plugged into eqn. 7.18 gives:

$$
T(r, t)=\left(\frac{2 \alpha E a}{\pi \kappa t \hbar \omega}\right) \int_{0}^{t} d t \sum_{i} \frac{d Q i}{d t} e^{-\frac{r^{2}}{4 D t} \frac{e^{\frac{-\left(\omega_{0}^{2}+8 D t\right) r^{\prime 2}}{4 D t+\omega_{0}^{2}}}}{\left(8 D t+\omega_{0}^{2}\right)}}
$$

In the event that the waist of the Gaussian profile of the pump laser is much larger than the diffusion coefficient of the media (water $\mathrm{D}=0.282 \mathrm{~cm}^{2} \bullet \mathrm{s}^{-2}$, air $\mathrm{D}=0.176 \mathrm{~cm}^{2} \cdot \mathrm{s}^{-2}$ ) (Cussler 2009), then $\left(4 D t+\omega_{0}^{2}\right) \sim \omega_{0}^{2}$ and eqn. 7.21 simplifies to: 


$$
T(r, t)=\left(\frac{4 \alpha E a}{8 \pi \kappa \hbar \omega}\right) \int_{0}^{t} d t \sum_{i} \frac{d Q i}{d t} \frac{e^{-\frac{\omega_{0}^{2}(1+r 2)}{4 D t+\omega_{0}^{2}}}}{\left(8 D t+\omega_{0}^{2}\right)}
$$

Integration over the temperature gives the final solution for the heat diffusion due to a Gaussian heat pulse and additional processes.

$$
T(r, t)=\left(\frac{4 \alpha E a}{8 \pi \kappa \hbar \omega}\right) \sum^{i} Q i \frac{e^{-\frac{2 r^{2}}{8 D t+\omega_{0}^{2}}}}{\left(8 D t+\omega_{0}^{2}\right)}
$$

In which the first term is correlated to sample and instrumental parameters such as the absorption of the sample, path length of the sample (Z), power of pump laser, and energy. The summation term corresponds to the heat deposited by the $\mathrm{i}^{\text {th }}$ molecule at time $\mathrm{t}$ and position r, and the last term correlates to the heat diffusion of such heat deposition.

Furthermore, to calculate the deflection of the probe beam in PBD we must also derive the response of the index of refraction of the media due to changes in temperature. This can be done by considering that the change in index of refraction (n) as a function of time can be due to at least three factors: i) changes in solvent density due to temperature change, ii) change due to photo-products which can affect the index of refraction, iii) and changes in molar volumes of the solvated species.

$$
\Delta n_{\text {total }}=\Delta n_{\text {thermal }}+\Delta n_{\text {chemistry }}+\Delta n_{\text {volume }}
$$

Under conditions such that the probe laser is not sensitive to changes in the chemical composition of the media, i.e. the absorption of the sample does not overlap with the wavelength of the laser, then $\Delta n_{\text {chemistry }}=0$. Under this condition, using:

$$
\Delta n(r, t)_{\text {thermal }} \sim\left(\frac{d n}{d T}\right) \Delta T(r, t)
$$

And 


$$
\Delta n_{\text {volume }}=\int \frac{d n}{d V} d V=\int \frac{d n}{d \rho} \frac{d \rho}{d V} d V
$$

Equation 7.24 simplifies to:

$$
\Delta n_{\text {total }}=\left(\frac{d n}{d T}\right) \Delta T(r, t)+\int \frac{d n}{d \rho} \frac{d \rho}{d V} d V
$$

The $\Delta T(r, t)$ term on the right has been derived in eqn. 7.23 , whereas the second term on the right can be derived from the Claussius-Mossoti relation (also known the the Feynman relation) (Feynman, Leighton \& Sands 1979, Böttcher et al. 1978).

$$
\begin{aligned}
& \rho \alpha=\frac{3\left(n^{2}-1\right)}{\left(n^{2}+2\right)} \\
& \frac{d n}{d \rho} \frac{d \rho}{d V}=\frac{-\left(n^{2}+2\right)\left(n^{2}-1\right)}{6 n V}
\end{aligned}
$$

Where the number of particles per unit volume is $\rho, \alpha$ is the polarizability of the particle, and $\mathrm{n}$ is the index of refraction. Applying the chain rule on eqn. 7.78 leads to 7.29 , and plugging into eqn. 7.27 together with eqn. 7.23 , yields.

$$
\Delta n_{\text {total }}=\left(\frac{d n}{d T}\right)\left(\frac{\alpha}{\pi \hbar \omega}\right)\left(\frac{E a}{\rho C p}\right) \sum_{i} Q_{i}+\int \frac{-\left(n^{2}+2\right)\left(n^{2}-1\right)}{6 n V} d V
$$

In the event that the volume changes are small enough, so that:

$$
\int_{V i}^{V f} \frac{1}{V} d V \sim \Delta V
$$

Then eqn. 7.30 simplifies to:

$$
\Delta n_{\text {total }}=\left(\frac{d n}{d T}\right)\left(\frac{\alpha}{\pi \hbar \omega}\right)\left(\frac{E a}{\rho C p}\right) \sum_{i} Q_{i}+\frac{-\left(n^{2}+2\right)\left(n^{2}-1\right)}{6 n} \Delta V
$$

Which can be rearranged so that:

$$
\Delta n_{\text {total }}=A E_{a} \phi\left\{\frac{d n}{d T}\left(\frac{1}{\rho C p}\right) \sum_{i} Q_{i}+\frac{-\left(n^{2}+2\right)\left(n^{2}-1\right)}{6 n} \Delta V\right\}
$$

Where the constant A contains instrumental parameters, such as absorption, alignment and path length of the sample. Here it was assumed that $\frac{d n}{d T}$ does not change with temperature, 
the quantum yield $(\phi)$ of the reaction was also included. For a process in which all the energy absorbed is released as heat $(\phi=1)$, then $\sum_{i} Q_{i}=E_{h v}$ and if there are no associated structural or chemical changes upon photoexcitation of the chromophore, the eqn. 7.33 reduces to:

$$
\Delta n_{\text {total }}=A E_{a}\left\{\frac{d n}{d T}\left(\frac{1}{\rho C p}\right) E_{h v}\right\}
$$

Even though the angle of deviation in PBD can be calculate by taking the path integral of this equation, a better approach is to measure the deviation of the beam and take the ratio of this same signal with a compound satisfying the conditions that lead to eqn. 7.34. In this scenario, the compound satisfying eqn. 7.34 is called a reference sample, thus we can write:

$$
S=\text { Sample }=A_{S} E_{a} \phi_{S}\left\{\frac{d n}{d T}\left(\frac{1}{\rho C p}\right) \sum_{i} Q_{i}+\frac{-\left(n^{2}+2\right)\left(n^{2}-1\right)}{6 n} \Delta V\right\}
$$

And,

$$
R=\text { Reference }=A_{R} E_{a}\left\{\frac{d n}{d T}\left(\frac{1}{\rho C p}\right) E_{h v}\right\}
$$

Where the left hand side is equal to the PBD signal. Under conditions so that the alignment of the PBD instrument as well as the absorption at the excitation wavelength of both the sample and reference is the same $\left(A_{s}=A_{r}\right)$, taking the ratio of eqn. 7.35 and 7.36 leads to:

$$
\frac{S}{R}=\frac{\Phi_{s}}{E_{h v}}\left[\sum_{i} Q_{i}+\frac{-\left(n^{2}+2\right)\left(n^{2}-1\right)}{6 n}\left[\left(\frac{d n}{d T}\right)\left(\frac{1}{\rho C p}\right)\right]^{-1} \Delta V\right]
$$

In this equation, $E_{h v}$ and $\Phi_{s}$ are temperature independent constants while the term $\left[\left(\frac{d n}{d T}\right)\left(\frac{1}{\rho C p}\right)\right]^{-1}$ is a temperature dependent term which can be calculated from known parameter values for water, or other solvents. The term $-\left(n^{2}+2\right)\left(n^{2}-1\right) / 6 n V=\epsilon$ for water can be considered constant as a function of temperature, with a value of $\epsilon=-0.365$. 
Thus, a plot $\frac{S}{R} E_{h v}$ vs $\epsilon\left[\left(\frac{d n}{d T}\right)\left(\frac{1}{\rho C p}\right)\right]^{-1}$ follows a linear trend with intercept equal to $\Phi_{s} \sum_{i} Q_{i}$ and slope equal to $\Phi_{s} \Delta V$. If the quantum yield of the reaction is known, then the enthalpy change $\Delta H=\Phi_{s} \sum_{i} Q_{i}$ and volume change $\Delta V$ can be calculated. In addition, the temporal dependence of $\Delta H$ and $\Delta V$ can be determined in the range of $10 \mu$ s to $\sim 100 \mathrm{~ms}$. The PBD measurements were performed on a home-build instrument described below. The samples were held in a $1.0 \times 0.5 \mathrm{~cm}$ or $3 \times 3 \times 5 \mathrm{~mm}$ cuvette which were placed inside a temperature controlled holder (TC 125, Quantum Northwest). Illumination of the sample was done with a Nd: YAG laser using the $355 \mathrm{~nm}$ line. The pulse of this source lasts about 5 ns and the power was kept between $600 \mu \mathrm{J}$ to $2400 \mu \mathrm{J}$ (Minilite, Continuum, CA). The laser probe beam (635 nm, 5 mW output from He-Ne Laser, Edmund Optics) was aligned so that it is nearly collinear to the pump beam and crossed in the center of the sample cell. Mirror were used to reflects and align the probe beam onto the center of a split diode. The detector is of our own design and the signal output was digitized by a $400 \mathrm{MHz}$ digitizer (WaveSurfer, LeCroy).

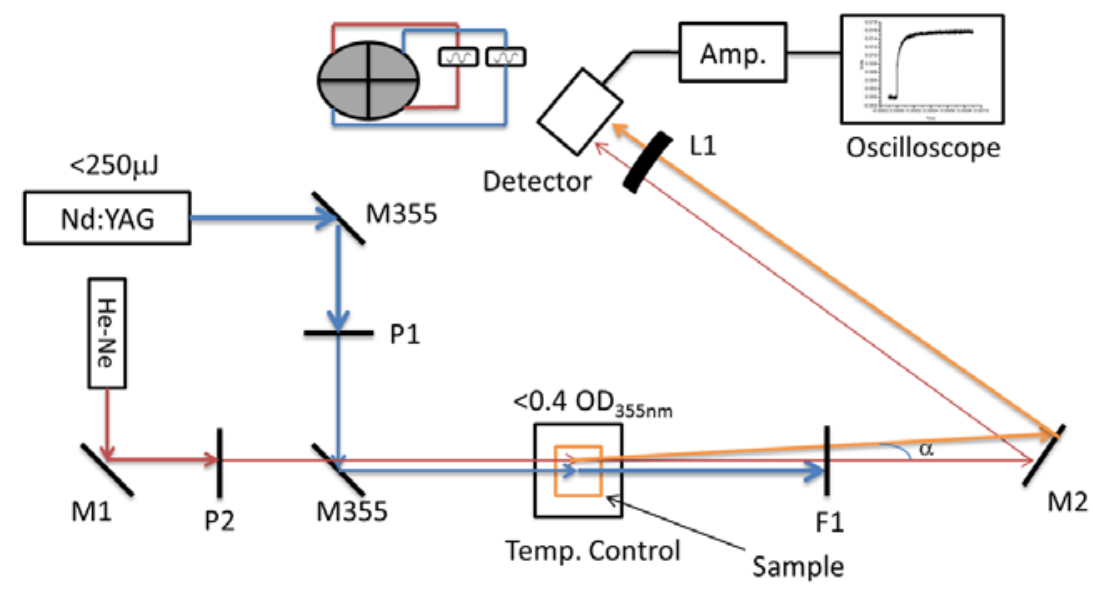

Figure 7.3. Experimental set-up for the photo-thermal beam deflection measurements in the collinear configuration. 
The instrumental design is shown in Figure 7.3, where $\mathrm{M}_{1}$ and $\mathrm{M}_{2}$ represent flat mirrors used to focus the probe beam on the center of a position sensitive detector, $\mathrm{M}_{355}$ represent high energy $\mathrm{Nd}$ :YAG laser mirrors, $\mathrm{L}_{1}$ represents a convex lens positioned in front of a detector, $\mathrm{P}_{1}$ and $\mathrm{P}_{2}$ represents pinholes to shape the pump and probe beam diameter, respectively. a $500 \mathrm{~nm}$ long pass filter is represented by $\mathrm{F}_{1}$.

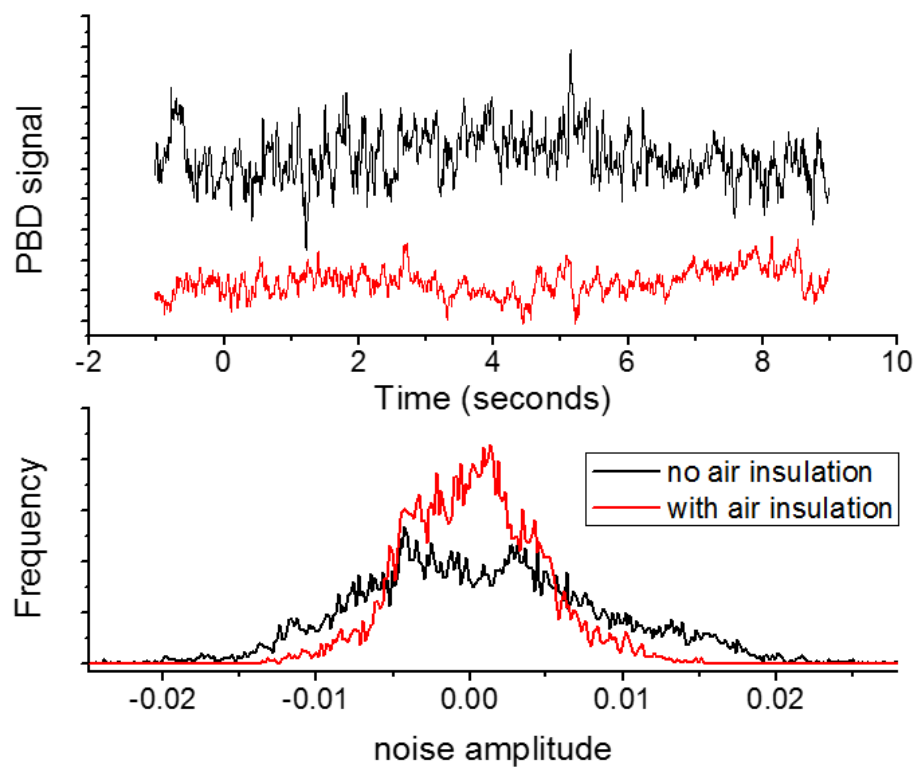

Figure 7.4. (Top) PBD noise on long timescales with and without air insulation. (Bottom) The PBD amplitude distribution of the noise.

The probe beam travels through a refractive index gradient created by a temperature gradient in a medium is deflected by an angle $\alpha$. The deflection angle is measured using a position sensitive detector. We have recently modified this set-up to include vibration damping columns as well as an acrylic enclosure. These additions have resulted in significant decrease in vibration noise and probe beam drifts due to changes in air temperature. Air currents, such as those of nearby AC vents, can create temperature gradients which can effectively induce deflection of the probe beam. In addition, a strong airflow can carry dust particles that when crossing the probe beam laser will reduce the 
intensity and shape of the beam arriving at the detector. As shown in figure 7.4, these artifacts can be significantly reduced by isolation from air current and reduction of vibrational noise.

Figure 7.5. (a) PBD signal amplitude of $\mathrm{Na}_{2}\left(\mathrm{CrO}_{4}\right)_{2}$ in $20 \mathrm{mM}$ MOPS pH 7.4 and $100 \mathrm{mM}$
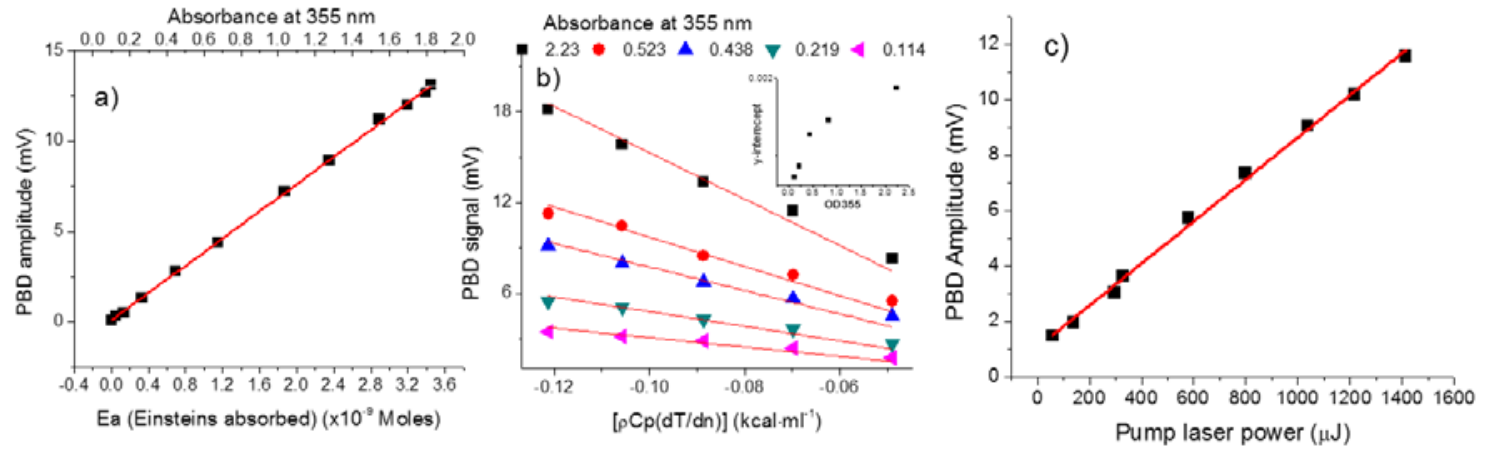

$\mathrm{NaCl}$ as a function of absorbance at $355 \mathrm{~nm}$. (b) Linear plots of the PBD data as a function of $\left[\left(\frac{d n}{d T}\right)\left(\frac{1}{\rho C p}\right)\right]^{-1}$ at different optical absorbance at $355 \mathrm{~nm}$. The inset shows the deviation from 0-interecept as a function of optical density at $355 \mathrm{~nm}$. (c) PBD signal amplitude of the same sample as a function of pump laser power at an absorbance of 0.701 at $355 \mathrm{~nm}$.

Additional experiments were carried out to characterize the linearity of the PBD measurements as a function of laser power and optical density of the sample. In figure 7.5a, the amplitude of the PBD signal as a function of absorbance at $355 \mathrm{~nm}$ is shown. The linearity of this plot shows that no multiphoton process is observed for $\mathrm{Na}_{2}\left(\mathrm{CrO}_{4}\right)_{2}$ at this laser power $(1200 \mu \mathrm{J})$ and temperature $\left(20^{\circ} \mathrm{C}\right)$. In contrast, plots of the sample signal as a function of $\left[\left(\frac{d n}{d T}\right)\left(\frac{1}{\rho C p}\right)\right]^{-1}$ shows small deviations from zero y-intercept at increasing absorbance. This deviation likely arises from changes in ionic strength of the solvent due to the large concentrations of $\mathrm{Na}^{+}$and $\mathrm{CrO}^{-}$ions. This increase in ionic strength would likely need to be corrected by adjusting the $\frac{d n}{d T}$ value. Lastly, analysis of the PBD signal amplitude as a function of pump laser power shows no deviation from linearity in the range between $60 \mu \mathrm{J}$ and $1400 \mu \mathrm{J}$. Indicating that at these powers, no multiphoton absorption is 
observed for $\mathrm{Na}_{2}(\mathrm{CrO} 4)_{2}$ in solution. These experiments highlight the robustness of the PBD set-up and must be carried out in the event that a new reference compound if used or if high concentrations of analyte are necessary.

\subsection{Results}

\subsubsection{Data analysis and calculations}

A typical PBD signal for sodium chromate $\left(\mathrm{Na}_{2}\left(\mathrm{CrO}_{4}\right)_{2}\right)$ in aqueous solution is shown in Figure 7.6. In this plot, a fast rise upon illumination with the 355nm pump laser is observed, followed by a slow decay due to heat diffusion. As the temperature increases, the PBD signal increases, and becomes minimal at about $\sim{ }^{\circ} \mathrm{C}$. At this temperature the $\left(\frac{d n}{d T}\right)$ term becomes zero and the first term in eqn. 7.35 is negligible, thus the signal measured corresponds to the volume change $(\Delta V)$.
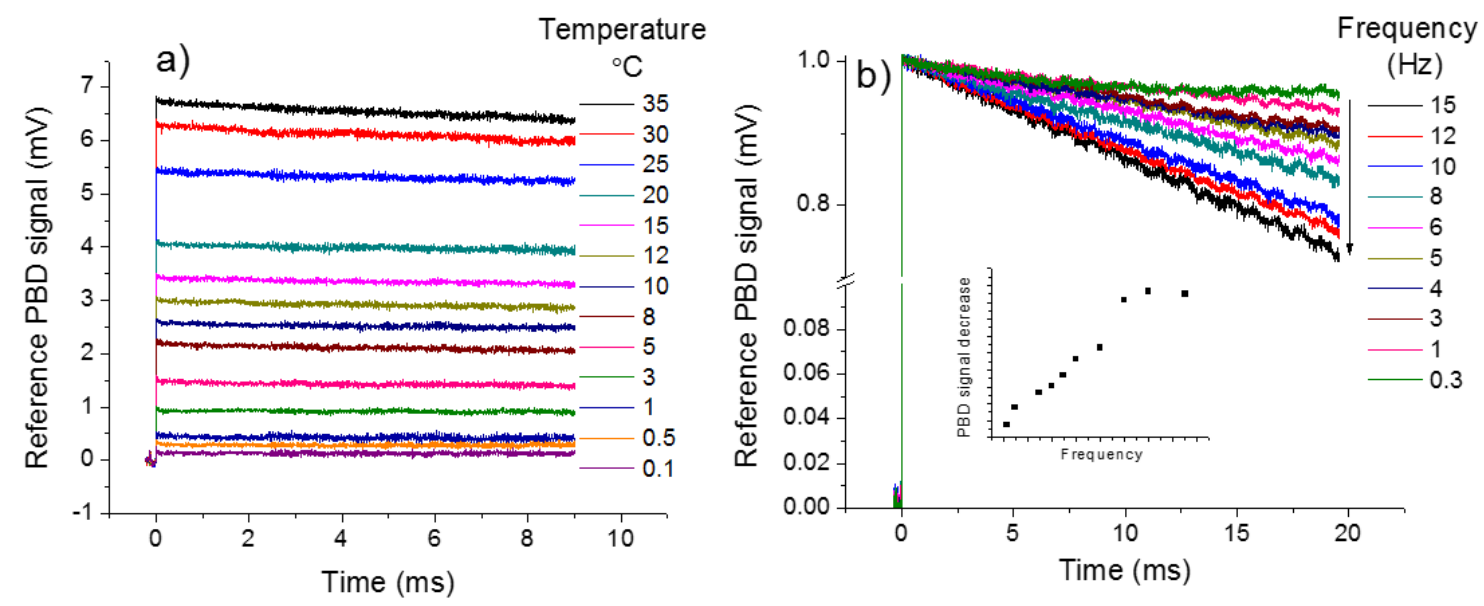

Figure 7.6. (a) $\mathrm{PBD}$ signal of $\mathrm{Na}_{2}\left(\mathrm{CrO}_{4}\right)_{2}$ in $20 \mathrm{mM}$ MOPS $\mathrm{pH} 7.4$ upon excitation with $1100 \mu \mathrm{J}$ power at $355 \mathrm{~nm}$. (b) PBD signal as a function of pump laser repetition rate at 20 ${ }^{\circ} \mathrm{C}$. Inset shows the magnitude of the heat diffusion effect as a function of frequency. 
In the timescales of $10 \mu$ s to $10 \mathrm{~ms}$ the PBD signal appears to be flat due to the negligible heat diffusion and disruption of the transient lens. However, it must be kept in mind that the slow diffusion of heat can lead to laser pump pileup. This can be observed in Figure 7.6b, where the diffusion effects become larger as the laser pump frequency is increased. As the frequency increases, the heat deposited increases due to pump pulse pileup and leads to a larger PBD amplitude decrease at times longer than 20 ms. This is an important aspect which must be taken into account, especially if the reference and sample signals are not measured using identical laser repetition rates or if processes with slow kinetics must be measured. Nonetheless, at low pump laser repetition rates and timescales shorter than $10 \mathrm{~ms}$, the PBD signal can be assumed to follow a square wave shape. Thus, when using eqn. 35 and 36, the difference between the pre-pulse signal and post pulse signal is taken to represent the PBD signal, Figure 7.7.

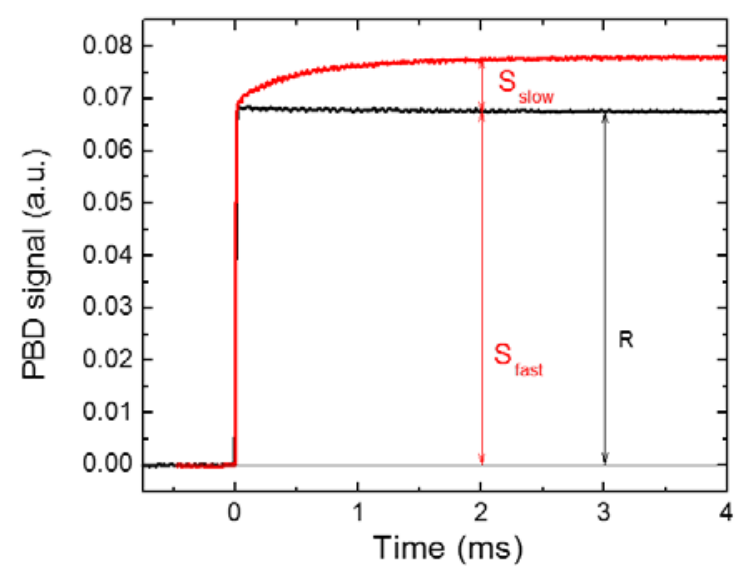

Figure 7.7. A representative trace highlighting the kinetics of a photo triggered processes and the amplitude of the slow and fast phases used to calculate the enthalpy and volume changes.

The change in PBD signal can be associated with fast processes (those than happen faster than $10 \mu \mathrm{s}$ ) and slow processes (processes with kinetics within $10 \mu \mathrm{s}$ and $10 \mathrm{~ms}$ ). In this case the volume and enthalpy changes for the process of interest are calculated using: 


$$
\begin{gathered}
\Delta H_{\text {fast }}=-\left(Q_{\text {fast }}-E_{h v}\right) / \phi \\
\Delta H_{\text {slow }}=-Q_{\text {slow }} / \phi \\
\Delta V=\Delta V_{\text {non-thermal }} / \phi
\end{gathered}
$$

Following Eqn. 7.37, a plot of PBD signal vs $\epsilon\left[\left(\frac{d n}{d T}\right)\left(\frac{1}{\rho C p}\right)\right]^{-1}$ for the reference compound should be linear with intercept at zero. However, as shown in Figure 7.8, small variances in the value of $\epsilon\left[\left(\frac{d n}{d T}\right)\left(\frac{1}{\rho C p}\right)\right]^{-1}$ can lead to significant deviations from the linear fit intercepting zero. In addition, the presence of salts or organic solvents can result in significant deviation from linearity due to large changes on the $\epsilon\left[\left(\frac{d n}{d T}\right)\left(\frac{1}{\rho C p}\right)\right]^{-1}$ term.

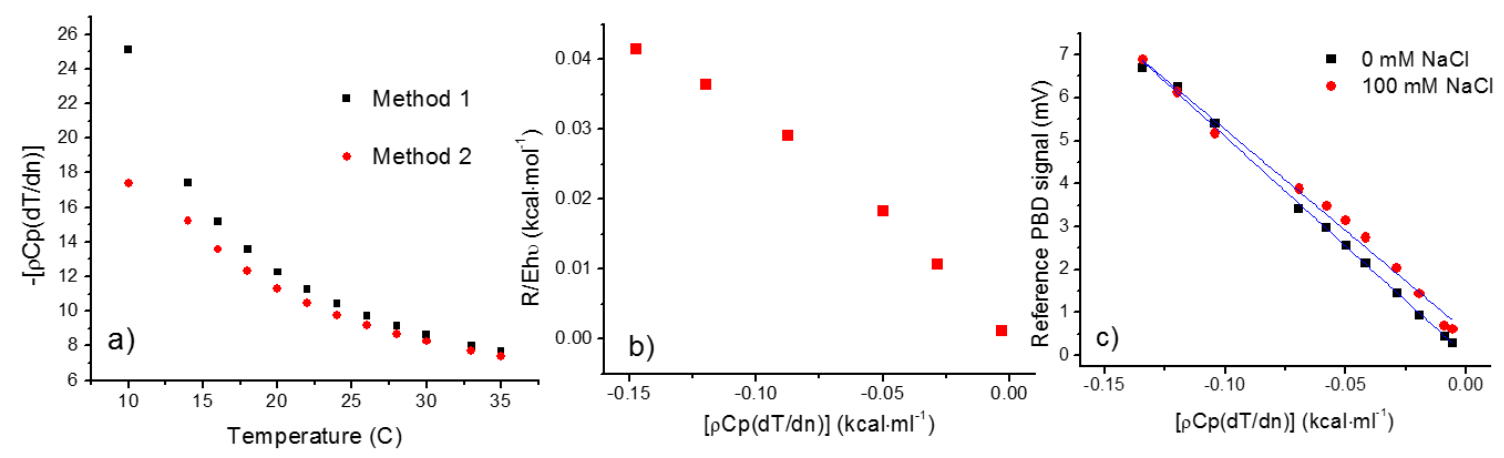

Figure 7.8. (a) Differences in calculated $\left[\left(\frac{d n}{d T}\right)\left(\frac{1}{\rho C p}\right)\right]^{-1}$ values. In method 1 , the values are obtained by fitting the $\left(\frac{d n}{d T}\right), \frac{1}{\rho}$, and $\frac{1}{C p}$ terms individually to a polynomial of rank 3. In method 2, the values of $\left(\frac{d n}{d T}\right), \frac{1}{\rho}$, and $\frac{1}{C p}$ are first multiplied and the trace fitted with polynomial of rank 5. These polynomials are used to calculate the $\left[\left(\frac{d n}{d T}\right)\left(\frac{1}{\rho C p}\right)\right]^{-1}$ at the temperature shown. (b) The deviation from linearity becomes more obvious at lower temperatures (near 0 on the x-axis). This plot was obtained using $\mathrm{Na}_{2}\left(\mathrm{CrO}_{4}\right)_{2}$ in $20 \mathrm{mM}$ MOPS pH $7.4100 \mathrm{mM} \mathrm{NaCl}$ with a laser power of $1100 \mu \mathrm{J}$. (c) Effect on the intercept due to increase in ionic strength, conditions are the same as in (b). 
An alternative approach is to rearrange eqn. 7.35 and 7.37 to obtain a linear equation of sample PBD signal as a function of the reference PBD signal as in eqn. 7.41. Thus a plot of sample PBD signal vs reference PBD signal divided by the laser energy would result in a linear plot with slope $=\phi_{s} \sum_{i} Q_{i}$. The volume change associated with the process can be recovered if the parameter $A_{s}$ is determined, which can be done by plotting the reference PBD signal vs $\epsilon\left[\left(\frac{d n}{d T}\right)\left(\frac{1}{\rho C p}\right)\right]^{-1}$. However, in the event that the volume changes are not necessary, then eqn. 7.41 provides a convenient approach to determine the enthalpy of a process without previous knowledge of the solvent thermal properties. Also, determining the enthalpy from the slope of the linear plot leads to less systematic error due to extrapolation of the linear fit. Another important advantage of this approach is that the linear fit has equal contributions from all temperatures, whereas the linear plot following eqn. 7.37 has much larger contribution from lower temperatures where the $\epsilon\left[\left(\frac{d n}{d T}\right)\left(\frac{1}{\rho C p}\right)\right]^{-1}$ values show the largest changes.

$$
\begin{aligned}
& S=\text { Sample }=\phi_{s} \frac{R}{E_{h v}} \sum_{i} Q_{i}+C \\
& C=A_{S} E_{a} \phi_{S} \frac{-\left(n^{2}+2\right)\left(n^{2}-1\right)}{6 n V} \Delta V
\end{aligned}
$$

In the event that no thermal information is known for the solvent to be used, then it is possible to find a temperature at which the $\epsilon\left[\left(\frac{d n}{d T}\right)\left(\frac{1}{\rho C p}\right)\right]^{-1}$ term approaches zero, at this point the PBD signal for the reference approaches zero, while that of the sample are directly proportional to $\Phi_{s} \Delta V$. This temperature can be determined measuring the PBD signal as a function of temperature, as in Figure 7.9c. At this temperature the PBD signal for the reference approaches zero, while that of the sample is directly proportional to $\Phi_{s} \Delta V$. 

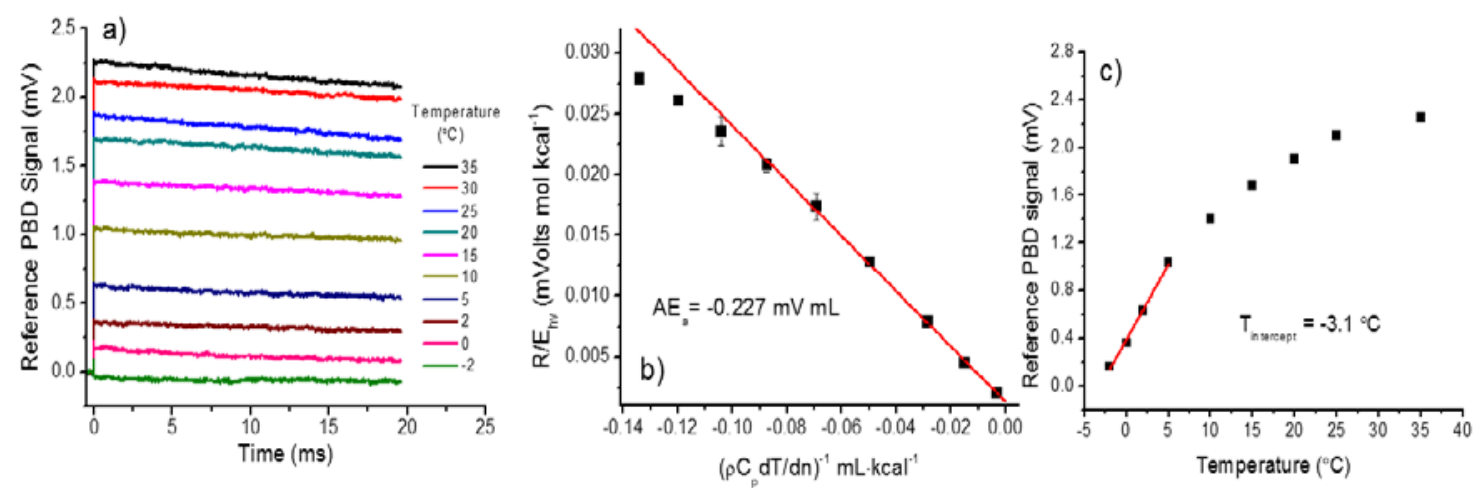

Figure 7.9. (a) PBD signal of $\mathrm{Na}_{2}\left(\mathrm{CrO}_{4}\right)_{2}$ (reference) in $20 \mathrm{mM}$ MOPS pH $7.4100 \mathrm{mM}$ NaCl. (b) Plot of the reference PBD signal as a function of $\left[\left(\frac{d n}{d T}\right)\left(\frac{1}{\rho C p}\right)\right]^{-1}$ used to determine the instrument constant $A E_{a}$. (c) Linear fit of the PBD signal as a function temperature used to identify the temperature at which the PBD signal for the reference is equal to 0 .

\subsubsection{Time-resolved thermodynamics of $\mathrm{Cu}^{2+}, \mathrm{Ca}^{2+}, \mathrm{Tb}^{3+}$, and $\mathrm{H}_{+}$release}

Photo-thermal beam deflection enables the determination of time-resolved volume and enthalpy changes for a wide range of photo-initiated processes, including the release of a bioactive molecule from cage compound complexes (Gonzalez, Miksovska 2014, Dhulipala et al. 2009). Cage compounds are molecules that are biologically inactive, but which can decompose upon irradiation with light to yield a biologically active by-product. The ability to control the spatiotemporal release of biologically active compounds has led to the development of numerous photosensitive caged compounds. Including caged adenosine triphosphate, inositol triphosphate, cyclic adenosine monophosphate, $\mathrm{Ca}^{2+}, \mathrm{H}^{+}$, and DNA, to name a few (Kaplan, Somlyo 1989, Ellis-Davies 2007). The main advantage of such compounds, is their ability to release the biologically active compound within milliseconds. Thus, caged compounds offer the opportunity to study ligand induced biological processes on timescales not accessible by other techniques. However, to be relevant to the study of biological processes, these caged compounds must release their 
cargo within millisecond timescales and this process must have a relative large quantum yield (Gurney 1994).
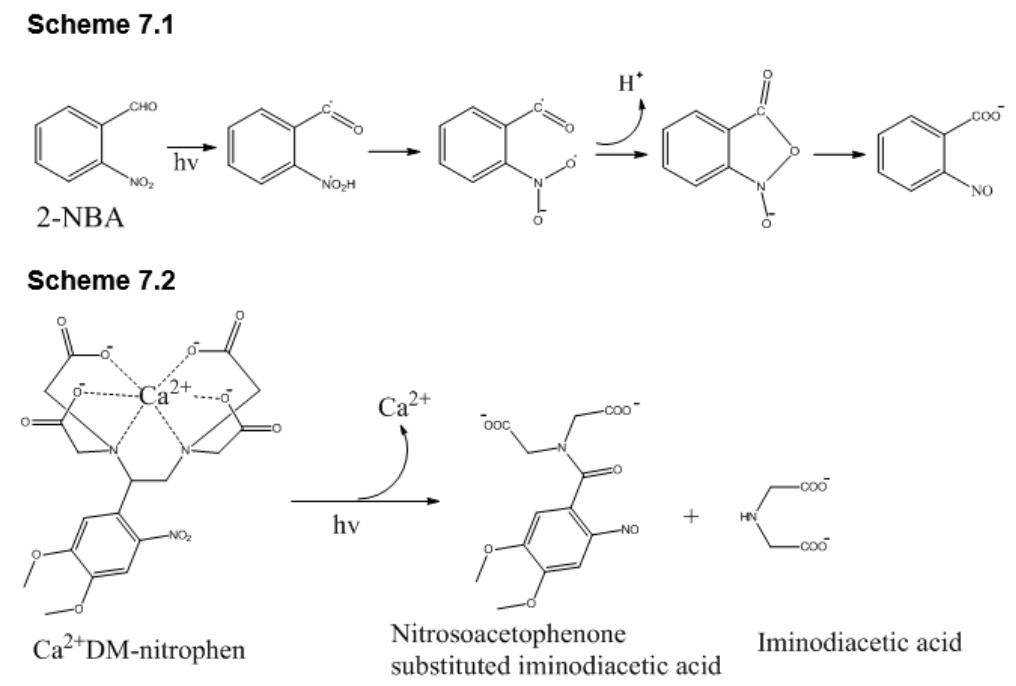

Scheme 7.3
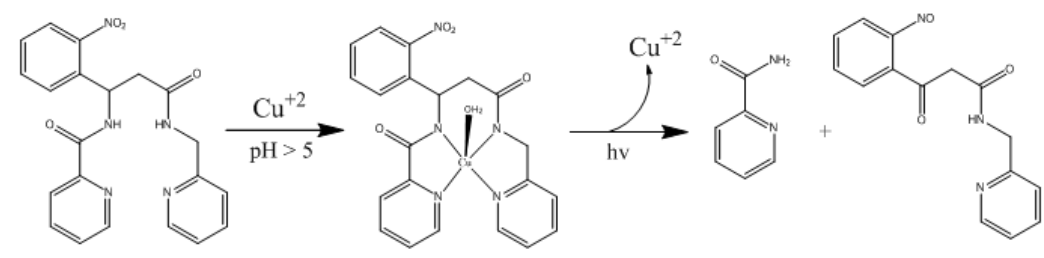

$\mathrm{H}_{2}$ Cage

$\left[\mathrm{Cu}\left(\mathrm{OH}_{2}\right)(\right.$ cage $\left.)\right]$

Table 7.1 Volume and enthalpy following photo-cleavage of caged compounds

\begin{tabular}{|c|c|c|c|c|}
\hline & $\begin{array}{c}\Delta \mathrm{V}_{1} \\
\left(\mathrm{~mL} \mathrm{~mol} \mathbf{m o l}^{-1}\right) \\
\end{array}$ & $\begin{array}{c}\Delta \mathrm{H}_{1} \\
\left(\mathrm{kcal} \mathrm{mol}^{-1}\right) \\
\end{array}$ & $\begin{array}{c}\Delta V_{2} \\
\left(\mathrm{~mL} \mathrm{~mol}^{-1}\right)\end{array}$ & $\begin{array}{c}\Delta \mathrm{H}_{2} \\
\left(\mathrm{kcal} \mathrm{mol}^{-1}\right) \\
\end{array}$ \\
\hline $2-\mathrm{NBA}^{\mathrm{a}}$ & $16 \pm 3$ & $-36 \pm 18$ & & \\
\hline DM-nitrophen $^{\text {b }}$ & $12 \pm 1$ & $63 \pm 6$ & & \\
\hline $\mathrm{Ca}^{2+} \mathrm{DM}$-nitrophen ${ }^{\mathrm{b}}$ & $-12 \pm 5$ & $-50 \pm 20$ & $9 \pm 3$ & $-23 \pm 12$ \\
\hline $\mathrm{Tb}^{3+} \mathrm{DM}$-nitrophen ${ }^{\mathrm{b}}$ & $-11 \pm 2$ & $-53 \pm 21$ & & \\
\hline $\mathrm{H}_{2}$ Cage $^{\mathrm{c}}$ & $-29 \pm 1$ & $-2 \pm 5$ & $17 \pm 2$ & $-65 \pm 14$ \\
\hline $\mathrm{Cu}\left(\mathrm{OH}_{2}\right)$ Cage $^{\mathrm{c}, \mathrm{d}}$ & $20 \pm 5$ & $-390 \pm 94$ & $-15 \pm 3$ & $-33 \pm 9$ \\
\hline
\end{tabular}

a) Determined in water adjusted to $\mathrm{pH} 9.8$ with $\mathrm{NaOH}$. b) determined at $\mathrm{pH} 7.4$ in 20mM MOPS and $100 \mathrm{mM} \mathrm{NaCl}$. Determined at $\mathrm{pH} 7.1$ in 5mM MOPS. d) this data was obtained from a single PBD trial, errors are from the linear fit to the data. 
In this section we use PBD to determine the thermodynamics of $\mathrm{H}^{+}$release from the caged compound o-nitrobenzaldehyde, $\mathrm{Ca}^{2+}$ and $\mathrm{Tb}^{3+}$ from DM-nitrophen, and $\mathrm{Cu}^{2+}$ from a pyridyl-amide-nitrophenyl based compound (Scheme 7). The kinetics, enthalpy, and volume change associated with photo-cleavage of o-nitrobenzaldehyde to release a proton and 2-nitroso benzoic acid have been studied in (Bonetti, Vecli \& Viappiani 1997, Choi, Hirota \& Terazima 2001) using photoacoustic calorimetry and transient grating. Through implementation of PBD we have been able to determine the volume and enthalpy changes associated with this process, and the results are shown in Table 7.1. The measured value of $\Delta \mathrm{H}=-36 \pm 18 \mathrm{kcal} \cdot \mathrm{mol}^{-1}$ and $\Delta \mathrm{V}=16.1 \pm 3.2 \mathrm{~mL} \cdot \mathrm{mol}^{-1}$ at $\mathrm{pH} 9.8$ are in good agreement with those reported by Viapiani et al. (Bonetti, Vecli \& Viappiani 1997). Following these results, we have also determined the volume and enthalpy associated with photo-release of $\mathrm{Ca}^{2+}$ ions from the cage compound DM-nitrophen. As shown in scheme 7.2, illumination with a $355 \mathrm{~nm}$ laser pulse induces the photo-cleavage of a C-C bond to yield free $\mathrm{Ca}^{2+}$ and photoproducts. Photo-dissociation of the $\mathrm{Ca}^{2+} \mathrm{DM}$-nitrophen leads to a prompt $(<15 \mu \mathrm{s})$ reaction volume change that can be expressed as:

$$
\Delta V=\Delta V_{D M-n i t r o p h e n}+\Delta V_{C a^{2+}}^{\circ}
$$

where $\Delta V_{D M-n i t r o p h e n}$ corresponds to the volume change associated with the photolysis of DM-nitrophen and $\Delta V_{C a^{2+}}^{\circ}$ is the partial molar volume of $\mathrm{Ca}^{2+}$ ion. Using $\Delta V_{D M-n i t r o p h e n}$ $=12 \mathrm{ml} \cdot \mathrm{mol}^{-1}$, the partial molar volume of $\mathrm{Ca}^{2+}$ was determined to be $-24 \pm 5 \mathrm{~mL} \cdot \mathrm{mol}^{-1}$. A value similar to the partial molar volume determined using densometric techniques at $25{ }^{\circ} \mathrm{C}, \Delta V_{\mathrm{Ca}^{2+}}^{\circ}=-23 \mathrm{~mL} \cdot \mathrm{mol}^{-1}$ (Millero 1971) and $-20 \mathrm{~mL} \cdot \mathrm{mol}^{-1}$ as observed in transient 
grating measurements (Kondoh, Baden \& Terazima 2008). Similarly, the enthalpy change determined for the fast phase can be described as:

$$
\Delta H=\Delta H_{D M-n i t r o p h e n}+\Delta_{h y d} H_{C a^{2+}}^{\circ}
$$

where $\Delta H_{D M-n i t r o p h e n}$ corresponds to the enthalpy change due to the DM-nitrophen cleavage and $\Delta_{h y d} H_{\mathrm{Ca}^{2+}}^{\circ}$, corresponds to the enthalpy of hydration for $\mathrm{Ca}^{2+}$. Using $\Delta H_{D M-n i t r o p h e n}=63 \pm 6 \mathrm{kcal} \mathrm{mol}^{-1}$, we estimate the enthalpy of hydration to be $-113 \pm 20$ kcal $\mathrm{mol}^{-1}$ for $\mathrm{Ca}^{2}$.

The $400 \mu$ s phase likely corresponds to $\mathrm{Ca}^{2+}$ rebinding to unphotolysed DMnitrophen, which leads to a positive volume change of $9 \pm 3 \mathrm{~mL} \mathrm{~mol}^{-1}$ and a negative enthalpy change of $23 \pm 12 \mathrm{kcal} \mathrm{mol}^{-1}$. The observed volume increase is consistent with the positive volume change observed for $\mathrm{Ca}^{2+}$ complexation to EDTA $\left(\Delta \mathrm{V}=18.4 \mathrm{~mL} \mathrm{~mol}^{-}\right.$ $\left.{ }^{1}\right)$ or EGTA $\left(\Delta \mathrm{V}=13.3 \mathrm{~mL} \mathrm{~mol}^{-1}\right)$. In addition, the negative change in enthalpy determined for $\mathrm{Ca}^{2+}$ binding to un-photolyzed DM-nitrophen matches well the enthalpy change for $\mathrm{Ca}^{2+}$ binding to EDTA $\left(\Delta \mathrm{H}=-12 \mathrm{kcal} \mathrm{mol}^{-1}\right)$ as determined using ITC (Griko 1999).

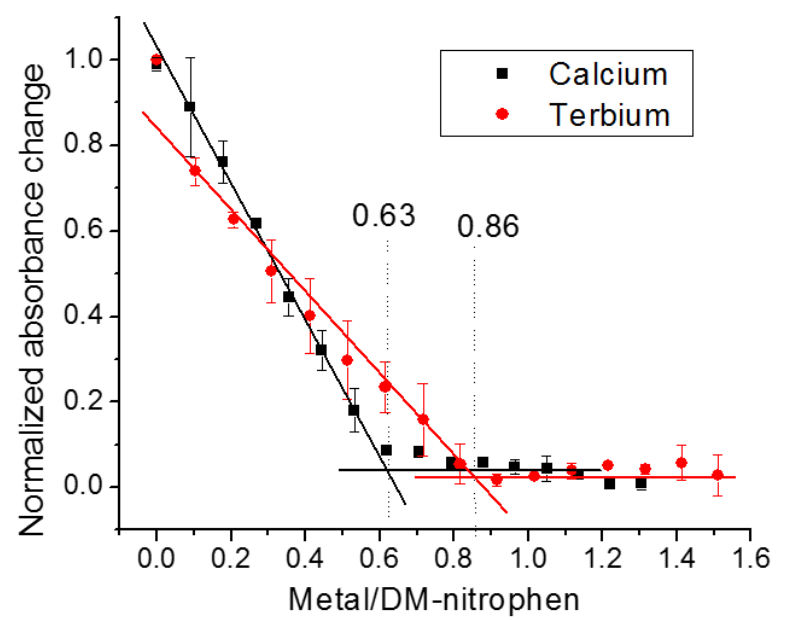

Figure 7.10. Titration curves for $\mathrm{Ca}^{2+}$ and $\mathrm{Tb}^{3+}$ binding to $\mathrm{DM}$-nitrophen. The titrations were conducted in $20 \mathrm{mM}$ MOPS $\mathrm{pH} 6.5$ and $100 \mu \mathrm{M}$ DM-nitrophen. The absorbance at $350 \mathrm{~nm}$ is plotted. 
In addition, we were interested in determining if DM-nitrophen is able to chelate $\mathrm{Tb}^{3+}$ ions and release these ions upon light illumination. Lanthanides have been widely reported to have similar coordination as $\mathrm{Ca}^{2+}$ ions, and are often used as $\mathrm{Ca}^{2+}$ biomimetics (Martin, Richardson 1979). However, there have been no reports of association between DM-nitrophen and lanthanides. To initially identify if such interaction is present, we have monitored the change in absorption of DM-nitrophen as a function of $\mathrm{Tb}^{3+}$ concentration (Figure 7.10). Addition of $\mathrm{Tb}^{3+}$ resulted in a sharp absorption decrease, up to an equimolar concentration. These changes are similar to those observed due to $\mathrm{Ca}^{2+}$ chelation, the deviation from 1:1 stoichiometry is likely due to about $5 \%$ error in the calculation of the $\mathrm{Tb}^{3+}$ stock concentration.

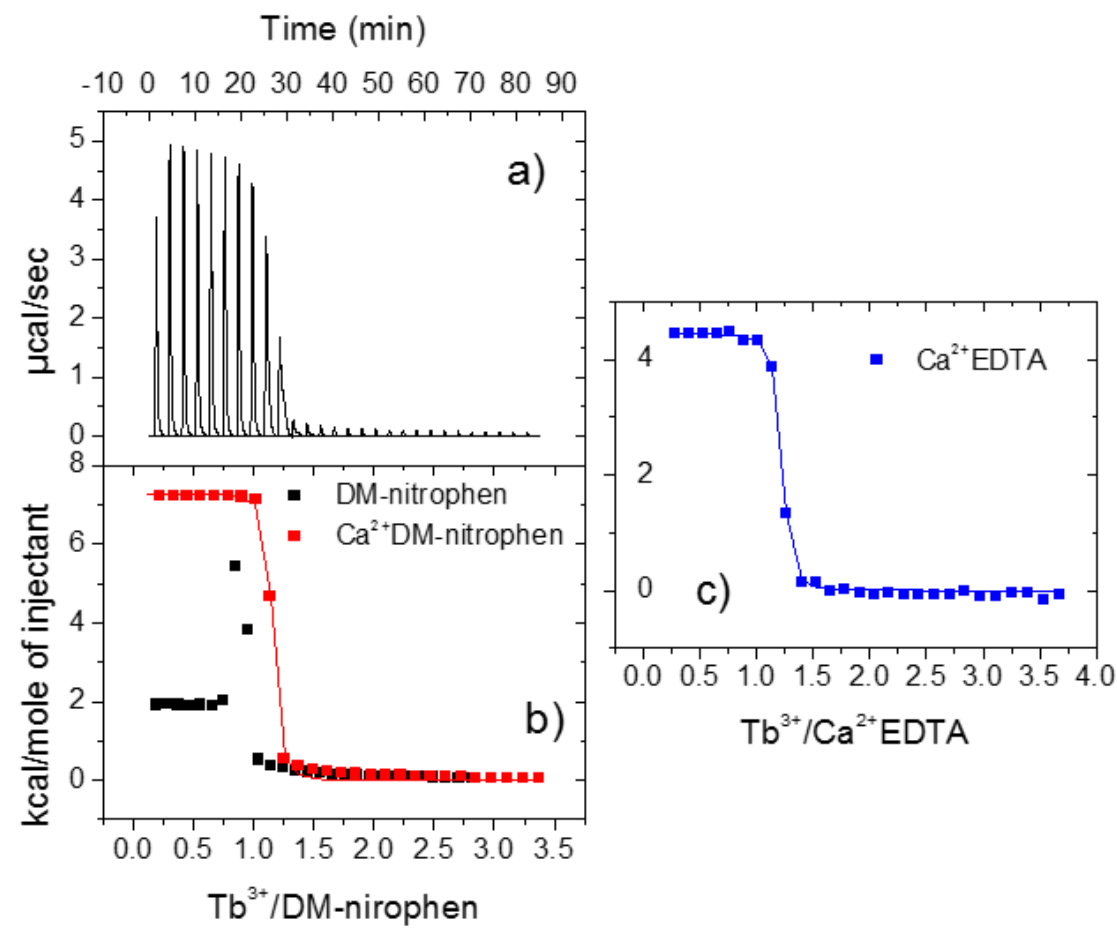

Figure 7.11. (a) Representative ITC isotherms for displacement of $\mathrm{Ca}^{2+}$ by $\mathrm{Tb}^{3+}$ upon binding to DM-nitrophen. (b) Heat due to $\mathrm{Tb}^{3+}$ binding to DM-nitrophen (black symbols), $\mathrm{Tb}^{3+}$ displacement of $\mathrm{Ca}^{2+}$ from DM-nitrophen (red symbols), and (c) from EDTA. The solid lines represent the best fit to the data using a one-site model. 
Isothermal titration calorimetry provides further evidence that $\mathrm{Tb}^{3+}$ is not only able to directly bind to DM-nitrophen, but that it can also displace $\mathrm{Ca}^{2+}$ ions (Figure 7.11). The data for the association of $\mathrm{Tb}^{3+}$ binding to $\mathrm{DM}$-nitrophen shows a one to one stoichiometry with an enthalpy of $\Delta \mathrm{H}=1.9 \pm 0.1 \mathrm{kcal} \cdot \mathrm{mol}^{-1}$. The complex isotherm observed in the absence of $\mathrm{Ca}^{2+}$ is likely due to contributions of $\mathrm{Tb}^{3+}$ binding to $\mathrm{Ca}^{2+}$ free DM-nitrophen (at low molar ratios) followed by $\mathrm{Tb}^{3+}$ displacement of DM-nitrophen bound to contaminating $\mathrm{Ca}^{2+}$. In the presence of $\mathrm{Ca}^{2+}$, the stoichiometry of 1:1 is maintained, but an increase enthalpy is observed $\left(\Delta \mathrm{H}=7.1 \pm 0.1 \mathrm{kcal} \bullet \mathrm{mol}^{-1}\right)$. The isotherm was also best fitted by a one-site model with $\mathrm{n}=1.1 \pm 0.1, \mathrm{~K}=14 \pm 4 \times 10^{6} \mathrm{M}^{-1}$, and $\mathrm{T} \Delta \mathrm{S}=16.8 \pm 0.3 \mathrm{kcal} \cdot \mathrm{mol}^{-}$ ${ }^{1}$. The more endothermic enthalpy due to ligand exchange on DM-nitrophen highlights the subtle differences between $\mathrm{Tb}^{3+}$ and $\mathrm{Ca}^{2+}$. These differences likely arise from distinct solvation shells around $\mathrm{Tb}^{3+}$ and $\mathrm{Ca}^{2+}$ in bulk water as well as a due to a distinct structural organization of the $\mathrm{Tb}^{3+}$ :DM-nitrophen complex (Martin, Richardson 1979). Similar isotherms and thermodynamic parameters were observed for $\mathrm{Tb}^{3+}$ displacement of $\mathrm{Ca}^{2+}$ from EDTA $\left(\mathrm{n}=1.2 \pm 0.1, \mathrm{~K}=6 \pm 4 \times 10^{7} \mathrm{M}^{-1}, \Delta \mathrm{H}=4.5 \pm 0.1 \mathrm{kcal} \bullet \mathrm{mol}^{-1}\right.$, and $\mathrm{T} \Delta \mathrm{S}=15$ $\pm 0.2 \mathrm{kcal} \cdot \mathrm{mol}^{-1}$ in $5 \mathrm{mM}$ TRIS $\mathrm{pH} 7.4$ and $100 \mathrm{mM} \mathrm{NaCl}$ ). Indicating that enthalpic and entropic increases upon displacement of $\mathrm{Ca}^{2+}$ by $\mathrm{Tb}^{3+}$ likely arise from solvation effects and not structural changes of the chelator. The association constant determined for $\mathrm{Tb}^{3+}$ displacement of $\mathrm{Ca}^{2+}$ together with the published affinity of DM-nitrophen for $\mathrm{Ca}^{2+}(\mathrm{K}=$ $\left.2.5 \times 10^{6} \mathrm{M}^{-1}\right)$ (Kaplan, Ellis-Davies 1988), can be used to calculate the affinity of $\mathrm{Tb}^{3+}$ ions for DM-nitrophen using (Sigurskjold 2000):

$$
K_{T b^{3+}}=K_{a p p}\left(1+K_{C a^{2+}}\left[\mathrm{Ca}^{2+}\right]\right)
$$


where $\mathrm{K}_{\mathrm{Ca}^{2+}}$ is the association constant of $\mathrm{Ca}^{2+}$ to DM-nitrophen, $\left[\mathrm{Ca}^{2+}\right]$ is the concentration of $\mathrm{Ca}^{2+}(1 \mathrm{mM})$, and $K_{a p p}$ is the association constant of $\mathrm{Tb}^{3+}$ determined in the presence of $\mathrm{Ca}^{2+}$. The association constant for $\mathrm{Tb}^{3+}$ binding to DM-nitrophen and EDTA is thus calculated to be $K_{T b^{3+}}=3.6 \times 10^{10} \mathrm{M}^{-1}$ and $K_{T b^{3+}} \sim 10^{14} \mathrm{M}^{-1}$., respectively The deviation for the association constant of $\mathrm{Tb}^{3+}$ binding to EDTA from the reported value $\left(\sim 10^{16} \mathrm{M}^{-1}\right)$ likely arises from the tight binding, which may not be easily determined under our conditions (Betts, Dahlinger 1959).

Furthermore, DM-nitrophen not only is able to chelate $\mathrm{Tb}^{3+}$ with high affinity, but PBD experiments show that $\mathrm{Tb}^{3+}$ can be released within microseconds after illumination with a $355 \mathrm{~nm}$ pulse of light. The PBD traces for the $\mathrm{Tb}^{3+} \mathrm{DM}$-nitrophen complex show that $\mathrm{Tb}^{3+}$ release from DM-nitrophen happens within $10 \mu$ s. Plotting the sample PBD signal and reference PBD signal following eqn. 7.37 and eqn. 7.41, allows the calculation of $\Delta \mathrm{V}$ to be $-11 \pm 2 \mathrm{~mL} \cdot \mathrm{mol}^{-1}$ and $\Delta \mathrm{H}=-53 \pm 21 \mathrm{kcal} \cdot \mathrm{mol}^{-1}$. These value are in good agreement with those obtained for $\mathrm{Ca}^{2+}$ photo-release (Table 7.1). However, unlike $\mathrm{Ca}^{2+}$, the release from $\mathrm{Tb}^{3+}$ was not accompanied by a slower rebinding processes. The lack of a slower rebinding process in the $\mathrm{Tb}^{3+} \mathrm{DM}$-nitrophen complex following photo-cleavage can facilitate the interpretation of $\mathrm{Ca}^{2+}$ binding experiment with kinetics within $10 \mu$ s and 10 ms. The results presented here supplement numerous reports highlighting the biophysical similarities between $\mathrm{Ca}^{2+}$ and lanthanides.

The last cage compound studied is a $\mathrm{Cu}^{2+}$ chelating photosensitive compound synthetized by the group of Dr. Kavalieratos at FIU (Ciesienski et al. 2008). The quantum yield of photolysis for each compound $\left(\Phi_{\mathrm{H} 2 \mathrm{Cage}}=0.73\right.$ and $\left.\Phi_{[\mathrm{Cu}(\mathrm{OH} 2)(\mathrm{cage})]}=0.32\right)$ were determined by Ciesienski (Ciesienski et al. 2008). As shown in scheme 7.3, illumination 
with $355 \mathrm{~nm}$ light induces the breakdown of two pyridyl-amide arms which are initially connected by the photosensitive nitrophenyl group. The application of this $\mathrm{Cu}^{2+}$ cage for fast induction of oxidative stress and trafficking of copper inside of the cell are highly dependent on the kinetics photo-release of this cage. Thus, in this section we study the kinetics and thermodynamics of breakdown of the ligand free cage $\left(\mathrm{H}_{2} \mathrm{Cage}\right)$ and of the copper complex $\left(\mathrm{Cu}\left(\mathrm{OH}_{2}\right)\right.$ Cage $)$.
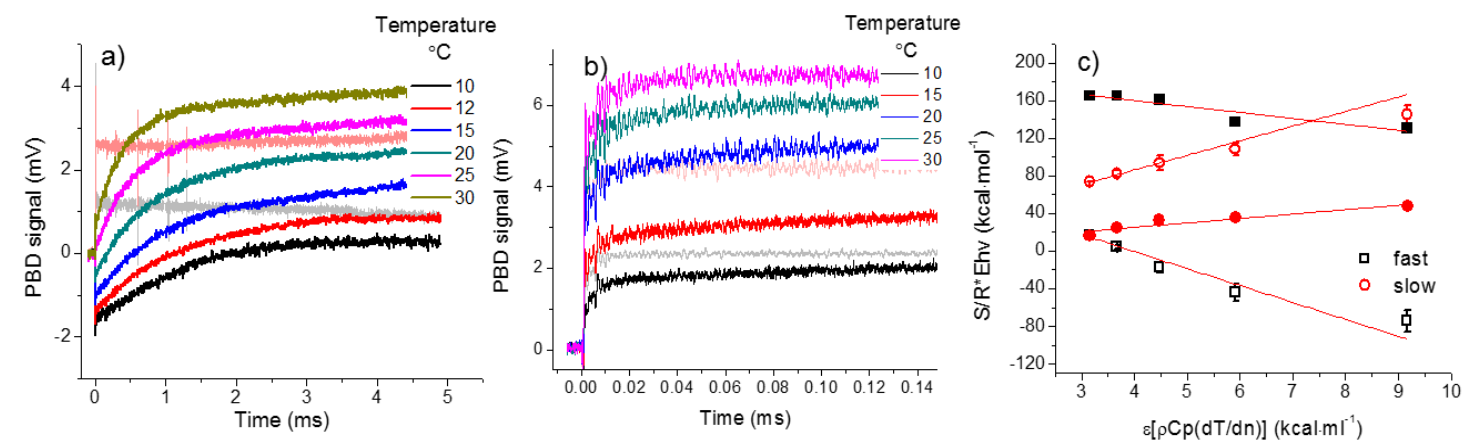

Figure 7.12. (a) PBD signal of $\mathrm{H}_{2}$ Cage (dark lines) in $20 \mathrm{mM}$ MOPS pH $7.1100 \mathrm{mM} \mathrm{NaCl}$ as a function of temperature. (b) PBD signal of $\mathrm{Cu}\left(\mathrm{OH}_{2}\right) \mathrm{Cage}$ at different temperatures. The reference PBD signal of $\mathrm{Na}_{2}\left(\mathrm{CrO}_{4}\right)_{2}$ (light lines) at $30{ }^{\circ} \mathrm{C}$ (red) and $10{ }^{\circ} \mathrm{C}$ (black) are shown for comparison in plots a and b. (c) Plot of the sample to reference PBD signal ratio as a function of $\epsilon\left[\left(\frac{d n}{d T}\right)\left(\frac{1}{\rho C p}\right)\right]^{-1}$ for the fast and slow phases of $\mathrm{H}_{2} \mathrm{Cage}$ and $\mathrm{Cu}(\mathrm{OH})_{2} \mathrm{Cage}$ used to determine $\Delta \mathrm{H}$ and $\Delta \mathrm{V}$, following eqn. 7.37. The solid red lines represent the linear fit of the data. Recovered parameters listed in Table 7.1.

Photothermal beam deflection traces for the $\mathrm{H}_{2}$ Cage are shown in Figure 7.12a/b together with the reference compound. The $\mathrm{H}_{2} \mathrm{Cage}$ and $\left[\mathrm{Cu}\left(\mathrm{OH}_{2}\right)\right.$ (cage)] photo-cleavage shows two distinct phases in the PBD signal. The fast phase occurs within the $10 \mu \mathrm{s}$ resolution of the instrument, while the slow phase shows a life time of around $850 \mu$ s for $\mathrm{H}_{2}$ Cage and $50 \mu$ s for $\left[\mathrm{Cu}\left(\mathrm{OH}_{2}\right)(\right.$ cage $\left.)\right]$ at $20^{\circ} \mathrm{C}$. We attribute the fast phase to the rapid population of the aci-nito intermediate and the slow phase to the subsequent decay of the aci-nitro intermediate, since its kinetics closely matched the aci-nitro intermediate lifetimes of both compounds, as determined using transient absorption spectroscopy at $435 \mathrm{~nm}$ (data 
not shown). No additional kinetics were observed up to $100 \mathrm{~ms}$ timescale on PBD, suggesting that the aci-nitro decay is the rate-limiting step for both $\mathrm{H}_{2}$ Cage and $\left[\mathrm{Cu}\left(\mathrm{OH}_{2}\right)(\right.$ cage $\left.)\right]$ photodissociation. The PBD traces shown in Figure 7.12 confirms that chelation of $\mathrm{Cu}^{2+}$ by the $\mathrm{H}_{2}$ Cage increases the aci-nitro intermediate 17 -fold.

The thermodynamic parameters were determined from a linear plot using eqn. 7.37 (Figure 7.12) and are listed in Table 7.1. The formation of the aci-nitro intermediate in the $\mathrm{H}_{2}$ Cage was accompanied by a contraction of $-29 \pm 1 \mathrm{~mL} \cdot \mathrm{mol}^{-1}$ and near zero enthalpy. The decay of this intermediate resulted in an expansion of $17 \pm 2 \mathrm{~mL} \cdot \mathrm{mol}^{-1}$ and $\Delta \mathrm{H}=-65$ $\pm 14 \mathrm{kcal} \cdot \mathrm{mol}^{-1}$. These volume and enthalpy changes are consistent with a fragmentation of covalent bonds. Fragmentation reactions create larger surface areas for solvent molecules to distribute around and the loss of the aci-nitro intermediate anion eliminates any electrostriction of the solvation sphere. The $\mathrm{Cu}(\mathrm{OH})_{2}$ Cage also shows two phases; however, the volume and enthalpy changes are markedly different. The fast phase shows a volume expansion of $20 \pm 5 \mathrm{~mL} \cdot \mathrm{mol}^{-1}$ and a large endothermic process with $\sim 400$

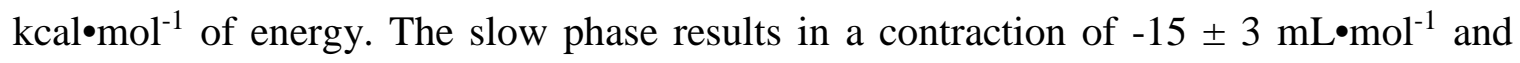
enthalpy change of $-33 \pm 9 \mathrm{kcal} \cdot \mathrm{mol}^{-1}$. Unlike the H2Cage, addition of Cu2+ results in a small but measurable absorption band near wavelength of the probe beam (632 nm). Absorption of the probe beam by the sample, or changes in the absorption band at this wavelength would lead to erroneous determination of the thermodynamic parameters by PBD. Thus, it is not possible to assign the $\Delta \mathrm{V}$ and $\Delta \mathrm{H}$ values to a chemical process, until the contribution of the absorption at $632 \mathrm{~nm}$ is verified to be negligible. An alternative approach is to employ a probe laser with longer wavelength than $632 \mathrm{~nm}$. Nonetheless, the 
data obtained from PBD allows facile determination of the activation enthalpy of $\mathrm{H}_{2} \mathrm{Cage}$ $\left(\Delta \mathrm{H}^{\ddagger}=7 \pm 1 \mathrm{kcal} \cdot \mathrm{mol}^{-1}\right)$ and $\mathrm{Cu}\left(\mathrm{OH}_{2}\right) \mathrm{Cage}\left(\Delta \mathrm{H}^{\ddagger}=12 \pm 01 \mathrm{kcal} \cdot \mathrm{mol}^{-1}\right)$ (Figure 7.13).

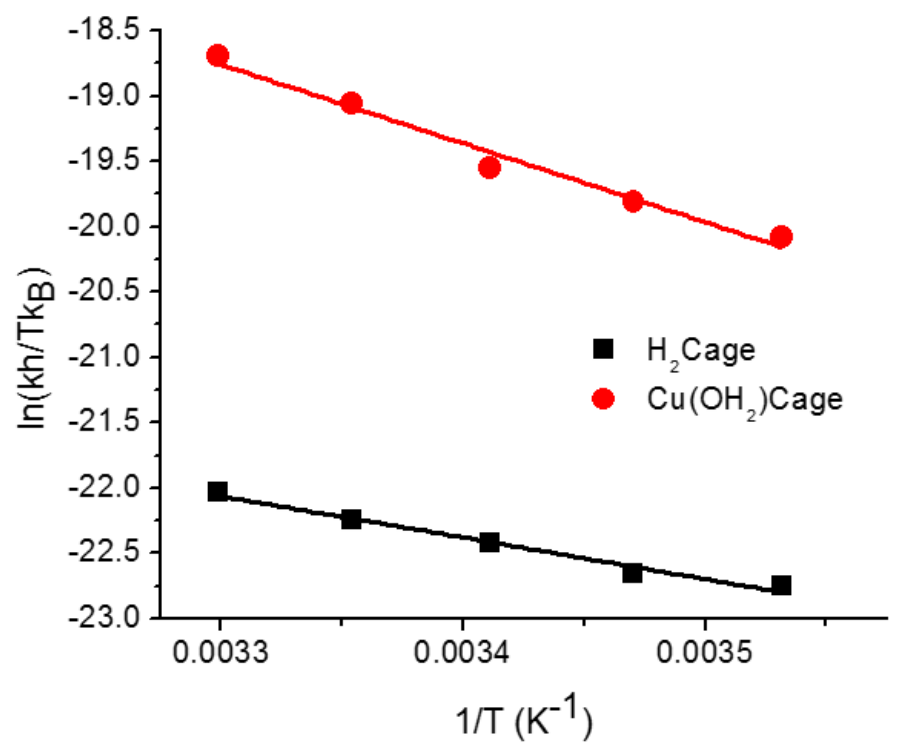

Figure 7.13. Eyring plot of $\mathrm{H}_{2}$ Cage and $\mathrm{Cu}\left(\mathrm{OH}_{2}\right)$ Cage aci-nitro intermediate decay as determined by PBD. Solid lines represent the best linear fit to the data.

Altogether, the experiments shown here demonstrate that PBD can be used to determine the kinetic and thermodynamics of photo-triggered reactions within the $10 \mu \mathrm{s}$ and $20 \mathrm{~ms}$ range. The data for DM-nitrophen confirm that this photosensitive compound is able to chelate $\mathrm{Tb}^{3+}$ and release it following illumination with a short $355 \mathrm{~nm}$ pulse of light. In addition, the thermodynamic parameters for release of $\mathrm{Tb}^{3+}$ and $\mathrm{Ca}^{2+}$ are very similar, in agreement with the idea that these two ions share similar biophysical properties in solution. Lastly, PBD data shows that the chelation of $\mathrm{Cu}^{2+}$ by the H2Cage compound induces a 17fold increase in the breakdown rate of this cage following illumination. Further studies are needed to fully determine the thermodynamic parameters of the $\mathrm{Cu}(\mathrm{OH} 2)$ Cage compound, but the data presented here show that the breakdown of this complex at about $50 \mu \mathrm{s}$ is well within the resolution of PBD. 


\subsubsection{Time-resolved thermodynamics of $\mathrm{Ca}^{2+}$ binding to DREAM constructs}

Following the application of PBD to study the photo-triggered release of $\mathrm{Ca}^{2+}$ from DM-nitrophen within $10 \mu \mathrm{s}$, this technique is also amenable to investigate the thermodynamics and dynamics associated with $\mathrm{Ca}^{2+}$ binding to DREAM. In figure 7.13 the PBD traces for DREAM( $\Delta 64)$, DREAM $(\Delta 160)$, and DM-nitrophen at $20{ }^{\circ} \mathrm{C}$ are shown. Due to the non-reversibility of the $\mathrm{Ca}^{2+}$ release process, the samples has to be exchanged after every pulse. To minimize the sample needed, a 3x3x5 mm (width-lengthheight) quartz cuvette was used ( $45 \mu \mathrm{L})$ and ten single shots were averaged. A sample concentration of $100 \mu \mathrm{M}$ for DREAM protein, $795 \mu \mathrm{M}$ DM-nitrophen, and $780 \mu \mathrm{M}$ calcium in 20mM HEPES pH 7.4, 1mM TCEP, $100 \mathrm{mM} \mathrm{NaCl}$ solution was used. Under these conditions DM-nitrophen shows a rebinding kinetic of about $350 \mu$ s (black trace in fig. 7.14). On the other hand, $\mathrm{Ca}^{2+}$ binding kinetics to $\operatorname{DREAM}(\Delta 64)$ and $\operatorname{DREAM}(\Delta 160)$ are slower, with $\tau=650 \mu$ s and $\tau=1270 \mu$ s, respectively. In the case of $\operatorname{DREAM}(\Delta 160)$ the association rate of $k_{\text {on }}=4.0 \mathrm{~s}^{-1} \bullet \mu \mathrm{M}^{-1}$ determined here is in good agreement with the calculated value of $\mathrm{k}_{\mathrm{on}}=4.0 \pm 0.1 \mathrm{~s}^{-1} \mu \mathrm{M}^{-1}$ from stopped-flow experiments. These results highlight the role of the N-terminus domain in regulating the kinetics of calcium binding at the C-terminus. Unfortunately, we observed that the $\mathrm{Ca}^{2+}$ rebinding kinetics to DMnitrophen following illumination varied among different manufacturers (Figure 7.14b). Thus the overlap of the DM-nitrophen and DREAM kinetics, as well as large variations in the DM-nitrophen kinetics prevented further deconvolution of these signals. 

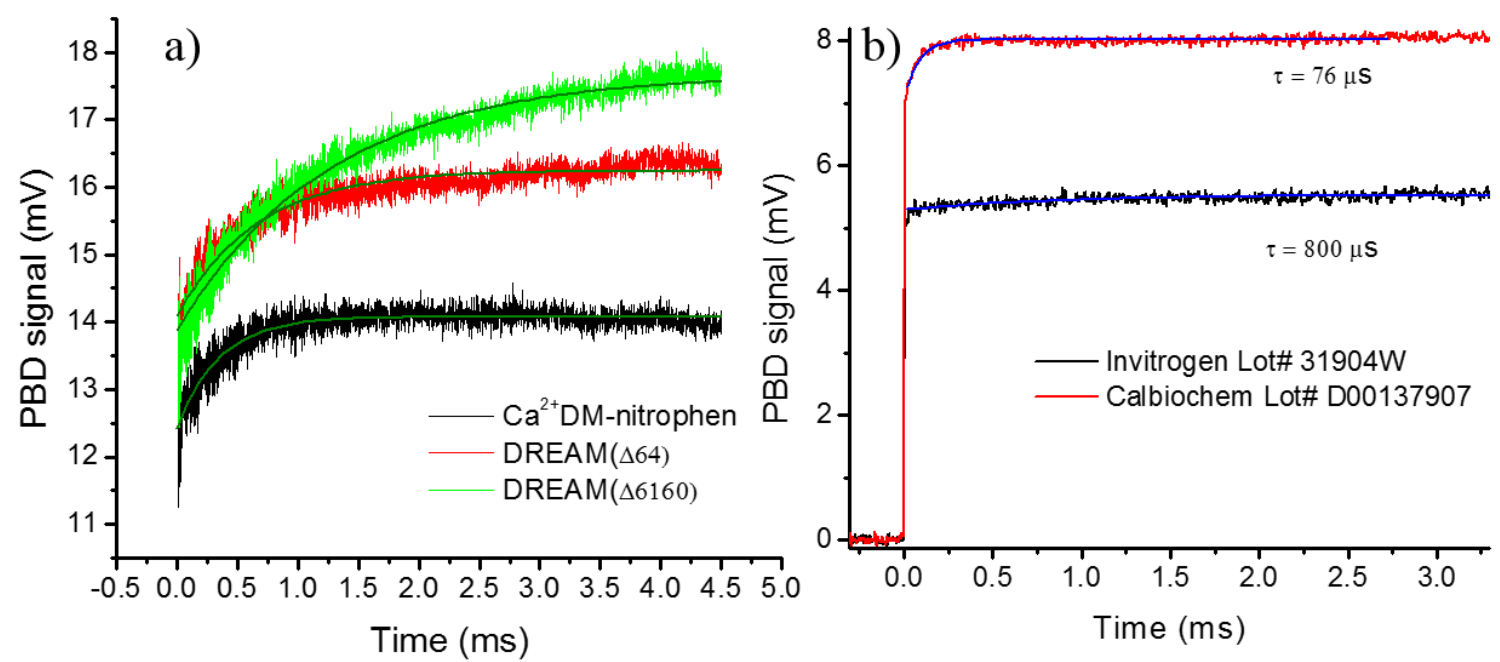

Figure 7.14. (a) PBD traces for DREAM constructs at $200 \mu \mathrm{M}$ concentration, solid green line represent best NLLS fit to an exponential decay. Trace for DREAM(1-256) not shown. (b) PBD traces of $\mathrm{Ca}^{2+} \mathrm{DM}$-nitrophen from different manufacturers.

\subsection{Summary}

In this section I presented the theory behind photothermal beam deflects as well as improvements on the PBD set up which decrease noise levels at longer timescales. The current PBD instrument is able to measure processes faster than $~ 40$ ms. However, at these timescales, much care must be taken to account for diffusion and vibrational effects. We demonstrate that PBD can be used to determine the kinetics and thermodynamics of ligand release from 2-NBA, DMnitrophen, and a novel copper cage. In addition, we presented the use of DM-nitrophen to cage and photo-release $\mathrm{Tb}^{3+}$. Kinetic analysis of the PBD data supports the idea that $\mathrm{Ca}^{2+}$ can bind to DREAM within milliseconds. However, further studies need to be carried out in order to determine the thermodynamics of this process. 


\section{ROLE OF AROMATIC AMINO ACIDS IN ALLOSTERIC REGULATION OF DREAM ACTIVITY}

\subsection{Background and significance}

The neuronal calcium sensors DREAM, KChIP1, KChIP 2, and KChIP 4 coassemble with the $\mathrm{K}_{\mathrm{V}}$ channels to enhance channel surface expression, facilitate channel assembly and regulate the functional gating properties of the channels. Despite numerous $\mathrm{X}$-ray structures of the $\mathrm{K}_{\mathrm{v}} 4 \mathrm{~T} 1$ domain in the presence and absence of the regulatory KChIPs, the mechanism by which these auxiliary proteins mediate channel gating remains unknown. Two fragments on the $\mathrm{K}_{\mathrm{v}} 4 \mathrm{~T} 1$ domain (amino acids 7-11 "site 1" and 71-90 "site 2") have been identified as being necessary for KChIP1 current regulation (Scannevin et al. 2004). The "site 1" interaction interface involves a large hydrophobic patch on the DREAM surface, while "site 2" interactions involves helix 2 at the KChIP N-terminus (Pioletti et al. 2006, Wang et al. 2007). Remarkably, we have shown that in the presence of calcium, $\operatorname{DREAM}(\Delta 65)$ exhibits a 26 -fold increase in affinity for site 1 , and approximately 50-fold increase for site 2 (Chapter 4). The fact that calcium binding at the C-terminus EF-hand 3 and EF-hand 4 of $\operatorname{DREAM}(\Delta 65)$ modulates binding of site 2 indicates that the structural changes coupled to calcium association are transmitted towards the DREAM N-terminus. The regulation of ligand binding at sites distant from the $\mathrm{Ca}^{2+}$ binding is also observed for DNA. However, DREAM interacts with DNA in a manner that opposes that of $\mathrm{K}_{\mathrm{v}} 4$ site 2, that is, $\mathrm{Ca}^{2+}$ induces the dissociation from DNA (Osawa et al. 2005, Carrion et al. 1999) (Figure 8.1). Nonetheless, these interactions highlight that $\mathrm{Ca}^{2+}$ binding to DREAM can induce long range structural reorganizations that can regulate ligand binding. The concept of regulation at a distance is not new, and has been widely 
observed in ligand binding proteins. The regulation of protein activity by binding of a ligand (effector) at a distinct site than that of the site involved in activity has been termed allosteric regulation.
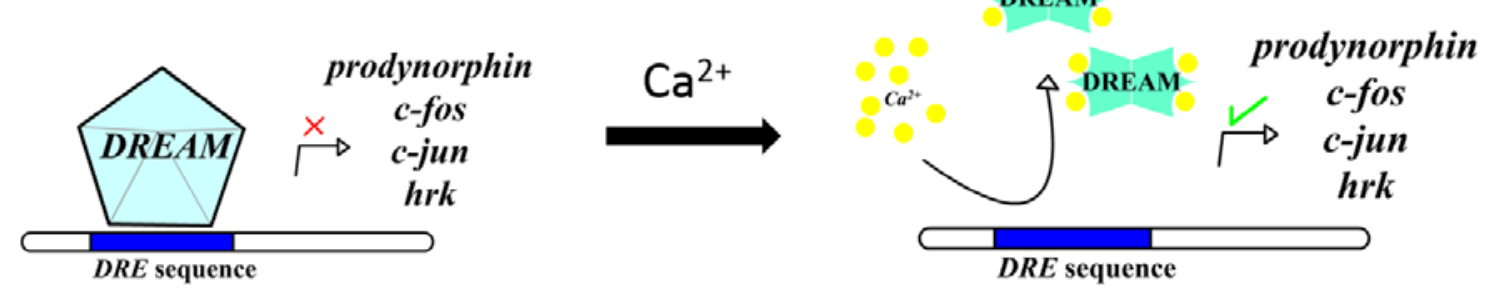

Figure 8.1. Calcium induced dissociation of DREAM from DNA. The $\mathrm{Ca}^{2+}$ free DREAM shown as a tetrameric blue pentagon, upon $\mathrm{Ca}^{2+}$ binding a dimer is formed (green shape). The dimeric form of DREAM has lower affinity for DNA. Dissociation of DREAM from DNA induces the expression of $D R E$ regulated genes.

Two widely studied models used to explain allosteric regulation in proteins are the Monod-Wyman-Changeux (WNC) (Monod, Wyman \& Changeux 1965) model and the Koshland-Nemethy-Filmer (KNF) model (Koshland Jr, Nemethy \& Filmer 1966). Both of these models follow the premise that proteins can populate two states, a relaxed state with high affinity for the ligand, and a tense state with lower affinity for the ligand. However, these models differ on the condition of whether all subunits of a protein actively populate only one state upon ligand binding (WNC, concerted) or whether ligand binding only results in local structural changes, allowing the protein complex to populate both states (KNF, model). However, the growing perspective that protein conformational dynamics and fluctuations control allosteric regulation offers a novel paradigm in understanding ligand binding. In view presented above, proteins do not behave in a binary fashion, but can rather populate a multitude of isoenergetic conformational states (Jardetzky 1996). 
These conformational states are highly dynamic and sensitive to perturbations resulting from ligand binding, which can induce a redistribution of the conformational space sampled by the protein (Cui, Karplus 2008, Tsai, Del Sol \& Nussinov 2009). However, experimental determination of allosteric mechanisms and pathways have proven to be elusive. Even after decades of research on the allosteric regulation of hemoglobin, the precise mechanism remains unresolved. Nonetheless, significant advances in molecular dynamics simulations have opened the opportunity to observe the the dynamic nature of proteins at the atomic level. Thus, it is now possible not only to study the role of protein dynamics in regulation of activity, but to dissect the pathways by which information propagates in these complex systems. The concept of dynamic regulation brings a shift in the classical view of the structure-function relationship of protein activity to a new focus in which structure-dynamic-function plays a major role.

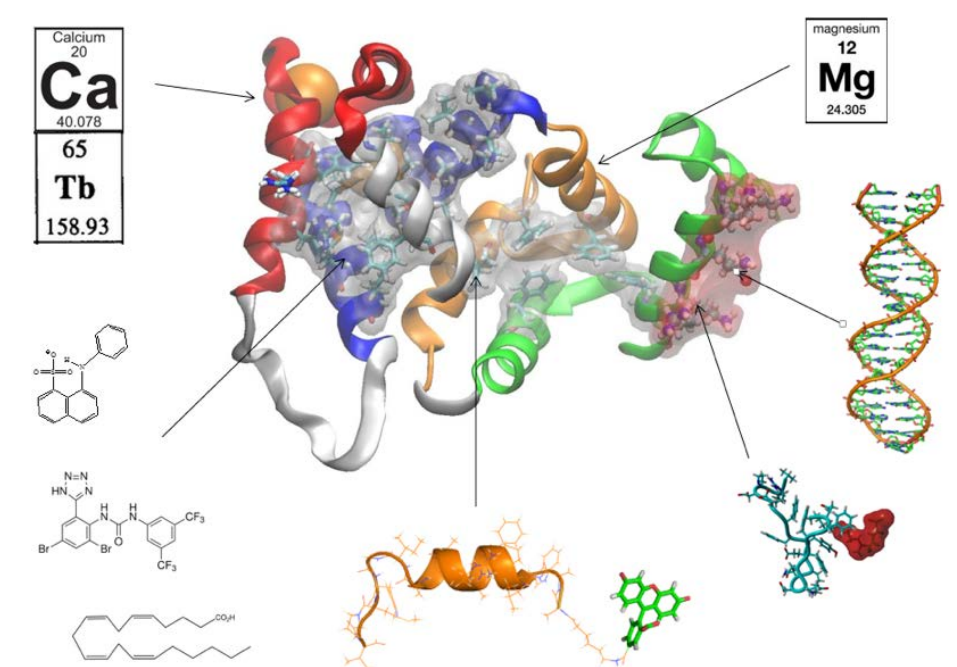

Figure 8.2. Association of ligand with DREAM (shown in the center as a cartoon model). The electrostatic and hydrophobic regions of DREAM are highlighted in a red surface or gray surface, respectively. Ligand known to interact with DREAM are shown, (counterclockwise) calcium, terbium(III), 1,8-ANS, NS5806, arachidonic acid, site 1 of $\mathrm{K}_{\mathrm{v}} 4.3$, site 2 of $\mathrm{K}_{\mathrm{v}} 4.3$, DRE DNA, and magnesium. 
In chapter 8 , we implement the combination of theoretical and experimental techniques with the aim of understanding how calcium modulates DREAM activity. More importantly, we seek to decipher how structural changes associated with $\mathrm{Ca}^{2+}$ binding at the C-terminus are propagated towards the $\mathrm{N}$-terminus. As presented in the previous chapters, DREAM is involved multiple biological events and can interacts with numerous small and large ligands (Figure 8.2). Therefore, understanding how structural information propagates across this protein would provide a new information which could potentially allow the development of approaches to control its activity. Knowledge of the molecular mechanism controlling structural changes could also be extrapolated to other neuronal calcium sensors and provide a fundamental understanding of $\mathrm{Ca}^{2+}$ binding and allostery.

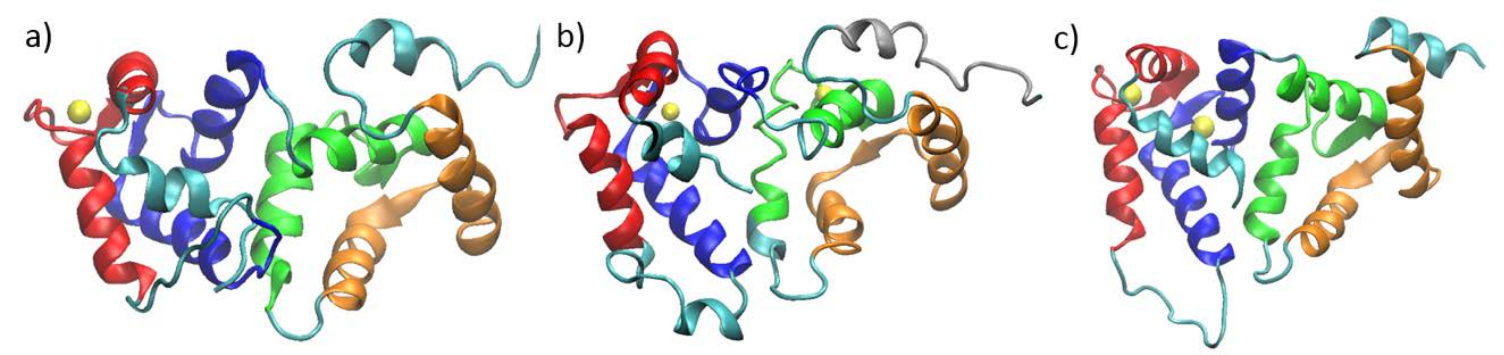

Figure 8.3. Cartoon representation of the three dimensional structures of (a) NCS1 (PDB: 1fpw), (b) recoverin (PDB: 1jsa), and (c) DREAM (PDB: 2jul) in the $\mathrm{Ca}^{2+}$ bound form.

\subsection{Results}

8.2.1 Calcium binding regulates structural reorganization of the N-terminus helices of EFhand 1

Elucidation of the structure of DREAM has provided valuable information in regards to the molecular organization of this protein. However, the NMR structures of DREAM(76-256) (PDB: 2jul) and DREAM(161-256) (PDB: 2e6w) have shown little deviation from the structure of recoverin or NCS-1 (Lusin et al. 2008, Yu et al. 2007a) 
(Figure 8.3). Nonetheless, these proteins are known to regulate different processes inside the cell and associate with different protein ligands. Furthermore, despite their similar structural organization, DREAM, recoverin, and NCS-1 all bind $\mathrm{Ca}^{2+}$ in different configurations. For instance, recoverin binds $\mathrm{Ca}^{2+}$ at EF-hand 2 and 3, KChIPs bind $\mathrm{Ca}^{2+}$ at EF-hand 3 and 4; while NCS-1 binds $\mathrm{Ca}^{2+}$ in EF-hands 2, 3, and 4. Nonetheless, close analysis of the structural organization of these proteins reveal a high degree of sequence and structural homology at the C-terminus containing EF-hand 3 and 4. Therefore, it is possible that the C-terminus domain represents a fundamental tertiary organization with allows these proteins to convert the biochemical energy of $\mathrm{Ca}^{2+}$ binding to structural information. In the hypothesis presented above, EF-hand 3 would play an essential structural role while EF-hand 2 and 4 function to relay structural information. We further hypothesize that in DREAM (and potentially other NCS proteins) structural information propagates in the form of structural and dynamic changes.
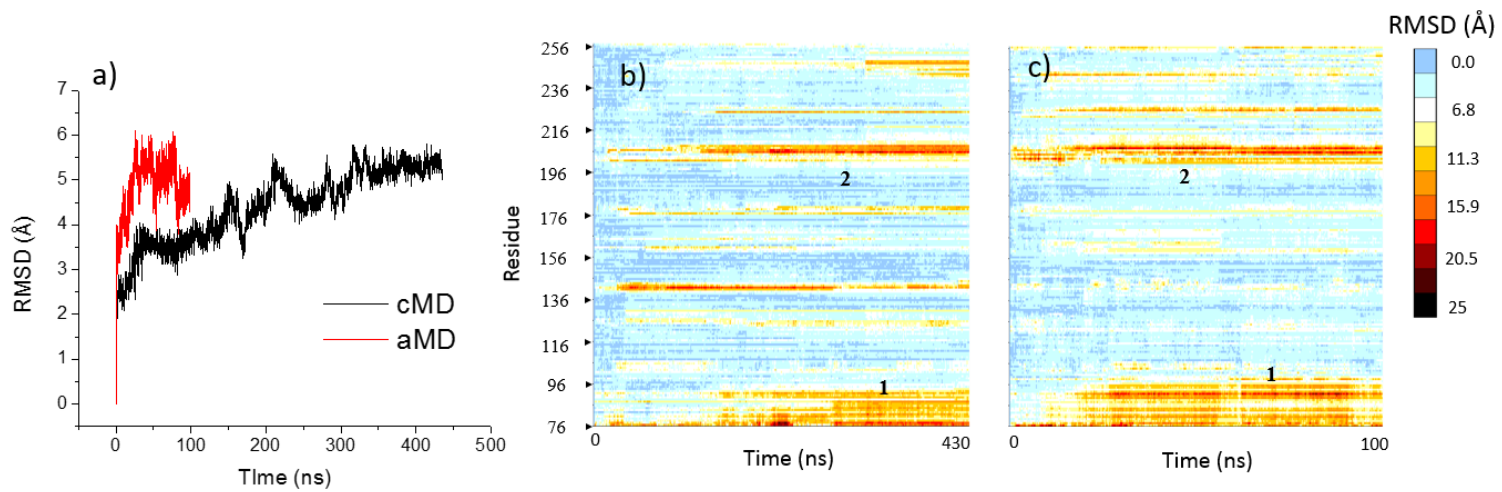

Figure 8.4. (a) Root mean square deviation (RMSD) of the alpha carbon $\left(\mathrm{C}_{\alpha}\right)$ DREAM (residues 76 to 256) as a function of simulation time. Notice that the classical simulation must undergo over 400 ns to reach a deviation similar to that observed using aMD. Heat map plot showing the RMSD of the $\mathrm{C}_{\alpha}$ of each residue as a function of simulation time for the cMD simulation (b) and for the aMD simulation (c). Note the similar patter or reorganization between residue 76 to 96 on both simulations (marked as 1). The region 2 corresponds to residues connecting the exiting helix of EF-hand 3 and entering helix of EFhand 4. 
To clearly understand the structural changes associated with $\mathrm{Ca}^{2+}$ binding to DREAM, we first must obtain a structural model of the protein in the $\mathrm{Ca}^{2+}$ free form. To obtain this model we conducted a 450 ns classical molecular dynamics (cMD) simulation and a 100 ns accelerated molecular dynamics (aMD) simulation. These simulations were initialized using the $\mathrm{Ca}^{2+}$ bound DREAM structure (PDB: 2jul), in which the $\mathrm{Ca}^{2+}$ ions have been removed. As shown in Figure 8.4a, the cMD simulation does not reach equilibrium even after $450 \mathrm{~ns}$, while the aMD simulation resulted in an equilibrated structure after about $20 \mathrm{~ns}$. The fast equilibration is expected, since the aMD approach allows the protein to sample a larger number of conformations by effectively lowering the energy barriers separating these states (Hamelberg, Mongan \& McCammon 2004). Both of the resulting DREAM structures in the absence of $\mathrm{Ca}^{2+}$ show an overall molecular reorganization of the $\mathrm{N}$ - and C-terminus, with an overall RMSD from the starting structure of $4.7 \AA$ for the aMD simulation and $5.5 \AA$ for the cMD simulation. However, analysis of the RMSF per residue as a function of simulation time reveal significant reorganization of the N-terminus domain near EF-hand 2 and 3 in both simulations (Figure 8.4b and c). Since the cMD simulation does not reach an equilibrium structure even after $450 \mathrm{~ns}$, we do not include it in further analysis; instead, we focus on the structure obtained from the aMD simulation. Following the simulation in the absence of $\mathrm{Ca}^{2+}$, the overall structure of DREAM becomes more compact, with an evident collapse of the hydrophobic cavity at the $\mathrm{N}$-terminus (Figure 8.5a and b). In addition, a reorientation of the $\mathrm{N}$-terminus with respect to the C-terminus is observed, likely triggered by the collapse of the $\mathrm{N}$-terminus hydrophobic cavity. 

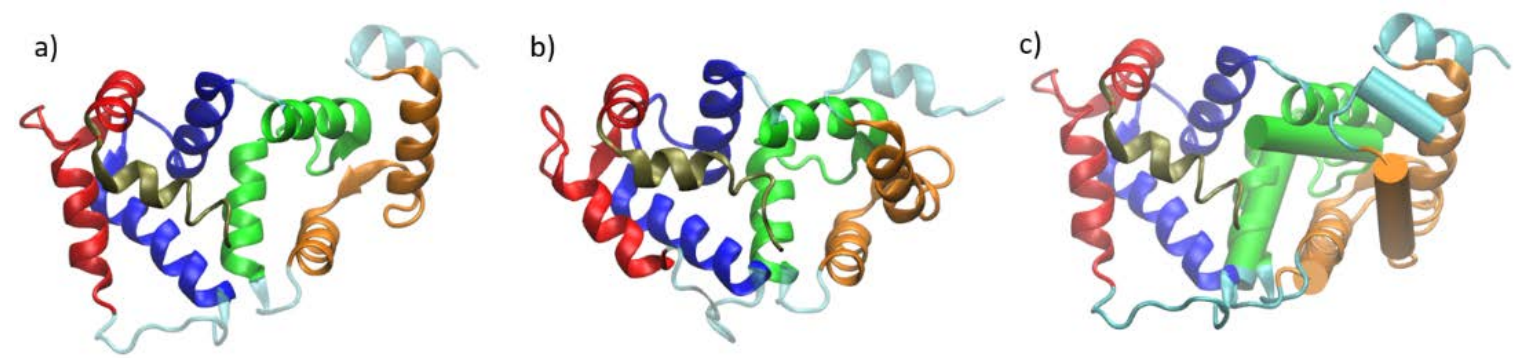

Figure 8.5. Global structural changes following 100 ns of aMD simulation. (a) Initial structure of DREAM following minimization and equilibration and (b) after $100 \mathrm{~ns}$ of aMD. (c) Overlaid of the initial structure shown in (a) with the N-terminus of DREAM after 100 ns of aMD simulation (b) represented as geometric cartoons. The C-terminus EFhand 3 and 4 were used in the alignment.

The most obvious changes at the $\mathrm{N}$-terminus are the reorganization of the entering helix of EF-hand 1, changing from a perpendicular orientation to a semi-parallel orientation with respect to the initial structure (Figure 8.6a), a similar rearrangement is observed for the EF-hand 2 (Figure 8.6b).

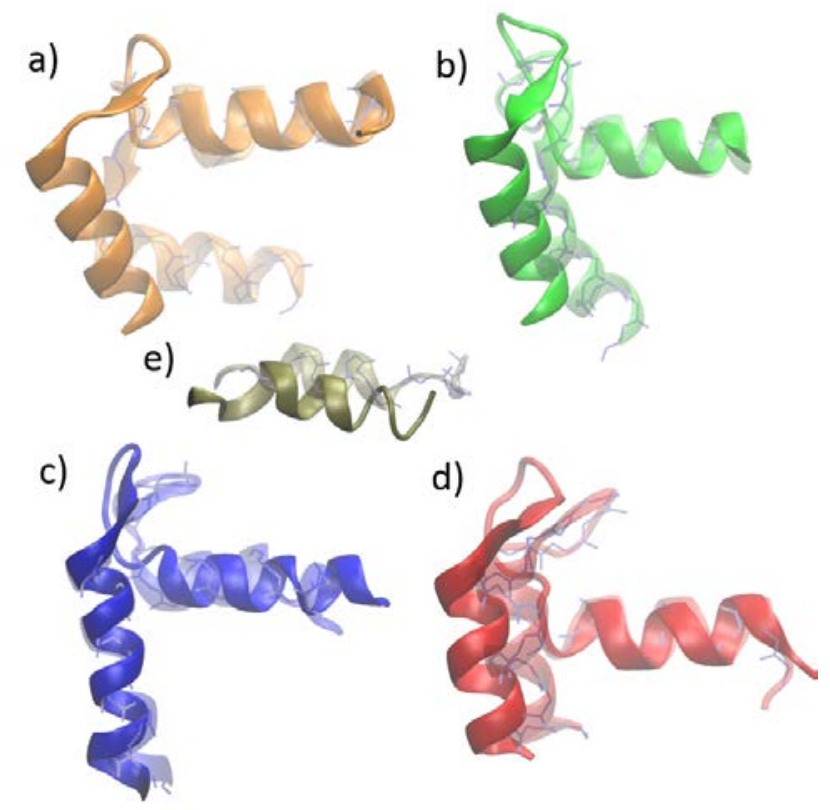

Figure 8.6. (a) Reorientation of the EF-hands of DREAM in the absence of $\mathrm{Ca}^{2+}$ before (shown in solid colors) and after 100 ns of aMD simulation (shown in diffuse color with solid lines representing the backbone atoms). All alignments were conducted using the first 12 amino acids of the entering helix (shown horizontal). The EF-hand 1 residues 89 to 122 (a), EF-hand 2 residues 128 to 160 (b), EF-hand 3 residue 163 to 198 (c), EF-hand 4 residue 210 to 242 (d), and the H10 helix residues 243 to 256. 
The reorientation of EF-hand 1 is accompanied by a collapse of the N-terminus hydrophobic cavity. However, at the C-terminus, a minimal reorganization of the helices is observed compared to the initial structure. The small reorganization is mainly localized on the EF-hand 3 and the H10 helix. A small bent of the entering helix of EF-hand 3 is observed (Figure 8.6c). The EF-hand 4 on the other hand, shows a minimal change in orientation of the entering and exiting helices (Figure 8.6d). As shown in Figure 8.6e, a slight shift in EF-hand 4 is also observed.
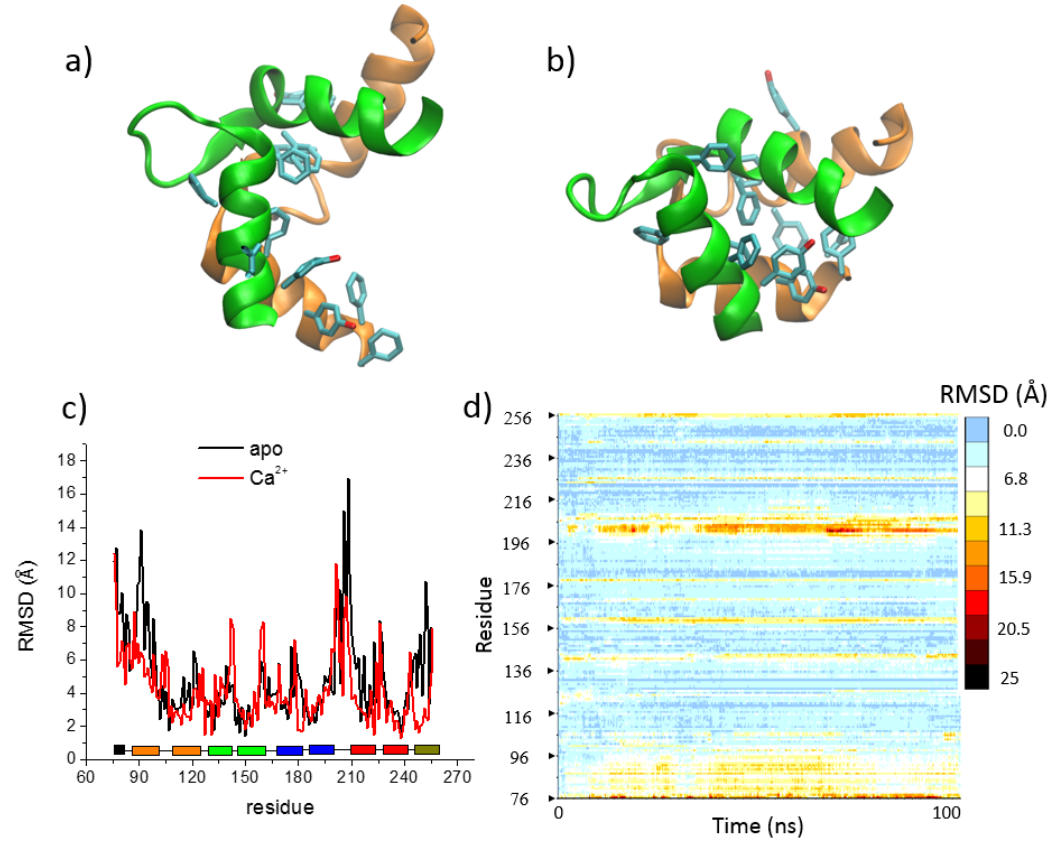

Figure 8.7. Structure of the N-terminus domain of DREAM (EF-hand 1 in orange and EFhand 2 in green) (a) before simulation and (b) after $100 \mathrm{~ns}$ of aMD simulation in the absence of $\mathrm{Ca}^{2+}$. Root mean square deviation per residue of the last $10 \mathrm{~ns}$ compared to the initial structure of $\mathrm{Ca}^{2+}$ bound DREAM (red) and $\mathrm{Ca}^{2+}$ free DREAM (black). The approximate positions of the EF-hand helices shown as colored squares, EF-hand 1 in orange, EF-hand 2 in green, EF-hand 3 in blue, and EF-hand 4 in red. Heat map representation of the RMSD per residue as a function of time for $\mathrm{Ca}^{2+}$ bound DREAM during $100 \mathrm{~ns}$ of aMD simulation.

At the residue level, the collapse of the N-terminus hydrophobic surface brings together numerous hydrophobic amino acids, namely Leu96, Phe100, Phe114, Ile117, Tyr118, Phe121, Phe122, Y151, Leu155, Leu158, and Leu159 (Figure 8.7a and b). 
Interestingly, the entering helix of EF-hand 1, on which residues known to interact with $\mathrm{K}_{\mathrm{v}} 4$ channels and DNA reside, shows the largest structural change. At the C-terminus, the structural changes observed in the presence or absence of $\mathrm{Ca}^{2+}$ are very similar, compare Figure 8.4c and 8.7d, with the most prominent change being due to motion of the long loop (residues 199 to 209) connecting EF-hand 3 and 4. As shown in Figure 8.6, the entering and exiting helices of EF-hands 3 and 4 do not move significantly; however, the metal binding loops of each hand undergo significant change in flexibility and structure (Figure 8.8). This change in structure and increase in flexibility is localized near Asn177 and Asp179 of EF-hand 3, which provide the $\mathrm{Y}$ and $\mathrm{Z}$ coordination to the $\mathrm{Ca}^{2+}$ metal (Figure 8.7a and b). Similarly, Asp223, Asn225, and Asp227 which provide the X, Y, and Z metal coordination of EF-hand 4 become significantly more flexible (Figure 8.8c and d).
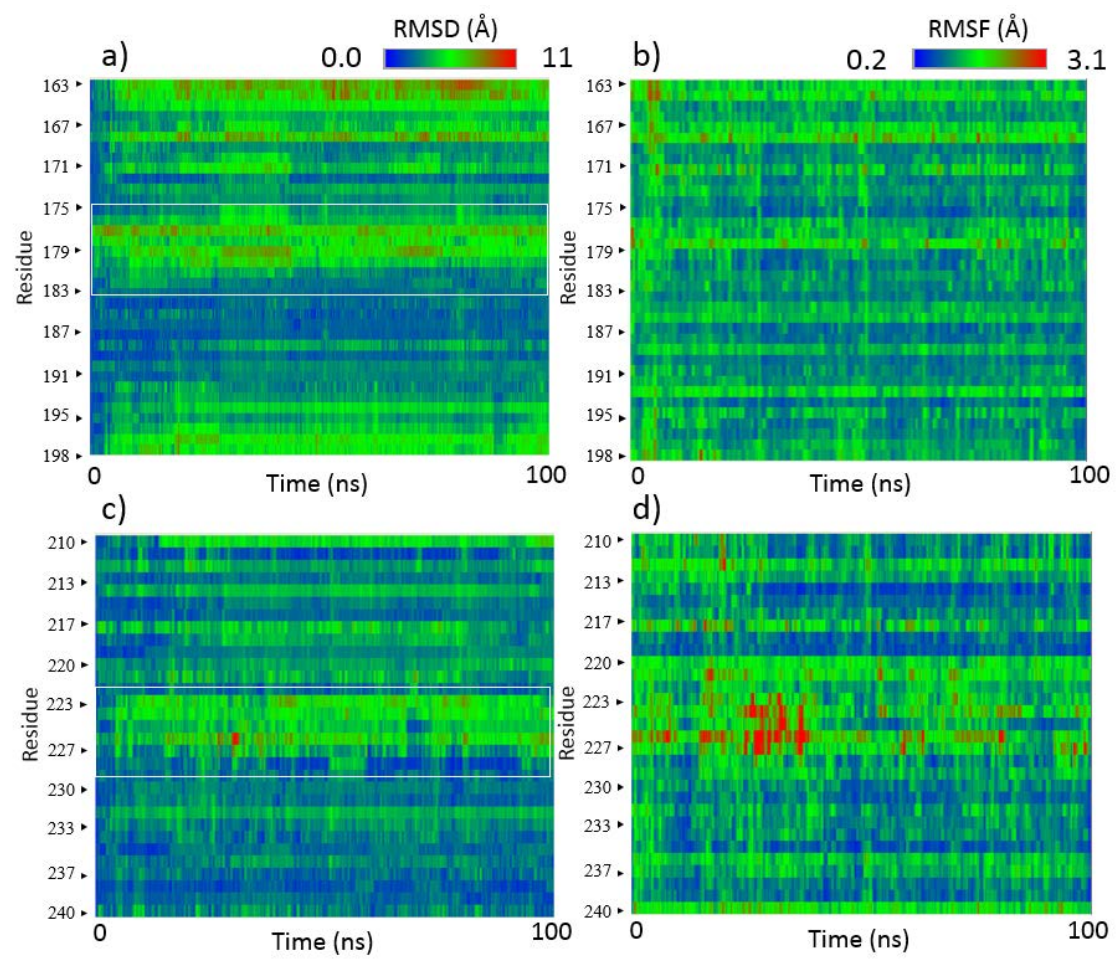

d)

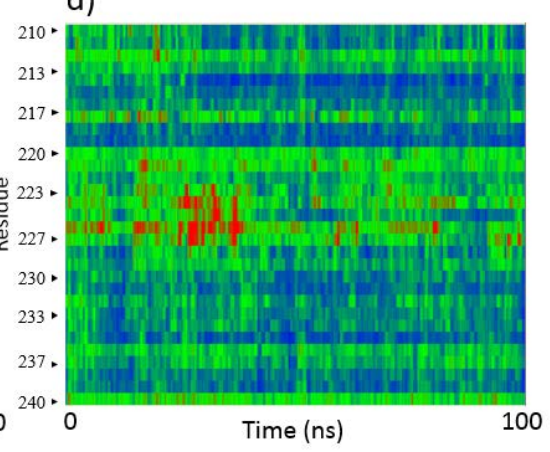

Figure 8.8. (a) Root mean square deviation (RMSD) and root mean square fluctuation (RMSF) of the EF-hand 2 (residues 163 to 198. RMSF calculated with a moving window of $1 \mathrm{~ns}$ with a step of $0.2 \mathrm{~ns}$. The RMSD (c) and RMSF(d) of EF-hand 4. White square highlights the N-terminus of the metal binding loop. 
These findings are in good agreement with a model in which the N-terminus amino acids of the EF-hand chelating loop are highly flexible in the absence of $\mathrm{Ca}^{2+}$, while metal binding induces a decrease in flexibility and large entropic gain which drives metal binding (Gifford, Walsh \& Vogel 2007b). Interestingly, the fluctuations in EF-hand 4 are much larger than those observed in EF-hand 3, which is in good agreement with the observed loss of entropic gain when EF-hand 4 is inactivated by the Glu234Gln mutation as discussed in Chapter 6. However, to test whether the the collapse of the N-terminus domain is a result of $\mathrm{Ca}^{2+}$ dissociation or the simulation conditions, we also performed aMD simulations of the $\mathrm{Ca}^{2+}$ bound DREAM protein. As shown in Figure 8.7c and d, an increase in the N-terminus RMSD similar to that shown in Figure 8.4b and c is observed even in the presence of $\mathrm{Ca}^{2+}$. However, the change is not as large as that observed for the $\mathrm{Ca}^{2+}$ free protein (compare Figure 8.4c and 8.7d). Nonetheless, in the $\mathrm{Ca}^{2+}$ bound state, DREAM is known to form dimers (Lusin et al. 2008), which may indicate that the observed changes at the N-terminus arise from intersubunit interactions. To determine if intersubunit interactions play a role in stabilizing the $\mathrm{N}$-terminus domain, we conducted additional aMD simulations of a DREAM dimer structure. The DREAM dimer structure was generated using the dimeric structure of KChIP1 (PDB: 1S6C) (Zhou et al. 2004b), the structure of the DREAM dimer is shown in Figure 8.9a. The resulting structure is similar to the previously proposed structure, in which Leu155, Leu156, and Leu159 stabilize the dimeric structure in a head to tail fashion (Lusin et al. 2008). Following energy minimization and equilibration, the dimeric structure of DREAM reaches equilibrium within 10 ns of aMD simulation (Figure 8.9b). In addition, the RMSD of the entering helix of EF-hand 1 as well as the heat map plot reveal that the overall structure of DREAM is much less flexible in 
the dimeric conformation (Figure $8.10 \mathrm{~b}$ and c). These are remarkable results, and they clearly indicate that both binding of $\mathrm{Ca}^{2+}$ at EF-hand 3 and EF-hand 4 as well as dimerization play a significant role in stabilizing the N-terminus EF-hand 1. More importantly, these results provide evidence for the presence of pathways which propagates structural signals from the $\mathrm{C}$ - to the $\mathrm{N}$-terminus in DREAM.

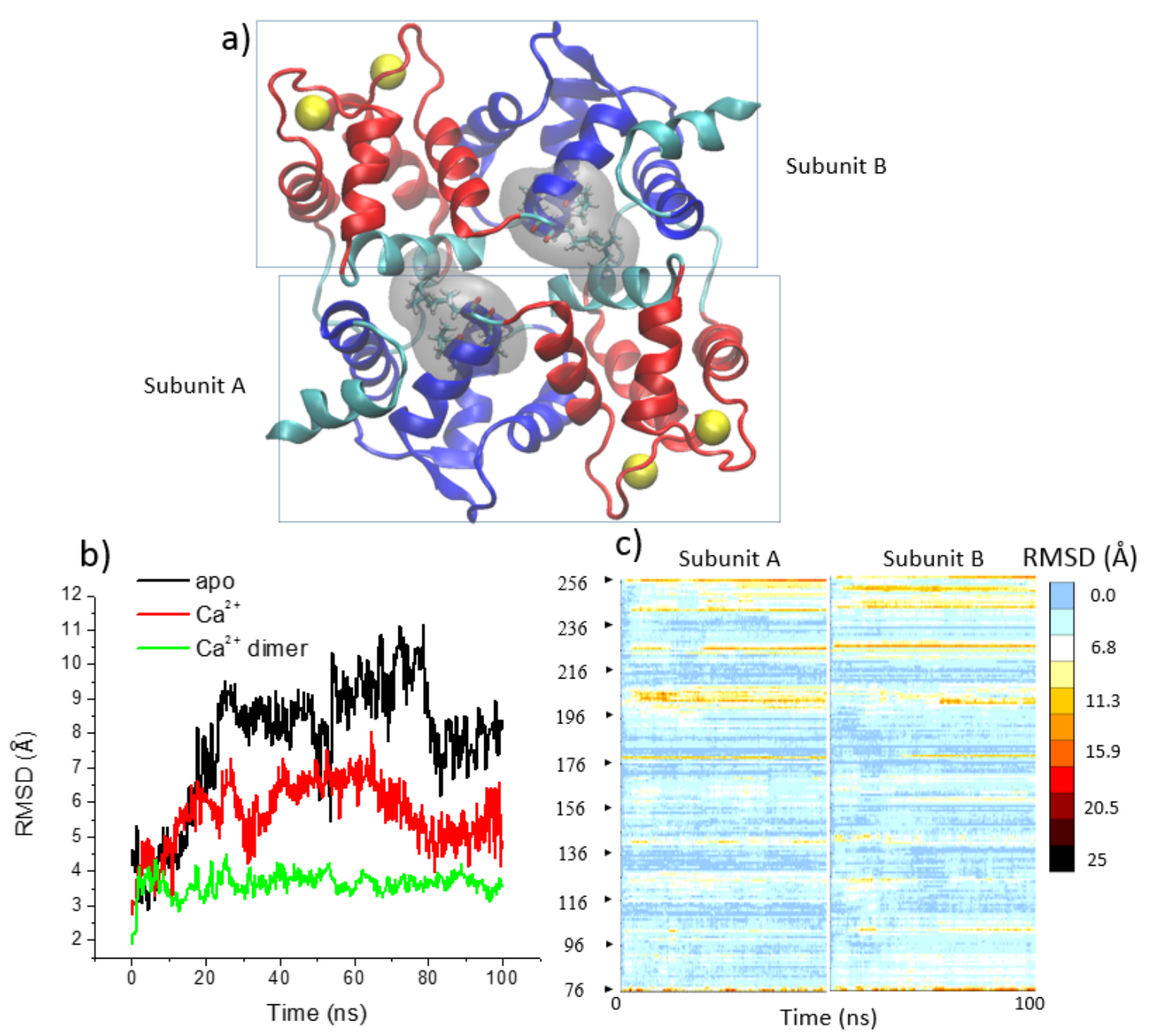

Figure 8.9. (a) Cartoon representation of the proposed DREAM dimer in which Leu155, Leu158, Leu159, and Leu251 are shown in licorice model with a diffuse surface. The Nterminus EF-hand 1 and 2 shown in blue and the C-terminus EF-hand 3 and 4 shown in red. (b) RMSD of the $C_{a}$ in EF-hand 1 (residues 89 to 102) as a function of aMD simulation time for the $\mathrm{Ca}^{2+}$ free and bound DREAM as well as the dimer of DREAM. (c) Heat map of the RMSD per residue as a function of simulation time for each DREAM subunit in the dimer protein. 
8.2.2 Molecular dynamics simulations reveal the role intersubunit and intrasubunit dynamic coupling

All members of the NCS subfamily are known to fold in a compact manner similar to that observed in recoverin (Figure 8.3), a domain organization significantly distinct than that observed in calmodulin. Also, unlike calmodulin, NCS proteins do not bind $\mathrm{Ca}^{2+}$ at the most N-terminal EF-hand 1. However, structural information on the $\mathrm{Ca}^{2+}$ free and bound recoverin seems to indicate that certain structural changes happen near the $\mathrm{N}$-terminus, where a myristoyl group resides (Ames et al. 1997). The similar compact structural organization of NCS proteins brings to question whether the ability to transduce signal from the C- to the N-terminus is a common feature among these proteins. Based on the results of the previous section and chapters, we propose that there are two pathways by which $\mathrm{Ca}^{2+}$ binding regulates structural changes at the $\mathrm{N}$-terminus. One is through direct intrasubunit structural changes and another which involves intersubunit communications. However, the nature of these structural changes are not obvious, since we have observed that besides local changes in flexibility (Figure 8.9), $\mathrm{Ca}^{2+}$ binding at EF-hand 3 and 4 does not lead to significant changes in the structure of the C-terminus. In this section, we test the hypothesis that monitoring of the dynamic fluctuation in DREAM provides useful information necessary to understand the mechanism controlling structural reorganization in this protein. 


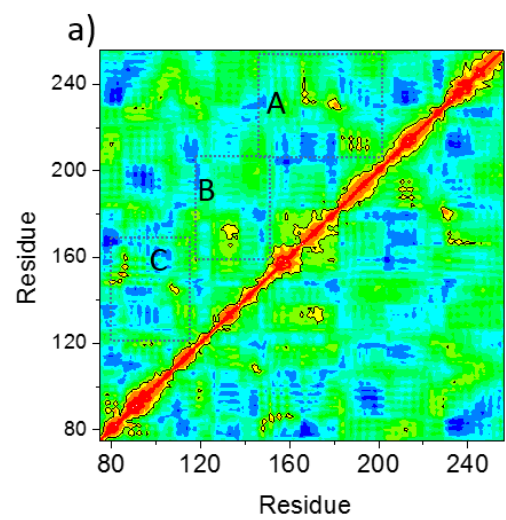

b)

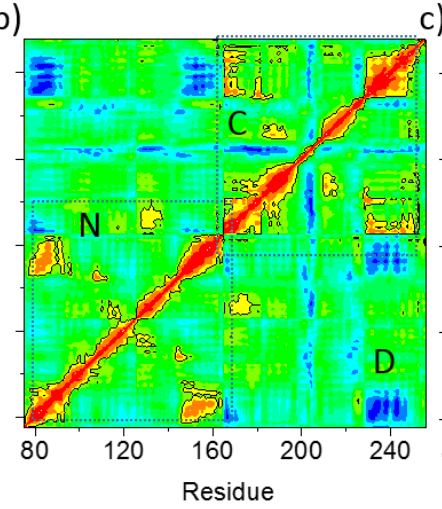

c)

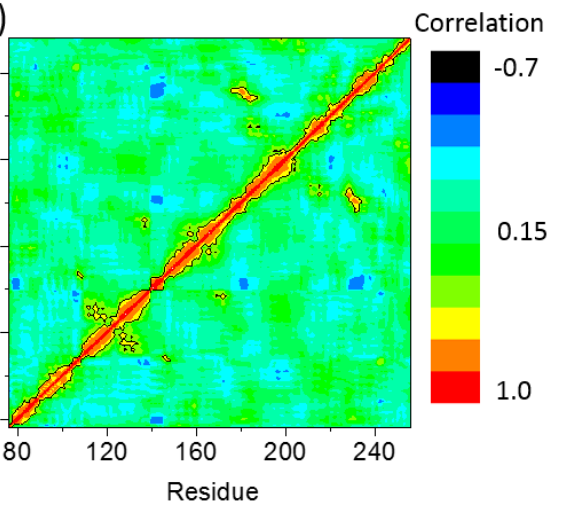

Figure 8.10. Residue cross correlation analysis of the cMD simulations for the (a) $\mathrm{Ca}^{2+}$ bound DREAM monomer, (b) $\mathrm{Ca}^{2+}$ bound DREAM dimer (only one subunit shown), and (c) $\mathrm{Ca}^{2+}$ free DREAM monomer. All the structure are based on the last $80 \mathrm{~ns}$ of a cMD simulation at which point the RMSD changed by less than $2 \AA$. The structure of the $\mathrm{Ca}^{2+}$ free DREAM was obtained after 100 ns of aMD simulation. Regions of interest are marked and described in the text.

We propose that the transmission of allosteric signal in DREAM is coupled to dynamic fluctuations of amino acids in the protein, these fluctuations effectively propagate information and induce structural changes at the N-terminus of DREAM. To test this hypothesis we have analyzed the cMD simulations of $\mathrm{Ca}^{2+}$ bound DREAM dimer and monomer as well as the $\mathrm{Ca}^{2+}$ free DREAM monomer using dynamic cross correlation of the alpha carbons between amino acids as a function of simulation time. The approach allows us to monitor all residues and identify cluster of amino acids (communities) in which dynamic fluctuations are highly correlated. These communities dissect the DREAM structure into segments that allows us to understand the pathways of signal transduction in DREAM protein. Figure 8.10 shows the residue-based dynamic cross correlation maps of DREAM as a function of metal binding and oligomerization state. These correlation maps were obtained by analyzing the equilibrium conformation of each state, namely, the last 80 ns of the simulation during which the RMSD did not change significantly. Therefore, these correlation maps represent fluctuations from equilibrium. Analysis of the cross correlation 
map of $\mathrm{Ca}^{2+}$ bound DREAM $(\Delta 65)$ shows the presence of widespread regions of correlated and anticorrelated motion, shown as blue and red in Figure 8.10a. In the map shown in Figure 8.10a we observe three region; region A connects amino acids at the C-terminus and region $\mathrm{C}$ connects amino acids at the C-terminus. These two dynamic domains are connected by a strong correlation between amino acids 130 to 137 at the $\mathrm{N}$-terminus and 168 to 174 at the C-terminus, highlighted as region B.

Furthermore, a clear widespread increase in dynamic coupling can be observed for the dimeric DREAM simulation (Figure 8.10b). The increase in correlation is present for regions $\mathrm{A}, \mathrm{B}$, and $\mathrm{C}$, which were already partially correlated in the $\mathrm{Ca}^{2+}$ bound DREAM monomer simulation. For instance, the region marked as C in Figure 8.10b corresponds to the C-terminus of DREAM and shows a strong increase in correlation, a similar increase if observe for the N-terminus. Interestingly, the anticorrelated region connecting the entering helix of EF-hand 1 (residues 80 to 102) to the exiting helix of EF-hand 4 (residue 230 to 242) is enhanced in the dimeric form as well, shown as region D. Therefore, these results suggest that dimerization strengthens already existing dynamic connections, likely enhancing the stability of the protein and transduction of dynamic information. On the other hand, the correlation map for $\mathrm{Ca}^{2+}$ free DREAM shows a clear overall decrease in correlation, both at the $\mathrm{N}$ - and C-terminus (Figure 8.10c). Regions $\mathrm{C}$ and $\mathrm{N}$ become significantly less correlated, while region B becomes completely uncorrelated. These results show a clear transition triggered by $\mathrm{Ca}^{2+}$ binding to DREAM, from a disordered protein conformation to a highly dynamically organized structure. 

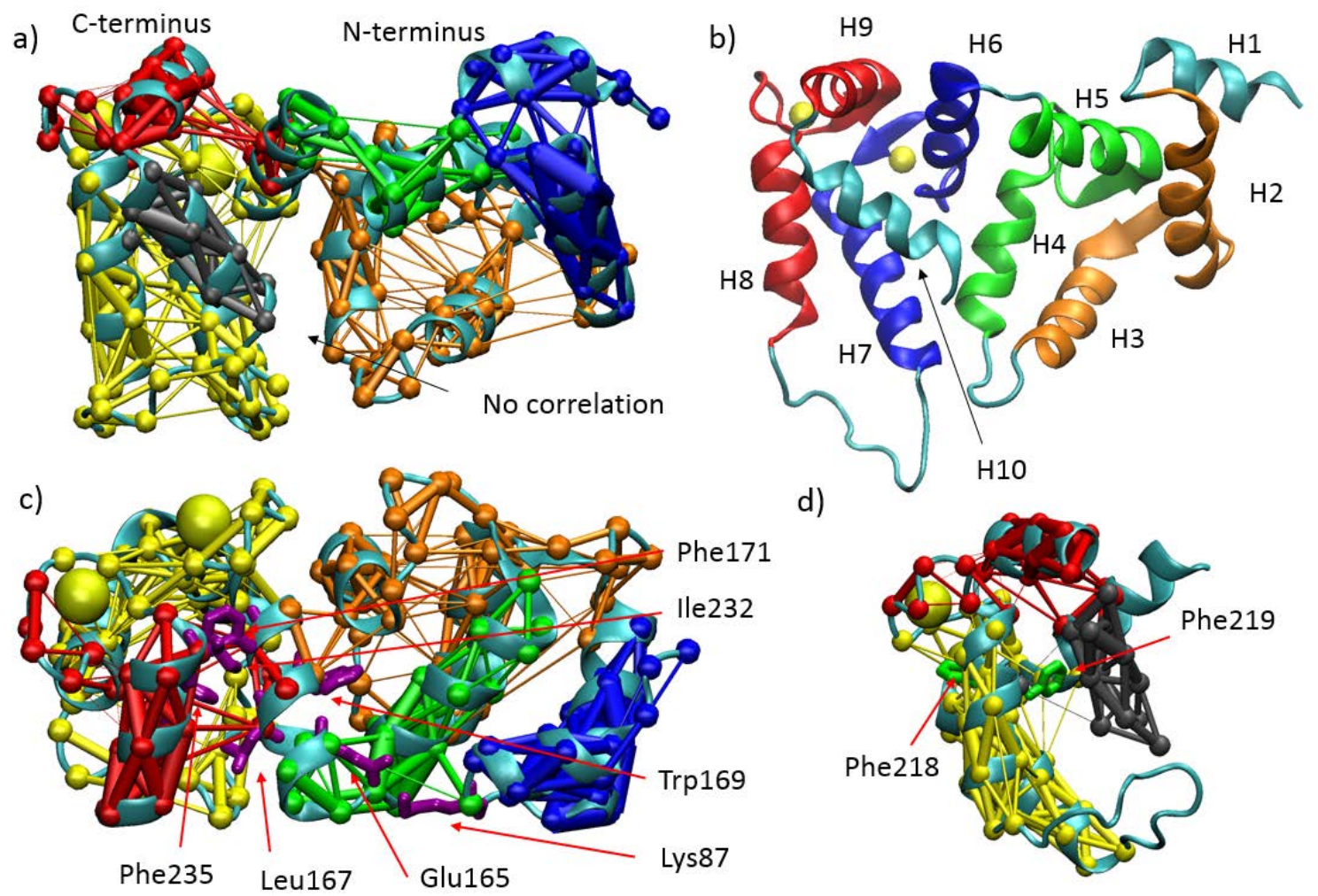

Figure 8.11. (a) Cartoon representation $\mathrm{Ca}^{2+}$ bound DREAM monomer with the colored communities shown as ball and stick colored cartoons. The structure was obtained following 100 ns of cMD simulation. (b) Cartoon representation of DREAM with EF-hands colored and helices labeled for reference to panel (a). (c) Structure of DREAM with overlaid dynamic networks highlighting the critical amino acids connecting the $\mathrm{N}$ - and Cterminus of DREAM (amino acids shown in purple licorice, H10 and gray network not shown for clarity). (d) Networks at the C-terminus showing the connection between the H10 helix (gray network) and the entering helix of EF-hand 4 (yellow network), critical amino acids shown in green licorice model.

To better illustrate the dynamic organization of DREAM, we have carried out analysis of the simulations discussed above using dynamic network analysis. This type of analysis allows us to build three dimensional networks which represent the cluster of amino acids whose motions are highly correlated. The resulting networks of $\mathrm{Ca}^{2+}$ bound DREAM monomer overlaid on the DREAM structure are shown in Figure 8.11. One of the most salient observation of Figure 8.11a is the particularly defined organization of the $\mathrm{N}$ - and C-terminus domains, each of which is subdivided in three communities. At the N-terminus, 
the blue community spans helix 1 and the entering helix of EF-hand 1, the orange community connects the exiting helix of EF-hand 2 and entering helix of EF-hand 3, while the green network contains the exiting helix of EF-hand 2 and the beginning portion of EFhand 3. The C-terminus shares a similar organization, with three main communities, the largest one being the yellow network connecting the exiting helix of EF-hand 3 and entering helix of EF-hand 4. The metal binding loops at the C-terminus also form a highly correlate community (shown in gray) which connects both loops and the entering helix of EF-hand 3. Lastly, the H10 helix is minimally coupled to the rest of the protein and forms a community shown in red. Overall, these communities highlight the role of the entering helix of EF-hand 3 in connecting both domains. The entering helix of EF-hand 3 (H6) seems to function as a dynamic hub through which fluctuations between the N- and Cterminus can be propagated. In Figure 8.11c the critical amino acids connecting the Nterminus (Trp169, Glu165, and Lys87) and the C-terminus (Leu165, Phe171, and Tyr174) are shown. Analysis of the amino acids connecting the communities, herein referred as critical nodes, identifies Trp169, Phe171, and Tyr174 as playing major roles. The Nterminus connection is dominated by interactions of Trp169 with Phe138 and Leu134. Additionally, the dynamic coupling in this domain is also extended by Glu165 and Lys87 which make a strong salt bridge spanning the entering helix of EF-hand 3 and entering helix of EF-hand 1 (green to blue network). At the C-terminus the predominant connection is that of Leu167 and Phe171 on the entering helix of EF-hand 3 interacting with Phe235 and Ile232 on the exiting helix of EF-hand 4. At the C-terminus, the H10 helix is coupled to the C-terminus core (yellow network) by the interaction between Phe252 on the H10 helix and Phe219 on the entering helix of EF-hand 4. Interestingly, Phe218 is strongly 
coupled to the EF-hand $3 \mathrm{Ca}^{2+}$ binding loop, connecting the yellow and the green network. Altogether, these dynamics networks highlight the role of hydrophobic amino acids, specially aromatic amino acids, in maintaining the dynamic coupling from the $\mathrm{N}$ - to the Cterminus.

C-terminus $\quad \mathrm{N}$-terminus

a)

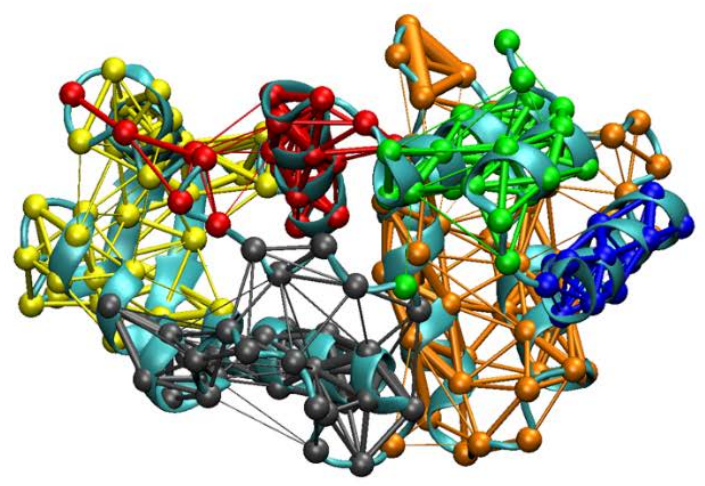

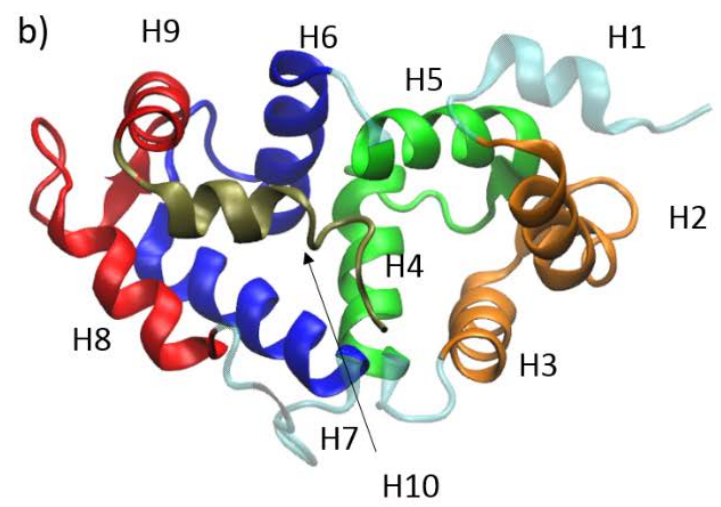

Figure 8.12. (a) Cartoon representation $\mathrm{Ca}^{2+}$ free DREAM monomer with the colored communities shown as ball and stick colored cartoons. The structure was obtained following $450 \mathrm{~ns}$ of cMD simulation. The communities were calculated using the last 80 ns of the cMD simulation. (b) Cartoon representation of DREAM with EF-hands colored and helices labeled for reference to panel (a).

Analysis of the $\mathrm{Ca}^{2+}$ free DREAM simulation shows that most of the communities are maintained, but the thinner edges connecting the nodes (representing alpha carbons) highlights a much weaker correlation (Figure 8.12). However, a strong reorganization of the networks at the C-terminus is observed, where the yellow and gray networks have rearranged. The yellow community now connects the entering and exiting helix of EF-hand 4 and partially the exiting helix of EF-hand 3. The gray network now connects the H10 helix and the exiting helix of EF-hand 3 and entering helix of EF-hand 4 while the red network is largely localized at the entering helix of EF-hand 3. These results allow us to propose that in the absence of $\mathrm{Ca}^{2+}$, the $\mathrm{H} 10$ helix becomes dynamically coupled to the core domain of DREAM through interactions with EF-hand 3. As described in Chapter 3 
and 4, binding of small hydrophobic ligands on DREAM is mediated by a hydrophobic cavity at the C-terminus. Therefore, it is likely that the mechanism being observed here, in which the H10 helix becomes free to move in the presence of $\mathrm{Ca}^{2+}$, but bound to the core domain of DREAM in the absence of $\mathrm{Ca}^{2+}$, is what regulates ligand binding. At the Nterminus, the three communities observed in the $\mathrm{Ca}^{2+}$ bound form of DREAM are maintained; however, the correlation strength is decreased significantly (judged by the thickness of the edges connecting the nodes).

a)

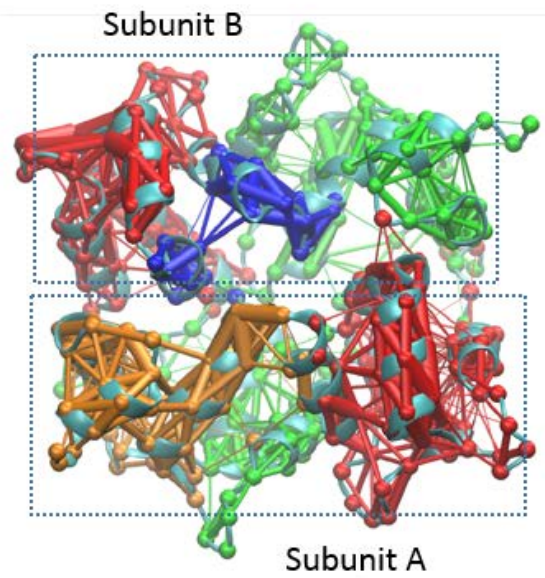

b)

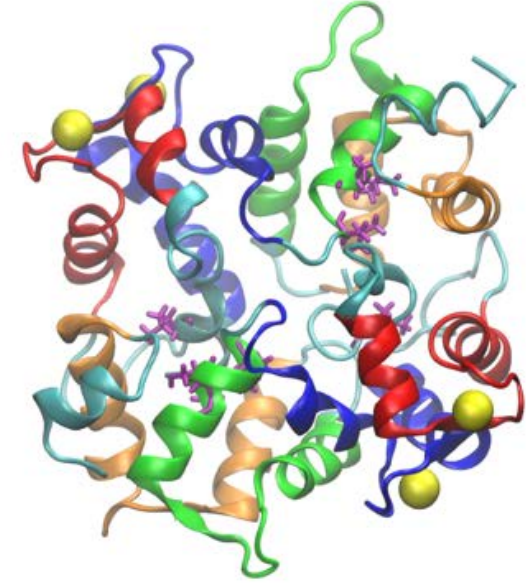

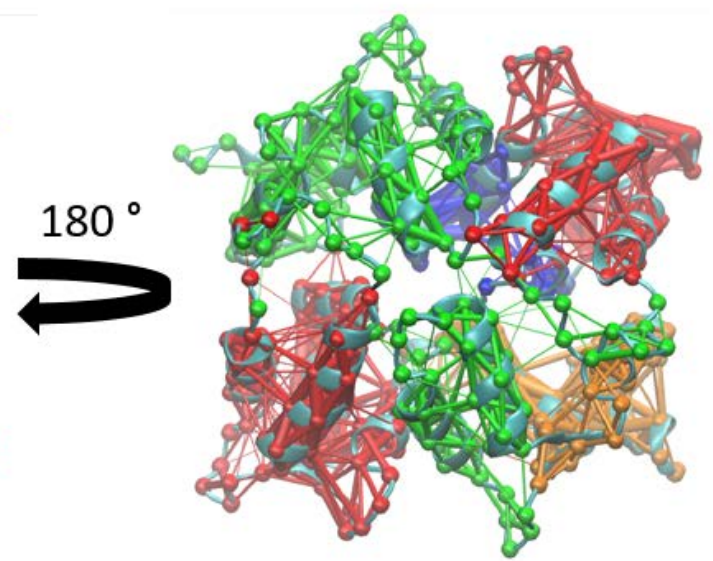

c)

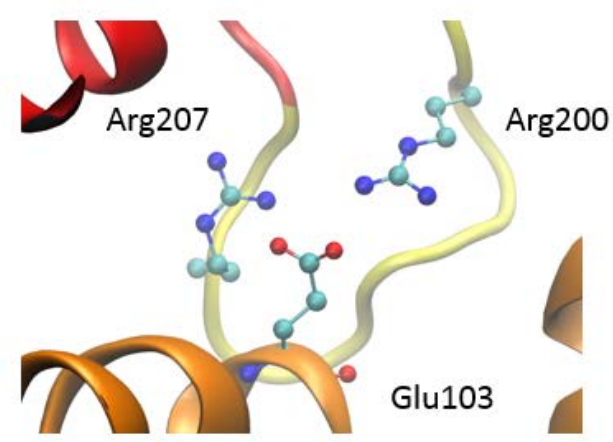

Figure 8.13. (a) Cartoon representation of the proposed DREAM dimer on which the communities have been overlaid using a ball and stick representation. Subunit A and B are highlighted in a dotted square. The figure on the right is rotated 180 degrees. (b) carton representation of DREAM with EF-hands colored and $\mathrm{Ca}^{2+}$ metal shown, LxxLL motif shown in purple licorice model. (c) Intersubunit salt bridges formed between DREAM subunits. 
The $\mathrm{Ca}^{2+}$ bound DREAM dimer shows the presence of much more extensive communities (Figure 8.13). The communities identified in the dimer are not symmetric, but in both subunit three well defined dynamic subdomains can be observed. The red community, is predominant in both subunit, and it includes both EF-hand 3, EF-hand 4, and the H10 helix. In addition, a community (shown in green) spanning the H1 helix, EFhand 1, and EF-hand 2 is found in subunit A. In subunit B, this community is smaller, only including the exiting helix of EF-hand 1 and entering helix of EF-hand 2 (shown in green). Despite their different organization, both subunits show strong intersubunit correlation between the red and green communities which is mediated by the long loop connecting EFhands 3 and 4 (Figure 8.13b and c). At the residue level, this intersubunit interaction is stabilized by a strong salt bridge between Glu103 on one subunit and Arg200 and Arg2007 on the adjacent subunit (Figure 8.13c). In addition a cluster of four aromatic amino acids (Phe121 and Phe122 on each subunit) form a stable connection between both green communities (not shown).

Analysis of the critical nodes connecting the network on each subunit revealed the role of Leu155, Leu156, Leu159, and Leu251 in stabilizing the dimeric structure of DREAM (Lusin et al. 2008). However, the interaction between the loop connecting EFhands 3 and 4 in one subunit with EF-hand 1 have not been previously identified. Overall, the model that emerges from these analyses is that the entering helix of EF-hand 3 plays an important role in connecting the dynamic motions of the N- and C-terminus. This observation may seem obvious at first, given that this helix is at the middle of the $\mathrm{N}$ - and C-terminus pair of EF-hands. However, a closer look at the structure of DREAM shows that the exiting helix of EF-hand 3 and the entering helix of EF-hand 2 are in adjacent to 
each other, and yet, no correlations was observed between these helices (Figure 8.11a). more importantly, the correlation between the entering helix of EF-hand 3 and the rest of the C-terminus is highly remodeled by binding of $\mathrm{Ca}^{2+}$ to EF-hand 3 and 4. Secondly, the dimeric DREAM shows enhancement of the dynamic coupling among residues at the Cterminus (red community in Figure 8.13). These simulation also highlight the role of charged residues at the flexible loop connecting EF-hands 3 and 4 in stabilizing the protein dimer. Indicating that the dimeric structure of DREAM is not only stabilized by hydrophobic interactions but also by the presence of salt bridges. Overall, these simulations have allowed us to identify the residues at the entering helix of EF-hand 3 and the flexible loop as playing a major role in connecting the $\mathrm{N}$ - and C-terminus. But more importantly,. The observation that specific aromatic amino acids play a role in connecting the dynamic subunits at the $\mathrm{N}$ - and $\mathrm{C}$-terminus provides an unique opportunity to investigate whether these amino acids are able to modulate the activity of this protein.

8.2.3 Dynamic network analysis reveals the role of key conserved aromatic amino acid at the C-terminus of DREAM

A closer inspection of the dynamic and structural changes triggered by removal of $\mathrm{Ca}^{2+}$ from DREAM during the cMD and aMD simulation of DREAM reveal and interesting reorganization of the C-terminus. As mentioned in the previous section, $\mathrm{Ca}^{2+}$ binding induces a strong reorganization of the C-terminus dynamic networks. These changes in dynamics show that upon binding of $\mathrm{Ca}^{2+}$, a strong dynamic coupling between then entering helix of EF-hand 3 (Figure 8.14a and b, purple helix) and the exiting helix of EFhand 4 (Figure 8.14a and b, cyan helix) is created. As shown in Figure 8.14c and d, we 
have observed that in the $\mathrm{Ca}^{2+}$ bound form, residue Phe235 is in close contact with Tyr174 and Phe171 at the entering helix of EF-hand 3. However, upon dissociation of $\mathrm{Ca}^{2+}$, Phe235 undergoes a rapid change in conformation so that it is closely stacked with Phe218 and Phe219 at the entering helix of EF-hand 4. The structural switching behavior of Phe235 is subtle, but the induced dynamic coupling is clearly shown by the dynamic network analysis.

a)

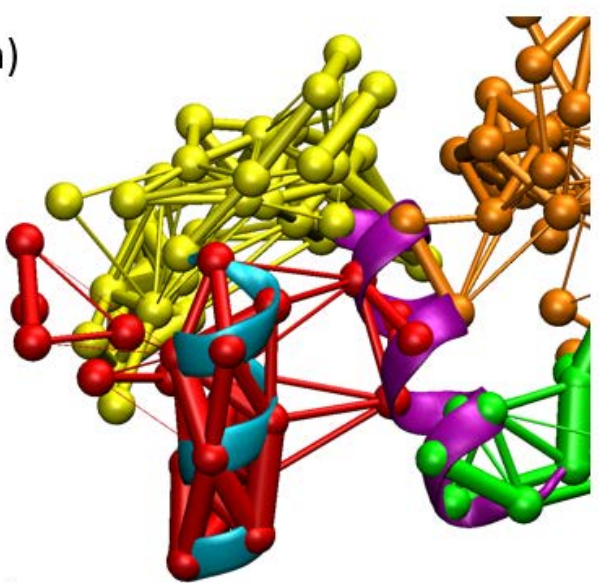

c)

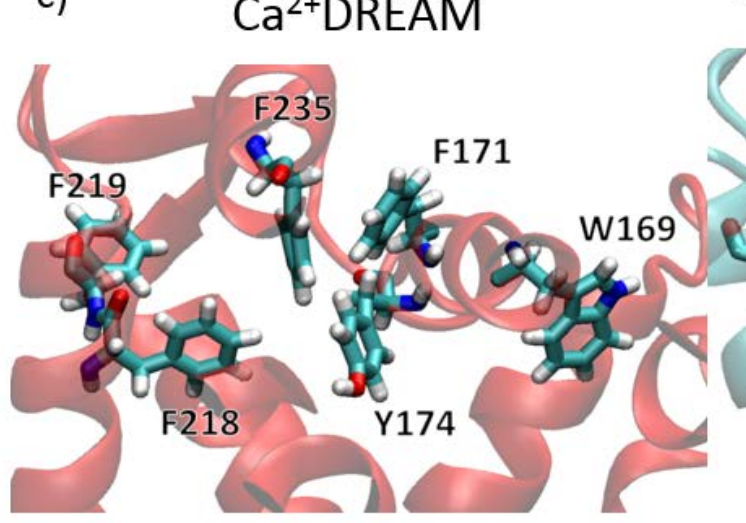

b)

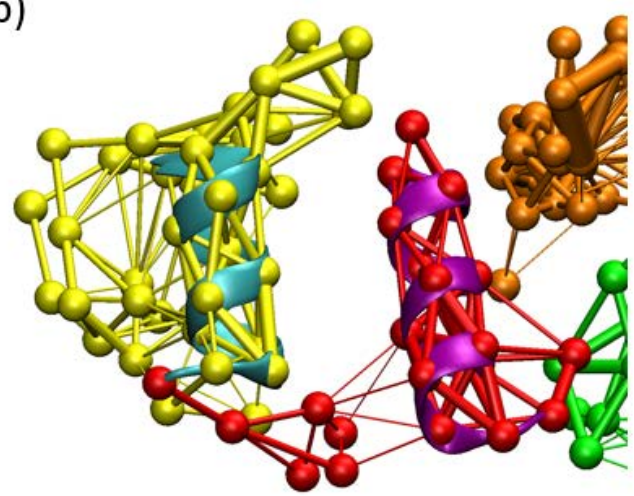

d)

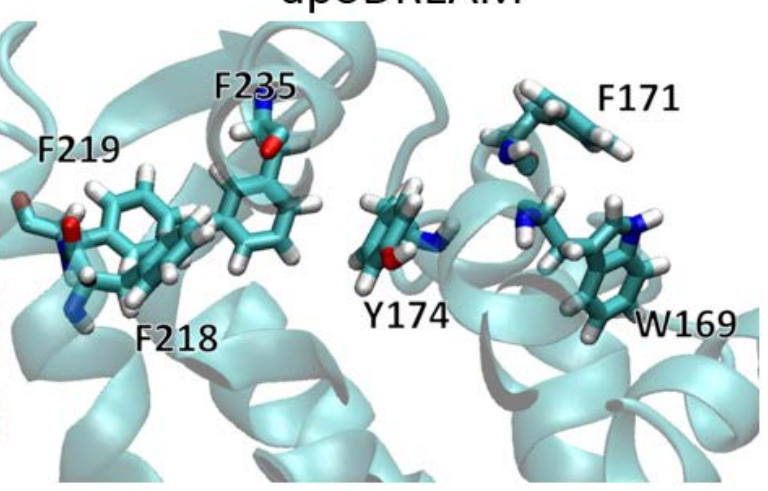

Figure 8.14. Dynamic networks at the C-terminus, highlighting the strong dynamic coupling between the exiting helix of EF-hand 4 (cyan) and the entering helix of EF-hand 3 (purple), (a) in the presence of $\mathrm{Ca}^{2+}$ and (b) in the absence of $\mathrm{Ca}^{2+}$. Reorganization of the C-terminus aromatic amino acids upon removal of $\mathrm{Ca}^{2+}$ from the EF-hands 3 and 4 of DREAM. 


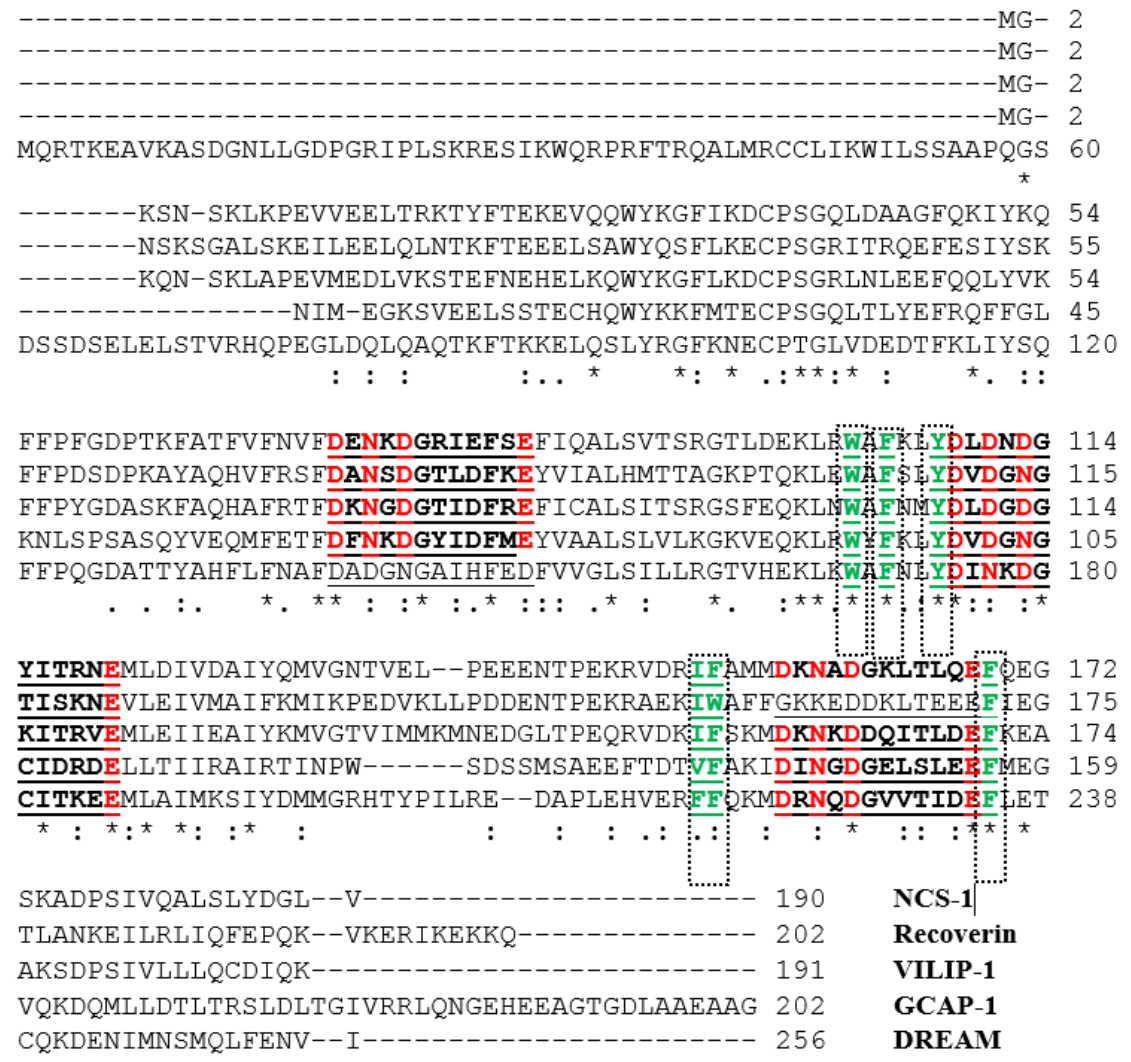

Figure 8.15. (a) Sequence alignment of mouse NCS-1, recoverin, VILIP-1, GCAP-1, and DREAM. Turn loops of EF-hand 1, 2, 3, and 4 are shown in bold and underlined. the negatively charged amino acids involved in metal coordination shown in red. The conserved aromatic amino acids are shown in bold underlined green letters. Asterisks indicate identical residues, semicolon indicates very similar residues, and the period is for somewhat similar residues. Amino acids involved in $\mathrm{K}_{\mathrm{v}} 4$ binding are shown in green.

Analysis of the amino acid sequence of proteins belonging to all five group of NCS proteins (recoverin, NCS-1, VILIP-1, GCAP1, and DREAM) shows that these five aromatic amino acids are highly conserved among NCS protein (Figure 8.15). Therefore, it is possible that the observed conformation shift of Phe235 may represent a fundamental conformation transition upon $\mathrm{Ca}^{2+}$ binding in this family of CaBPs. Interestingly, in Chapter 4, we describe that the small hydrophobic compound NS5806 interacts with DREAM via a stacking interaction between the rings of Phe218 and Tyr174. The stacking interaction between N25805, Phe218, and Tyr174 induces a structural change on DREAM 
which facilitates $\mathrm{K}_{\mathrm{v}} 4$ channel blinding, even in the absence of calcium. Thus, we speculate that stacking of NS5806 between Phe218 and Phe219 would effectively displace Phe235 and enhance the correlation of this amino acid with the entering helix of EF-hand 3. Therefore, it could be possible that the switching mechanism of Phe235 observed in these simulation is essential in regulating DREAM activity.

\subsubsection{Disruption of intrasubunit and intersubunit communication in DREAM by site} directed mutagenesis and a DREAM-NCS1 chimera

The analyses using molecular dynamic simulations have highlighted the role of the entering helix of EF- hand 3, the loop connecting EF-hand 3 and 4, and aromatic amino acids at the C-terminus of DREAM in regulating $\mathrm{Ca}^{2+}$ induced oligomerization and structural changes. These theoretical approaches were carried out to identify the mechanism by which $\mathrm{Ca}^{2+}$ regulates binding of ligands at the $\mathrm{N}$ - and C-terminus. Dynamic network analysis supports a model in which the $\mathrm{H} 10$ helix become dynamically coupled to EF-hand 3 in the absence of $\mathrm{Ca}^{2+}$, effectively eliminating the flexibility of this helix. This mechanism could explain the observed decreased in affinity for hydrophobic ligands binding at the C-terminus of DREAM. On the other hand, analysis of the simulation trajectory in section 8.2.1 and 8.2.2 show that propagation of structural changes from the C-terminus towards the N-terminus could potentially be mediated by intrasubunit and/or intersubunit couplings.

Therefore, to support these findings it is essential to develop experimental approaches that could verify the role of specific amino acids on $\mathrm{K}_{\mathrm{v}} 4$ and drug binding. In this section and the next, we present extensive biochemical characterization of a small 
library of DREAM mutants developed to understand the precise pathways of signal transduction in DREAM. Following results from the previous section, we have generated DREAM mutants in which Tyr174 and Phe235 were replaced by alanine in order to disrupt the intrasubunit pathways spanning the $\mathrm{N}$ - and $\mathrm{C}$-terminus. These amino acids were identified as critical amino acid coupling dynamic motions between the $\mathrm{N}$ - and $\mathrm{C}$-terminus. In addition, the single amino acid mutants Phe218Ala, and Phe219Ala have been generated to study the role of dynamic coupling at the C-terminus and the role of the transition shown in Figure 8.16.

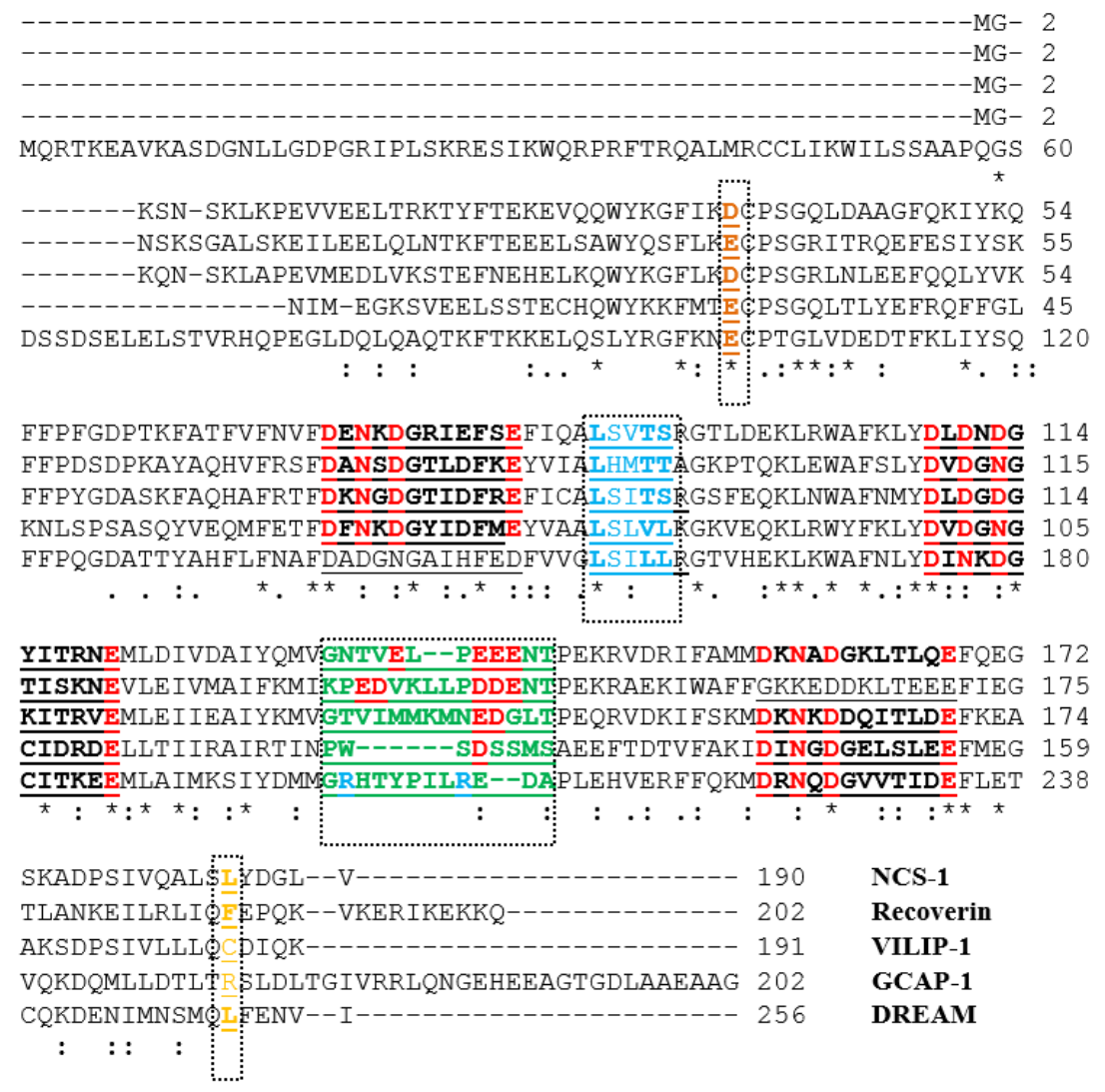

Figure 8.16. (a) Sequence alignment of mouse NCS-1, recoverin, VILIP-1, GCAP-1, and DREAM. Turn loops of EF-hand 1, 2, 3, and 4 are shown in bold and underlined. the negatively charged amino acids involved in metal coordination shown in red. The loop connecting the EF-hands 3 and 4 are shown in green bold and underlined, the respective negatively residue with whom these amino acids interacts is shown in bold underlined orange color. The LxxLL motif is shown in blue and the the amino acid with whom they interact is shown in orange. 
To investigate the role of intersubunit communications, we have generated a DREAM-NCS1 chimera in which the loop region connecting EF-hands 3 and 4 as well as the leucine rich motif in DREAM have been mutated to those present in NCS1. The neuronal calcium sensor-1 protein was selected because it is the only member of the NCS subfamily of $\mathrm{Ca}^{2+}$ binding proteins known to form monomers in the presence of $\mathrm{Ca}^{2+}(\mathrm{Lian}$ et al. 2011). As shown in Figure 8.16, the Leucine charged domain with motif LxxLL is present in DREAM and partially in GCAP-1, but not in NCS-1, recoverin, and VILIP-1. Also, the hydrophobic amino acid with which the LxxLL motif interacts, is only present in DREAM (Leu251), NCS-1, and recoverin. Thus, indicating that the LxxLL motif may not be the only regulator of dimerization. However, analysis of the charge density of the loop connecting EF-hands 3 and 4 reveal a gradual decrease in total charge, with NCS-1 (charge -4), recoverin (charge -3), VILIP-1 (charge -1), GCAP-1 (charge -1), and DREAM (charge 0). At the N-terminus, the negatively charged residue forming a salt bridge with the charged loop is well conserved among all NCS proteins (Figure 8.16 highlighted in orange). Therefore, it is probable that NCS-1 would experience a strong repulsion between the negatively charged loop connecting EF-hands 3 and 4 and Asp37 at the N-terminus, while DREAM would form a favorable salt bridge between Arg200, Arg2007, and Glu103. It is interesting to note that Glu103 corresponds to the $-\mathrm{Z}$ coordination of the $\mathrm{Ca}^{2+}$ binding loop of EF-hand 1. Therefore, it is possible that in NCS proteins, the structural changes induced at EF-hand 1 are not regulated by direct $\mathrm{Ca}^{2+}$ binding, but by intersubunit contact. On the basis of these observations we hypothesize that replacement of the loop residues of DREAM (residues 200 to 207, sequence: RHTYPILR) by the loop of NCS (residues 134 to 141, sequence: NTVELPEE) and of the LxxLL motif of DREAM by the LxxTS motif 
of NCS would result in inhibition of dimerization. The purpose of eliminating the dimerization of DREAM would serve to identify if whether the structural changes observed at the N-terminus of DREAM are triggered by intersubunit communications.

In, addition we have also expressed and purified a $\operatorname{DREAM}(\Delta 160)$ protein construct in which the N-terminus domain (EF-hands 1 and 2) have been deleted. The $\operatorname{DREAM}(\Delta 160)$ construct allows us to investigate the role of the N-terminus in modulating the stability of the C-terminus. It would also provide insight in the how the presence of EFhand 1 and 2 can affect the fluorescence of Trp169 residing at the entering helix of EFhand 3. Lastly, a mutant in which Phe252 has been replaced by alanine would allow us to investigate whether disruption of the critical node between Phe252 and Phe218 can enhance or inhibit association of ligands at the C-terminus hydrophobic cavity.

8.2.5 Mutation of the proposed aromatic amino acids and replacement of the LxLL and loop region of DREAM does not induce unfolding of the protein

Expression of these DREAM mutants in E. coli results in good protein yields, with quantities ranging from 10 to $15 \mathrm{mg}$ of protein per liter of TB media. To ensure that these protein mutants are in a folded conformation, we have measured the CD spectra of all the mutants (Figure 8.17). These spectra clearly show that all mutants have a significant degree of alpha helical content, as expected for a fully alpha helical protein. These data also show that addition of $\mathrm{Ca}^{2+}$ induces an increase in alpha helical content, or reorganization of the alpha helices, which results in an overall decrease in ellipticity. However, not all mutants showed this transition, indicating that these amino acids mutations have effectively affected the ability of $\mathrm{Ca}^{2+}$ to reorganize the protein structure. For instance, the 
DREAM(F235A) mutant (Figure 8.17e and inset) shows a reverse transition to that of $\operatorname{DREAM}(\Delta 65)$, in which $\mathrm{Ca}^{2+}$ binding induces a decrease in ellipticity. The small deviations in ellipticity likely arise from deviations in protein concentration or small differences in the overall structure of the proteins.

Figure 8.17. Far-UV circular dichroism spectra of DREAM mutants. (a) DREAM $(\Delta 65)$, (b) DREAM( 160$)$, (c) DREAM(Y174A), (d) DREAM(F218A), (e) DREAM(F235A),
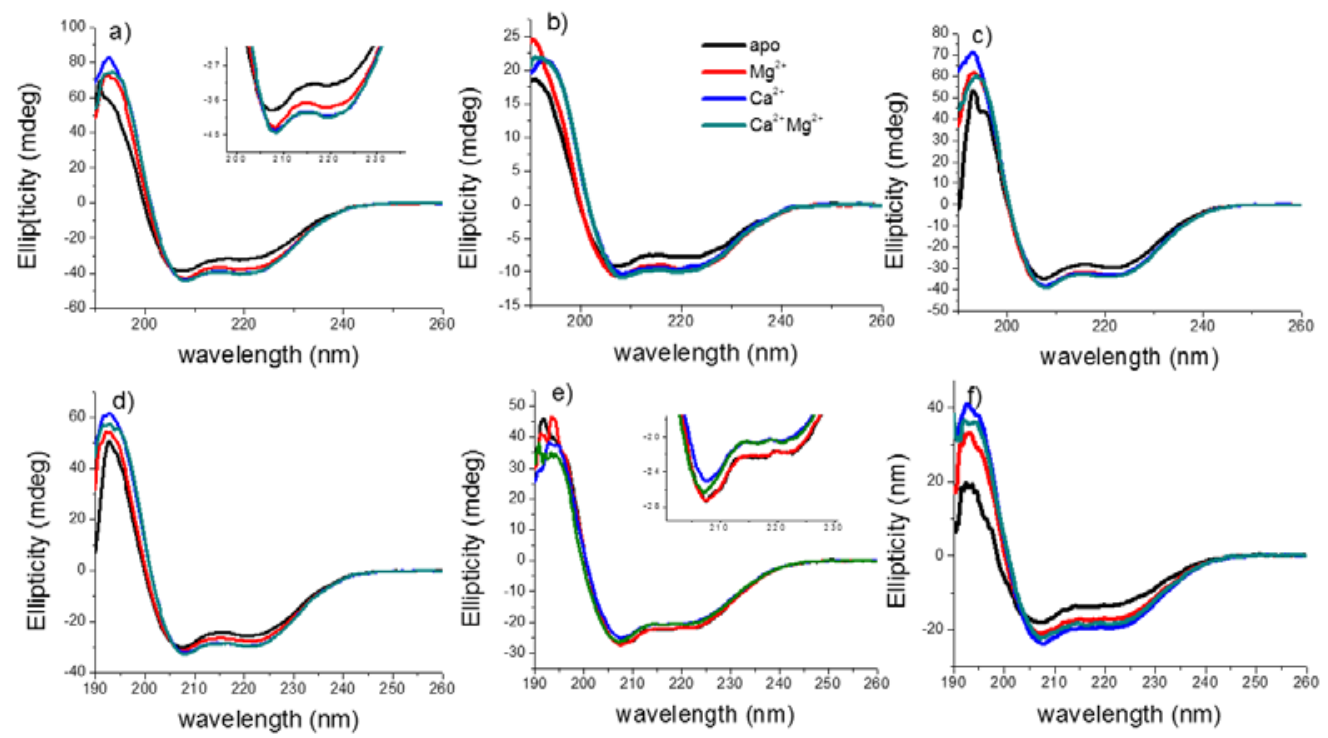

and (f) DREAM-NCS. The spectra are shown in the presence of $1 \mathrm{mM}$ EDTA (apo), $5 \mathrm{mM}$ $\mathrm{MgCl}_{2}$ and $1 \mathrm{mM}$ EGTA $\left(\mathrm{Mg}^{2+}\right), 1 \mathrm{mM} \mathrm{CaCl}_{2}\left(\mathrm{Ca}^{2+}\right)$, and $1 \mathrm{mM} \mathrm{CaCl} 2$ and $5 \mathrm{mM} \mathrm{MgCl}$ $\left(\mathrm{Mg}^{2+} \mathrm{Ca}^{2+}\right)$. The coloring scheme is the same for all spectra. The protein centration was kept near $10 \mu \mathrm{M}$ for all protein constructs in $5 \mathrm{mM}$ TRIS $\mathrm{pH}$ 7.4.

8.2.6 Mutation of Phe219, Phe235, or loop replacement induce a reversal of the transition in tryptophan fluorescence upon $\mathrm{Ca}^{2+}$ binding to DREAM

Another convenient approach to determine the extent of structural reorganization upon $\mathrm{Ca}^{2+}$ binding, is to monitor the intrinsic tryptophan fluorescence of these construct. All constructs contain a single tryptophan amino acid located at the entering helix of EFhand 3. The helix discussed above is important for coupling of the N- and C-terminus dynamic motions, and should be highly sensitive to local structural changes as a results of 
the mutations. As shown in Figure 8.18, all mutants show a clear tryptophan fluorescence near 330 nm; however, there are clear difference in the transition observed upon metal binding. The DREAM $(\Delta 65)$ shows the expected decrease in tryptophan fluorescence upon $\mathrm{Ca}^{2+}$ binding, while the DREAM $(\Delta 160)$ mutant shows a similar decrease transition. In addition, the $\operatorname{DREAM}(\Delta 160)$ construct shows an additional sensitivity to $\mathrm{Mg}^{2+}$, which induces an increase in fluorescence in the absence of $\mathrm{Ca}^{2+}$.

In contrast, DREAM(Y174A) (Figure 8.18c), shows a decrease in fluorescence upon $\mathrm{Ca}^{2+}$ binding to the apo protein, but a strong increase upon $\mathrm{Mg}^{2+}$ binding. The increase in fluorescence observed upon $\mathrm{Mg}^{2+}$ binding is eliminated once $\mathrm{Ca}^{2+}$ is added, indicating that both metal share the same metal biding site on this protein, or that the structural changes induced by $\mathrm{Ca}^{2+}$ eliminate those induced by $\mathrm{Mg}^{2+}$. The DREAM(F218A) and $\operatorname{DREAM}(\mathrm{F} 252 \mathrm{~A})$ mutants show a transition identical to that observed in the $\operatorname{DREAM}(\Delta 65)$ construct, indicating that this mutation does not induce significant changes in the Trp169 environment upon metal binding. On the other hand, the DREAM(F219A) mutant shows a inversed transition upon $\mathrm{Ca}^{2+}$ binding, in which $\mathrm{Ca}^{2+}$ binding to the protein induces an increase in tryptophan fluorescence (Figure 8.18e). The reversed transition is also observed in the DREAM(F235A) and DREAM-NCS mutants (Figure 8.19f and h). In all cases the presence of $\mathrm{Mg}^{2+}$ did not extensively affect the tryptophan fluorescence of the $\mathrm{Ca}^{2+}$ free or bound form, with exception of DREAM( $\Delta 160)$ and DREAM(Y174A) construct. Overall, these results reveal that the structural changes near the Trp169 residue are highly sensitive to mutations of aromatic amino acids at the C-terminus. 

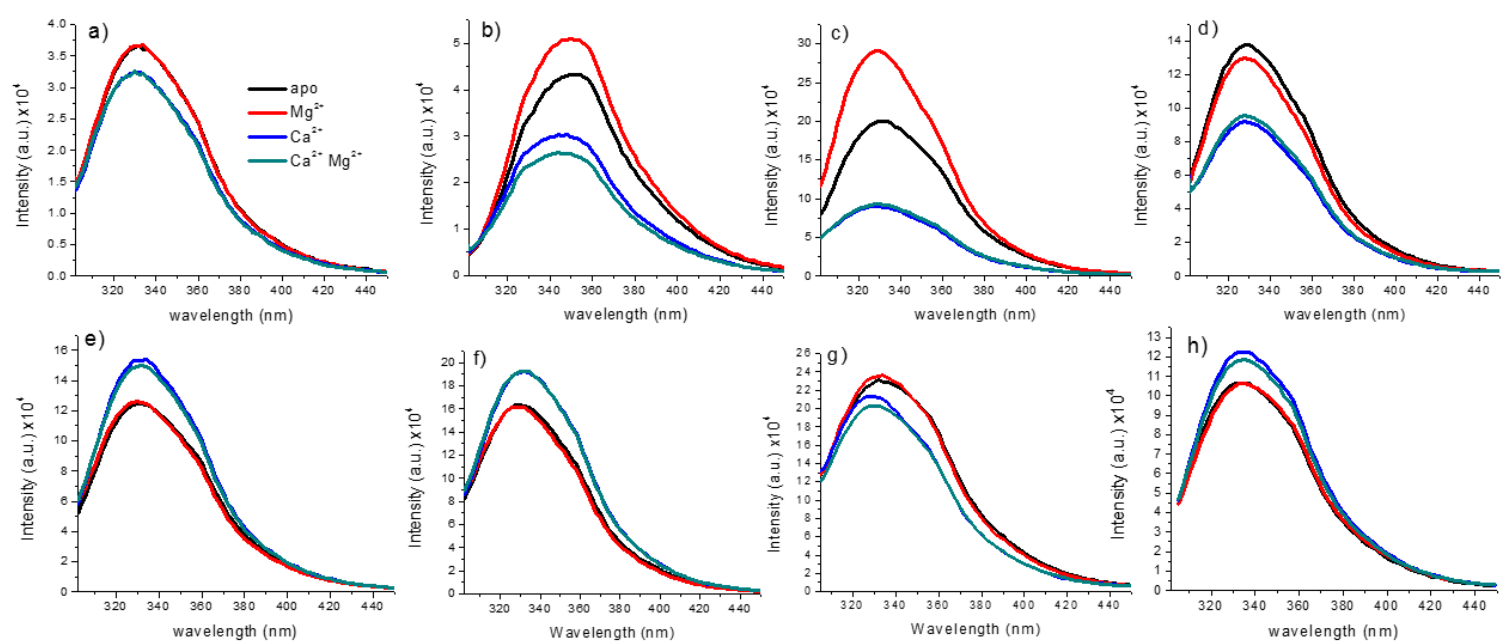

Figure 8.18. Tryptophan fluorescence spectra of DREAM mutants. (a) DREAM $(\Delta 65)$, (b) DREAM( 160$)$, (c) DREAM(Y174A), (d) DREAM(F218A), (e) DREAM(F219A), (f) DREAM(F235A), (g) DREAM(F252A), and (h) DREAM-NCS. The spectra are shown in the presence of $1 \mathrm{mM}$ EDTA (apo), $5 \mathrm{mM} \mathrm{MgCl}_{2}$ and $1 \mathrm{mM}$ EGTA $\left(\mathrm{Mg}^{2+}\right), 1 \mathrm{mM} \mathrm{CaCL} 2$ $\left(\mathrm{Ca}^{2+}\right)$, and $1 \mathrm{mM} \mathrm{CaCl} 2$ and $5 \mathrm{mM} \mathrm{MgCl} 2\left(\mathrm{Mg}^{2+} \mathrm{Ca}^{2+}\right)$. The coloring scheme is the same for all spectra. The protein concentration was kept near $10 \mu \mathrm{M}$ for all protein constructs in 5 mM TRIS pH 7.4.

Additionally, using frequency domain fluorescence and anisotropy decay of 1,8ANS bound to DREAM-NCS1 as described in chapter 3, we have determined that this

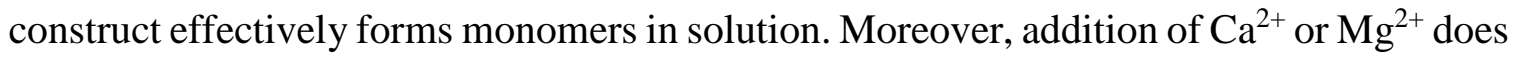
not induce the formation of the dimer. However, an increase in $\mathrm{Ca}^{2+} 1,8$-ANS fluorescence supports a model in which reorganization of the H10 helix and not dimerization mediate binding of small hydrophobic molecules such as 1,8-ANS, arachidonic acids, and NS5806.

The emission spectra of DREAM-NCS1 bound to ANS shows a transition towards stronger fluorescence upon binding of $\mathrm{Ca}^{2+}$ as well as in the presence of $\mathrm{Mg}^{2+}$. However, this increase in fluorescence is not additive, and addition of both metals leads to an intermediate fluorescence. Similarly, we observe that the fluorescence lifetime of 1,8-ANS bound to the hydrophobic cavity of DREAM $\left(\tau_{3}\right)$ increase in the presence of $\mathrm{Mg}^{2+}$ or $\mathrm{Ca}^{2+}$ Table 8.1). 


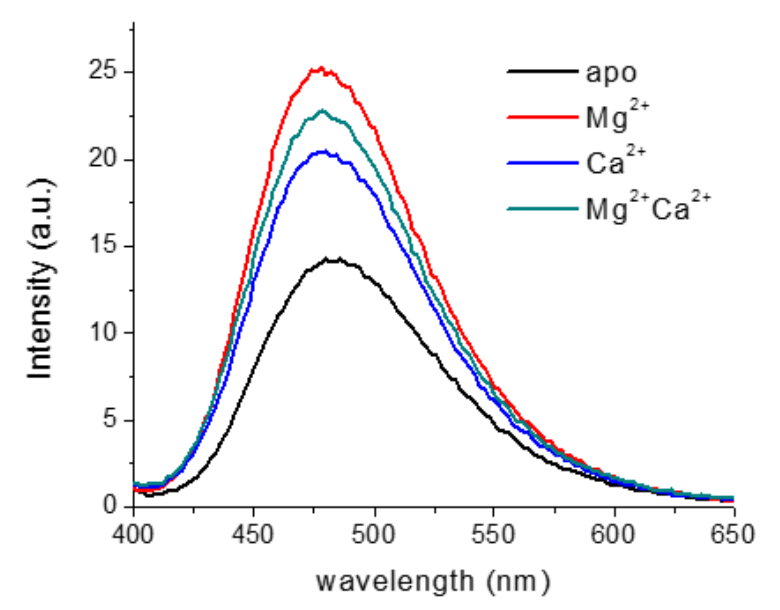

Figure 8.19. Fluorescence emission spectra of 1,8-ANS bound to DREAM-NCS1 in the presence of $\mathrm{Ca}^{2+}, \mathrm{Mg}^{2+}$, or both.

The pre-exponential parameter $\left(\alpha_{3}\right)$ also shows a strong increase from $50 \%$ in the metal free state, to about 60 to $70 \%$ upon binding of $\mathrm{Ca}^{2+}$ or $\mathrm{Mg}^{2+}$. The larger fraction of 1,8-ANS bound at the hydrophobic cavity, compared to that observed for $\operatorname{DREAM}(\Delta 65)$ in Chapter 3, indicates that this mutant is able to bind 1,8-ANS more readily than the wildtype protein. The anisotropy decay of 1,8-ANs bound to DREAM reveals the presence of at least three depolarization processes. A fast rotation of about $1.15 \mathrm{~ns}$ as well as a $\sim 11 \mathrm{~ns}$ and 52 ns rotational correlation times are observed regardless of the presence of $\mathrm{Ca}^{2+}$ or $\mathrm{Mg}^{2+}$. These rotational correlation times are identical to those observed in the $\operatorname{DREAM}(\Delta 65)$ construct and likely represent a local motion (1.2 ns), monomeric (11 ns), and tetrameric DREAM-NCS1 (52 ns). Moreover, the fact that these rotations do not change, but the intensity of 1,8-ANS increases significantly, indicates that this protein construct form monomers in solution and is able to induce exposure of a hydrophobic cavity upon $\mathrm{Ca}^{2+}$ binding. 
Table 8.1 Fluorescence and anisotropy decay parameters of 1,8-ANS bound DREAM-NCS1.

\begin{tabular}{|c|c|c|c|c|c|c|c|c|c|c|}
\hline & $f_{1}$ & $\tau_{2}$ & $f_{2}$ & $\tau_{3}$ & $f_{3}$ & $\alpha_{1}$ & $\theta_{2}$ & $\alpha_{1}$ & $\theta_{2}$ & $\chi^{2}$ \\
\hline apo & 0.06 & 5.8 & 0.41 & 13.8 & 0.5 & -0.38 & 6.0 & 0.3 & 0.74 & 0.3 \\
\hline $\mathrm{Mg}^{2+}$ & 0.03 & 6.3 & 0.32 & 16.5 & 0.64 & -0.31 & 11.2 & 0.57 & 0.39 & 1.19 \\
\hline $\mathrm{Ca}^{2+}$ & 0.02 & 6.6 & 0.37 & 17.5 & 0.6 & -0.2 & 14.3 & 0.68 & 0.4 & 1.46 \\
\hline $\mathrm{Mg}^{2+} \mathrm{Ca}^{2+}$ & 0.02 & 6.7 & 0.26 & 18.2 & 0.7 & -0.2 & 10.4 & 0.58 & 0.55 & 0.74 \\
\hline \multicolumn{11}{|c|}{$\begin{array}{l}\text { A value of } 0.28 \mathrm{~ns} \text { was fixed for the lifetime of unbound 1,8ANS as described in } \\
\text { Chapter } 3 \text {. A rotation of } 1.15 \mathrm{~ns} \text { and a third with } 52 \text { ns were set as linked variables } \\
\text { among all traces. A stoichiometric ration } 1: 1:, 20 \mu \mathrm{M} \text { DREAM-NCS1 and } 20 \mu \mathrm{M} \\
\text { 1,8-ANS in 20mM MOPS pH 7.4 1mM DTT was used throughout. }\end{array}$} \\
\hline
\end{tabular}

8.2.7 Aromatic amino acid mutation or loop replacement modulates the affinity of $\mathrm{Ca}^{2+}$ binding at EF-hand 3 and 4.

To determine whether mutations of the aromatic amino acid or the loop connecting EF-hand 3 and 4 affect $\mathrm{Ca}^{2+}$ binding, we have conducted calcium titrations while monitoring the change in tryptophan fluorescence. In addition, we have also studied the $\mathrm{Ca}^{2+}$ affinity of DREAM(E186Q) and DREAM(E234Q), whose EF-hand 3 and EF-hand 4 have been inactivated, respectively. Overall, we have observed that the constructs presented here either undergo a decrease or increase in tryptophan fluorescence upon $\mathrm{Ca}^{2+}$ binding. However, the magnitude of these transitions can be significantly different (Figure 8.17). In Figure 8.20 we present the normalized change in tryptophan fluorescence intensity of all mutants as a function of $\mathrm{Ca}^{2+}$ binding. The group of proteins showing a decrease in fluorescence include $\operatorname{DREAM}(\Delta 65), \quad \operatorname{DREAM}(\Delta 160), \quad \operatorname{DREAM}(Y 174 \mathrm{~A})$, DREAM(F218A), DREAM(F252A), and DREAM(E186Q). 

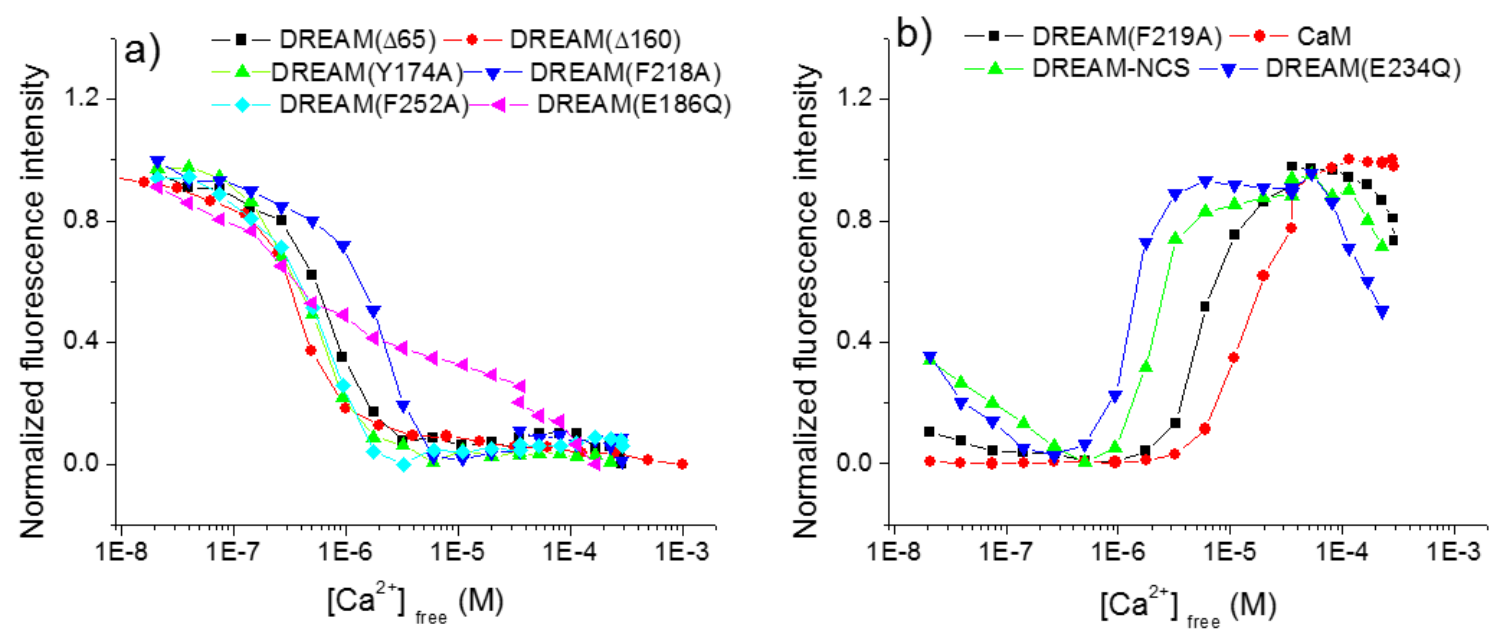

Figure 8.20. Calcium binding experiments monitoring the tryptophan fluorescence change upon $\mathrm{Ca}^{2+}$ binding to each DREAM mutants. (a) All the titration curves for DREAM constructs showing a decrease in tryptophan fluorescence similar to that of $\operatorname{DREAM}(\Delta 65)$ and (b) DREAM mutants whose change in fluorescence is reverse to that observed in the $\operatorname{DREAM}(\Delta 65)$ protein. Protein samples contained $5 \mu \mathrm{M}$ DREAM in $20 \mathrm{mM}$ MOPS pH 7.4 $1 \mathrm{mM}$ EGTA and $1 \mathrm{mM}$ NTA. The concentration of calcium free was determined using the known fluorescence transition and dissociation constants of Quin-2.

They all show a single sigmoidal isotherm, with exception of DREAM(E186Q), which shows a monotonous almost linear decrease. In terms of $\mathrm{Ca}^{2+}$ affinity, analysis of the data with a simple Hill equation reveals affinities ranging from $400 \mathrm{nM}$ to $2.1 \mu \mathrm{M}$, as shown in Table 8.2. The $\operatorname{DREAM}(\Delta 160)$ and $\operatorname{DREAM}(\Delta 65)$ construct show the strongest $\mathrm{Ca}^{2+}$ affinity, followed by DREAM(Y174A), DREAM(F218A), DREAM(F252A), and DREAM(E186Q). The Hill coefficient for all these constructs is near 2. The $\operatorname{DREAM}(\mathrm{E} 186 \mathrm{Q})$ showed a similar decrease as $\operatorname{DREAM}(\Delta 65)$, but the $\mathrm{Ca}^{2+}$ binding curve was markedly different. This construct showed the presence of strong binding site with Kd $\sim 10 \mu \mathrm{M}$ and $\mathrm{n}=1$ and an additional binding with a dissociation constant in the millimolar range. We propose that the additional binding site is due to $\mathrm{Ca}^{2+}$ binding to EF-hand 3 or 2, which likely happens at high $\mathrm{Ca}^{2+}$ concentrations. 


\begin{tabular}{lcc}
\hline \multicolumn{3}{l}{ Table 8.2 Equilibrium dissociation } \\
constants for $\mathrm{Ca}^{2+}$ binding to DREAM \\
\hline construct & $\begin{array}{c}\mathrm{Kd}_{\text {ss }} \\
(\mu \mathrm{M})^{\mathrm{a}}\end{array}$ & $\mathrm{n}$ \\
\cline { 2 - 3 } DREAM( $\Delta 65)$ & 0.4 & $2.2 \pm 0.2$ \\
DREAM( $\Delta 160)$ & 0.7 & $2.1 \pm 0.2$ \\
DREAM(E186Q) & $\sim 1.0$ & $0.8 \pm 0.1$ \\
DREAM(E234Q) & 1.3 & $4.0 \pm 0.4$ \\
DREAM(Y174A) & 0.5 & $1.8 \pm 0.2$ \\
DREAM(F218A) & 2.1 & $2.5 \pm 0.3$ \\
DREAM(F219A) & 6.0 & $2.4 \pm 0.3$ \\
DREAM(F235A) & $\mathrm{n} . \mathrm{d}$. & $\mathrm{n} . \mathrm{d}$. \\
DREAM(F252A) & 5.8 & $2.2 \pm 0.4$ \\
DREAM-NCS & 2.0 & $3.7 \pm 0.2$ \\
CaM & 15 & $2.0 \pm 0.1$ \\
\hline (a) Dissociation constants calculated from \\
steady-state fluorescence. Errors are less \\
than 0.1 units. Not determined (n.d.) \\
\hline
\end{tabular}

The group of DREAM construct characterized by an increase in tryptophan fluorescence upon $\mathrm{Ca}^{2+}$ binding included DREAM(F219A), DREAM-NCS, and DREAM(E234Q) (Figure 8.20b). These mutants show somewhat larger differences in affinities (Table 8.2), with dissociation constants ranging from $1.2 \mu \mathrm{M}$ to $6.0 \mu \mathrm{M}$. For comparison, the titration curve for calmodulin is shown, with a dissociation constant of 15 $\mu \mathrm{M}$ and Hill coefficient of 2.0 , which are similar to the reported values of 5 to $20 \mu \mathrm{M}$ (Kuboniwa et al. 1995). Remarkably, fitting of the DREAM(E234Q) titration yields a Hill coefficient larger than 1 , which is not expected on the basis that only one high affinity $\mathrm{Ca}^{2+}$ binding site is present. However, it is possible that because of the small change in fluorescence observed upon $\mathrm{Ca}^{2+}$ binding in this construct, additional effects such as changes in ionic strength and nonspecific binding of $\mathrm{Ca}^{2+}$ may contribute to the signal 
change. Similarly, fitting of the DREAM-NCS titration data yields a large Hill coefficient $(\mathrm{n}=3.7 \pm 0.2$ ), this mutant also shows a small tryptophan transition upon calcium binding. Despite the differences, these $\mathrm{Ca}^{2+}$ binding studies confirm that these protein construct are able to bind $\mathrm{Ca}^{2+}$ with affinities in micromolar range and bind $\mathrm{Ca}^{2+}$ with higher affinities than calmodulin. This strong binding affinity is a common characteristic among NCS proteins, which implicates that these constructs are properly folded (Burgoyne 2007)

8.2.8 Aromatic amino acids at the C-terminus provide significant stabilization of the Nand C-terminus domain of DREAM.

We carried out unfolding experiments to characterize the role of aromatic amino acids at the C-terminus as well as the role of the flexible loop connecting EF-hands 3 and 4 in stabilizing the structure of DREAM. In these experiments we have monitored the intrinsic tryptophan fluorescence of each construct as a function of increasing concentrations of $\mathrm{GuHCl}$, a compound known to destabilize the tertiary and secondary structure of proteins (Monera, Kay \& Hodges 1994). At increasing concentrations of GuHCl (up to 6.9 M) all of the DREAM mutants show a clear shift in the emission maxima of tryptophan (Figure 8.21). However, the profiles for the unfolding curve are highly dependent on metal binding as well as to specific mutations. In the absence of $\mathrm{Ca}^{2+}$ or $\mathrm{Mg}^{2+}$, most DREAM construct have an emission maxima near $340 \mathrm{~nm}$, with the exception of $\operatorname{DREAM}(\Delta 160)$ (maxima at $356 \mathrm{~nm}$ ), DREAM-NCS (maxima at $350 \mathrm{~nm}$ ), and $\operatorname{DREAM}(\mathrm{Y} 174 \mathrm{~A})$ (maxima at $346 \mathrm{~nm}$ ). The differences in emission spectra between Figure 8.21 and those shown in Figure 8.18 is because the spectra of Figure 8.18 have not been corrected for the PMT response of the instrument. An intermediate unfolded state of 
the protein is also clearly visible between 2 and $3 \mathrm{M} \mathrm{GuHCl}$, with an emission maxim of $350 \mathrm{~nm}$.
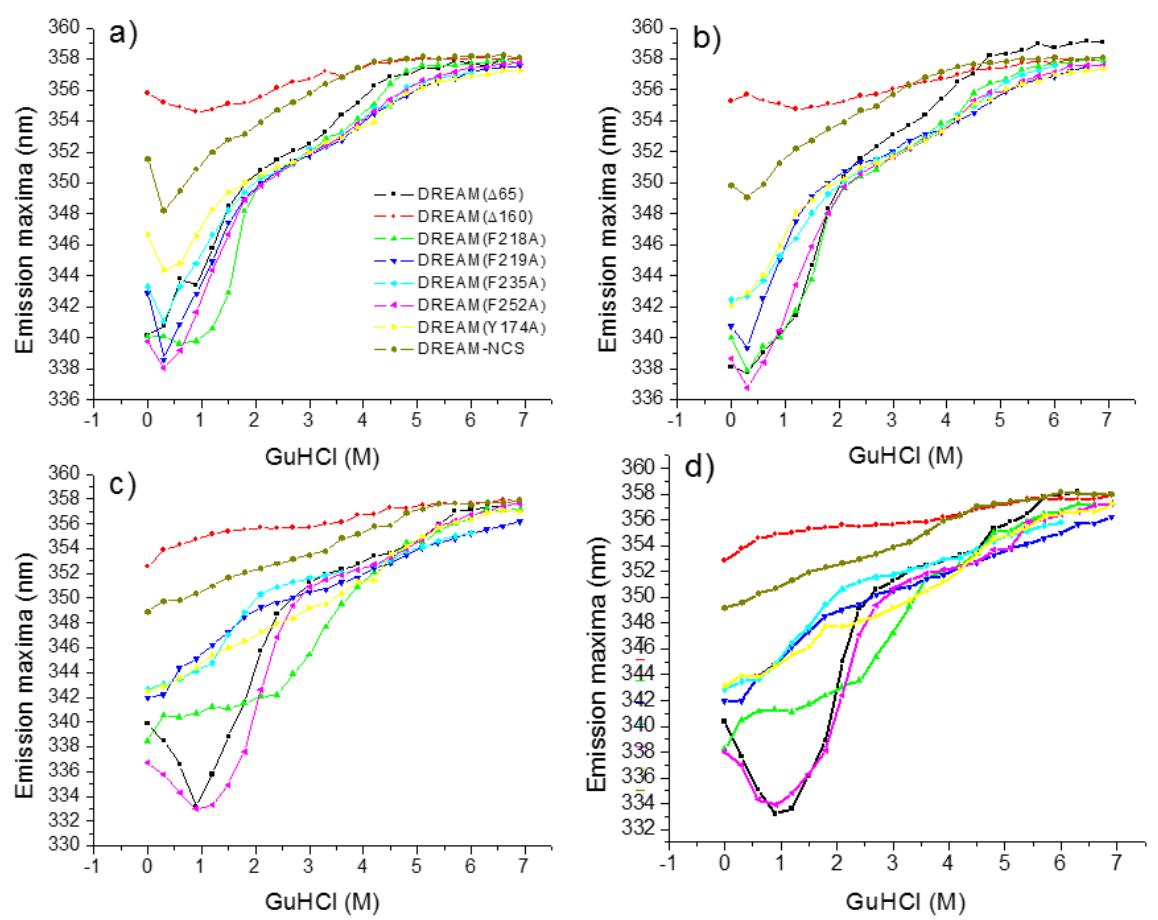

Figure 8.21. Unfolding experiment of DREAM constructs, in which the tryptophan fluorescence maxima was monitored as a function of guanidium hydrochloride ( $\mathrm{GuHCl})$ concentration. The protein was kept at $5 \mu \mathrm{M}$ for all protein construct and at all $\mathrm{GuHCl}$ concentrations.

Even though it may not be visible in Figure 8.21a, DREAM-NCS also shows the presence of an intermediate with maxima at $352 \mathrm{~nm}$, while the $\operatorname{DREAM}(\Delta 160)$ construct unfolds without populating an intermediate in the absence of $\mathrm{Ca}^{2+}$. At $6.9 \mathrm{M} \mathrm{GuHCl}$, all DREAM construct are in an unfolded state with emission maxima at $358 \pm 1 \mathrm{~nm}$. In the presence of excess $\mathrm{Mg}^{2+}$, the unfolding traces do not look remarkably different than Figure 8.21a, and similar emission maxima are observed (Figure 8.21b).

Upon binding of $\mathrm{Ca}^{2+}$, significantly different unfolding curves are recovered for most of the mutants (Figure 8.21c). The most obvious changes are observed for 
$\operatorname{DREAM}(\Delta 65)$ and $\operatorname{DREAM}(\mathrm{F} 252 \mathrm{~A})$, both of which show the presence of an additional blue shift upon addition of less than $1 \mathrm{M} \mathrm{GuHCl}$. The blue shift is followed by a red shift to $352 \mathrm{~nm}$ at which point an intermediate unfolded state can be observed. Also, upon binding of $\mathrm{Ca}^{2+}$, DREAM(F235A) unfolds through a a two-step process without populating a clear intermediate. In addition, upon $\mathrm{Ca}^{2+}$ binding, DREAM $(\Delta 160)$ unfolds through a three-step process with a partially stable intermediate at between 2 and $3 \mathrm{M} \mathrm{GuHCl}$ and emission maxima of $356 \mathrm{~nm}$. As observed before, addition of $5 \mathrm{mM} \mathrm{Mg}^{2+}$ to the Ca2+ bound proteins did not cause major changes in the unfolding curves (shown in Figure 8.21d).

The presence of an intermediate state becomes more obvious when the intensity of the tryptophan fluorescence is monitored as a function of GuHCl. As shown in Figure 8.22a and $b$, a clear redshift and increase in fluorescence is observed upon unfolding of $\mathrm{Ca}^{2+}$ free and bound DREAM $(\Delta 65)$. In the absence of $\mathrm{Ca}^{2+}$, figure 8.22a and c, show that DREAM unfolds without a significant increase in fluorescence intensity upon formation of the intermediate. In the presence of $\mathrm{Ca}^{2+}$, however, formation of the intermediate state (between $\mathrm{GuHCl}$ concentrations of $3 \mathrm{M}$ and $5 \mathrm{M}$ ) leads to an approximately two-fold enhancement in fluorescence intensity compared to the apo protein at the same $\mathrm{GuHCl}$ concentrations. In addition, in the presence of $\mathrm{Ca}^{2+}$, there is an additional initial process which triggers a blue shift of the tryptophan fluorescence, this process is not present in the absence of $\mathrm{Ca}^{2+}$. In the mutants, the brighter intermediate was only observed in the DREAM(F235A), DREAM(F219A), and DREAM(F252A) in addition to the $\operatorname{DREAM}(\Delta 65)$. 

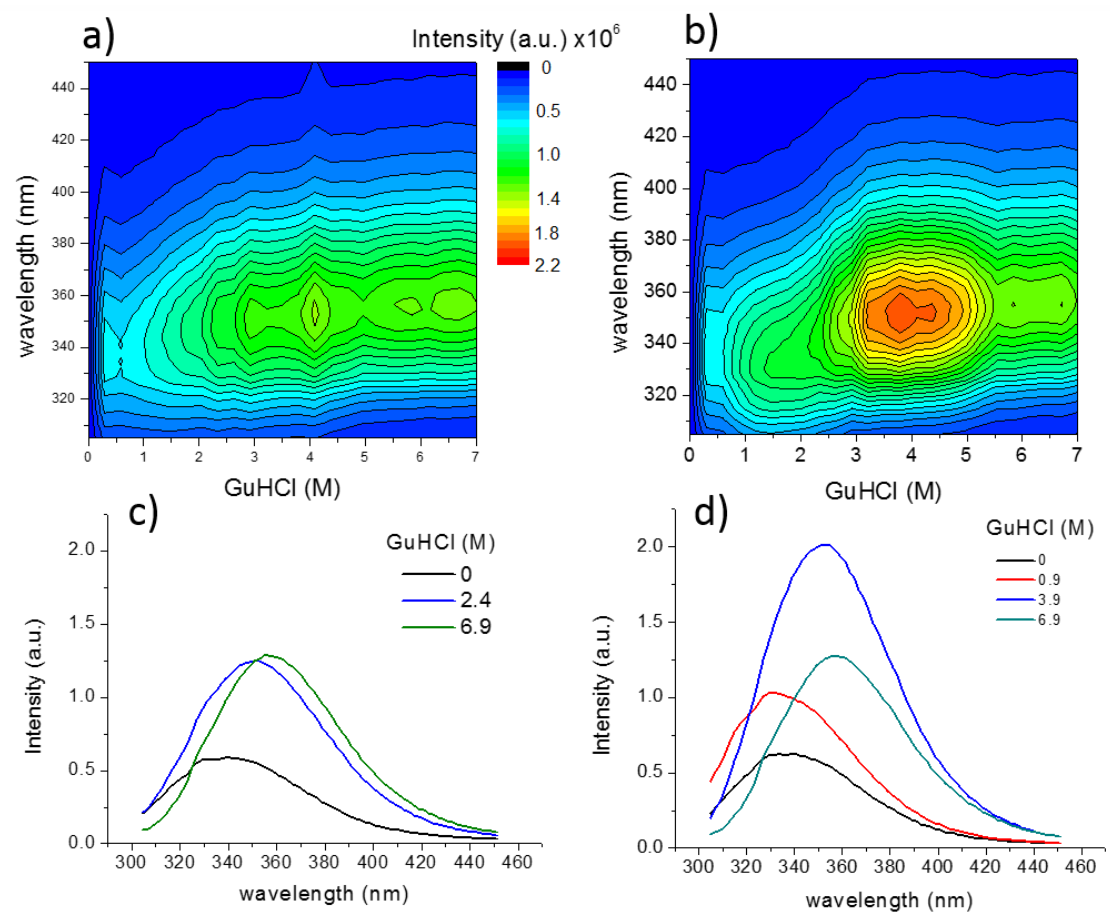

Figure 8.22. Tryptophan fluorescence emission spectra as a function of $\mathrm{GuHCl}$ for (a) $\mathrm{Ca}^{2+}$ free and (b) $\mathrm{Ca}^{2+}$ bound DREAM $(\Delta 65)$. The emission spectra of the native DREAM, intermediate, and unfolding protein in the absence of $\mathrm{Ca}^{2+}$ (c) and presence of $\mathrm{Ca}^{2+}$ is shown (d).

Even though there are very clear difference in the unfolding curves in the absence and presence of $\mathrm{Ca}^{2+}$, in order to quantify the magnitude of the effects of mutation and metal binding on stability, we have fitted the data in figure 8.21 using equation 8.4. Equation 8.4 allows the extraction of the energetics associated with unfolding of the protein. A representative unfolding dataset with the fitted curve is shown in Figure 8.23a and $\mathrm{b}$, and the recovered parameters for all constructs are shown Table 8.3 and 8.4. Most unfolding traces show the presence of a three-step unfolding pathways, with the exception of the $\mathrm{Ca}^{2+}$ free $\operatorname{DREAM}(\Delta 160)$ construct as well as the $\mathrm{Ca}^{2+}$ bound $\operatorname{DREAM}(\mathrm{F} 218 \mathrm{~A})$ construct. 

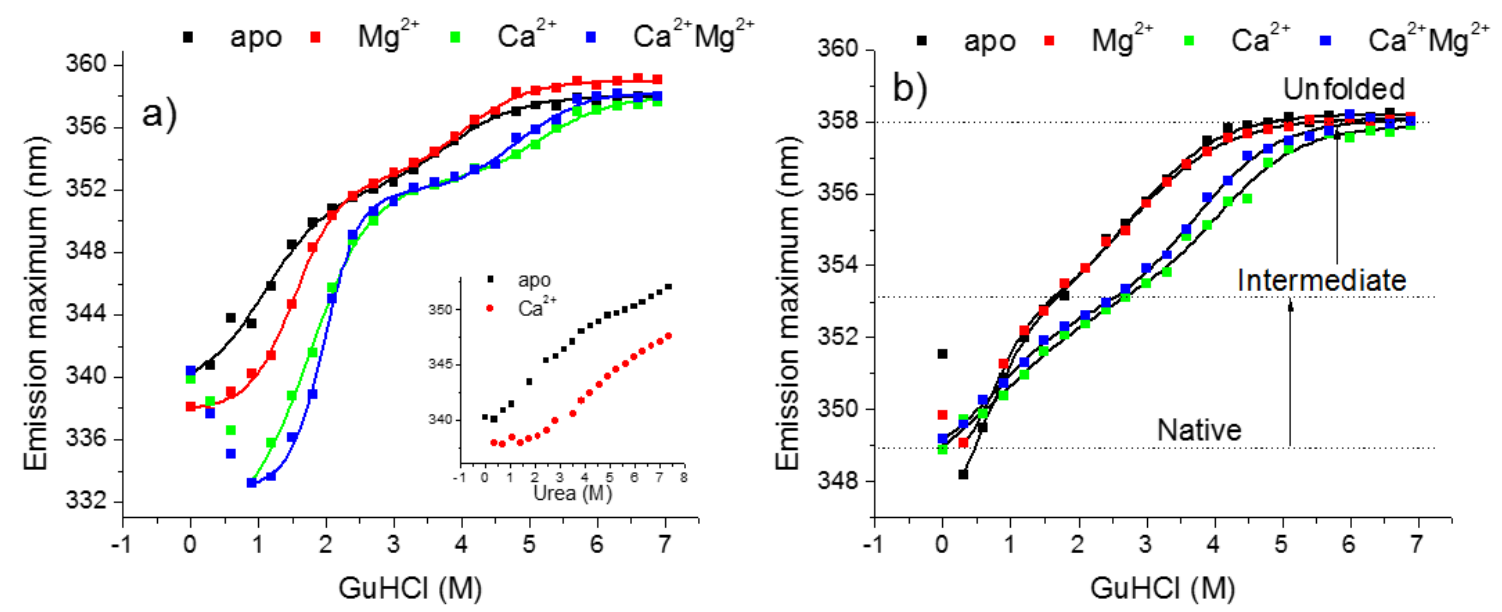

Figure 8.23. Representative unfolding trace of (a) DREAM ( $\Delta 65)$ and (b) DREAM-NCS in the apo, $\mathrm{Mg}^{2+}, \mathrm{Ca}^{2+}$, and $\mathrm{Mg}^{2+} \mathrm{Ca}^{2+}$ bound forms. Solid lines represent the best fit using equation 8.4.

As shown in Figure 8.24, the $\mathrm{Ca}^{2+}$ free and $\mathrm{Mg}^{2+}$ bound $\operatorname{DREAM}(\Delta 65)$ unfolding curve is well fitted by Eqn. 8.4. However, in the presence of $\mathrm{Ca}^{2+}$, the additional unfolding process happening at $\mathrm{GuHCl}$ concentrations below $1 \mathrm{M}$ cannot be properly fitted. Nonetheless, the initial blue shift of tryptophan fluorescence only occurs when using GuHCl, since unfolding DREAM with Urea did not show the presence of an initial blue shift (Figure 8.23a inset). It has been proposed that changes in protein structure at low concentrations of $\mathrm{GuHCl}$ are due to direct binding of $\mathrm{GuHCl}$ to the protein, whereas at higher GuHCl concertation the unfolding is driven by solvent effects (Zarrine-Afsar et al. 2006, Mason et al. 2009). Therefore for the traces showing the blue shift at low GuHCl concentration, we have decided to not fit the initial blue shift, since it may actually represent a binding effect. Thus for the $\mathrm{Ca}^{2+}$ bound $\operatorname{DREAM}(\Delta 65)$ and DREAM(F252A) mutants, only the data for $\mathrm{GuHCl}$ concentrations above $1 \mathrm{M}$ were fitted. 


\begin{tabular}{|c|c|c|c|c|c|c|c|c|}
\hline & \multicolumn{4}{|c|}{$\operatorname{DREAM}(\Delta 65)$} & \multicolumn{4}{|c|}{$\operatorname{DREAM}(\Delta 160)$} \\
\hline & apo & $\mathrm{Mg}^{2+}$ & $\mathrm{Ca}^{2+}$ & $\mathrm{Mg}^{2+} \mathrm{Ca}^{2+}$ & apo & $\mathrm{Mg}^{2+}$ & $\mathrm{Ca}^{2+}$ & $\mathrm{Mg}^{2+} \mathrm{Ca}^{2+}$ \\
\hline$\lambda_{\text {Native }}$ & 339 & 338 & 332 & 333 & 354 & 354 & 353 & 352 \\
\hline$\lambda_{\text {Intermediate }}$ & 352 & 352 & 352 & 352 & & & 356 & 356 \\
\hline$\lambda_{\text {Unfolded }}$ & 358 & 359 & 358 & 358 & 355 & 358 & 358 & 358 \\
\hline$\Delta \mathrm{G}_{\mathrm{I}}$ & $1.3 \pm 0.1$ & $2.7 \pm 0.1$ & $2.6 \pm 0.2$ & $4.4 \pm 0.2$ & $2.0 \pm 0.3$ & $1.9 \pm 0.2$ & $0.7 \pm 0.1$ & $0.3 \pm 0.1$ \\
\hline $\mathrm{m} \Delta \mathrm{G}_{\mathrm{I}}$ & 1.2 & 1.8 & 1.4 & 2.2 & 0.7 & 0.5 & 1.4 & 1.3 \\
\hline$\Delta \mathrm{G}_{\mathrm{U}}$ & $4.9 \pm 0.8$ & $4.6 \pm 0.3$ & $6.2 \pm 1.0$ & $5.8 \pm 0.6$ & & & $4.8 \pm 0.4$ & $5.4 \pm 0.4$ \\
\hline $\mathrm{mG}_{\mathrm{U}}$ & 1.3 & 1.1 & 1.2 & 1.2 & & & 1.2 & 1.2 \\
\hline \multirow[t]{3}{*}{$\Delta \mathrm{G}_{\text {TOTAL }}$} & 6.3 & 7.3 & 8.8 & 10.2 & 2.0 & 1.9 & 5.5 & 5.7 \\
\hline & \multicolumn{4}{|c|}{ DREAM(Y174) } & \multicolumn{4}{|c|}{ DREAM(F218A) } \\
\hline & apo & $\mathrm{Mg}^{2+}$ & $\mathrm{Ca}^{2+}$ & $\mathrm{Mg}^{2+} \mathrm{Ca}^{2+}$ & apo & $\mathrm{Mg}^{2+}$ & $\mathrm{Ca}^{2+}$ & $\mathrm{Mg}^{2+} \mathrm{Ca}^{2+}$ \\
\hline$\lambda_{\text {Native }}$ & 344 & 341 & 340 & 343 & 340 & 339 & 340 & 339 \\
\hline$\lambda_{\text {Intermediate }}$ & 350 & 350 & 349 & 348 & 350 & 350 & & \\
\hline$\lambda_{\text {Unfolded }}$ & 358 & 358 & 357 & 357 & 358 & 358 & 358 & 358 \\
\hline$\Delta \mathrm{G}_{\mathrm{I}}$ & $2.1 \pm 0.4$ & $1.5 \pm 0.2$ & $0.7 \pm 0.4$ & $1.8 \pm 0.4$ & $6.4 \pm 0.6$ & $3.6 \pm 0.4$ & $2.5 \pm 0.7$ & $2.5 \pm 0.2$ \\
\hline $\mathrm{m} \Delta \mathrm{G}_{\mathrm{I}}$ & 2.2 & 1.7 & 0.7 & 1.5 & 3.9 & 2.3 & 0.7 & 0.7 \\
\hline$\Delta \mathrm{G}_{\mathrm{U}}$ & $2.6 \pm 0.2$ & $2.8 \pm 0.2$ & $4.9 \pm 0.6$ & $3.5 \pm 0.3$ & $4.1 \pm 0.2$ & $3.8 \pm 0.4$ & & \\
\hline $\mathrm{mG}_{\mathrm{U}}$ & 0.7 & 0.7 & 1.6 & 0.8 & 1.1 & 0.9 & & \\
\hline$\Delta \mathrm{G}_{\text {Total L }}$ & 4.7 & 4.2 & 5.7 & 5.4 & 10.5 & 7.4 & 2.5 & 2.5 \\
\hline $\begin{array}{l}\text { Parameters } \\
\text { calculate } \\
\mathrm{kcal} \cdot \mathrm{mol}^{-1} \text {. }\end{array}$ & $\begin{array}{l}\text { Coverec } \\
\text { Total . E }\end{array}$ & g equat & and d & $\begin{array}{l}\text { own in } \\
n \text { unit }\end{array}$ & $\begin{array}{l}\text { 8. The } \\
\text { while th }\end{array}$ & $\begin{array}{l}\Delta \mathrm{G}_{\mathrm{I}} \mathrm{a} \\
\text { paramet }\end{array}$ & $\begin{array}{l}\Delta \mathrm{G}_{\mathrm{U}} \text { are } \\
\text { are show }\end{array}$ & to \\
\hline
\end{tabular}




\begin{tabular}{|c|c|c|c|c|c|c|c|c|}
\hline & \multicolumn{4}{|c|}{ DREAM(F235A) } & \multicolumn{4}{|c|}{ DREAM(F252A) } \\
\hline & apo & $\mathrm{Mg}^{2+}$ & $\mathrm{Ca}^{2+}$ & $\mathrm{Mg}^{2+} \mathrm{Ca}^{2+}$ & apo & $\mathrm{Mg}^{2+}$ & $\mathrm{Ca}^{2+}$ & $\mathrm{Mg}^{2+} \mathrm{Ca}^{2+}$ \\
\hline$\lambda_{\text {Native }}$ & 342 & 341 & 343 & 343 & 339 & 337 & 333 & 334 \\
\hline$\lambda_{\text {Intermediate }}$ & 349 & 351 & 352 & 351 & 349 & 350 & 351 & 351 \\
\hline$\lambda_{\text {Unfolded }}$ & 358 & 358 & 356 & 356 & 358 & 358 & 358 & 358 \\
\hline$\Delta \mathrm{G}_{\mathrm{I}}$ & $2.3 \pm 0.8$ & $1.4 \pm 0.1$ & $2.0 \pm 0.1$ & $2.7 \pm 0.2$ & $2.6 \pm 0.4$ & $2.4 \pm 0.4$ & $4.2 \pm 0.1$ & $4.2 \pm 0.3$ \\
\hline $\mathrm{m} \Delta \mathrm{G}_{\mathrm{I}}$ & 2.1 & 1.2 & 1.4 & 1.8 & 2.1 & 1.9 & 2.0 & 2.0 \\
\hline$\Delta \mathrm{G}_{\mathrm{U}}$ & $2.4 \pm 1.3$ & $3.9 \pm 0.6$ & $4.3 \pm 0.9$ & $4.3 \pm 1.0$ & $2.4 \pm 0.5$ & $2.7 \pm 1.0$ & $4.7 \pm 0.6$ & $4.6 \pm 0.7$ \\
\hline $\mathrm{mG}_{\mathrm{U}}$ & 0.7 & 0.9 & 0.9 & 0.9 & 0.6 & 0.7 & 0.9 & 0.9 \\
\hline \multirow[t]{3}{*}{$\Delta \mathrm{G}_{\text {TOTAL }}$} & 4.8 & 5.3 & 6.3 & 7.0 & 5.0 & 5.1 & 8.9 & 8.8 \\
\hline & \multicolumn{4}{|c|}{ DREAM(F219A) } & \multicolumn{4}{|c|}{ DREAM-NCS } \\
\hline & apo & $\mathrm{Mg}^{2+}$ & $\mathrm{Ca}^{2+}$ & $\mathrm{Mg}^{2+} \mathrm{Ca}^{2+}$ & apo & $\mathrm{Mg}^{2+}$ & $\mathrm{Ca}^{2+}$ & $\mathrm{Mg}^{2+} \mathrm{Ca}^{2+}$ \\
\hline$\lambda_{\text {Native }}$ & 339 & 340 & 340 & 341 & 346 & 348 & 347 & 348 \\
\hline$\lambda_{\text {Intermediate }}$ & 350 & 339 & 350 & 348 & 353 & 352 & 353 & 353 \\
\hline$\lambda_{\text {Unfolded }}$ & 358 & 363 & 357 & 359 & 358 & 358 & 358 & 358 \\
\hline$\Delta \mathrm{G}_{\mathrm{I}}$ & $2.0 \pm 0.1$ & $2.4 \pm 0.6$ & $0.8 \pm 0.1$ & $1.2 \pm 0.1$ & $0.8 \pm 0.5$ & $1.7 \pm 0.4$ & $0.5 \pm 0.6$ & $0.8 \pm 0.4$ \\
\hline $\mathrm{m} \Delta \mathrm{G}_{\mathrm{I}}$ & 1.7 & 2.7 & 1.0 & 1.3 & 1.43 & 2.29 & 0.65 & 1.09 \\
\hline$\Delta \mathrm{G}_{\mathrm{U}}$ & $2.6 \pm 0.4$ & $0.4 \pm 0.3$ & $2.9 \pm 0.3$ & $1.6 \pm 0.1$ & $2.7 \pm 0.4$ & $2.1 \pm 0.3$ & $4.0 \pm 1.2$ & $3.5 \pm 0.5$ \\
\hline $\mathrm{mG}_{\mathrm{U}}$ & 0.7 & 0.2 & 0.6 & 0.3 & 0.93 & 0.80 & 0.97 & 0.95 \\
\hline$\Delta \mathrm{G}_{\text {Total }}$ & 4.6 & 2.9 & 3.7 & 2.8 & 3.5 & 3.8 & 4.5 & 4.4 \\
\hline
\end{tabular}


We observe that in unfolding of the the $\mathrm{Ca}^{2+}$ free $\operatorname{DREAM}(\Delta 65)$ protein, the first unfolding process is associated with a $\Delta \mathrm{G}_{\mathrm{I}}$ of $1.3 \pm 0.1 \mathrm{kcal} \cdot \mathrm{mol}^{-1}$ and the second with $\Delta \mathrm{G}_{\mathrm{U}}$ $=4.9 \pm 0.8 \mathrm{kcal} \cdot \mathrm{mol}^{-1}$. In the presence of $\mathrm{Mg}^{2+}$, the first unfolding transitions shows an increase in stability, with $\Delta \mathrm{G}_{\mathrm{I}}=2.7 \pm 0.1 \mathrm{kcal} \cdot \mathrm{mol}^{-1}$, while the second remains unchanged at $\Delta \mathrm{G}_{\mathrm{U}}=4.6 \pm 0.3 \mathrm{kcal} \cdot \mathrm{mol}^{-1}$. A similar, but stronger, increase in stability of the protein is observed upon binding of $\mathrm{Ca}^{2+}$ to the apo protein $\left(\Delta \mathrm{G}_{\mathrm{I}}=2.6 \pm 0.2 \mathrm{kcal} \cdot \mathrm{mol}^{-1}\right.$ and $\Delta \mathrm{G}_{\mathrm{U}}=$ $\left.6.2 \pm 1.0 \mathrm{kcal} \cdot \mathrm{mol}^{-1}\right)$ and $\mathrm{Ca}^{2+}$ binding to the $\mathrm{Mg}^{2+}$ bound protein $\left(\Delta \mathrm{G}_{\mathrm{I}}=4.4 \pm 0.2 \mathrm{kcal} \cdot \mathrm{mol}^{-}\right.$ ${ }^{1}$ and $\left.\Delta \mathrm{Gu}=5.8 \pm 0.6 \mathrm{kcal} \cdot \mathrm{mol}^{-1}\right)$. The stabilization provided by metal binding is clearly observed on the total energy required to unfold the protein, which increase from 6.3 $\mathrm{kcal} \cdot \mathrm{mol}^{-1}$ in the apoform to $10.2 \mathrm{kcal} \cdot \mathrm{mol}^{-1}$ upon binding of $\mathrm{Mg}^{2+}$ and $\mathrm{Ca}^{2+}$.

The $\operatorname{DREAM}(\Delta 160)$ construct in the metal free form unfolds via two-step process which is insensitive to the presence of $\mathrm{Mg}^{2+}\left(\Delta \mathrm{G}_{\mathrm{I}}=2.0 \pm 0.3 \mathrm{kcal} \cdot \mathrm{mol}^{-1}\right.$ and $\Delta \mathrm{Gu}_{\mathrm{U}}=1.9 \pm$ $\left.0.2 \mathrm{kcal} \cdot \mathrm{mol}^{-1}\right)$. However, this construct is stabilized by about $3 \mathrm{kcal} \cdot \mathrm{mol}^{-1}$ upon binding of $\mathrm{Ca}^{2+}$, regardless of the presence of $\mathrm{Mg}^{2+}$. In addition, the $\mathrm{Ca}^{2+}$ bound form unfolds though a three-step process in in which the first step is marginally stable $\left(0.5 \mathrm{kcal} \cdot \mathrm{mol}^{-1}\right)$. The different emission maxima of the $\operatorname{DREAM}(\Delta 65)$ and $\operatorname{DREAM}(\Delta 160)$ intermediate structures (352 nm vs $356 \mathrm{~nm}$, respectively) as well as the different energetics, indicates that the initial $\mathrm{N} \rightarrow \mathrm{I}$ unfolding process in the $\mathrm{Ca}^{2+}$ bound form of these protein is not the same. Furthermore, the total energy required to unfold the EF-hands 3 and 4 present in the $\operatorname{DREAM}(\Delta 160)$ construct is identical to the necessary energy to transition from the intermediate to the unfolded state of the $\mathrm{Ca}^{2+}$ and $\mathrm{Mg}^{2+} \mathrm{Ca}^{2+}$ bound DREAM $(\Delta 65)$, compare $\Delta \mathrm{G}_{\mathrm{I}} \sim 6.0 \mathrm{kcal} \cdot \mathrm{mol}^{-1}$ for $\operatorname{DREAM}(\Delta 65)$ and $\Delta \mathrm{G}_{\text {Total }} \sim 5.6 \mathrm{kcal} \cdot \mathrm{mol}^{-1}$ for $\operatorname{DREAM}(\Delta 160)$. 
The DREAM(Y174A) construct was also characterized by a three-step unfolding pathway, in which the native, intermediate, and unfolded states have a similar tryptophan fluorescence maxima at $340 \mathrm{~nm}, 350 \mathrm{~nm}$, and $358 \mathrm{~nm}$. The $\mathrm{N} \rightarrow \mathrm{I}$ transition indicates that the native conformation is less stable than that observed in $\operatorname{DREAM}(\Delta 65)$ and only marginally affected by the presence of $\mathrm{Ca}^{2+}$ or $\mathrm{Mg}^{2+}$ (apo: $2.1 \pm 0.4 \mathrm{kcal} \cdot \mathrm{mol}^{-1}, \mathrm{Mg}^{2+}: 1.5$ $\left.\pm 0.2 \mathrm{kcal} \cdot \mathrm{mol}^{-1}, \mathrm{Ca}^{2+}: 0.7 \pm 0.4 \mathrm{kcal} \cdot \mathrm{mol}^{-1}, \mathrm{Ca}^{2+} \mathrm{Mg}^{2+}: 1.8 \pm 0.4 \mathrm{kcal} \cdot \mathrm{mol}^{-1}\right)$. The transition from the intermediate structure to the fully unfolded conformation $(\mathrm{I} \rightarrow \mathrm{U})$ shows a clear dependence on $\mathrm{Ca}^{2+}$ binding, but not $\mathrm{Mg}^{2+}$. On average, $\mathrm{Ca}^{2+}$ binding stabilizes the transition from the intermediate to the unfolded structure by about $1.2 \mathrm{kcal} \cdot \mathrm{mol}^{-1}$, which is much less than that observed for binding of $\mathrm{Mg}^{2+}$ and $\mathrm{Ca}^{2+}$ to $\operatorname{DREAM}(\Delta 65)$. Interestingly, the DREAM-NCS and DREAM(F235A) constructs showed similar insensitivity to the presence of $\mathrm{Mg}^{2+}$ as that observed in the DREAM(Y174A) mutant as well as a modest increase in stability upon $\mathrm{Ca}^{2+}$ binding. The DREAM-NCS construct was also less stable than the DREAM(Y174A) mutant, with a total energy of unfolding in the absence of $\mathrm{Ca}^{2+}$ of about $3.6 \mathrm{kcal} \cdot \mathrm{mol}^{-1}$ and $4.5 \mathrm{kcal} \cdot \mathrm{mol}^{-1}$ upon $\mathrm{Ca}^{2+}$ binding. On the other hand, DREAM(F235A), was slightly more stable in the presence of $\mathrm{Ca}^{2+}$ than the $\operatorname{DREAM}(\mathrm{Y} 174 \mathrm{~A})$ mutant $\left(\Delta \mathrm{G}_{\text {TOTAL }} \sim 5 \mathrm{kcal} \cdot \mathrm{mol}^{-1}\right.$ in the $\mathrm{Ca}^{2+}$ free and $\Delta \mathrm{G}_{\text {TOTAL }} \sim 6.7$ $\mathrm{kcal} \cdot \mathrm{mol}^{-1}$ in the $\mathrm{Ca}^{2+}$ bound state). The DREAM(F235A) construct had similar tryptophan emission wavelength for the native conformation ( $\lambda_{\text {Native }}$ on average $343 \mathrm{~nm}$ ), intermediate conformation ( $\lambda_{\text {Intermediate }}$ on average $\left.351 \mathrm{~nm}\right)$, and unfolded state $\left(\lambda_{\text {Unfolded }}\right.$ on average 357 $\mathrm{nm})$ as the DREAM $(\Delta 65)$. However, DREAM-NCS had an emission maxima in the native state of about $347 \mathrm{~nm}$ (also note the plot on Figure 8.21), while the emission of the intermediate and unfolded states were similar to those of DREAM $(\Delta 65)$. 
The mutation of Phe218 on the entering helix of EF-hand 4 is characterized by a remarkable reversal of the unfolding stabilities compared to the $\operatorname{DREAM}(\Delta 65)$ and $\operatorname{DREAM}(\Delta 160)$ constructs. In this case, DREAM(F218A) is more stable in the absence of $\mathrm{Ca}^{2+}$ or $\mathrm{Mg}^{2+}$, and $\mathrm{Ca}^{2+}$ binding triggers the transition from a three-state to a two-state unfolding pathway. The overall unfolding stability of the $\mathrm{Ca}^{2+}$ free DREAM(F218A) construct $\left(\Delta \mathrm{G}_{\text {TOTAL }}=10.5 \mathrm{kcal} \cdot \mathrm{mol}^{-1}\right)$ is comparable to that of the $\mathrm{Mg}^{2+} \mathrm{Ca}^{2+}$ bound $\operatorname{DREAM}(\Delta 65)$ protein $\left(\Delta \mathrm{G}_{\mathrm{TOTAL}}=10.2 \mathrm{kcal} \cdot \mathrm{mol}^{-1}\right)$. However, in DEAM(F218A), the first transition from native to intermediate is more stable $\left(\Delta \mathrm{G}_{\mathrm{I}}=6.4 \pm 0.6 \mathrm{kcal} \cdot \mathrm{mol}^{-1}\right)$ than the intermediate to unfolded state transition $\left(\Delta \mathrm{G}_{\mathrm{U}}=4.1 \pm 0.6 \mathrm{kcal} \cdot \mathrm{mol}^{-1}\right)$. Binding of $\mathrm{Mg}^{2+}$ to this mutant decreases the stability of both transition to about $3.7 \mathrm{kcal} \cdot \mathrm{mol}^{-1}$, while binding of $\mathrm{Ca}^{2+}$ to the metal free or $\mathrm{Mg}^{2+}$ bound DREAM(F218A) protein also leads to a significant decrease in stability, with $\Delta \mathrm{G}_{\mathrm{TOTAL}}=2.5 \mathrm{kcal} \cdot \mathrm{mol}^{-1}$.
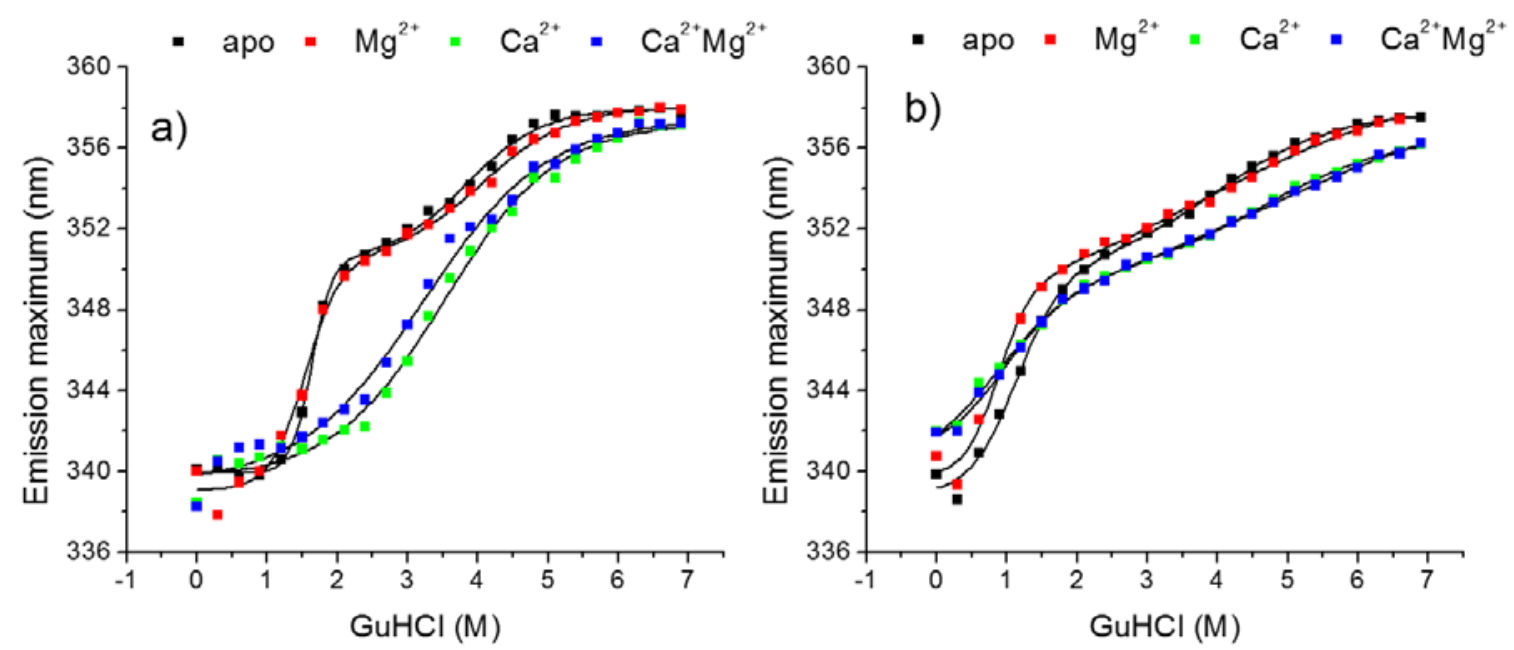

Figure 8.24. Unfolding plot of (a) DREAM (F218A) and (b) DREAM(F219A) in the apo, $\mathrm{Mg}^{2+}, \mathrm{Ca}^{2+}$, and $\mathrm{Mg}^{2+} \mathrm{Ca}^{2+}$ bound forms. Solid lines represent the best fit using equation 8.4. 


\begin{tabular}{|c|c|c|c|c|c|c|c|c|}
\hline & $\begin{array}{c}\text { DREAM } \\
(\Delta 65)\end{array}$ & $\begin{array}{c}\text { DREAM } \\
(\Delta 160)\end{array}$ & $\begin{array}{c}\text { DREAM } \\
\text { (Y174) }\end{array}$ & $\begin{array}{l}\text { DREAM } \\
\text { NCS }\end{array}$ & $\begin{array}{l}\text { DREAM } \\
\text { (F218A) }\end{array}$ & $\begin{array}{l}\text { DREAM } \\
\text { (F219A) }\end{array}$ & $\begin{array}{l}\text { DREAM } \\
\text { (F235A) }\end{array}$ & $\begin{array}{l}\text { DREAM } \\
\text { (F252A) }\end{array}$ \\
\hline $\begin{array}{l}\text { Unfolding } \\
\text { path in } \mathrm{Ca}^{2+} \\
\text { free }\end{array}$ & $\mathrm{N} \rightarrow \mathrm{I} \rightarrow \mathrm{U}$ & $\mathrm{I} \rightarrow \mathrm{U}$ & $\mathrm{N} \rightarrow \mathrm{I} \rightarrow \mathrm{U}$ & $\mathrm{N} \rightarrow \mathrm{I} \rightarrow \mathrm{U}$ & $\mathrm{N} \rightarrow \mathrm{I} \rightarrow \mathrm{U}$ & $\mathrm{N} \rightarrow \mathrm{I} \rightarrow \mathrm{U}$ & $\mathrm{N} \rightarrow \mathrm{I} \rightarrow \mathrm{U}$ & $\mathrm{N} \rightarrow \mathrm{I} \rightarrow \mathrm{U}$ \\
\hline $\begin{array}{l}\text { Unfolding } \\
\text { path in } \mathrm{Ca}^{2+} \\
\text { bound }\end{array}$ & $\mathrm{N} \rightarrow \mathrm{I} \rightarrow \mathrm{U}$ & $\mathrm{N} \rightarrow \mathrm{I} \rightarrow \mathrm{U}$ & $\mathrm{N} \rightarrow \mathrm{I} \rightarrow \mathrm{U}$ & $\mathrm{N} \rightarrow \mathrm{I} \rightarrow \mathrm{U}$ & $\mathrm{I}-->\mathrm{U}$ & $\mathrm{N} \rightarrow \mathrm{I} \rightarrow \mathrm{U}$ & $\mathrm{N} \rightarrow \mathrm{I} \rightarrow \mathrm{U}$ & $\mathrm{N} \rightarrow \mathrm{I} \rightarrow \mathrm{U}$ \\
\hline $\mathrm{Mg}^{2+}$ effect & $\begin{array}{l}\text { Stabilize } \\
\mathrm{N} \rightarrow \mathrm{I} \rightarrow \mathrm{U}\end{array}$ & none & none & none & $\begin{array}{c}\text { Destabilize } \\
\mathrm{N} \rightarrow \mathrm{I} \\
\text { in apo }\end{array}$ & $\begin{array}{c}\text { Destabilize } \\
\mathrm{I} \rightarrow \mathrm{U}\end{array}$ & none & none \\
\hline $\mathrm{Ca}^{2+}$ effect & $\begin{array}{c}\text { stabilize } \\
\mathrm{N} \rightarrow \mathrm{U}\end{array}$ & $\begin{array}{c}\text { Stabilize } \\
\mathrm{N} \rightarrow \mathrm{U}\end{array}$ & $\begin{array}{c}\text { Stabilize } \\
\mathrm{I} \rightarrow \mathrm{U}\end{array}$ & $\begin{array}{c}\text { Stabilize } \\
\mathrm{I} \rightarrow \mathrm{U}\end{array}$ & $\begin{array}{c}\text { Destabilize } \\
\mathrm{N} \rightarrow \mathrm{U}\end{array}$ & $\begin{array}{c}\text { Destabilize } \\
\mathrm{N} \rightarrow \mathrm{U}\end{array}$ & $\begin{array}{c}\text { weak } \\
\text { Stabilize } \\
\mathrm{N} \rightarrow \mathrm{I} \rightarrow \mathrm{U}\end{array}$ & $\begin{array}{l}\text { Stabilize } \\
\mathrm{N} \rightarrow \mathrm{I} \rightarrow \mathrm{U}\end{array}$ \\
\hline $\begin{array}{l}\text { Bright } \\
\text { Intermediate }\end{array}$ & YES & NO & NO & NO & NO & YES & YES & YES \\
\hline$\Delta \lambda_{\mathrm{N--}>\mathrm{U}}$ & 23 & 4 & 15 & 11 & 19 & 15 & 15 & 22 \\
\hline
\end{tabular}


Mutation of the amino acid adjacent of Phe218 in the DREAM(F219A) mutant leads to an overall decrease in stability of the protein, compared to the $\operatorname{DREAM}(\Delta 65)$ construct, mainly due to a decrease in stability of the $\mathrm{I} \rightarrow \mathrm{U}$ unfolding pathway. In the DREAM(F219A) mutant, we also observe a metal induced decrease in stability similar to DREAM(F218A); however, the decrease in stability due to $\mathrm{Ca}^{2+}$ or $\mathrm{Mg}^{2+}$ binding is restricted to the $\mathrm{N} \rightarrow \mathrm{I}$ or the $\mathrm{I} \rightarrow \mathrm{U}$ transition, respectively. The metal-selective decrease in stability seems to be additive, since in the presence of $\mathrm{Mg}^{2+}$ and $\mathrm{Ca}^{2+}$ the overall stability of the unfolding process decreases by half (from $\Delta \mathrm{G}_{\mathrm{TOTAL}}=4.6 \mathrm{kcal} \cdot \mathrm{mol}^{-1}$ to $\Delta \mathrm{G}_{\mathrm{TOTAL}}=$ $\left.2.8 \mathrm{kcal} \cdot \mathrm{mol}^{-1}\right)$.

Lastly, replacement of Phe252 on the H10 helix to alanine partially stabilized the transition from $\mathrm{N} \rightarrow \mathrm{I}$ in the presence of $\mathrm{Ca}^{2+}$, compared to the $\operatorname{DREAM}(\Delta 65)$ construct, while $\mathrm{Mg}^{2+}$ had no effect on the stability (apo: $\Delta \mathrm{G}_{\mathrm{I}}=2.6 \pm 0.4 \mathrm{kcal} \cdot \mathrm{mol}^{-1}, \Delta \mathrm{G}_{\mathrm{I}}=\mathrm{Mg}^{2+}$ : $\Delta \mathrm{G}_{\mathrm{I}}=2.4 \pm 0.4 \mathrm{kcal} \cdot \mathrm{mol}^{-1}, \Delta \mathrm{G}_{\mathrm{I}}=\mathrm{Ca}^{2+}: 4.2 \pm 0.1 \mathrm{kcal} \cdot \mathrm{mol}^{-1}, \mathrm{Ca}^{2+} \mathrm{Mg}^{2+}: 4.2 \pm 0.3$ $\mathrm{kcal} \cdot \mathrm{mol}^{-1}$ ). The second transition, from $\mathrm{I} \rightarrow \mathrm{U}$, was identical to the $\mathrm{N} \rightarrow \mathrm{I}$ transition in terms of energetics and metal binding effect. Overall, the energy required to unfold apoDREAM(F252A) ( $\left.\Delta \mathrm{G}_{\mathrm{TOTAL}}\right)$ was similar to that required to unfold the apoDREAM $(\Delta 65)$ construct; however, no increase in stability was observed upon binding of $\mathrm{Mg}^{2+}$. The summary of observations described above are listed in Table 8.4.

8.2.9 Amino acid mutations modulate the conformational dynamics of Trp169

As show in the previous sections, the position of Trp169 at the entering helix of EF-hand 3 is highly sensitive to amino acid mutations, $\mathrm{Ca}^{2+}$ binding, and protein unfolding. To better understand how these experiment observations correlate to the molecular 
organization of DREAM, in this section, we employ extensive biophysical characterization of tryptophan dynamics. In particular, we hypothesize that fluctuations of the rotameric states of Trp169 may help explain the observed changes in fluorescence due to amino acid mutation and calcium binding. In solution, tryptophan has been proposed to populate three rotameric states (the $\mathrm{g}^{+}, \mathrm{g}^{-}$, and t) (Figure 8.25). More importantly, the fluorescence decay of tryptophan has also been correlated to population of different rotameric states (Szabo, Rayner 1980, Clayton, Sawyer 1999). In solution, the $\mathrm{g}^{+}$is the most favored conformation, while $\mathrm{g}^{-}$is the least favored (Ross, Jameson 2008). However, in proteins, the structural organization of the backbone and other regions of the proteins may hinder or promote a specific rotameric conformation. Furthermore, it is possible that dynamic fluctuations in protein conformation may allow the population of multiple rotameric conformations depending on the protein structure and dynamics.

a)
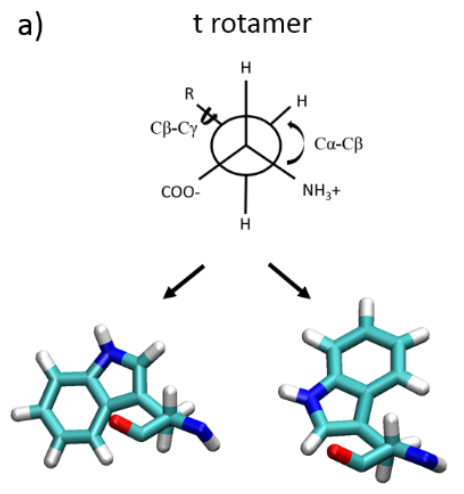

G- conformation

$\mathrm{G}+$ conformation b) $\quad g^{+}$rotamer
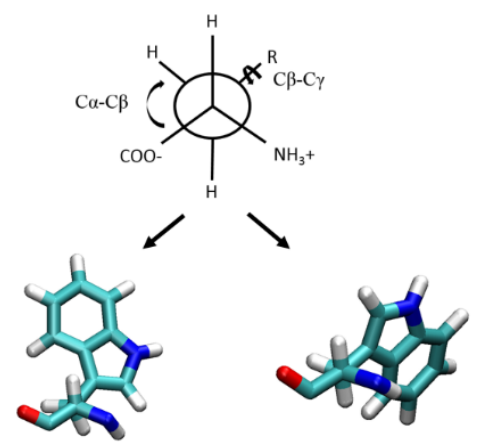

G- conformation G+ conformation

Figure 8.25. Rotameric states of tryptophan in proteins. (a) Newman projection of the $t$ rotamer and (b) $\mathrm{g}^{+}$rotamer with view along the $\mathrm{C} \alpha-\mathrm{C} \beta$ carbon bond. The corresponding indole conformations as observed in the aMD simulation of apoDREAM $(\Delta 160)$. The measured $\mathrm{C} \alpha-\mathrm{C} \beta$ and $\mathrm{C} \beta-\mathrm{C} \gamma$ angles are highlighted with black arrows.

Implementation of molecular dynamic simulations is ideal to investigate the structural organization of DREAM at the molecular level, and to define whether the 
experimental observations can be associated with multiple rotameric states of tryptophan. However, the rotameric transitions in DREAM may occur in the microsecond to millisecond timescales, which are not readily available through classical molecular dynamics. To overcome the computational costs of obtaining MD data with temporal resolution similar to that observed experimentally, we have employed accelerated molecular dynamics (aMD). The aMD simulation method allows us to enhance the conformational sampling of the protein by lowering the energy barrier separating multiple conformations of the protein (Hamelberg, Mongan \& McCammon 2004). The decrease in energetic barriers between multiple states is attained by increase of the potential energy wells by addition of an external energy. In our approach, the external energy is added to the dihedral angles of every amino acids. The energetic addition results in an enhanced sampling of rotameric states for all the amino acids as well as an increase in the conformational space that the protein can sample. As described in section 8.2.1, aMD effectively induced the transition from the $\mathrm{Ca}^{2+}$ bound structure of DREAM to a proposed structure of the apoprotein within 20 ns. Therefore, we propose to use this method not to only investigate the rotameric state of Trp169 in DREAM in the presence of $\mathrm{Ca}^{2+}$ but also in the absence of this metal.

In Figure 8.26 and 8.27 we show the population of rotameric states for the $\mathrm{Ca}^{2+}$ free and bound state of the DREAM constructs. In these figures we plot the $\mathrm{C}_{\beta}-\mathrm{C}_{\gamma}$ angle as a function of $C_{\alpha}-C_{\beta}$ angle, this was taken from the observation that the benzene of the indole ring of DREAM was able to rotate $180^{\circ}$ and point towards the nitrogen (angles less than $180^{\circ}$, herein referred as $\mathrm{D}^{+}$) or towards the carboxy group of the peptide bond (angles between $180^{\circ}$ and $360^{\circ}$, herein referred as D-). In these plots, the g+ rotamer of tryptophan 
would lay between $120^{\circ}$ and $239^{\circ}$, the g- between $240{ }^{\circ}$ and $360^{\circ}$, and the $t$ rotamer between $0^{\circ}$ to $119^{\circ}$ on the $\mathrm{x}$-axis. The $\mathrm{C}_{\alpha}-\mathrm{C}_{\beta}$ dihedral angle distribution in Figure 8.26 and 8.27 clearly show that no conformation was present with angles above $240^{\circ}$. This observation is expected, since a $\mathrm{g}^{-}$conformation for tryptophan forming part of an alpha helix would result in significant steric hindrance and distortion of the helix.
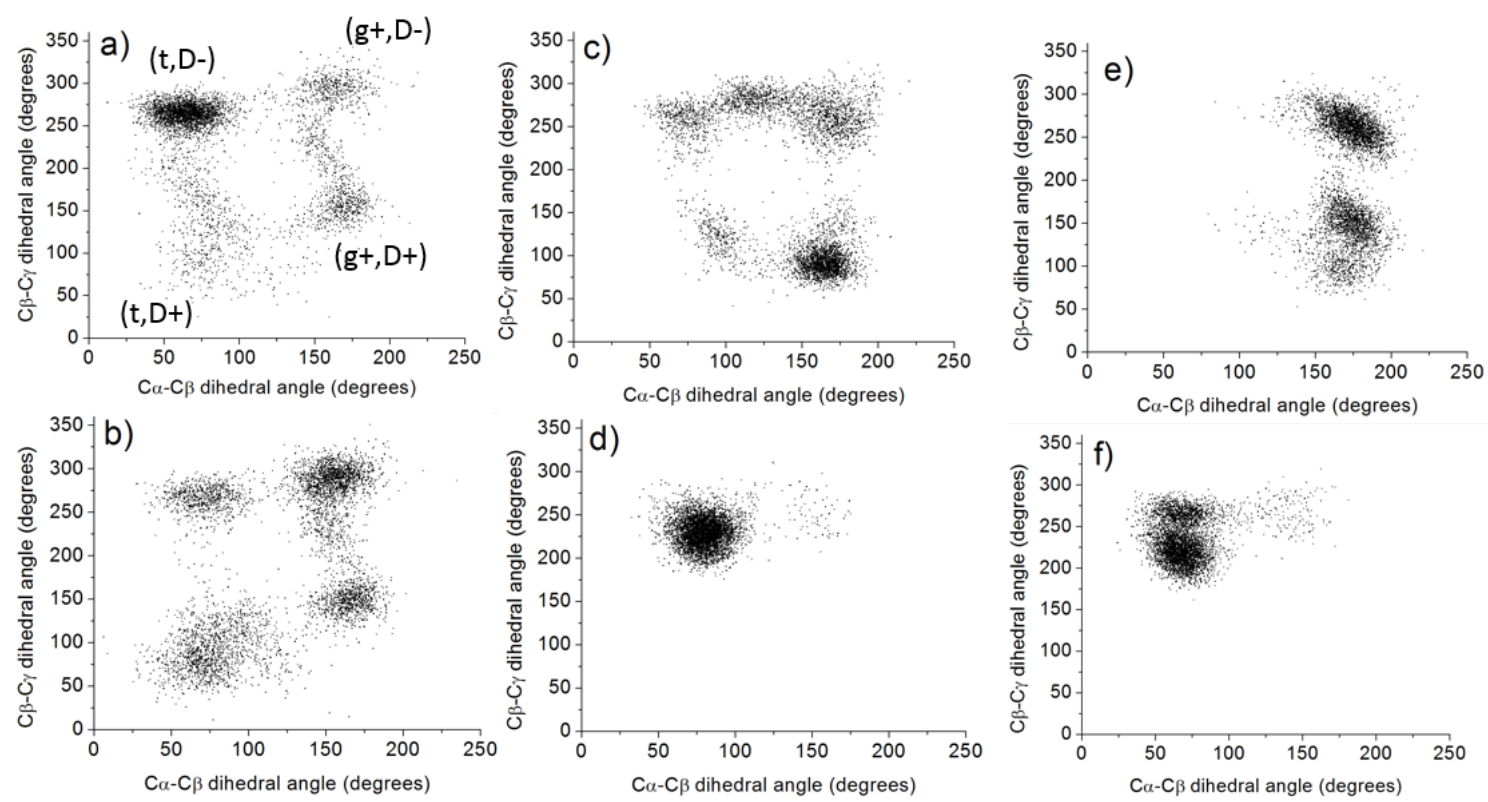

Figure 8.26. Plots of the $\mathrm{C}_{\beta}-\mathrm{C}_{\gamma}$ as a function of $\mathrm{C}_{\alpha}-\mathrm{C}_{\beta}$ dihedral angle of tryptophan 169 in the $\mathrm{Ca}^{2+}$ bound (a) DREAM $(\Delta 160)$, (c) $\operatorname{DREAM}(\Delta 65)$, and (e) DREAM(Y174A), as well as the $\mathrm{Ca}^{2+}$ free of the (b) DREAM( $\left.\Delta 160\right)$, (f) DREAM( $\Delta 65$ ), and (f) DREAM(Y174A). A total of 4000 points representing 20 ps each are plotted, obtained from the aMD simulations. The angles at which the $t$ or $\mathrm{g}^{+}$rotamers are present as well as the orientation of the indole ring in the $\mathrm{D}+$ or $\mathrm{D}$ - are show in in panel (a).

Thus, in DREAM, only the $\mathrm{g}^{+}$and $\mathrm{t}$ rotamers are present. On the other hand, the distribution of $\mathrm{C}_{\beta}-\mathrm{C}_{\gamma}$ angles reveals that a wide range of angles can be populated for the dihedral plane between the $\mathrm{C}_{\beta}$ and $\mathrm{C}_{\gamma}$ carbons, with angles between $50^{\circ}$ to $90^{\circ}$ and $200^{\circ}$ to $300^{\circ}$ being most common, depending on the protein construct. For instance, the $\operatorname{DREAM}(\Delta 160)$ construct shows the presence of $\mathrm{g}^{+}$and $\mathrm{t}$ rotamers with indole ring in the 
$\mathrm{D}+$ and $\mathrm{D}$ - conformation. In the presence of $\mathrm{Ca}^{2+}$ the predominant specie is the $\mathrm{t}$ rotamer with D- indole conformation, while in the absence of $\mathrm{Ca}^{2+}$ the $\mathrm{g}^{+}$rotamer with $\mathrm{D}$ - indole conformation is preferred (Figure 8.26a and b). The widespread distribution of Trp169 rotamers and indole orientation in the $\operatorname{DREAM}(\Delta 160)$ construct is likely because of the absence of the N-terminus domain, which significantly decrease the steric hindrance of tryptophan.
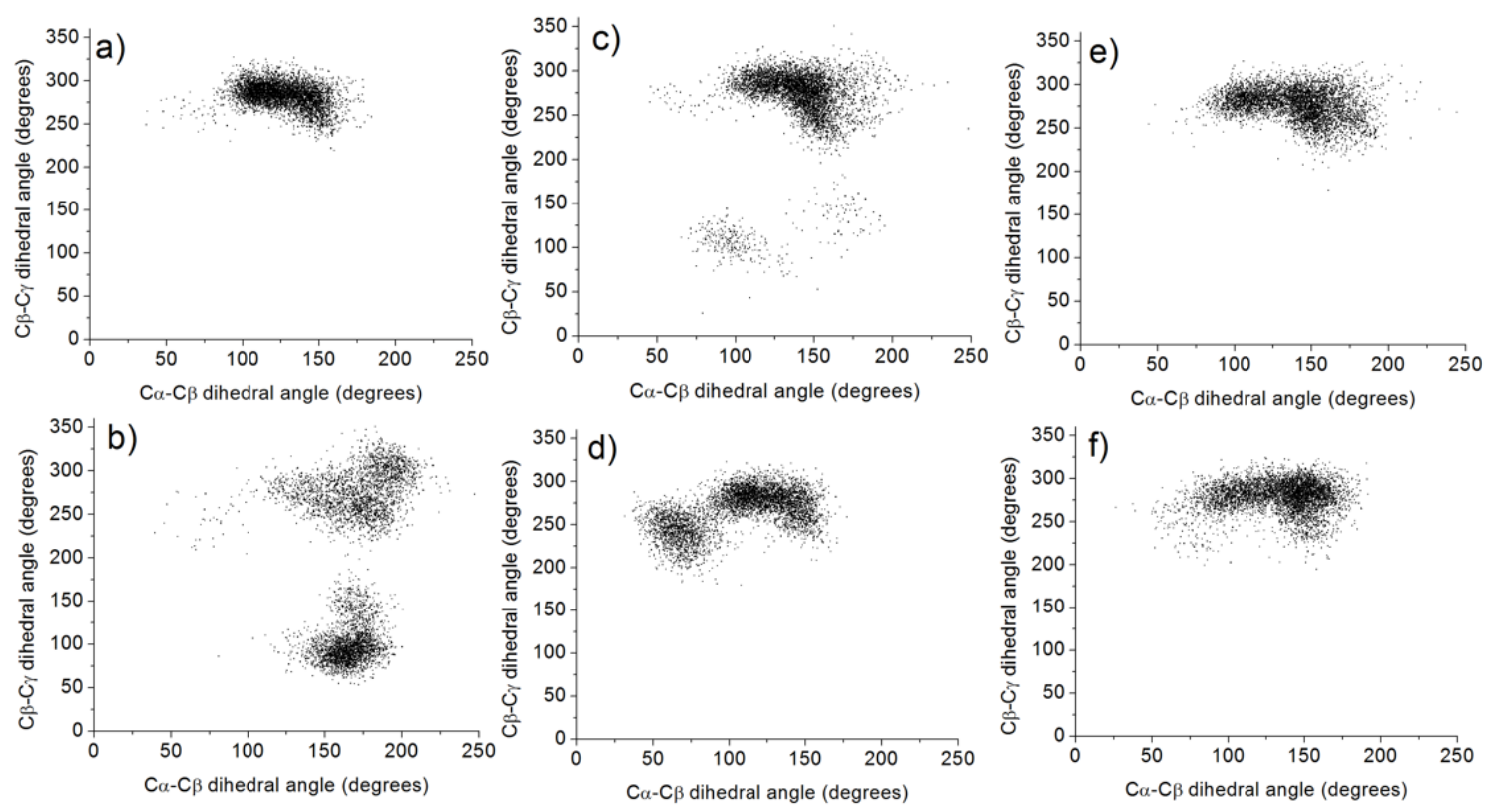

Figure 8.27. Plots of the $C \beta-C \gamma$ as a function of $C \alpha-C \beta$ dihedral angle of tryptophan 169 in the $\mathrm{Ca}^{2+}$ bound (a) DREAM(F218A), (c) DREAM(F219A), and (e) DREAM(F252A), as well as the $\mathrm{Ca}^{2+}$ free of the (b) DREAM(F218A), (d) DREAM(F219A), and (f) DREAM(F252A). A total of 4000 points representing 20 ps each are plotted, obtained from the aMD simulations.

In the DREAM $(\Delta 65)$ construct, the absence of $\mathrm{Ca}^{2+}$ results in the population of the $\mathrm{t}$ rotamer and indole in the $\mathrm{D}$ - conformation, while upon binding of $\mathrm{Ca}^{2+}$, a clear transition to the $\mathrm{g}^{+}$rotamer with $\mathrm{D}+$ conformation is observed. Interestingly, the $\mathrm{D}$ - rotameric state of Trp169 places the indole in direct interaction with Phe138 and Leu158, residing at the entering and exiting helices of EF-hand 2. The interaction is effectively eliminated when 
the indole is in the $\mathrm{D}+$ conformation (pointing towards the solvent). Similarly the DREAM(Y174A) mutant shows a similar transition, with Trp169 populating the trotamer with D- indole orientation in the absence of $\mathrm{Ca}^{2+}$, and the $\mathrm{g}^{+}$rotamer with $\mathrm{G}+$ indole orientation upon $\mathrm{Ca}^{2+}$ binding.

In contrast, the conformational distribution of Trp169 in the DREAM(F218A) mutant shows a transition distinct than that observed in the DREAM $(\Delta 65)$ construct. In DREAM(F218A), binding of $\mathrm{Ca}^{2+}$ induces a transition from a single conformation (the $\mathrm{g}^{+}$ rotamer and D- conformation) to two conformations of the same rotamer, with indole in the $\mathrm{D}+$ and $\mathrm{D}$ - conformation. The transition from organized (one conformation) to disorganized (two conformations) upon $\mathrm{Ca}^{2+}$ binding is opposite to that observed in the $\operatorname{DREAM}(\Delta 65)$ construct. Similarly, the DREAM(F219), DREAM(F235A), and DREAM(F252A) show population of mainly the g+ rotamer with indole in the Dorientation in the absence of $\mathrm{Ca}^{2+}$. However, upon $\mathrm{Ca}^{2+}$ binding, a small change in orientation of Trp169 towards the t rotamer with D- indole orientation is observed for these three constructs (Figure 8.27c, d, e, f, and 8.28a and b). As shown in Figure 8.28c and 8.28d, the t rotamer with D- indole conformation shows a strong hydrophobic interaction of Trp169 with the hydrophobic N-terminus, while the $\mathrm{g}^{+}$rotamer with the $\mathrm{D}+$ indole orientation shows the most solvent exposure. Analysis of the simulation trajectory also reveals that rotation of the indole ring induces the largest change solvent accessible surface area, from $\sim 365 \AA^{2}$ in the D+ conformation to $\sim 345 \AA^{2}$ in the D- conformation. Even though the transition from a $\mathrm{D}$ - to a $\mathrm{D}+$ conformation in the $\operatorname{DREAM}(\Delta 160)$ construct is not obvious, an increase in population with the D+ can be observed. However, in the 
$\operatorname{DREAM}(\Delta 65), \operatorname{DREAM}(\mathrm{Y} 174 \mathrm{~A})$, and DREAM(F218A) constructs a clear transition from

D- to the $\mathrm{D}+$ conformation can be observed upon $\mathrm{Ca}^{2+}$ binding.
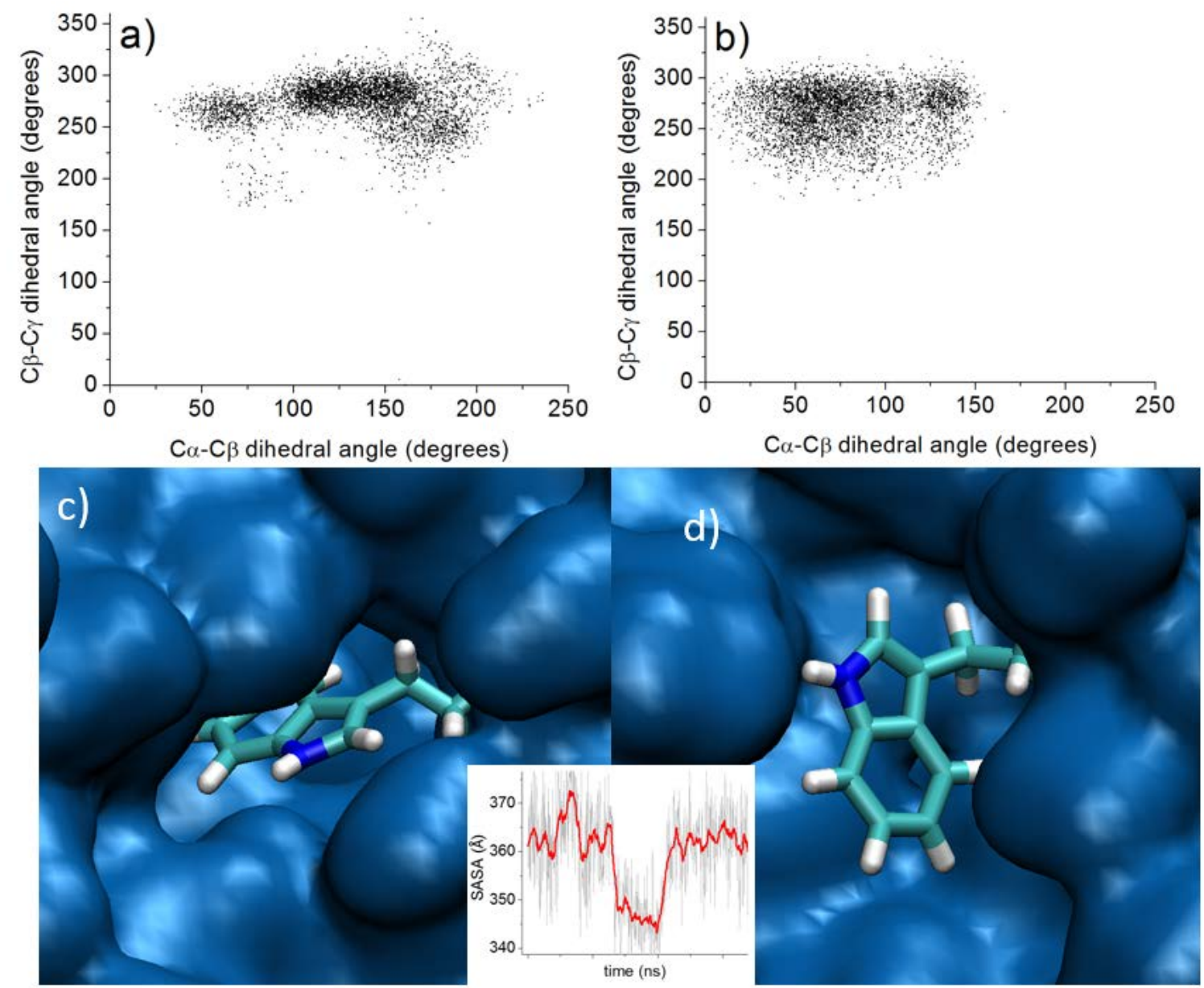

Figure 8.28. Plots of the $C \beta-C \gamma$ as a function of $C \alpha-C \beta$ dihedral angle of tryptophan 169 in the $\mathrm{Ca}^{2+}$ bound (a) and $\mathrm{Ca}^{2+}$ free (b) of the DREAM(F235A). Solvent exposure of Trp169 in the (c) t rotamer with D- indole orientation and in the (d) $\mathrm{g}^{+}$rotamer with D+ indole orientation. Inset shows the change in SASA as a function of time.

All these constructs show a decrease in tryptophan fluorescence (Figure 8.18) upon calcium binding, which allows us to propose that the decrease in tryptophan fluorescence observed upon $\mathrm{Ca}^{2+}$ binding is caused by the reorientation of the indole ring of Trp169. The reorientation towards the $\mathrm{D}+$ conformation effectively increases the solvent accessibility of tryptophan as well as its proximity to Lys168, which could induce 
quenching of the fluorescence. Consequently, the increase in fluorescence observed for DREAM(F219A) and DREAM(F235A) (both which show retention of the Dconformation regardless of metal biding) could potentially be the result of the lack of change in indole orientation as well as structural changes at the N-terminus which effectively enhances the Trp169 fluorescence. A similar transition could be present for DREAM-NCS1, but we have not yet performed the simulations. However, we have also observed that the DREAM(252A) construct shows a lack of indole reorientation and yet, it is characterized by a decrease in tryptophan fluorescence (Figure 8.18). The disagreement with the idea that reorientation of the indole ring is causing the decrease in fluorescence. Nonetheless, the fact that these constructs are locked into a D- conformation, in which the indole is deeply buried between Phe138 and Leu158, may indicate that there may be additional structural changes at the $\mathrm{N}$-terminus which induce the decrease in fluorescence. In addition, it is possible that the timescales necessary to observe $\mathrm{D}$ - to $\mathrm{D}+$ transition in the DREAM(F252A) mutant exceeds the time sampled by our simulations.

8.2.10 Interdomain fluctuations in DREAM highly regulate structural stability of the protein.

Analysis of the structural rearrangement of Trp169 in the DREAM constructs reveals a certain degree of correlation with the tryptophan fluorescence data. However, to investigate whether structural dynamics play a role in the stability of DREAM we have conducted extensive analysis of the dynamic fluctuations of the protein. In this section we seek to identify whether the dynamic fluctuations of the N-terminus and C-terminus of DREAM can be correlated with the unfolding energy of these constructs determined in section 8.2.8. We hypothesize that the changes in unfolding energy of the $\mathrm{N}$ - and C- 
terminus domain of DREAM are correlated with the interdomain dynamics measured in the aMD simulations.
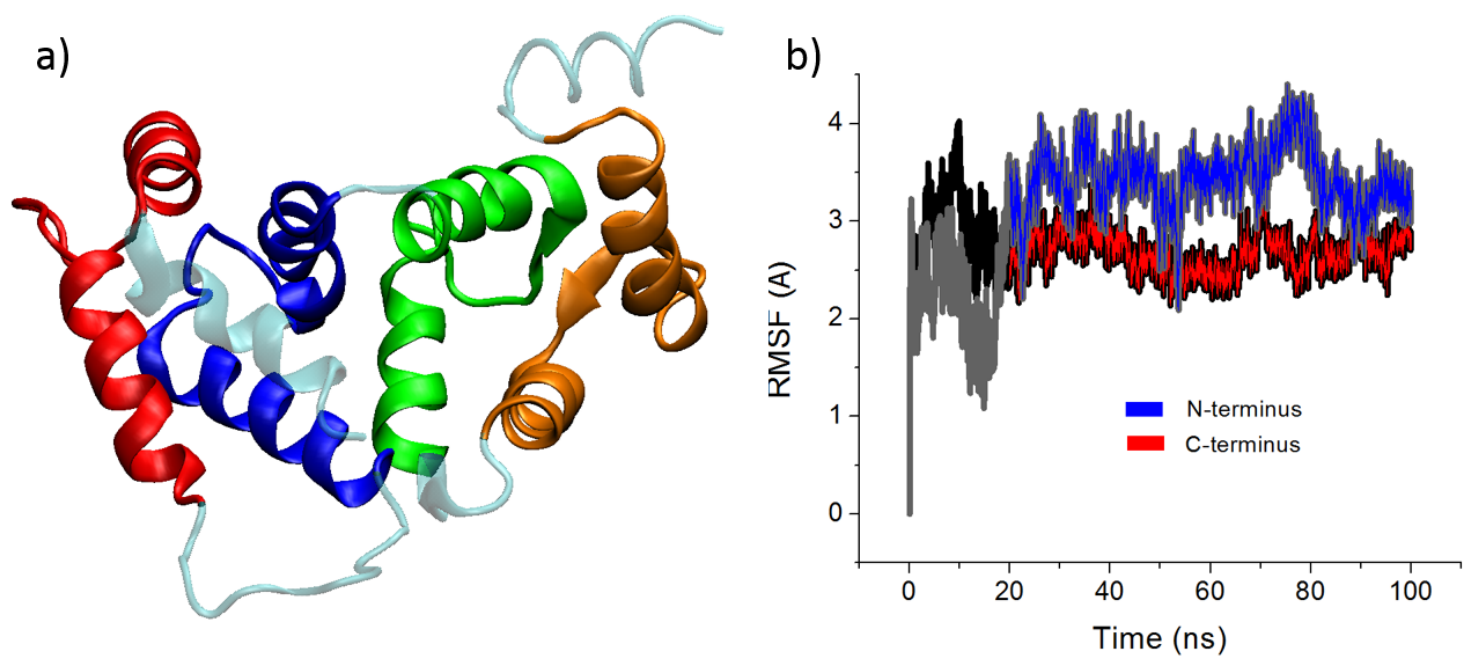

Figure 8.29 (a) Helices of DREAM included in the analysis of fluctuations. The EF-hands 1 and 2 are shown in orange and green, respectively, and form part of the $\mathrm{N}$-terminus. The EF-hands 3 and 4 are shown in blue and red, respectively, and form the C-terminus. The H1 helix, the connecting loop between EF-hand 3 and 4, and the H10 helix are not used in the calculations and are shown in diffuse cyan. (b) A representative RMSF plot of $\operatorname{apoDREAM}(\Delta 65) \mathrm{N}$ - and C-terminus as a function of simulation time, the last $80 \mathrm{~ns}$ of simulation time was used for all constructs.

To test the interdomain communication hypothesis, we have determined the $\mathrm{N}$ terminus fluctuations of the alpha carbons forming the EF-hands 1 and 2 as well as the fluctuations of the alpha carbons forming EF-hand 3 and 4 (Figure 8.29). The first helix (H1), the C-terminus loop connecting EF-hand 3 and 4, and the H10 helix have not been including in our analysis. However, preliminary calculation show that inclusion of these helices does not result in significant changes of the dynamics observed. Furthermore, exclusion of the EF-hand metal binding loops further enhances the difference in dynamics fluctuations described below (data not shown). These fluctuations are defined as the deviation of the alpha carbon, at a particular frame, from the averaged position of the same 
carbon in the average position of 1000 frames (10 ns). The moving window average allows us to identify fluctuations from equilibrium once the structure has reached its final conformation (Figure 8.29b). All our simulations reached equilibrium within 20 ns or sooner, and thus only the last 80 ns are included in our analysis.
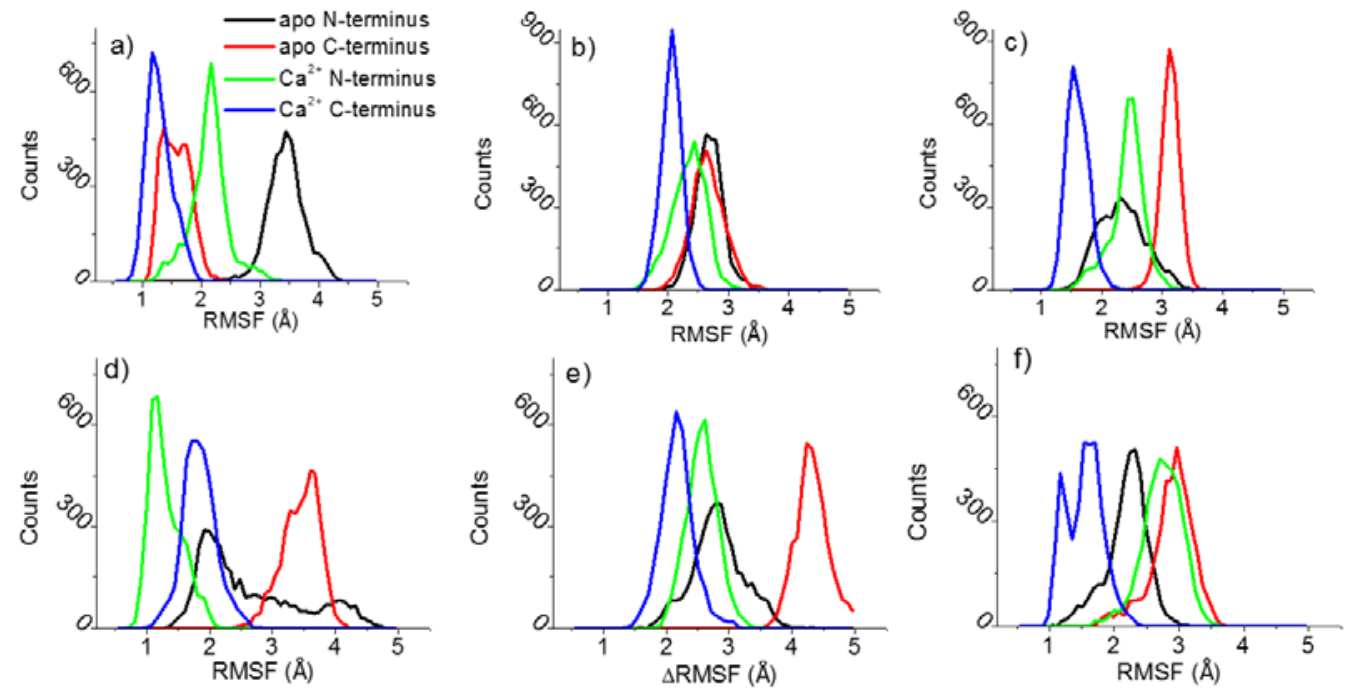

Figure 8.30. Plots of the root mean square fluctuations (RMSF) distribution for the last 80 ns of aMD simulation for $\mathrm{N}$ - and C-terminus of the (a) $\operatorname{DREAM}(\triangle 65)$, (b) DREAM(Y174A), (c) DREAM(F218A), (d) DREAM(F219A), (e) DREAM(F235A) and (f) DREAM(F252A) construct in the $\mathrm{Ca}^{2+}$ bound and free conformations.

Figure 8.30 shows the resulting distribution of root mean squared fluctuations (RMSF) obtained for all the twenty four simulations corresponding the $\mathrm{N}$ - and C-terminus of the apo and $\mathrm{Ca}^{2+}$ bound form of the mutants listed in the legend. The first clear observation is that the $\mathrm{N}$-terminus of the $\operatorname{DREAM}(\Delta 65)$ construct is significantly more flexible than the $\mathrm{C}$-terminus, and $\mathrm{Ca}^{2+}$ binding induces an increase in rigidity of both domains. However, in the absence of $\mathrm{Ca}^{2+}$, all the constructs show a larger flexibility of the C-terminus compared to the N-terminus. The only exception is the DREAM(Y174A) mutant, which shows similar flexibility of both domains, with RMSF magnitudes 
intermediate to that of DREAM $(\Delta 65)$. Nonetheless, $\mathrm{Ca}^{2+}$ binding still induces an increase in rigidity of both domains. An increase in rigidity upon $\mathrm{Ca}^{2+}$ binding compared to the $\mathrm{Ca}^{2+}$ free form is observed for all constructs, and in all cases the C-terminus is more rigid (compare blue trace to others in Figure 8.30). However, there was an exception to this trend, DREAM(F219A) was characterized by a larger rigidity of the N-terminus compared to the C-terminus, in both the apo and $\mathrm{Ca}^{2+}$ bound form (Figure 8.30d).

We also investigated whether the changes in flexibility of the protein, as quantified through backbone fluctuations, have any correlation to the experimentally determined stability of the protein. We determined the differences in fluctuations of the N- and Cterminus by subtracting the maximum of the RMSF distribution between the N- and Cterminus domain for both the apo and $\mathrm{Ca}^{2+}$ bound forms. We also calculated the difference between $\mathrm{N}$ - and C-terminus unfolding energy from table 8.3 and 8.4. The resulting values are plotted in Figure 8.31, where the difference in unfolding energy is plotted as a function of the difference in RMSF. A linear fit to the data reveals a certain degree of correlation, with $\mathrm{R}^{2}=0.68$, slope $=-1.16 \mathrm{kcal} \mathrm{mol}^{-1} \AA^{-1}$, and intercept of $1.8 \AA$. The linear relationship highlights the role of dynamic fluctuations in interdomain communications and stabilization of the protein. Moreover, it is likely that the deviations from linearity are due to small structural differences between the mutants. 


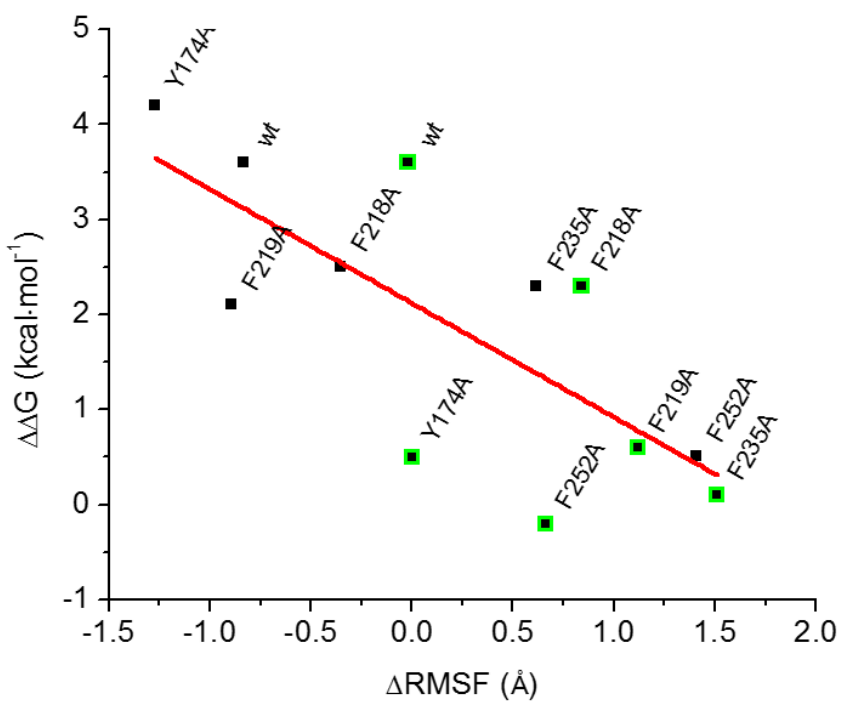

Figure 8.31. Plots of the difference in unfolding energy $\left(\Delta \Delta \mathrm{G}=\Delta \mathrm{G}_{\mathrm{I}}-\Delta \mathrm{G}_{\mathrm{U}}\right)$ as a function of the difference in RMSF ( $\left.\triangle \mathrm{RMSF}=\mathrm{RMSF}_{\mathrm{N} \text {-terminus }}-\mathrm{RMSF}_{\mathrm{C} \text {-terminus }}\right)$. The results for DREAM $(\Delta 65), \quad$ DREAM(Y174A), DREAM(F218A), DREAM(F219A), DREAM(F235A), and DREAM(F252A) are shown in the apo (symbols with green outline) and $\mathrm{Ca}^{2+}$ bound form.

8.2.11 Modulation of protein dynamics through mutation of hydrophobic amino acids effectively modulates allosteric regulation of DREAM

As a last confirmation of the essential role of aromatic amino acids and protein dynamics in regulation of the activity of DREAM, we are interested to determine whether the mutations described above can in fact regulate the ability of this protein to interact with Kv4 channels. In order to determine the ability of these DREAM construct to associate with $\mathrm{K}_{\mathrm{v}} 4$ channels, we have monitored their ability to interact with a fluorescently labeled synthetic peptide homologous to the site 2 of the Kv4.3 voltage gated potassium channel. This peptide was used in Chapter 4, and it showed a clear 50-fold increase in affinity upon binding to the $\mathrm{N}$-terminus of $\mathrm{Ca}^{2+}$ bound $\operatorname{DREAM}(\Delta 65)$ (Figure 8.33). 


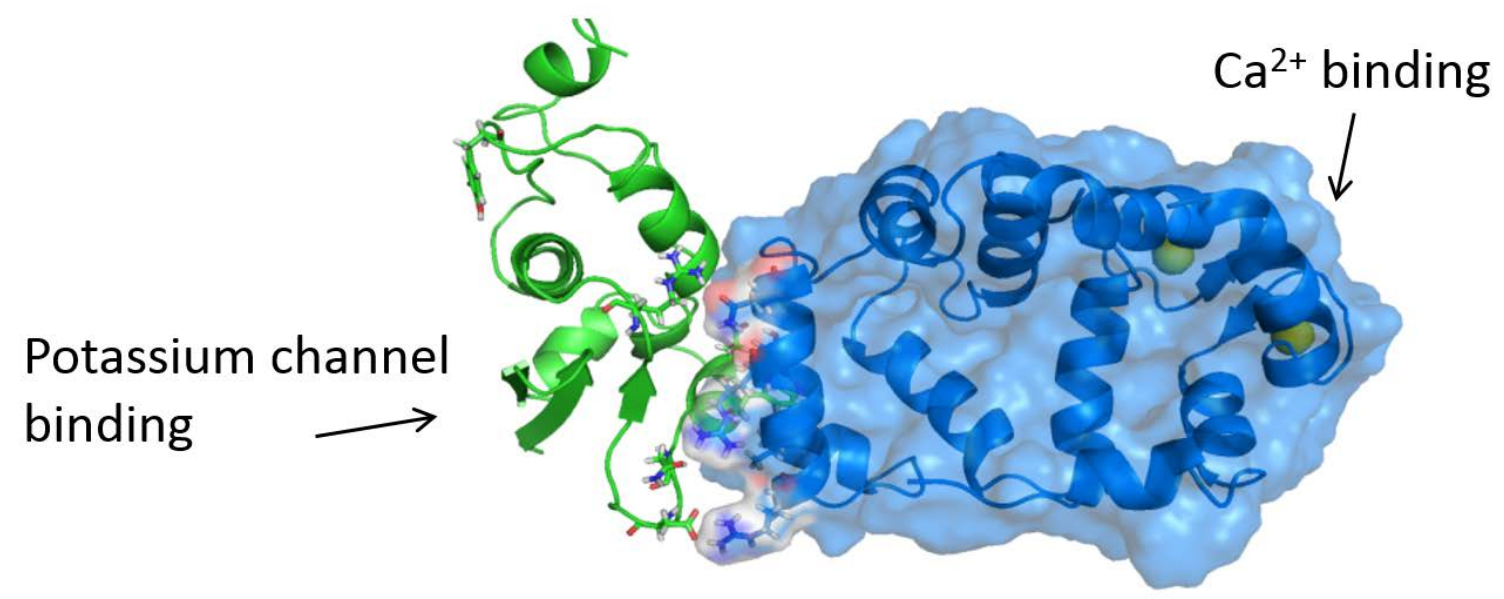

Figure 8.32. Binding site of the $\mathrm{K}_{\mathrm{V}} 4.3$ site 2 peptide at the N-terminus of DREAM.

The affinity in Chapter 4 was monitored through the increase in fluorescence of the dansylated N-terminus of this peptide. In Figure 8.34 we show the emission spectra of the DREAM constructs, DREAM(F219A) and DREAM(F252A) are not shown. In this figure, a clear increase in fluorescence upon binding of DREAM $(\Delta 65)$, DREAM(F218A), and DREAM(Y174A) is observed in the presence of $\mathrm{Ca}^{2+}$. However, the affinity was determined to be about $90 \pm 10 \mu \mathrm{M}$ for peptide binding to the DREAM(Y174A) mutant, in contrast to the affinity determined for the DREAM $(\Delta 65)$ construct of $10 \pm 1 \mu \mathrm{M}$. On the other hand, the DREAM(F218A) showed similar affinity to the site 2 peptide as $\operatorname{DREAM}(\Delta 65)$, with an affinity of $17 \pm 3 \mu \mathrm{M}$. Lastly, the DREAM(F235A) and DREAMNCS1 constructs showed no binding in the presence or absence of $\mathrm{Ca}^{2+}$ (Figure 8.33d and e). Altogether, these results show that the affinity between the $\mathrm{K}_{\mathrm{v}} 4.3$ peptide and the $\mathrm{N}$ terminus of DREAM can be modulated not only by mutations of aromatic amino acid at the C-terminus of DREAM but also by mutations which affect the oligomerization state of DREAM. 

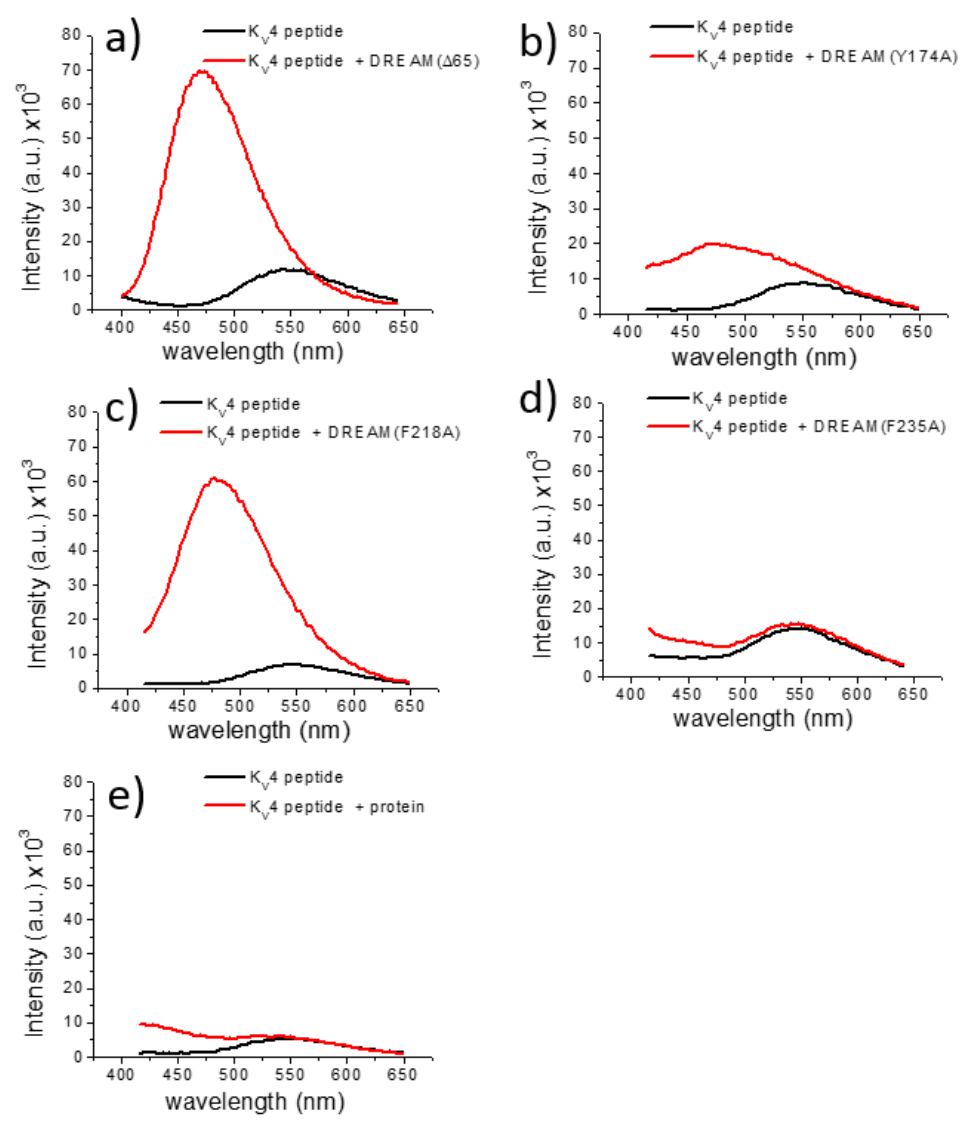

Figure 8.33. Emission spectra of dansylated $\mathrm{K}_{\mathrm{v}} 4.3$ site 2 peptide in the absence and in the presence of $\mathrm{Ca}^{2+}$ bound (a) DREAM( $\left.\Delta 65\right)$, (b) DREAM(Y174A), (c) DREAM(F218A), (d) DREAM(F235A), and (e) DREAM-NCS1.

\subsubsection{Coarse grained simulations reveal a potential mechanism for regulation of $\mathrm{K}_{\mathrm{v}} 4$} binding to DREAM.

The data presented above highlights the role of aromatic amino acids in transducing dynamic information to the $\mathrm{N}$-terminus. The simulations also reveal that the $\mathrm{N}$-terminus of DREAM can reorganize upon binding of $\mathrm{Ca}^{2+}$ at the $\mathrm{C}$-terminus. This reorganization of $\mathrm{Ca}^{2+}$ free monomeric DREAM results in the collapse of the N-terminus and a transition of the EF-hand 1 from a perpendicular orientation to a parallel alignment of the entering and exiting helix of this EFhand. Close analysis of the amino acids involved in stabilizing the KChIP1:Kv4.3 interaction 
(PDB: 2NZ0) reveals the presence of two salt bridges between Lys50 and Arg51 on KChIP1 and Glu77 and Asp78 on the Kv4.3, respectively (Liang et al. 2009). An additional stacking interaction between Tyr57 on KChIP1 and Phe73 on Kv4.3 is also present. Also, overlay of residue 86 to 242 corresponding to EF-hand 1 through 4 on DREAM (PDB: 2jul conformation 1) with the structure of KChIP1:Kv4.3 (PDB: 2nz0) reveals minimal structural differences (RMSD = $1.866 \AA$ between residues 50 to 201 in KChIP1 and residue 90 to 241 in DREAM).

a)

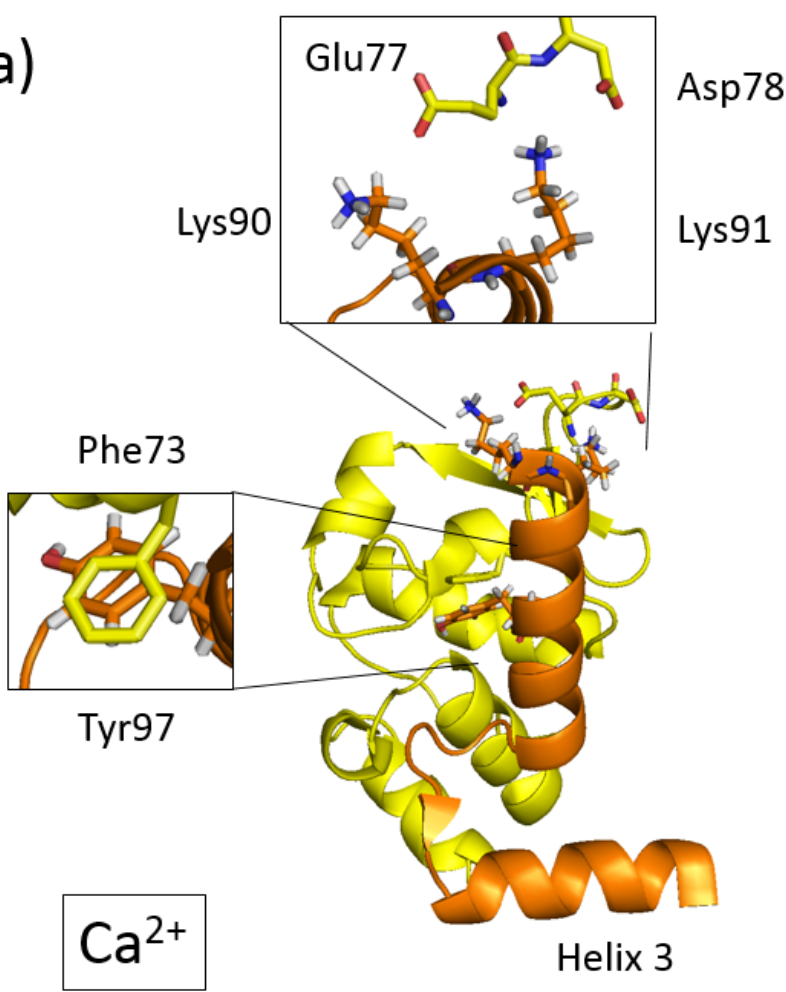

b)

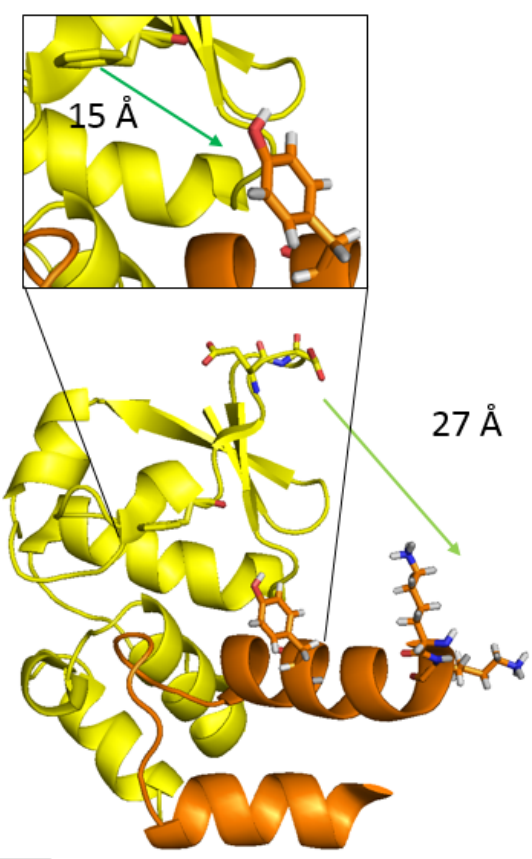

Helix 3

Figure 8.34. (a) Structural alignment of the EF-hand 1 exiting helix of the $\mathrm{Ca}^{2+}$ bound DREAM structure (PDB: 2jul) and the $\mathrm{Ca}^{2+}$ bound structure of KChIP1 bound to the T1-domain of Kv4.3, shown in yellow (PDB: 2nz0). The exiting helix of EF-hand 1 was used for aliment. (b) Alignment of the exiting helix of EF-hand 1 of $\mathrm{Ca}^{2+}$ free DREAM (structure obtained after 432 ns of cMD simulation) and the exiting helix of KChIP1 in complex with the Kv4.3 channel.

After aligning the structures, we observe that DREAM could potentially make the same contacts with Kv4.3 as KChIP1, where Lys90 and Lys91 on DREAM would form a salt bridge 
with Glu77 and Asp78 on Kv4.3, respectively. In DREAM, Tyr97 would form the stacking interaction with Phe73 on Kv4.3. Therefore, we investigated whether these amino acids (which reside on the entering helix of EF-hand 1) could potentially reorganize in the absence of $\mathrm{Ca}^{2+}$ bound at EF-hand 3 and 4 on DREAM. Analysis of the structure of DREAM following 432 ns of cMD simulation in the absence of $\mathrm{Ca}^{2+}$ reveals that Tyr97 undergoes a slight rotation of about $60^{\circ}$ towards the hydrophobic cavity at the N-terminus of DREAM (Figure 8.35a).
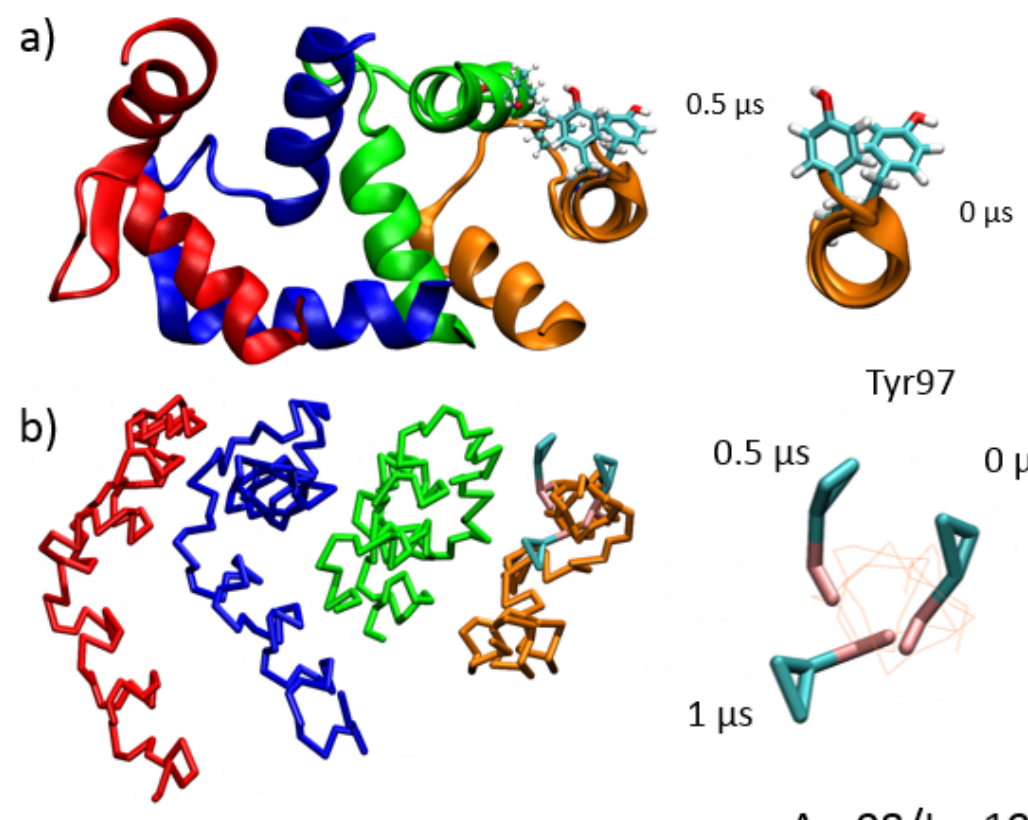

Tyr97
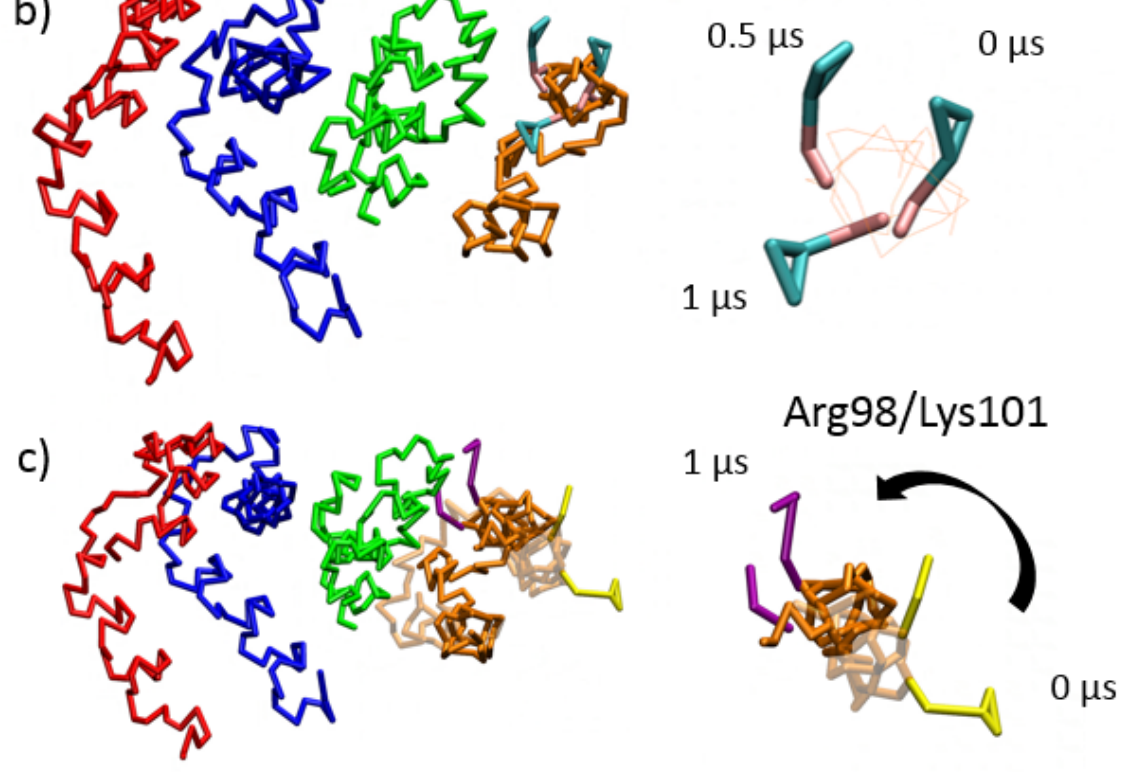

Figure 8.35. Structural reorganization of EF-hand 1 in the $\mathrm{Ca}^{2+}$ free form of DREAM obtained from: (a) $435 \mathrm{~ns}$ cMD simulation of apoDREAM and (b) $1 \mu$ s coarse-grained simulation. The inset shows the rotation Tyr97 into the hydrophobic cavity lining the N-terminus domain. The EF-hand 1 was aligned using the backbone atoms of residues 89 to 160. (c) Structure of DREAM after $1 \mu \mathrm{s}$ coarse-grained simulation showing the rotation of Arg98 and Lys101 in the absence of $\mathrm{Ca}^{2+}$. 
We speculate that in the absence of $\mathrm{Ca}^{2+}$, Tyr97 on DREAM becomes buried in the $\mathrm{N}$ terminus hydrophobic cavity of DREAM. To support this hypothesis, we carried out coarsegrained simulations of DREAM in the absence of $\mathrm{Ca}^{2+}$ in order to access structural changes which could occur at longer timescales than 500 ns. As shown in figure 8.35b, we observe that Tyr97 can undergo an almost $180^{\circ}$ rotation, and effectively become buried into the $\mathrm{N}$-terminus hydrophobic cavity. At this position, it interacts with residues Phe148 and Val152 at the exiting helix of EF-hand 2. In addition, we observe that Arg98 and Lys101 undergo a similar rotation; however, these amino acids do not make clear electrostatic interactions with the T1 domain (Figure 8.35c). The rotation of these amino acids may be responsible for modulation of binding of other ligands, such as DNA. On the other hand, Lys90 and Lys91 at the start of the entering helix of EF-hand 1 do not undergo any significant rotation, and remain solvent exposed. Thus, it could be possible that while the Tyr97:Phe73 interaction is disrupted by a helix rotation, the Lys90Lys91:Glu77Asp78 salt bridges are broken by a different mechanism. The structural change regulating the salt bridge interaction becomes obvious when the exiting helix of EF-hand 1 is aligned with the same helix of the KChIP1:Kv4.3 complex (Figure 8.34a). Alignment in the $\mathrm{Ca}^{2+}$ bound state shows that all these three interactions ( 2 salt bridges and $1 \pi$ - $\pi$ interaction) are present . However, alignment of the same helix in the DREAM structure obtained after 432 ns of cMD simulation in the absence of $\mathrm{Ca}^{2+}$ reveals that all three of these contacts are significantly altered. The disruption of these contacts is mainly mediated by the transition of EF-hand 1 from a perpendicular organization, to a parallel one (Figure 8.34b). The translation of the entering helix of EF-hand 1 effectively displaces Lys90 and Lys91 by about $27 \AA$ from Glu77 and Asp78, while Ty97 is displaced by about $15 \AA$ from Phe73. Altogether, the N-terminus structural reorganization of DREAM provide a clear description of the molecular mechanisms controlling Kv4.3 site 2 binding. Furthermore, the realignment of the 
entering helix of EF-hand 1 may also underline the mechanism by which DREAM associates with DNA. In this model, the antiparallel orientation of the helices in EF-hand 1 would form favorable contacts, probably between the DNA groves. The effect that dimerization has on the reorganization of the $\mathrm{N}$-terminus could also be a mechanism by which $\mathrm{Ca}^{2+}$ binding to DREAM would favor the dissociation from DNA.

\subsection{Summary}

In this chapter we have presented extensive biochemical and biophysical data supporting the role of global and domain dynamics in regulating the propagation of structural changes induced by $\mathrm{Ca}^{2+}$ binding to DREAM. In the first section, we demonstrate using long timescale classical and accelerated molecular dynamic simulations, that dissociation of $\mathrm{Ca}^{2+}$ from EF-hands 3 and 4 induces the collapse of the $\mathrm{N}$-terminus. The collapse of the $\mathrm{N}$-terminus is, to a lower extent, also observed in the $\mathrm{Ca}^{2+}$ bound structure of DREAM; however, modeling of DREAM as a $\mathrm{Ca}^{2+}$ bound dimer effectively eliminates structural changes at the $\mathrm{N}$-terminus during our simulation time. These results highlight the role of oligomerization and dynamic coupling between the $\mathrm{N}$ - and $\mathrm{C}$-terminus of DREAM. This intradomain interaction was also clearly observed upon analysis of the molecular dynamic simulations using cross correlation maps and dynamic network analysis. In this analysis approach, we identified that DREAM is dynamically organized in clusters of amino acids segregated at the $\mathrm{N}$ - and $\mathrm{C}$-terminus domains. We also show that the entering helix of EF-hand 3 plays a pivotal role of connecting the $\mathrm{N}$ - and C-terminus communities. The behavior of EF-hand 3 as a dynamic hub and the presence of numerous conserved amino acids on this helix, may indicate that the dynamic coupling between the $\mathrm{N}$ - and C-terminus observed here may be a widespread phenomenon in NCS proteins. Furthermore, we identified critical nodes connecting the N- and Cterminus as well as the H10 helix. These amino acids were shown to be highly conserved among 
NCS proteins, and could potentially represent a fundamental mechanism of dynamic reorganization in this group of EF-hand $\mathrm{Ca}^{2+}$ binding proteins. Using dynamic network analysis, we were also able to identify amino acids at the long loop connecting the EF-hands 3 and 4 which play a role in stabilizing the dimeric form of DREAM. The fact that DREAM has the least negatively charged loop, while NCS-1 has the most negatively charge loop seems to support the idea that modulation of the charges on this loop may help these protein to regulate their oligomerization inside the cell.

Making use of the information obtained in section 8.2.1 through 8.2.3 we generated a small library of DREAM mutants with the aim of selectively modulating the dynamic coupling between the $\mathrm{N}$ - and C-terminus through elimination of intersubunit and intrasubunit critical nodes. The hypothesis being, that by mutating these amino acid, we would effectively modify the dynamic coupling between the $\mathrm{N}$ - and C-terminus of DREAM and induce changes in the activity. However, to rule out the potential protein unfolding arising from the mutation of hydrophobic amino acids we carried out extensive biochemical characterization of the purified mutants. Based on the circular dichroism data, all the proteins showed a largely helical secondary structure. Furthermore, tryptophan fluorescence and $\mathrm{Ca}^{2+}$ binding studies demonstrate that these mutants are likely in a native-like conformation. This conformation is characterized by $\mathrm{Ca}^{2+}$ affinities in the low micromolar range, a common feature among NCS proteins (Burgoyne 2004). Altogether, supporting the idea that these proteins are functionally folded. However, in DREAM(F219A), DREAM(F235A), and DREAM-NCS1 we observed a reversal of the tryptophan fluorescence upon $\mathrm{Ca}^{2+}$ binding. This is an interesting finding since other NCS proteins, such as recoverin and NCS-1 are known to undergo an increase in tryptophan fluorescence upon $\mathrm{Ca}^{2+}$ binding (Hughes et al. 1998, Baldwin, Ames 1998). Interestingly, a similar reversal of tryptophan fluorescence was 
observed in a double mutant of myristoylated recoverin protein, where Ile52 and Tyr53 where mutated to alanine (Baldwin, Ames 1998). In this recoverin mutant, binding of $\mathrm{Ca}^{2+}$ induced a decrease of the tryptophan fluorescence. These findings highlight the sensitivity of tryptophan at the entering helix of EF-hand 3 (Trp169 in DREAM), to changes in protein conformation induced by mutations of amino acids at the $\mathrm{N}$ - or C-terminus domain.

Unfolding experiments reveal the presence of subtle but significant differences in the stability of some of the DREAM mutants. With the most remarkable observation made for the DREAM(F218A) construct, which showed a completely reversal of the stability upon $\mathrm{Ca}^{2+}$ binding. The DREAM(F219A) construct, despite having a mutation adjacent to DREAM(F218A), did not show such a change in stability. We also observe that the intermediate unfolded state of $\operatorname{DREAM}(\Delta 65)$, DREAM(F219A), DREAM(F235A), and DREAM(F252A) protein constructs is associated with a bright tryptophan fluorescence. Interestingly, the emission maxima of the bright intermediate were similar to those observed for the $\operatorname{DREAM}(\Delta 160)$ construct at $0 \mathrm{M} \mathrm{GuHCl}$. Indeed, we have determined that the quantum yield for tryptophan fluorescence of the $\operatorname{DREAM}(\Delta 160)$ mutant $(\Phi \sim 0.13)$ is similar to that of tryptophan in solution, while the quantum yield of native $\operatorname{DREAM}(\Delta 65)$ is near 0.08 (data not shown). The emission maxima of this bright intermediate $(\sim 352 \mathrm{~nm})$ is also identical to that of $\operatorname{DREAM}(\Delta 160)$ at $0 \mathrm{M} \mathrm{GuHCl}$ in the presence of $\mathrm{Ca}^{2+}$. These observation allows us to speculate that the intermediate state observed for some of the DREAM construct may represent a conformation in which the $\mathrm{N}$-terminus has been partially unfolded, and tryptophan is exposed to the solvent. Alternatively, it could be possible that the mutations have induced a complete decoupling of the tryptophan fluorescence to the N-terminus, as observed in the $\operatorname{DREAM}(\Delta 160)$ lacking this domain. Previous theoretical and single molecule studies have shown that the N-terminus domain of NCS1 unfolds first followed by the C-terminus 
(Heidarsson et al. 2013). This model being present in DREAM is supported by our data, in which we observe that the first transition in $\operatorname{DREAM}(\Delta 65)$ is stabilized by the presence of $\mathrm{Mg}^{2+}$, while the second unfolding transition is not. Also, association of $\mathrm{Ca}^{2+}$ induces a larger stabilization of the second transition. With this in mind, we have analyzed all our subsequent results on the basis that the first transition represents unfolding of the $\mathrm{N}$-terminus followed by unfolding of the C-terminus at $\mathrm{GuHCl}$ concentrations of about 3 to $4 \mathrm{M}$. The fact that the bright intermediate was only observed for some mutants, and only in the presence of $\mathrm{Ca}^{2+}$, indicates that the unfolding pathways of the Nterminus can be modified by modifications of the C-terminus.

Furthermore, to better understand the tryptophan transition observed upon $\mathrm{Ca}^{2+}$ binding and the reversal of the transition observed for the DREAM(F219A) and DREAM(F235A) mutants, we conducted analysis of the rotameric states of tryptophan at positon 169 on DREAM. We have identified that in the absence of the N-terminus, Trp169 can populate two rotameric states (the $t$ and $\mathrm{g}^{+}$), in each of which the indole ring can be oriented towards the carboxy (D-) or amino group $(\mathrm{D}+)$ of the peptide backbone. However, the presence of the N-terminus in the $\operatorname{DREAM}(\Delta 65)$ construct effectively restricts the rotameric state in the absence of $\mathrm{Ca}^{2+}$ to the trotamer with indole ring pointing towards the carboxy group of the backbone (D- conformation). Upon $\mathrm{Ca}^{2+}$ binding, a transition to the $\mathrm{g}^{+}$rotamer with the indole ring pointing towards the amide bond of the peptide bond is favored. The switching mechanism has not been studied extensively, but our findings show that the conformation orientation of the indole ring may play a major role in determining the transition in tryptophan fluorescence measured experimentally. In our model, we propose that transition from the D- to D+ induces a decrease in tryptophan fluorescence as a result of an increase in solvent quenching. In addition, in the D- conformation, Trp169 is observed to form hydrophobic contacts with Phe138 and Leu158. Therefore, it is possible that changes in the dynamics of the 
interaction in the absence of $\mathrm{Ca}^{2+}$ could stabilize the $\mathrm{N}$-terminus. It is also possible that the stacking observed between Trp169 and Leu158 could affect the stability of the dimer or binding of other proteins; Leu158 is known to form part of the LxxLL motif at the N-terminus of DREAM which has been proposed to mediate protein-protein interactions and oligomerization of DREAM (Lusin et al. 2008, Ledo et al. 2000). Therefore, in the model presented here, dynamic coupling between the exiting helix of EF-hand 4 and the entering helix of EF-hand 3 in the presence of $\mathrm{Ca}^{2+}$ (Figure 8.13), together with switching of Trp169 from the $\mathrm{D}$ - to the $\mathrm{D}+$ conformation (Figure 8.27), results in the modulation of a hydrophobic bridge spanning the $\mathrm{N}$ - and C-terminus.
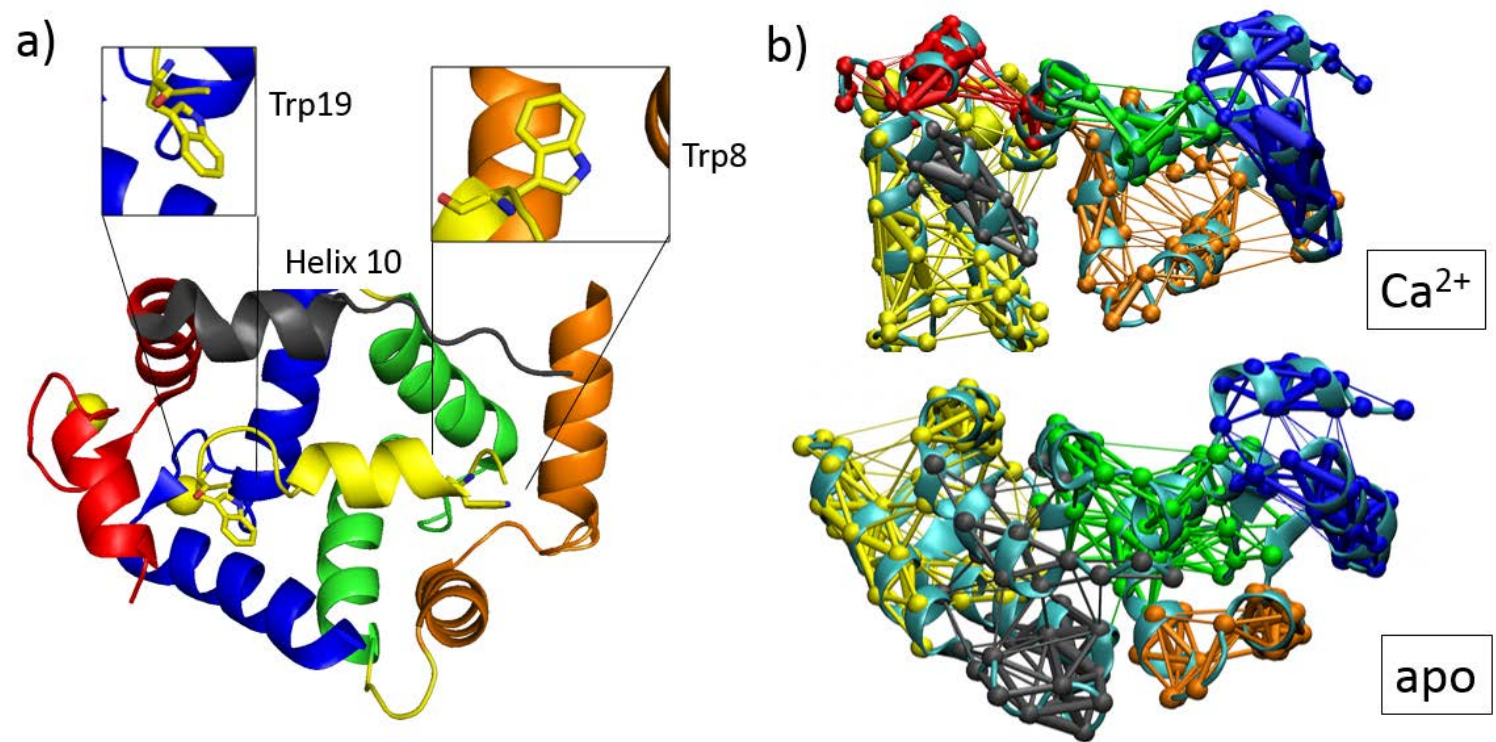

Figure 8.36. Summary of the mechanism by which binding of Kv4.3 site 1 at the hydrophobic surface of DREAM is regulated by $\mathrm{Ca}^{2+}$ binding and changes in structural dynamics. (a) Structure of KChIP1:Kv4.3 complex (PDB: 2nz0) highlighting the role of the $\mathrm{N}$ - and C-terminus hydrophobic cavity in ligand binding. (b) Dynamic network analysis which shows the collapse of the hydrophobic cavities on DREAM and the enhanced dynamic coupling of the H10 helix (black network).

All atom and coarse-grained simulations reveal a potential mechanism by which $\mathrm{Ca}^{2+}$ binding to DREAM and oligomerization changes may modulate $\mathrm{K}_{\mathrm{v}} 4$ binding. In the case of site 1 , we observe a clear transition in protein dynamics at the $\mathrm{C}$-terminus of DREAM upon $\mathrm{Ca}^{2+}$ binding. In the absence of $\mathrm{Ca}^{2+}$, the dynamic reorganization of Phe235 induces a decoupling of the H10 
helix. In the state described above, the H10 helix is flexible and vulnerable to displacement by the N-terminus (site 1) of Kv4.3, which results in the reorientation of the H10 helix as shown in Figure 8.36a. The displacement of H10 upon ligand binding is not a new concept (Liang et al. 2009, Wang, Chai 2009); however, the dynamic mechanisms controlling this displacement have not been described before. In addition, in section 8.2.12 we show that the entering helix of EF-hand 1 undergoes a rotation and displacement which effectively disrupt favorable contacts between the Nterminus of DREAM and Kv4.3 (Figure 8.34). The rotation is driven by the collapse of the Nterminus hydrophobic cavity, the magnitude of which depends on $\mathrm{Ca}^{2+}$ binding and dimerization of DREAM.

Finally, in Figure 8.32 we show that mutations affecting the intrasubunit critical nodes (DREAM(F218A), DREAM(F235A), and DREAM(Y174A)) can modulate binding of DREAM with the site 2 of $\mathrm{K}_{\mathrm{v}} 4.3$ channel. The observation allows us to postulate that structural changes propagated through these amino acids play a major role in inducing the proper conformation of the $\mathrm{N}$-terminus favorable to interact with Kv4.3 site 2. However, perturbation of intradomain dynamics does not seem to be the only important mechanism. In fact, we observe that DREAM-NCS1 has the lowest affinity for the site 2 peptide, indicating that oligomerization state may also play a major role in ligand selectivity. We have also observed that a site 1 peptide, homologous to the $\mathrm{N}$-terminus of $\mathrm{K}_{\mathrm{v}} 4.3$ can also induce the monomerization of DREAM upon binding. The observation would explain why the x-ray structure of Kv4.3 and KChIP1 shows the interaction of a single KChIP1 subunit per $\mathrm{N}$-terminus of the $\mathrm{T} 1$ subunit. Thus, it is possible that dimerization may allow binding to the site 2, while binding of site 1 induces the breakdown of the dimer into monomers. Alternatively, it is very likely that association of the site 1 peptide, which spans the $\mathrm{N}$ - and Cterminus of DREAM, prevents the collapse of the N-terminus and allows the protein complex to 
associate with site 2 . Thus, in chapter 8 we have provided extensive evidence for the role of protein dynamics in regulating structural changes at the N-terminus of DREAM. We have also laid the foundation for what could be a fundamental structural communication pathway in neuronal calcium sensors. More importantly, the approach taken in this section could potentially be applied to other more complex proteins in order to understand the mechanisms by which these proteins regulate ligand binding.

\section{CONCLUSIONS}

The hydrophobic cavity at the C-terminus of DREAM regulates binding of small hydrophobic ligands such as 1,8-ANS, 2,6-ANS, arachidonic acids, and NS5806. These interactions are mediated by numerous hydrophobic amino acids that connect the EF-hands 3 and 4. Using the solvent sensitive dyes 1,8-ANS and 2,6-ANS we show that binding of $\mathrm{Ca}^{2+}$ at EF-hands 3 and 4 induces the exposure of a hydrophobic surface on DREAM. In addition, using fluorescence anisotropy and the long lived fluorescent decay of ANS molecules, we show that DREAM can populate a dimer structure in the presence of $\mathrm{Ca}^{2+}$. Upon removal of $\mathrm{Ca}^{2+}$, rotations with correlation times of 12 ns and 50 ns were resolved. These rotations could represent a monomeric and tetrameric oligomerization state of DREAM. The bright fluorescence of 1,8-ANS upon binding to $\mathrm{Ca}^{2+}$ DREAM has allowed us to demonstrate, that the hydrophobic cavity at the C-terminus is a site relevant for binding of small hydrophobic ligands, such as arachidonic acids and NS5806. Also, we have shown that anisotropy decay measurement using 1,8-ANS can be successfully implemented to characterize the oligomerization state of this protein.

Interestingly, association of NS5806 at the C-terminus of DREAM induces a structural conformation able to associate with the hydrophobic N-terminus of $\mathrm{K}_{\mathrm{v}} 4.3$, 
independent of the presence of $\mathrm{Ca}^{2+}$. The activation of DREAM through interaction with this small hydrophobic ligand, indicates that the changes at the hydrophobic cavity at the C-terminus can result in global changes in the structure of DREAM. Furthermore, we demonstrate the residues Phe218 and Tyr174 play a major role in regulating binding and the activity of NS5806. Docking and molecular dynamics simulation allowed us to observe the effect of NS5806 binding at the C-terminus of DREAM. At the C-terminus site, NS5806 showed a clear stacking interaction with Phe218 and Phe219. The interaction effectively resulted in the displacement of the exiting helix of EF-hand 4. Overall, these findings were used to explain the observed increase in $\mathrm{K}^{+}$current and slowing of inactivation kinetics in cardiac cells upon injection of NS806. Remarkably, we were able to recover identical affinity constants for NS5806 binding to $\mathrm{Ca}^{2+}$ and apoDREAM as the EC50 for current activation and inactivation kinetics determined in vivo. Thus, indicating that the observed in vivo effects, are likely the result of direct interaction of NS5806 with KChIPs.

In addition, we have presented results which provide an insight into the potential structural organization of the flexible extended N-terminus of DREAM (residues 1 to 65). The N-terminus region of DREAM protein has largely been ignore in the literature, mainly because recombinant expression of DREAM proteins containing this flexible domain results in formation of insoluble inclusion bodies. Truncation of this $\mathrm{N}$-terminus region has been proposed to minimally perturb the DNA and Kv4 binding properties of DREAM (Osawa et al. 2005). Furthermore, DREAM contains a cleavage site, which in vivo could lead to the production of a DREAM construct similar to that of $\operatorname{DREAM}(\Delta 65)$. Nonetheless, the fact that the main difference among KChIPs lies at the N-terminus, 
indicates that the $\mathrm{N}$-terminus domain of DREAM may play role in regulating the activity of this protein. The model of DREAM and the biochemical results presented in Chapter 5 provide a renewed view on the potential role of this domain in regulating protein-protein and protein-DNA interactions. Furthermore, these results demonstrate an interesting interplay between a member of the calcium sensor subfamily (calmodulin) and another protein belonging to the neuronal calcium sensor subfamily (DREAM). The biological implication of this protein-protein complex remain to be studied. However, given the strong affinity of calmodulin for the N-terminus of DREAM, as well as the numerous posttranslational modification sequences present in this region, it is possible that the interaction of these two proteins is highly regulated in vivo.

To further understand the role of EF-hand 3 and 4 in regulating the activity of DREAM; in Chapter 6 we presented an extensive biochemical study of the interactions between terbium(III) and DREAM. Here, we show that $\mathrm{Tb}^{3+}$ can effectively bind at EFhands 3 and 4, displace $\mathrm{Ca}^{2+}$, and induce minimal disruption of the oligomerization and exposure of a hydrophobic cavity. Isothermal calorimetry data reveals that coordination of the $\mathrm{Ca}^{2+}$ ion by Glu234 in EF-hand 4 is associated with a larger enthalpic contribution than that due to coordination of $\mathrm{Ca}^{2+}$ by Glu186 at EF-hand 3. Indicating, that the structural reorganization triggered by $\mathrm{Ca}^{2+}$ binding at EF-hand 4, are likely much larger than those due to $\mathrm{Ca}^{2+}$ binding at EF-hand 3. Using the long lived luminescent properties of $\mathrm{Tb}^{3+}$, we observed that binding of $\mathrm{Mg}^{2+}$ at EF-hand 2, can result in structural reorganization of the environment near EF-hands 3 and 4. Thus highlighting the role of $\mathrm{Mg}^{2+}$ as a potential cofactor in stabilizing the structure of DREAM. 
In chapter 7, we described the theoretical and experimental details associated with the development of a photothermal beam deflection apparatus. We highlight different approaches to analyze the data as well as sample conditions to be considered when carrying out experiments. We further use this technique to study the ultrafast release of metal ions from caged compounds. The initial aim of this chapter was to employ the photosensitive $\mathrm{Ca}^{2+}$ chelator DM-nitrophen, in order to photo trigger the release of $\mathrm{Ca}^{2+}$ and monitor the photothermal profile due to $\mathrm{Ca}^{2+}$ binding to DREAM. However, the large variability of the purchased DM nitrophen stocks prevented the detailed analysis of the PBD signal in the presence of DM-nitrophen and DREAM. Also, the nonreversible photo release of $\mathrm{Ca}^{2+}$ from DM-nitrophen results in significant complications in terms of signal averaging. Nonetheless, we have shown that PBD can effectively be used to monitor the thermodynamics properties of metal photo release from caged compounds. We also demonstrate, that DM-nitrophen is able to form a highly stable complex with $\mathrm{Tb}^{3+}$ ions, and that photo release of this lanthanide results in enthalpy and volume changes identical to those observed for $\mathrm{Ca}^{2+}$.

Finally, in Chapter 8, we combine theoretical and experimental approaches to investigate the role of protein dynamics in regulation of DREAM activity. The aim of this chapter is to decipher the precise molecular reorganization induced by $\mathrm{Ca}^{2+}$ binding to DREAM, and how these changes regulate ligand binding. Following extensive studies, we show compelling evidence for a major role of the entering helix of EF-hand 3 of DREAM in regulating the $\mathrm{Ca}^{2+}$ induced structural changes. In particular, we show that the dynamic coupling between the helices at the C-terminus is strongly modulated by $\mathrm{Ca}^{2+}$ binding. More importantly, we identify that residue Phe218, Phe219, Phe235, Tyr 174, and Phe171 
play a pivotal role in regulating the apo to $\mathrm{Ca}^{2+}$ transition. Of particular importance, is the Phe235 residue, which is observed to switch between two conformations upon metal binding. In addition, we observe that a conformational switch of Trp169 could explain the mechanism by which dynamic signals are transduced from the C- towards the N-terminus. The role of the dynamic coupling identified in this section is also supported by unfolding experiments. In these experiments, we observe the presence of a linear relationship between the difference in stability of the $\mathrm{N}$ - and $\mathrm{C}$-terminus of DREAM, and the difference in fluctuations of these domains. Finally, we demonstrate that by mutation of the amino acids identified to play a major role in communication of dynamic fluctuations, we can effectively inhibit binding of DREAM to a peptide homologous to the human Kv4.3 channel. The fact that the mutations are located at the C-terminus and that peptide binding is mediated by the N-terminus, highlights the presence of a well-defined pathway by which these two domains communicate. Based on our results, we can effectively identify such pathway, as involving the dynamic coupling of hydrophobic (aromatic amino acids in particular) amino acids at the C-terminus, the entering helix of EF-hand 3, and the Nterminus. 


\section{LIST OF REFERENCES}

Adams, S.R., Campbell, R.E., Gross, L.A., Martin, B.R., Walkup, G.K., Yao, Y., Llopis, J. \& Tsien, R.Y. 2002, "New Biarsenical Ligands and Tetracysteine Motifs for Protein Labeling in Vitro and in Vivo: Synthesis and Biological Applications", Journal of the American Chemical Society, vol. 124, no. 21, pp. 6063-6076.

Adams, S.R. \& Tsien, R.Y. 2008, "Preparation of the membrane-permeant biarsenicals FlAsH-EDT 2 and ReAsH-EDT 2 for fluorescent labeling of tetracysteine-tagged proteins", Nature Protocols, vol. 3, pp. 1527+.

Agus, Z. \& Morad, M. 1991, "Modulation of cardiac ion channels by magnesium", Annual Review of Physiology, vol. 53, no. 1, pp. 299-307.

Amarillo, Y., De Santiago-Castillo, J.A., Dougherty, K., Maffie, J., Kwon, E., Covarrubias, M. \& Rudy, B. 2008, "Ternary Kv4.2 channels recapitulate voltage-dependent inactivation kinetics of A-type $\mathrm{K}^{+}$channels in cerebellar granule neurons", The Journal of Physiology, vol. 586, no. 8, pp. 2093-2106.

Ames, J.B., Hamasaki, N. \& Molchanova, T. 2002, "Structure and calcium-binding studies of a recoverin mutant (E85Q) in an allosteric intermediate state", Biochemistry, vol. 41, no. 18 , pp. 5776-5787.

Ames, J.B., Hendricks, K.B., Strahl, T., Huttner, I.G., Hamasaki, N. \& Thorner, J. 2000, "Structure and calcium-binding properties of Frq1, a novel calcium sensor in the yeast Saccharomyces cerevisiae", Biochemistry, vol. 39, no. 40, pp. 12149-12161.

Ames, J.B., Ishima, R., Tanaka, T., Gordon, J.I., Stryer, L. \& Ikura, M. 1997, "Molecular mechanics of calcium-myristoyl switches", Nature, vol. 389, no. 6647, pp. 198-202.

Ames, J.B. \& Lim, S. 2012, "Molecular structure and target recognition of neuronal calcium sensor proteins", Biochimica et Biophysica Acta (BBA)-General Subjects, vol. 1820, no. 8, pp. 1205-1213.

Ames, J.B., Tanaka, T., Stryer, L. \& Ikura, M. 1996, "Portrait of a myristoyl switch protein", Current opinion in structural biology, vol. 6, no. 4, pp. 432-438.

Ames, J.B., Levay, K., Wingard, J.N., Lusin, J.D. \& Slepak, V.Z. 2006, "Structural Basis for Calcium-induced Inhibition of Rhodopsin Kinase by Recoverin", The Journal of Biological Chemistry, vol. 281, no. 48, pp. 37237-37245.

An, W.F., Bowlby, M.R., Betty, M., Cao, J., Ling, H., Mendoza, G., Hinson, J.W., Mattsson, K.I., Strassle, B.W., Trimmer, J.S. \& Rhodes, K.J. 2000, "Modulation of Atype potassium channels by a family of calcium sensors", Nature, vol. 403, no. 6769, pp. 553-556. 
Anderson, D., Mehaffey, W.H., Iftinca, M., Rehak, R., Engbers, J.D.T., Hameed, S., Zamponi, G.W. \& Turner, R.W. 2010, "Regulation of neuronal activity by Cav3-Kv4 channel signaling complexes", Nature Neuroscience, vol. 13, no. 3, pp. 333-337.

Antz, C., Geyer, M., Fakler, B., Schott, M.K., Guy, H.R., Frank, R., Ruppersberg, J.P. \& Kalbitzer, H.R. 1997, "NMR structure of inactivation gates from mammalian voltagedependent potassium channels", Nature, vol. 385, no. 6613, pp. 272-275.

Astudillo, L., Bernad, S., Derrien, V., Sebban, P. \& Miksovska, J. 2010, "Probing the role of the internal disulfide bond in regulating conformational dynamics in neuroglobin", Biophysical Journal, vol. 99, no. 2, pp. L16-L18.

Austin, R.H., Stein, D.L. \& Wang, J. 1987, "Terbium luminescence-lifetime heterogeneity and protein equilibrium conformational dynamics", Proceedings of the National Academy of Sciences of the United States of America, vol. 84, no. 6, pp. 1541-1545.

Babich, A. \& Burkhardt, J.K. 2013, "Coordinate control of cytoskeletal remodeling and calcium mobilization during T-cell activation", Immunological Reviews, vol. 256, no. 1, pp. 80-94.

Badea, M. 1979, "Time-resolved fluorescence measurements.", Methods in Enzymology, vol. 61, pp. 378-425.

Bahring, R., Dannenberg, J., Peters, H.C., Leicher, T., Pongs, O. \& Isbrandt, D. 2001, "Conserved Kv4 N-terminal domain critical for effects of $\mathrm{Kv}$ channel-interacting protein 2.2 on channel expression and gating", The Journal of Biological Chemistry, vol. 276, no. 26, pp. 23888-23894.

Bähring, R. \& Covarrubias, M. 2011, "Mechanisms of closed-state inactivation in voltagegated ion channels", The Journal of Physiology, vol. 589, no. 3, pp. 461-479.

Baldwin, A.N. \& Ames, J.B. 1998, "Core mutations that promote the calcium-induced allosteric transition of bovine recoverin", Biochemistry, vol. 37, no. 50, pp. 1740817419.

Barbato, G., Ikura, M., Kay, L.E., Pastor, R.W. \& Bax, A. 1992, "Backbone dynamics of calmodulin studied by nitrogen-15 relaxation using inverse detected two-dimensional NMR spectroscopy: the central helix is flexible", Biochemistry, vol. 31, no. 23, pp. 5269-5278.

Barnett, M.W. \& Larkman, P.M. 2007, "The action potential", Practical Neurology, vol. 7, no. 3, pp. 192-197.

Bean, B.P. 2007, "The action potential in mammalian central neurons", Nature Reviews Neuroscience, vol. 8, no. 6, pp. 451-465. 
Beck, E.J., Bowlby, M., An, W.F., Rhodes, K.J. \& Covarrubias, M. 2002, "Remodelling inactivation gating of Kv4 channels by KChIP1, a small-molecular-weight calciumbinding protein", The Journal of Physiology, vol. 538, no. Pt 3, pp. 691-706.

Beechem, J.M. 1989, "A second generation global analysis program for the recovery of complex inhomogeneous fluorescence decay kinetics", Chemistry and Physics of Lipids, vol. 50, no. 3-4, pp. 237-251.

Beija, M., Afonso, C.A. \& Martinho, J.M. 2009, "Synthesis and applications of Rhodamine derivatives as fluorescent probes", Chemical Society Reviews, vol. 38, no. 8, pp. 24102433.

Bellucci, L., Corni, S., Di Felice, R. \& Paci, E. 2013, "The structure of neuronal calcium sensor-1 in solution revealed by molecular dynamics simulations", PloS one, vol. 8, no. 9, pp. e74383.

Berridge, M.J., Bootman, M.D. \& Lipp, P. 1998, "Calcium-a life and death signal", Nature, vol. 395, no. 6703, pp. 645-648.

Berridge, M.J., Bootman, M.D. \& Roderick, H.L. 2003, "Calcium signalling: dynamics, homeostasis and remodelling", Nature Reviews Molecular Cell Biology, vol. 4, no. 7, pp. 517-529.

Berridge, M.J., Lipp, P. \& Bootman, M.D. 2000, "The versatility and universality of calcium signalling", Nature reviews Molecular Cell Biology, vol. 1, no. 1, pp. 11-21.

Bett, G.C., Morales, M.J., Strauss, H.C. \& Rasmusson, R.L. 2006, "KChIP2b modulates the affinity and use-dependent block of Kv4.3 by nifedipine", Biochemical and Biophysical Research Communications, vol. 340, no. 4, pp. 1167-1177.

Betts, R. \& Dahlinger, O.F. 1959, "The heat and entropy of association of the complex ions formed by EDTA with the lanthanide elements in aqueous solution", Canadian Journal of Chemistry, vol. 37, no. 1, pp. 91-100.

Betzig, E., Patterson, G.H., Sougrat, R., Lindwasser, O.W., Olenych, S., Bonifacino, J.S., Davidson, M.W., Lippincott-Schwartz, J. \& Hess, H.F. 2006, "Imaging intracellular fluorescent proteins at nanometer resolution", Science (New York, N.Y.), vol. 313, no. 5793, pp. 1642-1645.

Bigelow, D.J. \& Inesi, G. 1991, "Frequency-domain fluorescence spectroscopy resolves the location of maleimide-directed spectroscopic probes within the tertiary structure of the calcium ATPase of sarcoplasmic reticulum", Biochemistry, vol. 30, no. 8, pp. 2113-2125. 
Bito, H., Deisseroth, K. \& Tsien, R.W. 1997, "Ca ${ }^{+2}$-dependent regulation in neuronal gene expression", Current Opinion in Neurobiology, vol. 7, no. 3, pp. 419-429.

Blackman, S.M., Piston, D.W. \& Beth, A.H. 1998, "Oligomeric state of human erythrocyte band 3 measured by fluorescence resonance energy homotransfer", Biophysical Journal, vol. 75, no. 2, pp. 1117-1130.

Boland, L.M. \& Drzewiecki, M.M. 2008, "Polyunsaturated fatty acid modulation of voltage-gated ion channels", Cell Biochemistry and Biophysics, vol. 52, no. 2, pp. 5984.

Bonetti, G., Vecli, A. \& Viappiani, C. 1997, "Reaction volume of water formation detected by time-resolved photoacoustics: photoinduced proton transfer between onitrobenzaldehyde and hydroxyls in water", Chemical Physics Letters, vol. 269, no. 3, pp. 268-273.

Böttcher, C.J.F., van Belle, O.C., Bordewijk, P. \& Rip, A. 1978, Theory of Electric Polarization, Elsevier Science Ltd.

Bourdeau, M.L., Laplante, I., Laurent, C.E. \& Lacaille, J.-. 2011, "KChIP1 modulation of Kv4.3-mediated A-type $\mathrm{K}+$ currents and repetitive firing in hippocampal interneurons", Neuroscience, vol. 176, no. 0, pp. 173-187.

Bowlby, M.R., Chanda, P., Edris, W., Hinson, J., Jow, F., Katz, A.H., Kennedy, J., Krishnamurthy, G., Pitts, K., Ryan, K., Zhang, H. \& Greenblatt, L. 2005, "Identification and characterization of small molecule modulators of KChIP/Kv4 function", Bioorganic \& Medicinal chemistry, vol. 13, no. 22, pp. 6112-6119.

Bowman-James, K., Bianchi, A. \& García-Espana, E. 2012, Anion coordination chemistry, John Wiley \& Sons.

Braslavsky, S.E. \& Heibel, G.E. 1992, "Time-resolved photothermal and photoacoustic methods applied to photoinduced processes in solution", Chemical Reviews, vol. 92, no. 6, pp. 1381-1410.

Braunewell, K. \& Gundelfinger, E.D. 1999, "Intracellular neuronal calcium sensor proteins: a family of EF-hand calcium-binding proteins in search of a function", Cell and tissue research, vol. 295, no. 1, pp. 1-12.

Braunewell, K. 2005, "The darker side of $\mathrm{Ca}^{2+}$ signaling by neuronal $\mathrm{Ca}^{2+}$-sensor proteins: from Alzheimer's disease to cancer", Trends in pharmacological sciences, vol. 26, no. 7, pp. 345-351. 
Brittain, H.G., Richardson, F.S. \& Martin, R.B. 1976, "Terbium(III) emission as a probe of calcium(II) binding sites in proteins", Journal of the American Chemical Society, vol. 98, no. 25, pp. 8255-8260.

Brooks, B.R., Bruccoleri, R.E., Olafson, B.D., States, D.J., Swaminathan, S. \& Karplus, M. 1983, "CHARMM: A program for macromolecular energy, minimization, and dynamics calculations", Journal of Computational Chemistry, vol. 4, no. 2, pp. 187217.

Brown, S.E., Martin, S.R. \& Bayley, P.M. 1997, "Kinetic Control of the Dissociation Pathway of Calmodulin-Peptide Complexes", Journal of Biological Chemistry, vol. 272, no. 6, pp. 3389-3397.

Bunzli, J. \& Eliseeva, S.V. 2011, "Basics of Lanthanide Photophysics" in Lanthanide Fluorescence, Springer Berlin Heidelberg, , pp. 1-45.

Burgoyne, R.D. 2007, "Neuronal calcium sensor proteins: generating diversity in neuronal $\mathrm{Ca}^{2+}$ signaling", Nature Reviews Neuroscience, vol. 8, no. 3, pp. 182-193.

Burgoyne, R.D. 2004, "The neuronal calcium-sensor proteins", Biochimica et Biophysica Acta (BBA)-Molecular Cell Research, vol. 1742, no. 1, pp. 59-68.

Burgoyne, R.D. \& Weiss, J.L. 2001, "The neuronal calcium sensor family of $\mathrm{Ca}^{2+}$-binding proteins", The Biochemical journal, vol. 353, no. Pt 1, pp. 1-12.

Burgoyne, R.D., O'Callaghan, D.W., Hasdemir, B., Haynes, L.P. \& Tepikin, A.V. 2004, "Neuronal $\mathrm{Ca}^{2+}$-sensor proteins: multitalented regulators of neuronal function", Trends in neurosciences, vol. 27, no. 4, pp. 203-209.

Buxbaum, J.D., Choi, E., Luo, Y., Lilliehook, C., Crowley, A.C., Merriam, D.E. \& Wasco, W. 1998, "Calsenilin: A calcium-binding protein that interacts with the presenilins and regulates the levels of a presenilin fragment", Nature medicine, vol. 4, no. 10, pp. 1177-1181.

Calloe, K., Cordeiro, J.M., Di Diego, J.M., Hansen, R.S., Grunnet, M., Olesen, S.P. \& Antzelevitch, C. 2009, "A transient outward potassium current activator recapitulates the electrocardiographic manifestations of Brugada syndrome", Cardiovascular research, vol. 81, no. 4, pp. 686-694.

Calloe, K., Soltysinska, E., Jespersen, T., Lundby, A., Antzelevitch, C., Olesen, S.P. \& Cordeiro, J.M. 2010, "Differential effects of the transient outward $\mathrm{K}(+)$ current activator NS5806 in the canine left ventricle", Journal of Molecular and Cellular Cardiology, vol. 48, no. 1, pp. 191-200. 
Callsen, B., Isbrandt, D., Sauter, K., Hartmann, L.S., Pongs, O. \& Bähring, R. 2005, "Contribution of $\mathrm{N}$ - and $\mathrm{C}$-terminal channel domains to $\mathrm{Kv}$ channel interacting proteins in a mammalian cell line", The Journal of Physiology, vol. 568, no. 2, pp. 397-412.

Carafoli, E. 2002, "Calcium signaling: A tale for all seasons", Proceedings of the National Academy of Sciences, vol. 99, no. 3, pp. 1115-1122.

Carrion, A.M., Link, W.A., Ledo, F., Mellstrom, B. \& Naranjo, J.R. 1999, "DREAM is a $\mathrm{Ca}^{2+}$-regulated transcriptional repressor", Nature, vol. 398, no. 6722, pp. 80-84.

Carrion, A.M., Mellstrom, B. \& Naranjo, J.R. 1998, "Protein Kinase A-Dependent Derepression of the Human Prodynorphin Gene via Differential Binding to an Intragenic Silencer Element", Molecular and Cellular Biology, vol. 18, no. 12, pp. 6921-6929.

Castro, G., Larson, B., Panilaitis, B. \& Kaplan, D. 2005, "Emulsan quantitation by Nile red quenching fluorescence assay", Applied Microbiology and Biotechnology, vol. 67, no. 6, pp. 767-770.

Cates, M.S., Berry, M.B., Ho, E.L., Li, Q., Potter, J.D. \& Phillips, G.N. 1999, "Metal-ion affinity and specificity in EF-hand proteins: coordination geometry and domain plasticity in parvalbumin", Structure, vol. 7, no. 10, pp. 1269-1278.

Chattopadhyaya, R., Meador, W.E., Means, A.R. \& Quiocho, F.A. 1992, "Calmodulin structure refined at 1.7 A resolution", Journal of Molecular Biology, vol. 228, no. 4, pp. 1177-1192.

Chaudhuri, D., Horrocks, W.D.,Jr, Amburgey, J.C. \& Weber, D.J. 1997, "Characterization of lanthanide ion binding to the EF-hand protein S100 beta by luminescence spectroscopy", Biochemistry, vol. 36, no. 32, pp. 9674-9680.

Chen, Y.F., Chen, Y.T., Chiu, W.T. \& Shen, M.R. 2013, "Remodeling of calcium signaling in tumor progression", Journal of Biomedical Science, vol. 20, pp. 23-0127-20-23.

Cheng, H.M., Pitcher, G.M., Laviolette, S.R., Whishaw, I.Q., Tong, K.I., Kockeritz, L.K., Wada, T., Joza, N.A., Crackower, M., Goncalves, J., Sarosi, I., Woodgett, J.R., Oliveira-dos-Santos, A.J., Ikura, M., van, d.K., Salter, M.W. \& Penninger, J.M. "DREAM Is a Critical Transcriptional Repressor for Pain Modulation", Cell, vol. 108, no. 1, pp. 31-43.

Chin, D. \& Means, A.R. 2000, "Calmodulin: a prototypical calcium sensor", Trends in Cell Biology, vol. 10, no. 8, pp. 322-328. 
Choi, J., Hirota, N. \& Terazima, M. 2001, "A pH-jump reaction studied by the transient grating method: photodissociation of o-nitrobenzaldehyde", The Journal of Physical Chemistry A, vol. 105, no. 1, pp. 12-18.

Choi, E.K., Zaidi, N.F., Miller, J.S., Crowley, A.C., Merriam, D.E., Lilliehook, C., Buxbaum, J.D. \& Wasco, W. 2001, "Calsenilin is a substrate for caspase-3 that preferentially interacts with the familial Alzheimer's disease-associated C-terminal fragment of presenilin 2", The Journal of Biological Chemistry, vol. 276, no. 22, pp. 19197-19204.

Chuang, S., Velkov, T., Horne, J., Porter, C.J.H. \& Scanlon, M.J. 2008, "Characterization of the Drug Binding Specificity of Rat Liver Fatty Acid Binding Protein", Journal of Medicinal Chemistry, vol. 51, no. 13, pp. 3755-3764.

Ciesienski, K.L., Haas, K.L., Dickens, M.G., Tesema, Y.T. \& Franz, K.J. 2008, "A photolabile ligand for light-activated release of caged copper", Journal of the American Chemical Society, vol. 130, no. 37, pp. 12246-12247.

Clapham, D.E. 2007, "Calcium Signaling", Cell, vol. 131, no. 6, pp. 1047-1058.

Clayton, A.H. \& Sawyer, W.H. 1999, "Tryptophan rotamer distributions in amphipathic peptides at a lipid surface", Biophysical journal, vol. 76, no. 6, pp. 3235-3242.

Collins, J.H. 1974, "Homology of myosin light chains, troponin-C and parvalbumins deduced from comparison of their amino acid sequences", Biochemical and Biophysical Research Communications, vol. 58, no. 1, pp. 301-308.

Collins, J.H., Potter, J.D., Horn, M.J., Wilshire, G. \& Jackman, N. 1973, "The amino acid sequence of rabbit skeletal muscle troponin C: gene replication and homology with calcium-binding proteins from carp and hake muscle", FEBS letters, vol. 36, no. 3, pp. 268-272.

Cook, W.J., Jeffrey, L.C., Cox, J.A. \& Vijay-Kumar, S. 1993, "Structure of a Sarcoplasmic Calcium-binding Protein from Amphioxus Refined at 2. $4 \AA$ Resolution", Journal of Molecular Biology, vol. 229, no. 2, pp. 461-471.

Cook, W.J., Walter, L.J. \& Walter, M.R. 1994, "Drug Binding by Calmodulin: Crystal Structure of a Calmodulin-Trifluoperazine Complex", Biochemistry, vol. 33, no. 51, pp. 15259-15265.

Cordeiro, J.M., Calloe, K., Moise, N.S., Kornreich, B., Giannandrea, D., Di Diego, J.M., Olesen, S.P. \& Antzelevitch, C. 2012, "Physiological consequences of transient outward $\mathrm{K}+$ current activation during heart failure in the canine left ventricle", Journal of Molecular and Cellular Cardiology, vol. 52, no. 6, pp. 1291-1298. 
Cordes, T. \& Blum, S.A. 2013, "Opportunities and challenges in single-molecule and single-particle fluorescence microscopy for mechanistic studies of chemical reactions", Nature chemistry, vol. 5, no. 12, pp. 993-999.

Corneliussen, B., Holm, M., Waltersson, Y., Onions, J., Hallberg, B., Thornell, A. \& Grundström, T. 1994, "Calcium/calmodulin inhibition of basic-helix-loop-helix transcription factor domains", Nature, 368, 760 - 764.

Costigan, M. \& Woolf, C.J. 2002, "No DREAM, No Pain: Closing the Spinal Gate", Cell, vol. 108, no. 3, pp. 297-300.

Cox, J.A., Durussel, I., Comte, M., Nef, S., Nef, P., Lenz, S.E. \& Gundelfinger, E.D. 1994, "Cation binding and conformational changes in VILIP and NCS-1, two neuronspecific calcium-binding proteins", The Journal of Biological Chemistry, vol. 269, no. 52, pp. 32807-32813.

Craig, T.A., Benson, L.M., Venyaminov, S.Y., Klimtchuk, E.S., Bajzer, Z., Prendergast, F.G., Naylor, S. \& Kumar, R. 2002, "The Metal-binding Properties of DREAM", Journal of Biological Chemistry, vol. 277, no. 13, pp. 10955-10966.

Crivici, A. \& Ikura, M. 1995, "Molecular and Structural Basis of Target Recognition by Calmodulin", Annual Review of Biophysics and Biomolecular Structure, vol. 24, no. 1, pp. 85-116.

Cui, Q. \& Karplus, M. 2008, "Allostery and cooperativity revisited", Protein science, vol. 17, no. 8, pp. 1295-1307.

Cui, Y.Y., Liang, P. \& Wang, K.W. 2008, "Enhanced trafficking of tetrameric Kv4.3 channels by KChIP1 clamping", Neurochemical research, vol. 33, no. 10, pp. 20782084.

Cushman, S.J., Nanao, M.H., Jahng, A.W., DeRubeis, D., Choe, S. \& Pfaffinger, P.J. 2000, "Voltage dependent activation of potassium channels is coupled to T1 domain structure", Nature Structural and Molecular Biology, vol. 7, no. 5, pp. 403-407.

Cussler, E.L. 2009, Diffusion: mass transfer in fluid systems, Cambridge university press.

Da Silva, A.C., Kendrick-Jones, J. \& Reinach, F.C. 1995, "Determinants of ion specificity on EF-hands sites. Conversion of the $\mathrm{Ca}^{2+} / \mathrm{Mg}^{2+}$ site of smooth muscle myosin regulatory light chain into a $\mathrm{Ca}\left({ }^{2+}\right)$-specific site", The Journal of biological chemistry, vol. 270 , no. 12 , pp. 6773-6778.

Darré, L., Machado, M.R., Brandner, A.F., González, H.C., Ferreira, S. \& Pantano, S. 2015, "SIRAH: A structurally unbiased coarse-grained force field for proteins with 
aqueous solvation and long-range electrostatics", Journal of Chemical Theory and Computation, vol. 11, no. 2, pp. 723-739.

Declercq, J., Evrard, C., Lamzin, V. \& Parello, J. 1999, "Crystal structure of the EF-hand parvalbumin at atomic resolution (0.91 $\AA$ ) and at low temperature (100 K). Evidence for conformational multistates within the hydrophobic core", Protein Science, vol. 8, no. 10, pp. 2194-2204.

Deisseroth, K., Heist, E.K. \& Tsien, R.W. 1998, "Translocation of calmodulin to the nucleus supports CREB phosphorylation in hippocampal neurons", Nature, vol. 392, no. 6672, pp. 198-202.

Deiters, E., Song, B., Chauvin, A., Vandevyver, C., Gumy, F. \& Bünzli, J. 2009, "Luminescent Bimetallic Lanthanide Bioprobes for Cellular Imaging with Excitation in the Visible-Light Range", Chemistry - A European Journal, vol. 15, no. 4, pp. 885900 .

Deschênes, I., DiSilvestre, D., Juang, G.J., Wu, R.C., An, W.F. \& Tomaselli, G.F. 2002, "Regulation of Kv4.3 Current by KChIP2 Splice Variants: A Component of Native Cardiac Ito", Circulation, vol. 106, no. 4, pp. 423-429.

Dhulipala, G., Rubio, M., Michael, K. \& Mikšovská, J. 2009, "Thermodynamic profile for urea photo-release from a N-(2-nitrobenzyl) caged urea compound", Photochemical \& Photobiological Sciences, vol. 8, no. 8, pp. 1157-1163.

Diwu, Z., Lu, Y., Zhang, C., Klaubert, D.H. \& Haugland, R.P. 1997, "Fluorescent Molecular Probes II. The Synthesis, Spectral Properties and Use of Fluorescent Solvatochromic Dapoxyl Dyes", Photochemistry and Photobiology, vol. 66, no. 4, pp. 424-431.

Dolinsky, T.J., Nielsen, J.E., McCammon, J.A. \& Baker, N.A. 2004, "PDB2PQR: an automated pipeline for the setup of Poisson-Boltzmann electrostatics calculations", Nucleic acids research, vol. 32, no. Web Server issue, pp. W665-7.

Drabikowski, W., Brzeska, H. \& Venyaminov SYu 1982, "Tryptic fragments of calmodulin. $\mathrm{Ca}^{2+}$ - and $\mathrm{Mg}^{2+}$-induced conformational changes.", The Journal of Biological Chemistry, vol. 257, no. 19, pp. 11584-11590.

Dweck, D., Reyes-Alfonso Jr., A. \& Potter, J.D. 2005, "Expanding the range of free calcium regulation in biological solutions", Analytical Biochemistry, vol. 347, no. 2, pp. 303-315.

Einstein, A. 1905, "Über die von der molekularkinetischen Theorie der Wärme geforderte Bewegung von in ruhenden Flüssigkeiten suspendierten Teilchen", Annalen der Physik, vol. 322, no. 8, pp. 549-560. 
Ellis-Davies, G.C. 2007, "Caged compounds: photorelease technology for control of cellular chemistry and physiology", Nature methods, vol. 4, no. 8, pp. 619-628.

Epps, D.E., Raub, T.J. \& Kezdy, F.J. 1995, "A General, Wide-Range Spectrofluorometric Method for Measuring the Site-Specific Affinities of Drugs Toward Human Serum Albumin", Analytical Biochemistry, vol. 227, no. 2, pp. 342-350.

Essmann, U., Perera, L., Berkowitz, M.L., Darden, T., Lee, H. \& Pedersen, L.G. 1995, "A smooth particle mesh Ewald method", The Journal of Chemical Physics, vol. 103, no. 19, pp. 8577-8593.

Evenäs, J., Malmendal, A. \& Akke, M. 2001, "Dynamics of the transition between open and closed conformations in a calmodulin C-terminal domain mutant", Structure, vol. 9, no. 3, pp. 185-195.

Eyring, H. 1935, "The Activated Complex in Chemical Reactions", The Journal of Chemical Physics, vol. 3, no. 2, pp. 107-115.

Falvey, D.E. 1997, "Photothermal Beam Deflection Calorimetry in Solution Photochemistry: Recent Progress and Future Prospects", Photochemistry and Photobiology, vol. 65, no. 1, pp. 4-9.

Fayer, M. 1982, "Dynamics of molecules in condensed phases: picosecond holographic grating experiments", Annual Review of Physical Chemistry, vol. 33, no. 1, pp. 63-87.

Fernandez-Lima, F., Kaplan, D. \& Park, M. 2011, "Note: Integration of trapped ion mobility spectrometry with mass spectrometry", Review of Scientific Instruments, vol. 82, no. 12, pp. 126106.

Fernandez-Lima, F., Kaplan, D.A., Suetering, J. \& Park, M.A. 2011, "Gas-phase separation using a trapped ion mobility spectrometer", International Journal for Ion Mobility Spectrometry, vol. 14, no. 2-3, pp. 93-98.

Feynman, R.P., Leighton, R.B. \& Sands, M. 1979, The Feynman lectures on physics, vol. 2: Mainly electromagnetism and matter, Addison-Wesley.

Fisher, J.R., Sharma, Y., Iuliano, S., Piccioti, R.A., Krylov, D., Hurley, J., Roder, J. \& Jeromin, A. 2000, "Purification of Myristoylated and Nonmyristoylated Neuronal Calcium Sensor-1 Using Single-Step Hydrophobic Interaction Chromatography", Protein Expression and Purification, vol. 20, no. 1, pp. 66-72.

Flaherty, K.M., Zozulya, S., Stryer, L. \& McKay, D.B. 1993, "Three-dimensional structure of recoverin, a calcium sensor in vision", Cell, vol. 75, no. 4, pp. 709-716. 
Foeger, N.C., Marionneau, C. \& Nerbonne, J.M. 2010, "Co-assembly of Kv4 a Subunits with K+ Channel-interacting Protein 2 Stabilizes Protein Expression and Promotes Surface Retention of Channel Complexes", The Journal of Biological Chemistry, vol. 285, no. 43, pp. 33413-33422.

Fontán-Lozano, Á., Romero-Granados, R., del-Pozo-Martín, Y., Suárez-Pereira, I., Delgado-García, J.M., Penninger, J.M. \& Carrión, Á.M. 2009, "Lack of DREAM Protein Enhances Learning and Memory and Slows Brain Aging", Current Biology, vol. 19, no. 1, pp. 54-60.

Gao, J., Mammen, M. \& Whitesides, G.M. 1996, "Evaluating Electrostatic Contributions to Binding with the Use of Protein Charge Ladders", Science, vol. 272, no. 5261, pp. 535-537.

Gasymov, O.K. \& Glasgow, B.J. 2007, "ANS fluorescence: Potential to augment the identification of the external binding sites of proteins", Biochimica et Biophysica Acta (BBA) - Proteins \& Proteomics, vol. 1774, no. 3, pp. 403-411.

Gebauer, M., Isbrandt, D., Sauter, K., Callsen, B., Nolting, A., Pongs, O. \& Bähring, R. 2004, "N-type Inactivation Features of Kv4.2 Channel Gating", Biophysical journal, vol. 86, no. 1, pp. 210-223.

George, S.E., Su, Z., Fan, D. \& Means, A.R. 1993, "Calmodulin-cardiac troponin C chimeras. Effects of domain exchange on calcium binding and enzyme activation", The Journal of biological chemistry, vol. 268, no. 33, pp. 25213-25220.

Ghosh, K.K., Burns, L.D., Cocker, E.D., Nimmerjahn, A., Ziv, Y., Gamal, A.E. \& Schnitzer, M.J. 2011, "Miniaturized integration of a fluorescence microscope", Nature Methods, vol. 8, no. 10, pp. 871-878.

Gifford, J., Walsh, M. \& Vogel, H. 2007a, "Structures and metal-ion-binding properties of the Ca2 -binding helix-loop-helix EF-hand motifs", Biochemistry Journal, vol. 405, pp. 199-221.

Gifford, J.L., Walsh, M.P. \& Vogel, H.J. 2007b, "Structures and metal-ion-binding properties of the $\mathrm{Ca}^{2+}$-binding helix-loop-helix EF-hand motifs", The Biochemical Journal, vol. 405, no. 2, pp. 199-221.

Gilli, R., Lafitte, D., Lopez, C., Kilhoffer, M., Makarov, A., Briand, C. \& Haiech, J. 1998, "Thermodynamic analysis of calcium and magnesium binding to calmodulin", Biochemistry, vol. 37, no. 16, pp. 5450-5456.

Girvan, M. \& Newman, M.E.J. 2002, "Community structure in social and biological networks", Proceedings of the National Academy of Sciences, vol. 99, no. 12, pp. 7821-7826. 
Gonzalez, W.G., Arango, A.S. \& Miksovska, J. 2015, "Amphiphilic Residues 29-44 of DREAM N-Termini Mediate Calmodulin:DREAM Complex Formation", Biochemistry, vol. 54, no. 28, pp. 4391-4403.

Gonzalez, W.G. \& Miksovska, J. 2014, "Submillisecond conformational changes in proteins resolved by photothermal beam deflection", Journal of Visualized experiments : JoVE, vol. (84):e50969. doi, no. 84, pp. e50969.

Gonzalez, W.G., Pham, K. \& Miksovska, J. 2014, "Modulation of the Voltage-gated Potassium Channel (Kv4.3) and the Auxiliary Protein (KChIP3) Interactions by the Current Activator NS5806", The Journal of Biological Chemistry, vol. 289, no. 46, pp. 32201-32213.

Gonzalez, W.G. \& Miksovska, J. 2014, "Application of ANS fluorescent probes to identify hydrophobic sites on the surface of DREAM", Biochimica et Biophysica Acta (BBA) - Proteins and Proteomics, vol. 1844, no. 9, pp. 1472-1480.

Gonzalez, W. \& Miksovska, J. 2013, "Modulation of the KV4.3-KChIP3 Interactions by $\mathrm{Ca}^{2+}$ and NS5806", Biophysical journal, vol. 104, no. 2, pp. 102a.

Gonzalez, W.G., Ramos, V., Diaz, M., Garabedian, A., Molano-Arevalo, J.C., FernandezLima, F. \& Miksovska, J. 2016, "Characterization of the Photophysical, Thermodynamic, and Structural Properties of the Terbium(III)-DREAM Complex", Biochemistry, vol. 55, no. 12, pp. 1873-1886.

Gotoh, H., Kajikawa, M., Kato, H. \& Suto, K. 1999, "Intracellular $\mathrm{Mg}^{+2}$ surge follows Ca ${ }^{+2}$ increase during depolarization in cultured neurons", Brain research, vol. 828, no. 1, pp. 163-168.

Grabarek, Z. 2006, "Structural basis for diversity of the EF-hand calcium-binding proteins", Journal of Molecular Biology, vol. 359, no. 3, pp. 509-525.

Grabarek, Z. 2005, "Structure of a trapped intermediate of calmodulin: calcium regulation of EF-hand proteins from a new perspective", Journal of Molecular Biology, vol. 346, no. 5, pp. 1351-1366.

Griko, Y.V. 1999, "Energetics of $\mathrm{Ca}^{2+}$-EDTA interactions: calorimetric study", Biophysical Chemistry, vol. 79, no. 2, pp. 117-127.

Gurney, A.M. 1994, "Flash photolysis of caged compounds", Microelectrode Techniques, pp. 389-406. 
Hagan, A.K. \& Zuchner, T. 2011, "Lanthanide-based time-resolved luminescence immunoassays", Analytical and Bioanalytical Chemistry, vol. 400, no. 9, pp. 28472864.

Hamelberg, D., Mongan, J. \& McCammon, J.A. 2004, "Accelerated molecular dynamics: a promising and efficient simulation method for biomolecules", The Journal of Chemical Physics, vol. 120, no. 24, pp. 11919-11929.

Hart, R.C., Bates, M.D., Cormier, M.J., Rosen, G.M. \& Conn, P.M. 1983, "Synthesis and characterization of calmodulin antagonistic drugs", Methods in enzymology, vol. 102, pp. 195-204.

Hawe, A., Sutter, M. \& Jiskoot, W. 2008, "Extrinsic fluorescent dyes as tools for protein characterization", Pharmaceutical Research, vol. 25, no. 7, pp. 1487-1499.

Heidarsson, P., Otazo, M., Bellucci, L., Mossa, A., Imparato, A., Paci, E., Corni, S., Di Felice, R., Kragelund, B. \& Cecconi, C. 2013, "Single-Molecule Folding Mechanism of an EF-Hand Neuronal Calcium Sensor", Structure, vol. 21, no. 10, pp. 1812-1821.

Heidorn, D.B. \& Trewhella, J. 1988, "Comparison of the crystal and solution structures of calmodulin and troponin C", Biochemistry, vol. 27, no. 3, pp. 909-915.

Henzl, M.T., Hapak, R.C. \& Likos, J.J. 1998, "Interconversion of the ligand arrays in the $\mathrm{CD}$ and EF sites of oncomodulin. Influence on $\mathrm{Ca}^{2+}$-binding affinity", Biochemistry, vol. 37, no. 25, pp. 9101-9111.

Hernandez, D.R., DeBord, J.D., Ridgeway, M.E., Kaplan, D.A., Park, M.A. \& FernandezLima, F. 2014, "Ion dynamics in a trapped ion mobility spectrometer", Analyst, vol. 139, no. 8, pp. 1913-1921.

Hess, B., Kutzner, C., Van Der Spoel, D. \& Lindahl, E. 2008, "GROMACS 4: algorithms for highly efficient, load-balanced, and scalable molecular simulation", Journal of Chemical Theory and Computation, vol. 4, no. 3, pp. 435-447.

Hodgkin, A.L. \& Huxley, A.F. 1952, "A quantitative description of membrane current and its application to conduction and excitation in nerve", The Journal of Physiology, vol. 117, no. 4, pp. 500-544.

Hogue, C.W., MacManus, J.P., Banville, D. \& Szabo, A.G. 1992, "Comparison of terbium (III) luminescence enhancement in mutants of EF hand calcium binding proteins", The Journal of Biological Chemistry, vol. 267, no. 19, pp. 13340-13347.

Holmqvist, M.H., Cao, J., Knoppers, M.H., Jurman, M.E., Distefano, P.S., Rhodes, K.J., Xie, Y. \& An, W.F. 2001, "Kinetic modulation of Kv4-mediated A-current by 
arachidonic acid is dependent on potassium channel interacting proteins", The Journal of Neuroscience : the official journal of the Society for Neuroscience, vol. 21, no. 12, pp. 4154-4161.

Holmqvist, M.H., Cao, J., Hernandez-Pineda, R., Jacobson, M.D., Carroll, K.I., Sung, M.A., Betty, M., Ge, P., Gilbride, K.J., Brown, M.E., Jurman, M.E., Lawson, D., Silos-Santiago, I., Xie, Y., Covarrubias, M., Rhodes, K.J., Distefano, P.S. \& An, W.F. 2002, "Elimination of fast inactivation in Kv4 A-type potassium channels by an auxiliary subunit domain", Proceedings of the National Academy of Sciences, vol. 99, no. 2, pp. 1035-1040.

Horrocks, W.D. \& Sudnick, D.R. 1979, "Lanthanide ion probes of structure in biology. Laser-induced luminescence decay constants provide a direct measure of the number of metal-coordinated water molecules", Journal of the American Chemical Society, vol. 101, no. 2, pp. 334-340.

Hu, K., Carroll, J., Fedorovich, S., Rickman, C., Sukhodub, A. \& Davletov, B. 2002, "Vesicular restriction of synaptobrevin suggests a role for calcium in membrane fusion", Nature, vol. 415, no. 6872, pp. 646-650.

Hudson, E.N. \& Weber, G. 1973a, "Synthesis and characterization of two fluorescent sulfhydryl reagents", Biochemistry, vol. 12, no. 21, pp. 4154-4161.

Hudson, E.N. \& Weber, G. 1973b, "Synthesis and characterization of two fluorescent sulfhydryl reagents", Biochemistry, vol. 12, no. 21, pp. 4154-4161.

Hughes, R.E., Hurley, J.B., Brzovic, P.S., Dizhoor, A.M. \& Klevit, R.E. 1998, "Ca2 dependent conformational changes in bovine GCAP-2", Protein Science, vol. 7, no. 12, pp. 2675-2680.

Hughes, R.E., Brzovic, P.S., Klevit, R.E. \& Hurley, J.B. 1995, "Calcium-dependent solvation of the myristoyl group of recoverin", Biochemistry, vol. 34, no. 36, pp. 11410-11416.

Humphrey, W., Dalke, A. \& Schulten, K. 1996, "VMD: Visual molecular dynamics", Journal of Molecular Graphics, vol. 14, no. 1, pp. 33-38.

Hung, H., Chen, Y., Liu, G., Lee, H. \& Chang, G. 2003, "Equilibrium protein foldingunfolding process involving multiple intermediates", Bulletin of Mathematical Biology, vol. 65, no. 4, pp. 553-570.

Hungerford, G., Hussain, F., Patzke, G.R. \& Green, M. 2010, "The photophysics of europium and terbium polyoxometalates and their interaction with serum albumin: a time-resolved luminescence study", Physical Chemistry Chemical Physics, vol. 12, no. 26, pp. 7266-7275. 
Ikura, M. 1996, "Calcium binding and conformational response in EF-hand proteins", Trends in biochemical sciences, vol. 21, no. 1, pp. 14-17.

Jackson, W.B., Amer, N.M., Boccara, A. \& Fournier, D. 1981a, "Photothermal deflection spectroscopy and detection", Applied Optics, vol. 20, no. 8, pp. 1333-1344.

Jackson, W.B., Amer, N.M., Boccara, A.C. \& Fournier, D. 1981b, "Photothermal deflection spectroscopy and detection", Applied Optics, vol. 20, no. 8, pp. 1333-1344.

Jahn, R. \& Scheller, R.H. 2006, "SNAREs-engines for membrane fusion", Nature Reviews Molecular Cell Biology, vol. 7, no. 9, pp. 631-643.

James, N.G., Ross, J.A., Štefl, M. \& Jameson, D.M. 2011, "Applications of phasor plots to in vitro protein studies", Analytical Biochemistry, vol. 410, no. 1, pp. 70-76.

Jardetzky, O. 1996, "Protein dynamics and conformational transitions in allosteric proteins", Progress in Biophysics and Molecular Biology, vol. 65, no. 3, pp. 171-219.

Jelesarov, I. \& Bosshard, H.R. 1999, "Isothermal titration calorimetry and differential scanning calorimetry as complementary tools to investigate the energetics of biomolecular recognition", Journal of Molecular Recognition : JMR, vol. 12, no. 1, pp. 3-18.

Jerng, H.H. \& Covarrubias, M. 1997, " $\mathrm{K}^{+}$channel inactivation mediated by the concerted action of the cytoplasmic N- and C-terminal domains", Biophysical Journal, vol. 72, no. 1, pp. 163-174.

Jerng, H.H., Shahidullah, M. \& Covarrubias, M. 1999, "Inactivation gating of Kv4 potassium channels: molecular interactions involving the inner vestibule of the pore", The Journal of General Physiology, vol. 113, no. 5, pp. 641-660.

Jerng, H.H. \& Pfaffinger, P.J. 2008, "Multiple Kv Channel-interacting Proteins Contain an N-terminal Transmembrane Domain That Regulates Kv4 Channel Trafficking and Gating", Journal of Biological Chemistry, vol. 283, no. 51, pp. 36046-36059.

Jordán, J., Galindo, M.F., Calvo, S., González-García, C. \& Ceña, V. 2000, "Veratridine induces apoptotic death in bovine chromaffin cells through superoxide production", British Journal of Pharmacology, vol. 130, no. 7, pp. 1496-1504.

Kääb, S., Dixon, J., Duc, J., Ashen, D., Näbauer, M., Beuckelmann, D.J., Steinbeck, G., McKinnon, D. \& Tomaselli, G.F. 1998, "Molecular Basis of Transient Outward Potassium Current Downregulation in Human Heart Failure: A Decrease in Kv4.3 mRNA Correlates With a Reduction in Current Density", Circulation, vol. 98, no. 14, pp. 1383-1393. 
Kaplan, J. \& Somlyo, A. 1989, "Flash photolysis of caged compounds: new tools for cellular physiology", Trends in neurosciences, vol. 12, no. 2, pp. 54-59.

Kaplan, J.H. \& Ellis-Davies, G.C. 1988, "Photolabile chelators for the rapid photorelease of divalent cations", Proceedings of the National Academy of Sciences, vol. 85, no. 17, pp. 6571-6575.

Kasturi, R., Vasulka, C. \& Johnson, J.D. 1993, "Ca ${ }^{2+}$, caldesmon, and myosin light chain kinase exchange with calmodulin.", The Journal of Biological Chemistry, vol. 268, no. 11, pp. 7958-7964.

Kekenes-Huskey, P.M., Metzger, V.T., Grant, B.J. \& Andrew McCammon, J. 2012, "Calcium binding and allosteric signaling mechanisms for the sarcoplasmic reticulum Ca2 ATPase", Protein Science, vol. 21, no. 10, pp. 1429-1443.

Kelly, S.M., Jess, T.J. \& Price, N.C. 2005a, "How to study proteins by circular dichroism", Biochimica et Biophysica Acta (BBA) - Proteins and Proteomics, vol. 1751, no. 2, pp. 119-139.

Kelly, S.M., Jess, T.J. \& Price, N.C. 2005b, "How to study proteins by circular dichroism", Biochimica et Biophysica Acta (BBA) - Proteins and Proteomics, vol. 1751, no. 2, pp. 119-139.

Kern, D. \& Zuiderweg, E.R. 2003, "The role of dynamics in allosteric regulation", Current opinion in structural biology, vol. 13, no. 6, pp. 748-757.

Kilhoffer, M., Gerard, D. \& Demaille, J.G. 1980, "Terbium binding to octopus calmodulin provides the complete sequence of ion binding", FEBS letters, vol. 120, no. 1, pp. 99103.

Kleinerman, M. 1969, "Energy Migration in Lanthanide Chelates", The Journal of chemical physics, vol. 51, no. 6, pp. 2370-2381.

Kojetin, D.J., Venters, R.A., Kordys, D.R., Thompson, R.J., Kumar, R. \& Cavanagh, J. 2006, "Structure, binding interface and hydrophobic transitions of Ca2 -loaded calbindin-D28K", Nature structural \& molecular biology, vol. 13, no. 7, pp. 641-647.

Kondoh, M., Baden, N. \& Terazima, M. 2008, "Photochemical reaction and diffusion of caged calcium studied by the transient grating", Chemical Physics Letters, vol. 462, no. 1 , pp. 58-63.

Korson, L., Drost-Hansen, W. \& Millero, F.J. 1969, "Viscosity of water at various temperatures", The Journal of physical chemistry, vol. 73, no. 1, pp. 34-39. 
Koshland Jr, D., Nemethy, G. \& Filmer, D. 1966, "Comparison of experimental binding data and theoretical models in proteins containing subunits", Biochemistry, vol. 5, no. 1, pp. 365-385.

Kosower, E.M. \& Kanety, H. 1983, "Intramolecular donor-acceptor systems. 10. Multiple fluorescences from 8-(N-phenylamino)-1-naphthalenesulfonates", Journal of the American Chemical Society, vol. 105, no. 20, pp. 6236-6243.

Kretsinger, R.H. \& Nockolds, C.E. 1973, "Carp muscle calcium-binding protein. II. Structure determination and general description", The Journal of biological chemistry, vol. 248, no. 9, pp. 3313-3326.

Krieger, E., Koraimann, G. \& Vriend, G. 2002, "Increasing the precision of comparative models with YASARA NOVA?a self-parameterizing force field", Proteins: Structure, Function, and Bioinformatics, vol. 47, no. 3, pp. 393-402.

Kuboniwa, H., Tjandra, N., Grzesiek, S., Ren, H., Klee, C.B. \& Bax, A. 1995, "Solution structure of calcium-free calmodulin", Nature Structural Biology, vol. 2, no. 9, pp. 768-776.

Kunjilwar, K., Qian, Y. \& Pfaffinger, P.J. 2013a, "Functional stoichiometry underlying KChIP regulation of Kv4.2 functional expression", Journal of Neurochemistry, vol. 126, no. 4, pp. 462-472.

Kunjilwar, K., Qian, Y. \& Pfaffinger, P.J. 2013b, "Functional stoichiometry underlying KChIP regulation of Kv4.2 functional expression", Journal of Neurochemistry, vol. 126, no. 4, pp. 462-472.

Kuroki, R. \& Yutani, K. 1998, "Structural and Thermodynamic Responses of Mutations at a $\mathrm{Ca}^{2+}$ Binding Site Engineered into Human Lysozyme", Journal of Biological Chemistry, vol. 273, no. 51, pp. 34310-34315.

Kuster, T., Staudenmann, W., Hughes, G.J. \& Heizmann, C.W. 1991, "Parvalbumin isoforms in chicken muscle and thymus. Amino acid sequence analysis of muscle parvalbumin by tandem mass spectrometry", Biochemistry, vol. 30, no. 36, pp. 88128816.

Ladant, D. 1995, "Calcium and membrane binding properties of bovine neurocalcin delta expressed in Escherichia coli", The Journal of Biological Chemistry, vol. 270, no. 7, pp. 3179-3185.

Laires, M.J., Monteiro, C.P. \& Bicho, M. 2004, "Role of cellular magnesium in health and human disease", Frontiers in Bioscience, vol. 9, no. 262, pp. 76. 
Lakowicz, J.R. 2010, Principles of Fluorescence Spectroscopy, 3rd ed., Springer, New York, NY USA.

Lakowicz, J.R., Cherek, H., Kuśba, J., Gryczynski, I. \& Johnson, M.L. 1993, "Review of fluorescence anisotropy decay analysis by frequency-domain fluorescence spectroscopy", Journal of Fluorescence, vol. 3, no. 2, pp. 103-116.

Laman, D.M. \& Falvey, D.E. 1996, "A photothermal beam deflection apparatus for the time-resolved kinetic study of fast photophysical and photochemical processes", Review of Scientific Instruments, vol. 67, no. 9, pp. 3260-3269.

Lang, F., Foller, M., Lang, K., Lang, P., Ritter, M., Vereninov, A., Szabo, I., Huber, S.M. \& Gulbins, E. 2007, "Cell volume regulatory ion channels in cell proliferation and cell death", Methods in Enzymology, vol. 428, pp. 209-225.

Larriba, C. \& Hogan Jr, C.J. 2013, "Ion mobilities in diatomic gases: measurement versus prediction with non-specular scattering models", The Journal of Physical Chemistry A, vol. 117, no. 19, pp. 3887-3901.

Larriba, C. \& Hogan, C.J. 2013, "Free molecular collision cross section calculation methods for nanoparticles and complex ions with energy accommodation", Journal of Computational Physics, vol. 251, pp. 344-363.

Larsen, R.W. \& Mikšovská, J. 2007, "Time resolved thermodynamics of ligand binding to heme proteins", Coordination Chemistry Reviews, vol. 251, no. 9-10, pp. 1101-1127.

Ledo, F., Carrion, A.M., Link, W.A., Mellstrom, B. \& Naranjo, J.R. 2000, "DREAMalphaCREM interaction via leucine-charged domains derepresses downstream regulatory element-dependent transcription", Molecular and Cellular Biology, vol. 20, no. 24, pp. 9120-9126.

Ledo, F., Kremer, L., Mellstrom, B. \& Naranjo, J.R. 2002, "Ca ${ }^{2+}$-dependent block of CREB-CBP transcription by repressor DREAM", The EMBO journal, vol. 21, no. 17, pp. 4583-4592.

Ledo, F., Link, W.A., Carrión, A.M., Echeverria, V., Mellström, B. \& Naranjo, J.R. 2000, "The DREAM-DRE interaction: key nucleotides and dominant negative mutants", Biochimica et Biophysica Acta (BBA) - Molecular Cell Research, vol. 1498, no. 2-3, pp. 162-168.

Lee, Y., Tanner, J.J., Larson, J.D. \& Henzl, M.T. 2004, "Crystal structure of a high-affinity variant of rat $\alpha$-parvalbumin", Biochemistry, vol. 43, no. 31, pp. 10008-10017. 
Lee, J.Y., Okumus, B., Kim, D.S. \& Ha, T. 2005, "Extreme conformational diversity in human telomeric DNA", Proceedings of the National Academy of Sciences of the United States of America, vol. 102, no. 52, pp. 18938-18943.

LeoÌ

Bohne, C., Boulanger, M.J. \& Hof, F. 2009, "Identification and Characterization of Binding Sites on S100A7, a Participant in Cancer and Inflammation Pathways", Biochemistry, vol. 48, no. 44, pp. 10591-10600.

León, R., Murray, J.I., Cragg, G., Farnell, B., West, N.R., Pace, T.C., Watson, P.H., Bohne, C., Boulanger, M.J. \& Hof, F. 2009, "Identification and characterization of binding sites on S100A7, a participant in cancer and inflammation pathways", Biochemistry, vol. 48, no. 44, pp. 10591-10600.

Levin, R.M. \& Weiss, B. 1977, "Binding of Trifluoperazine to the Calcium-Dependent Activator of Cyclic Nucleotide Phosphodiesterase", Molecular Pharmacology, vol. 13, no. 4, pp. 690-697.

Li, C., Pan, W., Braunewell, K.H. \& Ames, J.B. 2011, "Structural Analysis of Mg2+ and Ca2+ Binding, Myristoylation, and Dimerization of the Neuronal Calcium Sensor and Visinin-like Protein 1 (VILIP-1)", Journal of Biological Chemistry, vol. 286, no. 8, pp. 6354-6366.

Li, M., Lei, L., Jia, L., Ling, X., Zhang, J., Zhao, Y. \& Wang, K. 2014, "Interactions of KChIP4a and its mutants with Ca2+ or Kv4.3 N-terminus by affinity capillary electrophoresis", Analytical Biochemistry, vol. 449, no. 0, pp. 99-105.

Lian, L., Pandalaneni, S.R., Patel, P., McCue, H.V., Haynes, L.P., \& Burgoyne, R.D., 2011, "Characterisation of the Interaction of the C-Terminus of the Dopamine D2 Receptor with Neuronal Calcium Sensor-1", PLoS ONE, vol. 6, no. 11, pp. e27779.

Liang, P., Wang, H., Chen, H., Cui, Y., Gu, L., Chai, J. \& Wang, K. 2009, "Structural Insights into KChIP4a Modulation of Kv4.3 Inactivation", Journal of Biological Chemistry, vol. 284, no. 8, pp. 4960-4967.

Liao, Y.S., Chen, K.C. \& Chang, L.S. 2009, "Functional role of EF-hands 3 and 4 in membrane-binding of KChIP1", Journal of Biosciences, vol. 34, no. 2, pp. 203-211.

Linse, S., Brodin, P., Drakenberg, T., Thulin, E., Sellers, P., Elmden, K., Grundstroem, T. \& Forsen, S. 1987, "Structure-function relationships in EF-hand calcium-binding proteins. Protein engineering and biophysical studies of calbindin D9k", Biochemistry, vol. 26, no. 21, pp. 6723-6735.

Linse, S. \& Forsen, S. 1995, "Determinants that govern high-affinity calcium binding", Advances in Second Messenger and Phosphoprotein Research, vol. 30, pp. 89-151. 
Liss, B., Franz, O., Sewing, S., Bruns, R., Neuhoff, H. \& Roeper, J. 2001, "Tuning pacemaker frequency of individual dopaminergic neurons by Kv4.3L and KChip3.1 transcription", The EMBO journal, vol. 20, no. 20, pp. 5715-5724.

Liu, P., Huang, X., Zhou, R. \& Berne, B.J. 2005, "Observation of a dewetting transition in the collapse of the melittin tetramer", Nature, vol. 437, no. 7055, pp. 159-162.

Lundby, A., Jespersen, T., Schmitt, N., Grunnet, M., Olesen, S.P., Cordeiro, J.M. \& Calloe, K. 2010, "Effect of the I(to) activator NS5806 on cloned K(V)4 channels depends on the accessory protein KChIP2", British Journal of Pharmacology, vol. 160, no. 8, pp. 2028-2044.

Lusin, J.D., Vanarotti, M., Li, C., Valiveti, A. \& Ames, J.B. 2008, "NMR Structure of DREAM: Implications for $\mathrm{Ca}^{2+}$-Dependent DNA Binding and Protein Dimerization", Biochemistry, vol. 47, no. 8, pp. 2252-2264.

MacKerell, A.D., Feig, M. \& Brooks, C.L. 2004, "Extending the treatment of backbone energetics in protein force fields: Limitations of gas-phase quantum mechanics in reproducing protein conformational distributions in molecular dynamics simulations", Journal of Computational Chemistry, vol. 25, no. 11, pp. 1400-1415.

Markowitz, J.E., Liberti, W.A.,3rd, Guitchounts, G., Velho, T., Lois, C. \& Gardner, T.J. 2015, "Mesoscopic patterns of neural activity support songbird cortical sequences", PLoS biology, vol. 13, no. 6, pp. e1002158.

Marrink, S.J., Risselada, H.J., Yefimov, S., Tieleman, D.P. \& de Vries, A.H. 2007, "The MARTINI Force Field:â€\%o Coarse Grained Model for Biomolecular Simulations", The Journal of Physical Chemistry B, vol. 111, no. 27, pp. 7812-7824.

Martens, J.R., Kwak, Y. \& Tamkun, M.M. 1999, "Modulation of Kv Channel $\alpha / \beta$ Subunit Interactions", Trends in Cardiovascular Medicine, vol. 9, no. 8, pp. 253-258.

Martin, B. \& Richardson, F.S. 1979, "Lanthanides as probes for calcium in biological systems", Quarterly Reviews of Biophysics, vol. 12, no. 02, pp. 181-209.

Martin, M.P., Alam, R., Betzi, S., Ingles, D.J., Zhu, J.Y. \& Schonbrunn, E. 2012, "A novel approach to the discovery of small-molecule ligands of CDK2", Chembiochem : a European journal of chemical biology, vol. 13, no. 14, pp. 2128-2136.

Mason, P.E., Dempsey, C.E., Neilson, G.W., Kline, S.R. \& Brady, J.W. 2009, "Preferential interactions of guanidinum ions with aromatic groups over aliphatic groups", Journal of the American Chemical Society, vol. 131, no. 46, pp. 16689-16696. 
McCue, H.V., Haynes, L.P. \& Burgoyne, R.D. 2010, "The diversity of calcium sensor proteins in the regulation of neuronal function", Cold Spring Harbor perspectives in biology, vol. 2, no. 8, pp. a004085.

McDaniel, E.W. \& Mason, E.A. 1973, "Mobility and diffusion of ions in gases", .

Miao, Y., Feixas, F., Eun, C. \& McCammon, J.A. 2015, "Accelerated molecular dynamics simulations of protein folding", Journal of computational chemistry, vol. 36, no. 20, pp. 1536-1549.

Mikšovská, J., Day, J.H. \& Larsen, R.W. 2003, "Volume and enthalpy profiles of CO rebinding to horse heart myoglobin", Journal of Biological Inorganic Chemistry, vol. 8, no. 6, pp. 621-625.

Millero, F.J. 1971, "Molal volumes of electrolytes", Chemical reviews, vol. 71, no. 2, pp. $147-176$.

Moews, P.C. \& Kretsinger, R.H. 1975, "Refinement of the structure of carp muscle calcium-binding parvalbumin by model building and difference fourier analysis", Journal of Molecular Biology, vol. 91, no. 2, pp. 201-225.

Molano-Arevalo, J.C., Hernandez, D.R., Gonzalez, W.G., Miksovska, J., Ridgeway, M.E., Park, M.A. \& Fernandez-Lima, F. 2014, "Flavin adenine dinucleotide structural motifs: from solution to gas phase", Analytical Chemistry, vol. 86, no. 20, pp. 1022310230.

Monera, O.D., Kay, C.M. \& Hodges, R.S. 1994, "Protein denaturation with guanidine hydrochloride or urea provides a different estimate of stability depending on the contributions of electrostatic interactions", Protein Science, vol. 3, no. 11, pp. 19841991.

Monod, J., Wyman, J. \& Changeux, J. 1965, "On the nature of allosteric transitions: a plausible model", Journal of Molecular Biology, vol. 12, no. 1, pp. 88-118.

Morris, G.M., Goodsell, D.S., Halliday, R.S., Huey, R., Hart, W.E., Belew, R.K. \& Olson, A.J. 1998, "Automated docking using a Lamarckian genetic algorithm and an empirical binding free energy function", Journal of Computational Chemistry, vol. 19, no. 14, pp. 1639-1662.

Mulqueen, P., Tingey, J.M. \& Horrocks, W.D. 1985, "Characterization of lanthanide(III) ion binding to calmodulin using luminescence spectroscopy", Biochemistry, vol. 24, no. 23, pp. 6639-6645.

Mustafi, S.M., Mukherjee, S., Chary, K.V.R., Del Bianco, C. \& Luchinat, C. 2004, "Energetics and Mechanism of $\mathrm{Ca}^{2+}$ Displacement by Lanthanides in a Calcium Binding Proteinâ€ ", Biochemistry, vol. 43, no. 29, pp. 9320-9331. 
Nadal, M.S., Ozaita, A., Amarillo, Y., Vega-Saenz de Miera, E., Ma, Y., Mo, W., Goldberg, E.M., Misumi, Y., Ikehara, Y., Neubert, T.A. \& Rudy, B. 2003, "The CD26related dipeptidyl aminopeptidase-like protein DPPX is a critical component of neuronal A-type $\mathrm{K}^{+}$channels", Neuron, vol. 37, no. 3, pp. 449-461.

Nagai, M., Nagatomo, S., Nagai, Y., Ohkubo, K., Imai, K. \& Kitagawa, T. 2012, "NearUV Circular Dichroism and UV Resonance Raman Spectra of Individual Tryptophan Residues in Human Hemoglobin and Their Changes upon the Quaternary Structure Transition", Biochemistry, vol. 51, no. 30, pp. 5932-5941.

Nalefski, E.A. \& Falke, J.J. 1996, "The C2 domain calcium-binding motif: structural and functional diversity", Protein science : a publication of the Protein Society, vol. 5, no. 12, pp. 2375-2390.

Naraghi, M. \& Neher, E. 1997, "Linearized buffered $\mathrm{Ca}^{2+}$ diffusion in microdomains and its implications for calculation of [Ca2+] at the mouth of a calcium channel", The Journal of Neuroscience, vol. 17, no. 18, pp. 6961-6973.

Nelson, M.R. \& Chazin, W.J. 1998, "An interaction-based analysis of calcium-induced conformational changes in Ca2+ sensor proteins", Protein Science, vol. 7, no. 2, pp. 270-282.

Niwa, N. \& Nerbonne, J.M. 2010, "Molecular determinants of cardiac transient outward potassium current (Ito) expression and regulation", Journal of Molecular and Cellular Cardiology, vol. 48, no. 1, pp. 12-25.

Nystedt, J.M., Brandt, A., Vilim, F.S., Ziff, E.B. \& Panula, P. 2006, "Identification of transcriptional regulators of neuropeptide FF gene expression", Peptides, vol. 27, no. 5, pp. 1020-1035.

Obradovic, Z., Peng, K., Vucetic, S., Radivojac, P., Brown, C.J. \& Dunker, A.K. 2003, "Predicting intrinsic disorder from amino acid sequence", Proteins: Structure, Function, and Bioinformatics, vol. 53, no. S6, pp. 566-572.

Olaf, P. 2009, "Ins and outs of cardiac voltage-gated potassium channels", Current Opinion in Pharmacology, vol. 9, no. 3, pp. 311-315.

Osawa, M., Dace, A., Tong, K.I., Valiveti, A., Ikura, M. \& Ames, J.B. 2005, "Mg ${ }^{2+}$ and $\mathrm{Ca}^{2+}$ Differentially Regulate DNA Binding and Dimerization of DREAM", Journal of Biological Chemistry, vol. 280, no. 18, pp. 18008-18014.

Osawa, M., Tong, K.I., Lilliehook, C., Wasco, W., Buxbaum, J.D., Cheng, H.-.M., Penninger, J.M., Ikura, M. \& Ames, J.B. 2001, "Calcium-regulated DNA Binding and Oligomerization of the Neuronal Calcium-sensing Protein, 
Calsenilin/DREAM/KChIP3", Journal of Biological Chemistry, vol. 276, no. 44, pp. 41005-41013.

Ouyang, H., Larriba-Andaluz, C., Oberreit, D.R. \& Hogan Jr, C.J. 2013, "The collision cross sections of iodide salt cluster ions in air via differential mobility analysis-mass spectrometry", Journal of the American Society for Mass Spectrometry, vol. 24, no. 12, pp. 1833-1847.

Parul, D.A., Bokut, S.B., Milyutin, A.A., Petrov, E.P., Nemkovich, N.A., Sobchuk, A.N. \& Dzhagarov, B.M. 2000, "Time-resolved fluorescence reveals two binding sites of 1,8-ANS in intact human oxyhemoglobin", Journal of Photochemistry and Photobiology B: Biology, vol. 58, no. 2-3, pp. 156-162.

Patel, S.P., Campbell, D.L. \& Strauss, H.C. 2002, "Elucidating KChIP effects on Kv4.3 inactivation and recovery kinetics with a minimal KChIP2 isoform", The Journal of physiology, vol. 545, no. Pt 1, pp. 5-11.

Paterlini, M., Revilla, V., Grant, A. \& Wisden, W. 2000, "Expression of the neuronal calcium sensor protein family in the rat brain", Neuroscience, vol. 99, no. 2, pp. 205216.

Pham, K., Dhulipala, G., Gonzalez, W.G., Gerstman, B.S., Regmi, C., Chapagain, P.P. \& Miksovska, J. 2015, " $\mathrm{Ca}^{2+}$ and $\mathrm{Mg}^{2+}$ modulate conformational dynamics and stability of downstream regulatory element antagonist modulator", Protein Science, , pp. n/an/a.

Phillips, J.C., Braun, R., Wang, W., Gumbart, J., Tajkhorshid, E., Villa, E., Chipot, C., Skeel, R.D., Kale, L. \& Schulten, K. 2005, "Scalable molecular dynamics with NAMD", Journal of computational chemistry, vol. 26, no. 16, pp. 1781-1802.

Pintacuda, G., Park, A.Y., Keniry, M.A., Dixon, N.E. \& Otting, G. 2006, "Lanthanide Labeling Offers Fast NMR Approach to 3D Structure Determinations of Proteinâ^’Protein Complexes", Journal of the American Chemical Society, vol. 128, no. 11, pp. 3696-3702.

Pioletti, M., Findeisen, F., Hura, G.L. \& Minor,, Daniel L, 2006, "Three-dimensional structure of the KChIP1-Kv4.3 T1 complex reveals a cross-shaped octamer", Nat Struct Mol Biol, vol. 13, no. 11, pp. 987-995.

Polans, A., Baehr, W. \& Palczewski, K. 1996, "Turned on by Ca 2 ! The physiology and pathology of Ca 2 -binding proteins in the retina", Trends in neurosciences, vol. 19, no. 12, pp. 547-554.

Ramachandran, P.L., Craig, T.A., Atanasova, E.A., Cui, G., Owen, B.A., Bergen, H.R., Mer, G. \& Kumar, R. 2012, "The Potassium Channel Interacting Protein 3 
(DREAM/KChIP3) Heterodimerizes with and Regulates Calmodulin Function", Journal of Biological Chemistry, vol. 287, no. 47, pp. 39439-39448.

Rao, S.T., Satyshur, K.A., Greaser, M.L. \& Sundaralingam, M. 1996, "X-ray structures of $\mathrm{Mn}, \mathrm{Cd}$ and Tb metal complexes of troponin C", Acta crystallographica.Section D, Biological crystallography, vol. 52, no. Pt 5, pp. 916-922.

Raynal, P. \& Pollard, H.B. 1994, "Annexins: the problem of assessing the biological role for a gene family of multifunctional calcium-and phospholipid-binding proteins", Biochimica et Biophysica Acta (BBA)-Reviews on Biomembranes, vol. 1197, no. 1, pp. 63-93.

Reddy, P.P., Raghuram, V., Hradsky, J., Spilker, C., Chakraborty, A., Sharma, Y., Mikhaylova, M. \& Kreutz, M.R. 2014, "Molecular Dynamics of the Neuronal EFHand $\mathrm{Ca}^{2+}$-Sensor Caldendrin", PLoS ONE, vol. 9, no. 7, pp. e103186.

Rhoads, A.R. \& Friedberg, F. 1997, "Sequence motifs for calmodulin recognition", FASEB Journal, vol. 11, no. 5, pp. 331-340.

Rivas, M., Villar, D., González, P., Dopazo, X., Mellstrom, B. \& Naranjo, J. 2011, "Building the DREAM interactome", Science China Life Sciences, vol. 54, no. 8, pp. 786-792.

Robinson, G.W., Robbins, R.J., Fleming, G.R., Morris, J.M., Knight, A.E.W. \& Morrison, R.J.S. 1978, "Picosecond studies of the fluorescence probe molecule 8-anilino-1naphthalenesulfonic acid", Journal of the American Chemical Society, vol. 100, no. 23, pp. 7145-7150.

Rosati, B., Pan, Z., Lypen, S., Wang, H.S., Cohen, I., Dixon, J.E. \& McKinnon, D. 2001, "Regulation of KChIP2 potassium channel beta subunit gene expression underlies the gradient of transient outward current in canine and human ventricle", The Journal of Physiology, vol. 533, no. Pt 1, pp. 119-125.

Ross, J.A. \& Jameson, D.M. 2008, "Time-resolved methods in biophysics. 8. Frequency domain fluorometry: applications to intrinsic protein fluorescence", Photochemical \& Photobiological Sciences, vol. 7, no. 11, pp. 1301-1312.

Roy, R., Hohng, S. \& Ha, T. 2008, "A practical guide to single-molecule FRET", Nature Methods, vol. 5, no. 6, pp. 507-516.

Runnels, L.W. \& Scarlata, S.F. 1995, "Theory and application of fluorescence homotransfer to melittin oligomerization", Biophysical Journal, vol. 69, no. 4, pp. 1569-1583. 
Santoro, M.M. \& Bolen, D. 1988, "Unfolding free energy changes determined by the linear extrapolation method. 1. Unfolding of phenylmethanesulfonyl. alpha.-chymotrypsin using different denaturants", Biochemistry, vol. 27, no. 21, pp. 8063-8068.

Saver, J.L. 2006, "Time is brain--quantified", Stroke, vol. 37, no. 1, pp. 263-266.

Scannevin, R.H., Wang, K., Jow, F., Megules, J., Kopsco, D.C., Edris, W., Carroll, K.C., Lü, Q., Xu, W., Xu, Z., Katz, A.H., Olland, S., Lin, L., Taylor, M., Stahl, M., Malakian, K., Somers, W., Mosyak, L., Bowlby, M.R., Chanda, P. \& Rhodes, K.J. 2004, "Two N-Terminal Domains of Kv4 $\mathrm{K}^{+}$Channels Regulate Binding to and Modulation by KChIP1", Neuron, vol. 41, no. 4, pp. 587-598.

Schermelleh, L., Heintzmann, R. \& Leonhardt, H. 2010, "A guide to super-resolution fluorescence microscopy", The Journal of Cell Biology, vol. 190, no. 2, pp. 165-175.

Schulenberg, P.J., Gaertner, W. \& Braslavsky, S.E. 1995, "Time-resolved volume changes during the bacteriorhodopsin photocycle: A photothermal beam deflection study", The Journal of Physical Chemistry, vol. 99, no. 23, pp. 9617-9624.

Senguen, F.T. \& Grabarek, Z. 2012, "X-ray structures of magnesium and manganese complexes with the N-terminal domain of calmodulin: insights into the mechanism and specificity of metal ion binding to an EF-hand", Biochemistry, vol. 51, no. 31, pp. 6182-6194.

Sethi, A., Eargle, J., Black, A.A. \& Luthey-Schulten, Z. 2009, "Dynamical networks in tRNA:protein complexes", Proceedings of the National Academy of Sciences of the United States of America, vol. 106, no. 16, pp. 6620-6625.

Shepherd, C.M. \& Vogel, H.J. 2004, "A molecular dynamics study of $\mathrm{Ca}^{2+}$-calmodulin: evidence of interdomain coupling and structural collapse on the nanosecond timescale", Biophysical journal, vol. 87, no. 2, pp. 780-791.

Sigurskjold, B.W. 2000, "Exact analysis of competition ligand binding by displacement isothermal titration calorimetry", Analytical Biochemistry, vol. 277, no. 2, pp. 260266.

Someya, Y. \& Yui, H. 2010, "Fluorescence Lifetime Probe for Solvent Microviscosity Utilizing Anilinonaphthalene Sulfonate", Analytical Chemistry, vol. 82, no. 13, pp. 5470-5476.

Song, W.J., Tkatch, T., Baranauskas, G., Ichinohe, N., Kitai, S.T. \& Surmeier, D.J. 1998, "Somatodendritic depolarization-activated potassium currents in rat neostriatal cholinergic interneurons are predominantly of the A type and attributable to coexpression of Kv4.2 and Kv4.1 subunits", The Journal of Neuroscience, vol. 18, no. 9, pp. 3124-3137. 
Spencer, R.D. \& Weber, G. 1970, "Influence of Brownian Rotations and Energy Transfer upon the Measurements of Fluorescence Lifetime", The Journal of Chemical Physics, vol. 52, no. 4, pp. 1654-1663.

Steinberg, I.Z. 1971, "Long-range nonradiative transfer of electronic excitation energy in proteins and polypeptides", Annual Review of Biochemistry, vol. 40, pp. 83-114.

Stevens, F.C., Walsh, M., Ho, H.C., Teo, T.S. \& Wang, J.H. 1976, "Comparison of calcium-binding proteins. Bovine heart and brain protein activators of cyclic nucleotide phosphodiesterase and rabbit skeletal muscle troponin C", The Journal of biological chemistry, vol. 251, no. 15, pp. 4495-4500.

Strickland, E.H. 1974, "Aromatic contributions to circular dichroism spectra of proteins", CRC Critical Reviews in Biochemistry, vol. 2, no. 1, pp. 113-175.

Stryer, L. 1965, "The interaction of a naphthalene dye with apomyoglobin and apohemoglobin: a fluorescent probe of non-polar binding sites", Journal of Molecular Biology, vol. 13, no. 2, pp. 482-495.

Strynadka, N.C. \& James, M.N. 1989, "Crystal structures of the helix-loop-helix calciumbinding proteins", Annual Review of Biochemistry, vol. 58, no. 1, pp. 951-999.

Sun, Y., Brandmeier, B. \& Irving, M. 2006, "Structural changes in troponin in response to $\mathrm{Ca}^{2+}$ and myosin binding to thin filaments during activation of skeletal muscle", Proceedings of the National Academy of Sciences, vol. 103, no. 47, pp. 17771-17776.

Szabo, A. \& Rayner, D. 1980, "Fluorescence decay of tryptophan conformers in aqueous solution", Journal of the American Chemical Society, vol. 102, no. 2, pp. 554-563.

Szabò, I., Zoratti, M. \& Gulbins, E. 2010, "Contribution of voltage-gated potassium channels to the regulation of apoptosis", FEBS letters, vol. 584, no. 10, pp. 2049-2056.

Szebenyi, D.M. \& Moffat, K. 1986, "The refined structure of vitamin D-dependent calcium-binding protein from bovine intestine. Molecular details, ion binding, and implications for the structure of other calcium-binding proteins", The Journal of Biological Chemistry, vol. 261, no. 19, pp. 8761-8777.

Szmacinski, H., Jayaweera, R., Cherek, H. \& Lakowicz, J.R. 1987, "Demonstration of an associated anisotropy decay by frequency-domain fluorometry", Biophysical Chemistry, vol. 27, no. 3, pp. 233-241.

Takasaki, A., Hayashi, N., Matsubara, M., Yamauchi, E. \& Taniguchi, H. 1999, "Identification of the calmodulin-binding domain of neuron-specific protein kinase $\mathrm{C}$ substrate protein CAP-22/NAP-22. Direct involvement of protein myristoylation in 
calmodulin-target protein interaction", The Journal of Biological Chemistry, vol. 274, no. 17, pp. 11848-11853.

Takimoto, K., Yang, E. \& Conforti, L. 2002, "Palmitoylation of KChIP Splicing Variants Is Required for Efficient Cell Surface Expression of Kv4.3 Channels", Journal of Biological Chemistry, vol. 277, no. 30, pp. 26904-26911.

Tanaka, T., Amest, J.B., Harvey, T.S., Stryer, L. \& lkura, M. 1995, "Sequestration of the membrane-targeting myristoyl group of recoverin in the calcium-free state", Nature, vol. 376, no. 6539, pp. 444-447.

Togashi, D.M. \& Ryder, A.G. 2008, "A fluorescence analysis of ANS bound to bovine serum albumin: binding properties revisited by using energy transfer", Journal of Fluorescence, vol. 18, no. 2, pp. 519-526.

Tsai, C., Del Sol, A. \& Nussinov, R. 2009, "Protein allostery, signal transmission and dynamics: a classification scheme of allosteric mechanisms", Molecular Biosystems, vol. 5, no. 3, pp. 207-216.

Upadhyay, A., Bhatt, T., Tripathi, H.B. \& Pant, D.D. 1995, "Photophysics of 8anilinonaphthalene-1-sulphonate", Journal of Photochemistry and Photobiology A: Chemistry, vol. 89, no. 3, pp. 201-207.

Van Hoorick, D., Raes, A., Keysers, W., Mayeur, E. \& Snyders, D.J. 2003, "Differential modulation of Kv4 kinetics by KCHIP1 splice variants", Molecular and Cellular Neuroscience, vol. 24, no. 2, pp. 357-366.

Vigil, D., Gallagher, S.C., Trewhella, J. \& García, A.E. 2001, "Functional dynamics of the hydrophobic cleft in the N-domain of calmodulin", Biophysical Journal, vol. 80, no. 5, pp. 2082-2092.

Vijay-Kumar, S. \& Kumar, V.D. 1999, "Crystal structure of recombinant bovine neurocalcin", Nature Structural \& Molecular Biology, vol. 6, no. 1, pp. 80-88.

Wallace, R.W., Tallant, E.A., Dockter, M.E. \& Cheung, W.Y. 1982, "Calcium binding domains of calmodulin. Sequence of fill as determined with terbium luminescence.", Journal of Biological Chemistry, vol. 257, no. 4, pp. 1845-1854.

Wang, K. \& Chai, J. 2009, "Structural Basis for Auxiliary KChIP Modulation of Kv4 Channels" in Structure, Function, and Modulation of Neuronal Voltage-Gated Ion Channels, eds. V.K. Gribkoff \& L.K. Kaczmarek, John Wiley \& Sons, Inc., , pp. 343361. 
Wang, S., George, S.E., Davis, J.P. \& Johnson, J.D. 1998, "Structural determinants of Ca2 exchange and affinity in the $\mathrm{C}$ terminal of cardiac troponin C", Biochemistry, vol. 37, no. 41, pp. 14539-14544.

Wang, C.L., Aquaron, R.R., Leavis, P.C. \& Gergely, J. 1982, "Metal-binding properties of calmodulin", European Journal of Biochemistry / FEBS, vol. 124, no. 1, pp. 7-12.

Wang, G. \& Covarrubias, M. 2006, "Voltage-dependent gating rearrangements in the intracellular T1-T1 interface of a K+ channel", The Journal of General Physiology, vol. 127, no. 4, pp. 391-400.

Wang, H., Yan, Y., Liu, Q., Huang, Y., Shen, Y., Chen, L., Chen, Y., Yang, Q., Hao, Q., Wang, K. \& Chai, J. 2007, "Structural basis for modulation of Kv4 $\mathrm{K}^{+}$channels by auxiliary KChIP subunits", Nature neuroscience, vol. 10, no. 1, pp. 32-39.

Weber, G. 1952, "Polarization of the fluorescence of macromolecules. II. Fluorescent conjugates of ovalbumin and bovine serum albumin", The Biochemical Journal, vol. 51, no. 2, pp. 155-167.

Weiergräber, O.H., Senin, I.I., Philippov, P.P., Granzin, J. \& Koch, K. 2003, "Impact of N-terminal myristoylation on the Ca2 -dependent conformational transition in recoverin", Journal of Biological Chemistry, vol. 278, no. 25, pp. 22972-22979.

Whinnery, J.R. 1974, "Laser measurement of optical absorption in liquids", Accounts of Chemical Research, vol. 7, no. 7, pp. 225-231.

Witzel, K., Fischer, P. \& Bähring, R. 2012, "Hippocampal A-type current and Kv4.2 channel modulation by the sulfonylurea compound NS5806", Neuropharmacology, vol. 63, no. 8, pp. 1389-1403.

Woody, R.W. 2012, "Electronic Circular Dichroism of Proteins" in Comprehensive Chiroptical Spectroscopy John Wiley \& Sons, Inc., , pp. 473-497.

Xue, B., Dunbrack, R.L., Williams, R.W., Dunker, A.K. \& Uversky, V.N. 2010, "PONDRFIT: a meta-predictor of intrinsically disordered amino acids", Biochimica et Biophysica Acta, vol. 1804, no. 4, pp. 996-1010.

Y Cajal, S.R. 1995, Histology of the nervous system of man and vertebrates, Oxford University Press, USA.

Yamniuk, A. \& Vogel, H. 2004, "Calmodulin's flexibility allows for promiscuity in its interactions with target proteins and peptides", Molecular biotechnology, vol. 27, no. 1, pp. 33-57. 
Yap, K.L., Ames, J.B., Swindells, M.B. \& Ikura, M. 1999, "Diversity of conformational states and changes within the EF-hand protein superfamily", Proteins: Structure, Function, and Bioinformatics, vol. 37, no. 3, pp. 499-507.

Ye, Y., Lee, H., Yang, W., Shealy, S. \& Yang, J.J. 2005, "Probing site-specific calmodulin calcium and lanthanide affinity by grafting", Journal of the American Chemical Society, vol. 127, no. 11, pp. 3743-3750.

Yellen, G. 1998, "The moving parts of voltage-gated ion channels", Quarterly reviews of biophysics, vol. 31, no. 03, pp. 239.

Yu, L., Sun, C., Mendoza, R., Wang, J., Matayoshi, E.D., Hebert, E., Pereda-Lopez, A., Hajduk, P.J. \& Olejniczak, E.T. 2007a, "Solution structure and calcium-binding properties of EF-hands 3 and 4 of calsenilin", Protein Science, vol. 16, no. 11, pp. 2502-2509.

Yu, L., Sun, C., Mendoza, R., Wang, J., Matayoshi, E.D., Hebert, E., Pereda-Lopez, A., Hajduk, P.J. \& Olejniczak, E.T. 2007b, "Solution structure and calcium-binding properties of EF-hands 3 and 4 of calsenilin", Protein Science, vol. 16, no. 11, pp. 2502-2509.

Zaidi, N.F., Thomson, E.E., Choi, E., Buxbaum, J.D. \& Wasco, W. 2004, "Intracellular calcium modulates the nuclear translocation of calsenilin", Journal of Neurochemistry, vol. 89, no. 3, pp. 593-601.

Zarrine-Afsar, A., Mittermaier, A., Kay, L.E. \& Davidson, A.R. 2006, "Protein stabilization by specific binding of guanidinium to a functional arginine-binding surface on an SH3 domain", Protein Science, vol. 15, no. 1, pp. 162-170.

Zhang, M., Tanaka, T. \& Ikura, M. 1995, "Calcium-induced conformational transition revealed by the solution structure of apo calmodulin", Nature Structural Biology, vol. 2, no. 9, pp. 758-767.

Zhou, R., Huang, X., Margulis, C.J. \& Berne, B.J. 2004a, "Hydrophobic collapse in multidomain protein folding", Science (New York, N.Y.), vol. 305, no. 5690, pp. 16051609.

Zhou, W., Qian, Y., Kunjilwar, K., Pfaffinger, P.J. \& Choe, S. 2004b, "Structural Insights into the Functional Interaction of KChIP1 with Shal-Type K ${ }^{+}$Channels", Neuron, vol. 41, no. 4, pp. 573-586.

Zhu, Y., Wu, Y., Luo, Y., Zou, Y., Ma, B. \& Zhang, Q. 2014, "R102q mutation shifts the salt-bridge network and reduces the structural flexibility of human neuronal calcium sensor-1 protein", The Journal of Physical Chemistry B, vol. 118, no. 46, pp. 1311213122. 
Zicha, S., Xiao, L., Stafford, S., Cha, T.J., Han, W., Varro, A. \& Nattel, S. 2004, "Transmural expression of transient outward potassium current subunits in normal and failing canine and human hearts", The Journal of Physiology, vol. 561, no. 3, pp. 735748.

Zozulya, S. \& Stryer, L. 1992, "Calcium-myristoyl protein switch", Proceedings of the National Academy of Sciences, vol. 89, no. 23, pp. 11569-11573. 


\section{APPENDICES}

10.1 Appendix 1

The DNA sequence of the plasmids used throughout this work is shown below. In

all cases the $\mathrm{T} 7$ promoter was used as the primer for sequencing. In cases where a primer

was used to generate the mutant, the primer sequence is underlined, while the start and stop

codons are shown as bold letters.

$\operatorname{DREAM}(65-256)$ sequenced by Eurofins MWG operon NNNNNNNNNNNNNNNTTCCCTCTNAAANAANTTTGTNNANCTTTAAGAAGGAGATATAC ATATGGAACTGGAGTTATCCACGGTGCGCCATCAGCCAGAGGGCTTGGACCAGCTACAA GCTCAGACCAAGTTCACCAAGAAGGAGCTGCAGTCCCTTTACCGAGGCTTCAAGAATGA GTGTCCCACAGGCCTGGTGGATGAAGACACCTTCAAACTCATTTATTCCCAGTTCTTCC CTCAGGGAGATGCCACCACCTATGCACACTTCCTCTTCAATGCCTTCGATGCTGATGGG AACGGGGCCATCCACTTTGAGGACTTTGTGGTTGGGCTCTCCATCCTGCTTCGAGGGAC GGTCCATGAGAAGCTCAAGTGGGCCTTCAATCTCTATGACATTAACAAGGATGGTTGCA TCACCAAGGAGGAGATGCTGGCCATCATGAAGTCCATCTACGACATGATGGGCCGCCAC ACCTACCCCATCCTGCGGGAGGATGCACCCCTGGAGCATGTGGAGAGGTTCTTTCAGAA AATGGACAGGAACCAGGATGGAGTGGTGACCATTGATGAATTTCTGGAGACTTGTCAGA AGGATGAGAACATCATGAACTCCATGCAGCTGTTTGAGAACGTCATCTACCTCGAGCAC CACCACCACCACCACTAATGTTAATTAAGTTGGGCGTTCCTAGGCTGATAAAACAGAAT TTGCCTGGCGGCAGTAGCGCGGTGGTCCCACCTGACCCCATGCCGAACTCAGAAGTGAA ACGCCGTAGCGCCGATGGTAGTGTGGGGTCTCCCCATGCGAGAGTAGGGAACTGCCAGG CATCAAATAAAACGAAAGGCTCAGTCGAAAGACTGGGCCTTTCGTTTTATCTGTTGTTT GNCGGTGAACGCTCTCCTGAGNNGNNNAATCCGCCGGGAGCGGATTTGAACGTTGCGAA GCANGGCCNGGAAGGGGGNCGGANANAAAAAAAANAAAAANNNNNNNNNNNACNGACNN NNAATGATACNNNNTTATTANNNTATNNATAANNNNN

>DREAM_E186Q_T7 Sample_Name=DREAM_E186Q_T7 Chromat_id=3790104 Read_id $=3745990$ Version $=1$ Length $=1055$ NNNNNNNNNNNNNNNNNCNNNCTNNNNNNNTTTTGTTTAACTTTAAGAAGGAGATATACATATGG AACTGGAGTTATCNANGGTGCGCCATCAGCCAGAGGGCTTGGACCAGCTACAAGCTCAGACCAAG TTCACCAAGAAGGAGCTGCAGTCCCTTTACCGAGGCTTCAAGAATGAGTGTCCCACAGGCCTGGT GGATGAAGACACCTTCAAACTCATTTATTCCCAGTTCTTCCCTCAGGGAGATGCCACCACCTATG CACACTTCCTCTTCAATGCCTTCGATGCTGATGGGAACGGGGCCATCCACTTTGAGGACTTTGTG GTTGGGCTCTCCATCCTGCTTCGAGGGACGGTCCATGAGAAGCTCAAGTGGGCCTTCAATCTCTA TGACATTAACAAGGATGGTTGCATCACCAAGGAGCAGATGCTGGCCATCATGAAGTCCATCTACG ACATGATGGGCCGCCACACCTACCCCATCCTGCGGGAGGATGCACCCCTGGAGCATGTGGAGAGG TTCTTTCAGAAAATGGACAGGAACCAGGATGGAGTGGTGACCATTGATGAATTTCTGGAGACTTG TCAGAAGGATGAGAACATCATGAACTCCATGCAGCTGTTTGAGAACGTCATCTACCTCGAGCACC ACCACCACCACCACTAATGTTAATTAAGTTGGGCGTTCCTAGGCTGATAAAACAGAATTTGCCTG GCGGCAGTAGCGCGGTGGTCCCACCTGACCCCATGCCGAACTCNGAAGTGAAACGCCGTACCGCC 
GANGNTAGTGTGGGGTCTCCCCATGNNNGAGTAGGGAACTGCCAGGCATCAGATAAAAACGAAAG GCTCAGTCGAAAGACTGGGCCTTTCGTTTTATCTGTTGTTTGTCGGTGAACGCTCTCCTGAGTAG GACAAATCCGCCNGGANGCGGATTNGAACGTTGCGANGCAACNCCCNNGGAGGNTNNNNCNNNCT GCNNNNNNGTGNNNNGNNNNCCGNCNNNNNCTANANNNNANTATANNNNGNNNAGGNACTATACG ATGACTNCNNNNNNN

>DREAM_E234Q_T7 Sample_Name=DREAM_E234Q_T7 Chromat_id=4526469 Read_id $=4475245$ Version $=1$ Length $=1062$

GNNNNNNNNNNNNNNNNNNNNNNNNNNNNNNNNTCNNNCTAGNNTAATTTTGTTTAACTTTAAGA AGGAGATATACATATGGAACTGGAGTTATCNNCGGTGCGCCATCAGCCAGAGGGCTTGGACCAGC TACAAGCTCAGACCAAGTTCACCAAGAAGGAGCTGCAGTCCCTTTACCGAGGCTTCAAGAATGAG TGTCCCACAGGCCTGGTGGATGAAGACACCTTCAAACTCATTTATTCCCAGTTCTTCCCTCAGGG AGATGCCACCACCTATGCACACTTCCTCTTCAATGCCTTCGATGCTGATGGGAACGGGGCCATCC ACTTTGAGGACTTTGTGGTTGGGCTCTCCATCCTGCTTCGAGGGACGGTCCATGAGAAGCTCAAG TGGGCCTTCAATCTCTATGACATTAACAAGGATGGTTGCATCACCAAGGAGGAGATGCTGGCCAT CATGAAGTCCATCTACGACATGATGGGCCGCCACACCTACCCCATCCTGCGGGAGGATGCACCCC TGGAGCATGTGGAGAGGTTCTTTCAGAAAATGGACAGGAACCAGGATGGAGTGGTGACCATTGAT CAATTTCTGGAGACTTGTCAGAAGGATGAGAACATCATGAACTCCATGCAGCTGTTTGAGAACGT CATCTACCTCGAGCACCACCACCACCACCACTAATGTTAATTAAGTTGGGCGTTCCTAGGCTGAT AAAACAGAATTTGCCTGGCGGCAGTAGCGCGGTGGTCCCACCTGACCCCATGCCGAACTCAGAAG TGAAACGCCGTAGCGCCGATGGTAGTGTGGGGTCTCCCCATGCGAGAGTAGGGAACTGCCAGGCA TCAAATAAAACGAAAGGCTCAGTCGAAAGACTGGGCCTTTCGTTTTATCTGTTGTTNGTCGGTGA ACGCTCTCCTGAGTAGGACAANTCCGCCGGGAGCGGATTTGAACGTTGCGAANNAACGGCNNCNN NGGNTGGCGNTNNGNNNNNNNNNNNNTNNCNGCCANNGNTCNNNANNNCNNNNNNNCACANNNNN NGANNNNNNNNNNNNTNACNNN

>DREAM_Y174A_T7 Sample_Name=DREAM_Y174A_T7 Chromat_id=2855977 Read_id=2829164 Version=1 Length=929

NNNNNNNNNNNNNNNNNNNNNNCTNNNNNNNNTTTGTNNNNNTTNAAGAAGGAGATATACATATG GAACTGGAGTTNNCANCNNNGCGCCATCAGCCAGAGGGCTTGGACCAGCTACAAGCTCANACCAA GTTCACCAGGAAGGAGCTGCANTCCCTTTACCGAGGCTTCAAGAATGAGTGTCCCACAGGCCTGG TGGATGAAGACACCTTCAAACTCATTTATTCCCAGTTCTTCCCTCAGGGAGATGCCACCACCTAT GCACACTTCCTCTTCAATGCCTTCNATGCTGATGGGAACGGGGCCATCCACTTTGAGGACTTTGT GGTTGGGCTCTCCATCCTGCTTCGAGGGACGGTCCATGAGAAGCTCAAGTGGGCCTTCAATCTCG CGGACATTAACAAGGATGGTTGCATCACCAAGGAGGAGATGCTGGCCATCATGAAGTCCATCTAC GACATGATGGGCCGCCACACCTACCCCATCCTGCGGGAGGATGCACCCCTGGAGCATGTGGAGAG GTTCTTTCAGAAAATGGACAGGAACCAGGATGGAGTGGTGACCATTGATGAATTTCTGGAGACTT GTCAGAAGGATGAGAACATCATGAACTCCATGCAGCTGTTTGAGAACGTCATCTACCTCGAGCAC CACCACCACCACCACTAATGTTAATTAAGTTGGGCGTTCCTAGGCTGATAAAACAGAATTTGCCT GGCGGCAGTAGCGCGGTGGTCCCACCTGACCCCATGCCGAACTCAGAAGTGAAACGCCGTAGCGC CGATGGTAGTGTGGGGTCTCCCCATGCGAGAGTAGGGAACTGCCAGGCATCAAATAAAACGAAAG GCTCNNTCGAAAGACTGGGCCTTTCGNNTTATCTGTTGTTTGTCGGTGAACGCTCTCCTGAGTAG GACAANTCNNNCGGNAACG

>DREAM_F218A_T7 Sample_Name=DREAM_F218A_T7 Chromat_id=2855910 Read_id=2829077 Version=1 Length $=1038$ 
NNNNNNNNNNNNNNNNNNNNNNNNCTNNNNNANTTTNNNNTNNCNTTAAGAAGGAGATATACATA TGGAACTGGAGCTATNNNNGGNGCGCCATCAGCCAGAGGGCTTGGACCAGCTACNAGCTCAGACC AAGTTCACCAAGAAGGAGCTGCAGTCCCTTTACCGAGGCTTCAAGAATGAGTGTCCCACAGGCCT GGTGGATGAAGACACCTTCAAACTCATTTATTCCCAGTTCTTCCCTCAGGGAGATGCCACCACCT ATGCACACTTCCTCTTCAATGCCTTCGATGCTGATGGGAACGGGGCCATCCACTTTGAGGACTTT GTGGTTGGGCTCTCCATCCTGCTTCGAGGGACGGTCCATGAGAAGCTCAAGTGGGCCTTCAATCT CTATGACATTAACAAGGATGGTTGCATCACCAAGGAGGAGATGCTGGCCATCATGAAGTCCATCT ACGACATGATGGGCCGCCACACCTACCCCATCCTGCGGGAGGATGCACCCCTGGAGCATGTGGAG AGGGCGTTTCAGAAAATGGACAGGAACCAGGATGGAGTGGTGACCATTGATGAATTTCTGGAGAC TTGTCAGAAGGATGAGAACATCATGAACTCCATGCAGCTGTTTGAGAACGTCATCTACCTCGAGC ACCACCACCACCACCACTAATGTTAATTAAGTTGGGCGTTCCTAGGCTGATAAAACAGAATTTGC CTGGCGGCAGTAGCGCGGTGGTCCCACCTGACCCCATGCCGAACTCAGAAGTGAAACGCCGTAGC GCCGATGGTAGTGTGGGGTCTCCCCATGCGAGAGTAGGGAACTGCCAGGCATCAAATAAAACGAA AGGCTCAGTCGAAAGACTGGGCCTTTCGTTTATCTGNNGTTGTCGGGAACGCTNNCCTGAGTAGA ACAATCCGCCGGGAGCGGATTGAACGTTGCNAACAACGNNCCGGAGGNTNGCGGNCAGGACNNCC GCCNNAACTGCCAGCANNAATTANNCAGAAGGCATCCNNNCGATGGCNNTTTNNNNNNNNAAN

>DREAM_F219A_T7 Sample_Name=F219A_T7 Chromat_id=3997199 Read_id=3950666 Version=1 Length=1017 NNNNNNNNNNNNNNNNNNNNTCCCNTCTNNNNNNATTTTGTTNAACTTTAAGAAGGAGATATACA TATGGAACTGGAGTTATCCNCNGNGCGCCATCAGCCAGAGGGCTTGGACCAGCTACAAGCTCAGA CCAAGTTCACCAAGAAGGAGCTGCAGTCCCTTTACCGAGGCTTCAAGAATGAGTGTCCCACAGGC CTGGTGGATGAAGACACCTTCAAACTCATTTATTCCCAGTTCTTCCCTCAGGGAGATGCCACCAC CTATGCACACTTCCTCTTCAATGCCTTCGATGCTGATGGGAACGGGGCCATCCACTTTGAGGACT TTGTGGTTGGGCTCTCCATCCTGCTTCGAGGGACGGTCCATGAGAAGCTCAAGTGGGCCTTCAAT CTCTATGACATTAACAAGGATGGTTGCATCACCAAGGAGGAGATGCTGGCCATCATGAAGTCCAT CTACGACATGATGGGCCGCCACACCTACCCCATCCTGCGGGAGGATGCACCCCTGGAGCATGTGG AGAGGTTCGCGCAGAAAATGGACAGGAACCAGGATGGAGTGGTGACCATTGATGAATTTCTGGAG ACTTGTCAGAAGGATGAGAACATCATGAACTCCATGCAGCTGTTTGAGAACGTCATCTACCTCGA GCACCACCACCACCACCACTAATGTTAATTAAGTTGGGCGTTCCTAGGCTGATAAAACAGAATTT GCCTGGCGGCAGTAGCGCGGTGGTCCCACCTGACCCCATGCCGAACTCAGAAGTGAAACGCCGTA GCGCCGATGGTAGTGTGGGGTCTCCCCATGCGAGAGTAGGGAACTGCCAGGCATCAAATAAAACG AAAGGCTCAGTCGAAAGACTGGGCCTTTCGTTTTATCTGTTGTTTGTCGGTGAACGCTCTCCTGA GTAGGACAAATCCGCCGGGAGCGGATTTGAACGTTGCGAACCAACGGCCNNANGGNTGGCGGGCA GGNNNCCNGCCATAANCTGGCAGNNATCAANNNANGCNGAAG

>DREAM_F235A_T7 Sample_Name=DREAMF235A_T7 Chromat_id=3424601 Read_id=3384259 Version=1 Length=1037 NNNNNNNNNNNNNNNNNNNNNNNCCTCTAGNATNNNTTTGTTTAACTTTAAGAAGGAGATATACA TATGGAACTGGAGTTATCCACGGTGCGCCATCAGCCAGAGGGCTTGGACCAGCTACAAGCTCAGA CCAAGTTCACCAAGAAGGAGCTGCAGTCCCTTTACCGAGGCTTCAAGAATGAGTGTCCCACAGGC CTGGTGGATGAAGACACCTTCAAACTCATTTATTCCCAGTTCTTCCCTCAGGGAGATGCCACCAC CTATGCACACTTCCTCTTCAATGCCTTCGATGCTGATGGGAACGGGGCCATCCACTTTGAGGACT TTGTGGTTGGGCTCTCCATCCTGCTTCGAGGGACGGTCCATGAGAAGCTCAAGTGGGCCTTCAAT CTCTATGACATTAACAAGGATGGTTGCATCACCAAGGAGGAGATGCTGGCCATCATGAAGTCCAT CTACGACATGATGGGCCGCCACACCTACCCCATCCTGCGGGAGGATGCACCCCTGGAGCATGTGG AGAGGTTCTTTCAGAAAATGGACAGGAACCAGGATGGAGTGGTGACCATTGATGAAGCGCTGGAG 
ACTTGTCAGAAGGATGAGAACATCATGAACTCCATGCAGCTGTTTGAGAACGTCATCTACCTCGA GCACCACCACCACCACCACTAATGTTAATTAAGTTGGGCGTTCCTAGGCTGATAAAACAGAATTT GCCTGGCGGCAGTAGCGCGGTGGTCCCACCTGACCCCATGCCGAACTCAGAAGTGAAACGCCGTA GCGCCGATGGTAGTGTGGGGTCTCCCCATGCGAGAGTAGGGAACTGCCAGGCATCAAATAAAACG AAAGGCTCAGTCGAAAGACTGGGCCTTTCGTTTTATCTGTTGTTTGTCGGTGAACGCTCTCCTGA GTAGGACAAATCCGCCGGGAGCGGATTTGAACGTTGCGAAGCAACGGCCCGNANGGNTGGCGGNC NGNACGCCNGCCATAAACTGCNNNGNNNCAANTTANNNNAANGGCNATCCGNACGNNNGNCC

>DREAM_F252A_T7 Sample_Name=DREAM_K87A_T7 Chromat_id=4097482 Read_id=4050795 Version $=1$ Length $=1229$ NNNNNNNNNNNNNNTCCCNTCTANNNNNNTTTTGTTTAACTTTAAGAAGGAGATATACATATGGA ACTGGAGTTATCCACGGTGCGCCATCAGCCAGAGGGCTTGGACCAGCTACAAGCTCAGACCAAGT TCACCAAGAAGGAGCTGCAGTCCCTTTACCGAGGCTTCAAGAATGAGTGTCCCACAGGCCTGGTG GATGAAGACACCTTCAAACTCATTTATTCCCAGTTCTTCCCTCAGGGAGATGCCACCACCTATGC ACACTTCCTCTTCAATGCCTTCGATGCTGATGGGAACGGGGCCATCCACTTTGAGGACTTTGTGG TTGGGCTCTCCATCCTGCTTCGAGGGACGGTCCATGAGAAGCTCAAGTGGGCCTTCAATCTCTAT GACATTAACAAGGATGGTTGCATCACCAAGGAGGAGATGCTGGCCATCATGAAGTCCATCTACGA CATGATGGGCCGCCACACCTACCCCATCCTGCGGGAGGATGCACCCCTGGAGCATGTGGAGAGGT TCTTTCAGAAAATGGACAGGAACCAGGATGGAGTGGTGACCATTGATGAATTTCTGGAGACTTGT CAGAAGGATGAGAACATCATGAACTCCATGCAGCTGGCGGAGAACGTCATCTACCTCGAGCACCA CCACCACCACCACTAATGTTAATTAAGTTGGGCGTTCCTAGGCTGATAAAACAGAATTTGCCTGG CGGCAGTAGCGCGGTGGTCCCACCTGACCCCATGCCGAACTCAGAAGTGAAACGCCGTAGCGCCG ATGGTAGTGTGGGGTCTCCCCATGCGAGAGTAGGGAACTGCCAGGCATCAAATAAAACGAAAGGC TCAGTCGAAAGACTGGGCCTTTCGTTTTATCTGTTGTTTGTCGGTGAACGCTCTCCTGAGTAGGA CAAATCCGCCGGGAGCGGATTTGAACGTTGCGAANNAACGGCNGGNAAGGNTGGCGGNCAGGACG CCCGCCATAAACTGCCAGGCATCAAATTAAGCAGNNGGCNATCCTGACGGATGGCCTTNTNNNNT TNNNCAANCTCTTNGGTTNTTTTNNNNNNNNNTNNAANNNGNTTCNNCTNNNNANNNNNANNNNN CCCNTTGGGGNNTNAANNNGGACTTNNNGGGNTTTNNNNNAANNNNNNNNNNNNNCCGNNTNGNN AANGGNAACNNCCNNNNNNNCNNNNNNNNNNGNNNNNNNGNNNNNNCNNNNNNNNNNNN

>DREAM_K87A_T7 Sample_Name=DREAM_F252A_T7 Chromat_id=4097480 Read_id $=4050806$ Version=1 Length $=1072$

NNNNNNNNNNNNNNNNTNNNNNNNCTNNNNNNNTTTTGTTNATCTTTAAGAAGGAGATATACATA TGGAACTGGAGTTANCNCGGNGCGCCATCAGCCAGAGGGCTTGGACCAGCTACAAGCTCAGACCG CGTTCACCAAGAAGGAGCTGCAGTCCCTTTACCGAGGCTTCAAGAATGA $\overline{\text { GTGTCCCACAGGCCTG }}$ GTGGATGAAGACACCTTCAAACTCATTTATTCCCAGTTCTTCCCTCAGGGAGATGCCACCACCTA TGCACACTTCCTCTTCAATGCCTTCGATGCTGATGGGAACGGGGCCATCCACTTTGAGGACTTTG TGGTTGGGCTCTCCATCCTGCTTCGAGGGACGGTCCATGAGAAGCTCAAGTGGGCCTTCAATCTC TATGACATTAACAAGGATGGTTGCATCACCAAGGAGGAGATGCTGGCCATCATGAAGTCCATCTA CGACATGATGGGCCGCCACACCTACCCCATCCTGCGGGAGGATGCACCCCTGGAGCATGTGGAGA GGTTCTTTCAGAAAATGGACAGGAACCAGGATGGAGTGGTGACCATTGATGAATTTCTGGAGACT TGTCAGAAGGATGAGAACATCATGAACTCCATGCAGCTGTTTGAGAACGTCATCTACCTCGAGCA CCACCACCACCACCACTAATGTTAATTAAGTTGGGCGTTCCTAGGCTGATAAAACAGAATTTGCC TGGCGGCAGTAGCGCGGTGGTCCCACCTGACCCCATGCCGAACTCAGAAGTGAAACGCCGTAGCG CCGATGGTAGTGTGGGGTCTCCCCATGCGAGAGTAGGGAACTGCCAGGCATCAAATAAAACGAAA GGCTCAGTCGAAAGACTGGGCCTTTCGTTTTATCTGTTGTTTGTCGGTGAACGCTCTCCTGAGTA GGACAANTCCGCCGGGAGCGGATTNGAACGTTGCGANNCAACGGCCCGGAGGNTGGCGGNNAGGA CGCCNGCCTNAACTGCCAGGNNTCAANTANNNNGAAGGCATCCTGACGGATGGCNTTTNNGNNTT CTACNNNNNNTNGGTTATTNNNNANNNNTTNN 
>DREAM_E92A_T7 Sample_Name=E92A_T7 Chromat_id=3997196 Read_id=3950670 Version=1 Length=1013

NNNNNNNNNNNNNNNNNNNNNNNTCTNGNNTAATTTTGTTTAACTTNNAGAAGGAGATATACATA TGGAACTGGAGTTANCCNNGGNNCGCCATCAGCCAGAGGGCTTGGACCAGCTACAAGCTCCCACC AAGTTCACCAAGAAGGCGCTGCAGTCCCTTTACCGAGGCTTCAAGAATGAGTGTCCCACAGGCCT GGTGGATGAAGACACCTTCAAACTCATTTATTCCCAGTTCTTCCCTCAGGGAGATGCCACCACCT ATGCACACTTCCTCTTCAATGCCTTCGATGCTGATGGGAACGGGGCCATCCACTTTGAGGACTTT GTGGTTGGGCTCTCCATCCTGCTTCGAGGGACGGTCCATGAGAAGCTCAAGTGGGCCTTCAATCT CTATGACATTAACAAGGATGGTTGCATCACCAAGGAGGAGATGCTGGCCATCATGAAGTCCATCT ACGACATGATGGGCCGCCACACCTACCCCATCCTGCGGGAGGATGCACCCCTGGAGCATGTGGAG AGGTTCTTTCAGAAAATGGACAGGAACCAGGATGGAGTGGTGACCATTGATGAATTTCTGGAGAC TTGTCAGAAGGATGAGAACATCATGAACTCCATGCAGCTGTTTGAGAACGTCATCTACCTCGAGC ACCACCACCACCACCACTAATGTTAATTAAGTTGGGCGTTCCTAGGCTGATAAAACAGAATTTGC CTGGCGGCAGTAGCGCGGTGGTCCCACCTGACCCCATGCCGAACTCAGAAGTGAAACGCCGTAGC GCCGATGGTAGTGTGGGGTCTCCCCATGCGAGAGTAGGGAACTGCCAGGCATCAAATAAAACGAA AGGCTCAGTCGAAAGACTGGGCCTTTCGTTTTATCTNNNGTTTGTCGGTGAACGCTCTCCTGAGT AGGACAAATCCGCCGGGAGCGGATTTGAACGTTGCGAAGCAACGGCCNGGANGGNTGGCGGNNAG GANNCCNGCNNNAANNNGCNANNNNTCAATTNAANCNN

>CaM_A148C_T7 Sample_Name=CaM_A148C_T7 Chromat_id=2855911

Read_id=2829062 Version=1 Length $=743$

NNNNNNNNNNNNNNCNNNNNNNNNNNNNNNNTNANNNNNNGANNGAGTATACAATGGCTGACCAG CTTACTGGCGANNANAAGGGGGAATGGAAAGAAGCTTTCTCCCTATGGGACAAGGACGGGGATGG GACAATGACAACCAAGGAGCTGGGGACGGTGATGCGGTCTCTGGGGCAGAACCCCACAGAAGCAG ACCTGCAGGACATGATCAAGGAAGTAAATGCCAACGGCAATGCCCCGGTCGACTTCCCTGAATTC CTGACAAGGATGGCNACCCNAATGCAAGACACAGACAGTGAAGAAGAAATTAGAGAAGCGTTCCG NGTGTTTGATAAGGATGGCAATGGCTACATCAGTGCAGCATAGCTTCGCCACGTGATGACTAACC TTGGAGAGAAGTTAACAGATGAAGAGGTTGATGAAATGATCAGGGAAGCAGACATCGATGGGGAT GGTCAGGTAAACTACGAAGAGCTTGTACAAATGATGACATGCAAGTATGGGGATCCTCTNNAGTC GACCTGCAGCCCAAGCTTATCNNTGGCGTAATAGCGANNNGGCCNGCACCGATCGCCCTTCCCAA CNGTTGCGCAGCCTGAATGGCGAATGTACTCTCCCTGTATCGGCGCATTANNNGCNNNNGGTGNT NTNNTTACTCNCNNNNTNNNTGNTACACTTGNNNGCGNNNTTNTNNNNTGNNNNNTTCGCTTTCT TCCNNNCCTTTCTNNNNNCGANNGNCGG 


\subsection{Appendix 2}

The following scripts have been designed to work in MATLAB 2015b. Two inputs are necessary, the mz_table.csv and filename.csv files. The mz_table.csv file is an excel generated spreadsheet which contains three columns of data. Each row represents a charged ion species, the first column is the lowest $\mathrm{m} / \mathrm{z}$ value of this specie, and the second column is the highest $\mathrm{m} / \mathrm{z}$ value for the specie, while the third column is the charge of the ion. If background needs to be subtracted then a $\mathrm{m} / \mathrm{z}$ range in which the background is present must be placed in the first and second column, while the third column must contain a charged state of 0 . If no background is needed, then the scripts by default will not subtract any background. The second file, filename.csv, can be generated by right clicking the image of the $\mathrm{m} / \mathrm{z}$ spectra versus mobility obtained from the UIMF_Viewer software and selecting “Export Intensity Values (Complete Bin Resolution)”. The export command will generate a .csv spreadsheet that can be recognized by the script. Both the mz_table.csv and filename.csv files must be in the same folder. I recommend appending a numeric value (i e. $1,2,3,4$, or 5 ) at the end of the filename in order to be able to use the "timescale" option at line 11 of the first script.

\% Start of Script 1

$\%$ Signal extraction script for TIMS data version 2.0

\%This script was prepared in MATLAB R2011b, and may not work well with latest versions

\%this script has not been yet optimized but provides the basis for further development. \%Script designed by WGG 01/19/2016

$\%$ Load data from CSV matrix exported from Bruker software as .csv

$\%$ Create transpose matrices, so that $\mathrm{y}=$ mobility and $\mathrm{x}=$ mass to charge clear

clc \%\%\%\%\%\%\%\%\%\%\%\%\%\%\%\%\%\%\%\%\%\%\%\%\% timescale='1'; $\quad \%$ Only this part needs to be changed. \%\%\%\%\%\%\%\%\%\%\%\%\%\%\%\%\%\%\%\%\%\%\%\%\%\% 


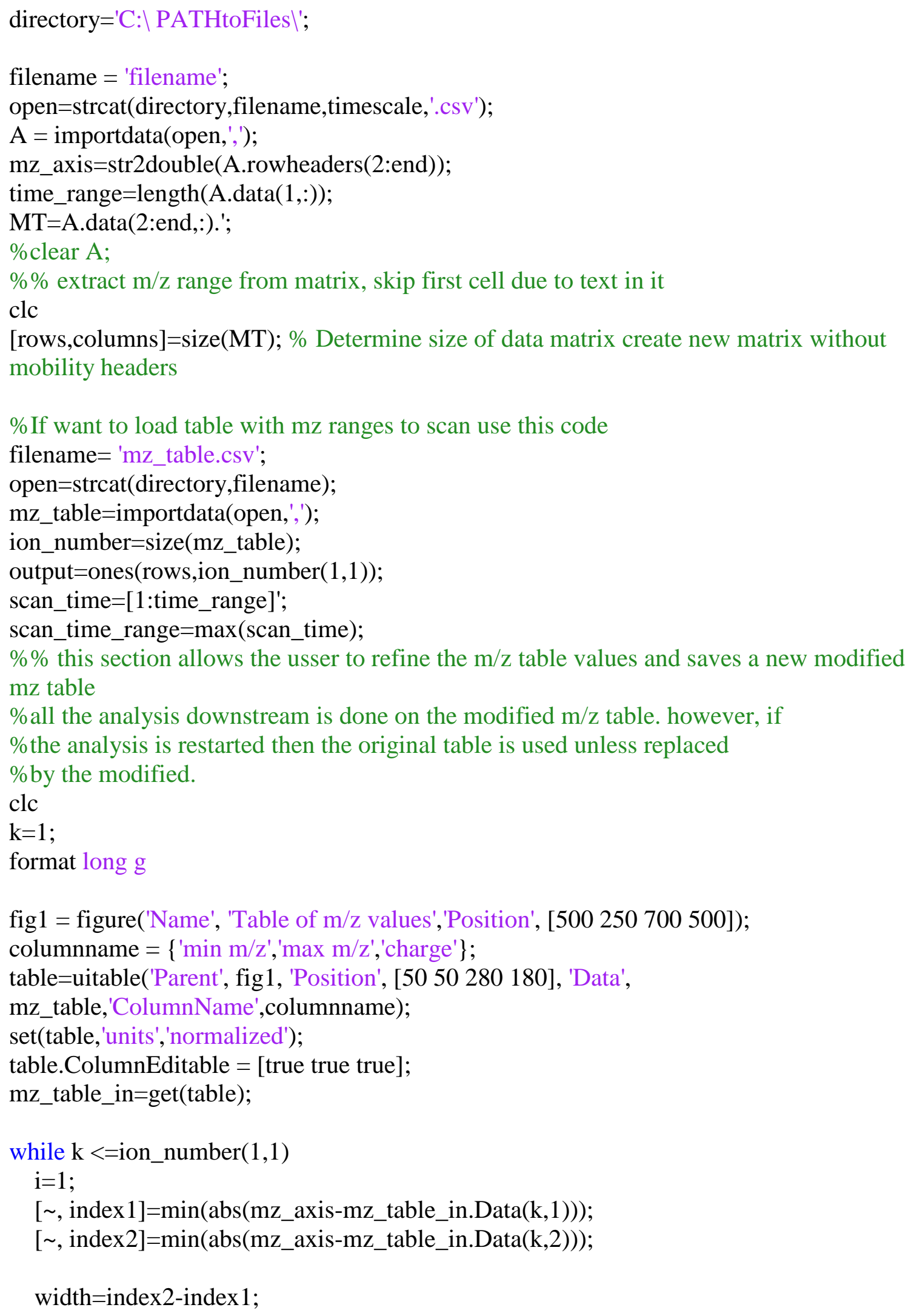




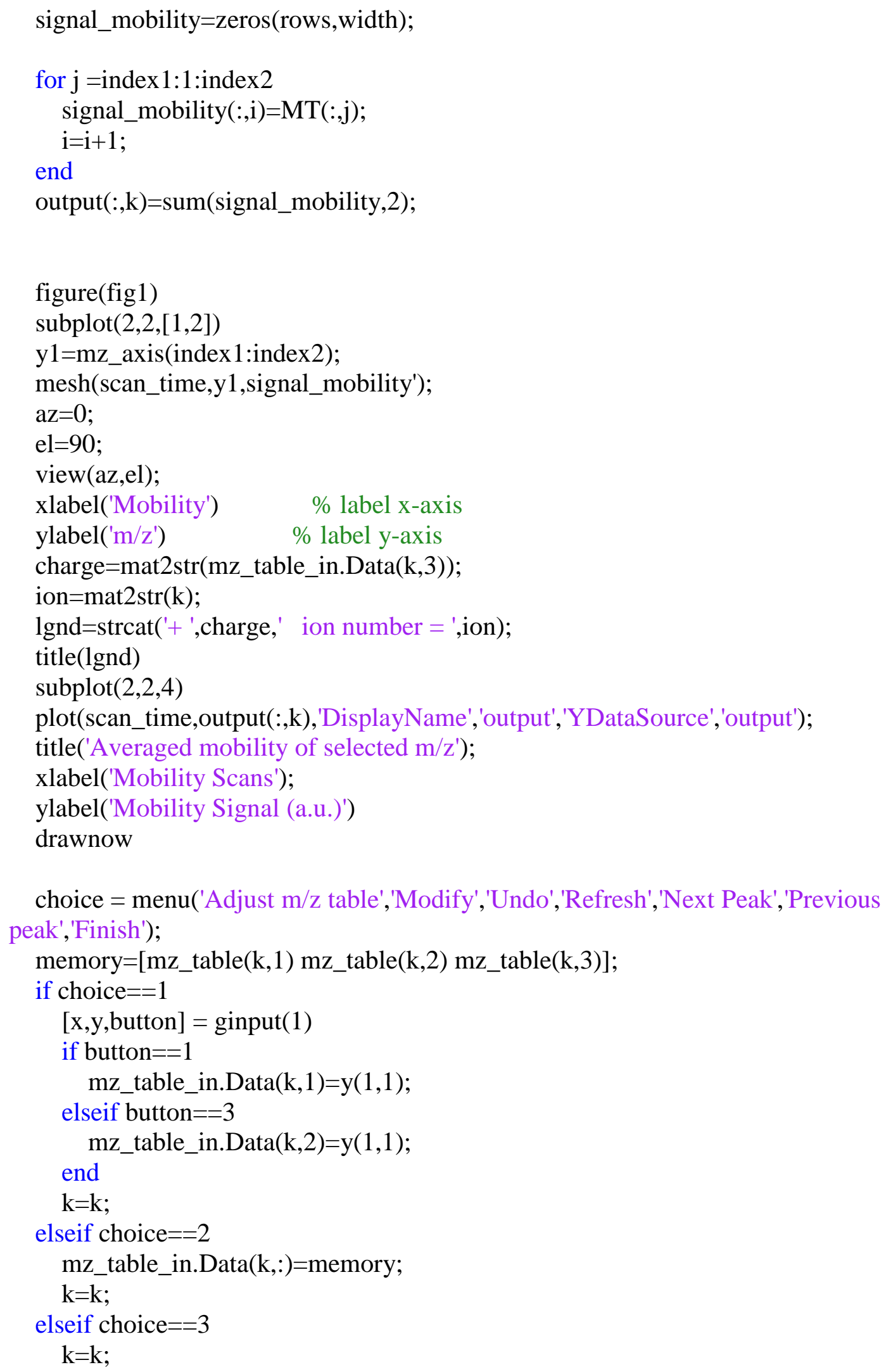




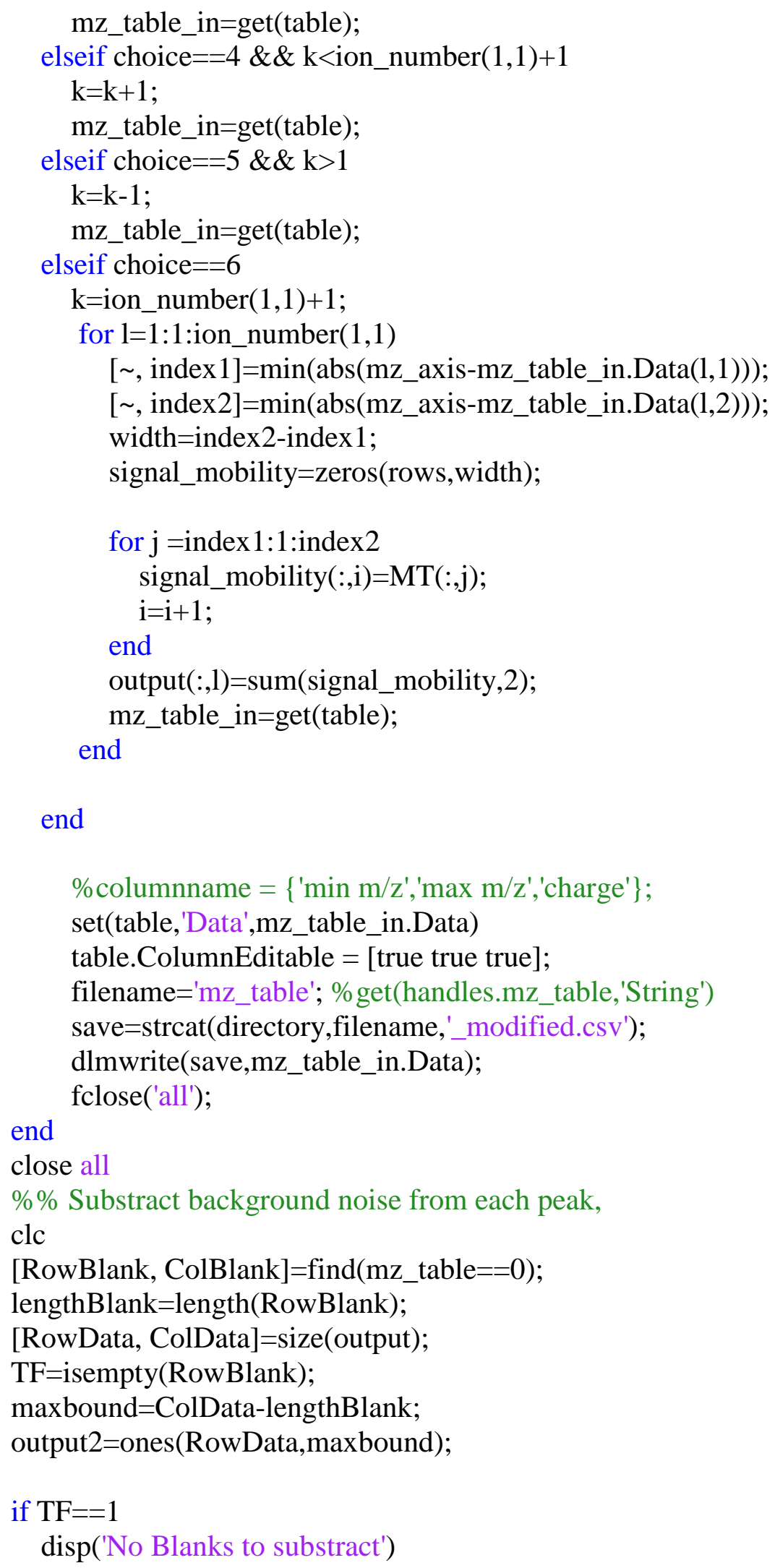




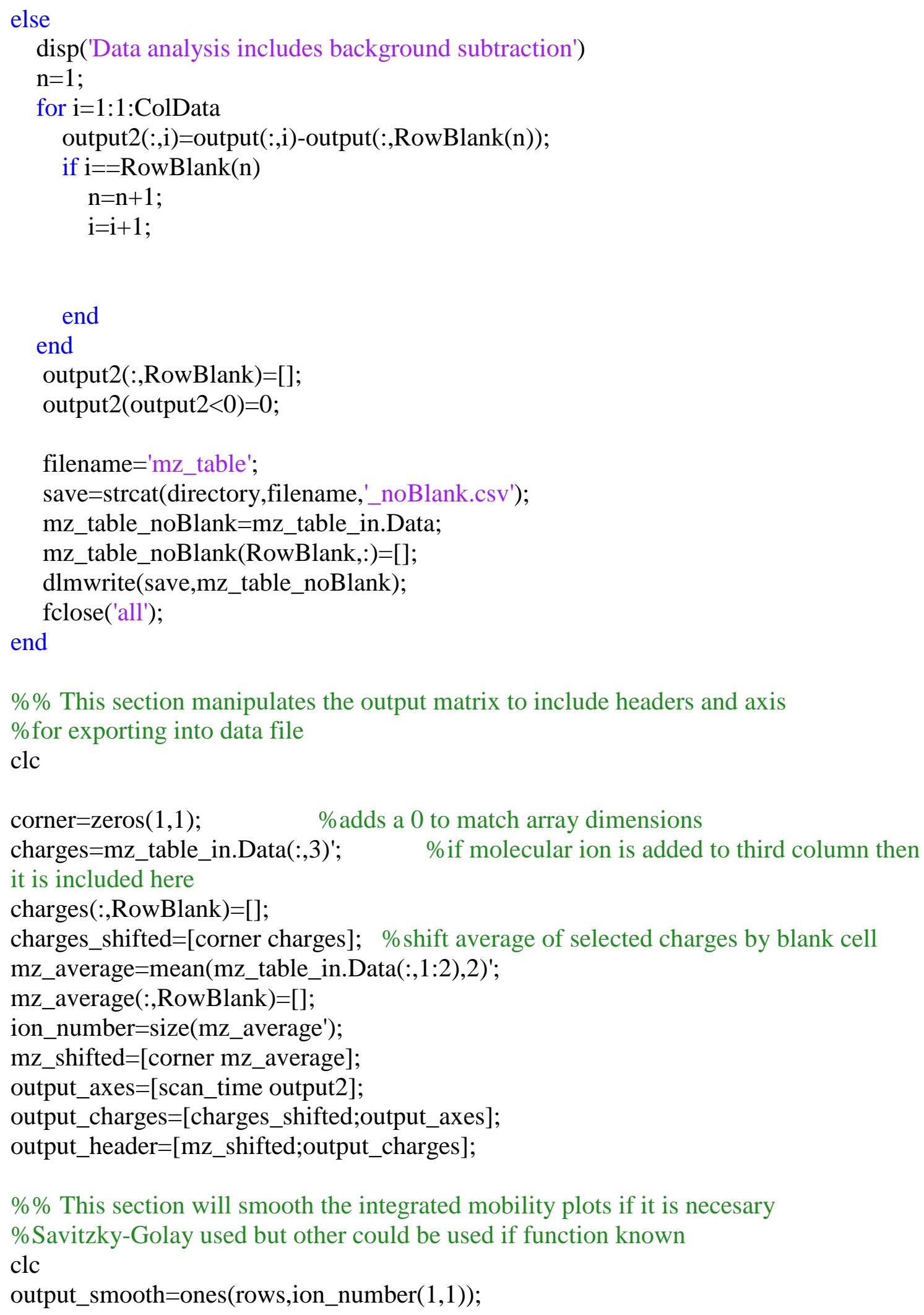




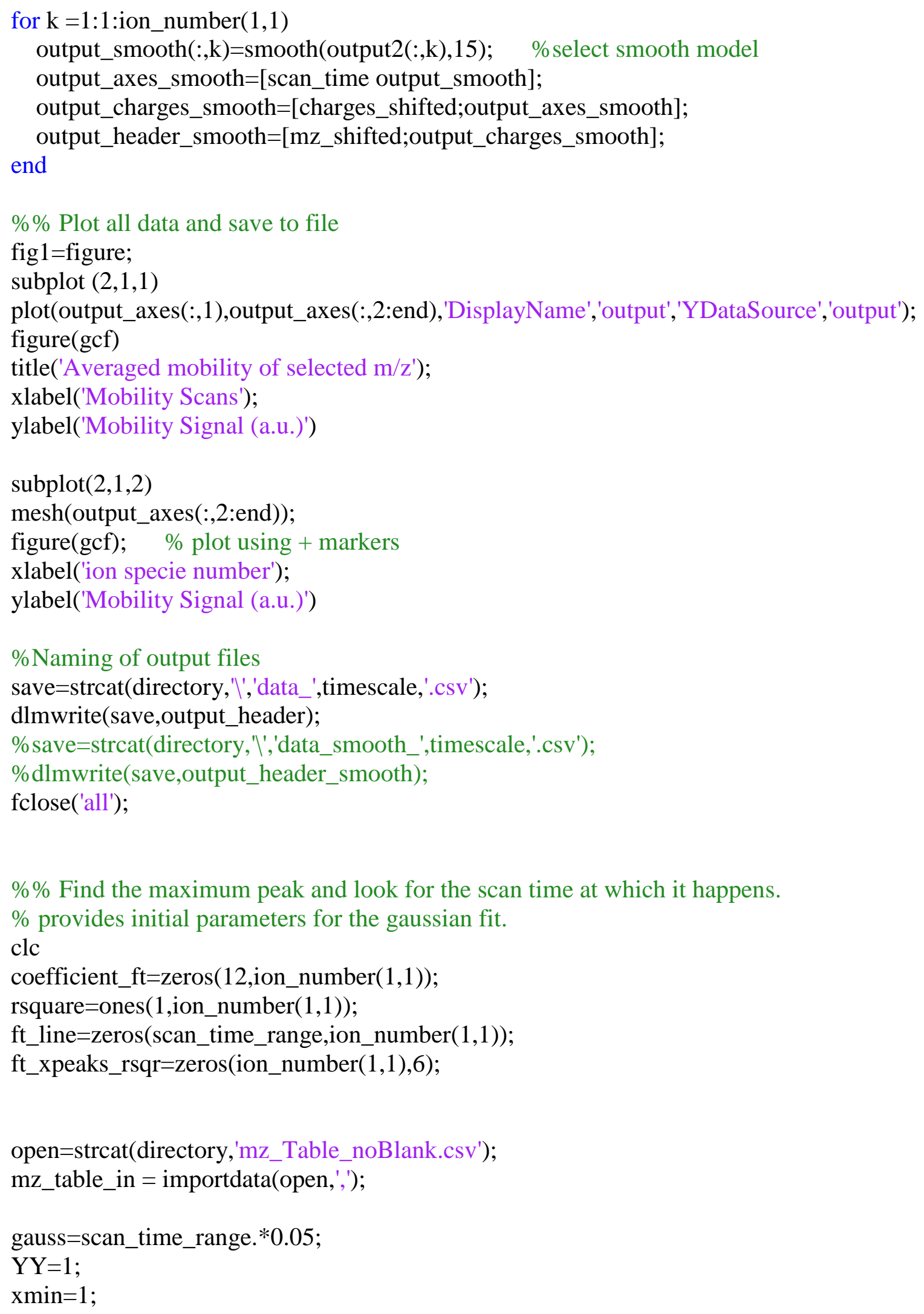


xmax=scan_time_range;

\%fig2=figure('Name', '3-D Mobility plot','Position', [20 60600 400]);

\%fig3=figure('Name','Fitting','Position', [600 601200 700]);

while $\mathrm{YY}<=$ ion_number(1,1)

\%xmin=str2num(get(handles.xmin,'String'));

\%xmax=str2num(get(handles.xmax,'String'));

[ , index1] $=\min \left(\right.$ abs $\left.\left(m z \_a x i s-m z \_t a b l e \_i n(Y Y, 1)\right)\right)$;

$\left[\sim\right.$, index2] $=\min \left(\operatorname{abs}\left(\mathrm{mz} \_a x i s-m z \_t a b l e \_i n(Y Y, 2)\right)\right)$;

$\mathrm{i}=1$;

width=index2-index1;

signal_mobility=zeros(rows,width);

for $\mathrm{j}=$ index1:1:index2

signal_mobility(:,i)=MT(:,j);

$\mathrm{i}=\mathrm{i}+1$;

end

fig2=figure('Name', '3-D Mobility plot','Position', [20 60600 400]);

$\mathrm{y}=\mathrm{mz} \_$axis(index1:index2);

mesh(scan_time,y,signal_mobility');

$\mathrm{az}=0$

el =90;

view(az, el);

xlabel('Mobility') \% label x-axis

ylabel('m/z') \% label y-axis

zlabel('Intensity') \% label z-axis

fig3=figure('Name','Fitting','Position', [600 601200 700]);

subplot $(2,1,1)$

plot(output_axes(xmin:xmax,1),output2(xmin:xmax,YY));

fig3_obj = datacursormode(fig3);

set(fig3_obj,'DisplayStyle','datatip','SnapToDataVertex','off','Enable','on')

ion=mat2str(YY);

charge $=$ mat2str(mz_table_in(YY,3));

lgnd=strcat('+ ',charge,' ion number:',ion);

legend(lgnd)

xlabel('Mobility - Scan')

ylabel('Signal')

title('Ion currently being fitted')

if $\mathrm{YY}<=$ ion_number(1,1)-1

subplot $(2,2,4)$ 
plot(output_axes(:,1),output2(:,YY+1));

ion=mat2str(YY+1);

charge $=$ mat2str(mz_table_in $(Y Y+1,3))$;

lgnd=strcat('+ ',charge,' ion number:',ion);

legend(lgnd)

xlabel('Mobility - Scan')

ylabel('Signal')

title('Next ion to be fitted')

end

columnname $=\{$ 'm/z','Peak 1','Peak 2','Peak3 3','Peak 4','R-square' $\}$;

$\mathrm{ft}$ xpeaks_rsqr=[mz_average' coefficient_ft(2,:)' coefficient_ft(5,:)'

coefficient_ft(8,:)' coefficient_ft(11,:)' rsquare'];

table2=uitable('Parent', fig3, 'Position', [120 50500 280], 'Data',

ft_xpeaks_rsqr,'ColumnName',columnname);

table2.ColumnEditable = [true true true];

ft_xpeaks_rsqr=get(table2);

choice = menu('Number of gaussians','1 Peaks','2 Peaks','3 Peaks','4 Peaks','Go Back 1 ion','Go Forward 1 ion','Redraw','Auto-Fit','Manual','Finish','Quit')

[xData, yData] = prepareCurveData(output_axes(xmin:xmax,1), output2(xmin:Xmax,YY));

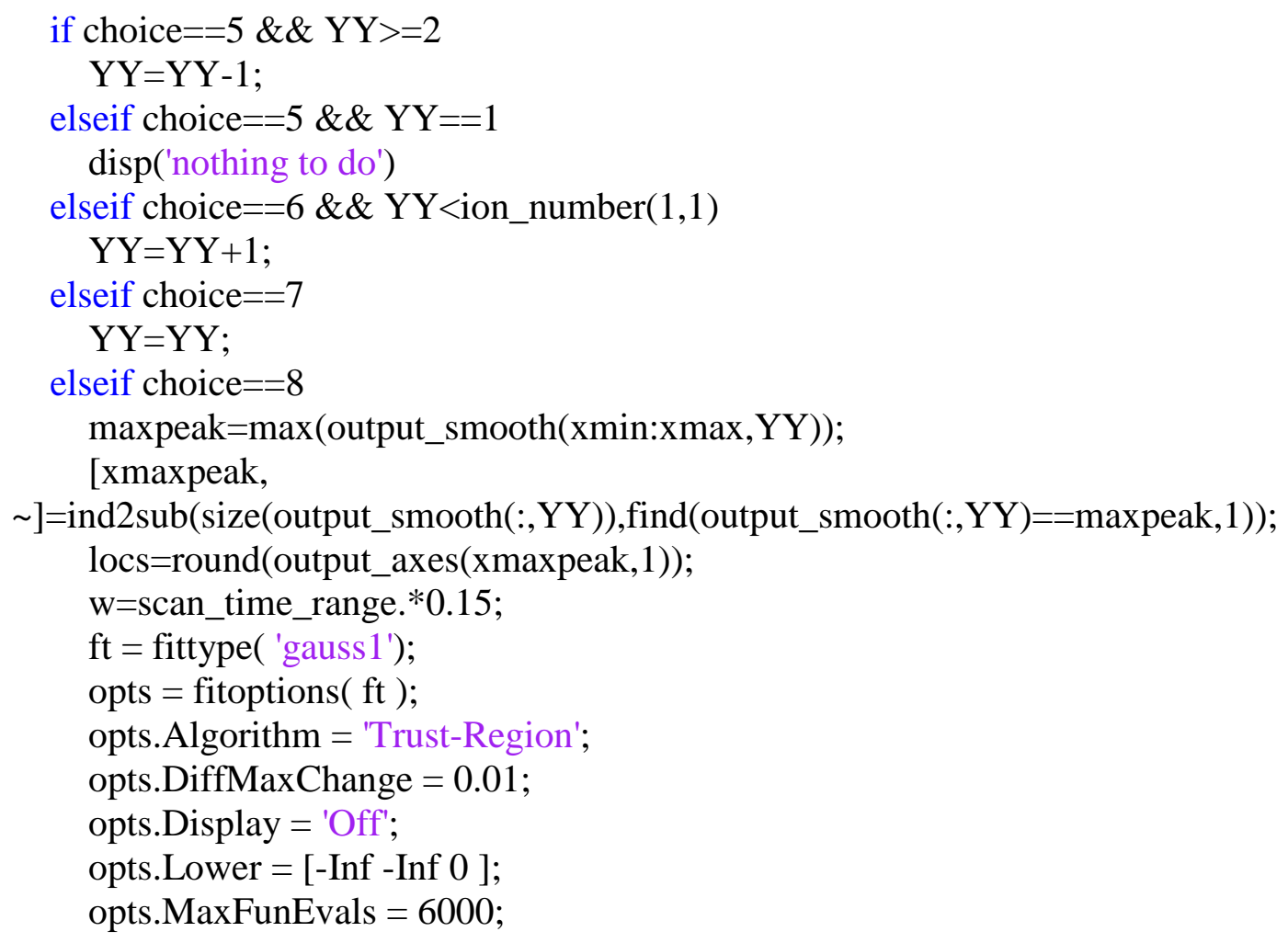




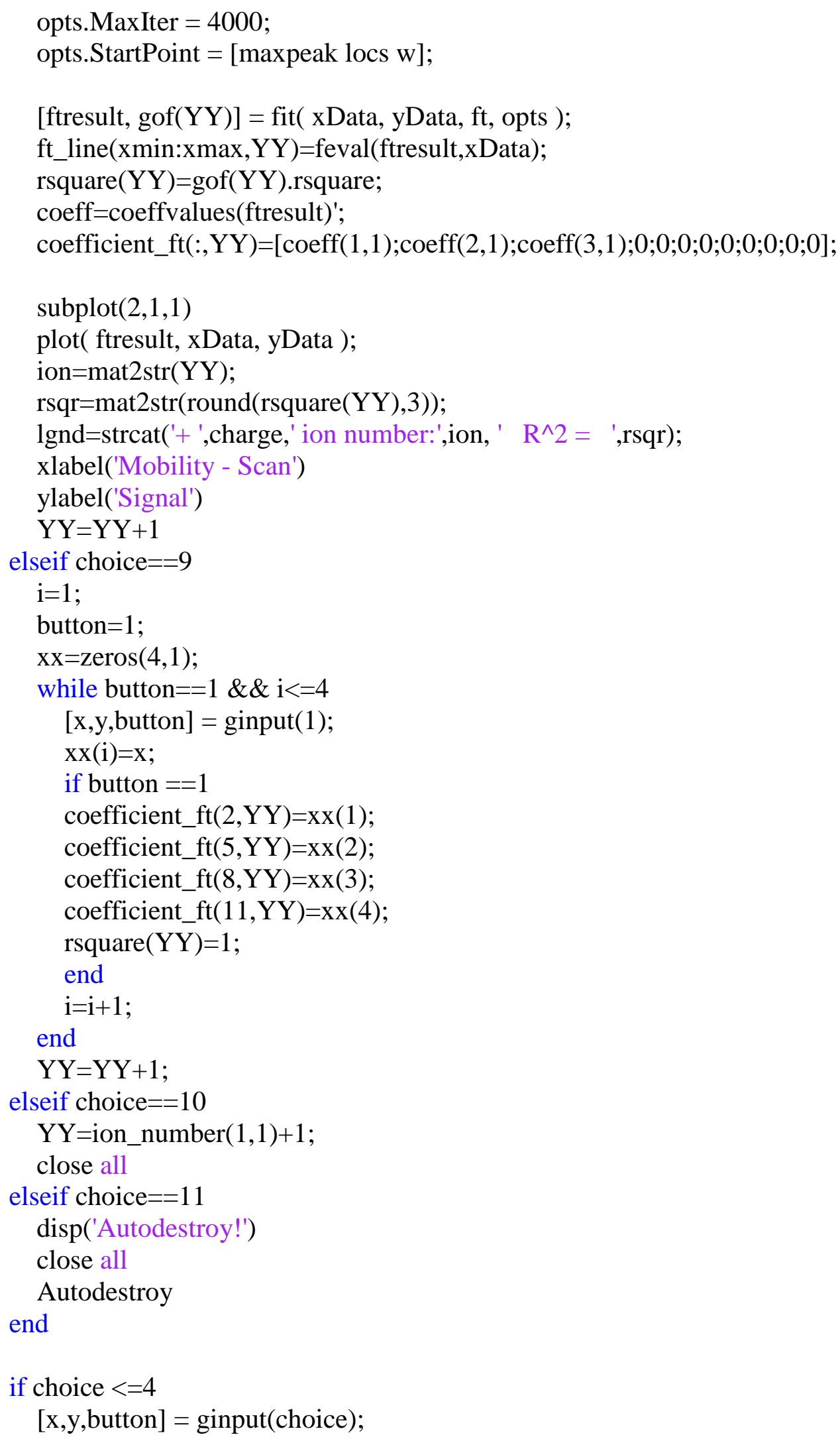




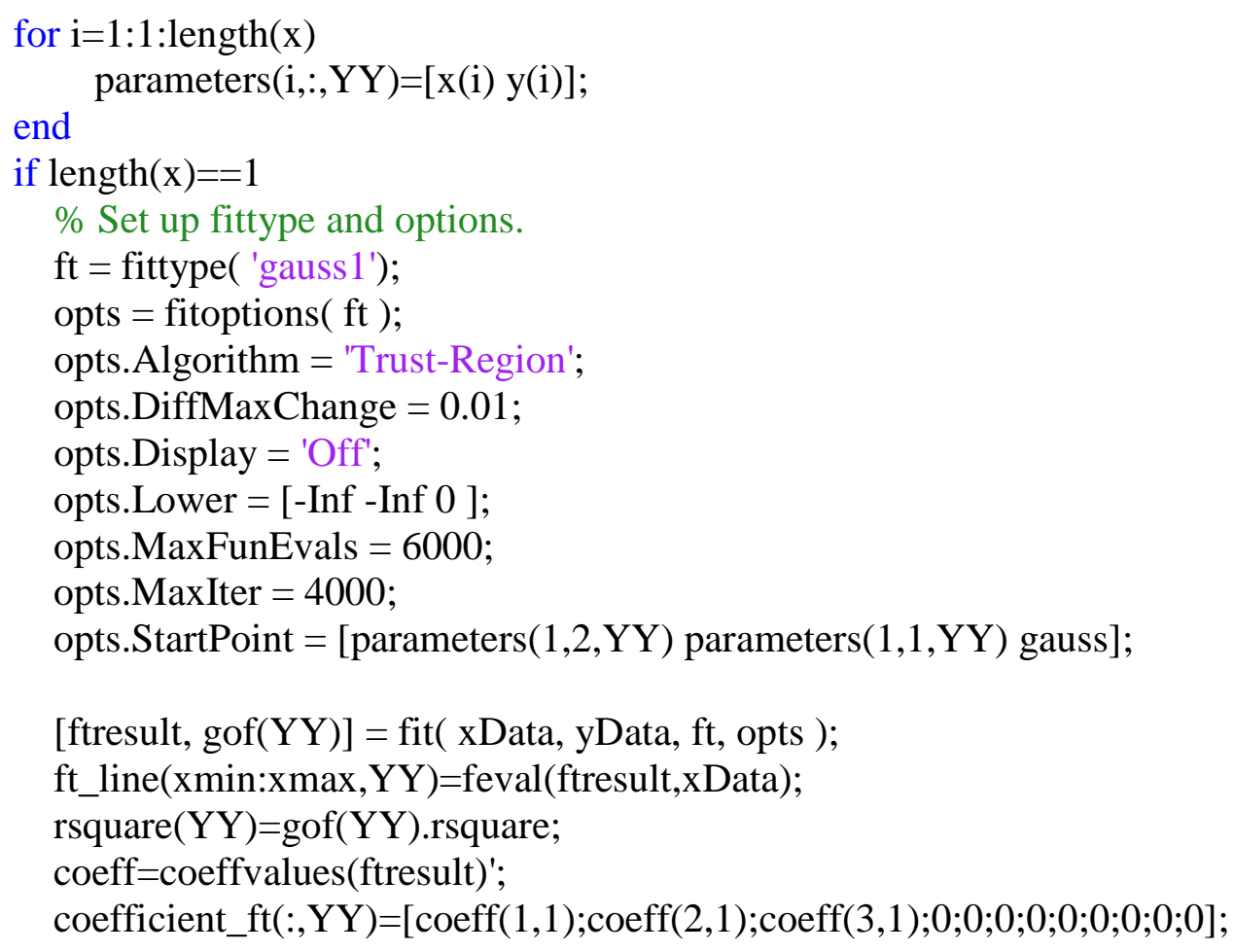

end

if length $(x)==2$

$\%$ Set up fittype and options. 


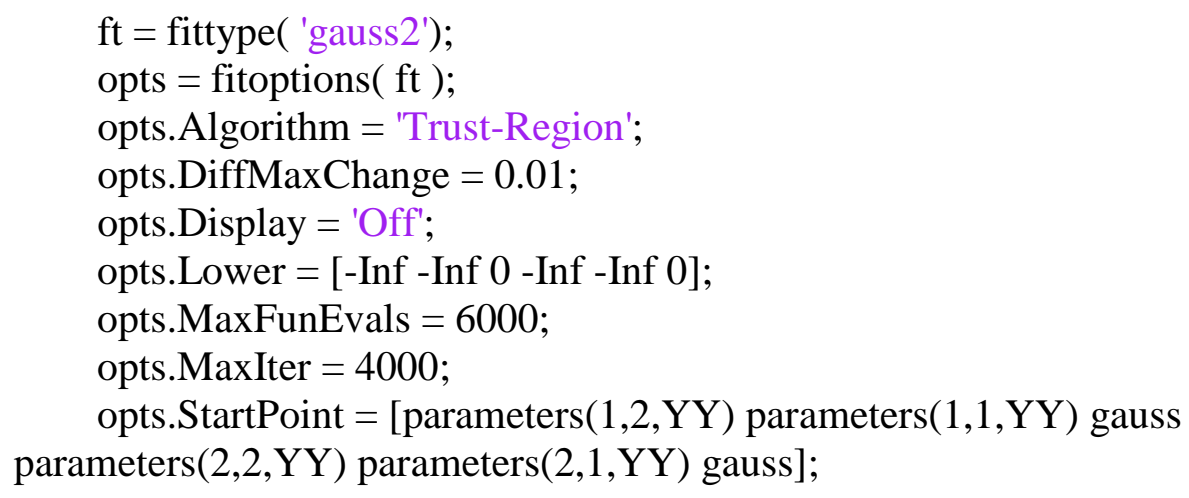

[ftresult, gof(YY)] = fit ( xData, yData, ft, opts );

ft_line(xmin:xmax, $Y Y)=$ feval(ftresult, $x$ Data);

rsquare $(Y Y)=\operatorname{gof}(Y Y)$.rsquare;

coeff=coeffvalues(ftresult)';

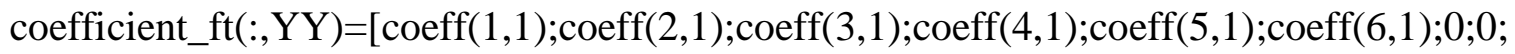
$0 ; 0 ; 0 ; 0]$

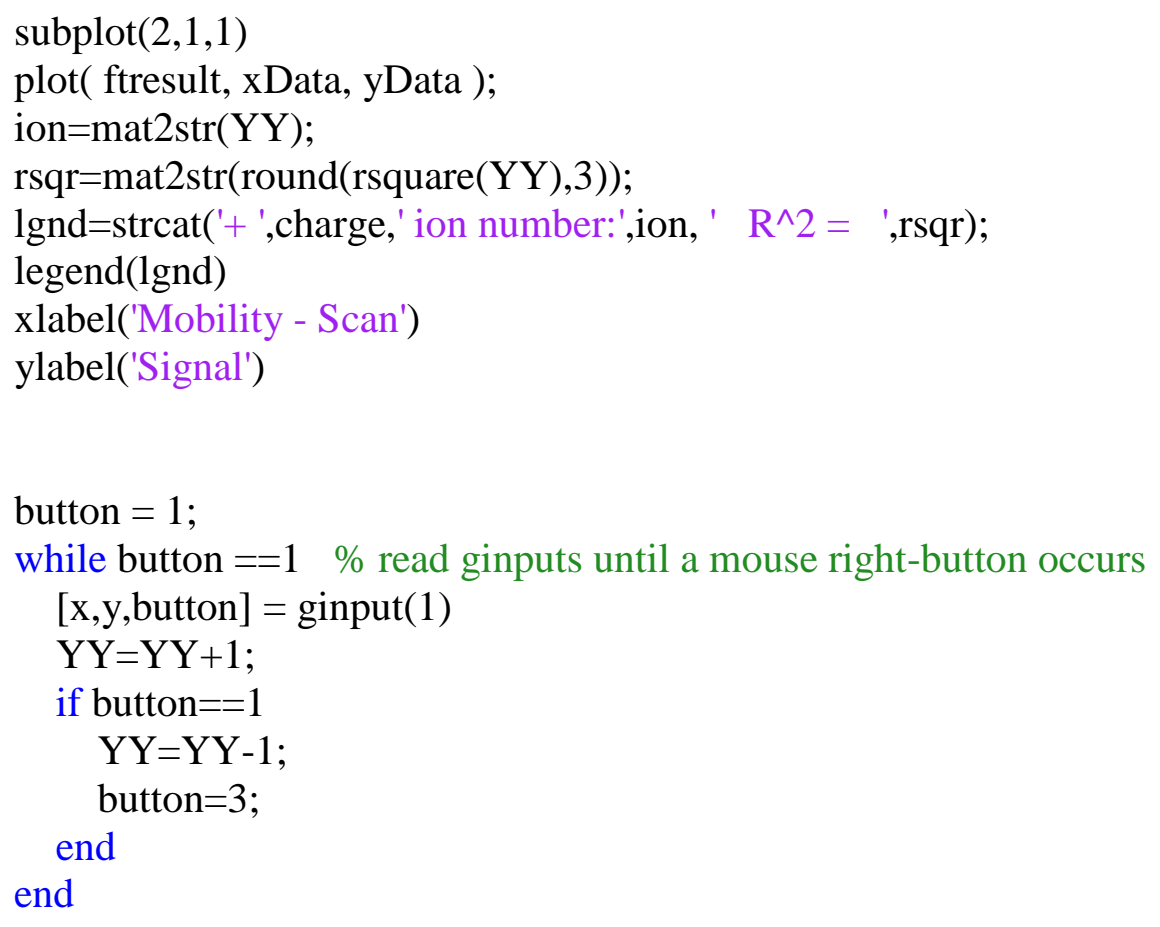

end

if length $(x)==3$

$\%$ Set up fittype and options.

$\mathrm{ft}=$ fittype( 'gauss3'); 
opts = fitoptions $(\mathrm{ft})$;

opts.Algorithm = 'Trust-Region';

opts.DiffMaxChange $=0.01$;

opts. Display = 'Off';

opts.Lower = [-Inf -Inf 0 -Inf -Inf 0 -Inf -Inf 0];

opts.MaxFunEvals = 6000;

opts. MaxIter = 4000;

opts.StartPoint $=$ [parameters $(1,2, Y Y)$ parameters $(1,1, Y Y)$ gauss

parameters $(2,2, Y Y)$ parameters $(2,1, Y Y)$ gauss parameters(3,2,YY) parameters(3,1,YY) gauss];

[ftresult, gof(YY)] = fit( $x$ Data, yData, ft, opts );

ft_line(xmin:xmax, $Y Y)=f e v a l(f t r e s u l t, x D a t a) ;$

rsquare $(Y Y)=\operatorname{gof}(Y Y)$.rsquare;

coeff=coeffvalues(ftresult)';

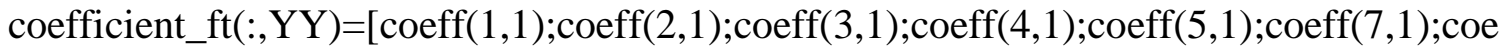
ff(8,1); $\operatorname{coeff}(9,1)$; $\operatorname{coeff}(6,1) ; 0 ; 0 ; 0]$;

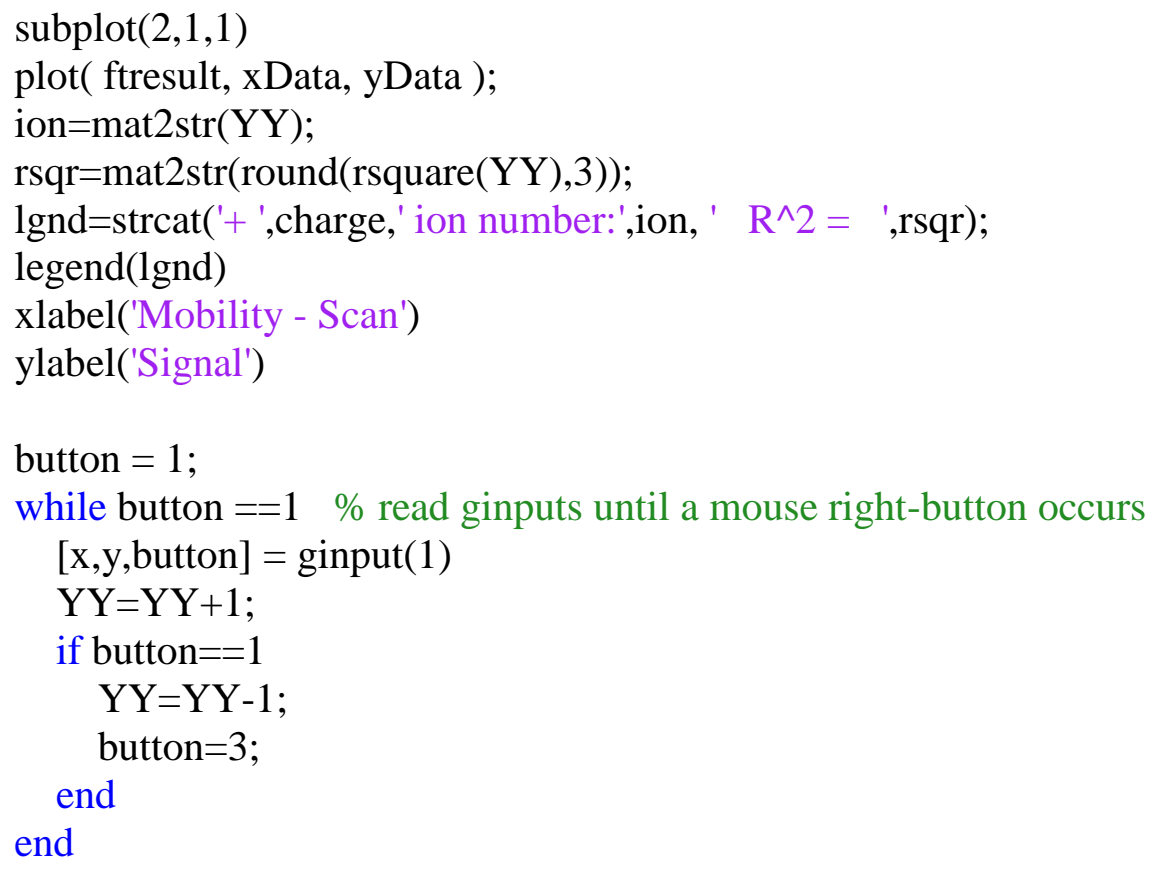


opts.Algorithm = 'Trust-Region';

opts.DiffMaxChange $=0.01$;

opts.Display = 'Off';

opts.Lower = [-Inf -Inf 0 -Inf -Inf 0 -Inf -Inf 0 -Inf -Inf 0];

opts. MaxFunEvals = 6000;

opts. MaxIter = 4000;

opts.StartPoint $=$ [parameters $(1,2, \mathrm{YY})$ parameters $(1,1, \mathrm{YY})$ gauss

parameters(2,2,YY) parameters(2,1,YY) gauss parameters(3,2,YY) parameters(3,1,YY) gauss parameters $(4,2, \mathrm{YY})$ parameters $(4,1, \mathrm{YY})$ gauss];

[ftresult, gof(YY)] = fit( $\mathrm{xData}, \mathrm{yData}, \mathrm{ft}$, opts );

$\mathrm{ft}$ _line(xmin:xmax, $\mathrm{YY})=$ feval(ftresult, $\mathrm{xData})$;

rsquare $(Y Y)=\operatorname{gof}(Y Y)$.rsquare;

coeff=coeffvalues(ftresult)';

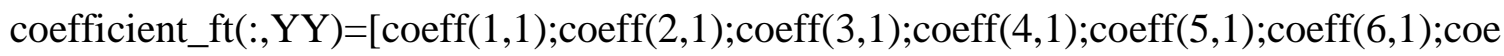
$\mathrm{ff}(7,1)$; $\operatorname{coeff}(8,1)$; $\operatorname{coeff}(9,1)$; $\operatorname{coeff}(10,1)$; $\operatorname{coeff}(11,1)$; $\operatorname{coeff}(12,1)]$;

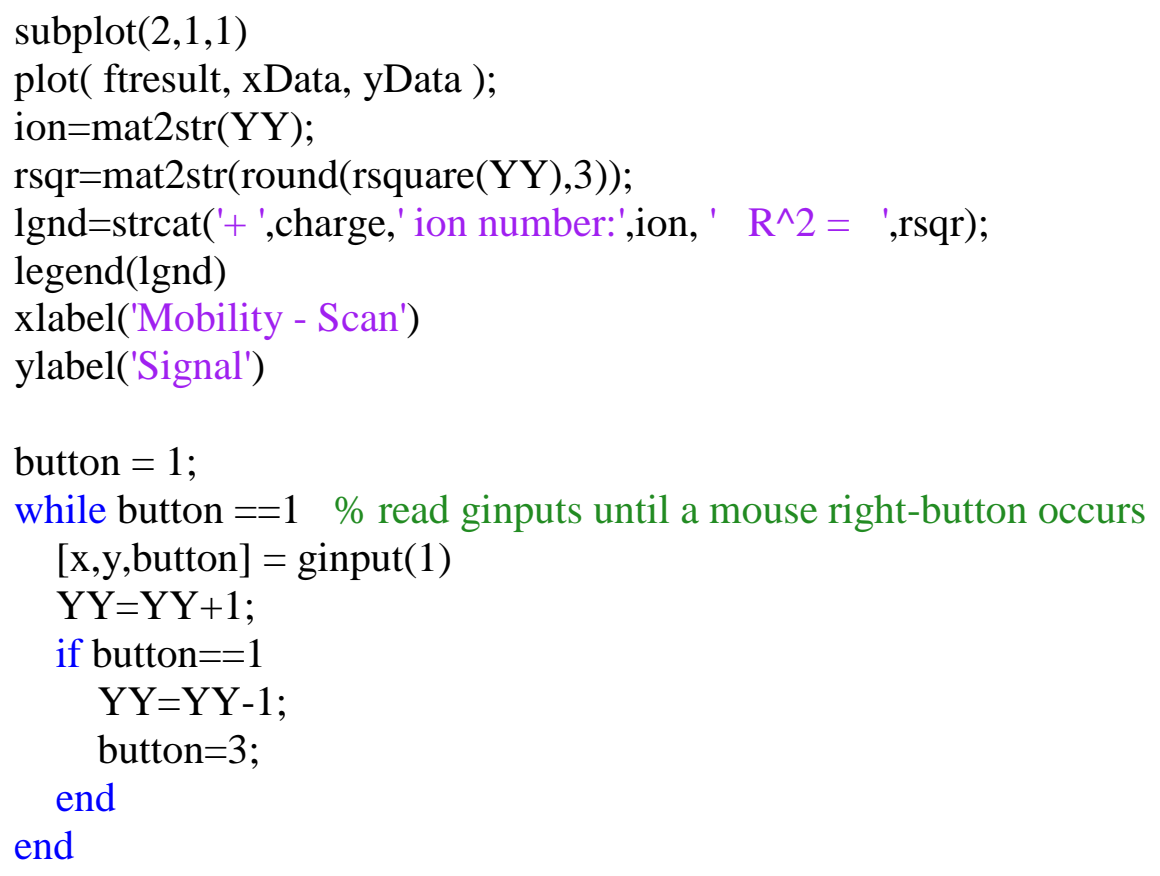


\%ft_line_header=[mz_shifted;ft_line_axes];

\%save=strcat(directory,"','fit_line_,'timescale,'.csv');

\%dlmwrite(save,ft_line_header);

\%fclose('all');

ft_xpeaks_rsqr=[mz_average' coefficient_ft(2,:)' coefficient_ft(5,:)' coefficient_ft(8,:)' rsquare'];

save=strcat(directory,"','mz_Peak_Rsqr_',timescale,'.csv');

dlmwrite(save,ft_xpeaks_rsqr);

fclose('all');

\%notification='No more data to analyze. Fits and peaks saved in files fit_file and mz_Peak_Rsqr';

\%set(handles.notification, 'String',notification);

\% End of Script 1

$\%$ Start of Script 2

$\%$ CCS calculator version 2.0

\%This script was prepared in MATLAB R2011b, and may not work well with latest versions

\%this script has not been yet optimized bu provides the basis for further

\%developmet.

\%Script designed by WGG 01/19/2016

$\%$ Data analsysis to extract CCS from TIMS experiments

\%this script requires the outputs from Singal_extraction_V2 to be present

\%The script is designed to analyze 5 timescales (100 200300400 and 500ms)

clc

clear

$\%$

directory='PATHtoFILEl';

for $\mathrm{mmm}=100: 100: 500$

timescale_export=mmm;

100, 200, 300 etc and F5

voltage $=-42$;

\%calibration=-125.8;

$\%<<<<<<<$ only need to change this number to

calibration=-101;

peak_number $=1$;

\%TIMS_ramp_duration=[400;650;800;1200;1600]; \%ramp times used

TIMS_ramp_duration=[705;1205;1905;2605;3405]; \%ramp times used

P0_average $=0 ; \quad \%$ if 0 then determined for each ion, otherwise obtained assuming a gaussian distribution of $\mathrm{P} 0$ vs ion

\%Import extracted data 


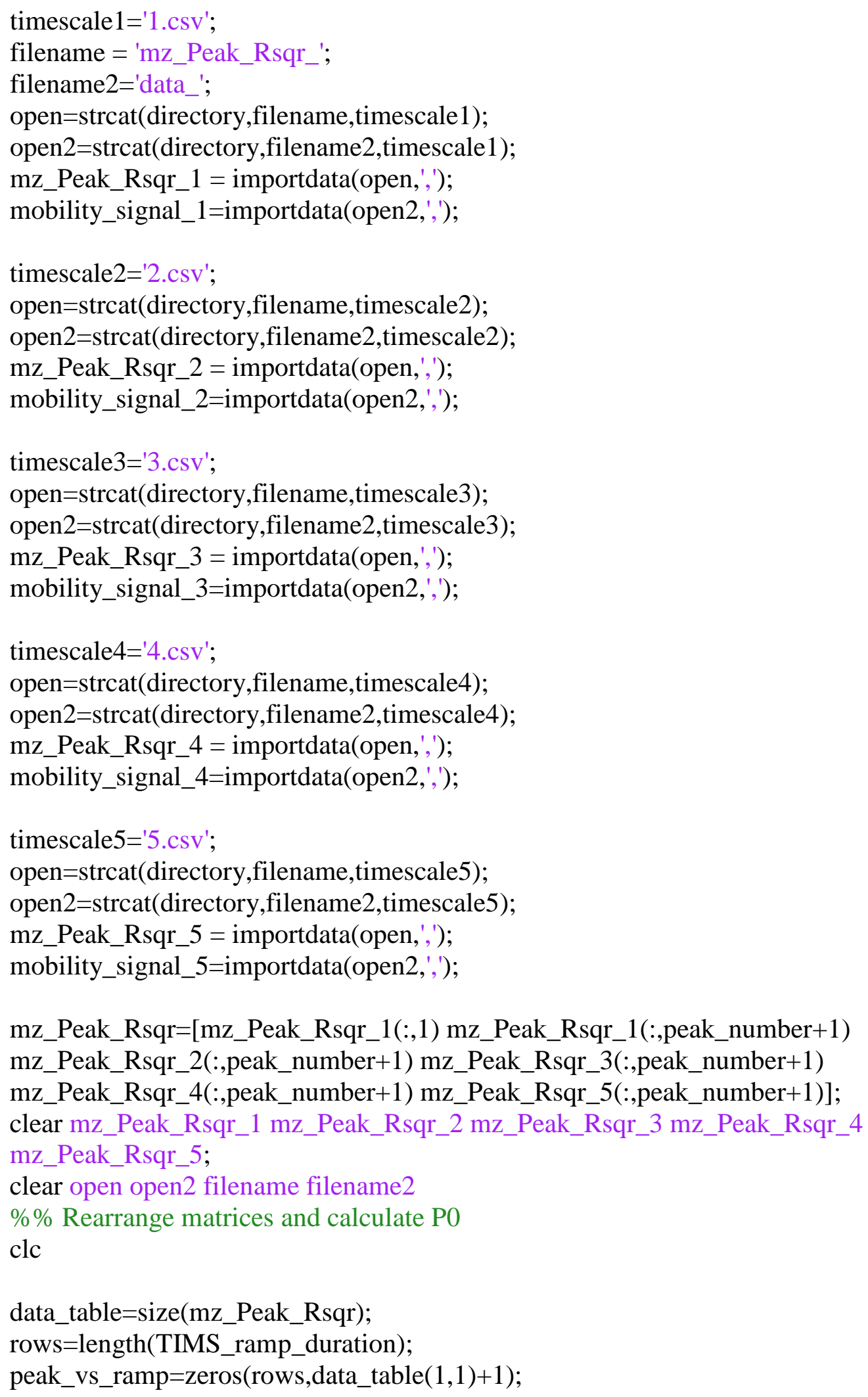




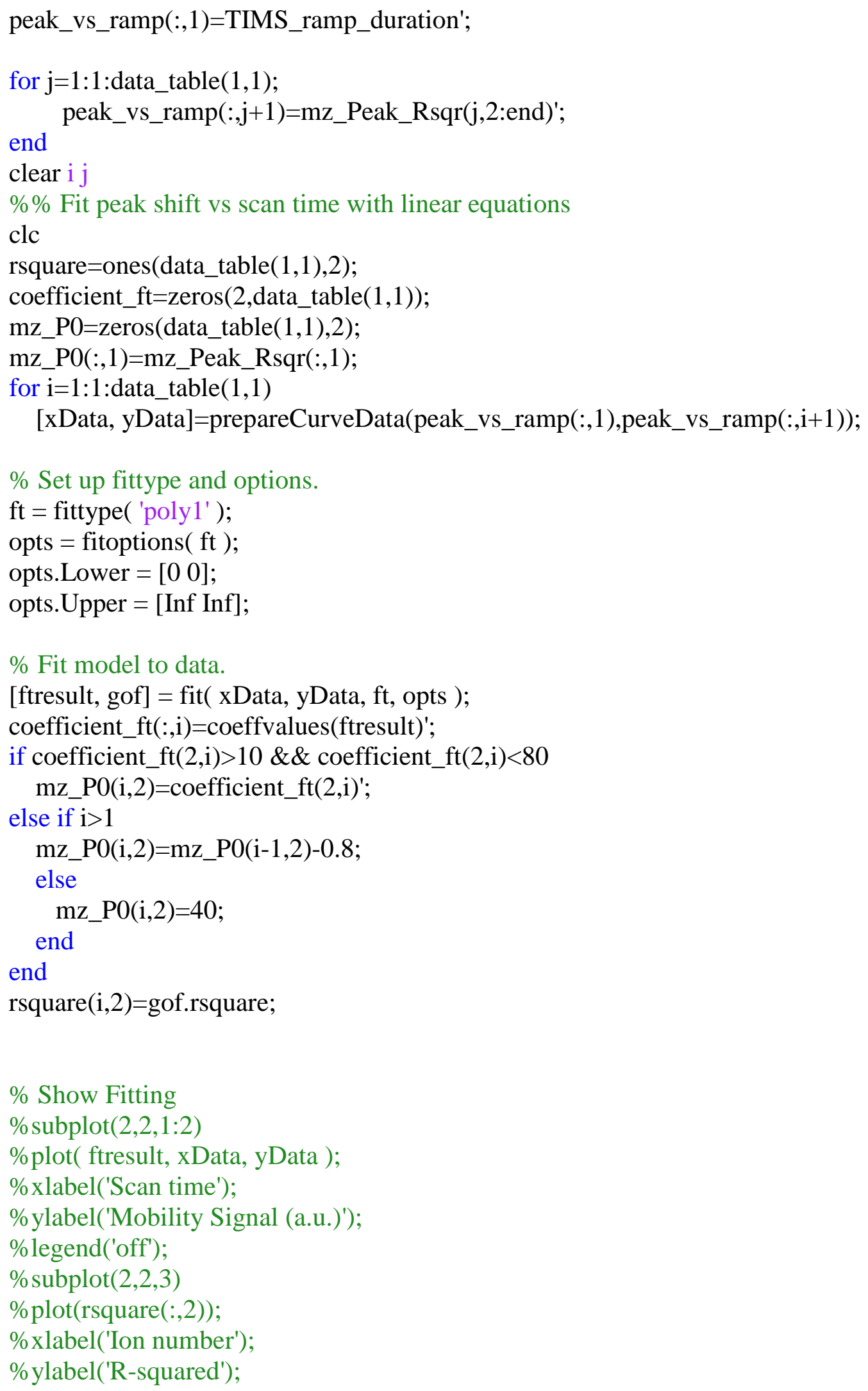




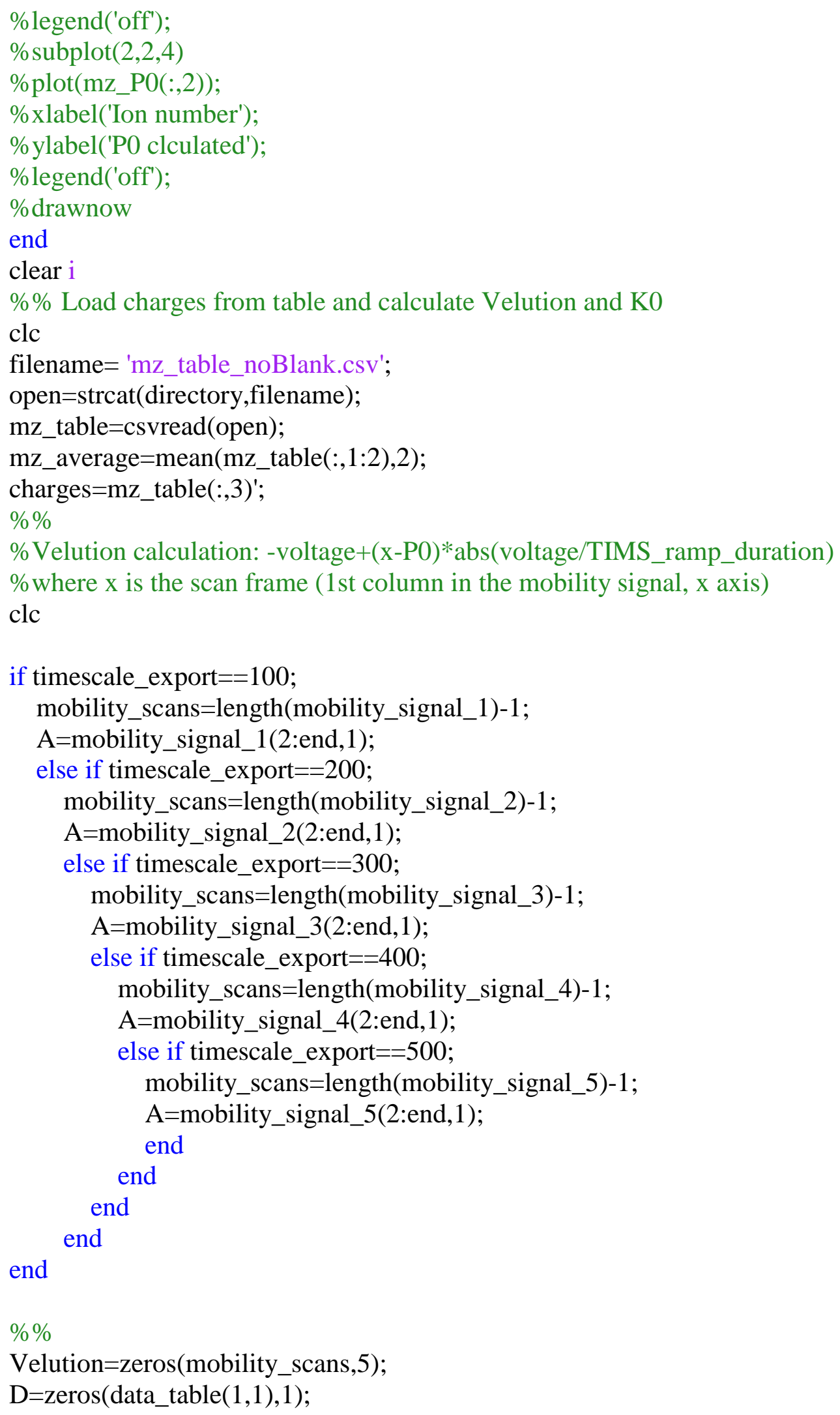




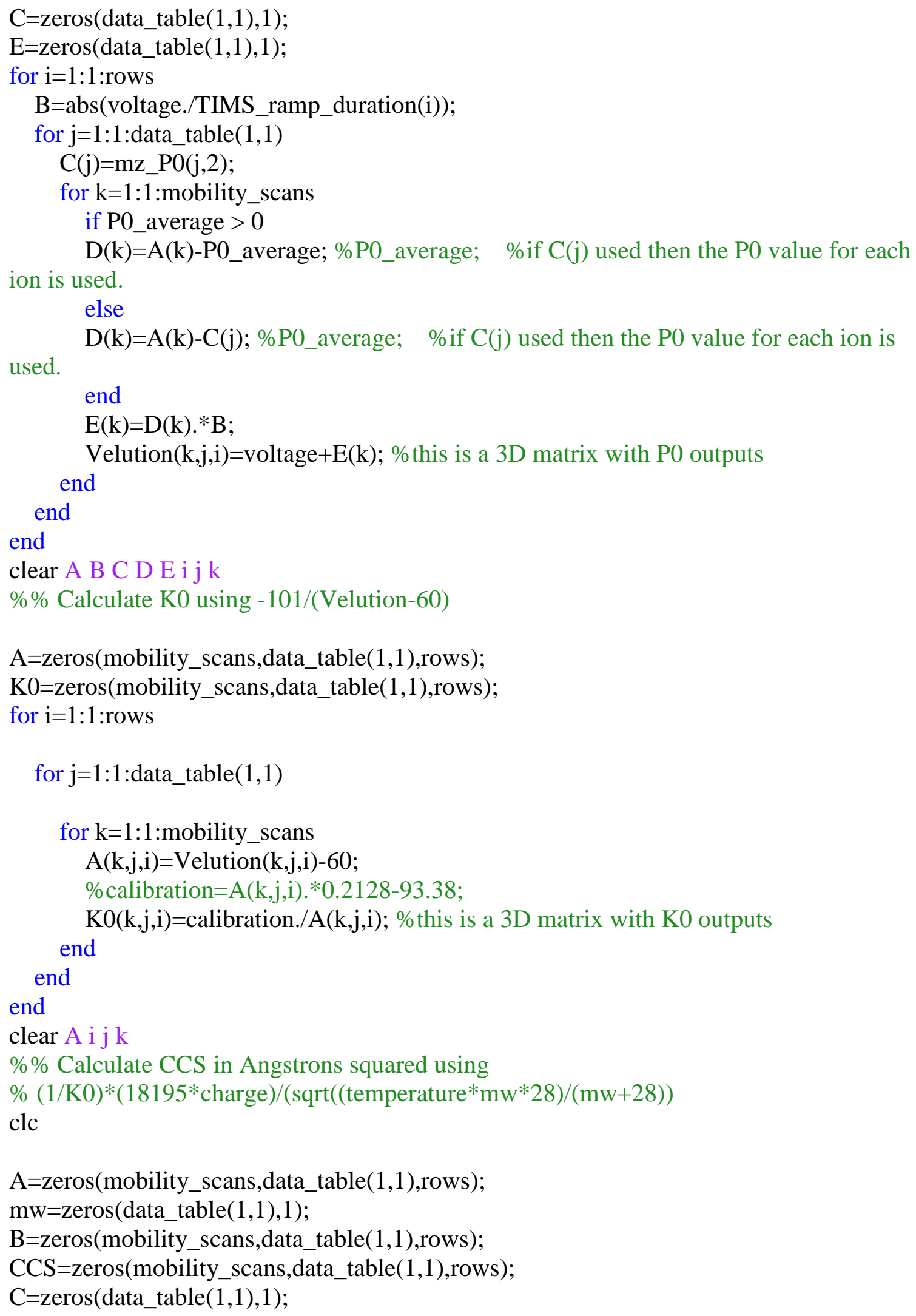




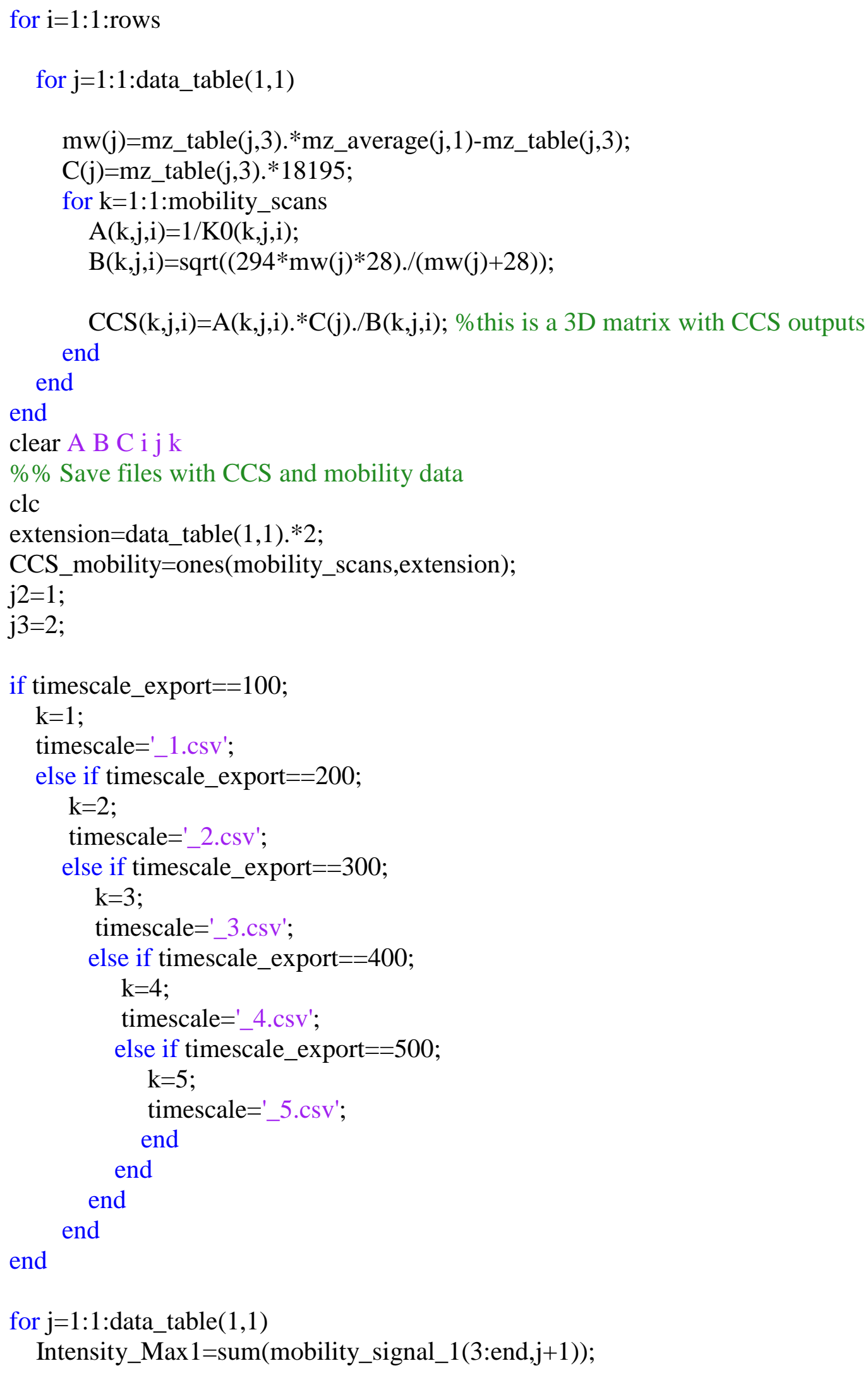


Intensity_Max2=sum(mobility_signal_2(3:end,j+1));

Intensity_Max3=sum(mobility_signal_3(3:end,j+1));

Intensity_Max4=sum(mobility_signal_4(3:end,j+1));

Intensity_Max5=sum(mobility_signal_5(3:end,j+1));

CCS_mobility(:,j2)=CCS(:,j,k); \%\%\%\%\%\%\%\%\%\%\% \%increase last axes for diff timescales

$\mathrm{j} 2=\mathrm{j} 2+2$

if timescale_export==100;

CCS_mobility(:,j3)=mobility_signal_1(2:end,j+1)./Intensity_Max1;

$\% \% \% \% \% \% \% \% \%$

else if timescale_export $==200$;

CCS_mobility(:,j3)=mobility_signal_2(2:end,j+1)./Intensity_Max2;

$\% \% \% \% \% \% \% \% \%$

else if timescale_export $==300$;

CCS_mobility(:,j3)=mobility_signal_3(2:end,j+1)./Intensity_Max3;

$\% \% \% \% \% \% \% \% \%$

else if timescale_export $==400$;

CCS_mobility(:,j3)=mobility_signal_4(2:end,j+1)./Intensity_Max4;

$\% \% \% \% \% \% \% \% \% \%$

else if timescale_export $==500$;

CCS_mobility(:,j3)=mobility_signal_5(2:end,j+1)./Intensity_Max5;

$\% \% \% \% \% \% \% \% \% \%$

end

end

end

end

end

$\mathrm{j} 3=\mathrm{j} 3+2$;

end

filename='Mobility_CCS';

if P0_average $>0$

P0set=num2str(P0_average);

save=strcat(directory,filename,'_',P0set,timescale);

dlmwrite(save,CCS_mobility);

fclose('all');

disp(strcat(filename,timescale));

else

pks=num2str(peak_number);

save=strcat(directory,filename,'_,'pks,timescale);

dlmwrite(save,CCS_mobility);

fclose('all');

disp(strcat(filename,timescale));

end 


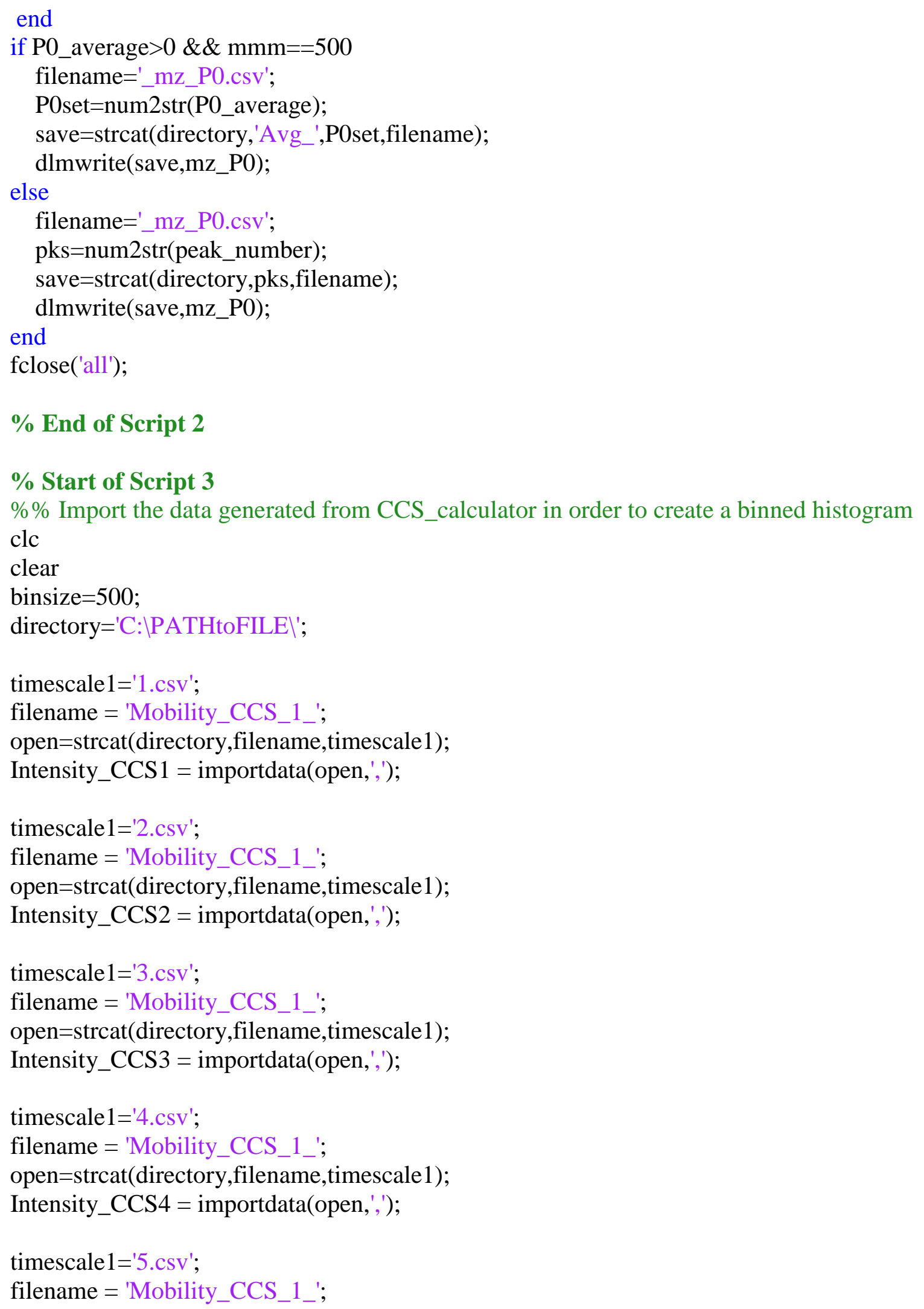




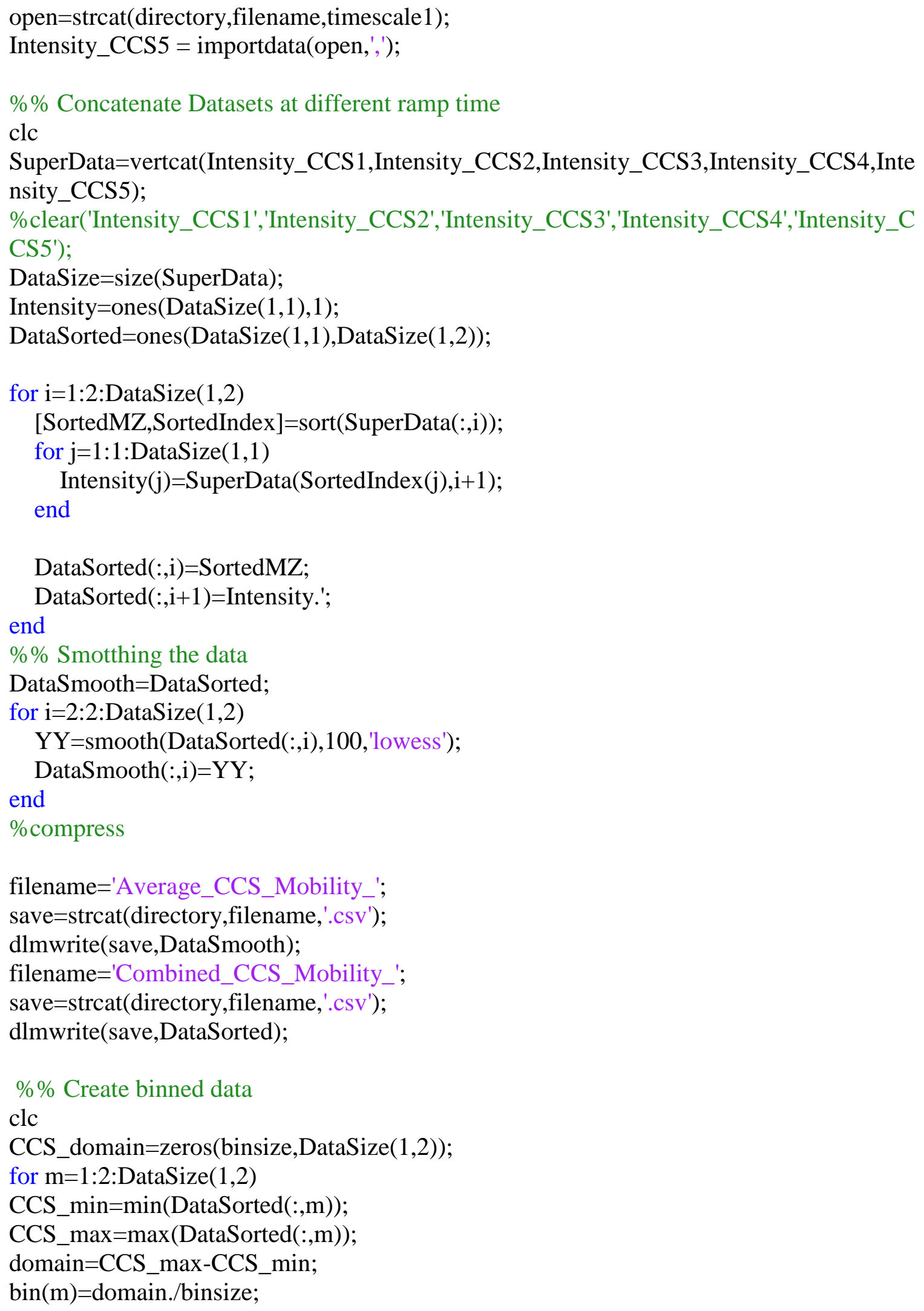




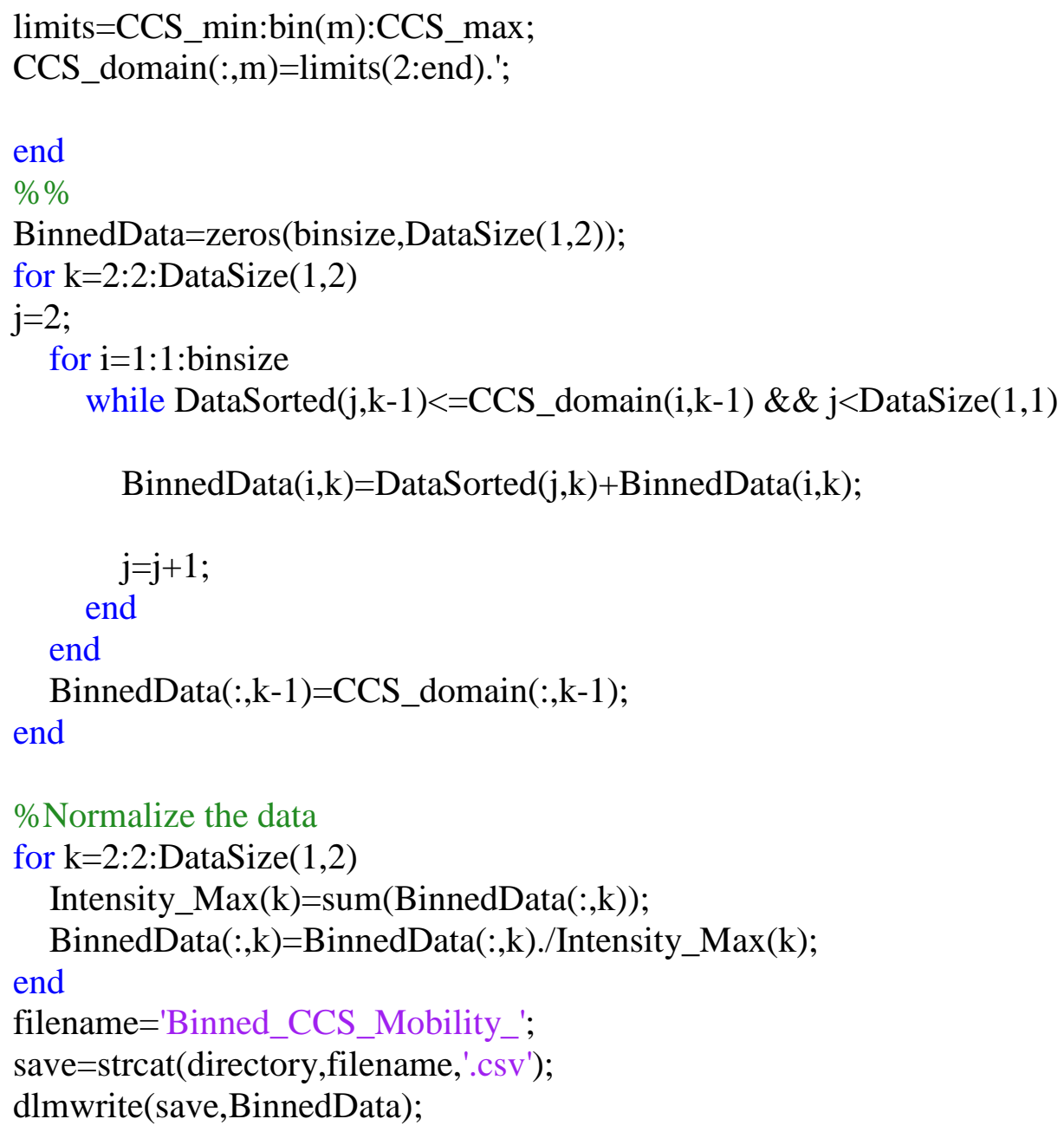

\subsection{Appendix 3}

Scripts used for preparing the system and performing the cMD and aMD simulations are shown below, note that these must be copied into independent configurations file for them to be run by NAMD.

\#\#\#\#\#\#\#\#\#\#\#\#\#\#\#\#\#\# MINIMIZATION\#\#\#\#\#\#\#\#\#\#\#\#\#\#\#\#\#\#\#\#

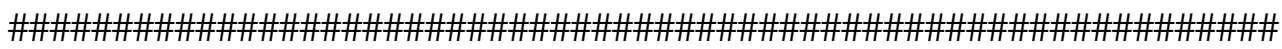
\#\# ADJUSTABLE PARAMETERS

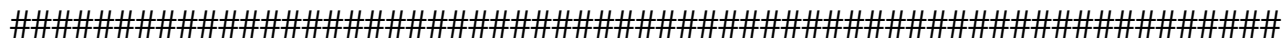




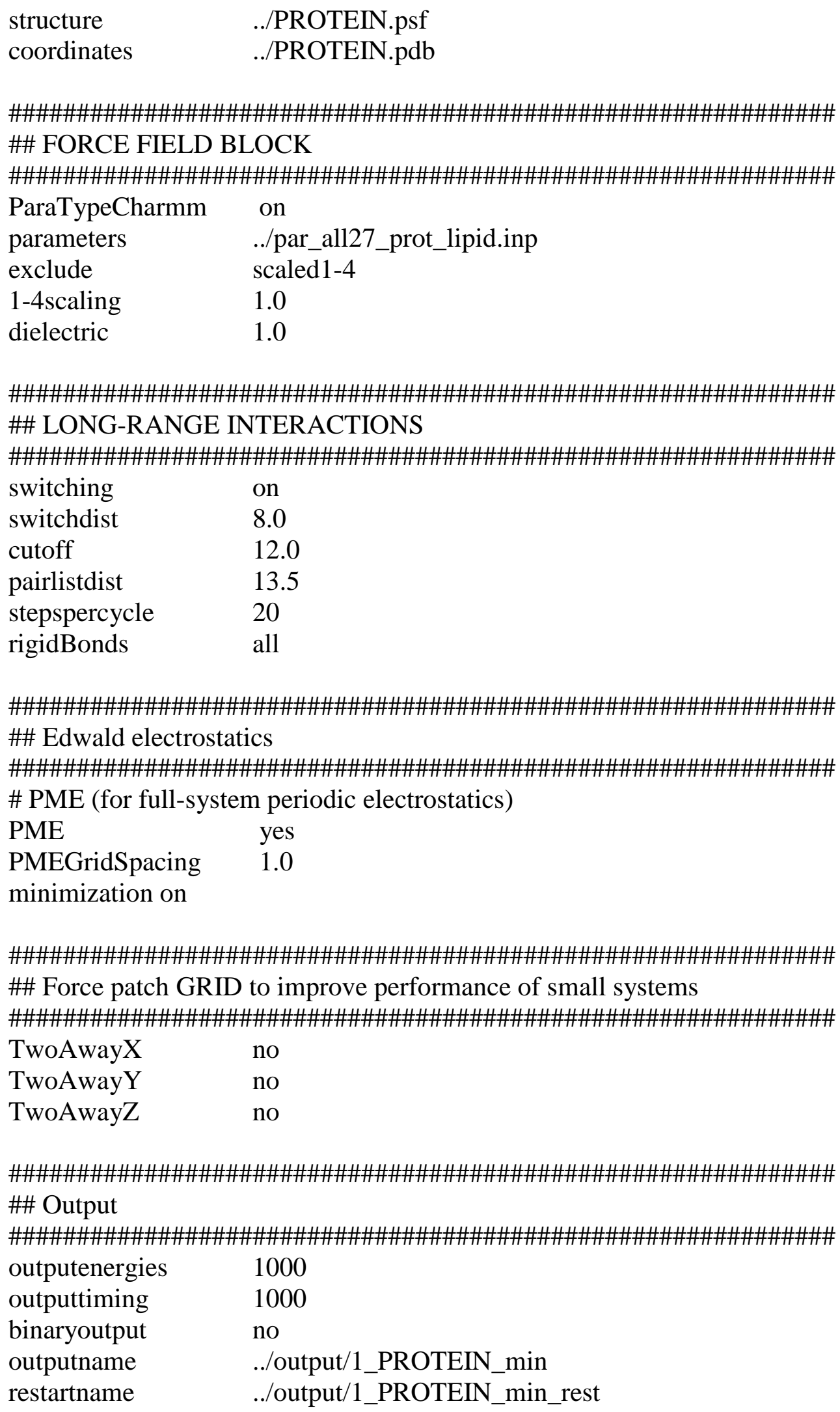




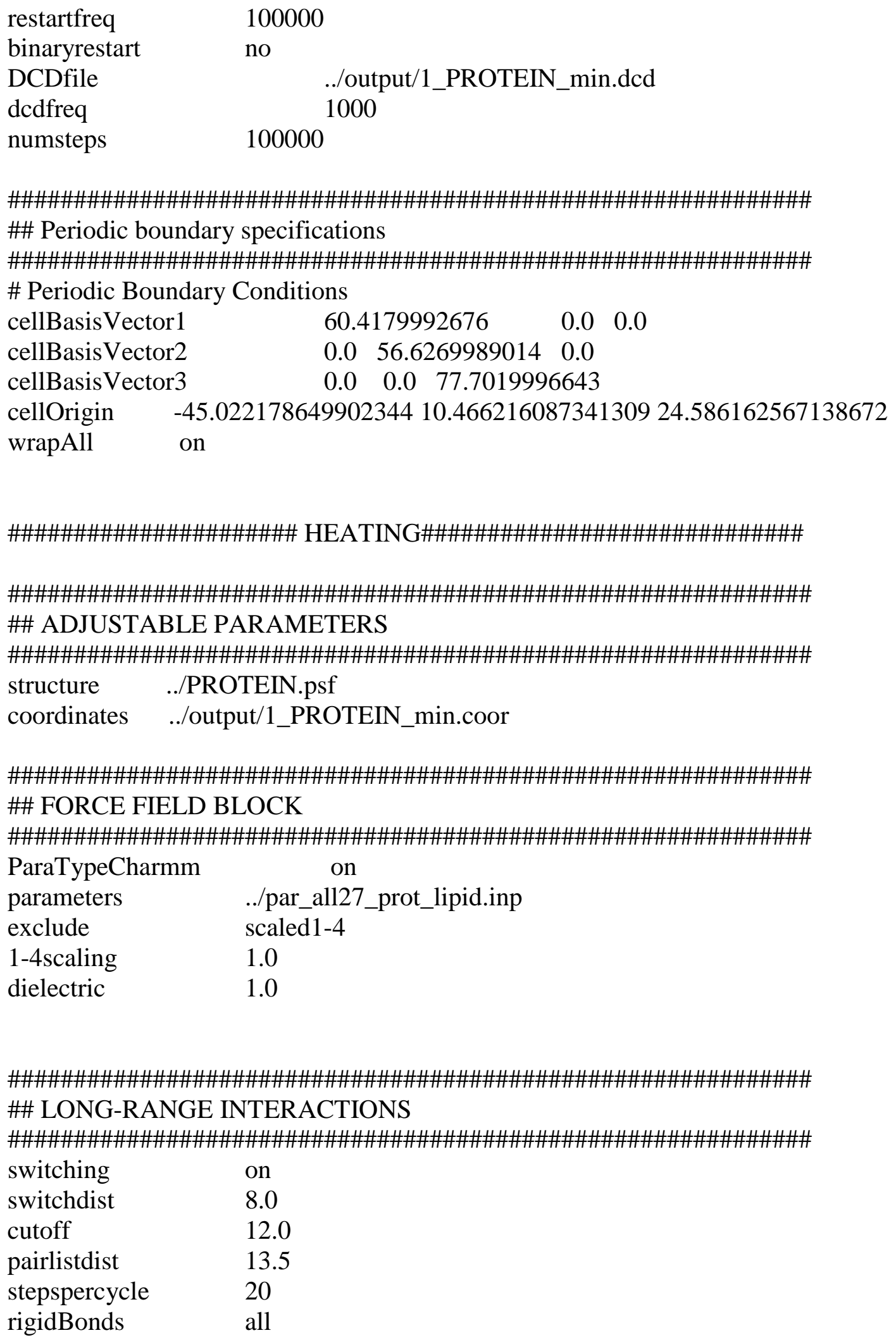




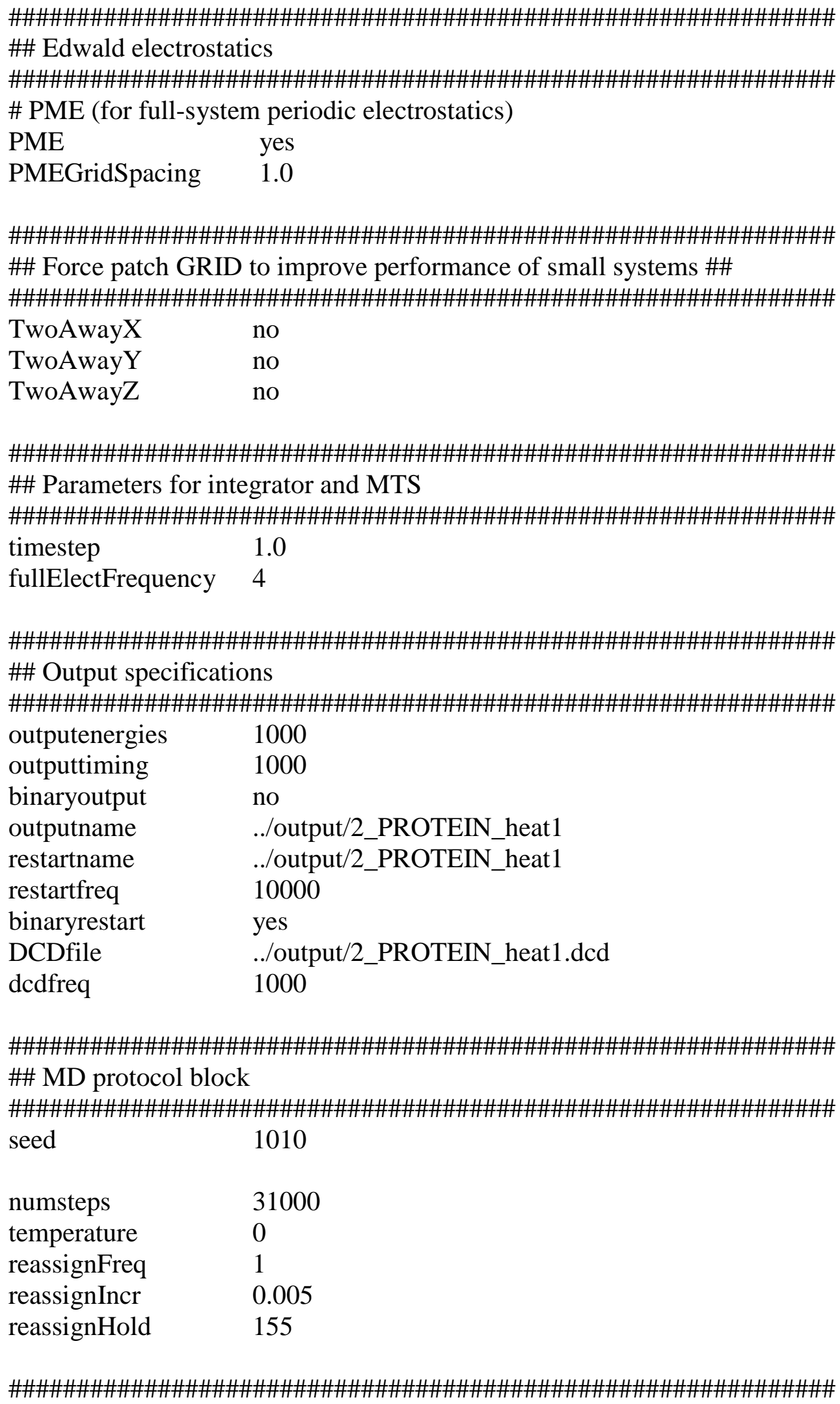




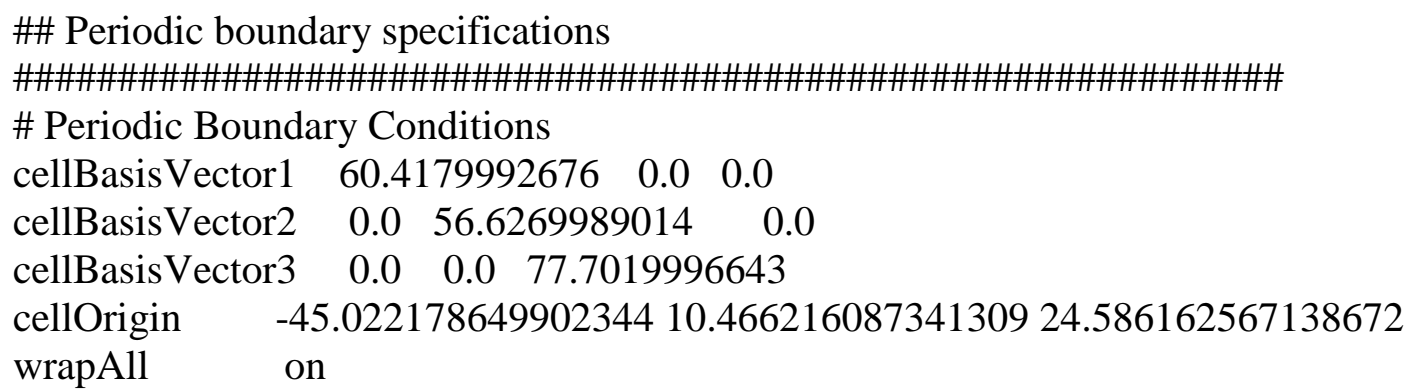




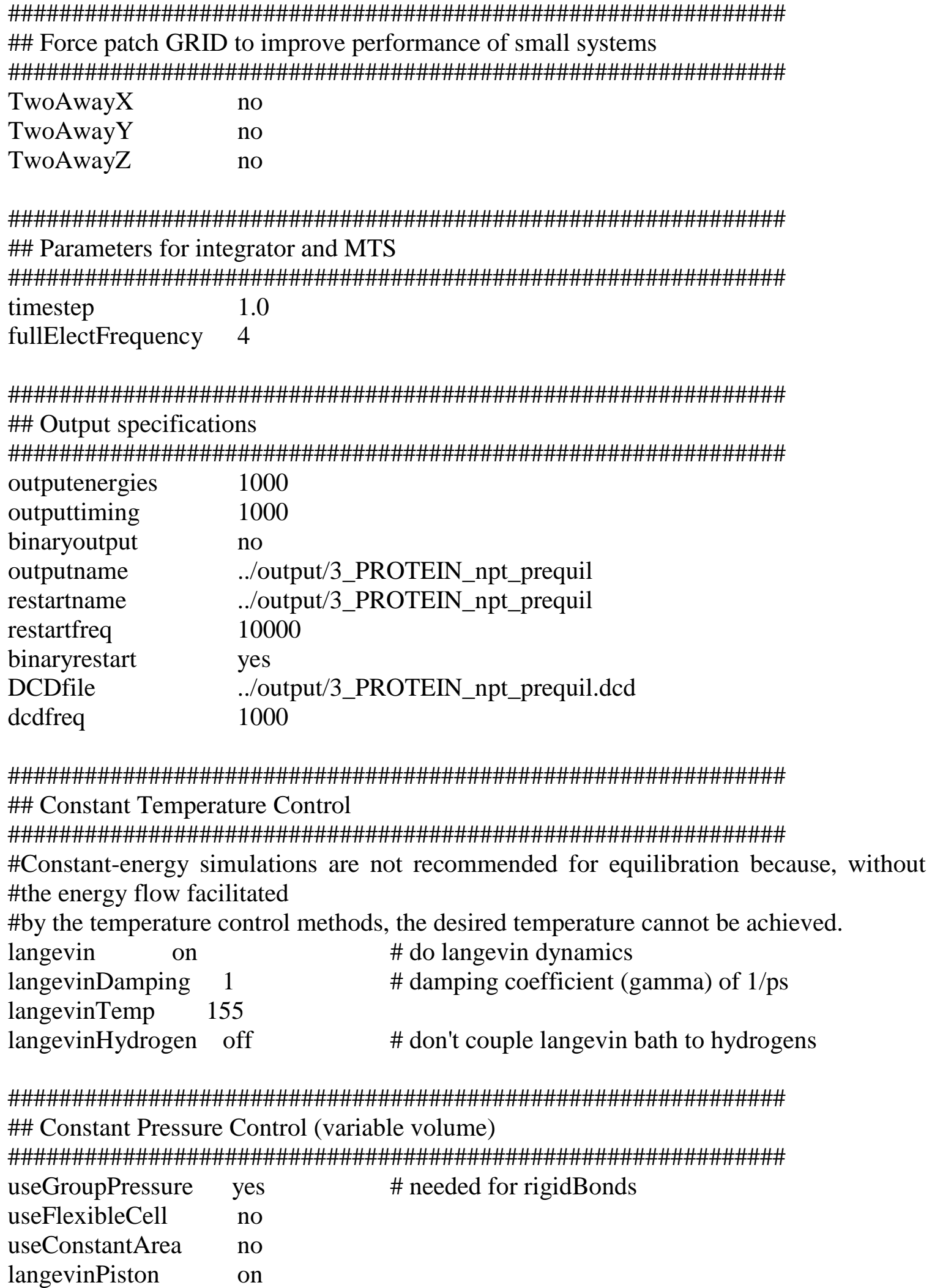




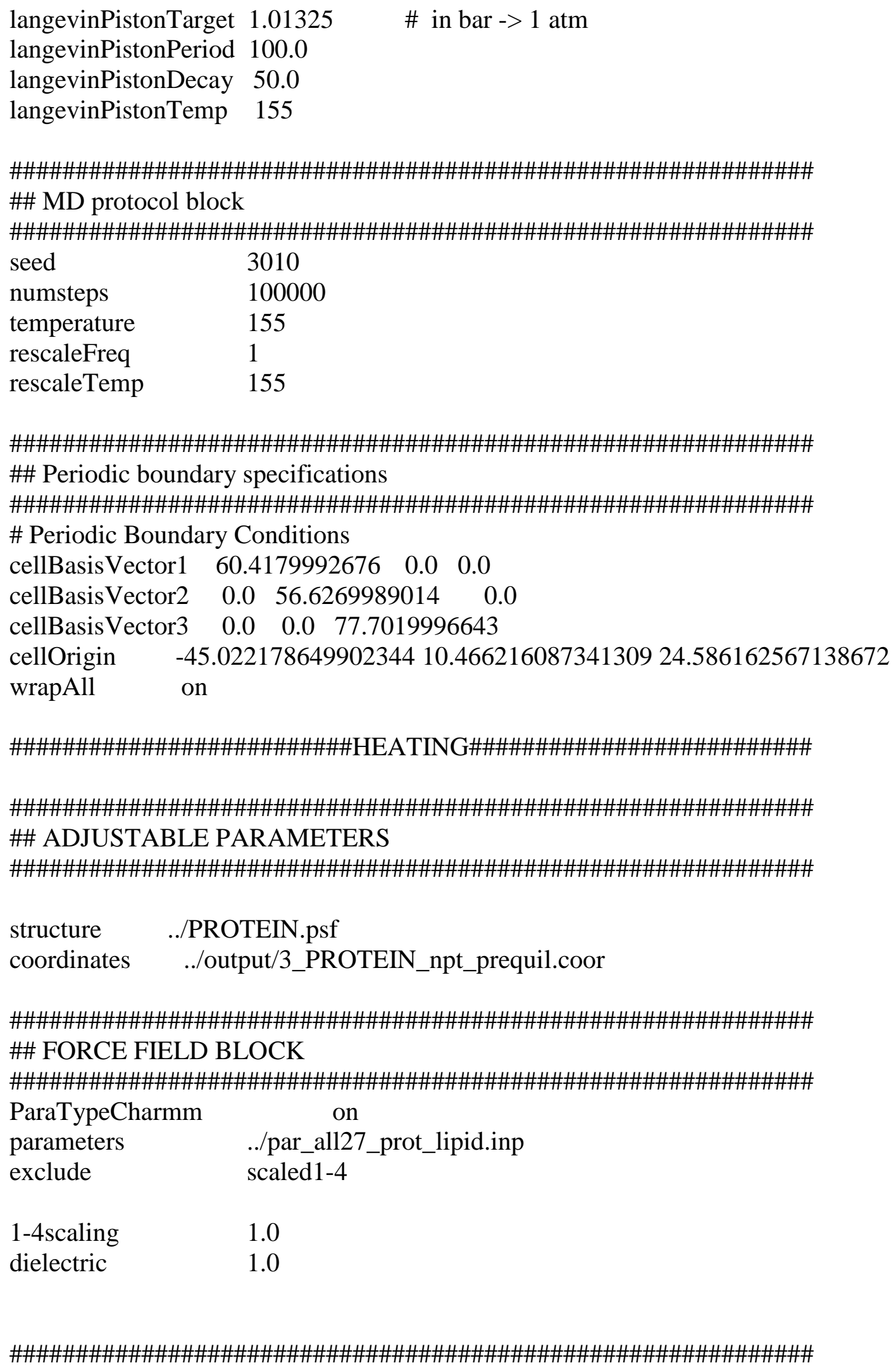




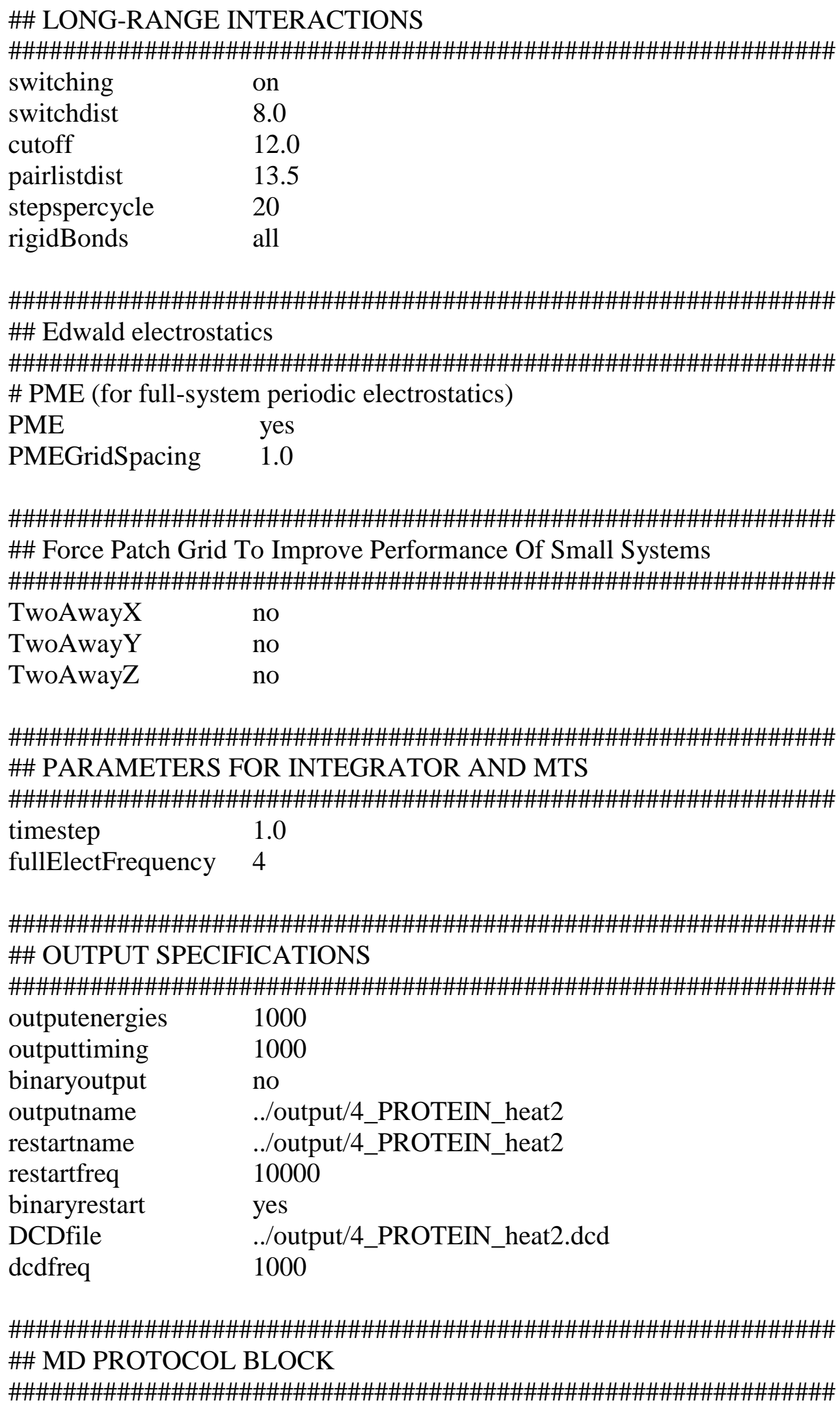




$\begin{array}{ll}\text { seed } & 1010 \\ \text { numsteps } & 31000 \\ \text { temperature } & 155 \\ \text { reassignFreq } & 1 \\ \text { reassignIncr } & 0.005 \\ \text { reassignHold } & 310\end{array}$

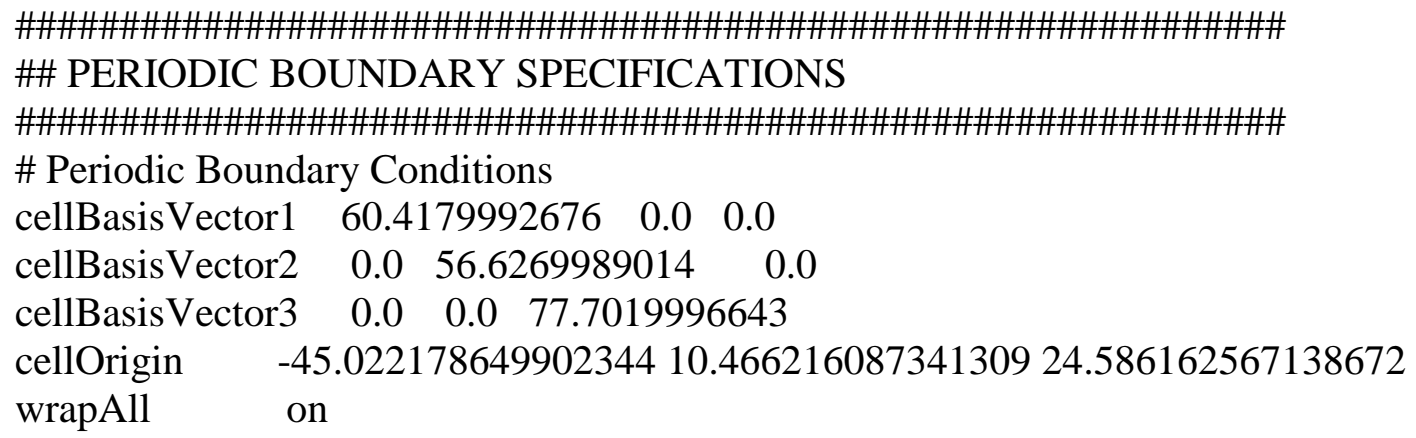




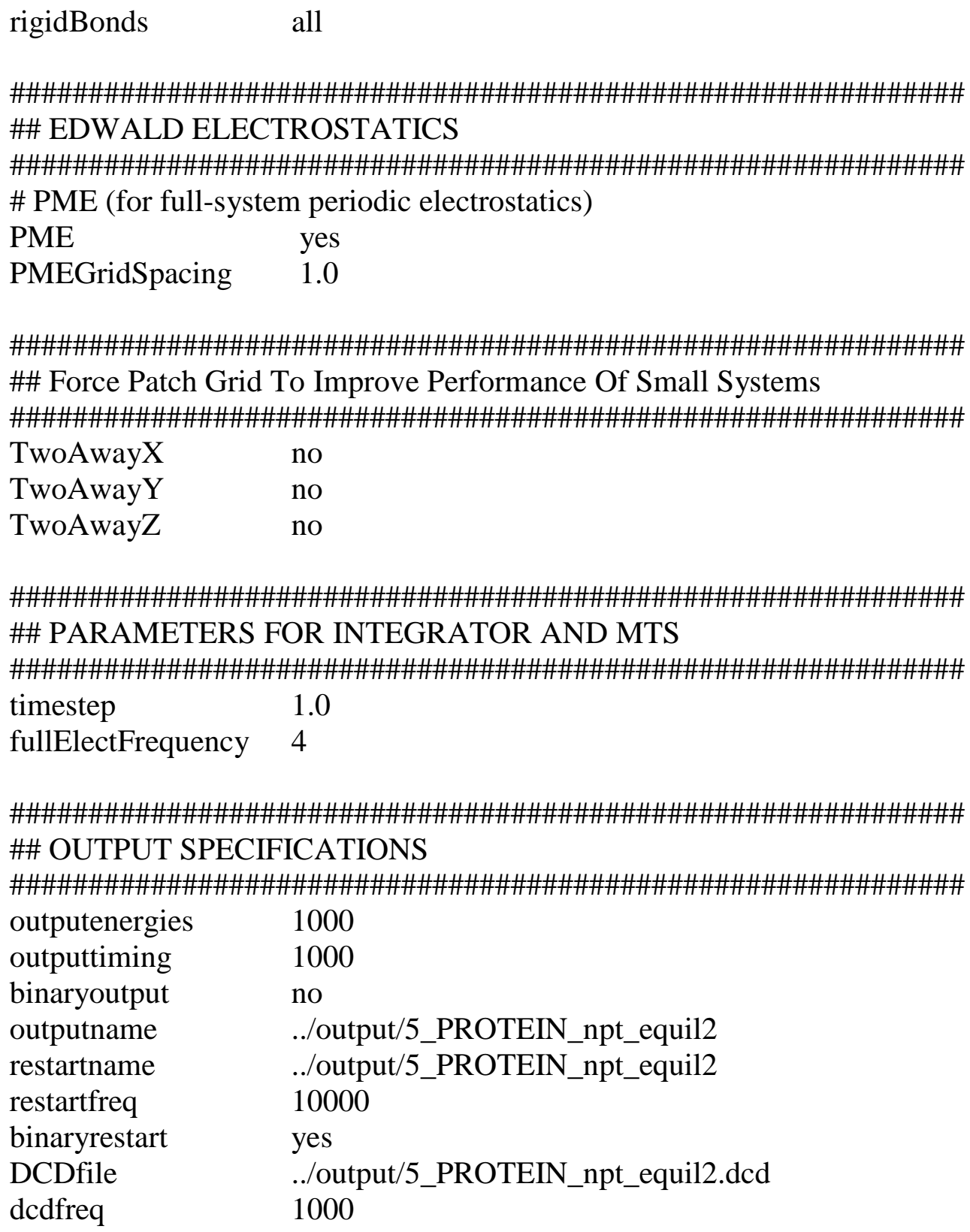




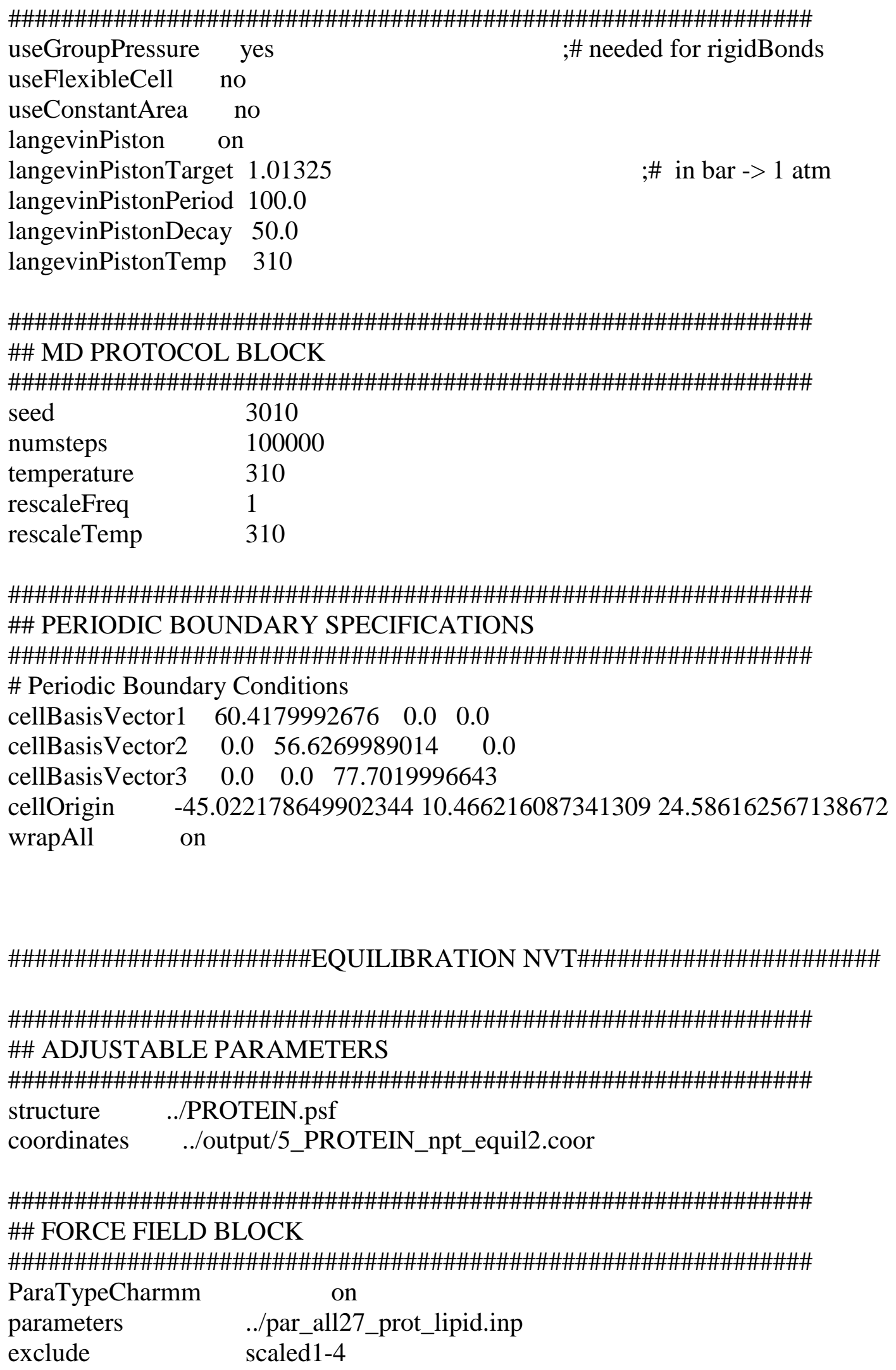




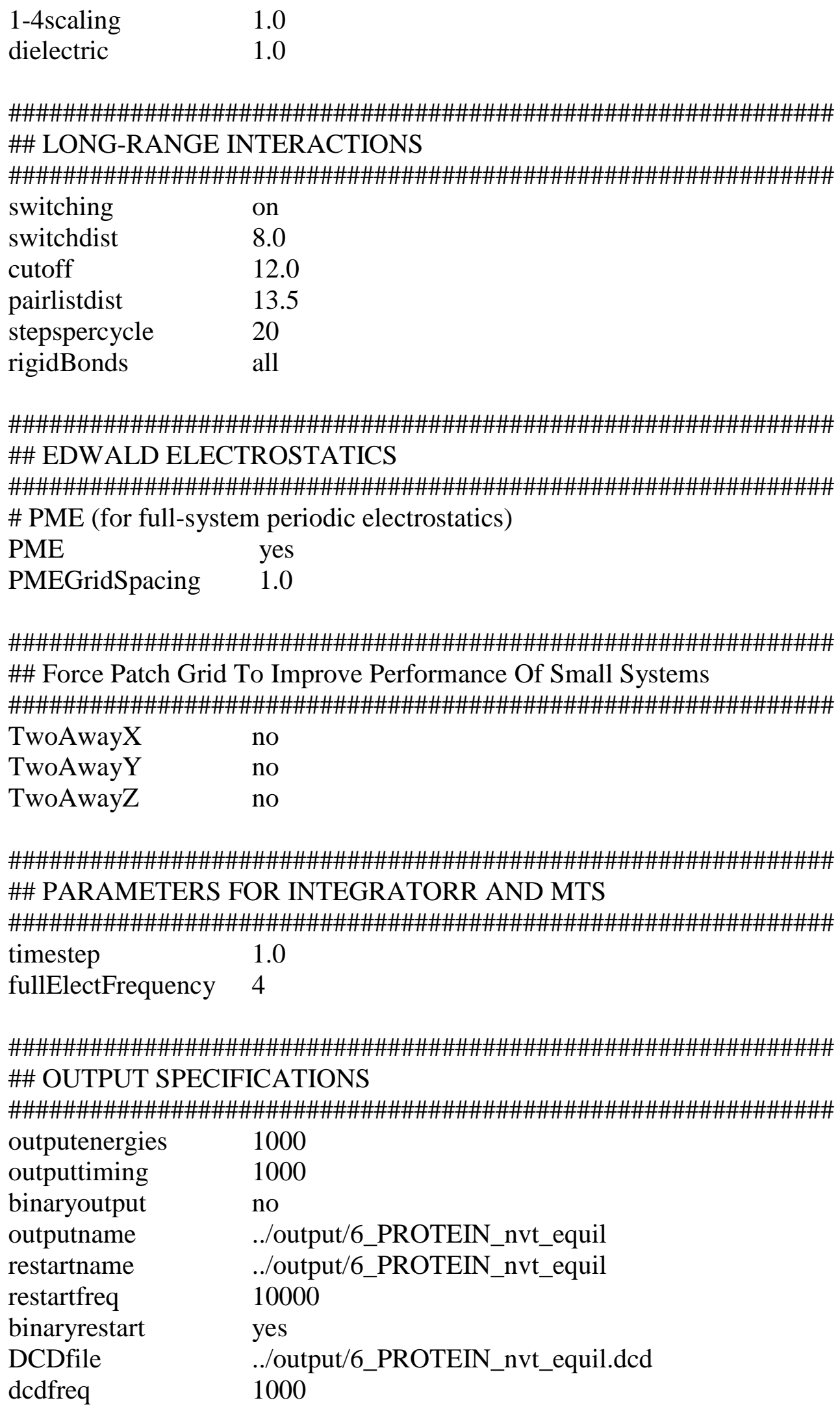




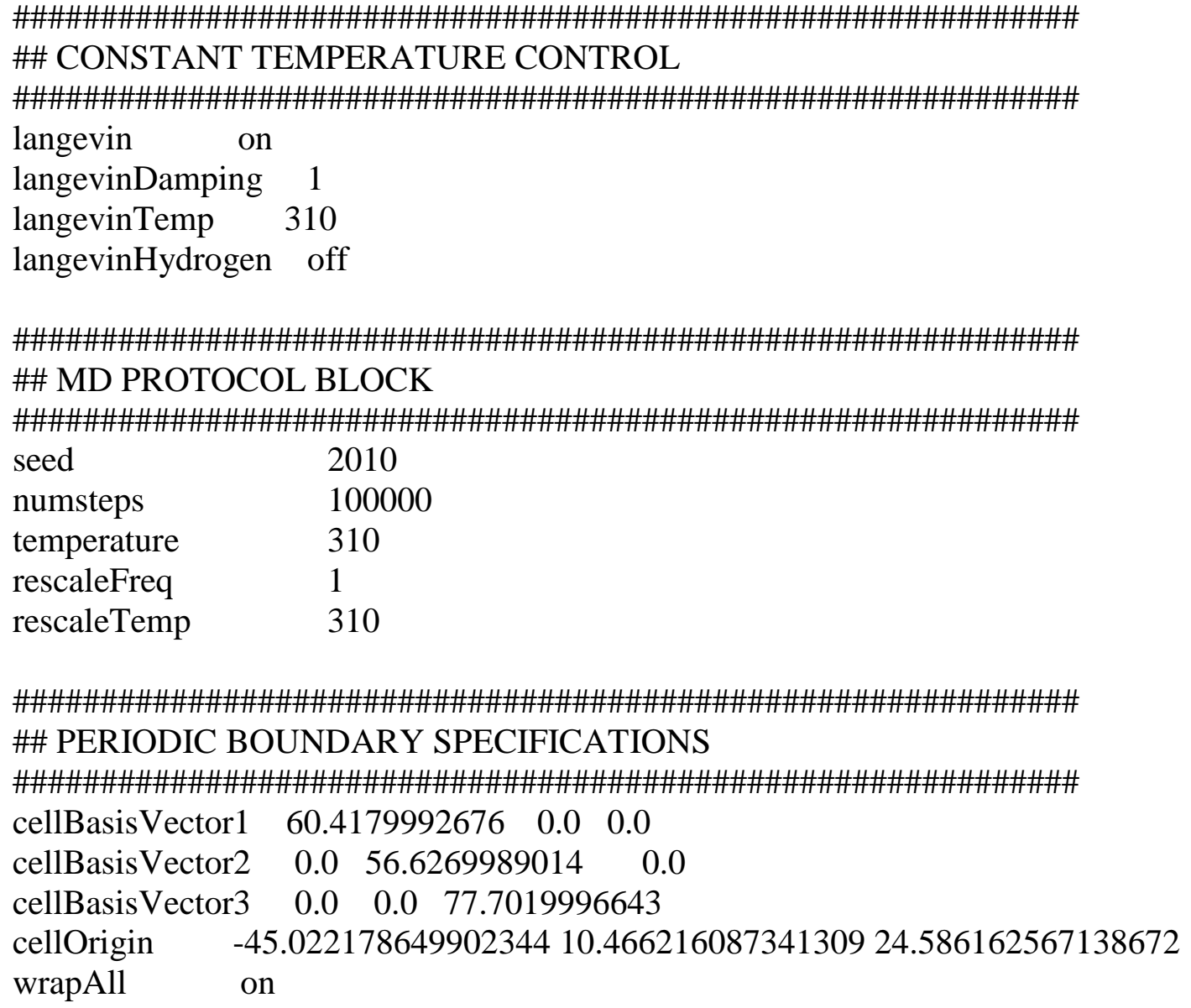




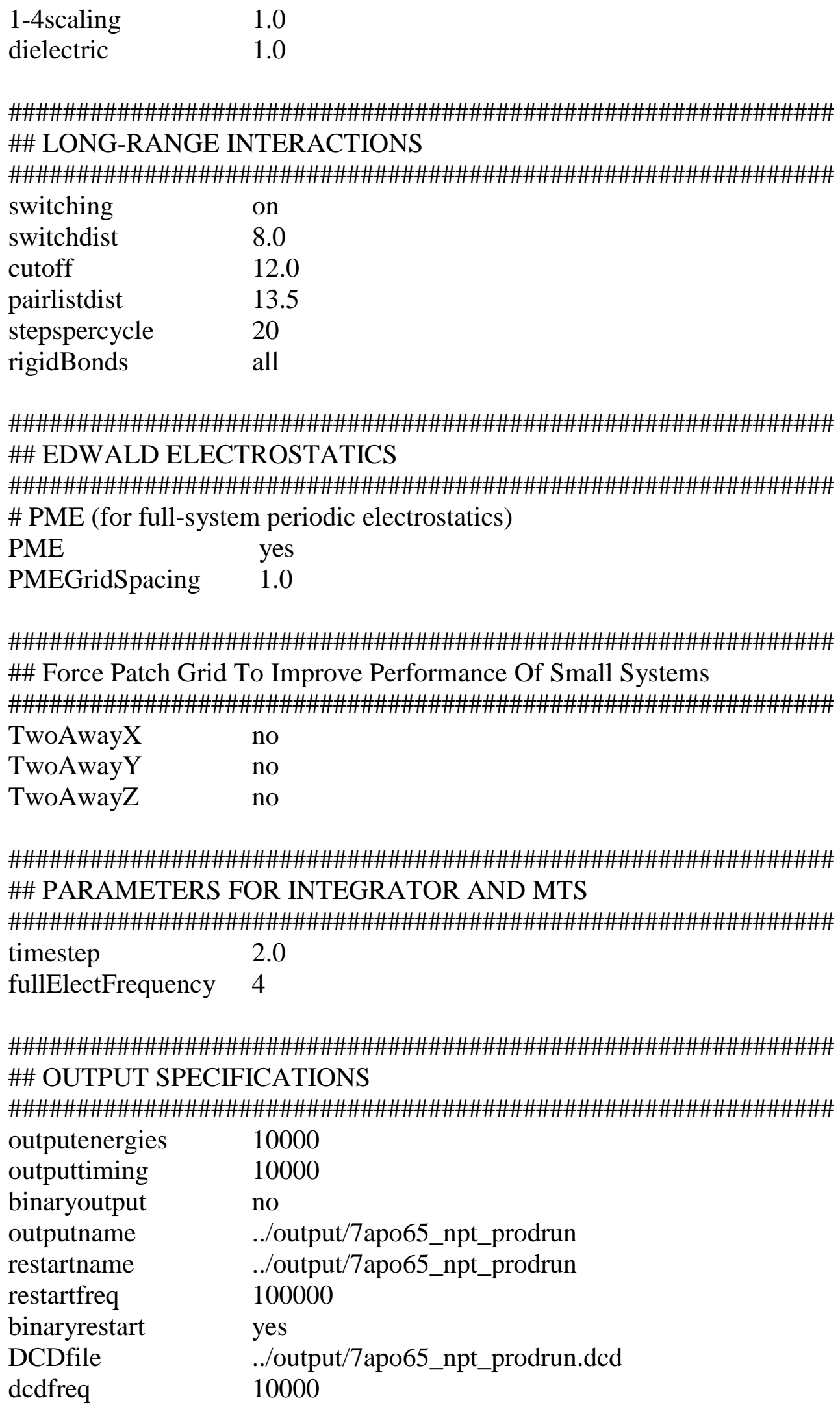




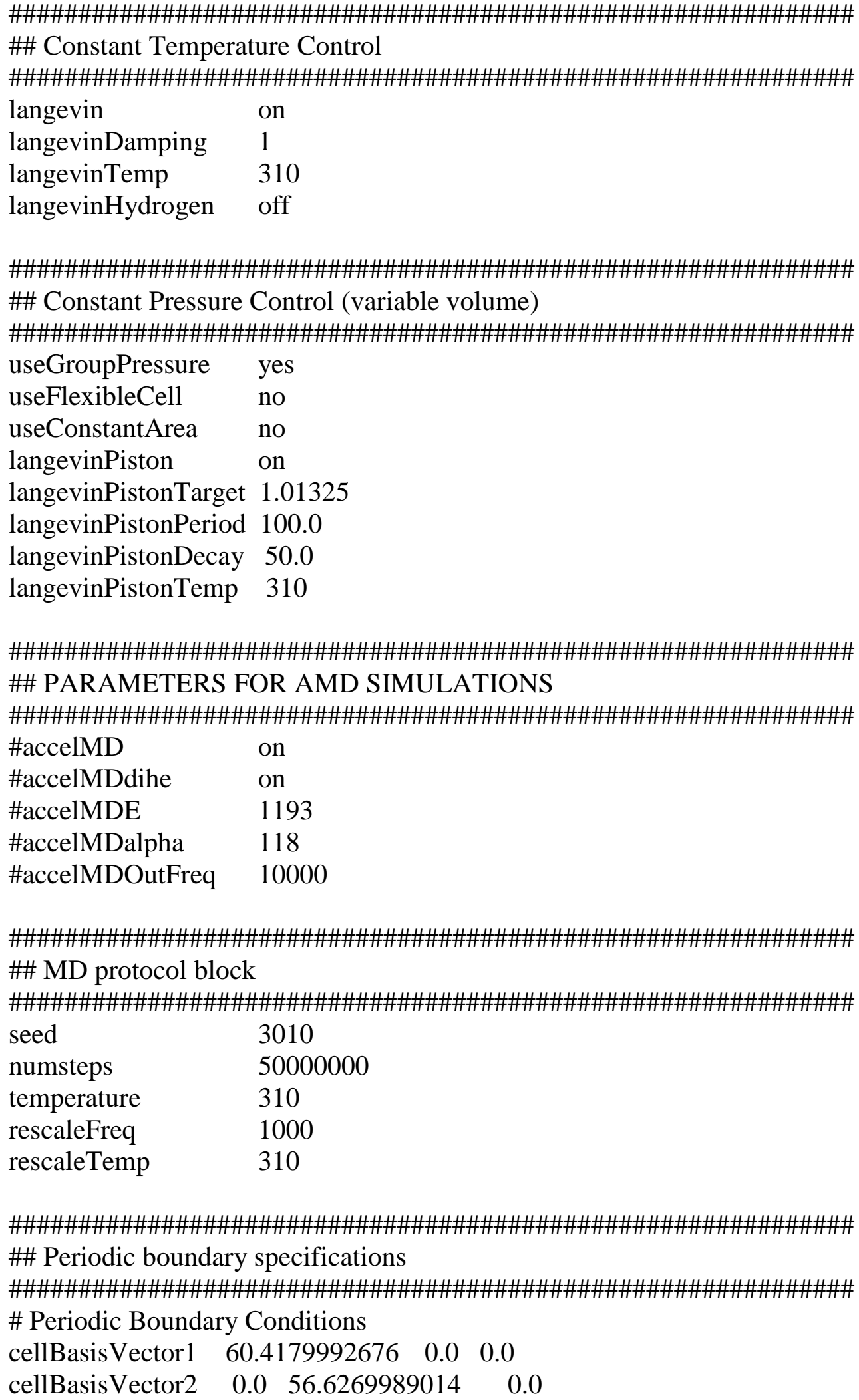


cellBasisVector3 $\quad 0.0 \quad 0.0 \quad 77.7019996643$

cellOrigin $\quad-45.02217864990234410 .46621608734130924 .586162567138672$

wrapAll

on 
VITA

WALTER GABRIEL GONZALEZ

EDUCATION AND SELECTED AWARDS

2010

Bachelors of Physics

Biophysics

Florida International University

Miami, Florida USA

$2011-2016$

Doctoral Candidate

Florida International University

Miami, Florida USA

ROLE OF AROMATIC RESIDUES IN MODULATING DYNAMICS AND ALLOSTERIC CONTROL IN DREAM/KCHIP3. (2015) LFD Workshop Award, $10^{\text {th }}$ LFD workshop University of California Irvin

ROLE OF DREAM PROTEIN DYNAMICS AND OLIGOMERIZATION ON LIGAND ASSOCIATION. (2015) Summer Research Award, NIGMS Biomedical Research Initiative.

PROTEIN-LIGAND INTERACTIONS AND ALLOSTERIC REGULATION OF DREAM PROTEIN ACTIVITY. (2015) MBRS RISE Graduate Fellowship, Florida International University.

CHARACTERIZATION OF THE PHOTOPHYSICAL, THERMODYNAMIC AND STRUCTURAL PROPERTIES OF THE TERBIUM(III)-KCHIP3 COMPLEX. (2015) Biophysical Society Travel Award, Biophysical Society.

MECHANISM OF CA ${ }^{2+}$ AND NS5806 INDUCED KCHIP3-KV4.3 ASSOCIATION. (2014) Summer Research Award, NIGMS Biomedical Research Initiative.

STRUCTURAL MODULATION OF KV4.3 T1 DOMAIN BY THE CALCIUM BINDING PROTEIN KCHIP3. (2013) Summer Research Award, NIGMS Biomedical Research Initiative.

$\mathrm{CA}^{2+}$ AND $\mathrm{MG}^{2+}$ INDUCED CONFORMATIONAL CHANGES IN DOWNSTREAM REGULATORY ANTAGONISTIC MODULATOR (DREAM) MEASURED BY EXTRINSIC HYDROPHOBIC PROBES. (2012) Graduate Research Award, FIU Dept. of Chemistry and Biochemistry. 
BINDING STUDY OF EXTRINSIC HYDROPHOBIC PROBES TO DREAM IN THE PRESENCE OF $\mathrm{MG}^{2+}$ AND $\mathrm{CA}^{2+}$. (2011) Graduate Poster Presentation $2^{\text {nd }}$ prize, ACS FAME.

\section{SELECTED PUBLICATIONS AND PRESENTATIONS}

Gonzalez Walter G; Ramos, Victoria; Diaz, Maurizio; and Miksovska, Jaroslava; (2016) Photophysics, thermodynamics and structural properties of the Terbium(III)-KChIP3 complex. (ACS Biochemistry in press).

Gonzalez, Walter G; Andres S. Arango; Miksovska, Jaroslava; (2015) Amphiphilic residues 29-44 of DREAM N-termini mediate the calmodulin:DREAM complex formation. Biochemistry; 54 (28), 4391-4403.

Gonzalez, Walter G; Pham, Khoa; Miksovska, Jaroslava; (2014) Modulation of the Voltage-gated Potassium Channel (Kv4. 3) and the Auxiliary Protein (KChIP3) Interactions by the Current Activator NS5806. Journal of Biological Chemistry; 289 (46): 32201-32213.

Gonzalez, Walter G; Miksovska, Jaroslava; (2014) Application of ANS fluorescent probes to identify hydrophobic sites on the surface of DREAM. Biochimica et Biophysica ActaProteins and Proteomics; 1844 (9): 1472-1480.

Gonzalez, Walter G; Miksovska, Jaroslava; (2014) Submillisecond Conformational Changes in Proteins Resolved by Photothermal Beam Deflection. Jove (Journal of Visualized Experiments); 8; e50969-e50969.

ROLE OF AROMATIC RESIDUES IN DYNAMIC NETWORKS IN DREAM/KCHIP3. (2016) Biophysical Society $60^{\text {th }}$ Annual Meeting; San Diego, California.

ALLOSTERIC NETWORK IN THE NEURONAL CALCIUM SENSOR PROTEIN DREAM. (2015) $13^{\text {h }}$ Raymond N. Castle Student Research Conference; Tampa, Florida.

INTERACTION-BASED ANALYSIS OF THE DREAM-CALMODULIN COMPLEX. (2015) South East Regional Meeting of the ACS; Nashville, Tennessee.

MODULATION OF THE KV4.3-KCHIP3 INTERACTIONS BY CA ${ }^{2+}$ AND NS5806. (2012) South East Regional Meeting of the ACS; Raleigh, North Carolina.

$\mathrm{CA}^{2+}$ AND $\mathrm{MG}^{2+}$ INDUCED CONFORMATIONAL CHANGES ON DREAM (DOWNSTREAM REGULATORY ANTAGONIST MODULATOR) MEASURED BY EXTRINSIC HYDROPHOBIC PROBES. (2011) South East Regional Meeting of the ACS; Richmond, Virginia. 